\title{
FUNCTION AND REGULATION OF CHEMORECEPTORS
}

EDITED BY: Dieter Wicher and Frédéric Marion-Poll PUBLISHED IN: Frontiers in Cellular Neuroscience

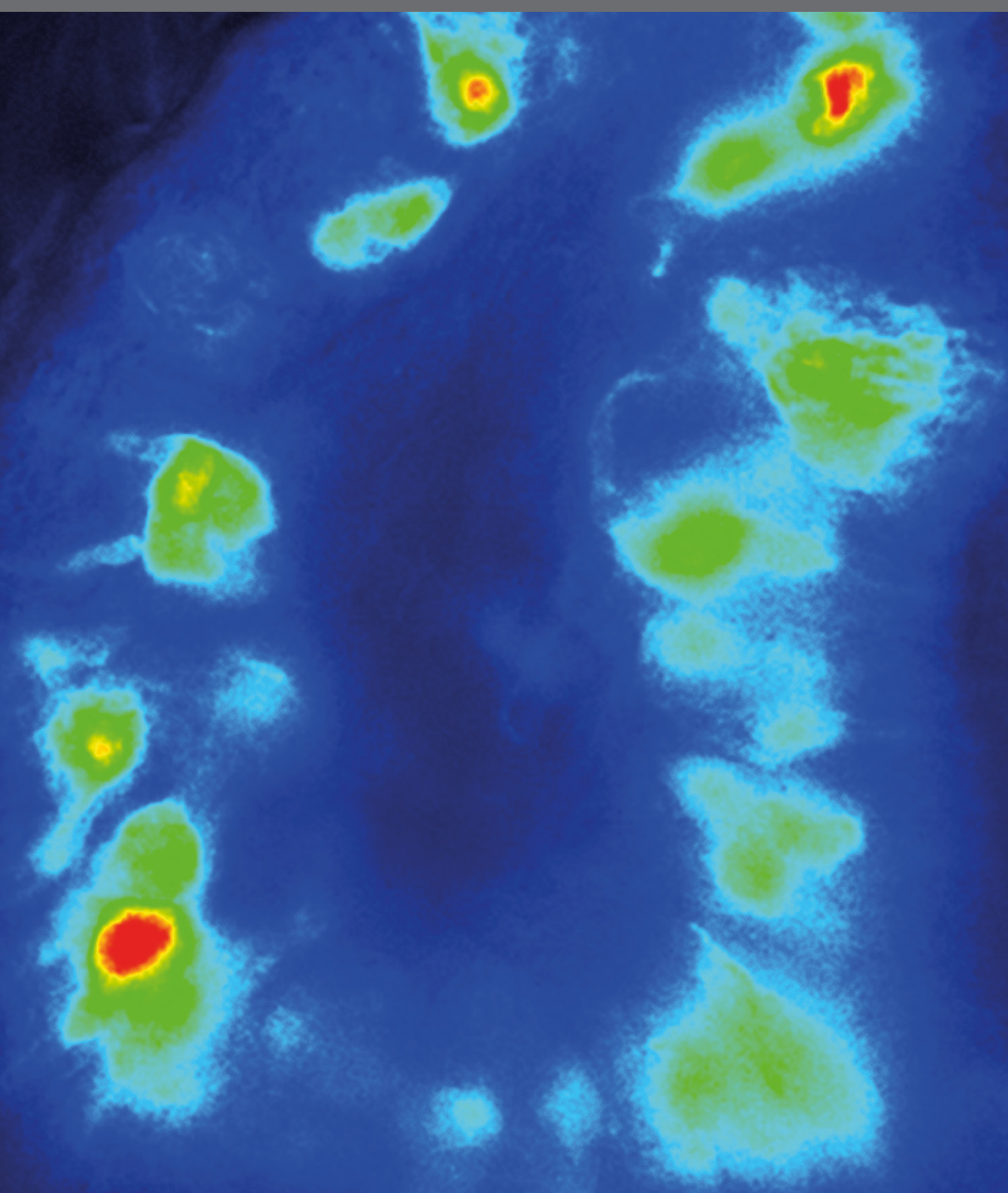

frontiers Research Topics 


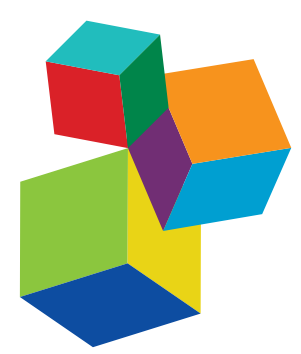

Frontiers Copyright Statement

C Copyright 2007-2019 Frontiers Media SA. All rights reserved.

All content included on this site, such as text, graphics, logos, button

icons, images, video/audio clips, downloads, data compilations and

software, is the property of or is

licensed to Frontiers Media SA

("Frontiers") or its licensees and/or subcontractors. The copyright in the text of individual articles is the property of their respective authors, subject to a license granted to Frontiers.

The compilation of articles constituting

this e-book, wherever published,

as well as the compilation of all other

content on this site, is the exclusive property of Frontiers. For the

conditions for downloading and

copying of e-books from Frontiers

website, please see the Terms for

Website Use. If purchasing Frontiers

e-books from other websites

or sources, the conditions of the website concerned apply.

Images and graphics not forming part

of user-contributed materials may not be downloaded or copied without permission

Individual articles may be downloaded and reproduced in accordance

with the principles of the CC-BY

licence subject to any copyright or other notices. They may not be re-sold as an e-book.

As author or other contributor you grant a CC-BY licence to others to reproduce your articles, including any

graphics and third-party materials supplied by you, in accordance with the Conditions for Website Use and subject to any copyright notices which you include in connection with your articles and materials.

All copyright, and all rights therein, are protected by national and international copyright laws.

The above represents a summary only.

For the full conditions see the Conditions for Authors and the Conditions for Website Use.

ISSN 1664-8714

ISBN 978-2-88945-748-9

DOI 10.3389/978-2-88945-748-9

\section{About Frontiers}

Frontiers is more than just an open-access publisher of scholarly articles: it is a pioneering approach to the world of academia, radically improving the way scholarly research is managed. The grand vision of Frontiers is a world where all people have an equal opportunity to seek, share and generate knowledge. Frontiers provides immediate and permanent online open access to all its publications, but this alone is not enough to realize our grand goals.

\section{Frontiers Journal Series}

The Frontiers Journal Series is a multi-tier and interdisciplinary set of open-access, online journals, promising a paradigm shift from the current review, selection and dissemination processes in academic publishing. All Frontiers journals are driven by researchers for researchers; therefore, they constitute a service to the scholarly community. At the same time, the Frontiers Journal Series operates on a revolutionary invention, the tiered publishing system, initially addressing specific communities of scholars, and gradually climbing up to broader public understanding, thus serving the interests of the lay society, too.

\section{Dedication to Quality}

Each Frontiers article is a landmark of the highest quality, thanks to genuinely collaborative interactions between authors and review editors, who include some of the world's best academicians. Research must be certified by peers before entering a stream of knowledge that may eventually reach the public - and shape society; therefore, Frontiers only applies the most rigorous and unbiased reviews.

Frontiers revolutionizes research publishing by freely delivering the most outstanding research, evaluated with no bias from both the academic and social point of view. By applying the most advanced information technologies, Frontiers is catapulting scholarly publishing into a new generation.

\section{What are Frontiers Research Topics?}

Frontiers Research Topics are very popular trademarks of the Frontiers Journals Series: they are collections of at least ten articles, all centered on a particular subject. With their unique mix of varied contributions from Original Research to Review Articles, Frontiers Research Topics unify the most influential researchers, the latest key findings and historical advances in a hot research area! Find out more on how to host your own Frontiers Research Topic or contribute to one as an author by contacting the Frontiers Editorial Office: researchtopics@frontiersin.org 


\section{FUNCTION AND REGULATION OF CHEMORECEPTORS}

Topic Editors:

Dieter Wicher, Max Planck Institute for Chemical Ecology, Germany Frédéric Marion-Poll, AgroParisTech, France

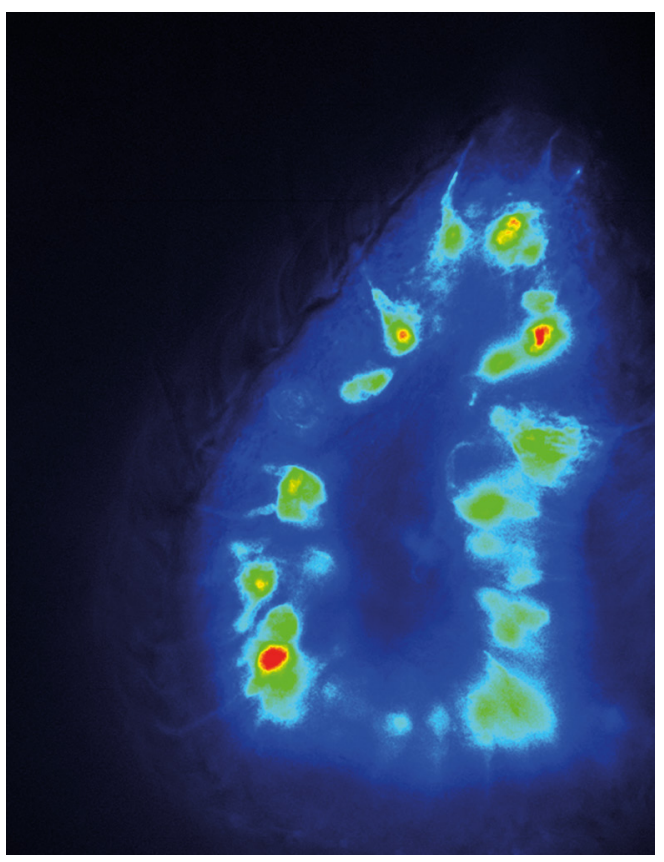

Olfactory sensory neurons in an open antenna preparation of the vinegar fly Drosophila melanogaster (cf. article 186). The neurons express the calcium-sensitive fluorescent dye GCaMP6f which was excited by $475 \mathrm{~nm}$ light exposure for $50 \mathrm{~ms}$.

Image: Kalpana Jain.

To perceive environmental chemical compounds and to convert these external signals into an intracellular message might be the oldest way for a living being to get information from the outside world. Chemoreceptors are proteins or protein complexes that detect molecules from the outside world either at distance (olfaction) or at close range (gustation). Chemoreceptors can operate as ionotropic or as metabotropic receptors. Ionotropic receptors form ion channels that are activated via ligand binding. Activation of a metabotropic receptor initiates an intracellular signaling cascade that could include a change of enzymatic activity, production of second messenger or activation of ion channels. The receptor performance has to be fine-tuned according to the actual physiological requirements and the presentation of the chemical signal. This Research Topic collects reports and reviews on structure and function of chemoreceptors in the animal kingdom, and how these receptors are regulated.

Citation: Wicher, D., Marion-Poll, F., eds. (2019). Function and Regulation of Chemoreceptors. Lausanne: Frontiers Media. doi: 10.3389/978-2-88945-748-9 


\section{Table of Contents}

05 Editorial: Function and Regulation of Chemoreceptors

Dieter Wicher and Frédéric Marion-Poll

08 The Two Main Olfactory Receptor Families in Drosophila, ORs and IRs: A Comparative Approach

Caroline Gomez-Diaz, Fernando Martin, Jose Manuel Garcia-Fernandez and Esther Alcorta

23 Tuning Insect Odorant Receptors

Dieter Wicher

30 Insect Pheromone Receptors - Key Elements in Sensing Intraspecific Chemical Signals

Jörg Fleischer and Jürgen Krieger

44 Current Source Density Analysis of Electroantennogram Recordings: A Tool for Mapping the Olfactory Response in an Insect Antenna Vincent E. J. M. Jacob

63 Using Pox-Neuro (Poxn) Mutants in Drosophila Gustation Research: A Double-Edged Sword

Yu-Chieh David Chen, Scarlet Jinhong Park, William W. Ja and Anupama Dahanukar

70 The Drosophila melanogaster $\mathrm{Na}^{+} / \mathrm{Ca}^{2+}$ Exchanger Calx Controls the $\mathrm{Ca}^{2+}$ Level in Olfactory Sensory Neurons at Rest and After Odorant Receptor Activation

Lorena Halty-deLeon, Bill S. Hansson and Dieter Wicher

79 The Diacylglycerol Analogues OAG and DOG Differentially Affect Primary Events of Pheromone Transduction in the Hawkmoth Manduca sexta in a Zeitgebertime-Dependent Manner Apparently Targeting TRP Channels Petra Gawalek and Monika Stengl

99 ATP and Odor Mixture Activate TRPM5-Expressing Microvillous Cells and Potentially Induce Acetylcholine Release to Enhance Supporting Cell Endocytosis in Mouse Main Olfactory Epithelium

Ziying Fu, Tatsuya Ogura, Wangmei Luo and Weihong Lin

$115 P / Q$ Type Calcium Channel Cav2.1 Defines a Unique Subset of Glomeruli in the Mouse Olfactory Bulb

Martina Pyrski, Mahbuba Tusty, Eugenia Eckstein, Livio Oboti,

Diego J. Rodriguez-Gil, Charles A. Greer and Frank Zufall

131 Synaptic Spinules in the Olfactory Circuit of Drosophila melanogaster Lydia Gruber, Jürgen Rybak, Bill S. Hansson and Rafael Cantera

138 Putative Neural Network Within an Olfactory Sensory Unit for Nestmate and Non-nestmate Discrimination in the Japanese Carpenter Ant: The Ultrastructures and Mathematical Simulation

Yusuke Takeichi, Tatsuya Uebi, Naoyuki Miyazaki, Kazuyoshi Murata, Kouji Yasuyama, Kanako Inoue, Toshinobu Suzaki, Hideo Kubo, Naoko Kajimura, Jo Takano, Toshiaki Omori, Ryoichi Yoshimura, Yasuhisa Endo, Masaru K. Hojo, Eichi Takaya, Satoshi Kurihara, Kenta Tatsuta, Koichi Ozaki and Mamiko Ozaki 
153 The Consequences of Preterm Birth and Chorioamnionitis on Brainstem Respiratory Centres: Implications for Neurochemical Development and Altered Functions by Inflammation and Prostaglandins

Vanesa Stojanovska, Suzanne L. Miller, Stuart B. Hooper and Graeme R. Polglase

168 Changes in the Peripheral Chemosensory System Drive Adaptive Shifts in Food Preferences in Insects

Ayako Wada-Katsumata, Hugh M. Robertson, Jules Silverman and Coby Schal 


\title{
Editorial: Function and Regulation of Chemoreceptors
}

\author{
Dieter Wicher ${ }^{1 *}$ and Frédéric Marion-Poll ${ }^{2,3}$ \\ ${ }^{1}$ Department of Evolutionary Neuroethology, Max Planck Institute for Chemical Ecology, Jena, Germany, ${ }^{2}$ AgroParisTech, \\ Université Paris-Saclay, Paris, France, ${ }^{3}$ UMR Evolution, Génomes, Comportement, Ecologie, CNRS, IRD, Univ Paris-Sud, \\ Université Paris-Saclay, Gif-sur-Yvette, France
}

Keywords: chemoreceptor, olfactory receptor, gustatory receptor, odorant receptor, ionotropic receptor, signal transduction

\section{Editorial on the Research Topic}

\section{Function and Regulation of Chemoreceptors}

To perceive environmental chemical compounds and to convert these external signals into an intracellular message might be the oldest way for a living being-from unicellular organisms to mammals - to get information from the outside world. The chemoreceptors are the first to receive an environmental signal and to convert it into message that can be interpreted and further processed in the organism. They are usually situated at the interface between organism and environment, in case multicellular organisms have developed specialized sensory organs, the receptors are localized at the dendrites of the chemosensory neurons. Chemoreceptors are proteins or protein complexes that bind molecules detected at distance and generally at low concentration (olfaction) or molecules detected at proximity and often at higher concentrations (gustation), respectively volatile and not volatile for organisms living in the aerial phase. Chemosensory systems are often highly sophisticated and allow the detection of a few molecules. The resolutions limits set by the noise of the chemosignals and quantified by the Berg-Purcel limit (Kaizu et al., 2014). According to this estimation, the resolution can be enhanced by increasing the receptive surface and expanding the detection time. Male moths, for example, have expanded antennae allowing them to detect extremely low pheromone concentrations (Hansson and Stensmyr, 2011).

Chemoreceptors may operate on ionotropic or on metabotropic basis. Ionotropic receptors are ion channels activated upon ligand binding. Activation of a metabotropic receptor affects intracellular chemical signaling, e.g., by stimulation or attenuation of enzymatic activity. This initiates an intracellular signaling cascade and results, for example, in the production of second messengers or to the activation of ion channels. During evolution, i.e., from bacteria to mammals, there was no preference for a certain type of chemoreceptor function (Wicher and Grosse-Wilde, 2017).

The present research topic deals with the function and regulation of chemoreception in the animal kingdom. The first part contains contributions dealing with structure and function of chemoreceptors and how the operation of receptors is affected by intracellular signaling within the chemosensory neurons. The second part contains papers on interneuronal communication and output control of sensory neurons including their synaptic transmission. Finally, the third part presents examples how developmental or environmental challenges affect the chemosensory apparatus of an organism.

Gomez-Diaz et al. present an overview on olfactory receptors in the model insect Drosophila. These receptors are grouped into two main families, the odorant receptors (ORs) and the ionotropic receptors (IRs). The authors compare structure, function, distribution and evolutionary history for members of these families. The sensitivity of ORs can be up-regulated by repeated odor stimulation at sub-threshold concentration (Getahun et al., 2013). The current view on function and regulation 
of insect ORs with special attention on the mechanisms involved in receptor sensitization is presented by Wicher.

A subtype of odorant receptors serving for intraspecific communication are the pheromone receptors (PRs). Fleischer and Krieger review the architecture of the pheromone detecting system, the expression of PRs, the diversity of ligands, and the transduction of the chemosignal.

When writing this Editorial, two very important studies on insect ORs were published. One, focusing on the evolution of this family (Brand et al., 2018) demonstrated that these ORs are present in ancestors of insect but do not occur in non-insect hexapodes. The other one reports the cryo-electron microscopy structure the insect odorant coreceptor Orco (Butterwick et al., 2018). While ORs in many animals form G protein coupled receptors, the insect OR proteins have a similar 7 -transmembrane structure but form ion channels, Butterwick et al. found that Orco channels form tetramers like members of the voltage-gated channel superfamily.

The contribution by Jacob develops a tool to map olfactory responses in the insect antenna. On basis of electroantennogram recordings the author performed a current source analysis to detect the origins of olfactory responses. The paper investigates under which conditions this technique can be applied in insect antenna. As for gustation, Chen et al. revisit a gustation mutant commonly used to evaluate the role of taste in different behaviors. This mutant, poxn, exhibits no external taste sensilla but Chen et al. demonstrate that while indeed external taste sensilla are inactivated, flies are not completely taste-blind and can still use their pharyngeal taste receptors to analyze foods.

A hallmark of OR function is the tuning of their odorant sensitivity, a process which involves intracellular $\mathrm{Ca}^{2+}$ signaling (Wicher, this topic). On the background of olfactory response regulation the study by Halty-deLeon et al. was aimed at testing the role of the $\mathrm{Na}^{+} / \mathrm{Ca}^{2+}$ exchanger CALX in $\mathrm{Ca}^{2+}$ management in Drosophila olfactory sensory neurons (OSNs).

The contribution by Gawalek and Stengl investigates the results of intracellular signaling initiated by hawkmoth pheromone receptor activation. Earlier studies have indicated that Manduca PRs operate as metabotropic receptors activating the $\mathrm{G}_{\mathrm{q}}$ dependent phospholipase $\mathrm{C}$ pathway (Stengl, 2010). In the present paper the authors suggest that downstream targets of pheromone signaling belong to the TRP channel family. Furthermore, depending on the activity state of the moths the authors observed that pheromone signaling targeted different second messenger-dependent ion channels.

$\mathrm{Fu}$ et al. investigate a mechanism important for the homeostasis of the neuronal network mediating odor responses and olfactory guided behavior in mice. The authors of this study show that ATP signaling and odor mixtures activate microvillous cells in the main olfactory epithelium which subsequently release acetylcholine. This release enhances the endocytotic activity of supporting cells required for the homeostasis of the multicellular network in the olfactory epithelium.

Mammalian olfactory sensory neurons send their axons into the first information processing center, the main olfactory bulb which is composed of glomeruli. In one glomerulus the input from sensory neurons expressing one receptor type is processed via synaptic contacts. To initiate synaptic transmission, the activation of voltage-gated $\mathrm{Ca}^{2+}$ channels is required to provide calcium ions for the fusion of transmitter-filled vesicles with the presynaptic plasma membrane. While in mice most of these $\mathrm{Ca}^{2+}$ channels belong to the N-type (CaV2.2), the study by Pyrski et al. describes a previously unknown olfactory subset of glomeruli expressing P/Q-type channels (CaV2.1).

The contribution presented by Gruber et al. is also related to synaptic transmission. Glomeruli are structures in the antennal lobe, the first olfactory processing center in insects, getting input from OSNs. In a certain glomerulus in the Drosophila the authors describe synaptic spinules. These are protrusions into a synaptic bouton of an OSN sent from another neuron, and they are assumed to release material into the host neuron. This process, triggered by synaptic activity is discussed as candidate mechanism for synapse remodeling and synaptic plasticity.

In their communication, Takeichi et al. study in detail the 3D disposition of olfactory dendrites in Japanese carpenter ants, which belong to basiconic sensilla used to discriminate the complex cuticular hydrocarbon profiles of different colonies. They show that these sensilla house up to 100 neurons with dendrites in very close apposition and structures which suggest the presence of gap junctions. In case future physiological studies confirm the function these structures as proposed in the mathematical model by the authors, interactions among peripheral sensory neurons might play role in pre-processing chemosensory information before it is transmitted to the central nervous system.

A complex example illustrating that a vulnerable state of an organism affects the performance of a chemosensory system is presented by Stojanovska et al. They review the problems arising for the respiratory control in preterm infants. The authors discuss the function of brainstem respiratory centers and how their function is affected due to preterm birth or under pathological conditions such as chorioamnionitis.

An ecologically important question is how an organism responds to changing conditions in the environment and what are the consequences for the chemosensory apparatus. In the German cockroach, Wada-Katsumata et al. exerted a strong selection pressure by feeding the animals with glucosecontaining toxic baits. Part of the animals displayed an adaptive chemosensory shift from feeding stimulation by glucose to an aversive behavior. This represents an impressive example for a fast process of evolution.

This research topic covers a wide range of complexity of chemosensory information processing at different levels. It deals with chemoreceptor function and regulation at molecular and cellular level, it covers intercellular information processing and it gives examples for the interaction of the chemosensory apparatus and the whole organism.

\section{AUTHOR CONTRIBUTIONS}

All authors listed have made a substantial, direct and intellectual contribution to the work, and approved it for publication. 


\section{REFERENCES}

Brand, P., Robertson, H. M., Lin, W., Pothula, R., Klingeman, W. E., Jurat-Fuentes, J. L., et al. (2018). The origin of the odorant receptor gene family in insects. Elife 7:38340. doi: 10.7554/eLife.38340

Butterwick, J. A., Del Marmol, J., Kim, K. H., Kahlson, M. A., Rogow, J. A., Walz, T., et al. (2018). Cryo-EM structure of the insect olfactory receptor Orco. Nature 560, 447-452. doi: 10.1038/s41586-018-0420-8

Getahun, M. N., Olsson, S. B., Lavista-Llanos, S., Hansson, B. S., and Wicher, D. (2013). Insect odorant response sensitivity is tuned by metabotropically autoregulated olfactory receptors. PLoS ONE 8:e58889. doi: 10.1371/journal.pone.0058889

Hansson, B. S., and Stensmyr, M. C. (2011). Evolution of insect olfaction. Neuron 72, 698-711. doi: 10.1016/j.neuron.2011.11.003

Kaizu, K., De Ronde, W., Paijmans, J., Takahashi, K., Tostevin, F., and Ten Wolde, P. R. (2014). The Berg-Purcell limit revisited. Biophys. J. 106, 976-985. doi: 10.1016/j.bpj.2013.12.030
Stengl, M. (2010). Pheromone transduction in moths. Front. Cell Neurosci. 4:133. doi: 10.3389/fncel.2010.00133

Wicher, D., and Grosse-Wilde, E. (2017). "Chemoreceptors in evolution," in Evolution of Nervous Systems, 2nd Edn., ed J. Kaas (Oxford: Elsevier), 245-255. doi: 10.1016/B978-0-12-804042-3.00021-X

Conflict of Interest Statement: The authors declare that the research was conducted in the absence of any commercial or financial relationships that could be construed as a potential conflict of interest.

Copyright (๑) 2018 Wicher and Marion-Poll. This is an open-access article distributed under the terms of the Creative Commons Attribution License (CC BY). The use, distribution or reproduction in other forums is permitted, provided the original author(s) and the copyright owner(s) are credited and that the original publication in this journal is cited, in accordance with accepted academic practice. No use, distribution or reproduction is permitted which does not comply with these terms. 


\section{OPEN ACCESS}

Edited by:

Dieter Wicher,

Max-Planck-Institut für Chemische

Ökologie, Germany

Reviewed by:

Jürgen Krieger,

Martin Luther University of Halle-Wittenberg, Germany

Monika Stengl,

University of Kassel, Germany

*Correspondence:

Esther Alcorta

ealcorta@uniovi.es

tThese authors have contributed equally to this work

Received: 25 May 2018 Accepted: 23 July 2018 Published: 30 August 2018

Citation:

Gomez-Diaz C, Martin F, Garcia-Fernandez JM and Alcorta E (2018) The Two Main Olfactory Receptor Families in Drosophila, ORs and IRs: A Comparative Approach.

Front. Cell. Neurosci. 12:253. doi: $10.3389 /$ fncel.2018.00253

\section{The Two Main Olfactory Receptor Families in Drosophila, ORs and IRs: A Comparative Approach}

\author{
Carolina Gomez-Diaz ${ }^{1 \dagger}$, Fernando Martin ${ }^{1 \dagger}$, Jose Manuel Garcia-Fernandez ${ }^{2}$ \\ and Esther Alcorta ${ }^{1 *}$
}

${ }^{1}$ Department of Functional Biology, Faculty of Medicine, University of Oviedo, Oviedo, Spain, ${ }^{2}$ Department of Cellular Biology, Faculty of Medicine, University of Oviedo, Oviedo, Spain

Most insect species rely on the detection of olfactory cues for critical behaviors for the survival of the species, e.g., finding food, suitable mates and appropriate egg-laying sites. Although insects show a diverse array of molecular receptors dedicated to the detection of sensory cues, two main types of molecular receptors have been described as responsible for olfactory reception in Drosophila, the odorant receptors (ORs) and the ionotropic receptors (IRs). Although both receptor families share the role of being the first chemosensors in the insect olfactory system, they show distinct evolutionary origins and several distinct structural and functional characteristics. While ORs are seven-transmembrane-domain receptor proteins, IRs are related to the ionotropic glutamate receptor (iGluR) family. Both types of receptors are expressed on the olfactory sensory neurons (OSNs) of the main olfactory organ, the antenna, but they are housed in different types of sensilla, IRs in coeloconic sensilla and ORs in basiconic and trichoid sensilla. More importantly, from the functional point of view, they display different odorant specificity profiles. Research advances in the last decade have improved our understanding of the molecular basis, evolution and functional roles of these two families, but there are still controversies and unsolved key questions that remain to be answered. Here, we present an updated review on the advances of the genetic basis, evolution, structure, functional response and regulation of both types of chemosensory receptors. We use a comparative approach to highlight the similarities and differences among them. Moreover, we will discuss major open questions in the field of olfactory reception in insects. A comprehensive analysis of the structural and functional convergence and divergence of both types of receptors will help in elucidating the molecular basis of the function and regulation of chemoreception in insects.

Keywords: chemosensation, receptors, Drosophila, ORs, IRs, olfaction, taste

\footnotetext{
Abbreviations: CNVs, copy number variants; cVA, cis-vaccenyl acetate; ETS, E26 transformation-specific transcription factor; GR, gustatory receptor; GRN, gustatory receptor neuron; Indels, small insertions and deletions; IR, ionotropic receptor; OBP, odorant binding protein; OR, odorant receptor; ORCO, odorant receptor co-receptor; OSN, olfactory sensory neuron; SNMP1, sensory neuron membrane protein 1; SNPs, single-nucleotide polymorphisms; SSR, single-sensillum recording; TF, transcription factor.
} 


\section{INTRODUCTION}

Detection of chemical cues in the environment is essential for almost all animals to find food, mates, habitats or to avoid predators. Among all invertebrates, insect olfactory systems have extreme sensitivity and discrimination power to detect volatile chemicals related to their food, conspecifics and predators. In this regard, the olfactory system of Drosophila resembles the organizational principles of the olfactory system of vertebrates despite being simpler (Stocker, 2001; Vosshall and Stocker, 2007; Touhara and Vosshall, 2009). Thus, in both vertebrates and invertebrates, odorants bind to transmembrane odorant receptors (ORs) expressed in the cilia or dendrites of bipolar olfactory sensory neurons (OSNs). The OSNs send their axons to the brain, where they connect with second-order neurons in the glomeruli of the antennal lobe, in insects, or its homolog in vertebrates, the olfactory bulb. Furthermore, in both types of organisms, each OSN expresses one or very few olfactory molecular receptors, and the axons of OSNs that express the same receptor project to the same glomeruli in both the olfactory bulb and the antennal lobe (Su et al., 2009). These similarities and the fact that Drosophila is a widespread model organism in genetics and neurobiological research made its olfactory system an attractive and simple model to study olfaction (see, for example, the recent reviews by Wilson, 2013; Barish and Volkan, 2015; Carraher et al., 2015; Joseph and Carlson, 2015; Fleischer et al., 2018; Grabe and Sachse, 2018; Rimal and Lee, 2018).

In Drosophila adults, OSNs are housed inside hair-like structures called sensilla on the surface of the olfactory organs, the third antennal segment and the maxillary palps (Shanbhag et al., 1999). Each sensillum contains 1-4 OSNs (Shanbhag et al., 1999; Joseph and Carlson, 2015). Based on their morphology, sensilla are classified into four types: basiconic, trichoid, intermediate and coeloconic (Shanbhag et al., 1999; Lin and Potter, 2015). Additionally, they differ in regionalization (Shanbhag et al., 1999) and in the substances they detect (de Bruyne et al., 1999, 2001; Yao et al., 2005). These sensilla have pores in the cuticle that allow odorants to diffuse into the sensillum lymph and reach the dendrites of OSNs with the help of odorant binding proteins (OBPs) (Shanbhag et al., 2001; Leal, 2013). Then, odorants are recognized by specific transmembrane protein receptors in the dendrites of the OSNs. In insects, two main families of olfactory receptors have been described, the ORs and the ionotropic receptors (IRs), although a third family, the gustatory receptors (GRs), is involved in carbon dioxide detection (Jones et al., 2007; Kwon et al., 2007). Only one or very few of these olfactory receptors are expressed in each OSN (Vosshall et al., 2000; Benton et al., 2009), similar to what is observed in vertebrates (Buck, 2000). Both receptor families likely form heteromeric complexes between a specific receptor and a co-receptor needed for cellular trafficking and function (Larsson et al., 2004; Neuhaus et al., 2005; Benton et al., 2006, 2009; Silbering et al., 2011). However, although ORs and IRs share characteristics including their role as initial chemosensors for the insect olfactory system, research advances in the last decade have shown that they have distinct evolutionary origins (Robertson et al., 2003; Croset et al., 2010) and have several distinct structural and functional characteristics (Hallem et al., 2004; Benton et al., 2009).

In this review, we will present the research advances of the genetic basis, evolution, structure, functional response and regulation of both OR and IR chemosensory families. For this, we will use a comparative approach emphasizing the similarities and differences among them. Additionally, we will consider key open questions in the field of olfactory reception in insects. Thus, we will help in elucidating the molecular basis of the function and regulation of chemoreception in insects by performing a comprehensive analysis of the structural and functional convergence and divergence of both types of receptors.

\section{THE MOLECULAR BASIS OF CHEMOSENSATION IN DROSOPHILA: MOLECULAR STRUCTURE OF ORS AND IRs}

During the 1990s, the first attempts to discover the chemoreceptors in insects by sequence similarity failed because of their lack of homology with the G protein-coupled (GPCR) ORs of vertebrates (Buck and Axel, 1991) and nematodes (Troemel et al., 1995). Later, using difference cloning and mining of genome databases, a family of proteins with seven transmembrane domains with expression in the OSNs, the OR family, was discovered in Drosophila (Clyne et al., 1999b; Gao and Chess, 1999; Vosshall et al., 1999).

These Drosophila ORs show no obvious sequence homology with GPCRs and display an inverted topology with an intracellular $\mathrm{N}$-terminus and an extracellular C-terminus (Benton et al., 2006; Lundin et al., 2007; Smart et al., 2008). Structurally, ORs likely form heteromers composed of one odor-specific OR and another member of the OR family, the odorant receptor co-receptor (ORCO; previously known as OR83b) (Larsson et al., 2004; Neuhaus et al., 2005; Benton et al., 2006). ORCO is highly conserved across insect species (Krieger et al., 2003; Pitts et al., 2004; Jones et al., 2005; Smadja et al., 2009), and it is also necessary for the trafficking of ORs to the ciliary membrane in vivo (Larsson et al., 2004; Benton et al., 2006).

In contrast to GPCRs, ORs do not have conventional binding sites for $G$ proteins, and several studies have reported that OR-ORCO heteromers expressed in heterologous systems can act as odorant-gated ionotropic channels with ionic permeability to $\mathrm{Ca}^{2+}, \mathrm{Na}^{+}$and $\mathrm{K}^{+}$(Sato et al., 2008; Smart et al., 2008; Wicher et al., 2008; Nakagawa and Vosshall, 2009). However, one of these studies also reported a metabotropic component that is dependent on G proteins and the cAMP transduction cascade (Wicher et al., 2008). This metabotropic component in the function of the OR-ORCO heteromers has been either supported (Kain et al., 2008; Chatterjee et al., 2009; Deng et al., 2011; Ignatious Raja et al., 2014; Miazzi et al., 2016; Murmu and Martin, 2016) or argued against (Yao and Carlson, 2010) in several studies of G-proteins in genetically modified flies.

Additionally, it has been reported that the activity of ORCO is regulated by phosphorylation via protein kinase 
C (PKC), which is activated by the inositol 1,4,5-inositol triphosphate/diacyl glycerol (IP3/DAG) signal transduction cascade (Sargsyan et al., 2011). Two hypotheses have been proposed in Drosophila to explain these results: (a) it is possible that ORs may be mixed ionotropic-metabotropic receptors (Wicher, 2010); or (b) alternatively, ORs may be metabotropically modulated ionotropic receptors (Nakagawa and Vosshall, 2009). These two hypotheses raise two different putative structures of the odor-gated ionic channel that combine either four or two OR/ORCO heteromers. If, as for the other known superfamilies of ligand-gated ionic channels, the central pore is formed by the combination of four subunits (Carraher et al., 2015), then two putative structures could arise. In the first putative structure, four OR subunits would bind the odorants and produce fast opening of an ionic channel formed by four ORCO subunits. Additionally, the OR subunits interact with $G$ proteins that produce slower metabotropic transduction cascades that regulate the ORCO channel (Wicher, 2010). In the second structure, two OR and two ORCO subunits form the central pore of the ion channel, which would open when a ligand binds the OR subunits and could be regulated by metabotropic transduction cascades (Nakagawa and Vosshall, 2009). In some other insect species, a third hypothesis proposing only metabotropic signal transduction and ORCO functioning as a pacemaker channel controlling membrane potential has been suggested (Stengl, 2010).

While there are still no X-ray crystallography 3D structures for the Drosophila ORs, site-directed mutagenesis, resonance energy transfer and structural modeling efforts (Hopf et al., 2015), have started to provide information about the molecular structure of these seven-transmembrane-domain receptors (Carraher et al., 2015). For example, the second extracellular loop has been suggested to form a lid over the binding pocket, which is formed by the extracellular regions of some transmembrane helices, especially the third and to a less extent the sixth and seventh, of the OR subunits (Carraher et al., 2015). In addition, the interaction between the ORCO and OR receptor subunits through the final intracellular loop and the adjacent transmembrane helices might be important for transducing ligand binding into receptor activation (Benton et al., 2006; Kumar et al., 2013). Furthermore, channel gating could be regulated using phosphorylation sites (Sargsyan et al., 2011) and a calmodulin-binding site in the second intracellular loop of the ORCO subunits (Mukunda et al., 2014; Bahk and Jones, 2016).

Ten years after ORs have been identified, in 2009, a new family of olfactory receptors in Drosophila was discovered, an ionotropic glutamate receptor (iGluR)-related family of receptors, termed the IRs (Benton et al., 2009). The IRs are formed by an extracellular N-terminus, a highly variable ligand-binding domain with two lobes separated by an ion channel domain, and a short cytoplasmic C-terminus (Benton et al., 2009). Sixteen IRs out of the 66 discovered are expressed in antennal neurons, while the rest, named "divergent IRs", are expressed in other locations in the body (Benton et al., 2009; Sánchez-Alcañiz et al., 2018).
While most IRs lack glutamate-binding residues, members of the IR family of olfactory receptors show similarities with the iGluRs of vertebrates and are suggested to form ligand-gated ion channels (Benton et al., 2009; Abuin et al., 2011; Rytz et al., 2013). Although X-ray crystallography 3D structures for IRs are not yet available, some protein homology modeling has been performed (Prieto-Godino et al., 2016, 2017). Still, the exact molecular mechanism of IR activation by different specific odorants remains to be shown, as for ORs. Although the molecular structures of ORs and IRs are extremely dissimilar, they show some commonalities. Both ORs and IRs form functional heterodimeric complexes of a receptor and a coreceptor. However, while the OR functional unit consists of the highly conserved co-receptor ORCO and an odorant-specific OR, which provides the complex with its ligand specificity (Benton et al., 2006), IRs show more than one possible co-receptor-IR25, IR8a, IR76b and IR93a-and a specific IR that, as for ORs, gives the complex its odorant specificity (Benton et al., 2009; Silbering et al., 2011; Ai et al., 2013). For both receptor families, the co-receptors are needed both for odor-evoked electrophysiological neuronal responses and receptor trafficking to the ciliary membrane, the sensory compartment where odorant transduction takes place (Benton et al., 2009; Abuin et al., 2011; Silbering et al., 2011; Ai et al., 2013). IRs show reciprocal need for the co-receptor and the ligand-specific IR for dendrite trafficking (Benton et al., 2009; Abuin et al., 2011). Similarly, ORs need ORCO for trafficking to ciliary membranes (Larsson et al., 2004; Bahk and Jones, 2016). The transport of Drosophila ORs to and within the dendritic cilia is regulated by the hedgehog $(\mathrm{Hh})$ signal transducer smoothened (Smo) (Sanchez et al., 2016).

Intriguingly, antennal pheromone-sensing neurons in insects show the expression of another membrane-bound element, the sensory neuron membrane protein 1 (SNMP1) (Rogers et al., 1997, 2001; Jiang et al., 2016). SNMP1 is a CD36-related receptor whose involvement in sensing cis-vaccenyl acetate (cVA), a pheromone produced by males in Drosophila, has been extensively studied (e.g., Benton et al., 2007; Jin et al., 2008). SNMP1-together with the complex ORCO/OR67d and the odorant/pheromone binding protein, LUSH - is essential for cVA electrophysiological responses but not for trafficking the OR complex to the sensory cilia (Benton et al., 2007). However, SNMP1 might support the functional expression of DmORCO found in mammalian cell culture (Halty-deLeon et al., 2016). Moreover, it has been shown that SNMP1 is important for both rapid activation and termination of the cVA response (Li et al., 2014). Based on homology modeling and structure-function studies, it has been recently proposed that SNMP1 funnels hydrophobic pheromones through a putative ectodomain tunnel from the extracellular fluid to the membrane receptors (GomezDiaz et al., 2016). Although some members of the IR family, such as the IR20a clade, have been proposed as pheromone sensors (Koh et al., 2014), no evidence has been found for an equivalent to SNMP1 dedicated to pheromone sensing for the IRs.

Other members of the peripheral sensory system include the OBPs (Vogt and Riddiford, 1981). They are secreted by auxiliary cells in the antenna and show specific sensillar patterns 
(Shanbhag et al., 2001; Leal, 2013). Although the number of OBP genes in Drosophila is similar to the number of ORs and they both show similar patterns of evolution in some species (Kopp et al., 2008), there are also some OBPs found both in taste organs and antennal IR-expressing neurons (Galindo and Smith, 2001; Shanbhag et al., 2001). The specific role and action mechanism of OBPs in olfaction are still under debate; although there are some OBPs, such as LUSH, that have been linked to the detection of odorants by ORs (Xu et al., 2005; Swarup et al., 2011; GomezDiaz et al., 2013), no functional evidence has been found for the need of OBPs in IR-expressing neurons.

\section{THE GENETIC BASIS OF OR AND IR PROTEIN FAMILIES}

The OR protein family is encoded by 60 genes and a few pseudogenes in the Drosophila genome. It comprises 62 receptor proteins as Or46a and Or69a each give rise to two proteins by alternative splicing (Robertson et al., 2003). Some of the OR genes are clustered together in groups of two or three, probably because they are recent duplications, but most of them are widely dispersed in the genome (Robertson et al., 2003) (Figure 1). The IR receptor family is extremely divergent, showing an overall amino acid sequence identity of $10 \%-70 \%$. Similar to the ORs, these genes are distributed throughout the Drosophila genome, many as individual genes, although some form cluster arrays of few genes as e.g., in cytological regions 7 and 52 (Benton et al., 2009) (Figure 1).

Expression studies of OR genes have shown that 45 members of this family are present in the adult antenna and maxillary palp, while 25 are expressed in the larval olfactory system. Some ORs are expressed in both developmental stages, and some are exclusive to one, either larvae or adults (Couto et al., 2005; Fishilevich et al., 2005). In the antenna, each OR is expressed in 2-50 OSNs (Clyne et al., 1999b; Vosshall et al., 1999, 2000) in a stereotyped sensillar map in which each sensilla subtype is characterized by the expression of one or few ORs in each of the OSNs that innervate it. ORs are almost uniquely expressed in neurons housed in single-walled sensilla, such as basiconic, trichoid and intermediate sensilla (Shanbhag et al., 1999; Lin and Potter, 2015), while IRs are expressed in doublewalled coeloconic sensilla (Benton et al., 2009; Silbering et al., 2011, 2016). Thus, 18 sensilla types house 39 OSN classes that express OR members with ten basiconics, two trichoids, three intermediates in the antennae as well as three basiconic sensilla types on the palps (Couto et al., 2005; Lin and Potter, 2015) Although ORs and IRs are expressed on different sensory lineages in the antenna, there is one exception: in ac3 sensilla, IR76b is coexpressed with OR35a/ORCO (Benton et al., 2009). Additionally, one of the four OSNs of the ab1 basiconic sensilla expresses two GRs, Gr21a and Gr63a, both dedicated to CO2 sensing (Jones et al., 2007; Kwon et al., 2007). Similar to vertebrates, each OSN usually expresses only one type of specific OR (and ORCO), although there are some exceptions in which two or three ORs are expressed in the same OSNs, such as ab5 sensilla, where OR33b and OR47a, both present in larvae and adult, are expressed together (Fishilevich and Vosshall, 2005; Goldman et al., 2005).

Genomic analysis in Drosophila has revealed 66 IR genes, including nine putative pseudogenes (Benton et al., 2009; Croset et al., 2010). Extensive effort in expression analysis has shown that antennal neurons express 16 IRs, most of which are in neurons housed in coeloconic sensilla. Four IRs, IR20a, IR40a, IR64a and IR93a, are expressed not in the coeloconic neurons but in the arista and sacculus neurons (Benton et al., 2009; Silbering et al., 2016). By using transgenic reporters, it was revealed that out of the 44 non-antennal IRs, 32 were expressed in larvae and 27 in adults, where they were found in various organs, such as the antennae, labella, pharynx, legs and wings (Joseph and Carlson, 2015; Sánchez-Alcañiz et al., 2018). Similar to ORs, some of them are specific to either larvae or adults, such as the IR52 clade, expressed in foreleg taste neurons, which has been related to mating behavior (Koh et al., 2014). Neurons housed at coeloconic sensilla express from two to four IRs (Benton et al., 2009). Additionally, IRs do not seem to be expressed in the secondary olfactory organs, the maxillary palps, which only contain OR-expressing neurons.

The expression studies carried out on both types of receptors have also permitted the generation of a complete projection map of the axons of the OSNs to the 52 glomeruli of the antennal lobe in the brain, showing that every OSN that expresses a particular olfactory receptor sends axonal projections to the same glomerulus (Couto et al., 2005; Silbering et al., 2011; Grabe and Sachse, 2018). There is a spatial organization in which the afferents from the OSNs innervating each type of sensilla project to glomeruli in the same location in the antennal lobe. Thus, OSNs in the antennal basiconic sensilla project to the medial region of the antennal lobe, palp basiconic sensilla to the central-medial region, antennal trichoid sensilla to the lateral anterior region, and antennal coeloconic sensilla to the posterior region (Couto et al., 2005; Silbering et al., 2011). Therefore, projections of OR- and IR-expressing neurons are segregated although interconnected in the antennal lobe (Silbering et al., 2011).

The genetic control of the stereotyped expression of the ORs of both families in the OSNs is still under study. There is a relationship between the expression of olfactory receptors and the zonal localization of sensillum types/subtypes. Expression of a given receptor is restricted to an OSN class that is located in a particular sensillum subtype, and thus, all OSNs form a sensory map on the antenna (Vosshall et al., 1999, 2000; Couto et al., 2005; Benton et al., 2009; Silbering et al., 2011). Each sensillum subtype houses stereotypical clusters of 1-4 OSN identities that arise through asymmetric divisions from a single multipotent sensory organ precursor (SOP; Rodrigues and Hummel, 2008).

The adult olfactory organs develop from the larval antennal imaginal disc, where the various morphological types of sensilla arise in the pupae due to the action of a combination of proneural and helix-loop-helix transcription factors (TFs) (Fuss and Ray, 2009). Thus, the TF atonal is necessary for the development of the antennal coeloconic and palp basiconic sensilla (Gupta and Rodrigues, 1997), while amos and lozenge are required for the antennal basiconic and trichoid sensilla (and the few 


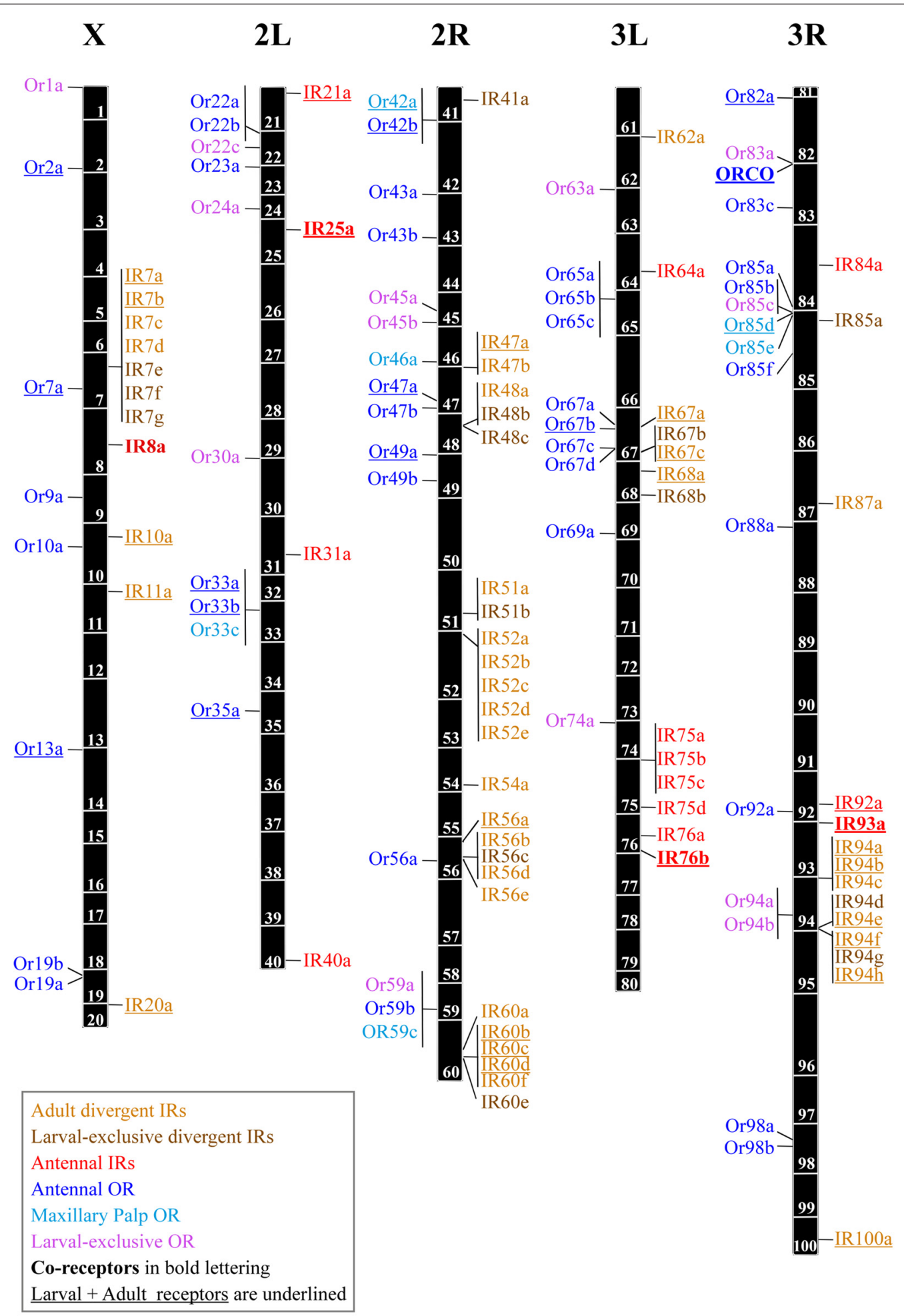

FIGURE 1 | Genomic locations of the odorant receptor (OR) and ionotropic receptor (IR) genes. The five major chromosome arms are drawn to scale, with OR genes shown left and IR genes right of each chromosome arm. Gene locations are based on data from Release 6 of the genome of $D$. melanogaster and the FlyBase database (release FB2018_02; Gramates et al., 2017) The names of the olfactory receptors are color-coded as follows: adult divergent IRs (light brown), larval-exclusive divergent IRs (brown), antennal IRs (red), antennal ORs (blue), maxillary palp ORs (light blue) and larval-exclusive ORs (magenta). Co-receptors are indicated in bold letters. Expression both in adults and larvae is indicated as underlined text (Couto et al., 2005; Fishilevich et al., 2005; Benton et al., 2009; Sánchez-Alcañiz et al., 2018). 
intermediate sensilla; Gupta et al., 1998; Goulding et al., 2000; zur Lage et al., 2003). In the antennal disc, the differential expression of Dachshund, Rotund, BarH1/H2, Bric-à-brac and Apterous patterns the antennal disc into seven concentric rings (Li et al., 2016; Hsieh et al., 2017). Each concentric ring will determine a subset of the subtypes of sensilla in the antenna; for example, the innermost ring determines the SOPs for the at2, ac3, ab2, ab3, ab4, ab6 and ab8 sensilla (Li et al., 2016; Hsieh et al., 2017). Finally, in each of these rings, the determined SOPs will develop into a particular sensilla subtype, and each OSN they house will specifically express one or few ORs (Barish and Volkan, 2015). In contrast to insect OR-expressing neurons, where most of them expresses a unique odorant-specific receptor type, and the co-receptor ORCO, IR-expressing neurons do show a more complicated receptor choice specification with some neurons expressing more than two IRs, needed for its functional response (Benton et al., 2009), but any information on the IR specification remains elusive. Conversely, in OR-expressing neurons, various studies have implicated several TFs in the OR choice specification of each OSN (Martin et al., 2013). Thus far, five TFs have been implicated in the regulation of the ORs expressed in the palps (Clyne et al., 1999a; Ray et al., 2007; Tichy et al., 2008; Bai and Carlson, 2010; Song et al., 2012), while in the antennae, at least nine TFs are involved in the control of OR expression (Jafari et al., 2012; Song et al., 2012). Recently, a genetic immortalization method has been used to elaborate a fate map of all olfactory lineages and to identify Pointed, a E26 transformation-specific transcription factor (ETS) family member, as a determinant of the Or67d pheromonesensing neuron development (Chai et al., 2018). Additionally, in a sensillum, a cluster of OSNs is asymmetrically differentiated from a single SOP into two classes in a manner dependent on differential Notch activity in their sibling precursors. In this way, Notch-ON and Notch-OFF specify olfactory receptor expression and axonal targeting of the different OSNs housed in a single sensillum (Endo et al., 2007). This pathway is dependent on the co-repressor Atrophin, which regulates Histone 3 acetylation to determine the OR expressed in any OSN (Alkhori et al., 2014). Apart from the TFs that regulate the expression of the ORs, mutagenesis of the upstream regulatory sequences of four OR genes has identified particular sequence motifs that act positively or negatively to dictate expression in the proper subset of OSNs (Miller and Carlson, 2010).

\section{EVOLUTION OF BOTH FAMILIES OF CHEMORECEPTORS}

Although the IR family is related to the iGluRs that mediate synaptic communication in vertebrate and invertebrate nervous systems (Benton et al., 2009), the insect OR receptors are not related to the ORs found in vertebrates and nematodes and have evolved independently (Robertson et al., 2003; Benton et al., 2006).

In the case of the OR family, via comparative genomic and transcriptomic analyses, several related but highly divergent genes have been found in many insect genomes (Grosse-Wilde et al., 2011; Kanost et al., 2016; de Fouchier et al., 2017), ranging from the seven ORs found in the human body louse (Pelletier et al., 2015), 79 ORs in the malaria mosquito (Fox et al., 2001; Hill et al., 2002), 163 in the honey bee (Robertson and Wanner, 2006), 256 in the red flour beetle (Engsontia et al., 2008; Dippel et al., 2016), to the more than 350 ORs found in some ant species (Zhou et al., 2012). In social insects, the chemosensory protein repertoire shows an OR-specific expansion (up to $450 \mathrm{OR}$ candidates found in antennal transcriptomes and genome-wide analysis) that does not seem to affect the IR or the GR families (Robertson and Wanner, 2006; Zhou et al., 2012; McKenzie et al., 2014; Oxley et al., 2014; Pitts et al., 2017). This expansion has been hypothesized to be linked to the strong diversification of flowering plants as food sources and to the enhanced needs for discrimination between nestmates and non-nestmates and for reproductive division of labor in social insects, although direct evidence for this in different species is still scarce (e.g., Sharma et al., 2015; Pask et al., 2017). Comparative phylogenetic analyses of these expanded ORs have allowed the identification of some OR subfamilies, as e.g., pheromone receptors, and to study their evolutionary origin and expansion in insect lineages (Missbach et al., 2014; Koenig et al., 2015; de Fouchier et al., 2017). In the case of ORCO, highly conserved homologs have been found in several insect orders, such as Lepidoptera, Diptera, Coleoptera, Hymenoptera, Hemiptera and Orthoptera (Krieger et al., 2003; Pitts et al., 2004; Smadja et al., 2009; Yang et al., 2012). However, in some cases, when the genomes of primitive not winged insects have been investigated, either no OR or ORCO was found (as in one member of the order Archaeognatha), or only a few gene homologs of ORCO were identified (as a species of the order Zygentoma; Missbach et al., 2014). Additionally, in the crustacean Daphnia pulex, which shares a common ancestor with insects, despite the fact that members of the GR family were identified, no ORs were found (Peñalva-Arana et al., 2009). Likewise, ORs are absent in the genomes of other arthropods, such as spiders (Vizueta et al., 2017). These data suggest that the OR family is exclusive to insects and probably evolved when insects developed flight, with the evolution of ORCO first and the other OR subfamilies later (Missbach et al., 2014). It is thought that both the ORs and GRs are part of a superfamily of chemosensory receptors (Robertson et al., 2003) and that the OR family evolved from the GR family, which can be found in all arthropods, when the insects became terrestrial organisms and started to fly.

In contrast to the insect-specific origin of the OR family, comparative genomic analysis across many animal groups has revealed an ancient Protostome origin for the IR family (Croset et al., 2010; Rytz et al., 2013). While antennal IRs are conserved and show orthologs in many different insect species, the genomic analysis of non-antennal IRs, originally named "divergent IRs", indicates a great expansion in Diptera and shows unclear orthologous relationships in other insects, forming phylogenetic species-specific clades across insects. It has been suggested that the enormous expansion of this chemosensory family arose from non-allelic homologous recombination and retroposition (Croset et al., 2010). Most IRs exist as single-copy highly conserved orthologs, but there are some cases where non-allelic homologous recombination and ancient duplication 
events played a large role in IR evolution, as is the case for the IR75 cluster (Croset et al., 2010).

Olfactory receptor families contain various pseudogenes. In Drosophila sechellia, IR75a encodes an expected pseudogene, with a premature stop codon, but it has been shown that it is a "pseudo-pseudogene", meaning that the receptor remains functional due to translational read-through of the premature termination codon (Prieto-Godino et al., 2016), although the exact mechanism of the read-through remains unknown. Another IR receptor, IR31a, showed characteristics of this pseudo-pseudogenization along with OR35a (Prieto-Godino et al., 2016), but whether this is a common feature also in the OR family remains to be shown.

Chemoreceptor families in Drosophila are extremely useful models for studying how selection acts over organisms in a changing environment because they show rapid adaptation over short timescales, which seems to be a function of relaxed constraints (Arguello et al., 2016). For example, Drosophila sechellia is attracted to hexanoic acid, present in the noni fruit, while $D$. melanogaster is not. This shift in preference is mainly driven by a single amino acid change in the IR75b protein, which together with some changes in the promoter and trans-acting loci, tunes this receptor in D. sechellia to hexanoic acid (PrietoGodino et al., 2017), allowing this species to adapt to its specific ecological niche.

By analysis of genome-wide data, including single-nucleotide polymorphisms (SNPs), copy number variants (CNVs) and small insertions and deletions (indels), of chemosensory families from various Drosophila melanogaster populations (from ancestral-like African populations to subsequent populations that inhabit different niches) and comparison with other large families, it has been demonstrated that chemosensory receptors do not show high rates of adaptive divergence between species but show genome-wide signals of recent selection within D. melanogaster (Arguello et al., 2016). Additionally, they display patterns of adaptive mutations that could predict diverse effects on protein function (Arguello et al., 2016).

\section{FUNCTIONAL REGULATION OF NEURONAL RESPONSE}

The responses of the OSNs could be modified by several external and internal factors that affect the olfactory receptor function at various levels, from genetic expression to functional interaction. For example, the environmental temperature affects olfactory behavior in Drosophila (Riveron et al., 2009) and also modifies antennal electrical responses of OR-expressing OSNs, as shown in electroantennograms (EAGs) and single-sensillum recordings (SSRs) (Martin et al., 2011). Correspondingly, in microarray transcriptomic studies using third antennal segments of high-temperature-acclimated flies, there were changes in the expression levels of several ORs and IRs (Riveron et al., 2013). Although high temperature produced significant overexpression only in four out of the 16 antennal IRs, the same tendency was shown in the rest of the IRs analyzed (Figure 2) (Riveron et al., 2013). However, for the ORs, significant changes for nine members of the family were found, four of them displaying overexpression and the other four and ORCO showing downregulation (Figure 2).

Additionally, internal signals can regulate the responses of OSNs expressing ORs. For example, in a lepidopteran, the crepuscular hawk moth, Manduca sexta, there is circadian control through octopamine (OA) over the olfactory metabotropic transduction of pheromones (Schendzielorz et al., 2015). OA could act on ORCO, which has been suggested to be a hormone-controlled pacemaker channel controlling spontaneous activity, threshold and temporal resolution of pheromone detection (Stengl, 2010; Stengl and Funk, 2013). In this species, no evidence of ORCO-based ionotropic signal transduction cascade has been found (Nolte et al., 2013, 2016).

Furthermore, the internal amino acid state can modulate yeast taste neurons. A common subset of the population of IR76band IR25-expressing neurons in the proboscis is required for yeast sensing (Steck et al., 2018). The response of these gustatory receptor neurons (GRNs) is directly modulated by the internal amino acid state, while the reproductive state modulates yeast feeding downstream of the receptor neurons (Steck et al., 2018).

Olfactory neuronal responses driven by both types of chemoreceptors can be differentially regulated. For example, the OR-expressing OSNs strongly adapt to odors in electrophysiological recordings of the whole antenna (electroantennograms, EAGs) (Störtkuhl et al., 1999), individual sensilla, (single-sensillum recordings, SSRs) (Nagel and Wilson, 2011; Martelli et al., 2013) or single OSNs (whole-cell patch clamp) (Cao et al., 2016). In contrast, IR-expressing OSNs showed no adaptation both in sensillum recordings (Abuin et al., 2011) and in whole cell patch clamp (Cao et al., 2016), indicating that the two types of neurons might use distinct odor transduction mechanisms. The adaptation in the OR/ORCO receptors seems to be mediated by odorant-induced phosphorylation changes of the serine 289 of ORCO (Guo and Smith, 2017). Additionally, there is a decrease in the spike amplitude in the SSR of OSNs expressing ORs during odor stimulation that has been related to its concentration (Martin and Alcorta, 2016).

Moreover, some effort has been made in describing the functional dynamics and latencies of the responses to odors in OR-expressing OSNs in Drosophila (Martelli et al., 2013) because a pure ionotropic response does not involve amplification and is believed to be faster than a metabotropic response. Although direct evidence for response latencies in ORs and IRs in Drosophila is still missing, these latencies have been investigated in EAGs of other insect species, such as the orange spotted cockroach (Blaptica dubia), hissing cockroaches (Gromphadorhina portentosa), locusts (Schistocerca americana), honey bees (Apis mellifera) and moths (Manduca sexta), showing latencies as short as $2 \mathrm{~ms}$ (Szyszka et al., 2014).

\section{FUNCTIONAL PROFILES OF ORs AND IRs}

From the perspective of sensory modalities that involve these two peripheral sensory systems, it seems that the OR receptor family is exclusively used in olfaction, while the IR family covers 

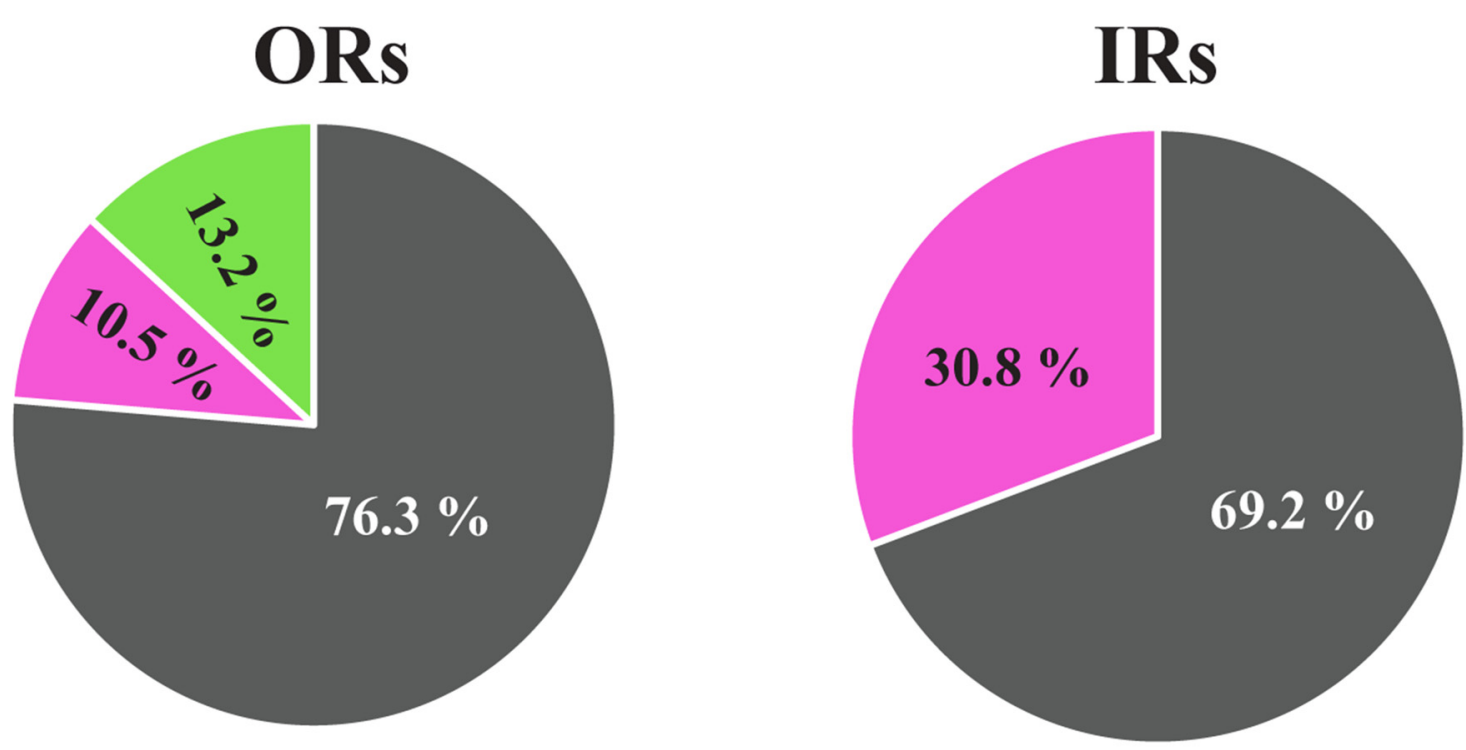

no change

upregulated

downregulated

FIGURE 2 | Changes in the antennal expression levels of OR and IR genes in response to high temperature. Percentage of detected genes that show up- and down-regulated gene expression under heat treatment condition (shifting from $21^{\circ} \mathrm{C}$ to $30^{\circ} \mathrm{C}$ ) using Affymetrix microarrays. Only significant changes with a false discovery rate (FDR) $<0.1$ were considered. Data adapted from Riveron et al. (2013).

both chemosensory modalities, olfaction and taste, and even non-chemosensory ones (Table 1).

Several studies have been carried out to establish the odorant response profiles of OR receptors using electrophysiological measurements, such as SSRs, obtained either by their ectopic expression in an empty neuron (Hallem et al., 2004; Hallem and Carlson, 2006) or directly in native OSNs (Clyne et al., 1997; de Bruyne et al., 1999, 2001). These studies involved panels of 100 odorants at most, although a computer simulation study with 240,000 odorants was partially validated in functional assays (Boyle et al., 2013). Likewise, similar studies have been performed to determine the odorant response profiles of the IR receptor family (Benton et al., 2009; Abuin et al., 2011; Silbering et al., 2011). All available Drosophila odorant response data have been combined to a single consensus response matrix linking odorants to olfactory receptors in the DoOR database (Galizia et al., 2010; Münch and Galizia, 2016).

Both chemosensory families are involved in food odor sensing, detecting a vast array of chemicals. While ORs are highly tuned to esters and alcohols (Hallem et al., 2004; Hallem and Carlson, 2006), IRs are highly tuned to amines and acids (Benton et al., 2009; Abuin et al., 2011; Silbering et al., 2011; Min et al., 2013). Usually, the ORs are broadly tuned to several compounds, while the IRs are more narrowly tuned to a few compounds (Silbering et al., 2011).

While some ORs, especially those expressed in trichoid sensilla, have been shown to be responsive to pheromones (Benton et al., 2007; Kurtovic et al., 2007; van der Goes van Naters and Carlson, 2007; Stengl, 2010), only a few IRs have been linked to pheromone sensing either indirectly (Grosjean et al., 2011) or through the IR20a clade (Koh et al., 2014). Neurons that express members of the IR20a clade are mostly located in the proboscis, pharynx, legs and wing margin of Drosophila. They send their axonal projections to taste centers in the brain that do not overlap with bitter-sensing neurons. Some of these members are activated by odors from conspecific females and are adjacent to a neural circuit for sexual behavior, the fru+ neurons (Koh et al., 2014). Additionally, IR52c and IR52d show sexually dimorphic expression in leg taste neurons (Koh et al., 2014), but their specific ligands are still unknown.

Some other sensory modalities, such as taste, while multimodal in most cases, seem to be exclusively mediated by GRs, IRs or a combination of both (Vosshall and Stocker, 2007; Liman et al., 2014; Sánchez-Alcañiz et al., 2018). To date, there is no evidence involving ORs in taste sensation. Using transgenic reporters, it has recently been shown that most IRs are expressed in diverse populations of peripheral sensory neurons of gustatory organs in both larvae and adults (Sánchez-Alcañiz et al., 2018). In general, taste seems to require the co-receptors IR25a and IR76b but not IR8a (SánchezAlcañiz et al., 2018). In fact, it has been shown that IR25a and IR7b are necessary in female sour-detecting GRNs for oviposition preference in acid-containing food (Chen and Amrein, 2017).

In Drosophila, long-range attraction to polyamines, pungentsmelling compounds required in numerous cellular and organismal processes, is mediated by IR76b and IR41a, while 


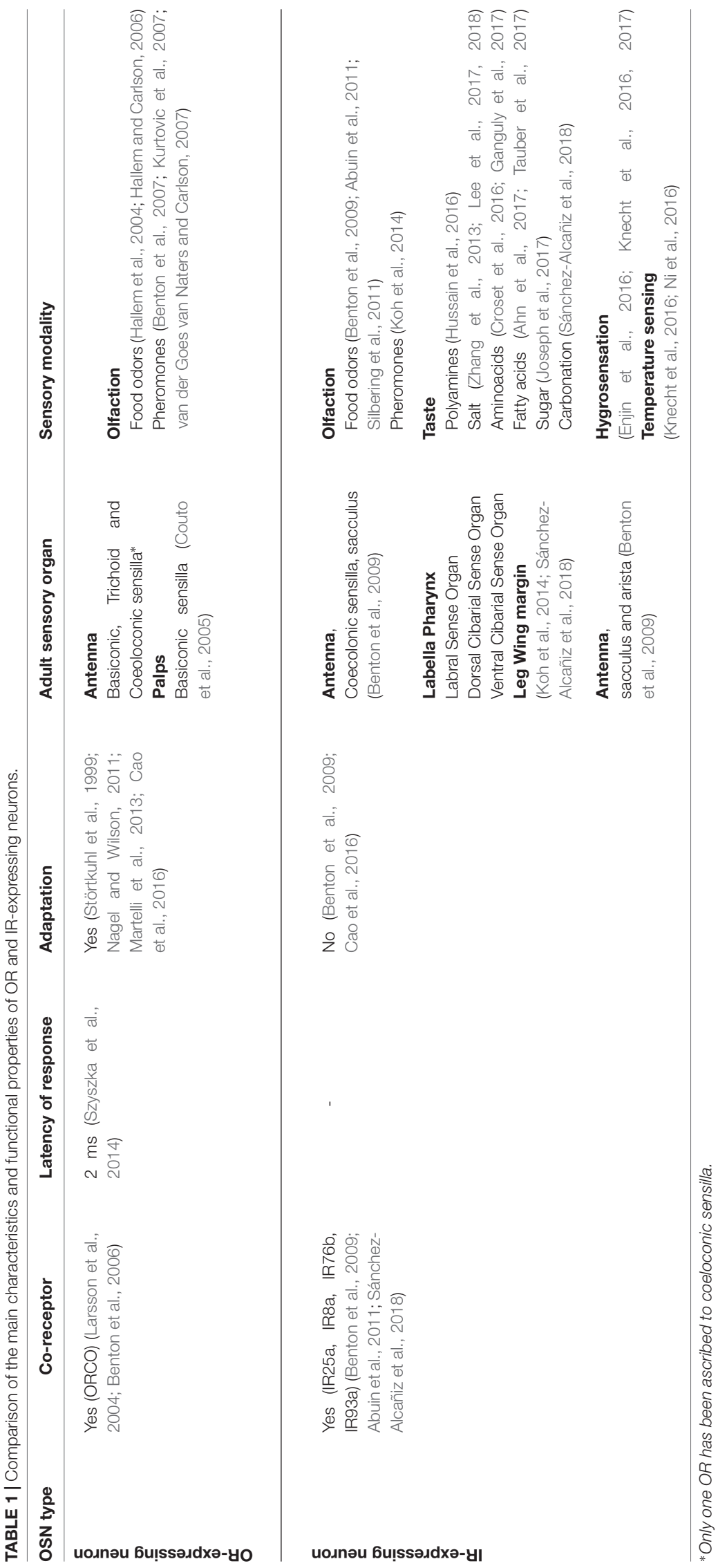


short-range attraction, which stimulates egg-laying behavior in polyamine rich-medium, seems to be a multimodal stimulus sensation mediated by IR76b and GR66a bitter-receptor neurons (Hussain et al., 2016). This mechanism seems highly conserved, as it is also found in mosquitoes (Hussain et al., 2016).

Flies use GRNs to respond to different concentrations of salt (Vosshall and Stocker, 2007; Liman et al., 2014). While attractive at low concentrations, salt can be harmful at higher concentrations. Strikingly, a highly conserved IR in insect genomes, IR76b, was shown to be a leak $\mathrm{Na}^{+}$channel that detects low salt and drives the salt-induced attractive pathway, while other GRNs would drive salt-aversive behavior (Zhang et al., 2013). This system could act as a bimodal switch for behavioral salt attraction and aversion (Zhang et al., 2013). Recent reports on $\mathrm{Na}^{+}$sensing in Drosophila showed that IR76b-sensing GRNs in both L- and S-bristles are required for repulsion (Lee et al., 2017), contrary to the previous idea of IR76b directing only attraction to low $\mathrm{Na}^{+}$(Zhang et al., 2013). More research will be needed to elucidate this open question. In addition to $\mathrm{Na}^{+}$ sensing, excessive $\mathrm{Ca}^{2+}$ taste avoidance is also important for avoiding toxic levels of this mineral in the food. This avoidance requires three members of the IR family-IR25a, IR62a and IR76b-expressed in GRNs in the labella, although the ectopic expression of these three elements is not sufficient to confer $\mathrm{Ca}^{2+}$ sensitivity, indicating that some other elements are needed (Lee et al., 2018).

Little evidence of a functional role for IRs has been gathered in larvae of Drosophila, but recently, it has been shown that larvae lacking IR76b displayed highly reduced behavioral attraction to some amino acids, while those lacking IR25a show no effect in attraction to them (Croset et al., 2016). Using functional imaging, it was shown that only a subset of IR76b-expressing gustatory neurons respond to some amino acids. In these IR76bexpressing cells, increases in calcium levels were observed upon presentation of nine amino acids; however, this subset did not correspond precisely with the stimuli that trigger preference behavior (Croset et al., 2016). These nine amino acids also elicited responses in IR60c-expressing neurons, although these neurons are not required for amino acid preference but rather mediate, together with IR76b, feeding suppression by high concentrations of amino acids (Croset et al., 2016). In adults, IR76b has been postulated as necessary for the post-mating female preference for amino acids by tarsal taste neurons (Ganguly et al., 2017). Additionally, it was suggested that IR20a blocks the IR76b salt-sensing activity and facilitates a mutually exclusive role of IR76b in both salt and amino acid sensing. Co-expression of IR20a confers amino acid sensitivity to sweetsensing neurons but not to L-type sensilla (Ganguly et al., 2017). Thus, indicating either that some other factor is needed or that the switching mechanism mediated by IR20a could be replaced.

Although little is known about fatty acid detection in insects, it has recently been linked to the IR family of chemoreceptors in Drosophila (Ahn et al., 2017; Tauber et al., 2017). By using either $\mathrm{Ca}^{2+}$ imaging in sweet-sensing GRNs on tarsal sensilla preparations or behavioral assays (Proboscis extension response, PER) using IR25a and IR76b mutants, the requirement of IR25a and IR76b in fatty acid detection was shown (Ahn et al., 2017). Moreover, both RNAi knockdown of IR56d in sweet-sensing neurons (Ahn et al., 2017) and functional imaging (Tauber et al., 2017) linked this receptor to their detection. Neurons that co-express Gr64f and IR56d are activated by medium-chain fatty acids being sufficient for reflexive feeding responses (Tauber et al., 2017). Fatty acids also elicit responses in bitter-sensing GRNs, but their molecular basis remains unknown (Ahn et al., 2017).

A healthy metabolism requires the control of sugar consumption. In Drosophila, it has been shown, by an optogenetic approach, that overconsumption of sugar could be avoided by activation of a circuit that inhibits sucrose feeding depending on IR60b (Joseph et al., 2017). IR60b is co-expressed in a neuron in the pharynx together with IR94f and IR94h but not with any sweet-sensing GRs (Joseph et al., 2017), although the roles of IR94h and IR94f remain elusive. While alternative explanations for the role of IR60b have been hypothesized (Szyszka and Galizia, 2018), they need further experimental analysis.

Carbonation, a non-nutritious product of microbial fermentation, has been shown to be detected in Drosophila by IR56d-expressing taste neurons together with IR25a and IR76b co-receptors (Sánchez-Alcañiz et al., 2018). Using transgenic reporters, it has been shown that IR56d is expressed in two different neuronal populations: the one in the taste pegs is dedicated to carbonation and fatty acid detection (but not activated by sucrose), while the other one, in taste bristles, is dedicated to sugar and fatty acid sensing (Tauber et al., 2017; Sánchez-Alcañiz et al., 2018). Although carbonation is modestly behaviorally attractive in an IR56d-dependent manner, IR56d seems to be necessary but not sufficient for this attraction (Sánchez-Alcañiz et al., 2018).

In conclusion, different cell-specific IR subunit combinations seem to be the basis for different taste qualities.

Although not the topic of this review, it should be mentioned that very recently, it was discovered that the IR family of receptors, unlike the OR family, covers other sensory modalities beyond chemosensation, such as hygrosensation (Enjin et al., 2016; Knecht et al., 2016, 2017) and temperature sensing (Knecht et al., 2016; Ni et al., 2016) in both adults and larvae (Sánchez-Alcañiz et al., 2018). Moreover, IR25 has been proposed as a temperature sensor that impacts the temperature-dependent resetting of the circadian clock (Chen et al., 2015).

\section{DISCUSSION AND CLOSING REMARKS}

Despite the recent increase in knowledge about the main receptor families in olfaction in Drosophila (see reviews by Wilson, 2013; Carraher et al., 2015; Joseph and Carlson, 2015; Fleischer et al., 2018; Rimal and Lee, 2018), there are still many open questions that remain to be answered.

For example, finding the ligands for orphan receptors in both chemosensory families will shed light on the different modalities that they subserve. Additionally, experiments identifying the transduction mechanisms used by the two types 
of chemoreceptors will also help understanding the exquisite sensitivity and specificity of these receptors. Also, accurate $\mathrm{X}$-ray crystallographic structures will help in solving some of these standing issues such as the exact composition of olfactory receptor heteromers or their ligand binding sites.

Although this review has been focused mainly on adult Drosophila, these two families of receptors are also present in larvae. However, few studies focus on larval chemosensory modalities. Twenty-five members of the OR family are expressed in the dorsal organ, the olfactory receptor organ in larvae (Fishilevich et al., 2005) and several studies have characterized their olfactory responses via behavioral tests (Fishilevich et al., 2005; Gomez-Marin et al., 2011) and electrophysiology measures (Hoare et al., 2008; Mathew et al., 2013). As we already mentioned, recent studies on larval IRs (Croset et al., 2016; Sánchez-Alcañiz et al., 2018) have shown their involvement in different taste modalities. Because there are both larval-specific ORs and larval-specific IRs, it could be hypothesized that there might be some larval-exclusive sensory modalities adaptations that have not been investigated yet. Further research on both families of larval chemosensory receptors will be needed to answer this question.

Importantly, much evidence of both expression and functional roles is coming from the use of Gal-4 lines, which are a extremely useful tool in the field but also show some caveats. Surely, the generation of more knockout mutants for the different receptors will answer some of the controversies caused by the caveats of using RNAi knockdown strategies.

Another question that will be addressed in the future is that the most ecologically relevant ligands may not have been found yet. Olfactory receptors are considered narrowly or broadly tuned based on analysis of ligands that may not be relevant at all for the fly (Bohbot and Pitts, 2015). Few works have linked olfactory ecology to structural and regulatory genetic

\section{REFERENCES}

Abuin, L., Bargeton, B., Ulbrich, M. H., Isacoff, E. Y., Kellenberger, S., and Benton, R. (2011). Functional architecture of olfactory ionotropic glutamate receptors. Neuron 69, 44-60. doi: 10.1016/j.neuron.2010.11.042

Ahn, J.-E., Chen, Y., and Amrein, H. (2017). Molecular basis of fatty acid taste in Drosophila. Elife 6:e30115. doi: 10.7554/eLife.30115

Ai, M., Blais, S., Park, J.-Y., Min, S., Neubert, T. A., and Suh, G. S. B. (2013). Ionotropic glutamate receptors IR64a and IR8a form a functional odorant receptor complex in vivo in Drosophila. J. Neurosci. 33, 10741-10749. doi: 10.1523/JNEUROSCI.5419-12.2013

Alkhori, L., Oest, A., and Alenius, M. (2014). The corepressor Atrophin specifies odorant receptor expression in Drosophila. FASEB J. 28, 1355-1364. doi: 10.1096/fj.13-240325

Arguello, J. R., and Benton, R. (2017). Open questions: tackling Darwin's "instincts": the genetic basis of behavioral evolution. BMC Biol. 15:26. doi: 10.1186/s12915-017-0369-3

Arguello, J. R., Cardoso-Moreira, M., Grenier, J. K., Gottipati, S., Clark, A. G., and Benton, R. (2016). Extensive local adaptation within the chemosensory system following Drosophila melanogaster's global expansion. Nat. Commun. 7:ncomms11855. doi: 10.1038/ncomms11855

Bahk, S., and Jones, W. D. (2016). Insect odorant receptor trafficking requires calmodulin. BMC Biol. 14:83. doi: 10.1186/s12915-016-0306-X changes in the chemoreceptor families (Prieto-Godino et al., 2017), but in upcoming years, new genome-editing technologies and the advancement of next-generation sequencing in insect species other than Drosophila will shed light on the function and evolution of both the OR and IR families (Arguello and Benton, 2017), and such work will have repercussions for controlling pests and diseases transmitted by insect vectors (van der Goes van Naters and Carlson, 2006; Crava et al., 2016; Benton, 2017).

In this review, we have focused on the main peripheral chemosensory systems at the receptor level (Table 1), but the interaction between OR- and IR-related circuits in both first relay and higher processing brain centers (Grabe and Sachse, 2018) is mainly unexplored and of outstanding interest for elucidating the behavioral output of the individual to chemical cues.

\section{AUTHOR CONTRIBUTIONS}

All the authors listed made substantial contributions to this review. CG-D, FM and EA drafted the initial manuscript. All authors critically read, corrected and approved the final version of the manuscript.

\section{FUNDING}

This study was supported by the Spanish Ministry of Economy and Competitiveness (SAF2013-48759-P, EA and BFU201785882-P, EA/CG-D), the Gobierno del Principado de Asturias (GRUPIN14-012; EA), the University of Oviedo (PAPI17PEMERG-2; CG-D) and FEDER Funds.

\section{ACKNOWLEDGMENTS}

We apologize to those scientists whose work has not been cited due to space constraints.

Bai, L., and Carlson, J. R. (2010). Distinct functions of acj6 splice forms in odor receptor gene choice. J. Neurosci. 30, 5028-5036. doi: 10.1523/JNEUROSCI. 6292-09.2010

Barish, S., and Volkan, P. C. (2015). Mechanisms of olfactory receptor neuron specification in Drosophila. Wiley Interdiscip. Rev. Dev. Biol. 4, 609-621. doi: $10.1002 /$ wdev.197

Benton, R. (2017). The neurobiology of gustation in insect disease vectors: progress and potential. Curr. Opin. Insect Sci. 20, 19-27. doi: 10.1016/j.cois.2017. 02.003

Benton, R., Sachse, S., Michnick, S. W., and Vosshall, L. B. (2006). Atypical membrane topology and heteromeric function of Drosophila odorant receptors in vivo. PLoS Biol. 4:e20. doi: 10.1371/journal.pbio.0040020

Benton, R., Vannice, K. S., Gomez-Diaz, C., and Vosshall, L. B. (2009). Variant ionotropic glutamate receptors as chemosensory receptors in Drosophila. Cell 136, 149-162. doi: 10.1016/j.cell.2008.12.001

Benton, R., Vannice, K. S., and Vosshall, L. B. (2007). An essential role for a CD36-related receptor in pheromone detection in Drosophila. Nature 450, 289-293. doi: 10.1038/nature06328

Bohbot, J. D., and Pitts, R. J. (2015). The narrowing olfactory landscape of insect odorant receptors. Front. Ecol. Evol. 3:39. doi: 10.3389/fevo.2015.00039

Boyle, S. M., McInally, S., and Ray, A. (2013). Expanding the olfactory code by in silico decoding of odor-receptor chemical space. Elife 2:e01120. doi: 10.7554/eLife.01120 
Buck, L. B. (2000). The molecular architecture of odor and pheromone sensing in mammals. Cell 100, 611-618. doi: 10.1016/s0092-8674(00)80698-4

Buck, L. B., and Axel, R. (1991). A novel multigene family may encode odorant receptors: a molecular basis for odor recognition. Cell 65, 175-187. doi: 10.1016/0092-8674(91)90418-x

Cao, L.-H., Jing, B.-Y., Yang, D., Zeng, X., Shen, Y., Tu, Y., et al. (2016). Distinct signaling of Drosophila chemoreceptors in olfactory sensory neurons. Proc. Natl. Acad. Sci. U S A 113, E902-E911. doi: 10.1073/pnas.1518329113

Carraher, C., Dalziel, J., Jordan, M. D., Christie, D. L., Newcomb, R. D., and Kralicek, A. V. (2015). Towards an understanding of the structural basis for insect olfaction by odorant receptors. Insect Biochem. Mol. Biol. 66, 31-41. doi: 10.1016/j.ibmb.2015.09.010

Chai, P. C., Cruchet, S., Wigger, L., and Benton, R. (2018). Sensory neuron lineage mapping and manipulation in the Drosophila olfactory system. bioRxiv [Preprint]. doi: 10.1101/312074

Chatterjee, A., Roman, G., and Hardin, P. E. (2009). Go contributes to olfactory reception in Drosophila melanogaster. BMC Physiol. 9:22. doi: 10.1186/14726793-9-22

Chen, Y., and Amrein, H. (2017). Ionotropic receptors mediate Drosophila oviposition preference through sour gustatory receptor neurons. Curr. Biol. 27, 2741.e4-2750.e4. doi: 10.1016/j.cub.2017.08.003

Chen, C., Buhl, E., Xu, M., Croset, V., Rees, J. S., Lilley, K. S., et al. (2015). Drosophila ionotropic receptor $25 \mathrm{a}$ mediates circadian clock resetting by temperature. Nature 527, 516-520. doi: 10.1038/nature16148

Clyne, P. J., Certel, S. J., de Bruyne, M., Zaslavsky, L., Johnson, W. A., and Carlson, J. R. (1999a). The odor specificities of a subset of olfactory receptor neurons are governed by Acj6, a POU-domain transcription factor. Neuron 22, 339-347. doi: 10.1016/s0896-6273(00)81094-6

Clyne, P. J., Warr, C. G., Freeman, M. R., Lessing, D., Kim, J. H., and Carlson, J. R. (1999b). A novel family of divergent seven-transmembrane proteins: candidate odorant receptors in Drosophila. Neuron 22, 327-338. doi: 10.1016/S08966273(00)81093-4

Clyne, P. J., Grant, A., O'Connell, R., and Carlson, J. R. (1997). Odorant response of individual sensilla on the Drosophila antenna. Invert. Neurosci. 3, 127-135. doi: $10.1007 / \mathrm{bf} 02480367$

Couto, A., Alenius, M., and Dickson, B. J. (2005). Molecular, anatomical, and functional organization of the Drosophila olfactory system. Curr. Biol. 15, 1535-1547. doi: 10.1016/j.cub.2005.07.034

Crava, C. M., Ramasamy, S., Ometto, L., Anfora, G., and Rota-Stabelli, O. (2016). Evolutionary insights into taste perception of the invasive pest Drosophila suzukii. G3 6, 4185-4196. doi: 10.1534/g3.116.036467

Croset, V., Rytz, R., Cummins, S. F., Budd, A., Brawand, D., Kaessmann, H., et al. (2010). Ancient protostome origin of chemosensory ionotropic glutamate receptors and the evolution of insect taste and olfaction. PLoS Genet. 6:e1001064. doi: 10.1371/journal.pgen.1001064

Croset, V., Schleyer, M., Arguello, J. R., Gerber, B., and Benton, R. (2016). A molecular and neuronal basis for amino acid sensing in the Drosophila larva. Sci. Rep. 6:34871. doi: 10.1038/srep34871

de Bruyne, M., Clyne, P. J., and Carlson, J. R. (1999). Odor coding in a model olfactory organ: the Drosophila maxillary palp. J. Neurosci. 19, 4520-4532. doi: 10.1523/JNEUROSCI.19-11-04520.1999

de Bruyne, M., Foster, K., and Carlson, J. R. (2001). Odor coding in the Drosophila antenna. Neuron 30, 537-552. doi: 10.1016/s0896-6273(01)00289-6

de Fouchier, A., Walker, W. B. III., Montagné, N., Steiner, C., Binyameen, M., Schlyter, F., et al. (2017). Functional evolution of Lepidoptera olfactory receptors revealed by deorphanization of a moth repertoire. Nat. Commun. 8:15709. doi: $10.1038 /$ ncomms 15709

Deng, Y., Zhang, W., Farhat, K., Oberland, S., Gisselmann, G., and Neuhaus, E. M. (2011). The stimulatory $G \alpha_{s}$ protein is involved in olfactory signal transduction in Drosophila. PLoS One 6:e18605. doi: 10.1371/journal.pone. 0018605

Dippel, S., Kollmann, M., Oberhofer, G., Montino, A., Knoll, C., Krala, M., et al. (2016). Morphological and transcriptomic analysis of a beetle chemosensory system reveals a gnathal olfactory center. BMC Biol. 14:90. doi: 10.1186/s12915016-0304-Z

Endo, K., Aoki, T., Yoda, Y., Kimura, K.-I., and Hama, C. (2007). Notch signal organizes the Drosophila olfactory circuitry by diversifying the sensory neuronal lineages. Nat. Neurosci. 10, 153-160. doi: 10.1038/nn1832
Engsontia, P., Sanderson, A. P., Cobb, M., Walden, K. K. O., Robertson, H. M., and Brown, S. (2008). The red flour beetle's large nose: an expanded odorant receptor gene family in Tribolium castaneum. Insect Biochem. Mol. Biol. 38, 387-397. doi: 10.1016/j.ibmb.2007.10.005

Enjin, A., Zaharieva, E. E., Frank, D. D., Mansourian, S., Suh, G. S. B., Gallio, M., et al. (2016). Humidity sensing in Drosophila. Curr. Biol. 26, 1352-1358. doi: 10.1016/j.cub.2016.03.049

Fishilevich, E., Domingos, A. I., Asahina, K., Naef, F., Vosshall, L. B., and Louis, M. (2005). Chemotaxis behavior mediated by single larval olfactory neurons in Drosophila. Curr. Biol. 15, 2086-2096. doi: 10.1016/j.cub.2005. 11.016

Fishilevich, E., and Vosshall, L. B. (2005). Genetic and functional subdivision of the Drosophila antennal lobe. Curr. Biol. 15, 1548-1553. doi: 10.1016/j.cub.2005. 07.066

Fleischer, J., Pregitzer, P., Breer, H., and Krieger, J. (2018). Access to the odor world: olfactory receptors and their role for signal transduction in insects. Cell. Mol. Life Sci. 75, 485-508. doi: 10.1007/s00018-017-2627-5

Fox, A. N., Pitts, R. J., Robertson, H. M., Carlson, J. R., and Zwiebel, L. J. (2001). Candidate odorant receptors from the malaria vector mosquito Anopheles gambiae and evidence of down-regulation in response to blood feeding. Proc. Natl. Acad. Sci. U S A 98, 14693-14697. doi: 10.1073/pnas.261432998

Fuss, S. H., and Ray, A. (2009). Mechanisms of odorant receptor gene choice in Drosophila and vertebrates. Mol. Cell. Neurosci. 41, 101-112. doi: 10.1016/j. mcn.2009.02.014

Galindo, K., and Smith, D. P. (2001). A large family of divergent Drosophila odorant-binding proteins expressed in gustatory and olfactory sensilla. Genetics $159,1059-1072$.

Galizia, C. G., Münch, D., Strauch, M., Nissler, A., and Ma, S. (2010). Integrating heterogeneous odor response data into a common response model: a DoOR to the complete olfactome. Chem. Senses 35, 551-563. doi: 10.1093/chemse/ bjq042

Ganguly, A., Pang, L., Duong, V.-K., Lee, A., Schoniger, H., Varady, E., et al. (2017). A molecular and cellular context-dependent role for Ir76b in detection of amino acid taste. Cell Rep. 18, 737-750. doi: 10.1016/j.celrep.2016.12.071

Gao, Q., and Chess, A. (1999). Identification of candidate Drosophila olfactory receptors from genomic DNA sequence. Genomics 60, 31-39. doi: 10.1006/geno.1999.5894

Goldman, A. L., Van der Goes van Naters, W., Lessing, D., Warr, C. G., and Carlson, J. R. (2005). Coexpression of two functional odor receptors in one neuron. Neuron 45, 661-666. doi: 10.1016/j.neuron.2005.01.025

Gomez-Diaz, C., Bargeton, B., Abuin, L., Bukar, N., Reina, J. H., Bartoi, T., et al. (2016). A CD36 ectodomain mediates insect pheromone detection via a putative tunnelling mechanism. Nat. Commun. 7:11866. doi: $10.1038 /$ ncomms11866

Gomez-Diaz, C., Reina, J. H., Cambillau, C., and Benton, R. (2013). Ligands for pheromone-sensing neurons are not conformationally activated odorant binding proteins. PLoS Biol. 11:e1001546. doi: 10.1371/journal.pbio.1001546

Gomez-Marin, A., Stephens, G. J., and Louis, M. (2011). Active sampling and decision making in Drosophila chemotaxis. Nat. Commun. 2:441. doi: $10.1038 /$ ncomms1455

Goulding, S. E., zur Lage, P., and Jarman, A. P. (2000). amos, a proneural gene for Drosophila olfactory sense organs that is regulated by lozenge. Neuron 25 , 69-78. doi: 10.1016/s0896-6273(00)80872-7

Grabe, V., and Sachse, S. (2018). Fundamental principles of the olfactory code. Biosystems 164, 94-101. doi: 10.1016/j.biosystems.2017.10.010

Gramates, L. S., Marygold, S. J., Santos, G. D., Urbano, J.-M., Antonazzo, G., Matthews, B. B., et al. (2017). FlyBase at 25: looking to the future. Nucleic Acids Res. 45, D663-D671. doi: 10.1093/nar/gkw1016

Grosjean, Y., Rytz, R., Farine, J.-P., Abuin, L., Cortot, J., Jefferis, G. S. X. E., et al. (2011). An olfactory receptor for food-derived odours promotes male courtship in Drosophila. Nature 478, 236-240. doi: 10.1038/nature10428

Grosse-Wilde, E., Kuebler, L. S., Bucks, S., Vogel, H., Wicher, D., and Hansson, B. S. (2011). Antennal transcriptome of Manduca sexta. Proc. Natl. Acad. Sci. U S A 108, 7449-7454. doi: 10.1073/pnas.1017963108

Guo, H., and Smith, D. P. (2017). Odorant receptor desensitization in insects. J. Exp. Neurosci. 11:1179069517748600. doi: 10.1177/1179069517748600

Gupta, B. P., Flores, G. V., Banerjee, U., and Rodrigues, V. (1998). Patterning an epidermal field: Drosophila Lozenge, a member of the AML-1/Runt family of 
transcription factors, specifies olfactory sense organ type in a dose-dependent manner. Dev. Biol. 203, 400-411. doi: 10.1006/dbio.1998.9064

Gupta, B. P., and Rodrigues, V. (1997). Atonal is a proneural gene for a subset of olfactory sense organs in Drosophila. Genes Cells 2, 225-233. doi: 10.1046/j. 1365-2443.1997.d01-312.x

Hallem, E. A., and Carlson, J. R. (2006). Coding of odors by a receptor repertoire. Cell 125, 143-160. doi: 10.1016/j.cell.2006.01.050

Hallem, E. A., Ho, M. G., and Carlson, J. R. (2004). The molecular basis of odor coding in the Drosophila antenna. Cell 117, 965-979. doi: 10.1016/j.cell.2004. 05.012

Halty-deLeon, L., Miazzi, F., Kaltofen, S., Hansson, B. S., and Wicher, D. (2016). The mouse receptor transporting protein RTP1S and the fly SNMP1 support the functional expression of the Drosophila odorant coreceptor Orco in mammalian culture cells. J. Neurosci. Methods 271, 149-153. doi: 10.1016/j. jneumeth.2016.07.005

Hill, C. A., Fox, A. N., Pitts, R. J., Kent, L. B., Tan, P. L., Chrystal, M. A., et al. (2002). G protein coupled receptors in Anopheles gambiae. Science 298, 176-178. doi: 10.1126/science.1076196

Hoare, D. J., McCrohan, C. R., and Cobb, M. (2008). Precise and fuzzy coding by olfactory sensory neurons. J. Neurosci. 28, 9710-9722. doi: 10.1523/JNEUROSCI.1955-08.2008

Hopf, T. A., Morinaga, S., Ihara, S., Touhara, K., Marks, D. S., and Benton, R. (2015). Amino acid coevolution reveals three-dimensional structure and functional domains of insect odorant receptors. Nat. Commun. 6:6077. doi: $10.1038 /$ ncomms 7077

Hsieh, Y.-W., Alqadah, A., and Chuang, C.-F. (2017). Mechanisms controlling diversification of olfactory sensory neuron classes. Cell. Mol. Life Sci. 74, 3263-3274. doi: 10.1007/s00018-017-2512-2

Hussain, A., Zhang, M., Uecpunar, H. K., Svensson, T., Quillery, E., Gompel, N., et al. (2016). Ionotropic chemosensory receptors mediate the taste and smell of polyamines. PLoS Biol. 14:e1002454. doi: 10.1371/journal.pbio. 1002454

Ignatious Raja, J. S., Katanayeva, N., Katanaev, V. L., and Galizia, C. G. (2014). Role of $\mathrm{G}(\mathrm{o} / \mathrm{i})$ subgroup of $\mathrm{G}$ proteins in olfactory signaling of Drosophila melanogaster. Eur. J. Neurosci. 39, 1245-1255. doi: 10.1111/ejn.12481

Jafari, S., Alkhori, L., Schleiffer, A., Brochtrup, A., Hummel, T., and Alenius, M. (2012). Combinatorial activation and repression by seven transcription factors specify Drosophila odorant receptor expression. PLoS Biol. 10:e1001280. doi: 10.1371/journal.pbio.1001280

Jiang, X. C., Pregitzer, P., Grosse-Wilde, E., Breer, H., and Krieger, J. (2016). Identification and characterization of two "sensory neuron membrane proteins" (SNMPs) of the desert locust, schistocerca gregaria (Orthoptera: Acrididae). J. Insect Sci. 16:33. doi: 10.1093/jisesa/iew015

Jin, X., Ha, T. S., and Smith, D. P. (2008). SNMP is a signaling component required for pheromone sensitivity in Drosophila. Proc. Natl. Acad. Sci. U S A 105, 10996-11001. doi: 10.1073/pnas.0803309105

Jones, W. D., Cayirlioglu, P., Kadow, I. G., and Vosshall, L. B. (2007). Two chemosensory receptors together mediate carbon dioxide detection in Drosophila. Nature 445, 86-90. doi: 10.1038/nature05466

Jones, W. D., Nguyen, T. A. T., Kloss, B., Lee, K. J., and Vosshall, L. B. (2005). Functional conservation of an insect odorant receptor gene across 250 million years of evolution. Curr. Biol. 15, R119-R121. doi: 10.1016/j.cub.2005.02.007

Joseph, R. M., and Carlson, J. R. (2015). Drosophila chemoreceptors: a molecular interface between the chemical world and the brain. Trends Genet. 31, 683-695. doi: 10.1016/j.tig.2015.09.005

Joseph, R. M., Sun, J. S., Tam, E., and Carlson, J. R. (2017). A receptor and neuron that activate a circuit limiting sucrose consumption. Elife 6:e24992. doi: 10.7554/eLife.24992

Kain, P., Chakraborty, T. S., Sundaram, S., Siddiqi, O., Rodrigues, V., and Hasan, G. (2008). Reduced odor responses from antennal neurons of $\mathrm{G}_{\mathrm{q}} \alpha$, phospholipase C $\beta$, and $r d g \mathrm{~A}$ mutants in Drosophila support a role for a phospholipid intermediate in insect olfactory transduction. J. Neurosci. 28, 4745-4755. doi: 10.1523/JNEUROSCI.5306-07.2008

Kanost, M. R., Arrese, E. L., Cao, X. L., Chen, Y. R., Chellapilla, S., Goldsmith, M. R., et al. (2016). Multifaceted biological insights from a draft genome sequence of the tobacco hornworm moth, Manduca sexta. Insect Biochem. Mol. Biol. 76, 118-147. doi: 10.1016/j.ibmb.2016. 07.005
Knecht, Z. A., Silbering, A. F., Cruz, J., Yang, L., Croset, V., Benton, R., et al. (2017). lonotropic Receptor-dependent moist and dry cells control hygrosensation in Drosophila. Elife 6:e26654. doi: 10.7554/eLife.26654

Knecht, Z. A., Silbering, A. F., Ni, L., Klein, M., Budelli, G., Bell, R., et al. (2016). Distinct combinations of variant ionotropic glutamate receptors mediate thermosensation and hygrosensation in Drosophila. Elife 5:e17879. doi: 10.7554/eLife.17879

Koenig, C., Hirsh, A., Bucks, S., Klinner, C., Vogel, H., Shukla, A., et al. (2015). A reference gene set for chemosensory receptor genes of Manduca sexta. Insect Biochem. Mol. Biol. 66, 51-63. doi: 10.1016/j.ibmb.2015. 09.007

Koh, T.-W., He, Z., Gorur-Shandilya, S., Menuz, K., Larter, N. K., Stewart, S., et al. (2014). The Drosophila IR20a clade of ionotropic receptors are candidate taste and pheromone receptors. Neuron 83, 850-865. doi: 10.1016/j.neuron.2014. 07.012

Kopp, A., Barmina, O., Hamilton, A. M., Higgins, L., Mcintyre, L. M., and Jones, C. D. (2008). Evolution of gene expression in the Drosophila olfactory system. Mol. Biol. Evol. 25, 1081-1092. doi: 10.1093/molbev/ msn055

Krieger, J., Klink, O., Mohl, C., Raming, K., and Breer, H. (2003). A candidate olfactory receptor subtype highly conserved across different insect orders. J. Comp. Physiol. A Neuroethol. Sens. Neural Behav. Physiol. 189, 519-526. doi: 10.1007/s00359-003-0427-x

Kumar, B. N., Taylor, R. W., Pask, G. M., Zwiebel, L. J., Newcomb, R. D., and Christie, D. L. (2013). A conserved aspartic acid is important for agonist (VUAA1) and odorant/tuning receptor-dependent activation of the insect odorant co-receptor (Orco). PLoS One 8:e70218. doi: 10.1371/journal.pone. 0070218

Kurtovic, A., Widmer, A., and Dickson, B. J. (2007). A single class of olfactory neurons mediates behavioural responses to a Drosophila sex pheromone. Nature 446, 542-546. doi: 10.1038/nature05672

Kwon, J. Y., Dahanukar, A., Weiss, L. A., and Carlson, J. R. (2007). The molecular basis of $\mathrm{CO} 2$ reception in Drosophila. Proc. Natl. Acad. Sci. U S A 104, 3574-3578. doi: 10.1073/pnas.0700079104

Larsson, M. C., Domingos, A. I., Jones, W. D., Chiappe, M. E., Amrein, H., and Vosshall, L. B. (2004). Or83b encodes a broadly expressed odorant receptor essential for Drosophila olfaction. Neuron 43, 703-714. doi: 10.1016/j.neuron. 2004.08.019

Leal, W. S. (2013). Odorant reception in insects: roles of receptors, binding proteins, and degrading enzymes. Annu. Rev. Entomol. 58, 373-391. doi: 10.1146/annurev-ento-120811-153635

Lee, Y., Poudel, S., Kim, Y., Thakur, D., and Montell, C. (2018). Calcium taste avoidance in Drosophila. Neuron 97, 67.e4-74.e4. doi: 10.1016/j.neuron.2017. 11.038

Lee, M. J., Sung, H. Y., Jo, H., Kim, H.-W., Choi, M. S., Kwon, J. Y., et al. (2017). Ionotropic receptor $76 \mathrm{~b}$ is required for gustatory aversion to excessive $\mathrm{Na}^{+}$in Drosophila. Mol. Cells 40, 787-795. doi: 10.14348/molcells.2017.0160

Li, Q., Barish, S., Okuwa, S., Maciejewski, A., Brandt, A. T., Reinhold, D., et al. (2016). A functionally conserved gene regulatory network module governing olfactory neuron diversity. PLoS Genet. 12:e1005780. doi: 10.1371/journal. pgen. 1005780

Li, Z., Ni, J. D., Huang, J., and Montell, C. (2014). Requirement for Drosophila SNMP1 for rapid activation and termination of pheromone-induced activity. PLoS Genet. 10:e1004600. doi: 10.1371/journal.pgen.1004600

Liman, E. R., Zhang, Y. V., and Montell, C. (2014). Peripheral coding of taste. Neuron 81, 984-1000. doi: 10.1016/j.neuron.2014.02.022

Lin, C.-C., and Potter, C. J. (2015). Re-classification of Drosophila melanogaster trichoid and intermediate sensilla using fluorescence-guided single sensillum recording. PLoS One 10:e0139675. doi: 10.1371/journal.pone.0139675

Lundin, C., Käll, L., Kreher, S. A., Kapp, K., Sonnhammer, E. L., Carlson, J. R., et al. (2007). Membrane topology of the Drosophila OR83b odorant receptor. FEBS Lett. 581, 5601-5604. doi: 10.1016/j.febslet.2007.11.007

Martelli, C., Carlson, J. R., and Emonet, T. (2013). Intensity invariant dynamics and odor-specific latencies in olfactory receptor neuron response. J. Neurosci. 33, 6285-6297. doi: 10.1523/JNEUROSCI.0426-12.2013

Martin, F., and Alcorta, E. (2016). Measuring activity in olfactory receptor neurons in Drosophila: focus on spike amplitude. J. Insect Physiol. 95, 23-41. doi: 10.1016/j.jinsphys.2016.09.003 
Martin, F., Boto, T., Gomez-Diaz, C., and Alcorta, E. (2013). Elements of olfactory reception in adult Drosophila melanogaster. Anat. Rec. 296, 1477-1488. doi: 10.1002/ar.22747

Martin, F., Riveron, J., and Alcorta, E. (2011). Environmental temperature modulates olfactory reception in Drosophila melanogaster. J. Insect Physiol. 57, 1631-1642. doi: 10.1016/j.jinsphys.2011.08.016

Mathew, D., Martelli, C., Kelley-Swift, E., Brusalis, C., Gershow, M., Samuel, A. D. T., et al. (2013). Functional diversity among sensory receptors in a Drosophila olfactory circuit. Proc. Natl. Acad. Sci. U S A 110, E2134-E2143. doi: 10.1073/pnas.1306976110

McKenzie, S. K., Oxley, P. R., and Kronauer, D. J. C. (2014). Comparative genomics and transcriptomics in ants provide new insights into the evolution and function of odorant binding and chemosensory proteins. BMC Genomics 15:718. doi: 10.1186/1471-2164-15-718

Miazzi, F., Hansson, B. S., and Wicher, D. (2016). Odor-induced cAMP production in Drosophila melanogaster olfactory sensory neurons. J. Exp. Biol. 219, 1798-1803. doi: 10.1242/jeb.137901

Miller, C. J., and Carlson, J. R. (2010). Regulation of odor receptor genes in trichoid sensilla of the Drosophila antenna. Genetics 186, 79-95. doi: 10.1534/genetics. 110.117622

Min, S., Ai, M., Shin, S. A., and Suh, G. S. B. (2013). Dedicated olfactory neurons mediating attraction behavior to ammonia and amines in Drosophila. Proc. Natl. Acad. Sci. U S A 110, E1321-E1329. doi: 10.1073/pnas.1215 680110

Missbach, C., Dweck, H. K. M., Vogel, H., Vilcinskas, A., Stensmyr, M. C., Hansson, B. S., et al. (2014). Evolution of insect olfactory receptors. Elife 3:e02115. doi: 10.7554/eLife.02115

Münch, D., and Galizia, C. G. (2016). DoOR 2.0-comprehensive mapping of Drosophila melanogaster odorant responses. Sci. Rep. 6:21841. doi: 10.1038/srep21841

Mukunda, L., Miazzi, F., Kaltofen, S., Hansson, B. S., and Wicher, D. (2014). Calmodulin modulates insect odorant receptor function. Cell Calcium 55, 191-199. doi: 10.1016/j.ceca.2014.02.013

Murmu, M. S., and Martin, J. R. (2016). Interaction between cAMP and intracellular $\mathrm{Ca}^{2+}$-signaling pathways during odor-perception and adaptation in Drosophila. Biochim. Biophys. Acta 1863, 2156-2174. doi: 10.1016/j.bbamcr. 2016.05.014

Nagel, K. I., and Wilson, R. I. (2011). Biophysical mechanisms underlying olfactory receptor neuron dynamics. Nat. Neurosci. 14, 208-216. doi: 10.1038/nn.2725

Nakagawa, T., and Vosshall, L. B. (2009). Controversy and consensus: noncanonical signaling mechanisms in the insect olfactory system. Curr. Opin. Neurobiol. 19, 284-292. doi: 10.1016/j.conb.2009.07.015

Neuhaus, E. M., Gisselmann, G., Zhang, W. Y., Dooley, R., Störtkuhl, K., and Hatt, H. (2005). Odorant receptor heterodimerization in the olfactory system of Drosophila melanogaster. Nat. Neurosci. 8, 15-17. doi: 10.1038/nn1371

Ni, L., Klein, M., Svec, K. V., Budelli, G., Chang, E. C., Ferrer, A. J., et al. (2016). The Ionotropic Receptors IR21a and IR25a mediate cool sensing in Drosophila. Elife 5:e13254. doi: 10.7554/eLife.13254

Nolte, A., Funk, N. W., Mukunda, L., Gawalek, P., Werckenthin, A., Hansson, B. S., et al. (2013). in situ tip-recordings found no evidence for an orco-based ionotropic mechanism of pheromone-transduction in Manduca sexta. PLoS One 8:e62648. doi: 10.1371/journal.pone.0062648

Nolte, A., Gawalek, P., Koerte, S., Wei, H., Schumann, R., Werckenthin, A., et al. (2016). No evidence for ionotropic pheromone transduction in the hawkmoth Manduca sexta. PLoS One 11:e0166060. doi: 10.1371/journal.pone.0166060

Oxley, P. R., Ji, L., Fetter-Pruneda, I., Mckenzie, S. K., Li, C., Hu, H., et al. (2014). The genome of the clonal raider ant Cerapachys biroi. Curr. Biol. 24, 451-458. doi: 10.1016/j.cub.2014.01.018

Pask, G. M., Slone, J. D., Millar, J. G., Das, P., Moreira, J. A., Zhou, X., et al. (2017). Specialized odorant receptors in social insects that detect cuticular hydrocarbon cues and candidate pheromones. Nat. Commun. 8:297. doi: 10.1038/s41467017-00099-1

Pelletier, J., Xu, P., Yoon, K. S., Clark, J. M., and Leal, W. S. (2015). Odorant receptor-based discovery of natural repellents of human lice. Insect Biochem. Mol. Biol. 66, 103-109. doi: 10.1016/j.ibmb.2015.10.009

Peñalva-Arana, D. C., Lynch, M., and Robertson, H. M. (2009). The chemoreceptor genes of the waterflea Daphnia pulex: many Grs but no Ors. BMC Evol. Biol. 9:79. doi: 10.1186/1471-2148-9-79
Pitts, R. J., Derryberry, S. L., Zhang, Z., and Zwiebel, L. J. (2017). Variant ionotropic receptors in the malaria vector mosquito anopheles gambiae tuned to amines and carboxylic acids. Sci. Rep. 7:40297. doi: 10.1038/srep40297

Pitts, R. J., Fox, A. N., and Zwiebel, L. J. (2004). A highly conserved candidate chemoreceptor expressed in both olfactory and gustatory tissues in the malaria vector Anopheles gambiae. Proc. Natl. Acad. Sci. U S A 101, 5058-5063. doi: 10.1073/pnas.0308146101

Prieto-Godino, L. L., Rytz, R., Bargeton, B., Abuin, L., Arguello, J. R., Dal Peraro, M., et al. (2016). Olfactory receptor pseudo-pseudogenes. Nature 539, 93-97. doi: 10.1038/nature19824

Prieto-Godino, L. L., Rytz, R., Cruchet, S., Bargeton, B., Abuin, L., Silbering, A. F., et al. (2017). Evolution of acid-sensing olfactory circuits in Drosophilids. Neuron 93, 661-667. doi: 10.1016/j.neuron.2016.12.024

Ray, A., Van Naters, W. V. D. G., Shiraiwa, T., and Carlson, J. R. (2007). Mechanisms of odor receptor gene choice in Drosophila. Neuron 53, 353-369. doi: 10.1016/j.neuron.2006.12.010

Rimal, S., and Lee, Y. (2018). The multidimensional ionotropic receptors of Drosophila melanogaster. Insect Mol. Biol. 27, 1-7. doi: 10.1111/imb.12347

Riveron, J., Boto, T., and Alcorta, E. (2009). The effect of environmental temperature on olfactory perception in Drosophila melanogaster. J. Insect Physiol. 55, 943-951. doi: 10.1016/j.jinsphys.2009.06.009

Riveron, J., Boto, T., and Alcorta, E. (2013). Transcriptional basis of the acclimation to high environmental temperature at the olfactory receptor organs of Drosophila melanogaster. BMC Genomics 14:259. doi: 10.1186/1471-216414-259

Robertson, H. M., and Wanner, K. W. (2006). The chemoreceptor superfamily in the honey bee, Apis mellifera: expansion of the odorant, but not gustatory, receptor family. Genome Res. 16, 1395-1403. doi: 10.1101/gr. 5057506

Robertson, H. M., Warr, C. G., and Carlson, J. R. (2003). Molecular evolution of the insect chemoreceptor gene superfamily in Drosophila melanogaster. Proc. Natl. Acad. Sci. U S A 100, 14537-14542. doi: 10.1073/pnas.23358 47100

Rodrigues, V., and Hummel, H. (2008). Development of the Drosophila olfactory system. Adv. Exp. Med. Biol. 628, 82-101. doi: 10.1007/978-0-387-78261-4_6

Rogers, M. E., Krieger, J., and Vogt, R. G. (2001). Antennal SNMPs (sensor neuron membrane proteins) of lepidoptera define a unique family of invertebrate CD36-like proteins. J. Neurobiol. 49, 47-61. doi: 10.1002/neu.1065

Rogers, M. E., Sun, M., Lerner, M. R., and Vogt, R. G. (1997). Snmp-1, a novel membrane protein of olfactory neurons of the silk moth Antheraea polyphemus with homology to the CD36 family of membrane proteins. J. Biol. Chem. 272, 14792-14799. doi: 10.1074/jbc.272.23.14792

Rytz, R., Croset, V., and Benton, R. (2013). Ionotropic Receptors (IRs): chemosensory ionotropic glutamate receptors in Drosophila and beyond. Insect Biochem. Mol. Biol. 43, 888-897. doi: 10.1016/j.ibmb.2013.02.007

Sanchez, G. M., Alkhori, L., Hatano, E., Schultz, S. W., Kuzhandaivel, A., Jafari, S., et al. (2016). Hedgehog signaling regulates the ciliary transport of odorant receptors in Drosophila. Cell Rep. 14, 464-470. doi: 10.1016/j.celrep.2015. 12.059

Sánchez-Alcañiz, J. A., Silbering, A. F., Croset, V., Zappia, G., Sivasubramaniam, A. K., Abuin, L., et al. (2018). An expression atlas of chemosensory ionotropic glutamate receptors identifies a molecular basis of carbonation detection. bioRxiv [Preprint]. doi: 10.1101/278804

Sargsyan, V., Getahun, M. N., Llanos, S. L., Olsson, S. B., Hansson, B. S., and Wicher, D. (2011). Phosphorylation via PKC regulates the function of the Drosophila odorant co-receptor. Front. Cell. Neurosci. 5:5. doi: 10.3389/fncel. 2011.00005

Sato, K., Pellegrino, M., Nakagawa, T., Nakagawa, T., Vosshall, L. B., and Touhara, K. (2008). Insect olfactory receptors are heteromeric ligand-gated ion channels. Nature 452, 1002-1006. doi: 10.1038/nature06850

Schendzielorz, T., Schirmer, K., Stolte, P., and Stengl, M. (2015). Octopamine regulates antennal sensory neurons via daytime-dependent changes in cAMP and IP3 levels in the hawkmoth manduca sexta. PLoS One 10:e0121230. doi: 10.1371/journal.pone.0121230

Shanbhag, S. R., Hekmat-Scafe, D., Kim, M. S., Park, S. K., Carlson, J. R., Pikielny, C., et al. (2001). Expression mosaic of odorant-binding proteins in Drosophila olfactory organs. Microsc. Res. Tech. 55, 297-306. doi: 10.1002/ jemt.1179 
Shanbhag, S. R., Muller, B., and Steinbrecht, R. A. (1999). Atlas of olfactory organs of Drosophila melanogaster-1. Types, external organization, innervation, and distribution of olfactory sensilla. Int. J. Insect Morphol. Embryol. 28, 377-397. doi: 10.1016/S0020-7322(99)00039-2

Sharma, K. R., Enzmann, B. L., Schmidt, Y., Moore, D., Jones, G. R., Parker, J., et al. (2015). Cuticular hydrocarbon pheromones for social behavior and their coding in the Ant Antenna. Cell Rep. 12, 1261-1271. doi: 10.1016/j.celrep.2015. 07.031

Silbering, A. F., Bell, R., Münch, D., Cruchet, S., Gomez-Diaz, C., Laudes, T., et al. (2016). Ir40a neurons are not DEET detectors. Nature 534, E5-E7. doi: $10.1038 /$ nature 18321

Silbering, A. F., Rytz, R., Grosjean, Y., Abuin, L., Ramdya, P., Jefferis, G. S. X. E., et al. (2011). Complementary function and integrated wiring of the evolutionarily distinct Drosophila olfactory subsystems. J. Neurosci. 31, 13357-13375. doi: 10.1523/JNEUROSCI.2360-11.2011

Smadja, C., Shi, P., Butlin, R. K., and Robertson, H. M. (2009). Large gene family expansions and adaptive evolution for odorant and gustatory receptors in the pea aphid, acyrthosiphon pisum. Mol. Biol. Evol. 26, 2073-2086. doi: 10.1093/molbev/msp116

Smart, R., Kiely, A., Beale, M., Vargas, E., Carraher, C., Kralicek, A. V., et al. (2008). Drosophila odorant receptors are novel seven transmembrane domain proteins that can signal independently of heterotrimeric G proteins. Insect Biochem. Mol. Biol. 38, 770-780. doi: 10.1016/j.ibmb.2008.05.002

Song, Y., Ori-Mckenney, K. M., Zheng, Y., Han, C., Jan, L. Y., and Jan, Y. N. (2012). Regeneration of Drosophila sensory neuron axons and dendrites is regulated by the Akt pathway involving Pten and microRNA bantam. Genes Dev. 26, 1612-1625. doi: 10.1101/gad.193243.112

Steck, K., Walker, S. J., Itskov, P. M., Baltaza, C., Moreira, J.-M., and Ribeiro, C. (2018). Internal amino acid state modulates yeast taste neurons to support protein homeostasis in Drosophila. Elife 7:e31625. doi: 10.7554/eLife.31625

Stengl, M. (2010). Pheromone transduction in moths. Front. Cell. Neurosci. 4:33. doi: $10.3389 /$ fncel.2010.00133

Stengl, M., and Funk, N. W. (2013). The role of the coreceptor Orco in insect olfactory transduction. J. Comp. Physiol. A Neuroethol. Sens. Neural Behav. Physiol. 199, 897-909. doi: 10.1007/s00359-013-0837-3

Stocker, R. F. (2001). Drosophila as a focus in olfactory research: mapping of olfactory sensilla by fine structure, odor specificity, odorant receptor expression and central connectivity. Microsc. Res. Tech. 55, 284-296. doi: 10.1002/ jemt.1178

Störtkuhl, K. F., Hovemann, B. T., and Carlson, J. R. (1999). Olfactory adaptation depends on the trp $\mathrm{Ca}^{2+}$ channel in Drosophila. J. Neurosci. 19, 4839-4846. doi: 10.1523/jneurosci.19-12-04839.1999

$\mathrm{Su}, \mathrm{C} .-\mathrm{Y} ., \mathrm{Menuz}, \mathrm{K}$, and Carlson, J. R. (2009). Olfactory perception: receptors, cells and circuits. Cell 139, 45-59. doi: 10.1016/j.cell.2009.09.015

Swarup, S., Williams, T. I., and Anholt, R. R. H. (2011). Functional dissection of Odorant binding protein genes in Drosophila melanogaster. Genes Brain Behav. 10, 648-657. doi: 10.1111/j.1601-183x.2011.00704.x

Szyszka, P., and Galizia, C. G. (2018). The role of the sucrose-responsive IR60b neuron for Drosophila melanogaster: a hypothesis. Chem. Senses 43, 311-312. doi: 10.1093/chemse/bjy020

Szyszka, P., Gerkin, R. C., Galizia, C. G., and Smith, B. H. (2014). High-speed odor transduction and pulse tracking by insect olfactory receptor neurons. Proc. Natl. Acad. Sci. U S A 111, 16925-16930. doi: 10.1073/pnas.1412051111

Tauber, J. M., Brown, E. B., Li, Y., Yurgel, M. E., Masek, P., and Keene, A. C. (2017). A subset of sweet-sensing neurons identified by IR56d are necessary and sufficient for fatty acid taste. PLoS Genet. 13:e1007059. doi: 10.1371/journal. pgen.1007059

Tichy, A. L., Ray, A., and Carlson, J. R. (2008). A new Drosophila POU gene, $\mathrm{pdm} 3$, acts in odor receptor expression and axon targeting of olfactory neurons. J. Neurosci. 28, 7121-7129. doi: 10.1523/JNEUROSCI.2063-08.2008

Touhara, K., and Vosshall, L. B. (2009). Sensing odorants and pheromones with chemosensory receptors. Annu. Rev. Physiol. 71, 307-332. doi: 10.1146/annurev.physiol.010908.163209

Troemel, E. R., Chou, J. H., Dwyer, N. D., Colbert, H. A., and Bargmann, C. I. (1995). Divergent 7 transmembrane receptors are candidate chemosensory receptors in C. elegans. Cell 83, 207-218. doi: 10.1016/0092-8674(95)90162-0 van der Goes van Naters, W., and Carlson, J. R. (2006). Insects as chemosensors of humans and crops. Nature 444, 302-307. doi: 10.1038/nature05403

van der Goes van Naters, W., and Carlson, J. R. (2007). Receptors and neurons for fly odors in Drosophila. Curr. Biol. 17, 606-612. doi: 10.1016/j.cub.2007. 02.043

Vizueta, J., Frías-López, C., Macías-Hernández, N., Arnedo, M. A., SánchezGracia, A., and Rozas, J. (2017). Evolution of chemosensory gene families in arthropods: insight from the first inclusive comparative transcriptome analysis across spider appendages. Genome Biol. Evol. 9, 178-196. doi: 10.1093/gbe/evw296

Vogt, R. G., and Riddiford, L. M. (1981). Pheromone binding and inactivation by moth antennae. Nature 293, 161-163. doi: 10.1038/293161a0

Vosshall, L. B., Amrein, H., Morozov, P. S., Rzhetsky, A., and Axel, R. (1999). A spatial map of olfactory receptor expression in the Drosophila antenna. Cell 96, 725-736. doi: 10.1016/s0092-8674(00)80582-6

Vosshall, L. B., and Stocker, R. E. (2007). Molecular architecture of smell and taste in Drosophila. Annu. Rev. Neurosci. 30, 505-533. doi: 10.1146/annurev.neuro. 30.051606 .094306

Vosshall, L. B., Wong, A. M., and Axel, R. (2000). An olfactory sensory map in the fly brain. Cell 102, 147-159. doi: 10.1016/s0092-8674(00) 00021-0

Wicher, D. (2010). Design principles of sensory receptors. Front. Cell. Neurosci. 4:25. doi: $10.3389 /$ fncel. 2010.00025

Wicher, D., Schäfer, R., Bauernfeind, R., Stensmyr, M. C., Heller, R., Heinemann, S. H., et al. (2008). Drosophila odorant receptors are both ligandgated and cyclic-nucleotide-activated cation channels. Nature 452, 1007-1011. doi: $10.1038 /$ nature06861

Wilson, R. I. (2013). Early olfactory processing in Drosophila: mechanisms and principles. Annu. Rev. Neurosci. 36, 217-241. doi: 10.1146/annurev-neuro062111-150533

Xu, P. X., Atkinson, R., Jones, D. N. M., and Smith, D. P. (2005). Drosophila OBP LUSH is required for activity of pheromone-sensitive neurons. Neuron 45, 193-200. doi: 10.1016/j.neuron.2004.12.031

Yang, Y., Krieger, J., Zhang, L., and Breer, H. (2012). The olfactory co-receptor orco from the migratory locust (Locusta migratoria) and the desert locust (Schistocerca gregaria): identification and expression pattern. Int. J. Biol. Sci. 8, 159-170. doi: 10.7150/ijbs.8.159

Yao, C. A., and Carlson, J. R. (2010). Role of G-proteins in odor-sensing and CO2-sensing neurons in Drosophila. J. Neurosci. 30, 4562-4572. doi: 10.1523/JNEUROSCI.6357-09.2010

Yao, C. A., Ignell, R., and Carlson, J. R. (2005). Chemosensory coding by neurons in the coeloconic sensilla of the Drosophila antenna. J. Neurosci. 25, 8359-8367. doi: 10.1523/JNEUROSCI.2432-05.2005

Zhang, Y. V., Ni, J., and Montell, C. (2013). The molecular basis for attractive salt-taste coding in Drosophila. Science 340, 1334-1338. doi: 10.1126/science. 1234133

Zhou, X., Slone, J. D., Rokas, A., Berger, S. L., Liebig, J., Ray, A., et al. (2012). Phylogenetic and transcriptomic analysis of chemosensory receptors in a pair of divergent ant species reveals sex-specific signatures of odor coding. PLoS Genet. 8:e1002930. doi: 10.1371/journal.pgen.1002930

zur Lage, P. I., Prentice, D. R. A., Holohan, E. E., and Jarman, A. P. (2003). The Drosophila proneural gene amos promotes olfactory sensillum formation and suppresses bristle formation. Development 130, 4683-4693. doi: 10.1242/dev. 00680

Conflict of Interest Statement: The authors declare that the research was conducted in the absence of any commercial or financial relationships that could be construed as a potential conflict of interest.

Copyright (๑) 2018 Gomez-Diaz, Martin, Garcia-Fernandez and Alcorta. This is an open-access article distributed under the terms of the Creative Commons Attribution License (CC BY). The use, distribution or reproduction in other forums is permitted, provided the original author(s) and the copyright owner(s) are credited and that the original publication in this journal is cited, in accordance with accepted academic practice. No use, distribution or reproduction is permitted which does not comply with these terms. 


\title{
Tuning Insect Odorant Receptors
}

\author{
Dieter Wicher* \\ Department of Evolutionary Neuroethology, Max Planck Institute for Chemical Ecology (MPG), Jena, Germany
}

Among the insect olfactory receptors the odorant receptors (ORs) evolved in parallel to the onset of insect flight. A special property of this receptor type is the capability to adjust sensitivity of odor detection according to previous odor contacts. This article presents a current view on regulatory processes affecting the performance of ORs and proposes a model of mechanisms contributing to OR sensitization.

Keywords: chemoreception, olfaction, ionotropic receptor, odorant receptor, receptor kinase, GPCR, intracellular signaling

\section{INTRODUCTION}

The performance of membrane proteins such as ion channels or receptors is dynamically adjusted according to changing physiological requirements. Olfactory receptors have to detect odors in a wide range of concentrations, from faint filaments at larger distance from the source to high concentrated and permanent presence near the source. In mammals, the olfactory receptors for general odors are G protein coupled receptors (GPCRs; Buck and Axel, 1991). For a comparison of vertebrate and insect olfaction see Kaupp (2010), for a recent review of insect olfactory receptors see Fleischer et al. (2018). Three types of receptor proteins detect volatile chemical information in insects. These are odorant receptors (ORs) which are restricted to insects, specific gustatory receptors (GRs) detecting carbon dioxide and receptors related to ionotropic glutamate receptors, called ionotropic receptors (IRs). The ORs evolved in parallel with the onset of insect flight (Missbach et al., 2014). Similar to GPCRs, insect ORs belong to the class of heptahelical transmembrane proteins. But compared with them, the OR proteins show an inverted orientation within the plasma membrane (Benton et al., 2006; Lundin et al., 2007; Smart et al., 2008). Analyzing the variation of insect OR protein amino acids during evolution revealed a model for transmembrane domain arrangement that is unrelated to GPCRs (Hopf et al., 2015).

An insect OR is a heteromeric construct formed by an odor-specific OrX protein and an ubiquitary odorant co-receptor, Orco (Larsson et al., 2004; Neuhaus et al., 2005). Heterologous coexpression of OrX and Orco proteins may in addition to the formation of ORs also lead to Orco homomers (German et al., 2013). It remains to be shown that the ciliar OSN membrane also comprises both types of constructs. At least for the soma membrane the insertion of Orco but not of Or22a/b proteins was demonstrated (Benton et al., 2006).

Experiments in heterologous expression systems supported the view that insect ORs primarily operate as ligand-gated channels (Sato et al., 2008; Wicher et al., 2008). An odor stimulation of sufficient strength produced-independent of G protein activity-an immediate transient response. The ORs form non-selective cation channels which are also permeable for $\mathrm{Ca}^{2+}$. At least some of these OR channels are constitutively active as their expression leads to an elevated level of free $\mathrm{Ca}^{2+}$, even in the absence of a stimulating odor. In addition to the fast ionotropic response there was a slowly developing OR current which relied on G protein function (Wicher et al., 2008). This finding raised the question whether there is also a role of metabotropic signaling in insect olfaction.

Intriguingly, when only Orco proteins are expressed they also form nonselective, $\mathrm{Ca}^{2+}$ permeable cation channels. These channels cannot be activated by odors but by cyclic nucleotides (Wicher et al., 2008). As in the case of ORs, it is presently unknown how the channels are composed of, either as dimers as the heptahelical channel rhodopsin (Müller et al., 2011; Kato et al., 2012), or as 
tetramer like conventional ion channels (Doyle et al., 1998). Orco dimer constructs have properties comparable to those of channels formed by native Orco proteins (Mukunda et al., 2014a).

Orco was found to be necessary for the insertion of the odor-specific receptor proteins into the plasma membrane (Larsson et al., 2004). An important signaling system during development is the hedgehog $(\mathrm{Hh})$ system (Briscoe and Thérond, 2013). The transport of Drosophila ORs to and within the dendritic cilia is regulated by the Hh pathway (Sanchez et al., 2016). The localization of the ORs depends on the distribution of the Hh signal transducer Smoothened (Smo). Smo knockdown flies showed reduced odor responses indicating less expression while mutants in the Smo repressor Patched (Ptc) display largely enhanced odor responses. As $\mathrm{Hh}$ is produced in the OSNs the tuning of OR distribution is an autoregulatory process (Sanchez et al., 2016). Orco proteins possess a putative calmodulin (CaM) binding domain that is well conserved among insect species (Mukunda et al., 2014b). Robust mutations within this region of Orco proteins disrupted the OR traffic to the ciliar membrane (Bahk and Jones, 2016).

\section{G PROTEINS}

That insect ORs-in spite of their inverted membrane topology - can interact with $G$ proteins has been demonstrated for heterologously expressed ORs. Activation of Drosophila Or43a receptor could be monitored when it was coexpressed with the promiscuous $\mathrm{G}$ protein $\alpha$ subunit $\mathrm{G}_{15}$ in Xenopus ooytes (Wetzel et al., 2001). In addition, pheromone-induced activation of the silkmoth Bombyx mori OR-1 and 3 (Grosse-Wilde et al., 2006) and Heliothis virescens HR13 (Grosse-Wilde et al., 2007) coexpressed with $\mathrm{G}_{15}$ in T-Rex293 cells was reflected by calcium signals upon activation of $\mathrm{IP}_{3}$ receptors upon PLC activation via $\mathrm{G}_{15}$.

In the antenna of Drosophila all subunits of heterotrimeric $\mathrm{G}$ proteins were shown to be expressed (Boto et al., 2010). According to immunohistochemical studies, $G_{s}, G_{i}$ and $G_{q} \alpha$ subunits could be detected in the OSNs. This also includes the sensilla along which $G_{i}$ and $G_{q}$ were found, whereas $G_{s}$ staining was seen at the basal segment (Boto et al., 2010). Expression of $G_{s}$ in fly sensilla was also reported, and $G_{s}$ proteins were found to be important for sensitive odor detection (Deng et al., 2011). In the antenna of $B$. mori the three $\alpha$ subunits $\mathrm{G}_{\mathrm{s}}, \mathrm{G}_{\mathrm{i}}$ and $\mathrm{G}_{\mathrm{q}}$ were detected (Miura et al., 2005) while in the mosquito Anopheles females only $\mathrm{G}_{\mathrm{q}}$ was found in certain sensilla (Rützler et al., 2006).

A role of $\mathrm{G}_{\mathrm{o}}$ in Drosophila olfactory reception was shown by expression of the inhibitor pertussis toxin (PTX). Electroantennogram responses and the rise in spike frequency upon odor stimulation were reduced when PTX was expressed in the OSNs (Chatterjee et al., 2009). An effect of $\mathrm{G}_{\mathrm{o} / \mathrm{i}}$ inhibition by PTX was also observed for heterologously expressed ORs (Or22a plus Orco; Ignatious Raja et al., 2014). Monitoring calcium responses in Or22a expressing OSNs to odor stimulation in intact Drosophila antenna revealed weaker responses when $\mathrm{G}_{\mathrm{o} / \mathrm{i}}$ were inhibited (Ignatious Raja et al., 2014). Also the involvement of
$\mathrm{G}_{\mathrm{s}}$ proteins in OR signal transduction was reported (Deng et al., 2011). In addition, other studies demonstrated the importance of $\mathrm{G}_{\mathrm{q}}$ proteins (Kain et al., 2008, 2009). Mutations in $d g q$, the gene encoding the Drosophila $\mathrm{G}_{\mathrm{q}} \alpha$ subunit caused reduced responses to odor stimulation.

In contrast to these findings, only tiny effects in Drosophila sensillum recordings were observed when manipulating the activity of G proteins (Yao and Carlson, 2010). Rather mild effects of $\mathrm{G}$ protein inhibition on $\mathrm{Ca}^{2+}$ responses were seen in heterologously expressed ORs (Smart et al., 2008).

\section{SECOND MESSENGER SYSTEMS}

Independent of $\mathrm{G}$ protein-coupled signal cascades $\mathrm{Ca}^{2+}$ is an ubiquitous messenger that regulates the activity of proteins and links such signaling cascades. OR activation leads to $\mathrm{Ca}^{2+}$ influx into OSNs. Prolonged odor stimuli lead to a $\mathrm{Ca}^{2+}$-induced adaptation of the odor response (Cao et al., 2016). On the other hand, CaM activity can enhance the OR response to moderate stimuli (Mukunda et al., 2014b).

Mutations in the cascade downstream $\mathrm{G}_{\mathrm{s}}$, i.e., in the adenylyl cyclase rutabaga and in the phosphodiesterase dunce affected the olfaction-guided behavior (Martín et al., 2001). Especially overexpression of dunce in specific OSNs which diminished the cAMP level in these cells produced severe phenotypes (Gomez-Diaz et al., 2004). A reduced cAMP level impairs the ability of flies to correctly detect an odor (Murmu and Martin, 2016). On the other hand, odor stimulation leads to enhanced cAMP production (Miazzi et al., 2016). That this effect was related to ORs had been suggested by the finding that odor stimulation of ORs expressed in HEK293 cells gave rise to enhanced cAMP production (Wicher et al., 2008). Artificially enhancing the cAMP concentration in Drosophila OSNs by injecting the membrane-permeable 8-bromo-cAMP or the adenylyl cyclase activator forskolin into the base of sensilla enhanced the odor-response and shifted the concentrationdependence towards lower odor concentration (Getahun et al., 2013). Similarly, in flies expressing a light-activated adenylyl cyclase in OSNs the spike activity could be enhanced by light exposure (Deng et al., 2011).

The signaling cascade downstream $\mathrm{G}_{\mathrm{q}}$ also plays a role in odorant signal processing of insects (Krieger and Breer, 1999; Kain et al., 2008). In the hawkmoth Manduca sexta, pheromone stimuli are detected via PLC-dependent signaling (review, Stengl, 2010). Short and faint pheromone presentation causes an immediate increase spike activity in the receptor neuron which is accompanied by a transient rise in $\mathrm{IP}_{3}$ (Breer et al., 1990; Boekhoff et al., 1993). In cultured receptor neurons, $\mathrm{IP}_{3}$ perfusion opened a $\mathrm{Ca}^{2+}$ channel, the $\mathrm{Ca}^{2+}$ rise in turn activated further types of ion channels (Stengl et al., 1992; Stengl, 1993, 1994). While the pheromone signal transduction in Manduca seems to employ solely metabotropic mechanisms (Nolte et al., 2013, 2016), heterologously expressed pheromone receptors of the silkmoth Bombyx mori were found to act as ligand-gated channels (Sato et al., 2008). This indicates that pheromone signals might be processed via ionotropic and/or metabotropic mechanisms. 
In Drosophila, norpA mutants that express a PLC enzyme with impaired function show reduced odorant responses (RiesgoEscovar et al., 1995). An attenuation of odor responses was also observed in plc21 mutants which express another defective PLC protein (Kain et al., 2008). Mutants in stmbhA, a gene encoding a putative $\mathrm{PIP}_{2}$-DAG lipase, show a markedly reduced electroantennogram response to odor stimulation (Kain et al., 2009). Thus, a disturbed $\mathrm{PIP}_{2}$ cleavage and regeneration cycle negatively affects odor information processing in insect OSNs.

$\mathrm{Ca}^{2+}$ signaling is employed by various pathways necessary for appropriate odor perception. One aspect of the $\mathrm{G}_{\mathrm{q}}$ signaling cascade are $\mathrm{Ca}^{2+}$ signals produced when the $\mathrm{PIP}_{2}$ cleavage product $\mathrm{IP}_{3}$ activates $\mathrm{IP}_{3}$ receptors $\left(\mathrm{IP}_{3} \mathrm{Rs}\right)$ which release $\mathrm{Ca}^{2+}$ from the endoplasmic reticulum. In mutants with disrupted RyR and $\mathrm{IP}_{3} \mathrm{R}$ signaling the adaptation to odor signals is impaired (Murmu et al., 2011; Murmu and Martin, 2016). A role of intracellular stores for odor signal amplification was also observed in vitro (Ignatious Raja et al., 2014). In mammals, a broad dynamic range of the OSNs in terms of odor concentration relies on a proper function of mitochondria (Fluegge et al., 2012).

There is also a crosstalk between the $\mathrm{G}_{\mathrm{s}}$ signaling cascade and intracellular $\mathrm{Ca}^{2+}$ signaling. Given that Orco proteins form cyclic nucleotide-activated ion channels permeable to $\mathrm{Ca}^{2+}$ (Wicher et al., 2008), an enhanced cAMP production may stimulate a $\mathrm{Ca}^{2+}$ influx into the OSNs.

Depending on the situation alternative messenger systems may be recruited while others are switched off. In Manduca pheromone receptor neurons, strong stimuli activate receptor guanylyl cyclases which lead to prolonged adaptation of neuronal activity. Furthermore, in the activity state the basal cAMP level is elevated, e.g., by octopaminergic signaling (Flecke et al., 2010), whereas the cGMP level is low, while at rest the cGMP level rises and the cAMP level drops (review see Stengl, 2010).

Second messenger signaling usually takes place within the sensory neuron. For silkmoth sex pheromone receptors an extracellular modulation has been observed (Nakagawa and Touhara, 2014). Extracellularly presented cyclic nucleotides were seen to weakly activate the Bombyx Or1/Orco complex and to inhibit the response to the sex pheromone bombykol.

\section{SENSITIZATION OF OR RESPONSE}

Stimulation of ORs with highly diluted odor below the detection threshold does not enhance the activity of the OSN (Figure 1A). However, when after a couple of seconds the same stimulus is presented again, the OSN can now respond with transiently enhanced spike frequency (Getahun et al., 2013). Similarly, an enhanced response after repeated gentle stimulation also leads to a rise in the intracellular $\mathrm{Ca}^{2+}$ concentration (Figure 1B, Mukunda et al., 2016). Thus, there must be an up-regulation of OR sensitivity during the interval between these stimuli. This sensitization could be mimicked by upregulation of cAMP production with the adenylyl cyclase activator forskolin. On the other hand, the OR sensitization can be suppressed by inhibition of cAMP
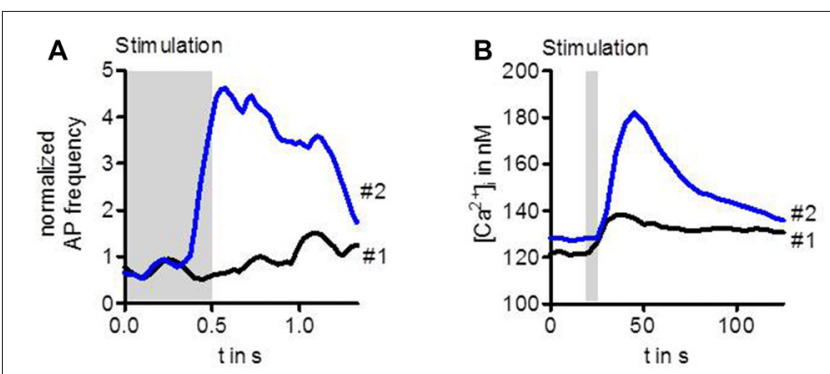

FIGURE 1 | Sensitization observed in the activity of Drosophila OSNs (A) and in $\mathrm{Ca}^{2+}$ level of cultured cells expressing odorant receptors (ORs) (B). The first weak odor stimulation did not enhance the spike frequency in OSNs

expressing Or22a. However, the same stimulus repeated $20 \mathrm{~s}$ later elicited a robust increase in OSN activity (A). Similarly, a first stimulation of HEK293 cells expressing Or22a + Orco enhanced the intracellular $\mathrm{Ca}^{2+}$ concentration $\left[\mathrm{Ca}^{2+}\right]_{\mathrm{i}}$ only slightly whereas the second stimulus led to a strong rise (B). For experimental information see Getahun et al. (2013) (A) and Mukunda et al. (2016) (B)

production (Getahun et al., 2013). Another way to mimick sensitization is to activate protein kinase C (PKC; Getahun et al., 2013).

Thus a main player in the sensitization process seems to be a protein affected by cAMP and PKC. A known target for cAMP and PKC is Orco. Heterologously expressed Orco proteins form ion channels activated by cyclic nucleotides (Wicher et al., 2008). Orco activation by cAMP requires a certain level of phosphorylation by PKC (Sargsyan et al., 2011). The Orco PKC site S289 was seen to be specifically important for OR sensitivity (Guo et al., 2017).

With strong intracellular $\mathrm{Ca}^{2+}$ buffering that inactivates $\mathrm{PKC}$, no Orco activation by cyclic nucleotides could be observed (Sato et al., 2008; Jones et al., 2011). On the other hand, PKC phosphorylation can activate Orco even in the absence of cAMP (Sargsyan et al., 2011). An Orco mutant that cannot be phosphorylated by $\mathrm{PKC}$ is insensitive to cAMP, i.e., the ion channel formed by Orco cannot be activated by cAMP (Sargsyan et al., 2011). In flies expressing this modified Orco protein the OR sensitivity is not enhanced by repeated odor stimulation at subthreshold concentration (Getahun et al., 2013). Also a forskolin-induced stimulation of cAMP production did not enhance the odor response as it was observed in wt flies.

When a rise in the cAMP level may sensitize ORs, the question arises whether an odor stimulus could initiate cAMP production. Using flies in which the OR-expressing OSNs coexpress a cAMP reporter, it was found that indeed odor stimulation caused an increase in cAMP concentration (Miazzi et al., 2016). Interestingly, in OSNs that lack an odor-specific OR protein but express Orco, odor stimuli did not change the cAMP level but Orco activation by the synthetic agonist VUAA1 let to a rise in CAMP. This might be due to activation of a $\mathrm{Ca}^{2+}$ dependent adenylyl cyclase as depolarization had the same effect (Miazzi et al., 2016).

These results are compatible with the following model of OR sensitization (Figure 2). An odor stimulus too weak to robustly activate the OR channel leads to OrX-dependent and/or 


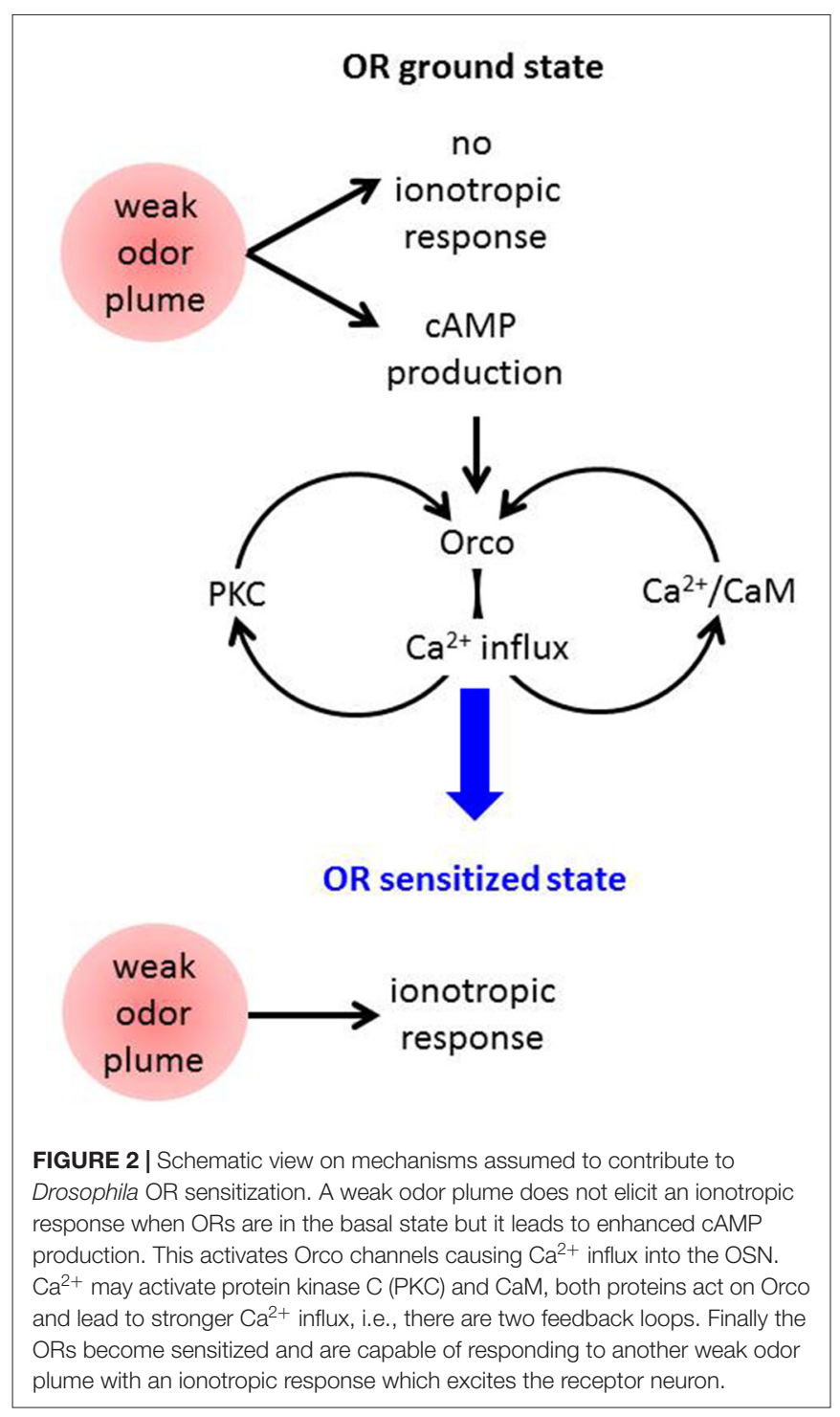

$\mathrm{Ca}^{2+}$-dependent cAMP production (Miazzi et al., 2016). cAMP in turn activates Orco which causes a cation influx including $\mathrm{Ca}^{2+}$ import. This may activate two feedback loops. First, $\mathrm{Ca}^{2+}$-activated calmodulin (CaM) can bind to Orco and enhance the $\mathrm{Ca}^{2+}$ influx (Mukunda et al., 2014b). The requirement of $\mathrm{CaM}$ function for OR sensitization has already been shown (Mukunda et al., 2016). And second, $\mathrm{Ca}^{2+}$ may activate PKC enzymes to phosphorylate Orco which also enhances the ion flow through these channels (Sargsyan et al., 2011). Taken together, the parallel signaling loops via $\mathrm{PKC}$ and $\mathrm{CaM}$ initiated by cAMP-induced $\mathrm{Ca}^{2+}$ influx through Orco both amplify the $\mathrm{Ca}^{2+}$ influx further until the ORs are tuned to the deserved sensitivity. In terms of this model also other sources of intracellular $\mathrm{Ca}^{2+}$ signals, e.g., from intracellular stores might initiate these loops. Even Orco may provide such signal as it was seen to show constitutive activity (Wicher et al., 2008).

In addition to improve the performance of Orco, CaM can also modify the function of the OR constructs which depends on the odor-specific OrX protein (Mukunda et al., 2014b). In this study it was, for example, observed that CaM markedly prolonged the current through the Drosophila geosmin receptor Or56a/Orco that detects the presence of harmful microbes (Stensmyr et al., 2012).

Among the insect olfactory receptors the ability to become sensitized by repetitive stimulation is restricted to ORs and was not observed with IRs (Getahun et al., 2013). The equipment of flying insects with tunable ORs might have qualified these animals to detect faint odor plumes during flight (Getahun et al., 2016). There are certainly many more mechanisms that contribute to receptor sensitization such as an enhanced OR expression level at a circadian time when flies are highly sensitive to odor cues (Tanoue et al., 2008).

\section{DESENSITIZATION AND ADAPTATION OF OR RESPONSE}

To appropriately process strong and/or maintained odor stimuli the insect olfactory system has to be able to downregulate the response in use-dependent manner. Long lasting stimulation and repetitive stimulation of sufficient strength leads to an adaptation of the OR response which is described by the Weber-Fechner relation (Nagel and Wilson, 2011; Cao et al., 2016). Under these conditions, the odor response becomes reduced and delayed. The $\mathrm{Ca}^{2+}$ influx during stimuli orchestrates the adaptive regulation of odor response (Cao et al., 2016).

One mechanism contributing to adaptation, a downregulation of Orco expression, was observed at elevated temperatures which cause enhanced odor concentration in the gas phase (Riveron et al., 2013) or upon excessive ethanol exposure (Morozova et al., 2006). Another way to reduce the OR sensitivity is Orco dephosphorylation at S289, as observed for prolonged odor exposure (Guo et al., 2017).

An adapting response also allowed to perceive turbulent odor filaments (Gorur-Shandilya et al., 2017). The processing of such stimuli is performed in two steps, first in the adaptation to the average odor strength which delays the response, and second in accelerating the onset of spiking. This in conjunction allows the correctly timed perception of odor plumes independent of their intensity (Gorur-Shandilya et al., 2017; Jacob et al., 2017).

\section{ORCO CHANNEL: PACEMAKER OR REGULATOR?}

A role of Orco as pacemaker channel controlling the activity of OSNs was suggested recently (Stengl and Funk, 2013). Depolarizing ion channels opening in the range of the resting membrane potential are capable of shifting the membrane potential towards the threshold for action potential generation. As Orco proteins form cation channels activated by cyclic nucleotides and/or phosphorylation by $\mathrm{PKC}$, its activation depolarizes the OSN membrane and thus should act as pacemaker (Stengl, 2010; Stengl and Funk, 2013). For Manduca pheromone receptors such a role is compatible with experimental findings (Nolte et al., 2013, 2016). 
In Drosophila OSNs, the background activity is determined by the type of expressed OrX receptor protein (Hallem et al., 2004). The $\Delta$ halo mutant, an ab3A neuron lacking Or22a (Dobritsa et al., 2003), the spontaneous firing rate is very low which indicates a weak or missing pacemaker role of Orco (Hallem et al., 2004). Expression of OrX proteins led to a considerably enhanced spiking. The spike frequency varied between a few $\mathrm{Hz}$ for Or59b or Or22a and $>60 \mathrm{~Hz}$ for Or47b (Hallem et al., 2004). Orco stimulation in Drosophila ab3A neurons with cAMP did not enhance their spontaneous activity (Getahun et al., 2013). However, odor stimulation of Or22a gave rise to a pacemaker activity and accelerates OSN spiking. A strong stimulation of OSN activity was also observed by administration of the synthetic OR agonist VUAA1 (Getahun et al., 2013). Although VUAA1 is capable of activating Orco, it is more efficiently in activation ORs (Jones et al., 2011). These observations support the above notion that in Drosophila OSNs OR activation but not Orco activation produces a pacemaker activity.

The missing pacemaker role of Orco in Drosophila OSNs is surprising insofar as heterologously expressed Orco proteins

\section{REFERENCES}

Bahk, S., and Jones, W. D. (2016). Insect odorant receptor trafficking requires calmodulin. BMC Biol. 14:83. doi: 10.1186/s12915-016-0306-x

Benton, R., Sachse, S., Michnick, S. W., and Vosshall, L. B. (2006). Atypical membrane topology and heteromeric function of Drosophila odorant receptors in vivo. PLoS Biol. 4:e20. doi: 10.1371/journal.pbio.0040020

Boekhoff, I., Seifert, E., Göggerle, S., Lindemann, M., Krüger, B.-W., and Breer, H. (1993). Pheromone-induced second-messenger signaling in insect antennae. Insect Biochem. Mol. Biol. 23, 757-762. doi: 10.1016/0965-1748(93)90063-x

Boto, T., Gomez-Diaz, C., and Alcorta, E. (2010). Expression analysis of the 3 G-protein subunits, $G \alpha, G \beta$, and $G \gamma$, in the olfactory receptor organs of adult Drosophila melanogaster. Chem. Senses 35, 183-193. doi: 10.1093/chemse/bjp095

Breer, H., Boekhoff, I., and Tareilus, E. (1990). Rapid kinetics of second messenger formation in olfactory transduction. Nature 345, 65-68. doi: 10.1038/345065a0

Briscoe, J., and Thérond, P. P. (2013). The mechanisms of Hedgehog signalling and its roles in development and disease. Nat. Rev. Mol. Cell Biol. 14, 416-429. doi: $10.1038 / \mathrm{nrm} 3598$

Buck, L., and Axel, R. (1991). A novel multigene family may encode odorant receptors: a molecular basis for odor recognition. Cell 65, 175-187. doi: 10.1016/0092-8674(91)90418-X

Cao, L. H., Jing, B. Y., Yang, D., Zeng, X., Shen, Y., Tu, Y., et al. (2016). Distinct signaling of Drosophila chemoreceptors in olfactory sensory neurons. Proc. Natl. Acad. Sci. U S A 113, E902-E911. doi: 10.1073/pnas.1518329113

Chatterjee, A., Roman, G., and Hardin, P. E. (2009). Go contributes to olfactory reception in Drosophila melanogaster. BMC Physiol. 9:22. doi: 10.1186/14726793-9-22

Deng, Y., Zhang, W., Farhat, K., Oberland, S., Gisselmann, G., and Neuhaus, E. M. (2011). The stimulatory $\mathrm{G} \alpha_{\text {s }}$ protein is involved in olfactory signal transduction in Drosophila. PLoS One 6:e18605. doi: 10.1371/journal.pone.0018605

Dobritsa, A. A., van der Goes van Naters, W., Warr, C. G., Steinbrecht, R. A., and Carlson, J. R. (2003). Integrating the molecular and cellular basis of odor coding in the Drosophila antenna. Neuron 37, 827-841. doi: 10.1016/s08966273(03)00094-1

Doyle, D. A., Morais Cabral, J., Pfuetzner, R. A., Kuo, A., Gulbis, J. M., Cohen, S. L., et al. (1998). The structure of the potassium channel: molecular basis of $\mathrm{K}^{+}$conduction and selectivity. Science 280, 69-77. doi: 10.1126/science.280. 5360.69

Flecke, C., Nolte, A., and Stengl, M. (2010). Perfusion with cAMP analogue affects pheromone-sensitive trichoid sensilla of the hawkmoth Manduca sexta in a time-dependent manner. J. Exp. Biol. 213, 842-852. doi: 10.1242/jeb.032839 form spontaneously active channels (Sargsyan et al., 2011). And such leaky channels are known to lead to oscillations of the resting membrane potential which facilitates the triggering of action potentials (Stengl, 2010). Probably the number of Orco channels in the ciliar membrane might be too low to provide an efficient pacemaker conductance. By contrast, the $\mathrm{Ca}^{2+}$ influx into the receptor neurons activated by Orco activation would be sufficient to act as intracellular messenger. By this means, $\mathrm{Ca}^{2+}$-dependent proteins such as PLC, PKC or CaM could be activated, thereby facilitating OR sensitization.

\section{AUTHOR CONTRIBUTIONS}

DW wrote the manuscript.

\section{ACKNOWLEDGMENTS}

This study was supported by the Max Planck Society.

Fleischer, J., Pregitzer, P., Breer, H., and Krieger, J. (2018). Access to the odor world: olfactory receptors and their role for signal transduction in insects. Cell. Mol. Life Sci. 75, 485-508. doi: 10.1007/s00018-017 $-2627-5$

Fluegge, D., Moeller, L. M., Cichy, A., Gorin, M., Weth, A., Veitinger, S., et al. (2012). Mitochondrial $\mathrm{Ca}^{2+}$ mobilization is a key element in olfactory signaling. Nat. Neurosci. 15, 754-762. doi: 10.1038/ nn.3074

German, P. F., van der Poel, S., Carraher, C., Kralicek, A. V., and Newcomb, R. D. (2013). Insights into subunit interactions within the insect olfactory receptor complex using FRET. Insect Biochem. Mol. Biol. 43, 138-145. doi: 10.1016/j. ibmb.2012.11.002

Getahun, M. N., Olsson, S. B., Lavista-Llanos, S., Hansson, B. S., and Wicher, D. (2013). Insect odorant response sensitivity is tuned by metabotropically autoregulated olfactory receptors. PLoS One 8:e58889. doi: 10.1371/journal. pone.0058889

Getahun, M. N., Thoma, M., Lavista Llanos, S., Keesey, I., Fandino, R. A. Knaden, M., et al. (2016). Intracellular regulation of the insect chemoreceptor complex impacts odor localization in flying insects. J. Exp. Biol. 219, 3428-3438. doi: 10.1242/jeb.143396

Gomez-Diaz, C., Martin, F., and Alcorta, E. (2004). The cAMP transduction cascade mediates olfactory reception in Drosophila melanogaster. Behav. Genet. 34, 395-406. doi: 10.1023/b:bege.0000023645.02710.fe

Gorur-Shandilya, S., Demir, M., Long, J., Clark, D. A., and Emonet, T. (2017). Olfactory receptor neurons use gain control and complementary kinetics to encode intermittent odorant stimuli. Elife 6:27670. doi: 10.7554/eLife. 27670

Grosse-Wilde, E., Gohl, T., Bouche, E., Breer, H., and Krieger, J. (2007). Candidate pheromone receptors provide the basis for the response of distinct antennal neurons to pheromonal compounds. Eur. J. Neurosci. 25, 2364-2373. doi: 10.1111/j.1460-9568.2007.05512.x

Grosse-Wilde, E., Svatos, A., and Krieger, J. (2006). A pheromone-binding protein mediates the bombykol-induced activation of a pheromone receptor in vitro. Chem. Senses 31, 547-555. doi: 10.1093/chemse/bjj059

Guo, H., Kunwar, K., and Smith, D. (2017). Odorant receptor sensitivity modulation in Drosophila. J. Neurosci. 37, 9465-9473. doi: 10.1523/JNEUROSCI.1573-17.2017

Hallem, E. A., Ho, M. G., and Carlson, J. R. (2004). The molecular basis of odor coding in the Drosophila antenna. Cell 117, 965-979. doi: 10.1016/j.cell.2004. 05.012

Hopf, T. A., Morinaga, S., Ihara, S., Touhara, K., Marks, D. S., and Benton, R. (2015). Amino acid coevolution reveals three-dimensional structure and 
functional domains of insect odorant receptors. Nat. Commun. 6:6077. doi: $10.1038 /$ ncomms7077

Ignatious Raja, J. S., Katanayeva, N., Katanaev, V. L., and Galizia, C. G. (2014). Role of Go/i subgroup of $G$ proteins in olfactory signaling of Drosophila melanogaster. Eur. J. Neurosci. 39, 1245-1255. doi: 10.1111/ejn. 12481

Jacob, V., Monsempes, C., Rospars, J. P., Masson, J. B., and Lucas, P. (2017). Olfactory coding in the turbulent realm. PLoS Comput. Biol. 13:e1005870. doi: 10.1371/journal.pcbi.1005870

Jones, P. L., Pask, G. M., Rinker, D. C., and Zwiebel, L. J. (2011). Functional agonism of insect odorant receptor ion channels. Proc. Natl. Acad. Sci. U S A 108, 8821-8825. doi: 10.1073/pnas. 1102425108

Kain, P., Chakraborty, T. S., Sundaram, S., Siddiqi, O., Rodrigues, V., and Hasan, G. (2008). Reduced odor responses from antennal neurons of $G_{q} \alpha$, phospholipase $\mathrm{C} \beta$, and $\operatorname{rdgA}$ mutants in Drosophila support a role for a phospholipid intermediate in insect olfactory transduction. J. Neurosci. 28, 4745-4755. doi: 10.1523/JNEUROSCI.5306-07.2008

Kain, P., Chandrashekaran, S., Rodrigues, V., and Hasan, G. (2009). Drosophila mutants in phospholipid signaling have reduced olfactory responses as adults and larvae. J. Neurogenet. 23, 303-312. doi: 10.1080/016770608023 72494

Kato, H. E., Zhang, F., Yizhar, O., Ramakrishnan, C., Nishizawa, T., Hirata, K., et al. (2012). Crystal structure of the channelrhodopsin light-gated cation channel. Nature 482, 369-374. doi: 10.1038/nature10870

Kaupp, U. B. (2010). Olfactory signalling in vertebrates and insects: differences and commonalities. Nat. Rev. Neurosci. 11, 188-200. doi: 10.1038/ nrn2789

Krieger, J., and Breer, H. (1999). Olfactory reception in invertebrates. Science 286, 720-723. doi: 10.1126/science.286.5440.720

Larsson, M. C., Domingos, A. I., Jones, W. D., Chiappe, M. E., Amrein, H., and Vosshall, L. B. (2004). Or83b encodes a broadly expressed odorant receptor essential for Drosophila olfaction. Neuron 43, 703-714. doi: 10.1016/j.neuron. 2004.08.019

Lundin, C., Käll, L., Kreher, S. A., Kapp, K., Sonnhammer, E. L., Carlson, J. R., et al. (2007). Membrane topology of the Drosophila OR83b odorant receptor. FEBS Lett. 581, 5601-5604. doi: 10.1016/j.febslet.2007.11.007

Martín, F., Charro, M. J., and Alcorta, E. (2001). Mutations affecting the cAMP transduction pathway modify olfaction in Drosophila. J. Comp. Physiol. A 187, 359-370. doi: 10.1007/s003590100208

Miazzi, F., Hansson, B. S., and Wicher, D. (2016). Odor induced cAMP production in Drosophila melanogaster olfactory sensory neurons. J. Exp. Biol. 219, 1798-1803. doi: 10.1242/jeb.137901

Missbach, C., Dweck, H. K., Vogel, H., Vilcinskas, A., Stensmyr, M. C., Hansson, B. S., et al. (2014). Evolution of insect olfactory receptors. Elife 3:e02115. doi: 10.7554/eLife.02115

Miura, N., Atsumi, S., Tabunoki, H., and Sato, R. (2005). Expression and localization of three $\mathrm{G}$ protein $\alpha$ subunits, Go, Gq, and Gs, in adult antennae of the silkmoth (Bombyx mori). J. Comp. Neurol. 485, 143-152. doi: 10.1002/cne. 20488

Morozova, T. V., Anholt, R. R., and Mackay, T. F. (2006). Transcriptional response to alcohol exposure in Drosophila melanogaster. Genome Biol. 7:R95. doi: 10.1186/gb-2006-7-10-r95

Mukunda, L., Lavista Llanos, S., Hansson, B. S., and Wicher, D. (2014a). Dimerisation of the Drosophila odorant coreceptor Orco. Front. Cell. Neurosci. 8:261. doi: $10.3389 /$ fncel.2014.00261

Mukunda, L., Miazzi, F., Kaltofen, S., Hansson, B. S., and Wicher, D. (2014b). Calmodulin modulates insect odorant receptor function. Cell Calcium 55, 191-199. doi: 10.1016/j.ceca.2014.02.013

Mukunda, L., Miazzi, F., Sargsyan, V., Hansson, B. S., and Wicher, D. (2016). Calmodulin affects sensitization of Drosophila melanogaster odorant receptors. Front. Cell. Neurosci. 10:28. doi: 10.3389/fncel.2016.00028

Müller, M., Bamann, C., Bamberg, E., and Kühlbrandt, W. (2011). Projection structure of channelrhodopsin-2 at 6 A resolution by electron crystallography. J. Mol. Biol. 414, 86-95. doi: 10.1016/j.jmb.2011.09.049

Murmu, M. S., and Martin, J. R. (2016). Interaction between cAMP and intracellular $\mathrm{Ca}^{2+}$-signaling pathways during odor-perception and adaptation in Drosophila. Biochim. Biophys. Acta 1863, 2156-2174. doi: 10.1016/j.bbamcr. 2016.05.014
Murmu, M. S., Stinnakre, J., Real, E., and Martin, J. R. (2011). Calciumstores mediate adaptation in axon terminals of olfactory receptor neurons in Drosophila. BMC Neurosci. 12:105. doi: 10.1186/1471-2202-12-105

Nagel, K. I., and Wilson, R. I. (2011). Biophysical mechanisms underlying olfactory receptor neuron dynamics. Nat. Neurosci. 14, 208-216. doi: 10.1038/ nn. 2725

Nakagawa, T., and Touhara, K. (2014). Extracellular modulation of the silkmoth sex pheromone receptor activity by cyclic nucleotides. PLoS One 8:e63774. doi: 10.1371/journal.pone.0063774

Neuhaus, E. M., Gisselmann, G., Zhang, W., Dooley, R., Störtkuhl, K., and Hatt, H. (2005). Odorant receptor heterodimerization in the olfactory system of Drosophila melanogaster. Nat. Neurosci. 8, 15-17. doi: 10.1038/ nn1371

Nolte, A., Funk, N. W., Mukunda, L., Gawalek, P., Werckenthin, A., Hansson, B. S., et al. (2013). in situ tip-recordings found no evidence for an Orco-based ionotropic mechanism of pheromone-transduction in Manduca sexta. PLoS One 8:e62648. doi: 10.1371/journal.pone.0062648

Nolte, A., Gawalek, P., Koerte, S., Wei, H., Schumann, R., Werckenthin, A., et al. (2016). No evidence for ionotropic pheromone transduction in the hawkmoth Manduca sexta. PLoS One 11:e0166060. doi: 10.1371/journal.pone. 0166060

Riesgo-Escovar, J., Raha, D., and Carlson, J. R. (1995). Requirement for a phospholipase $\mathrm{C}$ in odor response: overlap between olfaction and vision in Drosophila. Proc. Natl. Acad. Sci. U S A 92, 2864-2868. doi: 10.1073/pnas.92. 7.2864

Riveron, J., Boto, T., and Alcorta, E. (2013). Transcriptional basis of the acclimation to high environmental temperature at the olfactory receptor organs of Drosophila melanogaster. BMC Genomics 14:259. doi: 10.1186/1471-216414-259

Rützler, M., Lu, T., and Zwiebel, L. J. (2006). G $\alpha$ encoding gene family of the malaria vector mosquito Anopheles gambiae: expression analysis and immunolocalization of AG $\alpha \mathrm{q}$ and $\mathrm{AG} \alpha \mathrm{o}$ in female antennae. J. Comp. Neurol. 499, 533-545. doi: 10.1002/cne.21083

Sanchez, G. M., Alkhori, L., Hatano, E., Schultz, S. W., Kuzhandaivel, A., Jafari, S., et al. (2016). Hedgehog signaling regulates the ciliary transport of odorant receptors in Drosophila. Cell Rep. 14, 464-470. doi: 10.1016/j.celrep.2015. 12.059

Sargsyan, V., Getahun, M. N., Llanos, S. L., Olsson, S. B., Hansson, B. S., and Wicher, D. (2011). Phosphorylation via PKC regulates the function of the Drosophila odorant coreceptor. Front. Cell. Neurosci. 5:5. doi: 10.3389/fncel. 2011.00005

Sato, K., Pellegrino, M., Nakagawa, T., Nakagawa, T., Vosshall, L. B., and Touhara, K. (2008). Insect olfactory receptors are heteromeric ligand-gated ion channels. Nature 452, 1002-1006. doi: 10.1038/nature06850

Smart, R., Kiely, A., Beale, M., Vargas, E., Carraher, C., Kralicek, A. V., et al. (2008). Drosophila odorant receptors are novel seven transmembrane domain proteins that can signal independently of heterotrimeric G proteins. Insect Biochem. Mol. Biol. 38, 770-780. doi: 10.1016/j.ibmb.2008.05.002

Stengl, M. (1993). Intracellular-messenger-mediated cation channels in cultured olfactory receptor neurons. J. Exp. Biol. 178, 125-147.

Stengl, M. (1994). Inositol-trisphosphate-dependent calcium currents precede cation currents in insect olfactory receptor neurons in vitro. J. Comp. Physiol. A 174, 187-194. doi: 10.1007/bf00193785

Stengl, M. (2010). Pheromone transduction in moths. Front. Cell. Neurosci. 4:133. doi: $10.3389 /$ fncel.2010.00133

Stengl, M., and Funk, N. W. (2013). The role of the coreceptor Orco in insect olfactory transduction. J. Comp. Physiol. A Neuroethol. Sens. Neural. Behav. Physiol. 199, 897-909. doi: 10.1007/s00359-013-0837-3

Stengl, M., Zufall, F., Hatt, H., and Hildebrand, J. G. (1992). Olfactory receptor neurons from antennae of developing male Manduca sexta respond to components of the species-specific sex pheromone in vitro. J. Neurosci. 12, 2523-2531.

Stensmyr, M. C., Dweck, H. K., Farhan, A., Ibba, I., Strutz, A., Mukunda, L. et al. (2012). A conserved dedicated olfactory circuit for detecting harmful microbes in Drosophila. Cell 151, 1345-1357. doi: 10.1016/j.cell.2012. 09.046

Tanoue, S., Krishnan, P., Chatterjee, A., and Hardin, P. E. (2008). G protein-coupled receptor kinase 2 is required for rhythmic olfactory 
responses in Drosophila. Curr. Biol. 18, 787-794. doi: 10.1016/j.cub.2008. 04.062

Wetzel, C. H., Behrendt, H. J., Gisselmann, G., Störtkuhl, K. F., Hovemann, B., and Hatt, H. (2001). Functional expression and characterization of a Drosophila odorant receptor in a heterologous cell system. Proc. Natl. Acad. Sci. U S A 98, 9377-9380. doi: 10.1073/pnas.151103998

Wicher, D., Schäfer, R., Bauernfeind, R., Stensmyr, M. C., Heller, R., Heinemann, S. H., et al. (2008). Drosophila odorant receptors are both ligandgated and cyclic-nucleotide-activated cation channels. Nature 452, 1007-1011. doi: 10.1038/nature06861

Yao, C. A., and Carlson, J. R. (2010). Role of G-proteins in odor-sensing and $\mathrm{CO}_{2}$-sensing neurons in Drosophila. J. Neurosci. 30, 4562-4572. doi: 10.1523/JNEUROSCI.6357-09.2010
Conflict of Interest Statement: The author declares that the research was conducted in the absence of any commercial or financial relationships that could be construed as a potential conflict of interest.

The handling editor is currently co-organizing a Research Topic with the author DW, and confirms the absence of any other collaboration.

Copyright (c) 2018 Wicher. This is an open-access article distributed under the terms of the Creative Commons Attribution License (CC BY). The use, distribution or reproduction in other forums is permitted, provided the original author(s) and the copyright owner are credited and that the original publication in this journal is cited, in accordance with accepted academic practice. No use, distribution or reproduction is permitted which does not comply with these terms. 


\section{OPEN ACCESS}

Edited by:

Frédéric Marion-Poll,

AgroParisTech Institut des Sciences

et Industries du Vivant et de L'environnement, France

Reviewed by:

Angel Guerrero,

Instituto de Química Avanzada

de Cataluña (IQAC), Spain Peter Witzgall,

Swedish University of Agricultural

Sciences, Sweden

*Correspondence:

Jürgen Krieger

juergen.krieger@zoologie.uni-halle.de

Received: 30 August 2018

Accepted: 30 October 2018

Published: 20 November 2018

Citation:

Fleischer $J$ and Krieger J (2018) Insect Pheromone Receptors - Key Elements in Sensing Intraspecific

Chemical Signals.

Front. Cell. Neurosci. 12:425

doi: 10.3389/fncel.2018.00425

\section{Insect Pheromone Receptors - Key Elements in Sensing Intraspecific Chemical Signals}

\author{
Jörg Fleischer and Jürgen Krieger* \\ Department of Animal Physiology, Institute of Biology/Zoology, Martin Luther University Halle-Wittenberg, Halle, Germany
}

Pheromones are chemicals that serve intraspecific communication. In animals, the ability to detect and discriminate pheromones in a complex chemical environment substantially contributes to the survival of the species. Insects widely use pheromones to attract mating partners, to alarm conspecifics or to mark paths to rich food sources. The various functional roles of pheromones for insects are reflected by the chemical diversity of pheromonal compounds. The precise detection of the relevant intraspecific signals is accomplished by specialized chemosensory neurons housed in hair-like sensilla located on the surface of body appendages. Current data indicate that the extraordinary sensitivity and selectivity of the pheromone-responsive neurons (PRNs) is largely based on specific pheromone receptors (PRs) residing in their ciliary membrane. Besides these key elements, proper ligand-induced responses of PR-expressing neurons appear to generally require a putative co-receptor, the so-called "sensory neuron membrane protein 1" (SNMP1). Regarding the PR-mediated chemo-electrical signal transduction processes in insect PRNs, ionotropic as well as metabotropic mechanisms may be involved. In this review, we summarize and discuss current knowledge on the peripheral detection of pheromones in the olfactory system of insects with a focus on PRs and their specific role in the recognition and transduction of volatile intraspecific chemical signals.

Keywords: chemoreception, pheromone signaling, olfaction, odorant receptor, signal transduction

\section{INTRODUCTION}

Pheromone signals released from individuals to affect the behavior or physiology of conspecifics play a pivotal role for numerous animal species. In insects, pheromones trigger and control various critical processes such as mating, reproduction, aggregation and alarming as well as the division of labor in eusocial species (Wyatt, 2014). Pheromones are adequate stimuli of powerful chemosensory systems that enable insects to sensitively detect and

Abbreviations: $\mathrm{CHC}(\mathrm{s})$, cuticular hydrocarbon(s); cVA, cis-vaccenyl acetate; Deg-ENaCs, degenerin-epithelial sodium channels; DmelOr, odorant receptor from Drosophila melanogaster; GR(s), gustatory receptor(s); HEK, human embryonic kidney; $\mathrm{IP}_{3}$, inositol 1,4,5-trisphosphate; IR(s), ionotropic receptor(s); OBPs, odorant-binding proteins; OR(s), odorant receptor(s); Orco, OR co-receptor; OSN(s), olfactory sensory neuron(s); $\mathrm{PBP}(\mathrm{s})$, pheromone-binding protein(s); PKC, protein kinase $\mathrm{C}$; PLC $\beta$, phospholipase $\mathrm{C}$ type $\beta$; PR(s), pheromone receptor(s); PRN(s), pheromone-responsive neuron(s); SNMP1, sensory neuron membrane protein $1 ; \mathrm{SPC}(\mathrm{s})$, sex pheromone component(s); SPR(s), sex pheromone receptor(s). 
discriminate the relevant compounds in a complex chemical world that surrounds them (Deisig et al., 2014; Renou, 2014).

Volatile pheromone molecules are generally detected through specialized sensory neurons of the olfactory system located on the antennae (Hansson and Stensmyr, 2011), whereas non-volatile pheromones are usually received by contact chemoreception mediated by neurons of the gustatory system that predominantly reside on the proboscis and legs (Ebbs and Amrein, 2007; Joseph and Carlson, 2015; Kohl et al., 2015). In both chemosensory systems, the sensory neurons are located in hair-like cuticular structures named sensilla. While little is known about the processes mediating the detection of non-volatile pheromones in gustatory sensilla, studies conducted over the last two decades have considerably elucidated the elements and mechanisms of volatile pheromone signal detection in olfactory sensilla on the antenna (reviewed in Leal, 2013; Kohl et al., 2015; Montagne et al., 2015; Fleischer et al., 2018). The current data indicate a function of pheromone-binding proteins (PBPs) in taking up pheromones from the air and in transferring them across the sensillum lymph toward PRs residing in the ciliary membrane of PRNs. Insects receive olfactory signals through three main families of chemosensory receptor proteins: the odorant receptors (ORs), the gustatory receptors (GRs), and ionotropic receptors (IRs) (Montagne et al., 2015; Wicher, 2015; Fleischer et al., 2018). The large majority of hitherto identified insect PRs are members of the OR family. Additionally, in Drosophila, few GRs and IRs are involved in pheromone reception (Joseph and Carlson, 2015; Kohl et al., 2015). Like OR-expressing olfactory sensory neurons (OSNs) responding to general odorants, proper function of PRNs endowed with a PR type belonging to the OR family is supposed to require the OR co-receptor (Orco). The Orco protein is considered to form heteromeric complexes with ligand-binding ORs and to function as non-selective cation channel (Wicher, 2015; Butterwick et al., 2018; Wicher, 2018). In addition to PBPs and PRs, a CD36-related protein with two transmembrane domains named SNMP1 is necessary for fast and sensitive responses of PRNs (Jin et al., 2008; Li et al., 2014). SNMP1 has been suggested to interplay with PBPs and PRs in pheromone detection (Rogers et al., 2001a; Benton et al., 2007; Nichols and Vogt, 2008), but the mode of interaction is still cryptic.

Based on current data, most notably on Drosophila, complexes of a ligand-binding PR and Orco underlie ionotropic chemoelectrical signal transduction in PRNs (Benton et al., 2006; $\mathrm{Ha}$ and Smith, 2009). Yet, recent data obtained from moths indicate that in some insect species, metabotropic processes might be involved and that PRs activate G protein-mediated second messenger cascades, leading to opening of cation channels and depolarization of PRNs (Stengl, 2010; Nolte et al., 2016).

Nearly 70 years after identification of the first insect pheromone in the silkworm moth Bombyx mori (Butenandt et al., 1959), enormous progress has been made in understanding the primary processes in the peripheral detection of pheromones. On the molecular level, most notably genes encoding PBPs, PRs, and SNMP1 have been unraveled and deeper insights into the mechanism of the chemo-electrical signal transduction have been obtained. Some fundamental questions have been resolved mostly through studying model insects such as Drosophila and several moth species; however, many issues are a matter of discussion and await further investigation. In this review, we discuss data and concepts regarding the molecular basis of peripheral pheromone reception in insects. We will particularly focus on current knowledge on PRs and the role of olfactory key elements in the peripheral detection, transduction and discrimination of pheromone signals.

\section{INSECT PHEROMONES - BIOLOGICAL RELEVANCE AND DIVERSITY}

Per definition, pheromones are chemicals released by an individual and received by conspecifics in which they elicit specific reactions (Karlson and Lüscher, 1959). In insects, pheromones trigger and control various essential behaviors as well as pivotal physiological processes (Yew and Chung, 2015). Insect pheromone communication has fascinated scientists since centuries. The vital importance of a female-released scent for attracting male moths was realized already in the 19th century (Fabre, 1879), but it was not before the late 1950s that the first insect pheromone was chemically unraveled. This was $(\mathrm{Z}, \mathrm{E})$ 10,12-hexadecadienol named bombykol, the major component in the sex pheromone emitted by females of the silkworm moth B. mori to attract the males (Butenandt et al., 1959). Later bombykal, (Z,E)-10,12-hexadecadienal, was deciphered as second minor constituent of the female sex pheromone (Kaissling et al., 1978). To date, species-specific sex pheromone blends have been described for hundreds of moth species ${ }^{1}$; these pheromones serve as aphrodisiacs and/or attractants to signal the presence of potential mating partners and to indicate their reproductive status and fitness (Yew and Chung, 2015). Full biological activity of the blend is only provided when the components are present in the correct ratio (Vickers et al., 1991; Baker, 2008). Similar to sex pheromones, insects use aggregation pheromones to attract conspecifics; however, both sexes are affected (Wertheim et al., 2005). Aggregation pheromones facilitate cooperative exploitation of rich food sources (Prado and Tjallingii, 1997; Durisko et al., 2014), mate finding (Verhoef and Nagelkerke, 1977), and protection from dangers (Gamberale and Tullberg, 1998; Riipi et al., 2001). Contrary to attracting sex and aggregation pheromones, courtship inhibition pheromones prevent courtship behavior and repel conspecifics (Yew and Chung, 2015). Alarm pheromones, however, can induce dispersal on the one hand but also recruitment of conspecifics and aggression against an opponent on the other hand (Wilson and Regnier, 1971). In eusocial insects (wasps, bees, ants, and termites), pheromones are crucial for the establishment of a social hierarchy as well as suppression of reproduction in workers. Moreover, given pheromones allow kin recognition and may evoke aggression upon detection of foreign pheromone profiles (Yew and Chung, 2015; Leonhardt et al., 2016).

The various functional relevancies of pheromones for insect behavior and physiology are mirrored by the chemical diversity of pheromonal compounds, including hydrocarbons, acetate

\footnotetext{
${ }^{1}$ www.pherobase.com
} 
esters, alcohols, acids, epoxides, ketones, isoprenoids, and triacylglycerides (Yew and Chung, 2015). While some of the pheromonal substances appear to be rather species-specific, others are shared by different insect species (Dewhirst et al., 2010; Roelofs, 2016). Pheromone blends from different species with a partially overlapping composition are typical for sex pheromones released by female moths (de Bruyne and Baker, 2008; Roelofs, 2016) or pheromones allowing kin recognition in eusocial insects (Cervo et al., 2002; Ruther et al., 2002). In these cases, the distinctive combination and ratio of components renders the pheromone species-specific.

With respect to the biological relevance of insect pheromones, it is noteworthy that some of these substances are also detected by respective predatory insects and parasitoids in order to facilitate tracking of their victims. In turn, pheromones released by predatory insects can be received by their insect prey in which they evoke predator avoidance behavior (reviewed by Wyatt, 2014). Moreover, given plants produce and release insect sex pheromone substances to attract insect pollinators (reviewed by Schiestl, 2005). Thus, pheromonal substances can also function as allelochemicals that mediate interspecific communication. Consequently, in terms of chemical ecology, at least some insect pheromone compounds and their detection have a relevance that clearly goes beyond communication with conspecifics.

\section{ARCHITECTURE OF THE PERIPHERAL PHEROMONE DETECTION SYSTEM}

The detection of pheromones is mediated via chemosensory organs (Wyatt, 2014), although some pheromonal compounds seem to bypass conventional sensory organs and elicit behavioral or physiological responses via directly affecting target tissues (Koene and ter Maat, 2001). The majority of hitherto reported insect pheromones are volatile and detected via OSNs housed in olfactory sensilla (Figure 1A) that are mainly concentrated on the major olfactory organs, the antennae (Hansson and Stensmyr, 2011; Renou, 2014). Yet, in the fruit fly Drosophila melanogaster, some pheromones have been reported to stimulate gustatory/taste neurons located in sensilla on the labellum or legs (Lacaille et al., 2007; Miyamoto and Amrein, 2008; Moon et al., 2009; Inoshita et al., 2011). Contrary to aporous and uniporous sensillar types (e.g., mechanosensory and gustatory sensilla), the olfactory sensilla have numerous pores in their cuticle wall, giving pheromones and other odorants from the environment easy access to the inside of a sensillum (Steinbrecht, 1997).

According to their morphology, insect olfactory sensilla are classified into three main categories: coeloconic, basiconic, and trichoid (Steinbrecht, 1996; Stocker, 2001). Electrophysiological recordings from pheromone-responsive trichoid sensilla have revealed that this sensillum type generally comprises 1-3 OSNs (Meng et al., 1989; Almaas and Mustaparta, 1991; Kurtovic et al., 2007); however, in a sensillum with various neurons, not all OSNs necessarily are dedicated to pheromone detection (Almaas and Mustaparta, 1991; Baker et al., 2004). In moths, PRNs are typically clustered in the slender sensilla trichodea (Keil, 1989; Meng et al., 1989). Nonetheless, pheromone detection in insects is not restricted to the trichoid sensillum type. While no PRNs have been located to sensilla coeloconica yet, single sensillum recordings have identified PRNs also among the OSNs of the morphologically different sensilla basiconica. For example, in the desert locust Schistocerca gregaria, this sensillum type contains clusters of 20-50 OSNs, some of which respond to the courtship inhibition pheromone phenylacetonitrile (Ochieng' and Hansson, 1999; Seidelmann and Ferenz, 2002). More recently, in the ants Ooceraea biroi and Harpegnathos saltator, neurons detecting proposed pheromones were found among a larger number of OSNs located in the female-specific basiconic sensilla on the antennal club (McKenzie et al., 2016; Ghaninia et al., 2017). In contrast, in the beetle Monochamus galloprovincialis, basiconic sensilla house only 1-2 OSNs and were reported to contain a PRN tuned to an aggregation pheromone (Alvarez et al., 2015). Overall, the peripheral olfactory system of insects shows a remarkable morphological diversity (Hansson and Stensmyr, 2011) and it remains unclear whether a trichoid or basiconic sensillum architecture confers a functional advantage in detecting a particular class of pheromones.

Some insects show morphological specializations considered as evolutionary adaptation to sensitize pheromone reception. This is particularly obvious in species releasing pheromones in a sex-specific way (for instance moths or beetles). To increase the receptive surface, the antennae of the receiving sex (usually males in moths and females in beetles) are substantially enlarged and equipped with numerous, often strikingly long sensilla dedicated to the detection of pheromonal substances (Meng et al., 1989). A prime example for such a sexual dimorphisms are silk moth species of the genus Antheraea where only the males comprise extremely large feather-like antenna endowed with tens of thousands of long trichoid sensilla, most of which receive female-released pheromone components (Schneider et al., 1964; Meng et al., 1989).

\section{IDENTIFICATION OF THE FIRST PRS}

The chemical diversity and multicomponent composition of pheromones requires highly elaborated sensory systems for the precise detection and discrimination of species-specific pheromones. Typically, insect OSNs are endowed with a single type of olfactory receptor protein ("one receptor per neuron" rule) that confers responsiveness to cognate ligands (Vosshall et al., 2000; Dobritsa et al., 2003; Couto et al., 2005; Hallem and Carlson, 2006). For insect species employing multiple pheromones in chemical communication, this principle implies a larger repertoire of tuned PRNs equipped with distinct PRs.

The initial search for PRs was based on the notion that receptors for volatile pheromones belong to the family of insect ORs. Insect ORs were first identified in D. melanogaster (Clyne et al., 1999; Gao and Chess, 1999; Vosshall et al., 1999), providing the basis for the discovery of the first insect PRs in moths 5 years later (Krieger et al., 2004; Sakurai et al., 2004). By applying bioinformatics to screen Drosophila genome sequences for genes encoding proteins structurally 
A

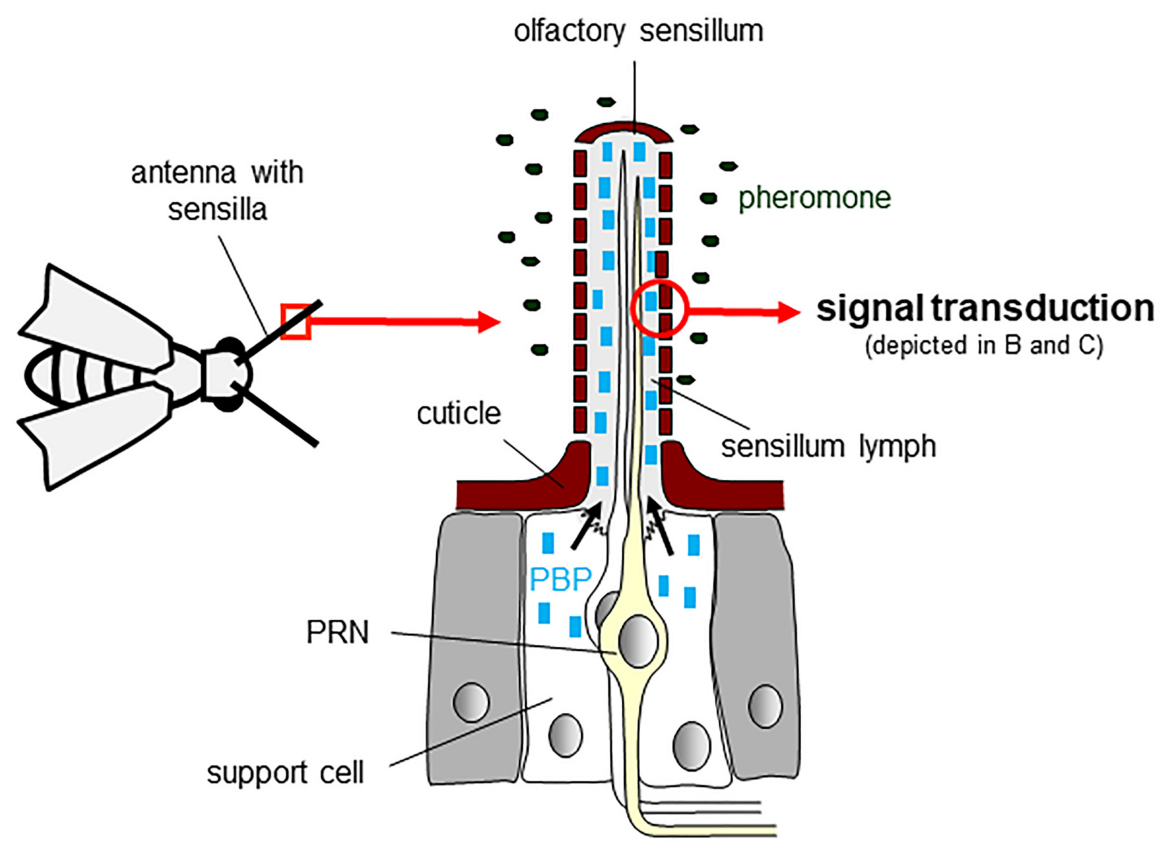

B

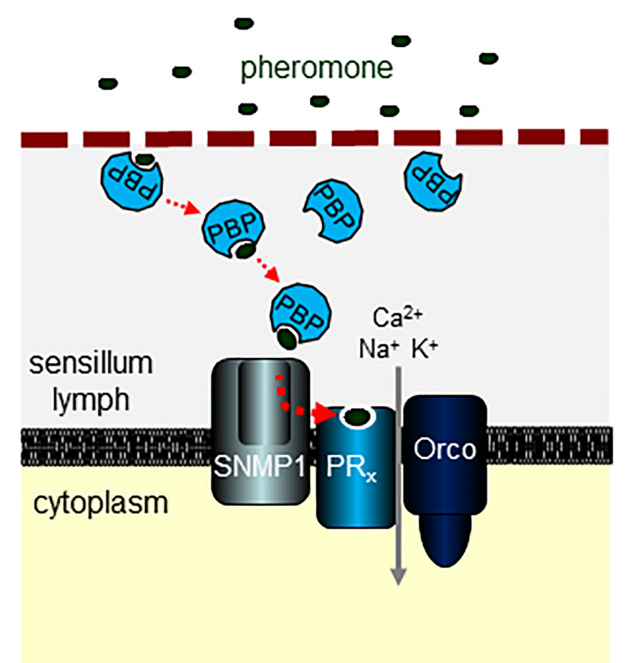

ionotropic
C

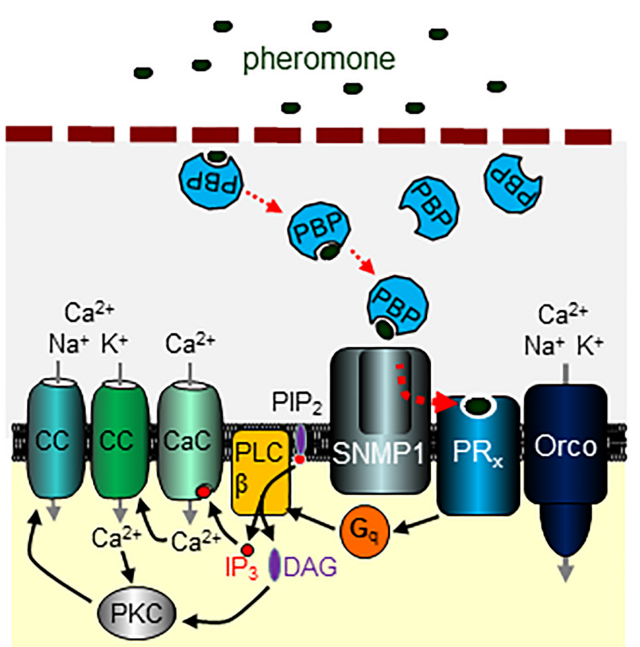

metabotropic

FIGURE 1 | Detection of volatile pheromones on the antenna. (A) The antenna of insect carries numerous hair-like extensions of the cuticle termed sensilla. Olfactory neurons extend their ciliary dendrites into the sensillum shaft that is filled with sensillum lymph. A subset of sensilla house pheromone-responsive neurons (PRNs). Support cells associated with the sensory neurons produce pheromone-binding proteins (PBPs) and secrete large quantities of PBPs into the aqueous sensillum lymph. (B,C) Volatile pheromones entering the sensillum through cuticle pores are supposed to be taken over by PBPs that solubilize the mostly hydrophobic molecules in the lymph and transfer them to a given pheromone receptor (PR $)$. Different models have been suggested for insect pheromone signal transduction. Based mainly on results from studies using Drosophila melanogaster (Sato et al., 2008; Touhara, 2009), a purely ionotropic mechanism has been proposed (B). After reaching the dendritic membrane of a PRN, the ligand-loaded PBP is supposed to interact with the sensory neuron membrane protein 1 (SNMP1). SNMP1 acts as co-receptor mediating the release of pheromones from PBPs and the transfer to the PR that forms a channel complex with the OR co-receptor (Orco). Binding of the pheromone to the $\mathrm{PR}_{\mathrm{x}}$ opens the channel complex leading to an influx of cations into the cell. In an alternative model (C) mostly based on data from moths, notably the hawk moth Manduca sexta (Stengl and Funk, 2013; Nolte et al., 2016), a role of the PRx/Orco complex as primary transduction channel is challenged. Instead, pheromone binding to the $P R_{x}$ is supposed to activate a $G$ protein $\left(G_{q}\right) /$ phospholipase $C$ type $\beta$ (PLC $\beta$ ) signaling pathway that via the breakdown of phosphatidylinositol 4,5-bisphosphate $\left(\mathrm{PIP}_{2}\right)$ into inositol 1,4,5-trisphosphate $\left(\mathrm{IP}_{3}\right)$ and diacylglycerol (DAG) induces opening of several ion channels in the plasma membrane. The rise in $\mathrm{IP}_{3}$ rapidly opens a calcium-selective ion channel $(\mathrm{CaC})$ evoking increased intracellular $\mathrm{Ca}^{2+}$ concentrations. This rapid rise in Ca ${ }^{2+}$ gates $\mathrm{Ca}^{2+}$-activated cation channels (CC) and increases the activity of protein kinase C (PKC). PKC is also activated by the rise of DAG. As a result, enhanced PKC activity leads to the opening of further CC. 
related to heptahelical mammalian ORs, a large family of Drosophila OR (DmelOr) genes was found. The corresponding DmelOr proteins were expressed in subsets of OSNs in the antennae and maxillary palps and conferred odorant sensitivity to OSNs (Clyne et al., 1999; Gao and Chess, 1999; Vosshall et al., 1999; Hallem et al., 2004; Hallem and Carlson, 2006). Subsequently, using the DmelOr sequences, bioinformatics and differential screening approaches to search genome and cDNA sequences of moths led to the discovery of OR genes in the tobacco budworm Heliothis virescens and the silkmoth B. mori (Krieger et al., 2004; Sakurai et al., 2004). In both moth species, a small subfamily of ORs was found to display more than $40 \%$ sequence identity, which is strikingly higher than the about $10-20 \%$ identity between other insect ORs. In accordance with a role in the detection of female sex pheromone components (SPCs), members of the conserved subfamilies were found to be expressed selectively in OSNs of male pheromone-responsive sensilla trichodea. Furthermore, functional analysis of these ORs in heterologous expression systems confirmed their responsiveness to distinct SPCs (Sakurai et al., 2004; Nakagawa et al., 2005; Grosse-Wilde et al., 2006, 2007; Wang et al., 2011). Matching the predictions from single sensillum recordings of $H$. virescens (Almaas and Mustaparta, 1991; Baker et al., 2004), expression of the OR types HR13 and HR6 responding to the major (HR13) and the minor (HR6) component of the female sex pheromone could be assigned to OSNs of pheromone-responsive trichoid sensilla classified as type $A$ and type $B$, respectively (Gohl and Krieger, 2006; Grosse-Wilde et al., 2007; Zielonka et al., 2017). Likewise, expression of the B. mori receptors BmOR1 and BmOR3 detecting the female-released SPCs bombykol and bombykal, respectively, was localized to the corresponding electrophysiologically characterized pairs of OSNs in sensilla trichodea of male silk moths (Kaissling et al., 1978; Krieger et al., 2005; Nakagawa et al., 2005).

\section{REPORTED PRS ACROSS INSECTS}

Since the initial discovery of insect PRs, advances in sequencing technologies and bioinformatics tools have rapidly increased the number of available insect genomes and gave access to the OR gene repertoires of many species. As a consequence, also the list of insects with described PRs has grown continuously. By utilizing homology-based search methods, genes encoding PRs for female SPCs were identified in various lepidopteran species. This was apparently facilitated by the high degree of conservation between moth sex pheromone receptors (SPRs) reflected in the characteristic clustering of moth SPRs in a "SPR clade" in phylogenetic trees of insect ORs (Engsontia et al., 2014; Koenig et al., 2015; Steinwender et al., 2015). However, it is important to recognize that not all ORs that group in the lepidopteran "SPR clade" are necessarily PRs. For example, the receptor types HR14 and HR16 from $H$. virescens mediate responses to pheromone compounds of other species that act as behavioral antagonist in $H$. virescens (Grosse-Wilde et al., 2007; Wang et al., 2011). Similarly, CpomOR3 and CpomOR6 of the codling moth
Cydia pomonella are activated by a plant-derived odorant and a pheromone antagonist, respectively (Cattaneo et al., 2017).

Beyond lepidopteran SPRs, a number of proven and candidate PRs with proposed roles in various social, sexual, and reproductive behaviors have been reported for dipteran ( $\mathrm{Ha}$ and Smith, 2006; Kurtovic et al., 2007), hymenopteran (Wanner et al., 2007; Pask et al., 2017), hemipteran (Liu F. et al., 2017; Zhang et al., 2017), and orthopteran species (Pregitzer et al., 2017). Amongst these, AmOR11 of the honey bee Apis mellifera (Hymenoptera) was identified as PR for the queen substance 9oxo-2-decenoic acid (9-ODA) that attracts workers to the queen, inhibits worker ovary development and acts as a sex pheromone by attracting drones during mating flights (Wanner et al., 2007). In the common bedbug Cimex lectularius (Hemiptera), several ORs detect different components of the aggregation pheromone (Liu F. et al., 2017). In another hemipteran species, Acyrthosiphon pisum, ApisOR5 mediates responses to the aphid alarm pheromone (E)- $\beta$-farnesene (Zhang et al., 2017).

Comprehensive studies have been conducted to identify pheromonal compounds and their respective PRs in the powerful genetic model D. melanogaster (Diptera) leading to a number of ORs implicated in various pheromone-driven behaviors of the vinegar fly (reviewed in Van der Goes van Naters, 2014; Kohl et al., 2015). DmelOr67d and DmelOr65a were found to detect the male-produced pheromone cis-vaccenyl acetate (cVA) that acts as aphrodisiac in females, inhibits courtship in males and promotes male/male aggression (Kurtovic et al., 2007; Wang and Anderson, 2010; Liu et al., 2011; Pitts et al., 2016). However, recent results indicate that Or65abc-expressing neurons are unresponsive to cVA (Pitts et al., 2016), challenging a role of DmelOr65a in cVA detection. DmelOr7 has been described to detect (Z)-9-tricosene, a pheromone released by males guiding aggregation and oviposition decisions in females (Lin et al., 2015). Receptors DmelOr69aB and DmelOr69aA are tuned to the pheromone $(\mathrm{Z})$-4-undecenal $(\mathrm{Z} 4-11 \mathrm{Al})$ that is released by female flies and induces flight attraction in both sexes. Intriguingly, these PR types are also activated by food odorants (Lebreton et al., 2017). In addition, DmelOr88a and DmelOr47b have been reported as PRs for fly-produced fatty acid methyl esters mediating copulation and attraction (Dweck et al., 2015); however, a conflicting study found little direct impact of the respective OSNs on courtship behaviors; instead, responses of these OSNs to a number of non-fly odors were observed (Pitts et al., 2016).

In Drosophila, also other receptors than ORs are considered as PRs. Notably, a small number of heptahelical GRs as well as members of the so-called pickpocket (Ppk) subfamily of degenerin-epithelial sodium channels (Deg-ENaCs) are required for pheromone-guided sexual behaviors (reviewed in Joseph and Carlson, 2015; Kohl et al., 2015). Neurons expressing these GRs and Deg-ENaCs are activated by cuticular hydrocarbons (CHCs) produced by either one or both sexes. Some of the identified CHCs have been shown to be volatile (Farine et al., 2012) suggesting sensory detection of pheromonal CHCs through the olfactory system as well as the taste system. While the Drosophila olfactory system seems to have some relevance for detecting volatile CHCs (Farine et al., 2012), all GRs and 
Deg-ENaCs implicated in the detection of pheromonal CHCs are expressed in neurons of gustatory sensilla on the labellum and the legs/tarsi (Bray and Amrein, 2003; Miyamoto and Amrein, 2008; Moon et al., 2009; Jeong et al., 2013). Thus, in Drosophila, pheromonal CHCs appear to be primarily sensed through contact chemoreception and the taste system. Interestingly, and contrary to the vinegar fly, in the ant $H$. saltator, a subfamily of ORs that is highly expressed in the antenna detects different CHCs supposed to be important in mediating eusocial behavior, including a candidate queen pheromone component (Pask et al., 2017; Slone et al., 2017). This finding suggests an outstanding importance of the ant olfactory system for the detection of CHC pheromones and may indicate different evolutionary adaptation to the detection of pheromonal CHCs in insects.

\section{SPECIAL ASPECTS OF PR EXPRESSION}

In insect species in which only one sex releases SPCs, PR expression is often biased, with exclusive or predominant expression in the non-releasing sex. This is particularly evident for PRs detecting the major component of the female-released sex pheromone blend in moths; these PRs are mainly expressed by males. In contrast, but in accordance with cVA-controlled behavior in both sexes of $D$. melanogaster, no sexual dimorphism in the expression was found for the receptor DmelOr67d detecting the male-released pheromone cVA (Kurtovic et al., 2007). Interestingly, cVA evokes in a DmelOr67d-dependent manner opposite behaviors in males versus females: while cVA elicits suppression of courtship in male flies, it promotes mating behavior in females (Ejima et al., 2007; Kurtovic et al., 2007; Datta et al., 2008).

Similar to Drosophila males, behavioral and electrophysiological studies provide evidence for "autodetection" of pheromones by females of various moth species, i.e., detection of SPCs released by themselves (Holdcraft et al., 2016). Contrary to what the term "autodetection" suggests, this ability presumably does not mainly serve detection of the compounds released by the pheromone-producing individual itself but rather the detection of SPCs released by conspecific females in the surrounding. Thus, sex pheromone information may be used by females to avoid places of high mating competition and unfavorable oviposition sites, thereby minimizing competition for ecological resources (Harari and Steinitz, 2013; Holdcraft et al., 2016). In line with this notion, expression of PRs for female-released SPCs has been reported for the antennae of female moths (Bengtsson et al., 2012; Sun et al., 2013; Holdcraft et al., 2016; Zielonka et al., 2017). For example, in female antennae of $H$. virescens that comprise trichoid sensilla tuned to the female-released SPC (Z)-9-tetradecenal (Hillier et al., 2006), OSNs expressing the cognate PR type HR6 are located to this sensillum type (Zielonka et al., 2017).

Intriguingly, in recent studies of several moth species, antennal OSNs of larvae were found to respond to female SPCs; moreover, the caterpillars were also attracted to food sources that contain such SPCs, suggesting that sex pheromones might serve as a relevant cue for larvae in food source selection (Poivet et al.,
2012; Jin et al., 2015; Zhu et al., 2016). While in Spodoptera littoralis, PBPs but no respective SPRs were identified in the larval antenna (Poivet et al., 2012, 2013), analysis of the sensilla from $H$. virescens caterpillars revealed responses to SPCs and expression of the PR types HR6 and HR13 for the major and the minor SPC in distinct OSNs of basiconic sensilla. In addition, coexpression of PRs with SNMP1 and expression of PBPs was found (Zielonka et al., 2016). This finding indicates that in moths, the responsiveness to pheromones in larval sensilla is based on the same molecular machinery as in the antenna of adults. However, the biological relevance of pheromone detection in the antenna of larvae needs further investigation.

Noteworthy, in moths, expression of SPRs is not confined to the antenna since RNA encoding these receptors has also been found in abdominal tissue from both sexes (Krieger et al., 2004; Widmayer et al., 2009). Detailed analyses of the abdomen from females of $H$. virescens have shown that HR6 and HR13 are expressed in sensilla surrounding the tip of the ovipositor. These findings have led to speculations that SPRs in the female abdomen might be involved in feedback mechanisms controlling the release of SPCs from pheromone glands (Widmayer et al., 2009).

\section{LIGAND SPECIFICITY OF PRS}

Ongoing collaborative projects like the $\mathrm{I} 5 \mathrm{~K}$ initiative that intends to sequence the genomes of 5000 arthropods will give access to the OR, IR, and GR gene repertoires and thus to candidate PR sequences of hundreds of nominated insect species. However, identification of PRs among the plethora of predicted olfactory receptor proteins in a given species will be a big challenge and will not only require detailed knowledge of pheromones but also appropriate and powerful functional expression systems for receptor deorphanization. Hitherto, three main in vivo heterologous expression systems are available, all of which have been applied successfully for PR characterization. These are (i) Xenopus oocytes coupled to voltage-clamp electrophysiology, (ii) mammalian or insect cell lines coupled to calcium imaging, and (iii) the so-called Drosophila "empty neuron" and T1 sensillum systems in combination with electrophysiological single sensillum recordings (Nakagawa et al., 2005; GrosseWilde et al., 2006; Kurtovic et al., 2007; Mitsuno et al., 2008; Forstner et al., 2009; Syed et al., 2010; Wanner et al., 2010; Zhang et al., 2016; Pask et al., 2017; reviewed in Montagne et al., 2015; Fleischer et al., 2018). In addition, a cell-free functional expression system involving OR synthesis in giant vesicles and patch clamp recordings has been reported (Hamada et al., 2014).

For the assessment of candidate PRs (and other ORs), the Xenopus oocyte system has been most widely applied (Nakagawa et al., 2005; Sun et al., 2013; Zhang et al., 2015, 2016; Liu F. et al., 2017). SPRs from moths functionally expressed in frog oocytes displayed a wide range of ligand specificities with receptors tuned to a single or to several components of female sex pheromones (Mitsuno et al., 2008; Wanner et al., 2010; Wang et al., 2011). Congruent results were described for moth SPRs analyzed in human embryonic kidney (HEK) cells (Grosse-Wilde et al., 2006, 2007; Forstner et al., 2009). Similarly, characterization of ORs 
of the common bedbug C. lectularius in frog oocytes revealed several ORs with partly overlapping tuning properties for distinct compounds of the multicomponent aggregation pheromone (Liu F. et al., 2017).

Candidate PRs of the ant $H$. saltator were characterized using the Drosophila "empty neuron" system (Pask et al., 2017). Systematic testing with a diverse panel of hydrocarbons revealed that most receptors are narrowly tuned, suggesting that in ants several PRs contribute to the detection and discrimination of different CHCs.

The Drosophila T1 sensillum system makes use of a given OSN type endogenously expressing the PR DmelOR67d (Ha and Smith, 2006), thus providing a sensillum environment and an OSN type equipped for pheromone detection. On the molecular level, this includes expression of a pheromone-transporting PBP and of SNMP1 shown to be required for proper function of Drosophila and moth PRs (Benton et al., 2007; Jin et al., 2008; Pregitzer et al., 2014). Several studies have proven the suitability of the Drosophila T1 sensillum for the characterization of PRs of other insects. For instance, replacement of DmelOr67d by the OR types SlitOR6 of S. littoralis (Montagne et al., 2012), BmOR1 of B. mori (Syed et al., 2010), or HR13 of H. virescens (Kurtovic et al., 2007) allowed to validate these ORs as narrowly tuned PRs for distinct SPCs.

Functional analyses using heterologous expression systems are the preferred tools for the assessment of the ligand specificity of insect PRs. Yet, whether the concentration of the stimulus experimentally applied in functional analyses of PRs complies with the natural pheromone concentrations detected by an insect is mostly unclear; however, it is a critical parameter for assessing the tuning of PRs. In addition, other experimental factors such as the set of compounds tested may affect the assessment of the receptor tuning (reviewed in Andersson et al., 2015). For PRs concluded to mediate the detection of several pheromonal compounds, a further aspect should be considered. In heterologous systems, the assessment of ligand specificities of PRs is usually conducted in the absence of the endogenous PBPs. Importantly, in the cases where PBPs have been employed in functional analyses of PRs in the Xenopus oocyte or the HEK cell system, more sensitive and specific responses were obtained (Grosse-Wilde et al., 2007; Forstner et al., 2009; Sun et al., 2013). This finding suggests that "ligand-matched" pairs of PBPs and PRs appear to underlie the overall reactivity of a pheromone detection system (Grosse-Wilde et al., 2006, 2007; Forstner et al., 2009). Moreover, PBPs appear to be far more than just solubilizers and transporters but also function as pre-filters enabling only distinct compounds to reach a PR. Thus, for a given PR classified as "broadly tuned" based on functional analyses in the absence of endogenous PBPs, the determined ligand spectrum may contain compounds that the PR protein in the ciliary membrane of a respective PRN would never face under natural conditions.

The existing data indicate that insects employ a range of narrowly and broadly tuned PRs for the detection of multicomponent pheromone blends and suggest combinatorial coding as the primary coding principle to perceive complex pheromone signals. Consequently, the pheromone detection process is not fundamentally different from the mechanisms insects use to analyze complex mixtures of odors originating from food sources, hosts or oviposition sites; these processes also employ specifically and broadly tuned ORs to detect and discriminate relevant odorants (Hallem and Carlson, 2006; Carey et al., 2010; Wang et al., 2010; Dweck et al., 2013).

In the current concept of pheromone detection, PRs that are specifically responsive to a single pheromone compound confer the ability to distinguish chemically very similar compounds, such as SPCs with different fatty acid chain length, same molecular backbone but different functional groups or stereoisomeric compounds (Zhang and Löfstedt, 2015). In male moths, narrowly tuned SPRs are crucial to discriminate conspecific sex pheromones from related molecules co-existing in the environment; thus, they are essential for precise mate recognition. The role of moth SPRs with a broader response spectrum and the determinants of their ligand selectivity are largely unclear. Probably, they represent a preadaptation to ensure effective tracking of female-released sex pheromone signals even if the composition of the blend undergoes slight changes (Gould et al., 2010; Heckel, 2010; Zhang and Löfstedt, 2015). Noteworthy, recent analyses of the SPR orthologs HassOR14b and HarmOr14b from the closely related moth species Helicoverpa assulta and Helicoverpa armigera showed that only few key amino acid residues appear to be sufficient to shift the ligand specificity between orthologous but differently tuned SPRs. In contrast, substitution of many other amino acid residues had no or only subtle effects (Yang et al., 2017). Based on these findings, it has been suggested that consecutive point mutations in key amino acids of SPRs during evolution may have been major drivers in the course of speciation.

\section{TRANSDUCTION MECHANISMS AND INTERPLAY OF PRs WITH OTHER SIGNALING PROTEINS}

\section{PBPs and Their Relevance for Pheromone Transport and Detection}

Pheromone detection is initiated when pheromonal substances enter olfactory sensilla via cuticular pores. In the aqueous lymph, pheromonal and odorous molecules bind to water-soluble odorant-binding proteins (OBPs) synthetized and released by support cells that surround OSNs (Figure 1A). OBPs are supposed to mediate solubilizing and subsequent transport to the relevant olfactory receptor proteins residing in the dendritic membrane of OSNs. For the specific binding and transport of pheromones to PRs, a subfamily of the OBPs, the PBPs, is regarded as essential. During the last decades, numerous PBPs from various insect species have been identified. For a more detailed review of PBPs, the reader is referred to articles that explicitly highlight this group of proteins (Pelosi et al., 2006; Fan et al., 2011; Vieira and Rozas, 2011; Leal, 2013; Brito et al., 2016). In brief, in vitro and in vivo studies using PRs from different moth species and the cognate pheromone compounds have demonstrated that the sensitivity as well as the specificity of pheromone-evoked signaling is enhanced in the presence of 
appropriate PBPs (Grosse-Wilde et al., 2006; Forstner et al., 2009; Chang et al., 2015). These observations are consistent with a role of PBPs in solubilizing and transporting pheromones. Yet, the precise role of PBPs for pheromone detection is uncertain. In this regard, it has been reported that in Drosophila flies mutant for the PBP type LUSH (OBP76a), the responsiveness to the LUSH-binding pheromone cVA is abolished and the spontaneous activity in cVA-sensitive antennal OSNs in the absence of the pheromone is diminished ( $\mathrm{Xu}$ et al., 2005). Introducing recombinant LUSH protein directly into cVA-responsive sensilla from LUSH mutant flies restored spontaneous activity (Xu et al., 2005). Moreover, detailed analyses indicate that LUSH is an inactive, extracellular ligand that is converted by cVA into an activator of PRNs (Laughlin et al., 2008). However, in a more recent study, activation of the relevant PRNs was induced by higher concentrations of cVA even in the absence of LUSH (Gomez-Diaz et al., 2013). Consequently, further studies are required to unravel the functional role of LUSH (and other PBPs) for pheromone detection.

\section{Role of SNMP1 in Pheromone Detection}

Besides an interplay of PRs and PBPs, a number of studies have demonstrated that in insects, sensitive pheromone signaling requires SNMP1 (Benton et al., 2007; Jin et al., 2008; Li et al., 2014; Pregitzer et al., 2014). SNMP1 was first discovered in the moth Antheraea polyphemus as a prominent protein in the dendritic membrane of PRNs (Rogers et al., 1997, 2001b). More recent studies revealed co-expression of several proven and candidate PRs with SNMP1 in OSNs (Krieger et al., 2002; Benton, 2007; Pregitzer et al., 2014, 2017) and suggest a localization of SNMP1 in close proximity to receptor proteins in the membrane (Benton et al., 2007; German et al., 2013). In cells expressing PRs, SNMP1 is required for highly sensitive responses and is important for rapid activation as well as termination of pheromone-induced activity (Benton, 2007; Li et al., 2014; Pregitzer et al., 2014). The specific function of SNMP1 in the pheromone signaling process is unclear. Already early, a role as co-receptor that may be involved in unloading pheromones from PBPs and passing the signal molecules to PRs has been postulated (Rogers et al., 1997; Vogt et al., 2009). This concept has recently been substantiated by demonstrating that SNMP1 may indeed bind pheromones to its large ectodomain and may forward ligands through this tunnellike domain to a PR (Gomez-Diaz et al., 2016). How SNMP1 interplays with PRs and PBPs is an open question. Furthermore, it is unknown whether and to what extend SNMP1 might be also involved in the sensitive detection of non-pheromonal compounds.

\section{lonotropic Versus Metabotropic Transduction Processes in Pheromone-Induced Signaling and the Uncertain Role of the OR Co-receptor Orco}

Insect OSNs positive for ORs - including PRs - seem to commonly co-express a non-canonical member of the OR family designated as Or83b or Orco (Krieger et al., 2003;
Larsson et al., 2004; Pitts et al., 2004; Jones et al., 2005). Over the past years, substantial evidence has been accumulated that Orco forms multimers of unknown stoichiometry with ORs and that Orco is crucial for dendritic localization, membrane targeting, and subsequent signaling of ORs (Larsson et al., 2004; Neuhaus et al., 2005; Benton et al., 2006). While no structural model for OR/Orco heteromers exists yet, the structure of Orco homomers has been elucidated recently by cryo-electron microscopy. The structure indicates a channel architecture, with four subunits symmetrically arranged around a central pore (Butterwick et al., 2018). With respect to pheromone detection, deletion or silencing of Orco expression has been reported to evoke a dramatic loss of OSN responsiveness to pheromonal compounds (Koutroumpa et al., 2016; Li et al., 2016; Liu Q. et al., 2017). Consistently, activation of PRs by appropriate pheromones in heterologous expression systems is also significantly higher upon co-expression of Orco (Nakagawa et al., 2005; Wanner et al., 2007). In fact, co-expression of the $B$. mori bombykol receptor BmOR1 with Orco in heterologous expression systems induced a considerable ligand-stimulated non-selective cation channel activity (Nakagawa et al., 2005; Sato et al., 2008). In this context, in addition to its function as a chaperon, in experiments using heterologous expression, Orco has been identified as a spontaneously opening $\mathrm{Ca}^{2+}$-permeable and unspecific cation channel (Wicher et al., 2008; Jones et al., 2011; Nolte et al., 2013). Thus, such observations have led to the concept that heteromeric complexes comprising Orco and ORs/PRs function as ligand-gated ion channels in which the binding of the ligand is exclusively mediated by the OR/PR protein (Figure 1B; Sato et al., 2008; Touhara and Vosshall, 2009; Wicher, 2015). Yet, the functional relevance of Orco in PRNs is still a matter of controversial discussion. In spite of the above described findings related to PRs from bees, $D$. melanogaster and B. mori, recent studies with PRs and/or PRNs from different moth species (including $H$. virescens and Manduca sexta) challenge the notion that pheromone-evoked signaling in OSNs is ionotropic and relies on Orco. Notably, in HEK cells expressing PRs but lacking Orco, pheromones elicited clear responses (Grosse-Wilde et al., 2006, 2007; Forstner et al., 2009). Furthermore, experimental findings of tip recordings from $M$. sexta pheromone-sensitive sensilla upon application of Orco agonists and antagonists argue against an involvement of Orco and ionotropic signaling in the primary transduction processes of pheromone detection. Instead, Orco seems to serve as a slower, second messengergated pacemaker channel that controls the membrane potential and hence affects the threshold and kinetics of pheromone-induced responses via changes of intracellular $\mathrm{Ca}^{2+}$ baseline concentrations (Nolte et al., 2013, 2016). Although the transduction cascade underlying pheromoneevoked signaling in moth OSNs is still elusive, it has been suggested that this process is largely metabotropic (Stengl and Funk, 2013; Nolte et al., 2016). This notion is in line with the observation that insect ORs - alike G protein-coupled receptors (GPCRs) - are heptahelical receptors although they share no sequence similarities with canonical GPCRs and 
show an inverted membrane topology with an intracellular N-terminus (Benton et al., 2006; Smart et al., 2008; Tsitoura et al., 2010). Consistent with the heptahelical structure of ORs/PRs and potential downstream $G$ protein-mediated signaling, earlier findings have revealed the synthesis of the second messenger substance inositol 1,4,5-trisphosphate $\left(\mathrm{IP}_{3}\right)$ in insect antennal tissue following exposure to pheromones (Boekhoff et al., 1990). Intriguingly, perfusing $M$. sexta OSNs with $\mathrm{IP}_{3}$ elicits a specific sequence of currents that is mimicked by exposure to pheromones (Stengl et al., 1992; Stengl, 1993; Stengl and Funk, 2013). Based on these findings, it has been proposed that in moth OSNs, pheromones elicit via PRs, G proteins, and phospholipase $\mathrm{C}$ type $\beta$ (PLC $\beta$ ) an increased formation of the second messengers $\mathrm{IP}_{3}$ and diacylglycerol, leading to the activation of $\mathrm{IP}_{3}$-gated $\mathrm{Ca}^{2+}$ channels and $\mathrm{Ca}^{2+}$-activated cation channels (Stengl and Funk, 2013). Additionally, activation of protein kinase C (PKC) by diacylglycerol might induce opening of PKC-activated cation channels (Figure 1C). Yet, future studies are urgently required to elucidate in more detail the metabotropic and ionotropic processes that underlie pheromone-evoked signaling in insect OSNs.

The electrical activity evoked in antennal OSNs upon binding of pheromones (or general odorants) to cognate olfactory receptors is transformed into a pattern of action potentials and transmitted via their axons to the primary olfactory center in the brain, a region of the insect deutocerebrum known as the antennal lobe (Martin et al., 2011; Renou, 2014). The axonal terminals of OSNs expressing a distinct PR (or other OR) converge on a single out of numerous spherical units called glomeruli, suggesting a receptor-based map of olfactory connectivity and coding (Vosshall et al., 2000; Couto et al., 2005; Sakurai et al., 2014). The size of the glomeruli appears to correlate with the number of OSNs expressing given receptors in the antennae (Grabe et al., 2016). This is most obvious in male moths comprising particular high numbers of antennal OSNs expressing PRs for female-released SPCs. Accordingly, in the antennal lobe, sex-specific clusters of enlarged glomeruli are found (termed macroglomeruli) that form the so-called macroglomerular complex (Hansson et al., 1992; Christensen and Hildebrand, 2002; Berg et al., 2014). Enlarged and malespecific glomeruli are not restricted to moths. Macroglomeruli have been also reported for bees, ants, and cockroaches (reviewed in Hansson and Anton, 2000; Galizia and Rossler, 2010); consequently, axonal convergence of OSNs endowed with PRs for sex pheromones on macroglomeruli might be a widespread trait in insects.

Yet, although the axonal projection of an individual OSN to a given glomerulus is apparently associated with the olfactory receptor expressed by this neuron, "normal" expression of an $\mathrm{OR} / \mathrm{PR}$ type is not required to navigate the axon to its target glomerulus (Dobritsa et al., 2003; Wang et al., 2003;

\section{REFERENCES}

Almaas, T. J., and Mustaparta, H. (1991). Heliothis virescens: response characteristics of receptor neurons in sensilla trichodea type 1 and type 2 . J. Chem. Ecol. 17, 953-972. doi: 10.1007/BF01395602
Sakurai et al., 2015). This finding indicates that it is not the receptor protein that determines targeting to the appropriate glomerulus; an observation that is in marked contrast to the vertebrate olfactory system (Komiyama and Luo, 2006).

\section{CONCLUSION AND FUTURE DIRECTIONS}

Research conducted in recent years has greatly advanced our understanding of the cellular and molecular processes that underlie pheromone reception in insects, but at the same time raised many open questions that will stimulate future investigations. On the molecular level, in a number of insects, an array of narrowly and broadly tuned PRs have been identified that mediate the recognition and coding of pheromone signals. In the coming years, genome sequencing and bioinformatics will give access to the olfactory receptor repertoires of a plethora of insect species that use pheromone communication. This will open the avenue to the discovery of PRs and other elements of pheromone reception in species that have yet not been accessible for a molecular analysis. In addition to PRs, current data indicate a crucial role of SNMP1 and PBPs in pheromone reception; however, how these proteins interplay in the process of pheromone signal recognition remains to be determined. To the same extent, the elements and mechanisms of pheromone signal transduction await further illumination, in particular with regard to the debated question whether distinct insect species use ionotropic, metabotropic, or both signaling processes to transduce intraspecific signals.

\section{AUTHOR CONTRIBUTIONS}

All authors listed have made a substantial, direct, and intellectual contribution to the work, and approved it for publication.

\section{FUNDING}

This work was supported by a grant to JK provided by the Deutsche Forschungsgemeinschaft (DFG), priority program SPP1392 (KR1786/4-2). We acknowledge the financial support by the "open access publication fund" of the Martin Luther University Halle-Wittenberg.

\section{ACKNOWLEDGMENTS}

We apologize to all colleagues whose work we could not include due to space limitations.

Alvarez, G., Ammagarahalli, B., Hall, D. R., Pajares, J. A., and Gemeno, C. (2015) Smoke, pheromone and kairomone olfactory receptor neurons in males and females of the pine sawyer Monochamus galloprovincialis (Olivier) (Coleoptera: Cerambycidae). J. Insect Physiol. 82, 46-55. doi: 10.1016/j.jinsphys.201 5.08 .004 
Andersson, M. N., Löfstedt, C., and Newcomb, R. D. (2015). Insect olfaction and the evolution of receptor tuning. Front. Ecol. Evol. 3:53. doi: 10.3389/fevo.2015. 00053

Baker, T. C. (2008). Balanced olfactory antagonism as a concept for understanding evolutionary shifts in moth sex pheromone blends. J. Chem. Ecol. 34, 971-981. doi: 10.1007/s10886-008-9468-5

Baker, T. C., Ochieng, S. A., Cosse, A. A., Lee, S. G., Todd, J. L., Quero, C., et al. (2004). A comparison of responses from olfactory receptor neurons of Heliothis subflexa and Heliothis virescens to components of their sex pheromone. J. Comp. Physiol. A 190, 155-165. doi: 10.1007/s00359-003-0483-2

Bengtsson, J. M., Trona, F., Montagne, N., Anfora, G., Ignell, R., Witzgall, P., et al. (2012). Putative chemosensory receptors of the codling moth, Cydia pomonella, identified by antennal transcriptome analysis. PLoS One 7:e31620. doi: 10.1371/journal.pone.0031620

Benton, R. (2007). Sensitivity and specificity in Drosophila pheromone perception. Trends Neurosci. 30, 512-519. doi: 10.1016/j.tins.2007.07.004

Benton, R., Sachse, S., Michnick, S. W., and Vosshall, L. B. (2006). Atypical membrane topology and heteromeric function of Drosophila odorant receptors in vivo. PLoS Biol. 4:e20. doi: 10.1371/journal.pbio.0040020

Benton, R., Vannice, K. S., and Vosshall, L. B. (2007). An essential role for a CD36related receptor in pheromone detection in Drosophila. Nature 450, 289-293. doi: $10.1038 /$ nature 06328

Berg, B. G., Zhao, X. C., and Wang, G. (2014). Processing of pheromone information in related species of heliothine moths. Insects 5, 742-761. doi: 10.3390/insects5040742

Boekhoff, I., Strotmann, J., Raming, K., Tareilus, E., and Breer, H. (1990). Odorantsensitive phospholipase C in insect antennae. Cell. Signal. 2, 49-56. doi: 10. 1016/0898-6568(90)90032-6

Bray, S., and Amrein, H. (2003). A putative Drosophila pheromone receptor expressed in male-specific taste neurons is required for efficient courtship. Neuron 39, 1019-1029. doi: 10.1016/S0896-6273(03)00542-7

Brito, N. F., Moreira, M. F., and Melo, A. C. (2016). A look inside odorantbinding proteins in insect chemoreception. J. Insect Physiol. 95, 51-65. doi: 10.1016/j.jinsphys.2016.09.008

Butenandt, A., Beckmann, R., Stamm, D., and Hecker, E. (1959). Über den Sexuallockstoff des Seidenspinners Bombyx mori, Reindarstellung und Konstitution. Z. Naturfor. 14, 283-284.

Butterwick, J. A., Del Marmol, J., Kim, K. H., Kahlson, M. A., Rogow, J. A., Walz, T., et al. (2018). Cryo-EM structure of the insect olfactory receptor Orco. Nature 560, 447-452. doi: 10.1038/s41586-018-0420-8

Carey, A. F., Wang, G., Su, C. Y., Zwiebel, L. J., and Carlson, J. R. (2010). Odorant reception in the malaria mosquito Anopheles gambiae. Nature 464, 66-71. doi: $10.1038 /$ nature 08834

Cattaneo, A. M., Gonzalez, F., Bengtsson, J. M., Corey, E. A., Jacquin-Joly, E., Montagne, N., et al. (2017). Candidate pheromone receptors of codling moth Cydia pomonella respond to pheromones and kairomones. Sci. Rep. 7:41105. doi: $10.1038 /$ srep41105

Cervo, R., Dani, F. R., Zanetti, P., Massolo, A., and Turillazzi, S. (2002). Chemical nestmate recognition in a stenogastrine wasp, Liostenogaster flavolineata (Hymenoptera Vespidae). Ethol. Ecol. Evol. 14, 351-363. doi: 10.1016/S00221910(01)00077-4

Chang, H., Liu, Y., Yang, T., Pelosi, P., Dong, S., and Wang, G. (2015). Pheromone binding proteins enhance the sensitivity of olfactory receptors to sex pheromones in Chilo suppressalis. Sci. Rep. 5:13093. doi: 10.1038/srep 13093

Christensen, T. A., and Hildebrand, J. G. (2002). Pheromonal and host-odor processing in the insect antennal lobe: how different? Curr. Opin. Neurobiol. 12, 393-399. doi: 10.1016/S0959-4388(02)00336-7

Clyne, P. J., Warr, C. G., Freeman, M. R., Lessing, D., Kim, J., and Carlson, J. R. (1999). A novel family of divergent seven-transmembrane proteins: candidate odorant receptors in Drosophila. Neuron 22, 327-338. doi: 10.1016/S08966273(00)81093-4

Couto, A., Alenius, M., and Dickson, B. J. (2005). Molecular, anatomical, and functional organization of the Drosophila olfactory system. Curr. Biol. 15, 1535-1547. doi: 10.1016/j.cub.2005.07.034

Datta, S. R., Vasconcelos, M. L., Ruta, V., Luo, S., Wong, A., Demir, E., et al. (2008). The Drosophila pheromone cVA activates a sexually dimorphic neural circuit. Nature 452, 473-477. doi: 10.1038/nature06808 de Bruyne, M., and Baker, T. C. (2008). Odor detection in insects: volatile codes. J. Chem. Ecol. 34, 882-897. doi: 10.1007/s10886-008-9485-4

Deisig, N., Dupuy, F., Anton, S., and Renou, M. (2014). Responses to pheromones in a complex odor world: sensory processing and behavior. Insects 5, 399-422. doi: 10.3390/insects5020399

Dewhirst, S. Y., Pickett, J. A., and Hardie, J. (2010). Aphid pheromones. Vitam. Horm. 83, 551-574. doi: 10.1016/S0083-6729(10)83022-5

Dobritsa, A. A., van der Goes van Naters, W., Warr, C. G., Steinbrecht, R. A., and Carlson, J. R. (2003). Integrating the molecular and cellular basis of odor coding in the Drosophila antenna. Neuron 37, 827-841. doi: 10.1016/S0896-6273(03) 00094- 1

Durisko, Z., Kemp, R., Mubasher, R., and Dukas, R. (2014). Dynamics of social behavior in fruit fly larvae. PLoS One 9:e95495. doi: 10.1371/journal.pone. 0095495

Dweck, H. K., Ebrahim, S. A., Kromann, S., Bown, D., Hillbur, Y., Sachse, S., et al. (2013). Olfactory preference for egg laying on citrus substrates in Drosophila. Curr. Biol. 23, 2472-2480. doi: 10.1016/j.cub.2013.10.047

Dweck, H. K. M., Ebrahim, S. A. M., Thoma, M., Mohamed, A. A. M., Keesey, I. W., Trona, F., et al. (2015). Pheromones mediating copulation and attraction in Drosophila. Proc. Natl. Acad. Sci. U.S.A. 112, 2829-2835. doi: 10.1073/pnas. 1504527112

Ebbs, M. L., and Amrein, H. (2007). Taste and pheromone perception in the fruit fly Drosophila melanogaster. Pflugers. Arch. 454, 735-747. doi: 10.1007/s00424-007-0246-y

Ejima, A., Smith, B. P. C., Lucas, C., van der Goes van Naters, W., Miller, C. J., Carlson, J. R., et al. (2007). Generalization of courtship learning in Drosophila is mediated by cis-vaccenyl acetate. Curr. Biol. 17, 599-605. doi: 10.1016/j.cub.200 7.01.053

Engsontia, P., Sangket, U., Chotigeat, W., and Satasook, C. (2014). Molecular evolution of the odorant and gustatory receptor genes in lepidopteran insects: implications for their adaptation and speciation. J. Mol. Evol. 79, 21-39. doi: 10.1007/s00239-014-9633-0

Fabre, J. H. (1879). Souvenirs Entomologiques, vol. 1-10. Paris: Delagrave.

Fan, J., Francis, F., Liu, Y., Chen, J. L., and Cheng, D. F. (2011). An overview of odorant-binding protein functions in insect peripheral olfactory reception. Genet. Mol. Res. 10, 3056-3069. doi: 10.4238/2011.December.8.2

Farine, J. P., Ferveur, J. F., and Everaerts, C. (2012). Volatile Drosophila cuticular pheromones are affected by social but not sexual experience. PLoS One 7:e40396. doi: 10.1371/journal.pone.0040396.

Fleischer, J., Pregitzer, P., Breer, H., and Krieger, J. (2018). Access to the odor world: olfactory receptors and their role for signal transduction in insects. Cell. Mol. Life Sci. 75, 485-508. doi: 10.1007/s00018-017-2627-5

Forstner, M., Breer, H., and Krieger, J. (2009). A receptor and binding protein interplay in the detection of a distinct pheromone component in the silkmoth Antheraea polyphemus. Int. J. Biol. Sci. 5, 745-757. doi: 10.7150/ijbs.5.745

Galizia, C. G., and Rossler, W. (2010). Parallel olfactory systems in insects: anatomy and function. Annu. Rev. Entomol. 55, 399-420. doi: 10.1146/annurev-ento112408-085442

Gamberale, G., and Tullberg, B. S. (1998). Aposematism and gregariousness: the combined effect of group size and coloration on signal repellence. Proc. R. Soc. B - Biol. Sci. 265, 889-894. doi: 10.1098/rspb.1998.0374

Gao, Q., and Chess, A. (1999). Identification of candidate Drosophila olfactory receptors from genomic DNA sequence. Genomics 60, 31-39. doi: 10.1006/ geno.1999.5894

German, P. F., van der, P. S., Carraher, C., Kralicek, A. V., and Newcomb, R. D. (2013). Insights into subunit interactions within the insect olfactory receptor complex using FRET. Insect Biochem. Mol. Biol. 43, 138-145. doi: 10.1016/j. ibmb.2012.11.002

Ghaninia, M., Haight, K., Berger, S. L., Reinberg, D., Zwiebel, L. J., Ray, A., et al. (2017). Chemosensory sensitivity reflects reproductive status in the ant Harpegnathos saltator. Sci. Rep. 7:3732. doi: 10.1038/s41598-017-03964-7

Gohl, T., and Krieger, J. (2006). Immunolocalization of a candidate pheromone receptor in the antenna of the male moth, Heliothis virescens. Invert. Neurosci. 6, 13-21. doi: 10.1007/s10158-005-0012-9

Gomez-Diaz, C., Bargeton, B., Abuin, L., Bukar, N., Reina, J. H., Bartoi, T., et al. (2016). A CD36 ectodomain mediates insect pheromone detection via a putative tunnelling mechanism. Nat. Commun. 7:11866. doi: 10.1038/ncomms11866 
Gomez-Diaz, C., Reina, J. H., Cambillau, C., and Benton, R. (2013). Ligands for pheromone-sensing neurons are not conformationally activated odorant binding proteins. PLoS Biol. 11:e1001546. doi: 10.1371/journal.pbio.1001546

Gould, F., Estock, M., Hillier, N. K., Powell, B., Groot, A. T., Ward, C. M., et al. (2010). Sexual isolation of male moths explained by a single pheromone response QTL containing four receptor genes. Proc. Natl. Acad. Sci. U.S.A. 107, 8660-8665. doi: 10.1073/pnas.0910945107

Grabe, V., Baschwitz, A., Dweck, H. K. M., Lavista-Llanos, S., Hansson, B. S., and Sachse, S. (2016). Elucidating the neuronal architecture of olfactory glomeruli in the Drosophila antennal lobe. Cell. Rep. 16, 3401-3413. doi: 10.1016/j.celrep. 2016.08.063

Grosse-Wilde, E., Gohl, T., Bouche, E., Breer, H., and Krieger, J. (2007). Candidate pheromone receptors provide the basis for the response of distinct antennal neurons to pheromonal compounds. Eur. J. Neurosci. 25, 2364-2373. doi: 10. 1111/j.1460-9568.2007.05512.x

Grosse-Wilde, E., Svatos, A., and Krieger, J. (2006). A pheromone-binding protein mediates the bombykol-induced activation of a pheromone receptor in vitro. Chem. Senses 31, 547-555. doi: 10.1093/chemse/bjj059

Ha, T. S., and Smith, D. P. (2006). A pheromone receptor mediates 11-cis-vaccenyl acetate-induced responses in Drosophila. J. Neurosci. 26, 8727-8733. doi: 10. 1523/JNEUROSCI.0876-06.2006

Ha, T. S., and Smith, D. P. (2009). Odorant and pheromone receptors in insects. Front. Cell. Neurosci. 3:10. doi: 10.3389/neuro.03.010.2009

Hallem, E. A., and Carlson, J. R. (2006). Coding of odors by a receptor repertoire. Cell 125, 143-160. doi: 10.1016/j.cell.2006.01.050

Hallem, E. A., Ho, M. G., and Carlson, J. R. (2004). The molecular basis of odor coding in the Drosophila antenna. Cell 117, 965-979. doi: 10.1016/j.cell.2004. 05.012

Hamada, S., Tabuchi, M., Toyota, T., Sakurai, T., Hosoi, T., Nomoto, T., et al. (2014). Giant vesicles functionally expressing membrane receptors for an insect pheromone. Chem. Commun. (Camb) 50, 2958-2961. doi: 10.1039/c3cc48216b

Hansson, B. S., and Anton, S. (2000). Function and morphology of the antennal lobe: new developments. Annu. Rev. Entomol. 45, 203-231. doi: 10.1146/ annurev.ento.45.1.203

Hansson, B. S., Ljungberg, H., Hallberg, E., and Lofstedt, C. (1992). Functional specialization of olfactory glomeruli in a moth. Science 256, 1313-1315. doi: $10.1126 /$ science. 1598574

Hansson, B. S., and Stensmyr, M. C. (2011). Evolution of insect olfaction. Neuron 72, 698-711. doi: 10.1016/j.neuron.2011.11.003

Harari, A. R., and Steinitz, H. (2013). The evolution of female sex pheromones. Curr. Zool. 59, 569-578. doi: 10.1093/czoolo/59.4.569

Heckel, D. G. (2010). Smells like a new species: gene duplication at the periphery. Proc. Natl. Acad. Sci. U.S.A. 107, 9481-9482. doi: 10.1073/pnas.1004511107

Hillier, N. K., Kleineidam, C., and Vickers, N. J. (2006). Physiology and glomerular projections of olfactory receptor neurons on the antenna of female Heliothis virescens (Lepidoptera: Noctuidae) responsive to behaviorally relevant odors. J. Comp. Physiol. A Neuroethol. Sens. Neural. Behav. Physiol. 192, 199-219. doi: 10.1007/s00359-005-0061-x

Holdcraft, R., Rodriguez-Saona, C., and Stelinski, L. L. (2016). Pheromone autodetection: evidence and implications. Insects 7:E17. doi: 10.3390/ insects7020017.

Inoshita, T., Martin, J. R., Marion-Poll, F., and Ferveur, J. F. (2011). Peripheral, central and behavioral responses to the cuticular pheromone bouquet in Drosophila melanogaster males. PLoS One 6:e19770. doi: 10.1371/journal.pone. 0019770

Jeong, Y. T., Shim, J., Oh, S. R., Yoon, H. I., Kim, C. H., Moon, S. J., et al. (2013). An odorant-binding protein required for suppression of sweet taste by bitter chemicals. Neuron 79, 725-737. doi: 10.1016/j.neuron.2013.06.025

Jin, R., Liu, N. Y., Liu, Y., and Dong, S. L. (2015). A larval specific OBP able to bind the major female sex pheromone component in Spodoptera exigua (Hubner). J. Integr. Agricult. 14, 1356-1366. doi: 10.1016/S2095-3119(14)60849-2

Jin, X., Ha, T. S., and Smith, D. P. (2008). SNMP is a signaling component required for pheromone sensitivity in Drosophila. Proc. Natl. Acad. Sci. U.S.A. 105, 10996-11001. doi: 10.1073/pnas.0803309105

Jones, P. L., Pask, G. M., Rinker, D. C., and Zwiebel, L. J. (2011). Functional agonism of insect odorant receptor ion channels. Proc. Natl. Acad. Sci. U.S.A. 108, 8821-8825. doi: 10.1073/pnas.1102425108
Jones, W. D., Nguyen, T. A. T., Kloss, B., Lee, K. J., and Vosshall, L. B. (2005). Functional conservation of an insect odorant receptor gene across 250 million years of evolution. Curr. Biol. 15, R119-R121. doi: 10.1016/j.cub.2005.02.007

Joseph, R. M., and Carlson, J. R. (2015). Drosophila chemoreceptors: a molecular interface between the chemical world and the brain. Trends Genet. 31, 683-695. doi: 10.1016/j.tig.2015.09.005

Kaissling, K.-E., Kasang, G., Bestmann, H. J., Stransky, W., and Vostrowsky, O. (1978). A new pheromone of the silkworm moth Bombyx mori. Naturwissenschaften 65, 382-384. doi: 10.1007/BF00439702

Karlson, P., and Lüscher, M. (1959). Pheromones - New term for a class of biologically active substances. Nature 183, 55-56. doi: 10.1038/183055a0

Keil, T. A. (1989). Fine structure of the pheromone-sensitive sensilla on the antenna of the hawkmoth, Manduca sexta. Tissue Cell 21, 139-151. doi: 10.1016/ 0040-8166(89)90028-1

Koene, J. M., and ter Maat, A. (2001). “Allohormones": a class of bioactive substances favoured by sexual selection. J. Comp. Physiol. A-Sens. Neural and Behav. Physiol. 187, 323-326. doi: 10.1007/s003590100214

Koenig, C., Hirsh, A., Bucks, S., Klinner, C., Vogel, H., Shukla, A., et al. (2015). A reference gene set for chemosensory receptor genes of Manduca sexta. Insect Biochem. Mol. Biol. 66, 51-63. doi: 10.1016/j.ibmb.2015.09.007

Kohl, J., Huoviala, P., and Jefferis, G. S. (2015). Pheromone processing in Drosophila. Curr. Opin. Neurobiol. 34, 149-157. doi: 10.1016/j.conb.2015.06.009

Komiyama, T., and Luo, L. (2006). Development of wiring specificity in the olfactory system. Curr. Opin. Neurobiol. 16, 67-73. doi: 10.1016/j.conb.2005. 12.002

Koutroumpa, F. A., Monsempes, C., Francois, M. C., de Cian, A., Royer, C., Concordet, J. P., et al. (2016). Heritable genome editing with CRISPR/Cas9 induces anosmia in a crop pest moth. Sci. Rep. 6:29620. doi: 10.1038/srep29620

Krieger, J., Grosse-Wilde, E., Gohl, T., and Breer, H. (2005). Candidate pheromone receptors of the silkmoth Bombyx mori. Eur. J. Neurosci. 21, 2167-2176. doi: 10.1111/j.1460-9568.2005.04058.x

Krieger, J., Grosse-Wilde, E., Gohl, T., Dewer, Y. M. E., Raming, K., and Breer, H. (2004). Genes encoding candidate pheromone receptors in a moth (Heliothis virescens). Proc. Natl. Acad. Sci. U.S.A. 101, 11845-11850. doi: 10.1073/pnas. 0403052101

Krieger, J., Klink, O., Mohl, C., Raming, K., and Breer, H. (2003). A candidate olfactory receptor subtype highly conserved across different insect orders. J. Comp. Physiol. A Neuroethol. Sens. Neural Behav. Physiol. 189, 519-526. doi: 10.1007/s00359-003-0427-x

Krieger, J., Raming, K., Dewer, Y. M. E., Bette, S., Conzelmann, S., and Breer, H. (2002). A divergent gene family encoding candidate olfactory receptors of the moth Heliothis virescens. Eur. J. Neurosci. 16, 619-628. doi: 10.1046/j.14609568.2002.02109.x

Kurtovic, A., Widmer, A., and Dickson, B. J. (2007). A single class of olfactory neurons mediates behavioural responses to a Drosophila sex pheromone. Nature 446, 542-546. doi: 10.1038/nature05672

Lacaille, F., Hiroi, M., Twele, R., Inoshita, T., Umemoto, D., Maniere, G., et al. (2007). An inhibitory sex pheromone tastes bitter for Drosophila males. PLoS One 2:e661. doi: 10.1371/journal.pone.0000661

Larsson, M. C., Domingos, A. I., Jones, W. D., Chiappe, M. E., Amrein, H., and Vosshall, L. B. (2004). Or83b encodes a broadly expressed odorant receptor essential for Drosophila olfaction. Neuron 43, 703-714. doi: 10.1016/j.neuron. 2004.08.019

Laughlin, J. D., Ha, T. S., Jones, D. N., and Smith, D. P. (2008). Activation of pheromone-sensitive neurons is mediated by conformational activation of pheromone-binding protein. Cell 133, 1255-1265. doi: 10.1016/j.cell.2008. 04.046

Leal, W. S. (2013). Odorant reception in insects: roles of receptors, binding proteins, and degrading enzymes. Annu. Rev. Entomol. 58, 373-391. doi: 10. 1146/annurev-ento-120811-153635

Lebreton, S., Borrero-Echeverry, F., Gonzalez, F., Solum, M., Wallin, E. A., Hedenstrom, E., et al. (2017). A Drosophila female pheromone elicits speciesspecific long-range attraction via an olfactory channel with dual specificity for sex and food. BMC Biol. 15:88. doi: 10.1186/s12915-017-0427-x

Leonhardt, S. D., Menzel, F., Nehring, V., and Schmitt, T. (2016). Ecology and evolution of communication in social insects. Cell 164, 1277-1287. doi: 10.1016/ j.cell.2016.01.035 
Li, Y., Zhang, J., Chen, D., Yang, P., Jiang, F., Wang, X., et al. (2016). CRISPR/Cas9 in locusts: successful establishment of an olfactory deficiency line by targeting the mutagenesis of an odorant receptor co-receptor (Orco). Insect Biochem. Mol. Biol. 79, 27-35. doi: 10.1016/j.ibmb.2016.10.003

Li, Z., Ni, J. D., Huang, J., and Montell, C. (2014). Requirement for Drosophila SNMP1 for rapid activation and termination of pheromone-induced activity. PLoS Genet. 10:e1004600. doi: 10.1371/journal.pgen.1004600

Lin, C. C., Prokop-Prigge, K. A., Preti, G., and Potter, C. J. (2015). Food odors trigger Drosophila males to deposit a pheromone that guides aggregation and female oviposition decisions. Elife 4:e08688. doi: 10.7554/eLife.08688.

Liu, F., Xiong, C., and Liu, N. (2017). Chemoreception to aggregation pheromones in the common bed bug, Cimex lectularius. Insect Biochem. Mol. Biol. 82, 62-73. doi: 10.1016/j.ibmb.2017.01.012

Liu, Q., Liu, W., Zeng, B., Wang, G., Hao, D., and Huang, Y. (2017). Deletion of the Bombyx mori odorant receptor co-receptor (BmOrco) impairs olfactory sensitivity in silkworms. Insect. Biochem. Mol. Biol. 86, 58-67. doi: 10.1016/j. ibmb.2017.05.007

Liu, W., Liang, X., Gong, J., Yang, Z., Zhang, Y. H., Zhang, J. X., et al. (2011). Social regulation of aggression by pheromonal activation of Or65a olfactory neurons in Drosophila. Nat. Neurosci. 14, 896-902. doi: 10.1038/nn.2836

Martin, J. P., Beyerlein, A., Dacks, A. M., Reisenman, C. E., Riffell, J. A., Lei, H., et al. (2011). The neurobiology of insect olfaction: sensory processing in a comparative context. Prog. Neurobiol. 95, 427-447. doi: 10.1016/j.pneurobio. 2011.09.007

McKenzie, S. K., Fetter-Pruneda, I., Ruta, V., and Kronauer, D. J. (2016). Transcriptomics and neuroanatomy of the clonal raider ant implicate an expanded clade of odorant receptors in chemical communication. Proc. Natl. Acad. Sci. U.S.A. 113, 14091-14096. doi: 10.1073/pnas.1610800113

Meng, L. Z., Wu, C. H., Wicklein, M., Kaissling, K.-E., and Bestmann, H.-J. (1989). Number and sensitivity of three types of pheromone receptor cells in Antheraea pernyi and A. polyphemus. J. Comp. Physiol. 165, 139-146. doi: 10.1007/BF00619188

Mitsuno, H., Sakurai, T., Murai, M., Yasuda, T., Kugimiya, S., Ozawa, R., et al. (2008). Identification of receptors of main sex-pheromone components of three Lepidopteran species. Eur. J. Neurosci. 28, 893-902. doi: 10.1111/j.1460-9568. 2008.06429.x

Miyamoto, T., and Amrein, H. (2008). Suppression of male courtship by a Drosophila pheromone receptor. Nat. Neurosci. 11, 874-876. doi: 10.1038/nn. 2161

Montagne, N., Chertemps, T., Brigaud, I., Francois, A., Francois, M. C., de Fouchier, A., et al. (2012). Functional characterization of a sex pheromone receptor in the pest moth Spodoptera littoralis by heterologous expression in Drosophila. Eur. J. Neurosci. 36, 2588-2596. doi: 10.1111/j.1460-9568.2012. 08183.x

Montagne, N., de Fouchier, A., Newcomb, R. D., and Jacquin-Joly, E. (2015). Advances in the identification and characterization of olfactory receptors in insects. Prog. Mol. Biol. Transl. Sci. 130, 55-80. doi: 10.1016/bs.pmbts.2014. 11.003

Moon, S. J., Lee, Y., Jiao, Y., and Montell, C. (2009). A Drosophila gustatory receptor essential for aversive taste and inhibiting male-to-male courtship. Curr. Biol. 19, 1623-1627. doi: 10.1016/j.cub.2009.07.061

Nakagawa, T., Sakurai, T., Nishioka, T., and Touhara, K. (2005). Insect sexpheromone signals mediated by specific combinations of olfactory receptors. Science 307, 1638-1642. doi: 10.1126/science.1106267

Neuhaus, E. M., Gisselmann, G., Zhang, W. Y., Dooley, R., Stortkuhl, K., and Hatt, H. (2005). Odorant receptor heterodimerization in the olfactory system of Drosophila melanogaster. Nat. Neurosci. 8, 15-17. doi: 10.1038/nn1371

Nichols, Z., and Vogt, R. G. (2008). The SNMP/CD36 gene family in diptera, hymenoptera and coleoptera: Drosophila melanogaster, D. pseudoobscura, Anopheles gambiae, Aedes aegypti, Apis mellifera, and Tribolium castaneum. Insect Biochem. Mol. Biol. 38, 398-415. doi: 10.1016/j.ibmb.2007.11.003

Nolte, A., Funk, N. W., Mukunda, L., Gawalek, P., Werckenthin, A., Hansson, B. S., et al. (2013). In situ tip-recordings found no evidence for an Orco-based ionotropic mechanism of pheromone-transduction in Manduca sexta. PLoS One 8:e62648. doi: 10.1371/journal.pone.0062648

Nolte, A., Gawalek, P., Koerte, S., Wei, H., Schumann, R., Werckenthin, A., et al. (2016). No evidence for ionotropic pheromone transduction in the hawkmoth Manduca sexta. PLoS One 11:e0166060. doi: 10.1371/journal.pone.0166060
Ochieng', S. A., and Hansson, B. S. (1999). Responses of olfactory receptor neurones to behaviourally important odours in gregarious and solitarious desert locust, Schistocerca gregaria. Physiol. Entomol. 24, 28-36. doi: 10.1046/j.1365-3032. 1999.00107.x

Pask, G. M., Slone, J. D., Millar, J. G., Das, P., Moreira, J. A., Zhou, X. F., et al. (2017). Specialized odorant receptors in social insects that detect cuticular hydrocarbon cues and candidate pheromones. Nat. Commun. 8:297. doi: 10. 1038/s41467-017-00099-1

Pelosi, P., Zhou, J. J., Ban, L. P., and Calvello, M. (2006). Soluble proteins in insect chemical communication. Cell. Mol. Life Sci. 63, 1658-1676. doi: 10.1007/ s00018-005-5607-0

Pitts, R. J., Fox, A. N., and Zwiebel, L. J. (2004). A highly conserved candidate chemoreceptor expressed in both olfactory and gustatory tissues in the malaria vector Anopheles gambiae. Proc. Natl. Acad. Sci. U.S.A. 101, 5058-5063. doi: 10.1073/pnas.0308146101

Pitts, S., Pelser, E., Meeks, J., and Smith, D. (2016). Odorant responses and courtship behaviors influenced by at4 neurons in Drosophila. PLoS One 11:e0162761. doi: 10.1371/journal.pone.0162761

Poivet, E., Gallot, A., Montagne, N., Glaser, N., Legeai, F., and Jacquin-Joly, E. (2013). A comparison of the olfactory gene repertoires of adults and larvae in the noctuid moth Spodoptera littoralis. PLoS One 8:e60263. doi: 10.1371/ journal.pone. 0060263

Poivet, E., Rharrabe, K., Monsempes, C., Glaser, N., Rochat, D., Renou, M., et al. (2012). The use of the sex pheromone as an evolutionary solution to food source selection in caterpillars. Nat. Commun. 3:1047. doi: 10.1038/ncomms2050

Prado, E., and Tjallingii, W. F. (1997). Effects of previous plant infestation on sieve element acceptance by two aphids. Entomol. Exp. Appl. 82, 189-200. doi: 10.1046/j.1570-7458.1997.00130.x

Pregitzer, P., Greschista, M., Breer, H., and Krieger, J. (2014). The sensory neurone membrane protein SNMP1 contributes to the sensitivity of a pheromone detection system. Insect. Mol. Biol. 23, 733-742. doi: 10.1111/imb.1 2119

Pregitzer, P., Jiang, X., Grosse-Wilde, E., Breer, H., Krieger, J., and Fleischer, J. (2017). In search for pheromone receptors: certain members of the odorant receptor family in the desert locust Schistocerca gregaria (Orthoptera: Acrididae) are co-expressed with SNMP1. Int. J. Biol. Sci. 13, 911-922. doi: 10.7150/ijbs.18402

Renou, M. (2014). "Pheromones and general odor perception in insects," in Neurobiology of Chemical Communication, Chap. 2, ed. C. Mucignat-Caretta (Boca Raton, FL: CRC Press).

Riipi, M., Alatalo, R. V., Lindstrom, L., and Mappes, J. (2001). Multiple benefits of gregariousness cover detectability costs in aposematic aggregations. Nature 413, 512-514. doi: 10.1038/35097061

Roelofs, W. L. (2016). "Reminiscence of the early days," in Pheromone Communication in Moths: Evolution, Behavior, and Application, Chap. 1, ed. J. D. Alisson and R. Carde (Oakland, CA: University of California Press).

Rogers, M. E., Krieger, J., and Vogt, R. G. (2001a). Antennal SNMPs (sensory neuron membrane proteins) of Lepidoptera define a unique family of invertebrate CD36-like proteins. J. Neurobiol. 49, 47-61. doi: 10.1002/neu.1065

Rogers, M. E., Steinbrecht, R. A., and Vogt, R. G. (2001b). Expression of SNMP1 in olfactory neurons and sensilla of male and female antennae of the silkmoth Antheraea polyphemus. Cell Tissue Res. 303, 433-446. doi: 10.1007/ s004410000305

Rogers, M. E., Sun, M., Lerner, M. R., and Vogt, R. G. (1997). Snmp-1, a novel membrane protein of olfactory neurons of the silk moth Antheraea polyphemus with homology to the CD36 family of membrane proteins. J. Biol. Chem. 272, 14792-14799. doi: 10.1074/jbc.272.23.14792

Ruther, J., Sieben, S., and Schricker, B. (2002). Nestmate recognition in social wasps: manipulation of hydrocarbon profiles induces aggression in the European hornet. Naturwissenschaften 89, 111-114. doi: 10.1007/s00114-001-0292-9

Sakurai, T., Mitsuno, H., Mikami, A., Uchino, K., Tabuchi, M., Zhang, F., et al. (2015). Targeted disruption of a single sex pheromone receptor gene completely abolishes in vivo pheromone response in the silkmoth. Sci. Rep. 5:11001. doi: $10.1038 /$ srep 11001

Sakurai, T., Nakagawa, T., Mitsuno, H., Mori, H., Endo, Y., Tanoue, S., et al. (2004). Identification and functional characterization of a sex pheromone receptor in the silkmoth Bombyx mori. Proc. Natl. Acad. Sci. U.S.A. 101, 16653-16658. doi: 10.1073/pnas.0407596101 
Sakurai, T., Namiki, S., and Kanzaki, R. (2014). Molecular and neural mechanisms of sex pheromone reception and processing in the silkmoth Bombyx mori. Front. Physiol. 5:125. doi: 10.3389/fphys.2014.00125

Sato, K., Pellegrino, M., Nakagawa, T., Nakagawa, T., Vosshall, L. B., and Touhara, K. (2008). Insect olfactory receptors are heteromeric ligand-gated ion channels. Nature 452, 1002-1006. doi: 10.1038/nature06850

Schiestl, F. P. (2005). On the success of a swindle: pollination by deception in orchids. Naturwissenschaften 92, 255-264. doi: 10.1007/s00114-005-0636-y

Schneider, D., Lacher, V., and Kaissling, K.-E. (1964). Die Reaktionsweise und das Reaktionspektrum von Riechzellen bei Antheraea pernyi (Lepidotera, Saturniidae). Zeitschr. Vergl. Physiol. 48, 632-662. doi: 10.1007/BF003 33745

Seidelmann, K., and Ferenz, H. J. (2002). Courtship inhibition pheromone in desert locusts, Schistocerca gregaria. J. Insect Physiol. 48, 991-996. doi: 10.1016/S00221910(02)00178-6

Slone, J. D., Pask, G. M., Ferguson, S. T., Millar, J. G., Berger, S. L., Reinberg, D., et al. (2017). Functional characterization of odorant receptors in the ponerine ant, Harpegnathos saltator. Proc. Natl. Acad. Sci. U.S.A. 114, 8586-8591. doi: 10.1073/pnas.1704647114

Smart, R., Kiely, A., Beale, M., Vargas, E., Carraher, C., Kralicek, A. V., et al. (2008). Drosophila odorant receptors are novel seven transmembrane domain proteins that can signal independently of heterotrimeric G proteins. Insect Biochem. Mol. Biol. 38, 770-780. doi: 10.1016/j.ibmb.2008.05.002

Steinbrecht, R. A. (1996). Structure and function of insect olfactory sensilla. Ciba Found. Symp. 200, 158-174.

Steinbrecht, R. A. (1997). Pore structures in insect olfactory sensilla: a review of data and concepts. Int. J. Insect Morphol. Embryol. 26, 229-245. doi: 10.1016/ S0020-7322(97)00024-X

Steinwender, B., Thrimawithana, A. H., Crowhurst, R. N., and Newcomb, R. D. (2015). Pheromone receptor evolution in the cryptic leafroller species, Ctenopseustis obliquana and C. herana. J. Mol. Evol. 80, 42-56. doi: 10.1007/ s00239-014-9650-z

Stengl, M. (1993). Intracellular-messenger-mediated cation channels in cultured olfactory receptor neurons. J. Exp. Biol. 178, 125-147.

Stengl, M. (2010). Pheromone transduction in moths. Front. Cell. Neurosci. 4:133. doi: $10.3389 /$ fncel.2010.00133

Stengl, M., and Funk, N. W. (2013). The role of the coreceptor Orco in insect olfactory transduction. J. Comp. Physiol. A Neuroethol. Sens. Neural Behav. Physiol. 199, 897-909. doi: 10.1007/s00359-013-0837-3

Stengl, M., Zufall, F., Hatt, H., and Hildebrand, J. G. (1992). Olfactory receptor neurons from antennae of developing male Manduca sexta respond to components of the species-specific sex pheromone in vitro. J. Neurosci. 12, 2523-2531. doi: 10.1523/JNEUROSCI.12-07-02523.1992

Stocker, R. F. (2001). Drosophila as a focus in olfactory research: mapping of olfactory sensilla by fine structure, odor specificity, odorant receptor expression, and central connectivity. Microsc. Res. Technol. 55, 284-296. doi: 10.1002/jemt. 1178

Sun, M., Liu, Y., Walker, W. B., Liu, C., Lin, K., Gu, S., et al. (2013). Identification and characterization of pheromone receptors and interplay between receptors and pheromone binding proteins in the diamondback moth, Plutella xyllostella. PLoS One 8:e62098. doi: 10.1371/journal.pone.006 2098

Syed, Z., Kopp, A., Kimbrell, D. A., and Leal, W. S. (2010). Bombykol receptors in the silkworm moth and the fruit fly. Proc. Natl. Acad. Sci. U.S.A. 107, 9436-9439. doi: $10.1073 /$ pnas. 1003881107

Touhara, K. (2009). Insect olfactory receptor complex functions as a ligand-gated ionotropic channel. Ann. N. Y. Acad. Sci. 1170, 177-180. doi: 10.1111/j.17496632.2009.03935.x

Touhara, K., and Vosshall, L. B. (2009). Sensing odorants and pheromones with chemosensory receptors. Annu. Rev. Physiol. 71, 307-332. doi: 10.1146/ annurev.physiol.010908.163209

Tsitoura, P., Andronopoulou, E., Tsikou, D., Agalou, A., Papakonstantinou, M. P., Kotzia, G. A., et al. (2010). Expression and membrane topology of Anopheles gambiae odorant receptors in lepidopteran insect cells. PLoS One 5:e15428. doi: 10.1371 /journal.pone. 0015428

Van der Goes van Naters, W. (2014). "Drosophila pheromones: from reception to perception," in Neurobiology of Chemical Communication, Chap. 6, ed. C. Mucignat-Carettach (Boca Raton, FL: CRC Press). doi: 10.1201/b16511-7
Verhoef, H. A., and Nagelkerke, C. J. (1977). Formation and ecological significance of aggregations in collembola: an experimental study. Oecologia 31, 215-226. doi: $10.1007 / \mathrm{Bf} 00346922$

Vickers, N. J., Christensen, T. A., Mustaparta, H., and Baker, T. C. (1991). Chemical communication in heliothine moths. III. Flight behavior of male Helicoverpa zea and Heliothis virescens in response to varying ratios of intraand interspecific pheromone compounds. J. Comp. Physiol. A 169, 275-280. doi: 10.1007/BF00206991

Vieira, F. G., and Rozas, J. (2011). Comparative genomics of the odorant-binding and chemosensory protein gene families across the Arthropoda: origin and evolutionary history of the chemosensory system. Genome Biol. Evol. 3, 476490. doi: $10.1093 /$ gbe/evr033

Vogt, R. G., Miller, N. E., Litvack, R., Fandino, R. A., Sparks, J., Staples, J., et al. (2009). The insect SNMP gene family. Insect Biochem. Mol. Biol. 39, 448-456. doi: 10.1016/j.ibmb.2009.03.007

Vosshall, L. B., Amrein, H., Morozov, P. S., Rzhetsky, A., and Axel, R. (1999). A spatial map of olfactory receptor expression in the Drosophila antenna. Cell 96, 725-736. doi: 10.1016/S0092-8674(00)80582-6

Vosshall, L. B., Wong, A. M., and Axel, R. (2000). An olfactory sensory map in the fly brain. Cell 102, 147-159. doi: 10.1016/S0092-8674(00)00021-0

Wang, G., Carey, A. F., Carlson, J. R., and Zwiebel, L. J. (2010). Molecular basis of odor coding in the malaria vector mosquito Anopheles gambiae. Proc. Natl. Acad. Sci. U.S.A. 107, 4418-4423. doi: 10.1073/pnas.0913392107

Wang, G., Vasquez, G. M., Schal, C., Zwiebel, L. J., and Gould, F. (2011). Functional characterization of pheromone receptors in the tobacco budworm Heliothis virescens. Insect Mol. Biol. 20, 125-133. doi: 10.1111/j.1365-2583.2010.01045.x

Wang, J. W., Wong, A. M., Flores, J., Vosshall, L. B., and Axel, R. (2003). Twophoton calcium imaging reveals an odor-evoked map of activity in the fly brain. Cell 112, 271-282. doi: 10.1016/S0092-8674(03)00004-7

Wang, L., and Anderson, D. J. (2010). Identification of an aggression-promoting pheromone and its receptor neurons in Drosophila. Nature 463, 227-231. doi: $10.1038 /$ nature 08678

Wanner, K. W., Nichols, A. S., Allen, J. E., Bunger, P. L., Garczynski, S. F., Linn, C. E., et al. (2010). Sex pheromone receptor specificity in the European corn borer moth, Ostrinia nubilalis. PLoS One 5:e8685. doi: 10.1371 /journal.pone. 0008685

Wanner, K. W., Nichols, A. S., Walden, K. K., Brockmann, A., Luetje, C. W., and Robertson, H. M. (2007). A honey bee odorant receptor for the queen substance 9-oxo-2-decenoic acid. Proc. Natl. Acad. Sci. U.S.A. 104, 14383-14388. doi: 10.1073/pnas.0705459104

Wertheim, B., van Baalen, E. J., Dicke, M., and Vet, L. E. (2005). Pheromonemediated aggregation in nonsocial arthropods: an evolutionary ecological perspective. Annu. Rev. Entomol. 50, 321-346. doi: 10.1146/annurev.ento.49. 061802.123329

Wicher, D. (2015). Olfactory signaling in insects. Prog. Mol. Biol. Transl. Sci. 130, 37-54. doi: 10.1016/bs.pmbts.2014.11.002

Wicher, D. (2018). Tuning insect odorant receptors. Front. Cell. Neurosci. 12:94. doi: 10.3389/fncel.2018.00094

Wicher, D., Schafer, R., Bauernfeind, R., Stensmyr, M. C., Heller, R., Heinemann, S. H., et al. (2008). Drosophila odorant receptors are both ligand-gated and cyclic-nucleotide-activated cation channels. Nature 452, 1007-1011. doi: 10. 1038/nature06861

Widmayer, P., Heifetz, Y., and Breer, H. (2009). Expression of a pheromone receptor in ovipositor sensilla of the female moth (Heliothis virescens). Insect Mol. Biol. 18, 541-547. doi: 10.1111/j.1365-2583.2009.00894.x

Wilson, E. O., and Regnier, F. E. (1971). Evolution of alarm-defense system in formicine ants. Am. Natural. 105, 279-289. doi: 10.1086/282724

Wyatt, T. D. (2014). Pheromones and Animal Behavior. Cambridge: Cambridge Univ Press.

Xu, P. X., Atkinson, R., Jones, D. N. M., and Smith, D. P. (2005). Drosophila OBP LUSH is required for activity of pheromone-sensitive neurons. Neuron 45 , 193-200. doi: 10.1016/j.neuron.2004.12.031

Yang, K., Huang, L. Q., Ning, C., and Wang, C. Z. (2017). Two single-point mutations shift the ligand selectivity of a pheromone receptor between two closely related moth species. Elife 6:e29100. doi: 10.7554/eLife.29100

Yew, J. Y., and Chung, H. (2015). Insect pheromones: an overview of function, form, and discovery. Prog. Lipid Res. 59, 88-105. doi: 10.1016/j.plipres.2015. 06.001 
Zhang, D. D., and Löfstedt, C. (2015). Moth pheromone receptors: gene sequences, function, and evolution. Front. Ecol. Evol. 3:105. doi: 10.3389/fevo.2015.00105

Zhang, D. D., Wang, H. L., Schultze, A., Fross, H., Francke, W., Krieger, J., et al. (2016). Receptor for detection of a type II sex pheromone in the winter moth Operophtera brumata. Sci. Rep. 6:18576. doi: 10.1038/srep1 8576

Zhang, J., Yan, S., Liu, Y., Jacquin-Joly, E., Dong, S., and Wang, G. (2015). Identification and functional characterization of sex pheromone receptors in the common cutworm (Spodoptera litura). Chem. Senses 40, 7-16. doi: 10.1093/ chemse/bju052

Zhang, R., Wang, B., Grossi, G., Falabella, P., Liu, Y., Yan, S., et al. (2017) Molecular basis of alarm pheromone detection in aphids. Curr. Biol. 27, 55-61. doi: 10.1016/j.cub.2016.10.013

Zhu, J., Ban, L., Song, L. M., Liu, Y., Pelosi, P., and Wang, G. (2016). General odorant-binding proteins and sex pheromone guide larvae of Plutella xylostella to better food. Insect Biochem. Mol. Biol. 72, 10-19. doi: 10.1016/j.ibmb.2016. 03.005
Zielonka, M., Breer, H., and Krieger, J. (2017). Molecular elements of pheromone detection in the female moth, Heliothis virescens. Insect Sci. 25, 389-400. doi: 10.1111/1744-7917.12434

Zielonka, M., Gehrke, P., Badeke, E., Sachse, S., Breer, H., and Krieger, J. (2016). Larval sensilla of the moth Heliothis virescens respond to sex pheromone components. Insect Mol. Biol. 25, 666-678. doi: 10.1111/imb.12253

Conflict of Interest Statement: The authors declare that the research was conducted in the absence of any commercial or financial relationships that could be construed as a potential conflict of interest.

Copyright (c) 2018 Fleischer and Krieger. This is an open-access article distributed under the terms of the Creative Commons Attribution License (CC BY). The use, distribution or reproduction in other forums is permitted, provided the original author(s) and the copyright owner(s) are credited and that the original publication in this journal is cited, in accordance with accepted academic practice. No use, distribution or reproduction is permitted which does not comply with these terms. 


\section{OPEN ACCESS}

Edited by:

Dieter Wicher

Max-Planck-Institut für chemische

Ökologie, Germany

Reviewed by:

Paul Szyszka,

Universität Konstanz, Germany

Shannon Bryn Olsson,

National Centre for Biological

Sciences, India

*Correspondence:

Vincent E. J. M. Jacob

vincent.jacob@cirad.fr

Received: 22 May 2018

Accepted: 13 August 2018

Published: 05 September 2018

Citation:

Jacob VEJM (2018) Current Source

Density Analysis of

Electroantennogram Recordings: A

Tool for Mapping the Olfactory

Response in an Insect Antenna.

Front. Cell. Neurosci. 12:287.

doi: 10.3389/fncel.2018.00287

\section{Current Source Density Analysis of Electroantennogram Recordings: A Tool for Mapping the Olfactory Response in an Insect Antenna}

\author{
Vincent E. J. M. Jacob* \\ CIRAD, UMR PVBMT, St. Pierre, La Réunion, France
}

The set of chemosensory receptors expressed by the olfactory receptor neurons lying in an insect's antennae and maxillary palps define the ability of this insect to perceive the volatile chemicals of its environment. The main two electrophysiological methods of antennal recordings for studying the range of chemicals that activate chemosensory receptors have limitations. Single-sensillum recording (SSR) samples a subset of olfactory receptor neurons and therefore does not reveal the full capacity of an insect to perceive an odor. Electroantennography (EAG), even if less resolutive than SSRs, is sometimes preferred since it samples the activity of a large number of the olfactory receptor neurons. But, at least in flies, the amplitude of the EAG signal is not directly correlated with the degree of sensitivity of the insect to the olfactory compound. Such dual methodology was also used to study mammalian brains, and the current source density (CSD) analysis was developed to bridge the gap between the cellular and the population recordings. This paper details the use of a similar approach adapted to the study of olfactory responses within insects with bulbous antennae. The EAG was recorded at multiple antennal positions and the CSD that generates the EAG potentials were estimated. The method measures the activation of olfactory receptor neurons (ORNs) across the antennae and thus it quantifies the olfactory sensitivity of the insect. It allows a rapid mapping of olfactory responses and thus can be used to guide further SSRs or to determine that two chemicals are detected by independent ORNs. This study further explored biases resulting from a limited number of recording positions or from an approximation of the antennal geometry that should be considered for interpreting the CSD maps. It also shows that the CSD analysis of EAGs is compatible with a gas chromatograph stimulator for analyzing the response to complex odors. Finally, I discuss the origin of the EAG signal in light of the CSD theory.

Keywords: insect, olfaction, electroantennogram, olfactory receptor neurons, antenna, current source density analysis 


\section{INTRODUCTION}

Insects live in an environment filled with numerous volatile compounds, but they detect and interact with only a few of them (Clavijo Mccormick et al., 2014) via their chemosensory receptors that includes olfactory receptors (ORs), ionotropic receptors (IRs) and gustatory receptors (GRs). The olfactory organs of the insects, antennae and maxillary palps, are covered with many olfactory sensilla, which house the dendrites of the olfactory receptor neurons (ORNs) (Shanbhag et al., 1999). The chemosensory receptors are located in the membrane of the ORN dendrites and are responsible for the activation of the ORNs by selective compounds (Joseph and Carlson, 2015).

Deciphering how insects perceive their chemical environment requires investigating the functions of the chemosensory receptors in the living insects. Two main types of antennal recordings were used over the past 60 years for this purpose (Olsson and Hansson, 2013): electroantennography (EAG) (Schneider, 1957a,b), which consists in recording field potentials across the antenna, and single-sensillum recording (SSR) (Einzelnen, 1962; Schneider and Boeckh, 1962), which consists in recording the spiking activity elicited by the ORNs of a single sensillum. SSR has some advantages over EAG. The signal is easier to interpret since it consists in the timing of the action potentials that travel through the antennal nerve and deliver the detected sensory information to antennal lobe neurons in the brain. Thus, SSR reliably measures the ORN output. Furthermore, some labeled-line behaviors are directly correlated with the activity level of a class of ORNs (Stensmyr et al., 2012; Dweck et al., 2013, 2015; Mansourian et al., 2016). However, the arbitrary sampling of individual sensilla provides an incomplete access to the functions of the olfactory organ. First, exploring the antennal responsiveness requires pooling the data from many individuals. Therefore, the interindividual variability cannot be estimated. Secondly, some classes of ORNs could be completely disregarded. An exhaustive exploration of the insect sensitivity to chemicals requires to sample all the different classes of chemosensory receptors expressed in an insect, and this information is solely available in Drosophila and demands significant experimental effort (Dweck et al., 2013, 2015, 2016; Nowotny et al., 2014; Ronderos et al., 2014; Münch and Galizia, 2016). Finally, the number of ORNs expressing the same chemosensory receptor is an important coding parameter that is difficult to estimate through SSR. It affects the latency of response and the sensitivity of second-order olfactory neurons (Bhandawat et al., 2007; Rospars et al., 2014).

Many researchers in chemical ecology use EAG rather than SSR because it provides a quick overview of the insect sensitivity to chemicals. The EAG signal results from the summation of the activity of many ORNs, thus combining different classes of chemosensory receptors. The amplitude of the EAG response depends both on the receptor potential response of individual ORNs (Kaissling, 1986; Lucas and Renou, 1992) and on the density of responsive ORNs in the vicinity of the recording electrode (Bigiani et al., 1989; Crnjar et al., 1989). It is tempting to consider that some evolutionary mechanisms should improve the detection of ecologically important chemicals through the involvement of more ORNs with an increased sensitivity, resulting in a large EAG response. However, experimental factors like the position of the recording electrode affects differently the amplitude of response to diverse chemicals (Bigiani et al., 1989; Crnjar et al., 1989; Biasazin et al., 2014; Jacob et al., 2017b). A small EAG response cannot be interpreted as a poor ability of the insect to detect a chemical. Obviously, neither SSR nor EAG are fully satisfying for quantitatively exploring the sensitivity of many insects to the constituents of their chemical environment.

The EAG is related to the local field potential (LFP) recordings in brain tissues that are known to depend on the complex properties of the propagation of electrical fields through the extracellular medium (Bédard and Destexhe, 2012). As in LFP, the contribution of an individual ORN to the EAG signal is inversely proportional to its distance to the recording electrode (Nagai, 1983b), so the EAG samples the activity of a subpopulation of antennal neurons. This consideration has a direct consequence for studying Drosophila melanogaster since morphological, functional and molecular observations over the last 20 years showed that each responsive class of ORN is confined in a sub-region of the funiculus (the third antennal segment; Couto et al., 2005) and this was also observed in other Muscomorpha species (Olsson et al., 2006; Tait et al., 2016). Clearly, an individual EAG recording misses or underestimates the response to some compounds in these species (Jacob et al., 2017b). Therefore, to estimate the sensitivity of an insect to olfactory compounds one must screen the antenna with varying the position of the EAG electrode (Biasazin et al., 2014; Jacob et al., 2017b). Since the pioneer work of Walter Pitts (Pitts, 1952), the methods of current source density (CSD) analysis (Nicholson, 1973; Mitzdorf, 1985; Buzsáki et al., 2012) have been developed to infer the single-cell activity from the population recordings. It consists in recording the LFPs at multiple positions and modeling the electrical field to localize the activated neurons (see Pettersen et al., 2006; Potworowski et al., 2012) for recent CSD models). In a recent paper we showed that CSD analysis can be applied to the EAG recorded in different Muscomorpha species and results in reproducible maps of antennal activation (Jacob et al., 2017b). This paper outlines a model of insect antenna used for analyzing the CSD from multiple EAG recordings. New experimental and modeled data are included here to enhance the interpretation of the CSD estimated by the model and to explore the limits of the method. CSD maps were found to correlate with response maps derived from ORN response data. However, an improper electrode sampling or a rough estimation of the antennal geometry were shown to bias the estimated CSD. As a proof of concept, example recordings are shown in several species of fruit flies and in the moth Chilo sacchariphagus, suggesting that CSD analysis can be used in various insect orders. In addition, the CSD maps were coupled with a gas chromatograph (GC), thus it is suited for future chemical ecology research and might be useful in the discovering of new attractants for insect pests.

\section{MATERIAL AND METHODS \\ Insects Used in This Study}

EAG datas were collected on sexually mature females of the species Zeugodacus cucurbitae, Ceratitis catoirii, Neoceratitis Cyanescens, Drosophila melanogaster, and the moth Chilo 
sacchariphagus. Antenna were measured on D. melanogaster (standard wild-type laboratory strain Canton Special, CS), on C. sacchariphagus, and on six tephritid species, namely Ceratitis capitata, C. catoirii, N. cyanescens, Bactrocera zonata, Z. cucurbitae, and Dacus demmerezi. C. capitata, C. catoirii, and B. zonata were reared on artificial diet (Duyck and Quilici, 2002), as well as D. melanogaster. $N$. cyanescens was reared on potato (Solanum tuberosum), and Z. cucurbitae and D. demmerezi were reared on zucchini (Cucurbita pepo). A C. sacchariphagus larva was collected in a sugar cane field then reared and the adult was studied after emergence. Insects were reared at $25 \pm 1^{\circ} \mathrm{C}$ and with $65 \pm 10 \%$ relative humidity and a 12:12 h light:dark photoperiod.

\section{Odor Delivery System}

A humidified air stream $(23 \mathrm{ml} / \mathrm{s}$, air speed $60 \mathrm{~cm} / \mathrm{s})$, filtered through a charcoal filter, was continuously delivered to the insect antenna through a $7-\mathrm{mm}$ glass tube held at $4 \mathrm{~mm}$. Stimuli were applied by inserting a Pasteur pipette $15 \mathrm{~cm}$ upstream containing a small piece of filter paper loaded with $1 \mu \mathrm{l}$ of a volatile compound diluted at $10^{-2}$ in paraffin oil. The compounds used in this paper were Z3-hexenyl acetate, linalool, methyl salicylate, ethyl butyrate or E2-hexenal. A puff of air $(200 \mathrm{~ms}, 5 \mathrm{ml} / \mathrm{s}$ ) was delivered through the pipette with an electro-valve (LHDA1233215-H, Lee Company, France) controlled by a digital output module (NI 9472, National Instr., Nanterre, France) and the software Labview (National Instr.). A control pipette was loaded with $1 \mu \mathrm{l}$ of paraffin oil. Control stimulations were applied twice, before and after a sequence of stimulation with each chemical applied in random order. Time intervals of $1 \mathrm{~min}$ were applied between consecutive puffs to limit the neuronal adaptation to chemicals.

Alternatively, the antenna was stimulated with a gas chromatograph (Clarus 580 GC, Perkin-Elmer, USA) injected with a mixture of Z3-hexenyl acetate, linalool, methyl salicylate, ethyl butyrate, pentyl acetate, ethyl acetate, and 1-octen-3-ol each diluted at $10^{-3}$ in hexane. The output capillary of the GC went through a $3 \mathrm{~m}$ transfer line (Antelia, Dardilly, France) heated at $250^{\circ} \mathrm{C}$ and its tip was inserted into the $7 \mathrm{~mm}$ glass tube instead of the Pasteur pipette.

\section{Electrophysiology}

Insects were secured in a plastic tube, and the head was fixed with dental wax, leaving the antennae exposed. Both the reference and the recording electrode were glass capillary electrodes (tip diameter 1-2 $\mu \mathrm{m}$, filled with $120 \mathrm{mM} \mathrm{NaCl}, 5 \mathrm{mM} \mathrm{KCl}$, $1 \mathrm{mM} \mathrm{CaCl}_{2}, 4 \mathrm{mM} \mathrm{MgCl}_{2}$, and $\left.10 \mathrm{mM} \mathrm{HEPES}\right)$. The reference electrode was inserted in the right eye, and the recording electrode was leaned against the left antenna without insertion. The same procedure was used for the moth C. sacchariphagus. The EAG was recorded consecutively while displacing the recording electrode in $N=4$ or 7 regularly interleaved positions, between 0 (adjacent to the basis of the arista in flies) and 1 (funiculus tip) along the proximo-distal axis. The recording position was set manually and was always on the middle axis of the lateral side of the funiculus. For the experiments with four antennal positions, the positions were explored in a random order. For the experiments with seven recording positions, the anatomical order (proximal to distal or distal to proximal) was used to assure that the spacing was as regular as possible. The signal was amplified (x200), low-pass filtered $(1 \mathrm{kHz})$ with a DAGAN Ex-1 amplifier (Minneapolis, Minnesota, USA), and was digitized at $500 \mathrm{~Hz}$ (NI 9215, National instr.) with Labview software. For quantifying the EAG response amplitude, the EAG was filtered with a Gaussian convolution of $20 \mathrm{~ms}$ width, and response to control was subtracted. Amplitude was defined as the maximum negative peak in the $0.5 \mathrm{~s}$ following stimulation minus the average value in the $0.5 \mathrm{~s}$ preceding stimulation.

\section{Current Source Density (CSD) Analysis}

The field potentials recorded in the neuronal tissues are caused by current sinks and sources surrounding the activated neurons. Current sources correspond to positive electric charges while current sinks correspond to negative charges. In the antennal surface, the activation of the ORNs result in a current sink near the dendrites that induce a negative potential recorded by the EAG electrode (Figure 10; Kaissling, 1986, 1995). The location of the current sinks in an insect antenna, and therefore of the activated ORNs, can be estimated from the spatial distribution of field potentials on the antennal surface (Figure 1A). To do so, the method used in this paper was adapted from the inverse method proposed by Pettersen and colleagues (Pettersen et al., 2006). It consists in three steps: (1) calculate the linear function that transforms a given distribution of CSD into the resulting spatial distribution of EAGs, (2) deduce the inverse linear function that transforms a given distribution of EAGs into the CSD it originates from, and (3) apply the inverse function to recorded EAGs.

The spatial distribution of potentials is estimated from EAGs recorded at $N$ positions on the antenna $(N=4$ or 7 ), and accordingly the current sources were estimated in $N$ compartments, each compartment corresponding to the antennal surface surrounding one recording position. The CSD distribution is approximated with the hypothesis that the CSD is constant on each compartment. This assumption is necessary to get a unique solution of CSD distribution from a given distribution of EAGs, but may result in potential biases addressed in the result section. Intuitively, the EAG recorded at a position on the antenna depends strongly of the nearby current sources, and weakly on the distant ones. For each antennal position $x_{i}$, the EAG potential recorded at this position was noted $\phi_{i}$ and the current source around this position was noted $C_{i}$. Each antennal compartment contributes additively to the potential $\phi_{i}$ as written in the Equation (1).

$$
\phi_{i}=\sum_{j} F_{i j}^{*} C_{j}
$$

The linear coefficients $F_{i j}$ describe the influence of the current source at the position $x_{j}$ to the EAG recorded at $x_{i}$, and decreases with the distance between $x_{i}$ and $x_{j}$. In matrix formulation:

$$
\phi=F^{*} C \text {. }
$$

The next section explains how to calculate directly the coefficients $F_{i j}$ trough the electrostatic forward solution applied to a model 


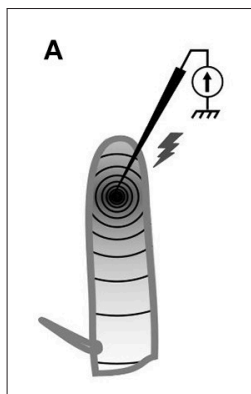

current source (CSD)
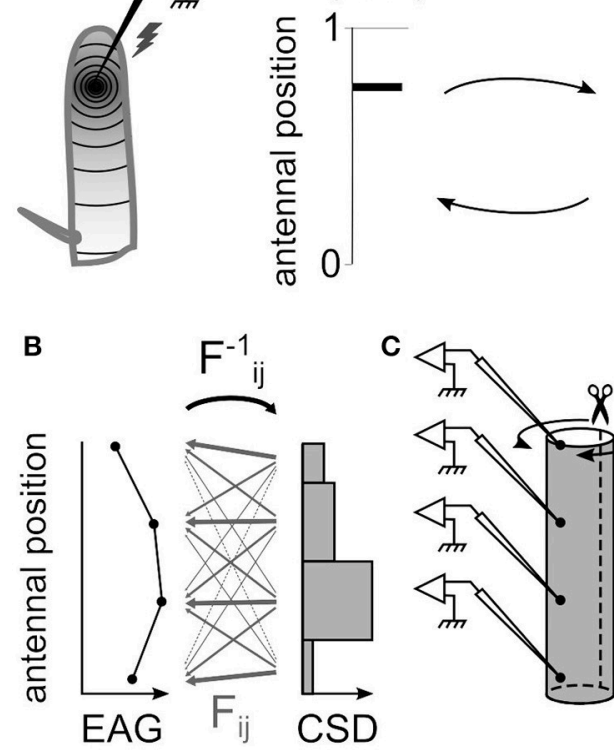

D1

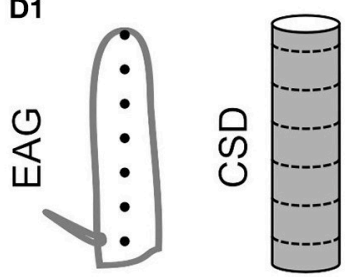

c

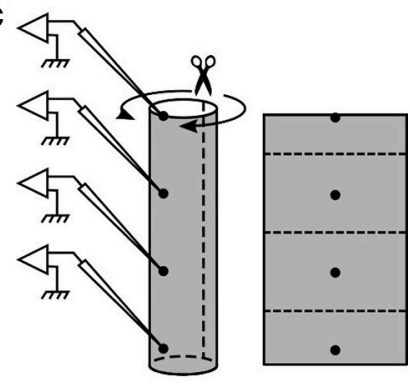

D2

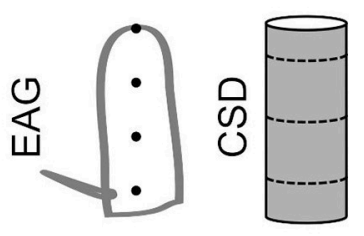

FIGURE 1 | The antennal model for estimating current source density. (A) Electrostatic principles on the insect antenna. Left, a source of current at a given position on the funiculus is schematized. A static source of current at a specific antennal locus (middle) induces an electric field along the antenna that gets weaker with the distance from the source (right). The distribution of current sources and the distribution of potentials can be reciprocally deduced from each other. (B) For a model with four EAG positions, the CSD is estimated in four compartments. The EAG linearly depends on the CSD, and the $4 \times 4$ linear coefficients $F_{i j}$ are symbolized with gray arrows. The thickness of each arrow symbolize the strength of the coefficient: it is small for the current sources far from the recording site, and it is large for the current sources close to the recording site. The reciprocal coefficients $F^{-1}$ ij could be detailed in the same way. (C) The model of funiculus is a cylinder with the recording positions located on the surface (left). The current sources are on the surface only, and only the surface is considered conductive. Hence, the surface of the cylinder was virtually unfolded (right) and subdivided in the same number of compartments than the number of recording sites. (D) The dimensions of the cylinder that models the funiculus was different for each species. The figures show a model for an elongated funiculus with seven recording positions (D1) and for a globular funiculus with four recording positions (D2).

of antenna. Assuming constant electrical conductivity, a point source current $I_{\mathrm{c}}$ spreads uniformly in all directions and generates a potential $\phi$ at distance $r$ from the source given by Equation (3) where $\sigma$ is the conductivity of the medium.

$$
\phi=I_{\mathrm{c}} / 4 \pi \sigma r
$$

A uniform current source with any geometrical shape can be considered as the sum of the point source currents that constitute the shape. How it affects the electric potential at a given position and distance can then be estimated by integrating spatially Equation (3). This general framework from Pettersen et al. (2006) was applied to the specific geometry of the antennal model. The geometry of the antenna was approximated with a cylinder (Figure 1C). With such approximation, to model can be applied to different insects with minimal measurements of the antennal dimensions. As an alternative, cone-like shapes were tested with varying the diameter of each compartment. The current sinks are generated by the dendritic activity of the ORNs located on the surface of the funiculus, so in our model they are located on the external surface of the cylinder. Because the amplitude of the signal drops as soon as the electrode penetrates the cuticle, the electric conductivity in the internal medium was assumed to be low compared to the conductivity on the antennal surface and was neglected. Therefore, the electric field propagates only through the surface of the funiculus. Thus, a simplified model was used with a $2 \mathrm{D}$ geometry corresponding to a virtual unfolding of the funiculus surface. Each current source was assumed to be uniform over a rectangular area surrounding an electrode position. The current sources were estimated at $N$ spatial positions, $N$ being the number of recording positions. Given this geometry and Equation (3), the potential $\psi_{i j}$ generated at electrode $x_{j}$ by current source $C_{i}$ around the electrode position $x_{i}$ is:

$$
\begin{gathered}
\psi_{i j}=\frac{1}{4 \pi \sigma} \int_{-q_{x_{i}-\frac{h}{2}}^{q}}^{q} \int_{x_{i}+\frac{h}{2}} \frac{1}{\sqrt{\left(x-x_{j}\right)^{2}+y^{2}}} d x d y * C_{i} \\
F_{i j}=\frac{1}{4 \pi \sigma} \int_{-q_{x_{i}-\frac{h}{2}}}^{q} \int_{\sqrt{\left(x-x_{j}\right)^{2}+y^{2}}}^{x_{i}+\frac{h}{2}} \frac{1}{\sqrt{(x)}} d y
\end{gathered}
$$

where $q$ is the width of the rectangular current source (circumference of the funiculus cross-section), and $h$ is the spacing between electrodes. The parameters $\mathrm{q}$ and $\mathrm{h}$ were estimated for each species (Figure 1D) by measuring the size of the funiculus of the left antenna (length, width, and thickness) with a light microscope; the average of 10 individuals was used for each species. Wherever unmentioned, the dimensions of $Z$. cucurbitae antennae were used. The circumference of the funiculus cross section was estimated from the approximation of Ramanujan (1914) for circumference of an ellipse $\pi *$ $(3(a+b)-\sqrt{(3 a+b)(a+3 b)})$, with $a$ and $b$ being the two radius of the ellipse (funiculus width/2 and funiculus thickness/2). The conductivity $\sigma$ was measured in 11 individuals with using two electrodes simultaneously on the antennal surface. In Equations (4,5), the median value $\sigma=10 \mathrm{~m} \Omega / \mathrm{mm}(25-75$ percentiles: 7-16 $\mathrm{m} \Omega / \mathrm{mm}$ ) was used. The parameter $\sigma$ has a multiplicative effect thus does not affect the relative amplitudes of the CSD, and in particular the spatial distribution of CSD is unaffected. Corrections on Equations $(4,5)$ were made for current sources at both extremities of the funiculus. At the distal end $x_{N}$, the electrode is located at the tip of the funiculus so 
that there is no neuron beyond the electrode position. Thus, $d x$ varied between $x_{N}-h / 2$ and $x_{N}$. Scanning electron microscopy experiments revealed that for the insect species of this paper, no olfactory sensilla lied more proximal than the arista where the electrode at the proximal end $x_{1}$ was located; as a consequence, $d x$ ranged from $x_{1}$ to $x_{1}+h / 2$. When a CSD model was adapted to a subset of the electrodes, the two outward electrodes were not necessarily located at the extremities of the funiculus. In such case the most proximal and most distal CSD compartments were extended up to the arista and to the distal tip of the funiculus with adjusting the integration window.

The coefficients $F_{i j}$ were calculated directly from Equation (5). Reciprocally, the CSD in each compartment is a linear combination of the $N$ amplitudes of EAG response (Figure 1B). The linear coefficients $F^{-1}$ ij for estimating the CSD distribution from the EAG signals can be directly calculated as the inverse of the matrix $F$. The best estimate of current sources $C_{i}$ when the potentials $\phi_{j}$ are known is then given by the reverse formula:

$$
\begin{array}{r}
C=F^{-1 *} \phi \\
C_{i}=\sum_{j} F_{i j}^{-1 *} \phi_{j}
\end{array}
$$

Mathematically, the spatial distribution of CSD amounts to a representation of the distribution of EAG signals observed through a change of coordinates. The CSD was estimated at each time point after stimulation. The area of the CSD response was quantified by reversing and integrating the CSD signal between 0 and $1.5 \mathrm{~s}$ after stimulation, and the amplitude of the CSD response was defined as the minimal CSD signal in the $500 \mathrm{~ms}$ interval preceding the stimulation minus the minimal CSD signal in the $500 \mathrm{~ms}$ interval following the stimulation. Without further indication, CSD response refers to the area of the CSD response in the text. The antennal activation was localized with the center of mass of the positive CSD responses, defined as the sum of response $\times$ position divided by the sum of responses.

\section{Cellular Functional Maps in D. melanogaster}

One-dimensional functional maps of $D$. melanogaster antenna in response to a given compound were estimated from the response levels of each basiconic and trichoid classes of antennal ORNs, the number sensilla in each class on a female D. melanogaster antenna, and their spatial distribution on the proximo-distal axis. The response levels of the antennal ORNs to methyl salicylate, linalool, ethyl acetate, 1-octen-3-ol, pentyl acetate and ethyl butyrate were extracted from the database DoOR (Münch and Galizia, 2016). The database didn't include the response to at1 ORNs, but small esters and polar compounds probably induce little response in these ORNs. The responses to coeloconic sensilla were not considered either but, since these sensilla are low in number and sparsely distributed, their contribution to the antennal responsiveness should be small. The number of sensilla per classes were reported in Grabe et al. (2016), which amount to 8 ab3, 39.825 ab1, 23 ab2, 14 ab4, 15 ab6, 34 ab5, 11.25 ab7, 18 ab8, 18 ab10, 24 ab9, 62.5 at1, 27 at3, 15 at2, and 19.5 at 4 . The spatial distributions of the different sensilla types on D. melanogaster antenna were estimated from de Bruyne et al. (2001), Grabe et al. (2016), Lin and Potter (2015), and parametrized as follow. The antennal proximo-distal position varied between 0 (departure of the arista) to 1 (tip of the funiculus). For each sensilla type, the proximo-distal spatial distribution was approximated by logit normal distributions that are bounded on the $[0,1]$ interval (Figure 3B). For basiconic sensilla, the centers of the distributions were regularly interleaved from 0.1 for ab1 to 0.85 for ab9 and had a standard deviation of 0.1 , with the exception of ab3 sensilla, the distribution of which was centered on 0.05 and had a standard deviation of 0.05 . For trichoid sensilla, the logit normal distributions were centered on positions 0.65 , $0.7,0.75$, and 0.8 and had a standard deviation of 0.15 . As an alternative, Gaussian distribution were tested and resulted in the same qualitative result. The distributions were expressed in sensilla density (number of sensilla per unit of antennal length). The response density was defined as the sum of the responses of each ORN multiplied by the corresponding number of sensilla and multiplied by the corresponding spatial distribution. In addition, 1,000 simulations of response density were obtained with attributing a random level of activation to each basiconic sensilla. The simulated response densities were used to calculate EAGs using a CSD model with 100 compartments and with the hypothesis that the responses densities are current sources. CSD were estimated backward using a model with four compartments.

\section{RESULTS}

A volatile compound activates a subset of the antennal ORNs that are not homogeneously distributed across the surface of the funiculus of the flies. Repeated stimulations of the antenna of $Z$. cucurbitae females with the same compound were applied while the EAG was recorded with an electrode located at different antennal positions. Figure 2A shows EAG responses recorded at seven positions on the antenna to puffs of Z3-hexenyl acetate. The positions were regularly interleaved and ranged along the entire proximo-distal axis of the funiculus surface. The response to Z3-hexenyl acetate was strong in the proximal region of the antenna and decreased gradually down to a small response distally. Then, a CSD model based on the geometry of the funiculus of $Z$. cucurbitae was built. It was used to estimate the spatial distribution of the CSD across the antenna using the EAG data. The antennal response consisted in current sinks (negative peaks of CSD) observed in the proximal third but not in the distal part of the funiculus (Figure 2B). This suggests that the small EAG response recorded with a distal electrode results from the activity of the distant ORNs localized proximally. In the literature, the CSD responses are frequently displayed as a space-time color map. Figures 2C,D shows the mean and standard deviation of response maps to a puff of Z3-hexenyl acetate from a population of seven individuals. The spatial extent of the CSD response is tighter than the spatial extent of the EAG response. Actually, the CSD analysis allows to delineate a sub-region of the funiculus where the activated ORNs lye. Note that the standard deviation is proportionally higher for the CSD map than for the EAG map. Figures $\mathbf{2 E - H}$ shows the same 

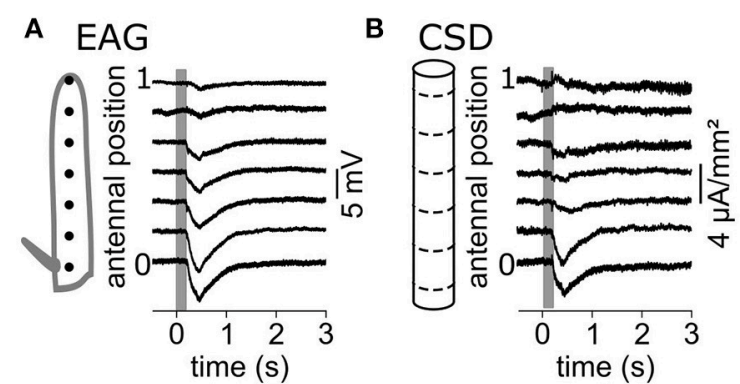

C

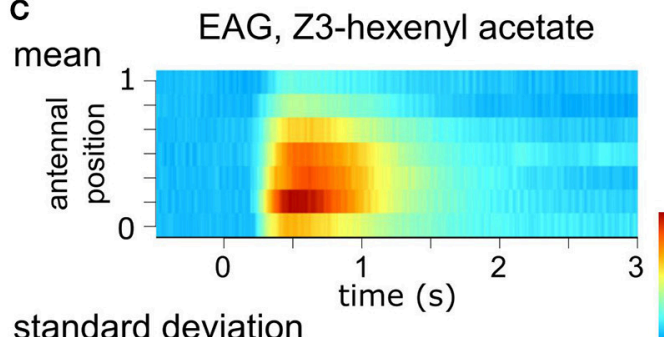

standard deviation

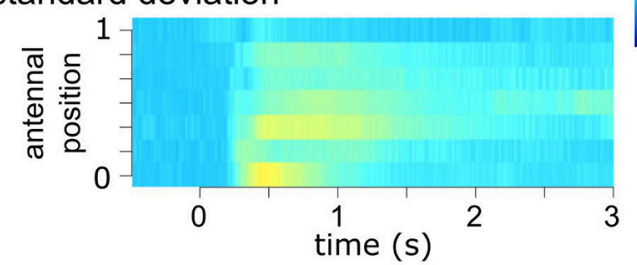

D

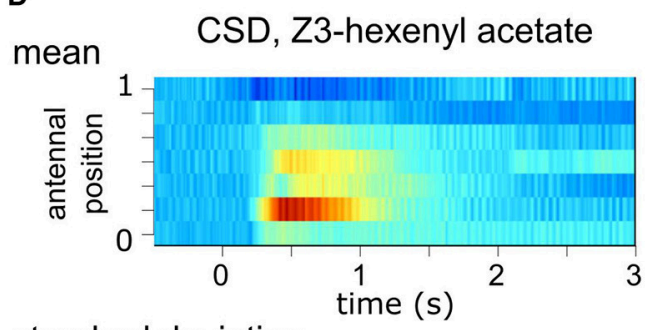

standard deviation

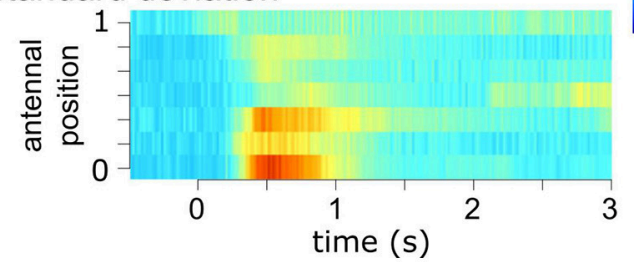

E EAG

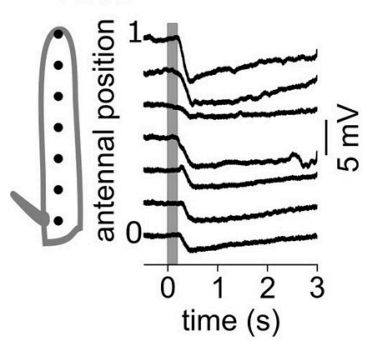

F CSD

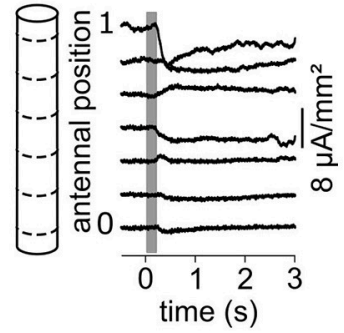

G

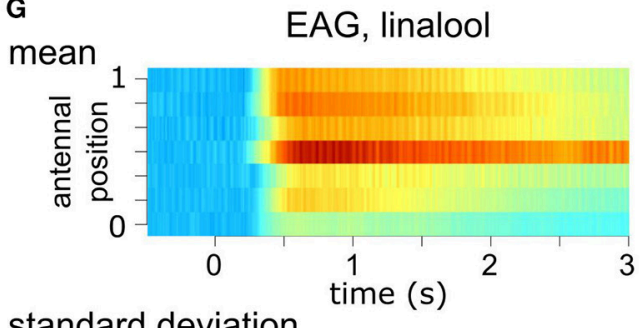

standard deviation

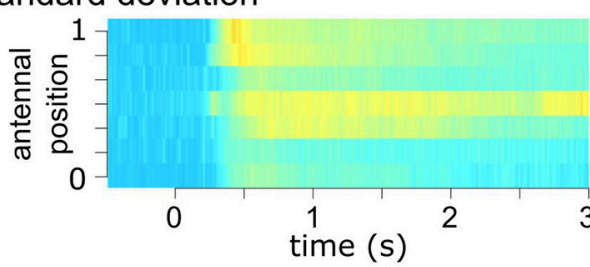

H

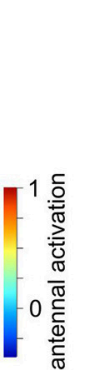

FIGURE 2 | Antennal response maps estimated with the CSD analysis. (A) EAGs were recorded in response to puffs of Z3-hexenyl acetate and with the electrode located at seven positions on the funiculus of a $Z$. cucurbitae female along the proximodistal axis. Gray area indicate the stimulation time. Antennal position varied between 0 (proximal position near the arista) and 1 (distal extremity of the funiculus). The scheme in the left represent the funiculus with the position of the electrodes. (B) A model of funiculus with seven compartments (scheme on the left) was used to estimate the CSD (right) from the EAG recording shown in (A). (C) Spatiotemporal maps of EAG recordings in response to puffs of Z3-hexenyl acetate. Both mean (top) and standard deviation (bottom) are plotted on the same color scale, normalized by the maximum EAG value ( $n=7$ Z. cucurbitae females). (D) Spatiotemporal maps of CSD response to puffs of Z3-hexenyl acetate. Values were normalized by the maximum CSD value. Same convention as in (C). (E) EAG responses of a female Z. cucurbitae to a puff of linalool. Same convention as in (A). (F) CSD response to a puff of linalool in the individual from (D). Same convention as in (B). (G) Spatiotemporal EAG maps of female $Z$. cucurbitae in response to puffs of linalool ( $n=7$ ). Same convention as in (C). (H) Spatiotemporal CSD maps of female Z. cucurbitae in response to puffs of linalool ( $n=7)$. Same convention as in (D). Data in this figure were included in Jacob et al. (2017b).

protocol performed with puffs of linalool. The EAG response to linalool stimulation was larger distally than proximally, and therefore had a different spatial distribution from the response to
Z3-hexenyl acetate stimulation. The CSD analysis revealed two distinct current sinks induced by linalool, one at the distal end and one in the middle region of the funiculus. This suggests that 

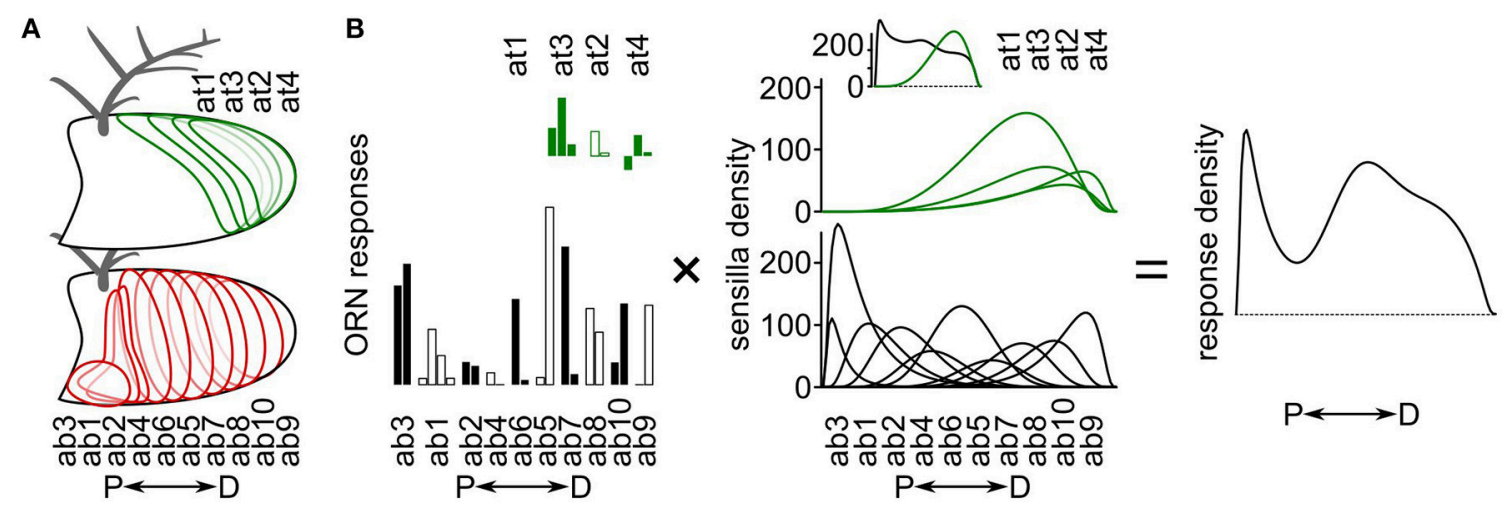

C methyl salicylate

linalool

ethyl acetate

1-octen-3-ol pentyl acetate ethyl butyrate
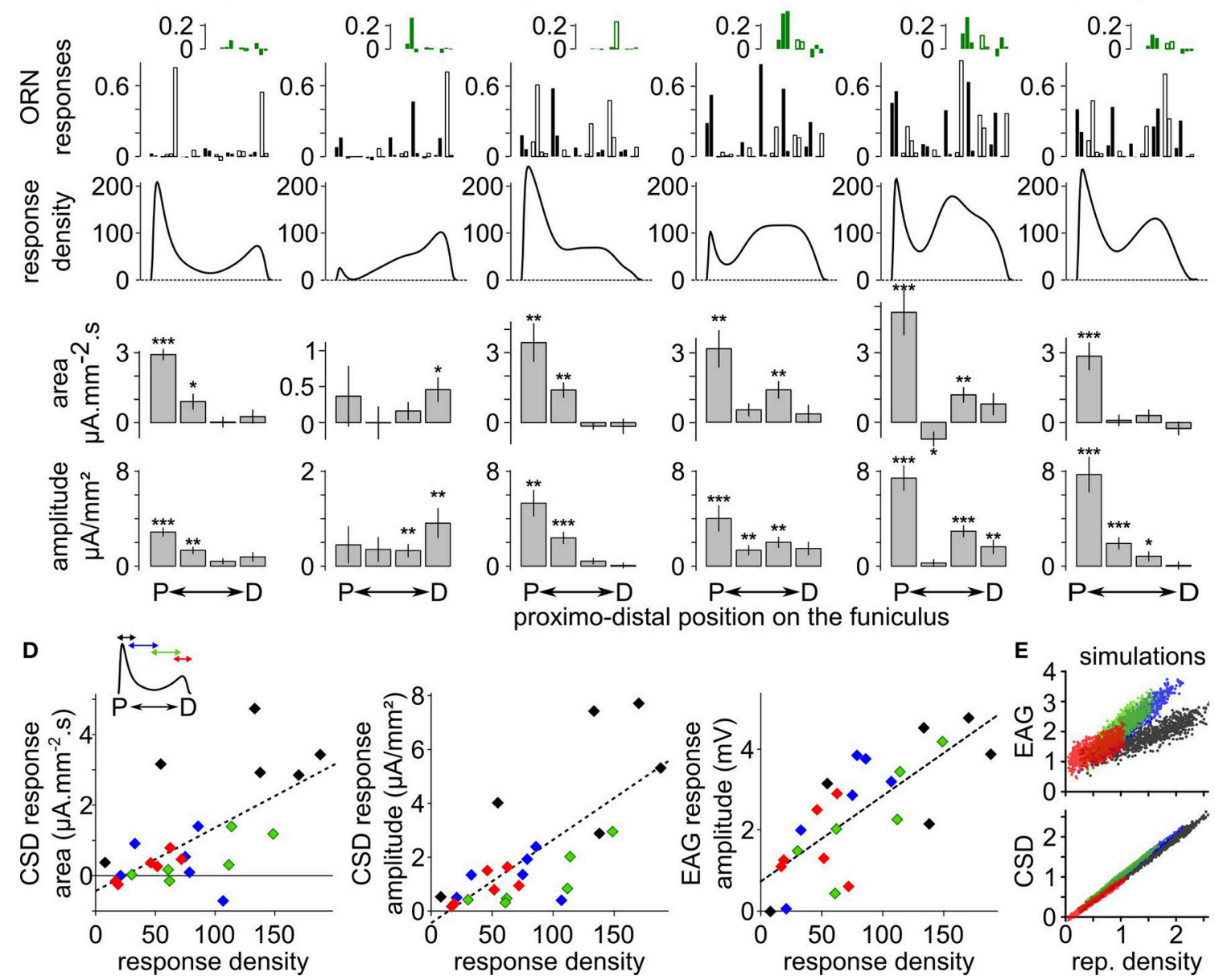

FIGURE 3 | Comparison between CSD maps and cellular maps in Drosophila melanogaster. (A) Drawings of a funiculus of $D$. melanogaster including the approximative distribution of each type of basiconic (red contours) and trichoid (green contour) sensilla. The name of the sensilla types are ordered from the most proximal to the most distal one (de Bruyne et al., 2001; Lin and Potter, 2015; Grabe et al., 2016). P, proximal; D, distal. (B) Method for estimating the response density across the antenna. The response density across the antenna (right) is defined as the sum of the response level of each ORN types (left) multiplied by the spatial density of the corresponding sensilla type (middle). Left, the response levels to a given stimulation are listed in a distal to proximal order. The response levels in consecutive sensilla types are alternatively indicated with filled and empty bars, and each sensilla type include two to four ORNs. Responses of trichoid sensilla (green) are indicated above responses to basiconic sensilla (black). Middle, the sensilla density for each sensilla type was approximated with a logit normal distribution weighted by the number of sensilla on the antenna (Grabe et al., 2016). The units are the number of sensilla per unit of antennal length. A uniform distribution with a value of 100 would mean that the antenna contains 100 sensilla. Inset: total density of basiconic and trichoid sensilla. (C) Functional maps in response to six chemicals. The ORN responses (up) were extracted from the database DoOR (Münch and Galizia, 2016) and are expressed in consensual scaled unit. The responses are shown in the same sensilla order as in panel (B). The resulting response densities along the proximo-distal axis are shown below. Down: the bar 
FIGURE 3 | graphs indicates the area and the amplitude of the CSD response (means $+\mathrm{SE},{ }^{\star \star \star} p<0.001,{ }^{\star \star} p<0.01,{ }^{*} p<0.05$, Wilcoxon's signed rank test, $n=10$ ). (D) Linear correlation between CSD maps and cellular maps. Inset: for each chemical, the curve of response density was averaged over four proximo-distal windows corresponding to the four compartments of the CSD model. The identity of the compartment/window is color coded (from proximal to distal: black, blue, green, and red). The scatterplots show the mean areas and amplitudes of the CSD responses in each antennal compartment and the mean amplitude of the EAG responses in the co-localized electrode in function of the mean response density along the same compartment. Dashed lines: linear regression curves. (E) EAG and CSD in function of the response density from simulated datasets. One dot of each color corresponds to one simulated response density built with allocating a random activation level to each basiconic sensilla. Units are arbitrary.

two populations of ORNs are activated by linalool in this species. SSRs would however be necessary to confirm it.

\section{CSD Maps Are Correlated With Functional Maps Estimated From Cellular Responses in Drosophila melanogaster}

The CSD response maps were compared with functional maps obtained at the cellular level in D. melanogaster. EAGs in response to methyl salicylate, linalool, ethyl acetate, 1-octen-3-ol, ethyl butyrate and pentyl acetate were recorded at four antennal positions of $10 \mathrm{D}$. melanogaster females and the CSD responses were calculated. Independently, functional maps in response to the same compounds were estimated using information available on the spatial distribution of each olfactory sensilla types on the antenna (de Bruyne et al., 2001; Lin and Potter, 2015; Grabe et al., 2016), on the number of each olfactory sensilla types (Grabe et al., 2016), and on the response levels of most classes of antennal ORNs extracted from the database DoOR (Münch and Galizia, 2016; Figures 3A,B). Responses of coeloconic sensilla and of at1 sensilla were not on the database, but would likely have a minor impact on the functional maps.

Qualitatively, spatial peaks in the CSD maps were located at the same loci than spatial peaks in the functional maps derived from the cellular responses (Figure 3C). Methyl salicylate induces a cellular response in the proximal part of the antenna. Accordingly, CSD analysis revealed a significant response in the two most proximal compartments (Wilcoxon's signed rank test, respectively $p<0.001$ and $p<0.05$ ). Linalool induces a cellular response in the distal part of the antenna, and CSD analysis revealed a significant response in the most distal compartment only (Wilcoxon's signed rank test $p<0.05$ ). The cellular response to ethyl acetate peaks proximally, and significant CSD responses were observed in the two proximal compartments only (Wilcoxon's signed rank test, respectively $p<0.01$ and $p<0.01$ ). 1 -octen-3-ol and pentyl acetate induces cellular responses at two antennal loci: a proximal peak, and a second medio-distal peak. Accordingly, significant CSD responses were observed both in the most proximal compartment (Wilcoxon's signed rank test, respectively $p<0.01$ and $p<0.001)$ and in the second-most distal compartment (Wilcoxon's signed rank test, respectively $p<0.01$ and $p<0.01$ ). Finally, ethyl butyrate induced a cellular response proximally and a significant CSD response was found in the most proximal compartment (Wilcoxon's signed rank test, $p<0.001$ ). However, secondary cellular responses induced distally by methyl salicylate and medially by ethyl butyrate were not associated with significant CSD responses. In addition, a significant negative CSD response was observed in the second proximal compartment in response to pentyl acetate (Wilcoxon's signed rank test, $p<0.05$ ), while this was not expected from the cellular responses.

Both the area $\left[F_{(1,22)}=15.53, p<0.001, r^{2}=0.41\right]$ and the amplitude $\left[F_{(1,22)}=24.92, p<0.0001, r^{2}=0.53\right]$ of CSD responses were significantly correlated with the cellular response density averaged within the corresponding antennal compartments (Figure 3D). EAGs recorded with electrodes positioned above each compartment also correlated significantly with the mean cellular response density within the compartment $\left[F_{(1,22)}=29.25, p<0.0001, r^{2}=0.57\right]$. To highlight the difference between EAG and CSD responses, a set of 1,000 response density curves were simulated with attributing a random response level to each basiconic sensilla. These response densities were considered as current source densities, and a CSD model with 100 compartments was used to calculate the resulting EAGs, then another CSD model with four compartments was used to estimate the CSD from four positions of EAGs. Figure 3E shows the relationship between EAGs, CSDs, and response densities in the simulated dataset. Again, both the EAG $\left[F_{(1,3998)}=4,371, p<10^{-15}, r^{2}=0.52\right]$ and the CSD $\left[F_{(1,3998)}=24,870, p<10^{-15}, r^{2}=0.98\right]$ were significantly correlated with the response density. By construction, the small jitter in the simulated CSD-response density relationship can only be due to the fact that response densities are defined at a smaller spatial scale than the four compartments of the CSD model. It resulted in two unexpected effects: for a response density of 0 , the CSD was slightly negative $\left(p<10^{-15}\right)$ and there was a significant effect of the position of the compartment $\left[F_{(3,3995)}=3,051, p<10^{-15}\right]$. The simulated EAG-response density relationship also depended significantly on the position of the electrode $\left[F_{(3,3995)}=1,942, p<10^{-15}\right]$, and the EAG was significantly positive for a subjacent response density of 0 $\left(p<10^{-15}\right)$. This effect was expected because the activity of distant ORNs contributes additively to the EAG signal.

Similar features were observed in the dataset of CSD responses to the six tested chemicals. Even if not significant, the intersection of the linear regression with a response density of 0 was slightly negative for the area $\left(-0.41 \mathrm{~mA} \cdot \mathrm{mm}^{-2} \cdot \mathrm{s}, p=0.345\right)$ and the amplitude $\left(-0.43 \mu \mathrm{A} \cdot \mathrm{mm}^{-2}, p=0.462\right)$ of CSD responses, and was positive for the EAG responses $(0.73 \mathrm{mV}$, $p=0.058$; Figure 3D). The effect of the antennal compartment was significant for the area $\left[F_{(3,19)}=9.0623, p<0.001\right]$ and amplitude $\left[F_{(3,19)}=6.0324, p<0.01\right]$ of the CSD responses, but not for the amplitude of the EAG $\left[F_{(3,19)}=0.7189\right.$, $p=0.55]$. More specifically, CSD responses in the most proximal compartment tended to be larger than in the third compartment for the same level of cellular responses. This effect 
was directly linked with the nature of the sensilla involved in the cellular response: the most proximal compartment elicited larger amplitudes of CSD responses to 1-octen-3-ol, pentyl acetate and ethyl butyrate than expected from the cellular response, but not for methyl salicylate, linalool and ethyl acetate. The first three compounds activate ab3 sensilla, but not the last three.

As a sum up, the CSD maps correlated with the functional maps derived from the activation of the ORNs. Still, some mismatches arose that might be due to inaccuracy of the electrode or sensilla positioning or inhomogeneous density of olfactory sensilla within the compartments of the CSD model.

\section{An Insufficient Sampling of EAGs Biases the CSD Estimates}

The activated ORNs might be located in regions smaller than one antennal compartment of the antennal model. How would it affect the estimation of the CSD? That question was addressed with testing artificial data sets. First, a test CSD distribution was generated with subdividing the antenna into 10 compartments and the current source was set at 0 or 1 on each compartment. Secondly, a 10-compartment model was used to directly calculate the spatial distribution of EAG induced by the test CSD distribution. Thirdly, a four-compartment model was fed with four positions of the calculated EAG and used to estimate backward the CSD distribution. Finally, the test and the estimated CSD distributions were compared to infer the biases induced by the inappropriate model compartmentalization. Figures $4 \mathrm{~A}-\mathrm{C}$ shows this approach performed on several test CSD distributions. If the location of the activated ORNs matches a compartment of the model, then the estimate is correct (case 1 and 7 in Figure 4). If the activated ORNs are distributed over an area larger than a compartment of the model (case 4 and 5), then the model detects a current source in each compartment that includes active ORNs. If the activated ORNs are distributed in an area smaller than a compartment of the model (cases 2, 3, and 6), then the model detects a current source in this compartment and mistakenly estimates a current source with a reverse polarity in the neighboring compartments. An inhibition of the neighboring compartments would result in the same distribution of EAGs, and therefore both cases cannot be disambiguated with four recording positions.

Next, a quantitative effect was tested with varying the spatial distribution of the same level of current sources within the most proximal compartment out of four (Figures 4D-F). Either the current sources were uniformly distributed within the first compartment with a value of 1 (case 8 in Figure 4), or the current sources lied in half of the proximal compartment only but had a value of 2 . This simulates the same number of active ORNs located closer to the proximal electrode. If the current sources were confined to the proximal half of the compartment (case 9 in Figure 4), the EAG in the proximal electrode was increased by $127 \%$ compared with case 8 , the EAG in the subsequent electrode was decreased to $89 \%$, and the estimated CSD was increased by $136 \%$. If the current sources were confined to the central half of the compartment (case 10 in Figure 4), which was simulated by dividing by two the diameter of the compartment in the test CSD

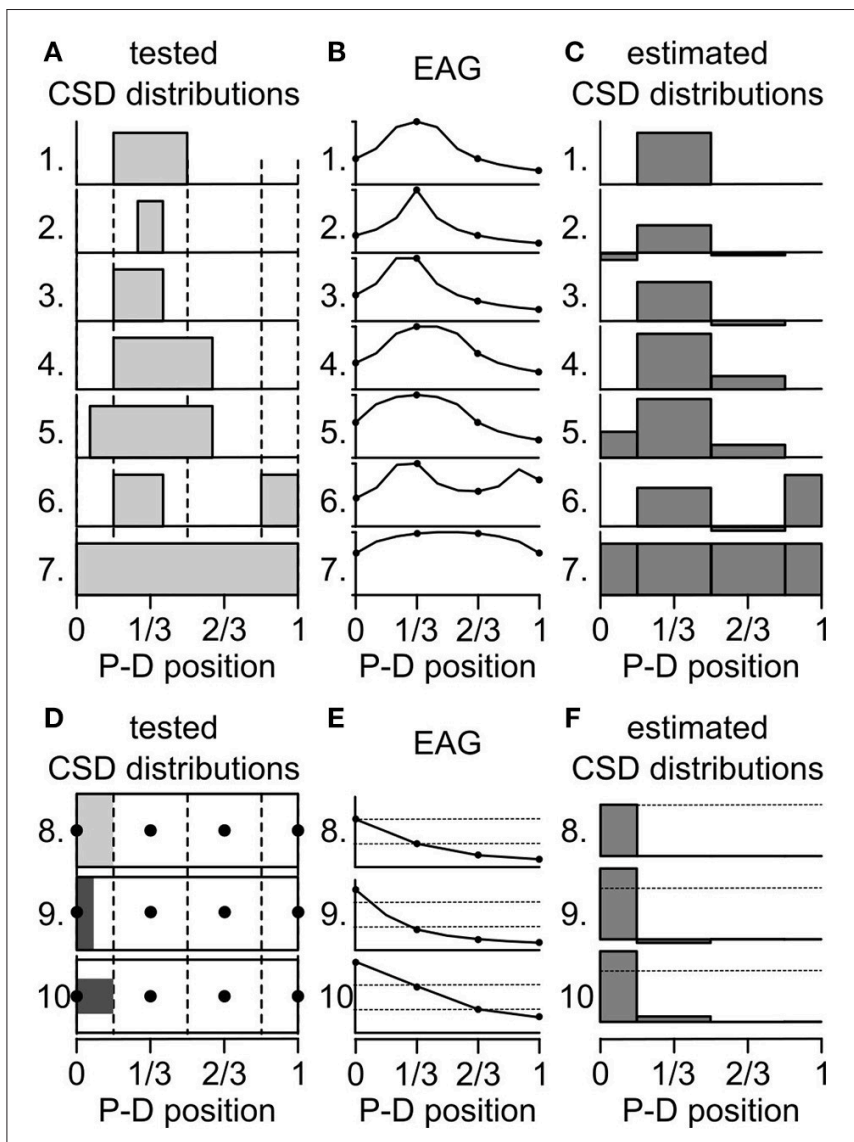

FIGURE 4 | Biased induced by the compartmentalization of the antenna. (A) Seven artificial CSD distributions were tested for qualitative biases. At each antennal position along the proximo-distal axis, the CSD was either set to 0 or to 1 (gray bars). The dashed lines delineate the four compartments of an antenna model. P-D, proximodistal. (B) The graphs show the EAG distributions that were predicted from the test CSD distributions from (A) labeled with the same numbers. The prediction was calculated with a model of antenna with 10 compartments. The amplitude of the EAG was normalized. Only four positions (dots) were used to calculate the CSD. (C) The graphs show the CSD distribution that were estimated with a model with four compartments applied on the EAG distributions from panel (B). (D) Three additional CSD distributions were tested for quantitative biases. The CSD distributions are shown as unfolded cylinders, the four compartments of the model are delineated by dotted lines and the position of the electrodes are indicated by black dots. The CSD was set to 1 (light gray) in the proximal compartment (8) or to 2 (dark gray) in one half of the proximal compartment: either the proximal half (9) or the central half (10). (E) EAG distributions predicted from the test CSD distributions from (D). The amplitudes of the EAG were not normalized. For comparison, dashed lines indicate the amplitudes predicted for the two most proximal electrodes in the distribution number 8. (F) CSD distribution estimated from the EAG in (E). For comparison, dashed lines indicate the amplitude of CSD estimated in the proximal compartment in the distribution number 8 .

model, the EAG in the proximal electrode was increased by $149 \%$ compared with case 8 , the EAG in the subsequent electrode was also increased by $200 \%$, and the estimated CSD in the proximal compartment was increased by $136 \%$. These observations can explain some mismatches observed between CSD maps and maps obtained from cellular recordings in D. melanogaster. In 
particular, ab3 sensilla densely fill a proximal region smaller than the first compartment of the antennal model (de Bruyne et al., 2001) that might be located only on the lateral surface of the funiculus where the electrode was set (Grabe et al., 2016). Their activation was associated with a strong CSD response in the proximal compartment and either a significant negative CSD response or an unexpected lack of activation of the subsequent compartment (Figure 3).

\section{Mapping the Antennal Response With Different Sets of Electrodes}

Using a subset of recording positions degrades the localization of evoked CSD responses. To test this effect, EAGs were recorded at seven positions of $Z$. cucurbitae funiculi, and the antenna were stimulated with puffs of methyl salicylate, Z3-hexenyl acetate and linalool. Accordingly, the CSD was estimated along a model of antenna with seven compartments. Methyl salicylate activated the proximal compartment, Z3-hexenyl acetate activated the proximal half of the funiculus with a maximum in the second compartment, and linalool activated mostly the medial and the distal compartments (Figure 5). For each individual, the spatial center of mass of the antennal activation was calculated to approximate the central location of the responding ORNs. It differed significantly between linalool and the other two compounds (paired Wilcoxon test, $p<0.05$ ).

Using seven recording positions is barely enough to get an accurate estimate of the position of the activated ORNs in this species, since current sinks were restricted to a single compartment of the model. Nevertheless, lowering the number of recording positions would result in a gain in time and thus increase the number of different stimuli that can be tested within a single individual. To test what spatial information can be inferred from a low number of recordings, the CSDs were estimated again from subsamples of the data and the resulting spatial information was compared to the complete data set. The subsamples included two, three or four positions and were chosen so that the positions were regularly interleaved. In each case the number of compartments of the CSD model matched the number of recording positions, and the length of the modeled antenna was kept identical by extending the first and the last compartment when required. Figure 5 shows the CSD maps estimated in response to puffs of methyl salicylate, Z3-hexenyl acetate and linalool. With four recording positions, most of the spatial features of the responses were still observed, i.e., response to methyl salicylate was maximal in the proximal compartment, response to Z3-hexenyl acetate was maximal in the second compartment, and response to linalool was maximal in the distal compartment. However, the response to linalool was not divided into two distinct current sinks. The response center of masses pointed to the same regions of the funiculus than for the whole data set, and again differed significantly between linalool and the other two odorants (paired Wilcoxon test, $p<0.05$ ). For testing three recording positions, the two extremities of the funiculus were either included or not. In both cases, the resulting activation of the antenna by Z3-hexenyl acetate and linalool were observed in the same loci than for the complete data set, and the response center of masses differed significantly between the two stimulations. With methyl salicylate stimulation however, the observed response was located in the same antennal loci than for the complete data set only if the most proximal recording position was included. Finally, while using two recording positions, the centers of mass of the CSD maps were biased toward one of the two positions. Still the spatial structures of the response resembled the one obtained with the complete data set. The response to Z3-hexenyl acetate was localized near the proximal electrode and the response to linalool near the distal electrode and the activation position significantly differed between the two (paired Wilcoxon test on center of masses, $p<0.05$ ). The response to methyl salicylate was located proximally only if the most proximal position was considered.

\section{The Modeled Geometry of the Antenna Affects the CSD Estimates}

Classically, the CSD was approximated by the second spatial derivative of the field potential. Hence the CSD could not be estimated at the position of the electrodes in the extremities (Vaknin et al., 1988). With EAG recorded at four positions, the CSD would be estimated only at the second and the third position with the respective linear coefficients $(-0.5,1,-0.5,0)$ and $(0$, $-0.5,1,-0.5)$. The model used in this study allows the estimation of the CSD at all the electrode positions. The coefficients $F_{\mathrm{ij}}^{-1}$ used to estimate the CSD distribution directly depend on the geometry of the antenna. Thus, the average length and width of the antenna shall be measured and used for calculating the CSD (Figure 1D). The size of the antenna varies between species, and its shape can be elongated or globular. To test the impact of the antennal geometry on the model, the length, width and thickness of the funiculus were measured for 10 individuals in seven fly species, namely Drosophila melanogaster, Ceratitis capitata, Ceratitis catoirii, Neoceratitis cyanescens, Bactrocera zonata, Zeugodacus cucurbitae, and Dacus demmerezi. The average values were used to model the antenna for each species. In these species, the length of the funiculus varied between 150 and $1,100 \mu \mathrm{m}$, and the ratio of width to length varied between 0.2 and 0.6 (Figure 6A). The largest antennae also tended to be the more elongated, with the exception of $Z$. cucurbitae vs. B. zonata. An additional antennal model was built from the measurement of the width $(125 \mu \mathrm{m})$ and the length $(13 \mathrm{~mm})$ of the antenna of a female C. sacchariphagus.

The coefficients for the different models are shown in Figures 6B-D. The coefficients were larger in the species with the smallest antennae, and the smallest species tend to have higher amplitudes of current source. However, the relative amplitudes of the current sources in the different compartments are not affected by the size of the antenna. On the contrary, the ratio of the width and the length of the funiculus affected the ratios between the different coefficients and therefore impacted the spatial distribution of the estimated CSD. The model calculated for the different species were used to calculate the EAG resulting from the same test distribution of current sources (Figure 6E). Intuitively, the ORNs located proximally should be less likely 


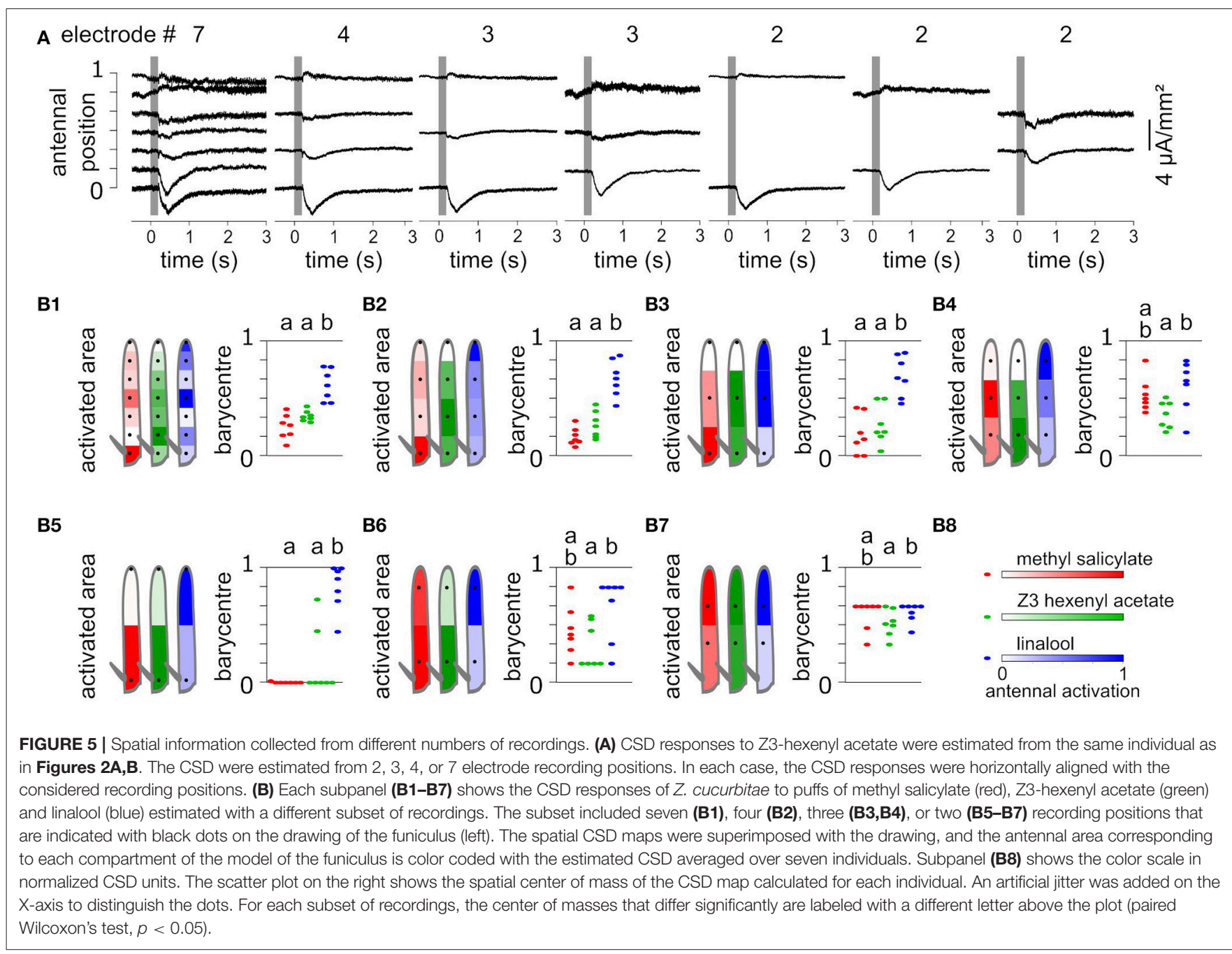

to polarize the distal end of an elongated funiculus than of a globular one, and this was indeed predicted by the models. Inversely, the models were used to estimate the CSD from a given distribution of EAG signals (Figure 6F). The position of the peak CSD response was always located in the same compartment. The relative amplitude of the CSD in the neighboring compartment varied between -18 and $20 \%$ of the peak CSD response.

Even if cylindric antennal model seems reasonable for fruit flies, insect antennae are frequently tapered. A better approximation of the antennal geometry should improve the accuracy of the CSD estimation. CSD distributions were estimated again with three virtual geometries: a cylindrical antenna based on $Z$. cucurbitae dimensions, a conical antenna with a proximo-distal decrease in diameter, and a conical antenna with a proximo-distal increase in diameter (Figure 6G). The average antennal diameter was the same in the three cases, and for the two conical models the largest diameter was three times larger than the smallest one. In each case the peak of the CSD response was located in the same antennal compartment, but its amplitude differed. In the other antennal compartments the CSD response was either positive or negative depending on the model. Generally, the level of CSD response in a compartment was negatively correlated with the surface of the compartment in the model. Thus, a bias due to an approximated geometry of the funiculus would affect marginally the level of CSD response, but should not affect the localization of the CSD peak. For example the CSD maps estimated for D. melanogaster had larger responses in the proximal compartment than in the distal ones (Figure 3). This observation might be due to the fact that the basiconic sensilla are sparser distally, but this feature would be less pronounced if a tapered antennal geometry was used.

\section{Examples of Ambiguous CSD Estimates}

EAG signals are mostly negative polarizations of the antenna and correspond to current sinks. Figure 7 shows two examples where current sources, i.e., with positive values, were observed. In response to a puff of ethyl butyrate, the funiculus of $C$. catoirii responded with a current sink in the second compartment, and small current sources were also observed in the first, the third and the fourth compartments (Figure 7A). In this case, the current sink is unambiguous, but not the current sources which can result from approximations of the antennal model. Either there is an 

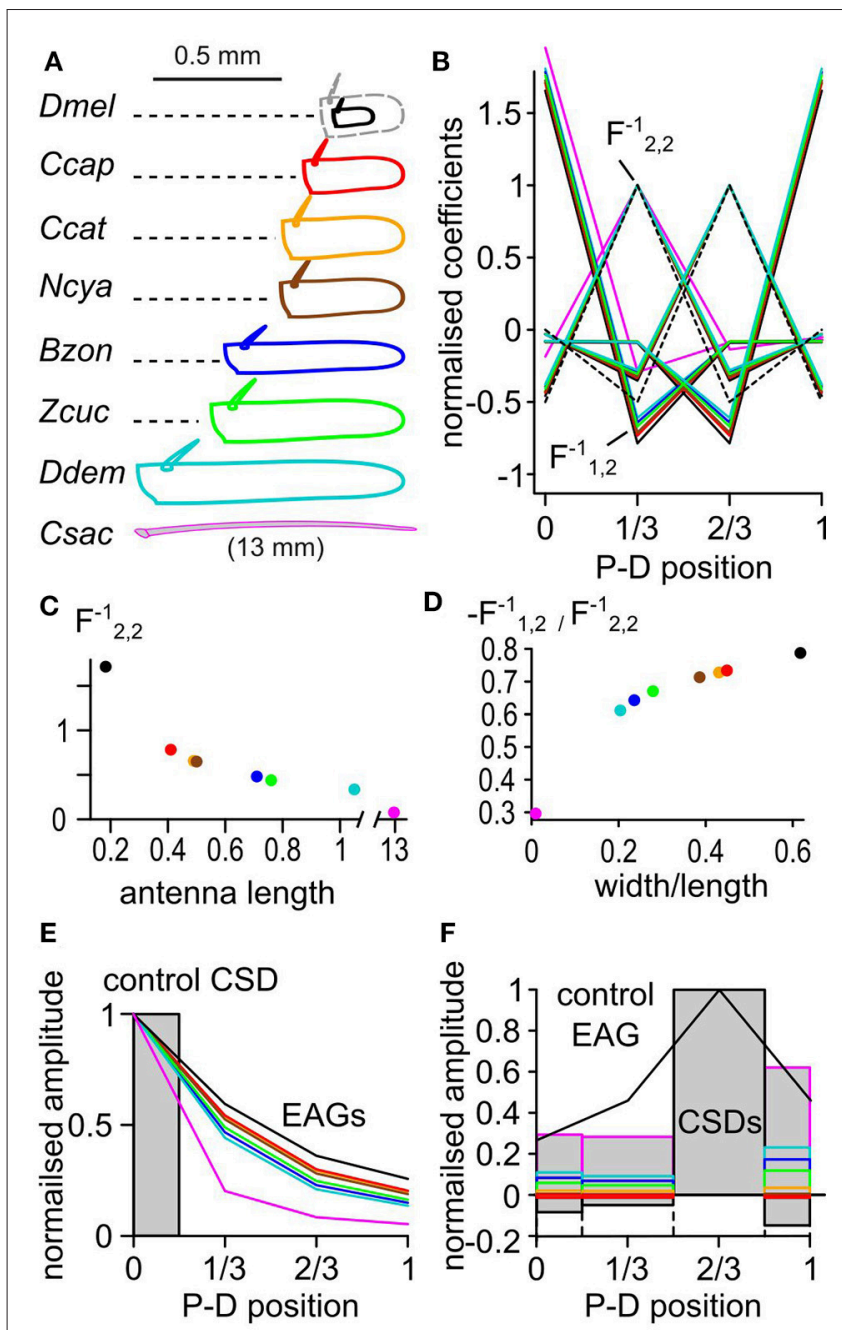

G
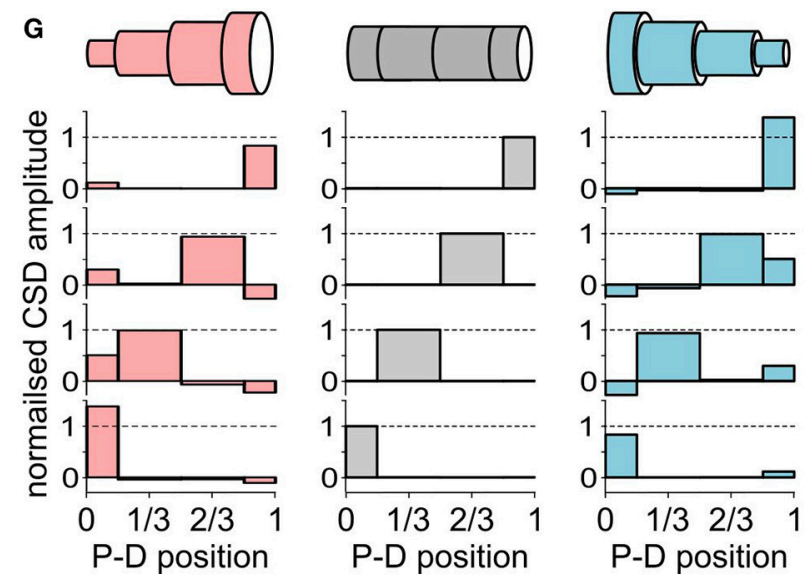

FIGURE 6 | The CSD model depends on the geometry of the antenna.

(A) The dimensions of the funiculus (length and width) were measured in seven insect species: D. melanogaster (Dmel), C. capitata (Ccap), C. catoirii (Ccat), N. cyanescens (Ncya), B. zonata (Bzon), Z. cucurbitae (Zcuc), and D. demmerezi (Ddem). The drawing of the funiculus was reshaped to match the average length and width for each species ( $n=10$ per species). Since D. melanogaster funiculus is small, it was proportionally enlarged (dotted gray

(Continued)
FIGURE 6 | lines) for comparison with the other species. In addition, the length and width of the antenna of a moth C. sacchariphagus (Csac) was also measured and included. For each panel, colors code for species. P-D, proximodistal. (B) Normalized linear coefficients of CSD models with four antennal compartments. Each line links the coefficients for calculating the CSD in one compartment from the four recorded EAGs. For clarity, only half of the coefficients calculated for $C$. sacchariphagus were included. For each line, the maximal coefficient is always for the EAG recorded at the same position as the CSD compartment. The dashed line represents the coefficients used in classical CSD models that had the value of $1,-0.5$, or 0 and were calculated only for the compartments 2 and 3. For each CSD model, the coefficient were normalized by $F_{2,2}^{-1}$ that is pinpointed by an arrow. $F_{1,2}^{-1}$ is the coefficient that varies the most between species. (C) Scatterplot of the coefficient $F_{2,2}^{-1}$ in function of the antennal length. (D) Scatterplot of the ratio between coefficients $F_{1,2}^{-1}$ and $F_{2,2}^{-1}$ in function of the ratio between width and length of the antenna. (E) Superimposed EAG distributions (lines) predicted from the same control CSD distribution (boxes) for the different species. (F)

Superimposed CSD distributions (boxes) estimated from the same EAG distribution (black line) for the different species. (G) Three antennal geometries (top) were used to estimate CSDs (bottom) from four different EAG distributions. Each horizontally aligned CSD map is estimated from the same EAG distribution. The antenna is modeled with a cylinder (middle, gray), a cone with a proximo-distal decreasing in diameter (right, light blue), or a cone with a proximo-distal increasing in diameter (left, light red). For each model, the average antennal diameter is the same. In the two conic models, the maximal diameter is three times larger than the minimal diameter.

actual current source, or the current sink covers a subregion of the second compartment of the model, or the geometry of the funiculus was improperly estimated. In response to E2-hexenal, the funiculus of $N$. cyanescens had a current sink in the distal compartment and a current source in the proximal compartment (Figure 7B). In this case, the EAG recorded in the first position also showed a transient positive polarization. This observation cannot result from the CSD model, and therefore it results most likely from an actual current source. As a conclusion, the experimenter should conclude about the existence of a secondary current source only if it is associated with a positive polarization of the antenna observed by EAG.

\section{Gas-Chromatography Coupled With CSD Analysis}

As a proof of concept that CSD analysis of EAG recorded at multiple positions can be used in the research in chemical ecology, EAG recordings were performed in a $Z$. cucurbitae individual while the odorants were provided through a GC. Figure 8 shows the EAG and CSD analysis in response to a mixture of seven synthetic components. Each component was isolated by GC and reached the antenna at a different time. The induced CSD maps revealed an excitation of the proximal compartment by ethyl acetate, Z3 hexenyl acetate and methyl salicylate, an excitation of the third compartment by pentyl acetate and 1-octen-3-ol, and an excitation of the distal compartment by linalool. This result is similar to the results obtained with direct stimulations of the antenna by these compounds (Jacob et al., 2017b). With this experimental setup, the CSD analysis can be used to analyze the insect sensitivity to complex odor mixtures, like the volatile compounds emitted by host plants or by conspecifics. 


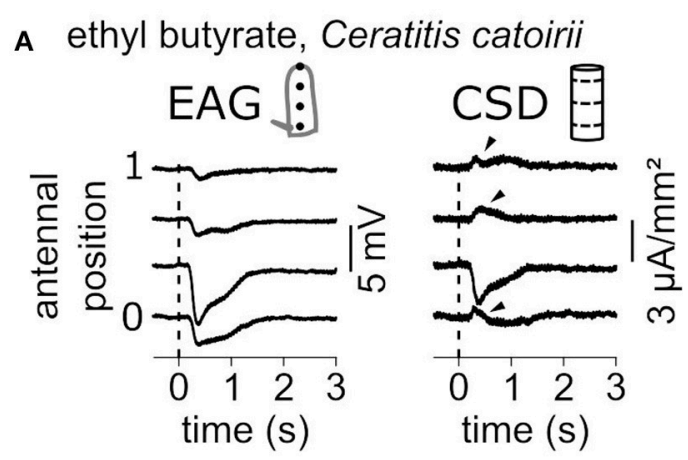

B E2 hexenal, Neoceratitis cyanescens

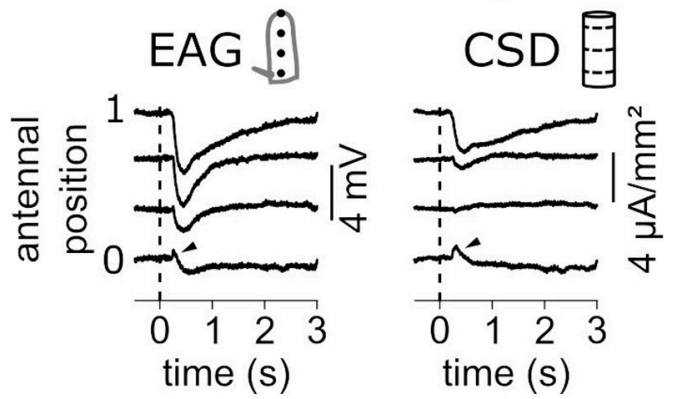

FIGURE 7 | Current sources are occasionally observed. (A) Antennal response of a C. catoirii female to puffs of ethyl butyrate. Left: The EAG responses were recorded at four antennal positions. The dash line indicated the onset of stimulation. Right: The CSD responses were estimated from the EAG recordings. Apparent current sources are pinpointed with arrowheads. (B) Antennal response of a N. cyanescens female to puffs of E2 hexenal. Same conventions as in (A). A transient positive response in the EAG signal is also pinpointed.

\section{CSD Mapping of a Moth Antenna}

To check if CSD analysis can be used in other insect orders, the EAG was recorded at four positions of the antenna of a female moth C. sacchariphagus, the cane sugar stem borer, stimulated with the green leaf volatile Z3-hexen-1-ol. The antenna was modeled with four antennal compartments and the distal one had a smaller diameter to fit the antennal dimension. The resulting CSD map was estimated (Figure 9). CSD responses were observed in each of the four antennal compartments, which amounted, respectively to $0.34,0.43,0.55$, and $0.50 \mu \mathrm{A} \cdot \mathrm{mm}^{-2} \cdot \mathrm{s}$ from the proximal to the distal segments. Further repetition of this protocol would be required to confirm that ORNs sensitive to Z3-hexen-1-ol are located all along the antenna of C. sacchariphagus, but this observation is in accordance with the current understanding of moth olfactory system which hypothesize that each antennal annulus is a repetition of the same ORNs distribution. In any case, this recording confirms that the CSD approach can also be used in insects with filamentous antenna.

\section{DISCUSSION}

The CSD analysis has been developed to localize the current sources and sinks that cause the field potentials in brain tissues
(Mitzdorf, 1985), including the mushroom body in insect brains (Kaulen et al., 1984). This paper shows that the CSD analysis applied to EAG recordings localizes current sinks induced by the olfactory stimulation of the insect antenna. Several indices suggest that the current sinks are co-localized with the activated ORNs. The amplitude of the EAG signal is correlated with the local density of olfactory sensilla at the electrode site (Bigiani et al., 1989; Crnjar et al., 1989). The spatial range of CSD responses to small polar molecules was accordant with the spatial distribution of basiconic sensilla across seven different species (Jacob et al., 2017b). The activation of conserved ORs resulted in conserved positions of current sinks between D. melanogaster and tephritids (Jacob et al., 2017b). Finally, this study shows that the maps of CSD responses to six compounds were spatially correlated with functional maps estimated from the number, response level and localization of the different ORNs in D. melanogaster.

Recording the EAG at many positions was found necessary to investigate in detail the olfactory response. First, while SSR and single-electrode EAG samples only a fraction of the antennal ORNs (Nagai, 1985; Jacob et al., 2017b), sampling EAG at several positions reveals the activity of most. In a previous paper, the responsiveness of six fruit flies species to seven chemicals broadly distributed in plant odors was tested (Jacob et al., 2017b). With $n=10$ individuals per species, single EAG revealed a significant response in $67 \%$ of the cases while using the four positions of recordings revealed a significant response in $97 \%$ of the cases. In addition, the peak amplitude of the CSD response can be used to quantify the olfactory responses as it correlates with the number of responsive ORNs multiplied by their response level. Hence the GC-multiple EAD approach combined with CSD analysis will be useful for chemical ecologists wishing to determine the olfactory sensitivity of an insect to the compounds of a natural blend. Secondly, the CSD analysis complements alternative methods (Hull and Cribb, 2001) suggesting that two chemicals from a blend activate different ORNs, and therefore are not conveying redundant sensory information. Finally the CSD analysis can be used by electrophysiologists to guide further SSR investigation into particular regions of the antenna for species whose olfactory system is not known. In Drosophila, functional maps can be explored by combining the exhaustive investigation of all the ORN classes with SSR performed in many insects (Crowley-Gall et al., 2016; Münch and Galizia, 2016) with the estimation of the number of ORNs in a class (Dekker et al., 2006; Grabe et al., 2016), an important property that might be developmentally regulated (Song et al., 2012). Combining SSR with CSD responses might be an interesting alternative for comparing different strains or species in this genus. In D. melanogaster, the more distal classes of basiconic sensilla have a sparser spatial distribution (Grabe et al., 2016). Accordingly, higher levels of CSD response were found for proximal ORNs than for distal ORNs with similar activation levels (Figure $\mathbf{3}$ ).

\section{Methodological Recommendations for Applying CSD Analysis to EAG Recordings}

A couple of potential experimental biases should be considered for the CSD analysis. (1) Each repetition of the same stimulation does not necessarily induce the same neuronal activation. In 


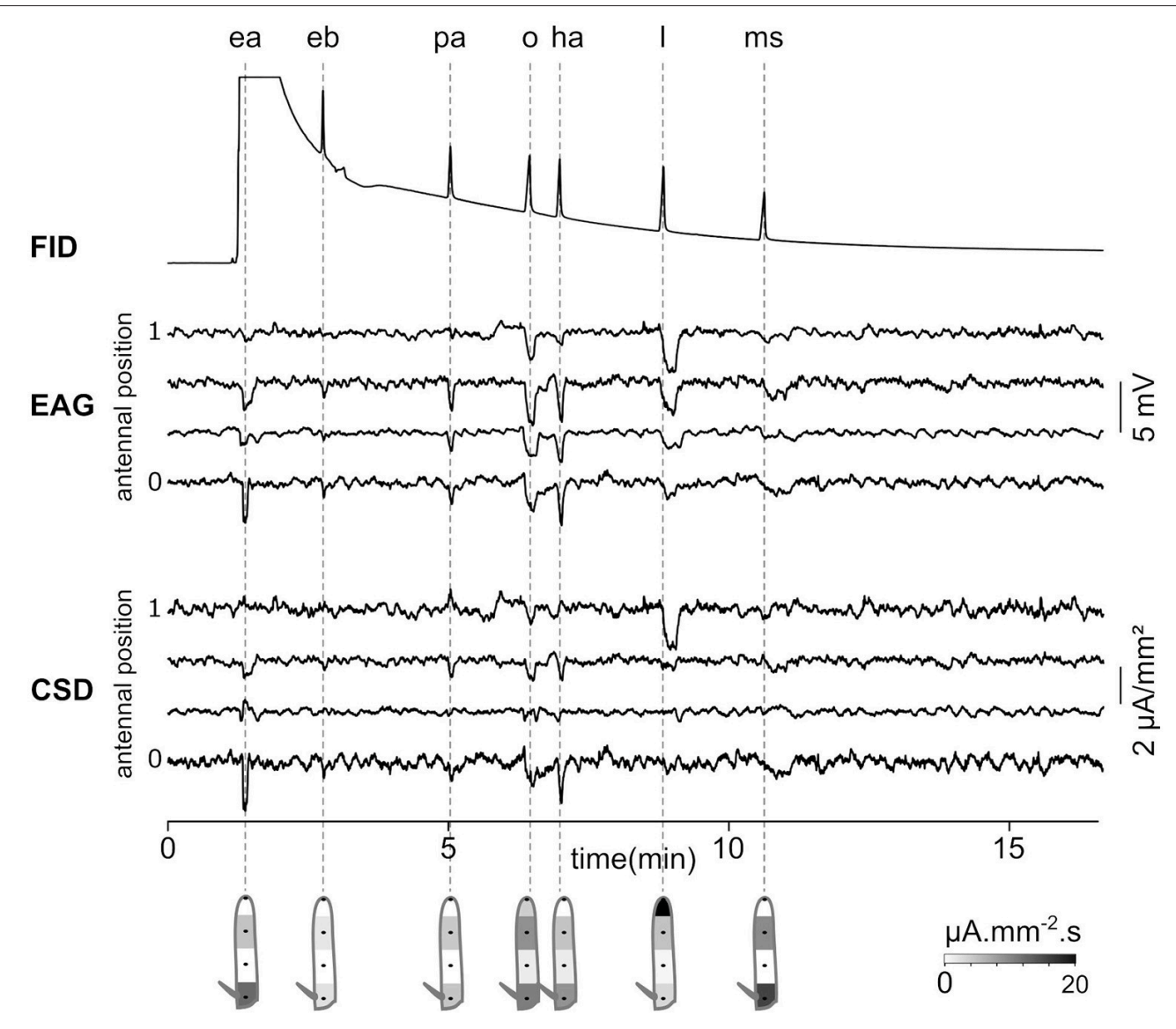

FIGURE 8 | Gas-chromatography coupled with an EAD-CSD. Top: Flame ionization detector (FID) signal recorded at the output of the gas-chromatograph injected with a $10^{-3}$ dilution of seven compounds in hexane. The compounds are ethyl acetate (ea), ethyl butyrate (eb), pentyl acetate (pa), 1-octen-3-ol (o), Z3-hexenyl acetate (ha), linalool (I), and methyl salicylate (ms). Times of peaks are indicated with dashed lines. Ethyl acetate was ejected at the same time as hexane, and therefore the peak is not visible in the FID signal. Middle: EAG was recorded at four positions of the funiculus of a Z. cucurbitae female. Bottom: the CSD response was estimated from EAG recordings. Under each response, the spatial maps were drawn from a temporal integration of CSD responses.

particular in ex vivo preparations the EAG response decreases with time due to a degradation of the biological tissues. Recording simultaneously at multiple antennal positions would improve the CSD estimation, and furthermore result in a gain in time allowing more complex protocols. (2) The recording electrode must be thin enough for having a good spatial resolution. The glass micropipettes I used were $1-2 \mu \mathrm{m}$ tip diameter. I would not recommend using large electrode contacts with the antenna through a drop of electrolytic gel of liquid for CSD analysis. In addition specific classes of olfactory sensilla might be covered by the electrode in such preparations and not be stimulated. (3) The reference electrode should not be in the vicinity of any olfactory sensilla, otherwise the EAG signal would result from a combination of the evoked potentials recorded by each electrode. Thus, the reference electrode should not be positioned at the base of the antenna but rather in the insect eye or body. (4) The experimenter should be careful about the quality of the electrode contacts and the precision of electrode positioning that might affect the CSD. (5) The model used in this paper hypothesize that the different ORN classes are organized in a 1-dimensionnal grid along the proximo-distal axis. However, some ORNs classes might lay mostly in the borders or the opposite surface of the funiculus. In tephritids, seven or more recording positions are required to estimate specific spatial features of the olfactory response of the antenna. Still, the rough analysis of the CSD resulting from three or four recording positions should be sufficient to localize the antennal activation. In any cases, using a proximal electrode near the arista might be of critical importance since the large basiconic or the clavate sensilla are specifically grouped in this locus both in Drosophila (Shanbhag et al., 1999) and in tephritides (Jacob et al., 2017b), while the other sensilla types are more broadly distributed. In this study a correct estimation and localization of $Z$. cucurbitae response to methyl salicylate required indeed a proximal recording.

\section{The Antennal Neural Tissues Are Ideal Generators of Field Potentials}

Despite of differences due to the propagation of the LFP signal through the brain tissue and of the EAG signal through the 

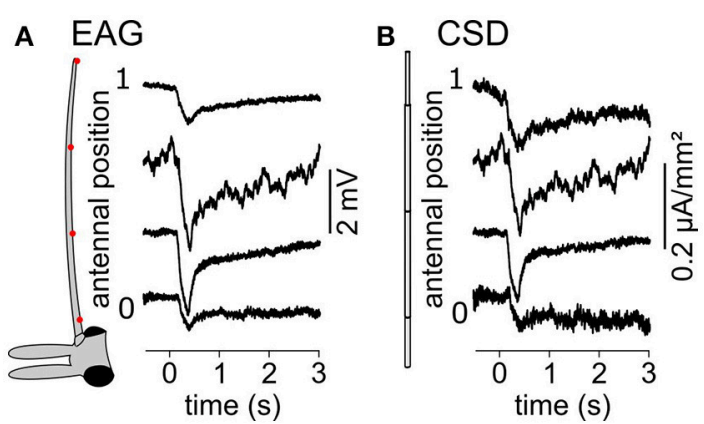

FIGURE 9 | CSD maps of a C. sacchariphagus antenna in response to Z3-hexen-1-ol stimulation. (A) EAG response was recorded at four antennal positions of the antenna of a female $C$. sacchariphagus. The drawing on the left of the head and one antenna of $C$. sacchariphagus indicates the positions of recording (red dots). (B) A four compartments antennal model (left) was used to estimate the CSD response at the four positions (right).

insect cuticle, the main principles are similar. A pharmacological disruption of spikes had no effect on the EAG signal (Nagai, 1985; Lucas and Renou, 1992; Nagel and Wilson, 2011), suggesting that the EAG essentially depends on the low frequency dendritic receptor currents as does the LFP. The LFP can be simultaneously recorded with action potentials of the cells within very close distances from the electrode (Roux et al., 2007). Similarly, an SSR electrode records simultaneously the action potentials of the ORNs within the sensillum and the sensillum potentials generated by many ORNs in the surrounding sensilla (Nagai, 1983a). In brain tissues, the difference in spatial range between action potential and field potential recordings is thought to be due to the non-homogeneous properties of the extracellular medium that selectively filters out the high frequency events (Bédard and Destexhe, 2012).

The extracellular current sinks and sources are induced by the transmembrane currents. The flow of cations into the dendrites, either due to synaptic activity of central neurons or to receptor activity of the ORN, induces a current sink, while at the same time a reverse capacitive current around the soma induces a current source (Figure 10A; Bazhenov et al., 2011). The neuron can therefore be considered as an electrical dipole (Figure 10B). A direct consequence is that field potentials are negative around the apical dendrites and positive in the vicinity of the soma (Nicholson, 1973). Most olfactory stimulations induce negative deflections of the EAG signal, which were hypothesized to correspond to the dendritic current sinks (Kaissling, 1995). Accordingly, the amplitude of the sensillum potential varies with the position of the electrode and reach a maximal negativity at mid-distance of the apical dendrite (Nagai, 1983a). Positive polarization of the antenna should result from the dendritic current sources that are expected from the odorevoked inhibition of the constitutive activity of the receptors that triggers orientation behaviors (Cao et al., 2017). However, this study showed that CSD analysis can be misled in reporting inexistent current sources due to an insufficient spatial sampling or to an approximation of the funiculus geometry.

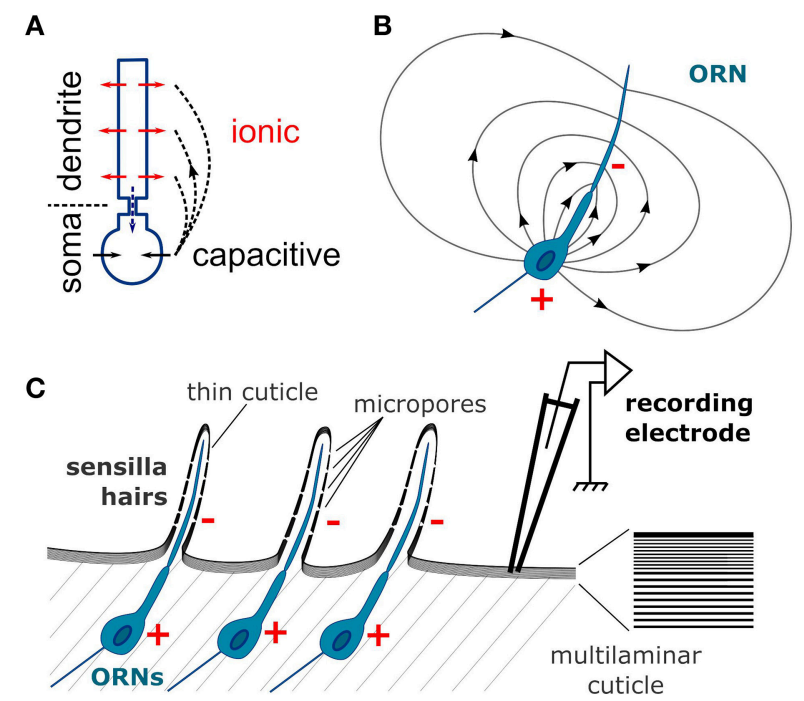

FIGURE 10 | Schematic depiction of the CSD theory applied to the insect antenna. (A) Conventional CSD model. Each neuron is depicted as two compartments, dendrite and soma. The neuron is activated via an ionic current (red arrows) in the dendrites that induces a current sink in the extracellular medium. At the same time a capacitive current in the soma (black arrows) induces a current source in the extracellular medium. The current flowing intracellularly between the compartments (dashed blue arrow) is equal and opposite to the current flowing in the extracellular medium (dashed black arrows). (B) Electric field generated by an ORN in a homogenously conductive medium. Since the ORN is asymmetric, it results in an electric dipole in the extracellular medium. The single dendrite is the negative pole (-) and the soma is the positive pole (+). (C) The organization of the neuronal tissue in the antenna is ideal for generating field potentials. Individual ORNs are perfectly aligned below the cuticle surface. The components of the electric fields perpendicular to the plane of alinement sum up for generating the EAG signal. Right: zoom on the cuticle microstructure. Since the cuticle has a multilaminar organization, it should have an anisotropic conductivity and the electric current should flow in the tangential direction.

The anatomical characteristics of a neuronal tissue have an impact on the quality of the LFP signal (Mitzdorf, 1985; Buzsáki et al., 2012). The neurons with more asymmetric somatodendritic architectures generate stronger electrical dipoles upon activation. In addition, if all the neurons are aligned on a plane with the same perpendicular orientation of their dendrites, the contributions of individual neurons to the field potential sum up; if not they cancel each other. Both conditions are optimal in insect antennae explaining the large amplitude of the EAG signals, in particular in D. melanogaster. The architecture of an ORN is strictly asymmetric: the single apical dendrite bathes in the sensillum lymph, while the cell body lies below the cuticle. All ORNs are perfectly aligned along the cuticular wall (Figure 10C). Kaissling hypothesized that the EAG signal results from the tangential orientation of the ORN dipoles with negative poles pointing to the distal part of the antenna and positive poles pointing proximally (Kaissling, 1995). However, each distal negative pole cancels out a proximal positive pole of a neighboring ORNs and the resulting signal should be almost zero. Alternatively, only the component of each dipole that is 
perpendicular to the cuticle surface should contribute to the EAG signal, as was theorized for brain tissues (Mitzdorf, 1985). Nonetheless the insect antenna is an ideal system for the CSD analysis, and has the additional advantage for modeling that the neuronal activity is spatially confined to the geometry of the funiculus surface that is easier to delineate than the geometry of brain tissues.

\section{How the Antennal Tissue Differs From Brain Tissues}

The classical models of CSD analysis required a uniform resistivity of the extracellular medium in every direction. This is obviously not the case in the external layers of the insect antennae, and the CSD model built in this paper hypothesized instead that the electric current flows in two dimensions along the antennal cuticle and does not penetrate the antenna. This conclusion was brought from two considerations. First, Nagaï bypassed the hemolymph by inserting a tungsten wire all along the antenna of the European corn borer, and this very invasive manipulation did not affect much the EAG properties (Nagai, 1985). Second, in flies the amplitude of the EAG signal drops down as soon as the electrode pierce through the cuticle, despite a decrease of resistance between the recording and reference electrodes due to the contact with the hemolymph. This could be explained if the locations of the current sinks, i.e., the dendrites of the ORNs, are insulated with a high resistivity barrier from the hemolymph but not from the electrode. The cuticle of insects has highly anisotropic mechanical properties (Klocke and Schmitz, 2011; Clark and Triblehorn, 2014), and has hypothetically an anisotropic conductivity as well. Such anisotropy emerges from the laminar microstructure of the insect cuticle (Figure 10C). The multiple layers of chitin and proteins (Vincent and Wegst, 2004; Andrew Jansen et al., 2016), parallel to the cuticular surface, could act as electric insulators that would preclude a perpendicular propagation of current, and would further improve a tangential propagation of current along the inter-laminar medium, a bit like insulation with myelin improves the current propagation along the axon of vertebrate neurons. In addition, the dendritic electric field could be conducted to the outside layers of the cuticle since it is thin on the sensilla and punctured by micropores (Mayo et al., 1987; Stocker, 1994; Shanbhag et al., 1999). Such assumption remains speculative, and I also analyzed the CSD with a 3-dimensional model of electric field which resulted in qualitatively similar results. Another hypothesis is that the resistance is uniform along the entire antennal surface. This might not be the case since the cuticle can be thinner in the distal region of the antenna (Nagai, 1985), so an estimation of the spatial distribution of cuticle conductivity might further improve the accuracy of the CSD model.

\section{On the Use of CSD Across the Insect Orders}

In this paper the CSD analysis was used to explore the response properties of species with bulbous antennae from the Diptera order. The CSD analysis revealed a map of olfactory selectivity and sensitivity. The CSD analysis is particularly useful in these species because the antenna includes only one compact olfactory segment, the funiculus. Each class of olfactory sensilla is unevenly distributed on the funiculus surface so that the olfactory sensitivity varies spatially. Recording EAG at multiple positions in moths also revealed localized ORN activity (Nagai, $1981,1983 b, 1985)$ and this study includes an example of CSD analysis performed in a moth species, thus it can be applied in various species of insects or other arthropods (Machon et al., 2016). The geometry of the modeled antenna should be adapted to each species to accurately estimate CSD and the experimenter might model a tapered antenna if necessary. In moth the micro-architecture of the antenna is more complex than in fruit flies (Sanes and Hildebrand, 1976) which might preclude a comparison of the amplitude of the CSD responses between species. In insects which filamenteous antennae like moths (Sanes and Hildebrand, 1976; Shields and Hildebrand, 2001; Ghaninia et al., 2014) or mosquitoes (Ghaninia et al., 2007), each annulus is thought to be a repetition of the same distribution of ORNs, and accordingly Z3-hexen-1-ol induced a CSD response in all the positions of a C. sacchariphagus antenna. Olfactory maps were described at the level of single antennal segment of moths (Ghaninia et al., 2014) but CSD analysis is not usable at this scale. In these species, the CSD analysis can still be used to determine the spatiotemporal patterns of activation of the ORNs that would result from the contact of the antenna with a natural odor plume (Celani et al., 2014). This information is relayed to the processing areas of an insect's brain (Nishino et al., 2018) and could be involved in the searching of the odor source (Lockey and Willis, 2015; Jacob et al., 2017a).

\section{CONCLUSION}

Most laboratories recorded the EAG with an electrode located at the distal end of the funiculus. Hence the activity of the proximal ORNs was frequently neglected, and part of the olfactory experience of the insect was not known. Occasionally, the EAG was recorded with an electrode capping the stump of the arista (Siciliano et al., 2014) that is located proximally. Recording the EAG in this way samples mostly the activity of the proximal ORNs. In chemical ecology studies, this situation resulted in discrepancies between ecological relevance and EAG responses to chemicals. For example, ethyl acetate, a major fruit compound also found in cuticular extracts, attracts C. capitata individuals (Jang et al., 1994; Casaña-Giner et al., 1999). However, this compound elicited only a low EAG response when recorded with a distal electrode (Light et al., 1988, 1992; Jang et al., 1989). This paper describes a method of recording and analysis that allows a deeper exploration of the olfactory sensitivity of insects with globular antennae. The CSD analysis has been extensively used in vertebrate brains and was proven useful in mapping the neuronal activity. This paper presented a model for adapting the CSD analysis to the particularities of the insect antenna. This method revealed for example that ethyl acetate activates actually the proximal region of C. capitata antennae (Jacob et al., 2017 b), reconciling behavioral and physiological studies. It is a 
promising tool for future research on the chemical ecology of arthropods.

\section{AUTHOR CONTRIBUTIONS}

VJ conceived and performed the experiments, did the modeling and analysis, and wrote the manuscript.

\section{FUNDING}

This work was funded by the Conseil Régional de la Réunion, the European Regional Development Fund (ERDF), the Centre

\section{REFERENCES}

Andrew Jansen, M., Singh, S. S., Chawla, N., and Franz, N. M. (2016). A multilayer micromechanical model of the cuticle of Curculio longinasus Chittenden, 1927 (Coleoptera: Curculionidae). J. Struct. Biol. 195, 139-158. doi: 10.1016/j.jsb.2016.05.007

Bazhenov, M., Lonjers, P., Skorheim, S., Bedard, C., and Destexhe, A. (2011). Nonhomogeneous extracellular resistivity affects the current-source density profiles of up-down state oscillations. Philos. Trans. R. Soc. A Math. Phys. Eng. Sci. 369, 3802-3819. doi: 10.1098/rsta.2011.0119

Bédard, C., and Destexhe, A. (2012). "Modeling local field potentials and their interaction with the extracellular medium," in Handbook of Neural Activity Measurement, eds. R. Brette and A. Destexhe (Cambridge: Cambridge University Press), 136-191.

Bhandawat, V., Olsen, S. R., Gouwens, N. W., Schlief, M. L., and Wilson, R. I. (2007). Sensory processing in the Drosophila antennal lobe increases reliability and separability of ensemble odor representations. Nat. Neurosci. 10, 1474-1482. doi: 10.1038/nn1976

Biasazin, T. D., Karlsson, M. F., Hillbur, Y., Seyoum, E., and Dekker, T. (2014). Identification of host blends that attract the African invasive fruit fly, Bactrocera invadens. J. Chem. Ecol. 40, 966-976. doi: 10.1007/s10886-014-0501-6

Bigiani, A., Scalera, G., Crnjar, R., Barbarossa, I. T., Magherini, P. C., and Pietra, P. (1989). Distribution and function of the antennal olfactory sensilla in Ceratitis capitata Wied. (Diptera, Trypetidae). Bol. di Zool. 56, 305-311. doi: $10.1080 / 11250008909355655$

Buzsáki, G., Anastassiou, C. A., and Koch, C. (2012). The origin of extracellular fields and currents-EEG, ECoG, LFP and spikes. Nat. Rev. Neurosci. 13, 407-420. doi: 10.1038/nrn3241

Cao, L. H., Yang, D., Wu, W., Zeng, X., Jing, B. Y., Li, M. T., et al. (2017). Odorevoked inhibition of olfactory sensory neurons drives olfactory perception in Drosophila. Nat. Commun. 8, 1-13. doi: 10.1038/s41467-017-01185-0

Casaña-Giner, V., Gandía-Balaguer, A., and Primo-Yúfera, E. (1999). Field trial of an attractant mixture for dipterous, including the pest Ceratitis capitata (Wiedemann) (Dipt., Tephritidae), in Valencia, Spain. J. Appl. Entomol. 123, 47-48. doi: 10.1046/j.1439-0418.1999.00329.x

Celani, A., Villermaux, E., and Vergassola, M. (2014). Odor landscapes in turbulent environments. Phys. Rev. X 4, 041015. doi: 10.1103/PhysRevX.4.041015

Clark, A. J., and Triblehorn, J. D. (2014). Mechanical properties of the cuticles of three cockroach species that differ in their wind-evoked escape behavior. PeerJ 2, e501. doi: $10.7717 /$ peerj.501

Clavijo Mccormick, A. C., Gershenzon, J., and Unsicker, S. B. (2014). Little peaks with big effects: establishing the role of minor plant volatiles in plant-insect interactions. Plant Cell Environ. 37, 1836-1844. doi: 10.1111/pce. 12357

Couto, A., Alenius, M., and Dickson, B. J. (2005). Molecular, anatomical, and functional organization of the Drosophila olfactory system. Curr. Biol. 15, 1535-1547. doi: 10.1016/j.cub.2005.07.034

Crnjar, R., Scalera, G., Liscia, A., Angioy, A. M., Bigiani, A., Pietra, P., et al. (1989). Morphology and EAG mapping of the antennal olfactory receptors in de Coopération International en Recherche Agronomique pour le Développement (CIRAD).

\section{ACKNOWLEDGMENTS}

The author thanks Jim Payet and Serge Glénac for rearing the Tephritidae species, and Richard Tibère for collecting and rearing C. sacchariphagus. Thanks to François Massol for discussion about the CSD model, and Philippe Lucas and Eric Jang for a critical reading of the manuscript. The author greatly acknowledges the Plant Protection Platform (3P, IBISA).
Dacus oleae. Entomol. Exp. Appl. 51, 77-85. doi: 10.1111/j.1570-7458.1989.tb0 1216.x

Crowley-Gall, A., Date, P., Han, C., Rhodes, N., Andolfatto, P., Layne, J. E., et al. (2016). Population differences in olfaction accompany host shift in Drosophila mojavensis. Proc. R. Soc. London Ser. B-Biological Sci. 283, 20161562. doi: 10.1098/rspb.2016.1562

de Bruyne, M., Foster, K., and Carlson, J. R. (2001). Odor coding in the Drosophila antenna. Neuron 30, 537-552. doi: 10.1016/S0896-6273(01)00289-6

Dekker, T., Ibba, I., Siju, K. P., Stensmyr, M. C., and Hansson, B. S. (2006). Olfactory shifts parallel superspecialism for toxic fruit in Drosophila melanogaster sibling, D. sechellia. Curr. Biol. 16, 101-109. doi: 10.1016/j.cub.2005.11.075

Duyck, P. F., and Quilici, S. (2002). Survival and development of different life stages of three Ceratitis spp. (Diptera: Tephritidae) reared at five constant temperatures. Bull. Entomol. Res. 92, 461-469. doi: 10.1079/BER20 02188

Dweck, H. K., Ebrahim, S. A., Khallaf, M. A., Koenig, C., Farhan, A., Stieber, R., et al. (2016). Olfactory channels associated with the Drosophila maxillary palp mediate short- and long-range attraction. Elife 5, e14925. doi: 10.7554/eLife.14925

Dweck, H. K., Ebrahim, S. A., Kromann, S., Bown, D., Hillbur, Y., Sachse, S., et al. (2013). Olfactory preference for egg laying on citrus substrates in Drosophila. Curr. Biol. 23, 2472-2480. doi: 10.1016/j.cub.2013. 10.047

Dweck, H. K., Ebrahim, S. A. M., Farhan, A., Hansson, B. S., and Stensmyr, M. C. (2015). Olfactory proxy detection of dietary antioxidants in Drosophila. Curr. Biol. 25, 455-466. doi: 10.1016/j.cub.2014.11.062

Einzelnen, A. N. (1962). Elektrophysiologische untersuchungen an einzelnen geruchsrezeptoren auf den antennen des Totengrabers (Necrophorus, Coleoptera). Z. Vgl. Physiol. 46, 212-248. doi: 10.1007/BF00341551

Ghaninia, M., Ignell, R., and Hansson, B. S. (2007). Functional classification and central nervous projections of olfactory receptor neurons housed in antennal trichoid sensilla of female yellow fever mosquitoes, Aedes aegypti. Eur. J. Neurosci. 26, 1611-1623. doi: 10.1111/j.1460-9568.2007.05786.x

Ghaninia, M., Olsson, S. B., and Hansson, B. S. (2014). Physiological organization and topographic mapping of the antennal olfactory sensory neurons in female hawkmoths, Manduca sexta. Chem. Senses 39, 655-671. doi: 10.1093/chemse/bju037

Grabe, V., Baschwitz, A., Dweck, H. K. M., Lavista-Llanos, S., Hansson, B. S., and Sachse, S. (2016). Elucidating the neuronal architecture of olfactory glomeruli in the Drosophila antennal lobe. Cell Rep. 16, 3401-3413. doi: 10.1016/j.celrep.2016.08.063

Hull, C. D., and Cribb, B. W. (2001). Olfaction in the queensland fruit fly, Bactrocera tryoni. I: Identification of olfactory receptor neuron types responding to environmental odors. J. Chem. Ecol. 27, 871-887. doi: 10.1023/A:1010374617409

Jacob, V., Monsempès, C., Rospars, J. P., Masson, J. B., and Lucas, P. (2017a). Olfactory coding in the turbulent realm. PLoS Comput. Biol. 13:e1005870. doi: 10.1371/journal.pcbi.1005870 
Jacob, V., Scolari, F., Delatte, H., Gasperi, G., Jacquin-Joly, E., Malacrida, A. R., et al. (2017b). Current source density mapping of antennal sensory selectivity reveals conserved olfactory systems between tephritids and Drosophila. Sci. Rep. 7, 15304. doi: 10.1038/s41598-017-15431-4

Jang, E. B., Light, D. M., Binder, R. G., Flath, R. A., and Carvalho, L. A. (1994). Attraction of female mediterranean fruit flies to the five major components of male-produced pheromone in a laboratory flight tunnel. J. Chem. Ecol. 20, 9-20. doi: 10.1007/BF02065987

Jang, E. B., Light, D. M., Flath, R. A., Nagata, J. T., and Mon, T. R. (1989). Electroantennogram responses of the Mediterranean fruit fly, Ceratitis capitata to identified volatile constituents from calling males. Entomol. Exp. Appl. 50, 7-19. doi: 10.1111/j.1570-7458.1989.tb02307.x

Joseph, R. M., and Carlson, J. R. (2015). Drosophila chemoreceptors: a molecular interface between the chemical world and the brain. Trends Genet. 31, 683-695. doi: 10.1016/j.tig.2015.09.005

Kaissling, K.-E. (1995). "Single unit and electroantennogram recordings in insect olfactory organs," in Experimental Cell Biology of Taste and Olfaction, eds. A. Spielman and J. Brand (Boca Raton, FL: CRC Press), 361-377.

Kaissling, K. E. (1986). Chemo-electrical transduction in insect olfactory receptors. Annu. Rev. Neurosci. 9, 121-145. doi: 10.1146/annurev.ne.09.030186.001005

Kaulen, P., Erber, J., and Mobbs, P. (1984). Current source-density analysis in the mushroom bodies of the honeybee (Apis mellifera carnica). J. Comp. Physiol. A 154, 569-582. doi: 10.1007/BF00610170

Klocke, D., and Schmitz, H. (2011). Water as a major modulator of the mechanical properties of insect cuticle. Acta Biomater. 7, 2935-2942. doi: 10.1016/j.actbio.2011.04.004

Light, D. M., Jang, E. B., and Dickens, J. C. (1988). Electroantennogram responses of the mediterranean fruit fly, Ceratitis capitata, to a spectrum of plant volatiles. J. Chem. Ecol. 14, 159-180. doi: 10.1007/BF01022539

Light, D. M., Jang, E. B., and Flath, R. A. (1992). Electroantennogram responses of the Mediterranean fruit fly, Ceratitis capitata, to the volatile constituents of nectarines. Entomol. Exp. Appl. 63, 13-26. doi: 10.1111/j.1570-7458.1992.tb02415.x

Lin, C. C., and Potter, C. J. (2015). Re-classification of Drosophila melanogaster trichoid and intermediate sensilla using fluorescence-guided single sensillum recording. PLoS ONE 10:e0139675. doi: 10.1371/journal.pone.0139675

Lockey, J. K., and Willis, M. A. (2015). One antenna, two antennae, big antennae, small: total antennae length, not bilateral symmetry, predicts odor-tracking performance in the American cockroach Periplaneta americana. J. Exp. Biol. 218, 2156-2165. doi: 10.1242/jeb.117721

Lucas, P., and Renou, M. (1992). Electrophysiological study of the effects of deltamethrin, bioresmethrin, and DDT on the activity of pheromone receptor neurones in two moth species. Pestic. Biochem. Physiol. 43, 103-115. doi: 10.1016/0048-3575(92)90024-T

Machon, J., Ravaux, J., Zbinden, M., and Lucas, P. (2016). New electroantennography method on a marine shrimp in water. J. Exp. Biol. 219, 3696-3700. doi: 10.1242/jeb.140947

Mansourian, S., Corcoran, J., Enjin, A., Löfstedt, C., Dacke, M., and Stensmyr, M. C. (2016). Fecal-derived phenol induces egg-laying aversion in Drosophila. Curr. Biol. 26, 2762-2769. doi: 10.1016/j.cub.2016. 07.065

Mayo, I., Anderson, M., Burguete, J., and Robles Chillida, E. (1987). Structure of superficial chemoreceptive sensilla on the third antennal segment of Ceratitis capitata (Wiedemann) (Diptera; Tephritidae). Int. J. Insect Morphol. Embryol. 16, 131-141. doi: 10.1016/0020-7322(87)90013-4

Mitzdorf, U. (1985). Current source-density method and application in cat cerebral cortex: investigation of evoked potentials and EEG phenomena. Physiol. Rev. 65, 37-100. doi: 10.1152/physrev.1985.65.1.37

Münch, D., and Galizia, C. G. (2016). DoOR 2. 0-Comprehensive mapping of Drosophila melanogaster odorant responses. Sci. Rep. 6, 21841. doi: 10.1038/srep21841

Nagai, T. (1981). Electroantennogram response gradient on the antenna of the European corn borer, Ostrinia nubilalis. J. Insect Physiol. 27, 889-894. doi: 10.1016/0022-1910(81)90090-1

Nagai, T. (1983a). On the relationship between the electroantennogram and simultaneously recorded single sensillum response of the European corn borer, Ostrinia nubilalis. Arch. Insect Biochem. Physiol. 1, 85-91. doi: 10.1002/arch.940010109
Nagai, T. (1983b). Spread of local electroantennogram response of the European corn borer, Ostrinia nubilalis. Pestic. Biochem. Physiol. 19, 291-298. doi: 10.1016/0048-3575(83)90057-3

Nagai, T. (1985). Summation and gradient characteristics of local electroantennogram response of the European corn borer, Ostrinia nubilalis. Pestic. Biochem. Physiol. 24, 32-39. doi: 10.1016/0048-3575(85)90110-5

Nagel, K. I., and Wilson, R. I. (2011). Biophysical mechanisms underlying olfactory receptor neuron dynamics. Nat. Neurosci. 14, 208-216. doi: 10.1038/nn.2725

Nicholson, C. (1973). Theoretical analysis of field potentials in anisotropic ensembles of neuronal elements. IEEE Trans. Biomed. Eng. 20, 278-288. doi: 10.1109/TBME.1973.324192

Nishino, H., Iwasaki, M., Paoli, M., Kamimura, I., Yoritsune, A., and Mizunami, M. (2018). Spatial receptive fields for odor localization. Curr. Biol. 28, 600-608.e3. doi: 10.1016/j.cub.2017.12.055

Nowotny, T., de Bruyne, M., Berna, A. Z., Warr, C. G., and Trowell, S. C. (2014). Drosophila olfactory receptors as classifiers for volatiles from disparate real world applications. Bioinspir. Biomim. 9, 046007. doi: 10.1088/1748-3182/9/4/046007

Olsson, S. B., and Hansson, B. S. (2013). "Electroantennogram and single sensillum recording in insect antennae," in Methods in Molecular Biology, vol. 1068, ed. K. Touhara (Berlin: Springer Science; Business Media, LLC), 157-177. doi: 10. 1007/978-1-62703-619-1_11

Olsson, S. B., Linn, C. E., and Roelofs, W. L. (2006). The chemosensory basis for behavioral divergence involved in sympatric host shifts. II: olfactory receptor neuron sensitivity and temporal firing pattern to individual key host volatiles. J. Comp. Physiol. A 192, 289-300. doi: 10.1007/s00359-0050066-5

Pettersen, K. H., Devor, A., Ulbert, I., Dale, A. M., and Einevoll, G. T. (2006). Current-source density estimation based on inversion of electrostatic forward solution: effects of finite extent of neuronal activity and conductivity discontinuities. J. Neurosci. Methods 154, 116-133. doi: 10.1016/j.jneumeth.2005.12.005

Pitts, W. (1952). "Investigations on synaptic transmission," in Cybernetics, Trans. 9th Conf. Josiah Macy Foundation, ed. H. von Foerster (New York, NY: Diaphanes), 159-166.

Potworowski, J., Jakuczun, W., Łeski, S., and Wójcik, D. K. (2012). Kernel current source density method. Neural Comput. 24, 541-575. doi: 10.1162/NECO_a_00236

Ramanujan, S. (1914). Modular equations and approximations to pi. Q. J. Pure Appl. Math. 45, 350-372.

Ronderos, D. S., Lin, C. C., Potter, C. J., and Smith, D. P. (2014). Farnesoldetecting olfactory neurons in Drosophila. J. Neurosci. 34, 3959-3968. doi: 10.1523/JNEUROSCI.4582-13.2014

Rospars, J. P., Grémiaux, A., Jarriault, D., Chaffiol, A., Monsempes, C., Deisig, N., et al. (2014). Heterogeneity and convergence of olfactory firstorder neurons account for the high speed and sensitivity of secondorder neurons. PLoS Comput. Biol. 10:e1003975. doi: 10.1371/journal.pcbi. 1003975

Roux, S. G., Cenier, T., Garcia, S., Litaudon, P., and Buonviso, N. (2007). A waveletbased method for local phase extraction from a multi-frequency oscillatory signal. J. Neurosci. Methods 160, 135-143. doi: 10.1016/j.jneumeth.2006 09.001

Sanes, J. R., and Hildebrand, J. G. (1976). Structure and development of antennae in a moth, Manduca sexta. Dev. Biol. 51, 282-299. doi: 10.1016/0012-1606(76)90144-5

Schneider, D. (1957a). Electrophysiological investigation on the antennal receptors of the silk moth during chemical and mechanical stimulation. Experientia 13, 89-91.

Schneider, D. (1957b). Elektrophysiologische untersuchungen von chemo-und mechanorezeptoren der antenne des seidenspinners Bombyx mori L. Z. Vgl. Physiol. 40, 8-41.

Schneider, D., and Boeckh, J. (1962). Rezeptorpotential und nervenimpulse einzelner olfaktorischer sensillen der insektenantenne. Z. Vgl. Physiol. 45, 405-412. doi: 10.1007/BF00340462

Shanbhag, S. ., Müller, B., and Steinbrecht, R. (1999). Atlas of olfactory organs of Drosophila melanogaster 1. Types, external organization, innervation and distribution of olfactory sensilla. Int. J. Insect Morphol. Embryol. 28, 377-397. doi: 10.1016/S0020-7322(99)00039-2 
Shields, V. D., and Hildebrand, J. G. (2001). Recent advances in insect olfaction, specifically regarding the morphology and sensory physiology of antennal sensilla of the female sphinx moth Manduca sexta. Microsc. Res. Tech. 55, 307-329. doi: 10.1002/jemt.1180

Siciliano, P., He, X. L., Woodcock, C., Pickett, J. A., Field, L. M., Birkett, M. A., et al. (2014). Identification of pheromone components and their binding affinity to the odorant binding protein CcapOBP83a-2 of the Mediterranean fruit fly, Ceratitis capitata. Insect Biochem. Mol. Biol. 48, 51-62. doi: 10.1016/j.ibmb.2014.02.005

Song, E., de Bivort, B., Dan, C., and Kunes, S. (2012). Determinants of the Drosophila odorant receptor pattern. Dev. Cell 22, 363-376. doi: 10.1016/j.devcel.2011.12.015

Stensmyr, M. C., Dweck, H. K., Farhan, A., Ibba, I., Strutz, A., Mukunda, L., et al. (2012). A conserved dedicated olfactory circuit for detecting harmful microbes in Drosophila. Cell 151, 1345-1357. doi: 10.1016/j.cell.2012.09.046

Stocker, R. F. (1994). The organization of the chemosensory cystem in Drosophila melanogaster: a review. Cell Tissue Res. 275, 3-26. doi: 10.1007/BF00 305372

Tait, C., Batra, S., Ramaswamy, S. S., Feder, J. L., and Olsson, S. B. (2016). Sensory specificity and speciation: a potential neuronal pathway for host fruitodour discrimination in Rhagoletis pomonella. Proc. R. Soc. London Ser. B Biol. Sci. 283, 1-9. doi: 10.1098/rspb.2016.2101

Vaknin, G., DiScenna, P. G., and Teyler, T. J. (1988). A method for calculating current source density (CSD) analysis without resorting to recording sites outside the sampling volume. J. Neurosci. Methods 24, 131-135. doi: 10.1016/0165-0270(88)90056-8

Vincent, J. F., and Wegst, U. G. (2004). Design and mechanical properties of insect cuticle. Arthropod. Struct. Dev. 33, 187-199. doi: 10.1016/j.asd.2004. 05.006

Conflict of Interest Statement: The author declares that the research was conducted in the absence of any commercial or financial relationships that could be construed as a potential conflict of interest.

Copyright (C) 2018 Jacob. This is an open-access article distributed under the terms of the Creative Commons Attribution License (CC BY). The use, distribution or reproduction in other forums is permitted, provided the original author(s) and the copyright owner(s) are credited and that the original publication in this journal is cited, in accordance with accepted academic practice. No use, distribution or reproduction is permitted which does not comply with these terms. 


\section{OPEN ACCESS}

Edited by:

Frédéric Marion-Poll,

AgroParisTech - Institut des Sciences

et Industries du Vivant et

de L'environnement, France

Reviewed by:

Michael Douglas Gordon,

The University of British Columbia,

Canada

Nilay Yapici,

Cornell University, United States

*Correspondence:

Anupama Dahanukar

anupama.dahanukar@ucr.edu

Received: 29 August 2018 Accepted: 08 October 2018 Published: 24 October 2018

Citation:

Chen $Y-C D$, Park SJ, Ja WW and Dahanukar A (2018) Using Pox-Neuro

(Poxn) Mutants in Drosophila

Gustation Research: A Double-Edged Sword. Front. Cell. Neurosci. 12:382. doi: 10.3389/fncel.2018.00382

\section{Using Pox-Neuro (Poxn) Mutants in Drosophila Gustation Research: A Double-Edged Sword}

\author{
Yu-Chieh David Chen ${ }^{1}$, Scarlet Jinhong Park², William W. Ja ${ }^{2}$ and \\ Anupama Dahanukar ${ }^{1,3 *}$
}

\begin{abstract}
${ }^{1}$ Interdepartmental Neuroscience Program, University of California, Riverside, Riverside, CA, United States, ${ }^{2}$ Department of Neuroscience, The Scripps Research Institute, Jupiter, FL, United States, ${ }^{3}$ Department of Molecular, Cell and Systems Biology, University of California, Riverside, Riverside, CA, United States
\end{abstract}

In Drosophila, Pox-neuro (Poxn) is a member of the Paired box (Pax) gene family that encodes transcription factors with characteristic paired DNA-binding domains. During embryonic development, Poxn is expressed in sensory organ precursor (SOP) cells of poly-innervated external sensory ( $p$-es) organs and is important for specifying $p$-es organ identity (chemosensory) as opposed to mono-innervated external sensory (m-es) organs (mechanosensory). In Poxn mutants, there is a transformation of chemosensory bristles into mechanosensory bristles. As a result, these mutants have often been considered to be entirely taste-blind, and researchers have used them in this capacity to investigate physiological and behavioral functions that act in a taste-independent manner. However, recent studies show that only external taste bristles are transformed in Poxn mutants whereas all internal pharyngeal taste neurons remain intact, raising concerns about interpretations of experimental results using Poxn mutants as tasteblind flies. In this review, we summarize the value of Poxn mutants in advancing our knowledge of taste-enriched genes and feeding behaviors, and encourage revisiting some of the conclusions about taste-independent nutrient-sensing mechanisms derived from these mutants. Lastly, we highlight that Poxn mutant flies remain a valuable tool for probing the function of the relatively understudied pharyngeal taste neurons in sensing meal properties and regulating feeding behaviors.

Keywords: Drosophila, Pox-neuro, pharyngeal taste, gustation, feeding behavior, taste-blind

\section{INTRODUCTION}

Taste is essential for insects to evaluate the palatability and nutritional content of food sources and to make important decisions on feeding, mating, and egg laying (Dethier, 1976; Scott, 2018). An understanding of the insect taste system may lead to the development of new strategies to control insect feeding behaviors, which constitute a significant economic and health burden each year. The vinegar fly, Drosophila melanogaster, has been a highly tractable model organism to explore the neurobiology of insect taste. With the powerful molecular genetic tools and robust behavioral assays in this model, scientists have explored how taste information is recognized and processed to control feeding behaviors. 
In Drosophila, there are two major types of external sensory bristles distinguished broadly as mono- or poly-innervated based on the number of neurons that are housed within. Mono-innervated external sensory (m-es) bristles, such as mechanosensory bristles, are distributed all over the body. Each is innervated by a single mechanosensory neuron, which extends its dendrite to the base of the shaft and detects deflection of the hair (Falk et al., 1976). Poly-innervated external sensory (p-es) bristles, such as taste bristles, are distributed in various parts of the body, including the labellum, distal segments of the legs, wing margins, and the ovipositor (Stocker, 1994; Liman et al., 2014; Freeman and Dahanukar, 2015). Within the taste bristles, there are multiple taste neurons (usually 2-4 in the labellar taste bristles) that extend their dendrites up to the tip of the hair shaft, close to a single pore through which tastants can enter the sensillum lymph. During development, sensory mother cells of different lineages generate different type of sensory organs, specified by sets of transcription factors (Ghysen and Dambly-Chaudiere, 2000). One such factor is Pox-neuro (Poxn), which is a transcription factor with a paired DNAbinding domain. During neurogenesis, Poxn is expressed in sensory mother cells that eventually give rise to $\mathrm{p}$-es organs of the peripheral nervous system (Dambly-Chaudiere et al., 1992). In Poxn mutants, all external chemosensory bristles are transformed into mechanosensory bristles (Dambly-Chaudiere et al., 1992; Awasaki and Kimura, 1997), offering a model with numerous possible uses in gustatory research.

The identification of candidate taste receptor genes in early 2000 was a major breakthrough in understanding the molecular and cellular basis of insect gustation (Clyne et al., 2000). By scanning for predicted structural properties of encoded proteins rather than specific DNA sequences, John Carlson's group at Yale University identified a transmembrane receptor family that shared no sequence similarity to any known proteins. Many members of this family were expressed in a major gustatory organ, the labellum, which informed its naming as the Gustatory receptor (Gr) gene family. Importantly, Poxn mutants were used to support taste-specific or taste-enriched expression of selected $G r$ genes. A comparison of $G r$ expression between wild-type and Poxn mutant flies uncovered that 18 of $19 \mathrm{Gr}$ transcripts were not detected in mutant labella (Clyne et al., 2000). This study was the first to demonstrate the utility of Poxn mutants for identifying the $G r$ gene family, which was quickly followed by further characterization of additional $G r$ members and analysis of their expression with transgenic reporters (Scott et al., 2001).

Subsequently, Poxn mutants and related genetic tools have been widely used for gustation research in Drosophila. In this mini-review, we examine how Poxn manipulations were used to reveal additional taste sensillum-enriched genes and discuss examples of how Poxn mutants have been utilized in behavioral research to dissect the involvement of gustatory sensory inputs as well as to identify taste-independent nutrient-sensing mechanisms. Finally, we highlight recent studies confirming that internal pharyngeal taste neurons remain intact in Poxn mutants (LeDue et al., 2015; Chen and Dahanukar, 2017), indicating that these mutants are not taste-blind. We suggest that the importance of pharyngeal input in driving feeding behaviors should be considered and explored further, and that some conclusions of previous studies should be reevaluated in light of these recent findings.

\section{Poxn MUTANTS AS A VALUABLE TOOL FOR IDENTIFYING TASTE SENSILLUM-ENRICHED GENES}

The absence of gustatory bristles in Poxn mutants enabled the identification of taste-related genes, whose expression was expected to be down-regulated in the mutants as compared to control flies. This rationale was validated through numerous studies and confirmed the value of Poxn as a tool for such molecular discoveries. For example, by using RT-PCR or microarray analysis of cDNA from taste organs in wild-type and Poxn mutants, many genes that were enriched in wildtype relative to Poxn mutants were identified as chemosensory receptor genes, including the $G r$ (Clyne et al., 2000; Ueno et al., 2001; Moon et al., 2009), ionotropic receptor (Ir) (Koh et al., 2014), and pickpocket (Ppk) (Cameron et al., 2010; Lu et al., 2012 , 2014) gene families. Similar strategies were used to reveal expression of other genes in external taste organs, including those that encode odorant-binding proteins (Koganezawa and Shimada, 2002; Jeong et al., 2013) and the adipokinetic hormone receptor (Bharucha et al., 2008), which led to the characterization of their roles in taste detection and feeding behavior.

More recently, molecular genetic tools derived from the Poxn locus were used to alter sensory bristles in a taste organ-specific manner. Taking advantage of the GAL4/UAS system (Brand and Perrimon, 1993) to induce tissue-specific RNAi, Raad et al. (2016) found that silencing of Poxn in wings caused all taste bristles in the anterior wing margin to be transformed into mechanosensory bristles, leaving those in other taste organs intact. Such targeted silencing of Poxn in specific tissues could be of value for dissecting roles of different taste organs in chemosensory behaviors. In addition, several Poxn-GAL4 transgenes synthesized with various Poxn enhancers are available and can be used to gain genetic access to the vast majority of taste neurons (Boll and Noll, 2002). Both GAL4 and UAS transgenic reagents of Poxn have been used to label or to knock down genes of interest in taste neurons. More recently, Poxn-GAL80 has also been generated for blocking GAL4 activity in most if not all taste neurons (Steck et al., 2018). Together, the Poxn molecular genetic toolkit (Table 1) has the necessary components for executing intersectional strategies to broadly manipulate taste hairs.

\section{Poxn MUTANTS ARE NOT TASTE-BLIND}

The Poxn mutant has been widely used to investigate the importance of taste sensory input in driving behaviors of interest (Table 2). The underlying assumption for many of these studies was the taste-blind feature of Poxn mutant flies. In instances where Poxn mutants exhibited behaviors similar to those of wildtype counterparts, the palpable conclusion was that the observed behaviors were generated by taste-independent mechanisms. 
TABLE 1 | The Poxn toolkit.

\begin{tabular}{|c|c|c|}
\hline Poxn tools & Purpose & Reference \\
\hline Poxn-GAL4 & Genetic access to most taste neurons via the GAL4/UAS system & $\begin{array}{l}\text { Boll and Noll, 2002; Bhandari et al., 2006; Mellert et al., 2010; Wang } \\
\text { et al., 2011; Liu et al., 2012; Starostina et al., 2012; Park et al., 2013; } \\
\text { Lu et al., 2014; Vijayan et al., 2014; Clowney et al., 2015; Yilmazer } \\
\text { et al., 2016; Chowdhury et al., 2017; Sovik et al., 2017; Kojima et al., } \\
\text { 2018; Steck et al., } 2018\end{array}$ \\
\hline Poxn-GAL80 & Block activity of the GAL4/UAS system in most taste neurons & Steck et al., 2018 \\
\hline UAS-POXn RNAi & Tissue-specific Poxn knockdown & Raad et al., 2016; Houot et al., 2017 \\
\hline Poxn-CD8::GFP & GFP expression under direct control of Poxn enhancer & Minocha et al., 2017 \\
\hline Anti-Poxn antibody & Poxn expression & Diaper et al., 2013 \\
\hline
\end{tabular}

TABLE 2 | Poxn mutants for behavioral research.

\begin{tabular}{|c|c|c|}
\hline Behaviors & Phenotype & Reference \\
\hline \multirow[t]{9}{*}{ Feeding } & Nutrient sensing & Dus et al., 2011, 2013; Abu et al., 2018 \\
\hline & Sugar & $\begin{array}{l}\text { Usui-Aoki et al., 2005; Sun et al., 2014; LeDue et al., 2015; Liu et al., 2015; } \\
\text { Murata et al., } 2017\end{array}$ \\
\hline & Bitter & Mitri et al., 2009; Chen and Dahanukar, 2017 \\
\hline & Salt & Kojima et al., 2018 \\
\hline & $\mathrm{pH}$ & Deshpande et al., 2015 \\
\hline & Yeast & Steck et al., 2018 \\
\hline & Water & Chen et al., 2010 \\
\hline & Ethanol & Devineni and Heberlein, 2009 \\
\hline & Fatty acid & Masek and Keene, 2013 \\
\hline \multirow[t]{2}{*}{ Social } & Aggregation pheromone detection & Lin et al., 2015 \\
\hline & Social interaction & Schneider et al., 2012; Schneider and Levine, 2014 \\
\hline \multirow[t]{2}{*}{ Reproductive } & Oviposition & $\begin{array}{l}\text { Joseph et al., 2009; Joseph and Heberlein, 2012; Hussain et al., 2016; } \\
\text { Verschut et al., } 2017\end{array}$ \\
\hline & Courtship & Boll and Noll, 2002; Krstic et al., 2009 \\
\hline \multirow[t]{3}{*}{ Others } & Grooming & Yanagawa et al., 2014, 2018 \\
\hline & Positional preference & Joseph et al., 2009; Joseph and Heberlein, 2012 \\
\hline & Starvation-induced hyperactivity & Yang et al., 2015 \\
\hline
\end{tabular}

However, several studies provided hints that internal pharyngeal taste organs are intact in Poxn mutants (Galindo and Smith, 2001; LeDue et al., 2015; Chen and Dahanukar, 2017). Analysis of odorant binding protein (OBP) expression revealed that Poxn mutants lose expression of external gustatory-specific OBPs but not of ones in the pharynx, such as OBP56b (Galindo and Smith, 2001). This study, published in 2001, was the first to posit a specific requirement for Poxn in cell fate determination of external but not internal taste organs. It was not until much later that a functional demonstration followed, in a study that found intact pharyngeal Gr43a taste neurons in Poxn mutants and proved their requirement for sugar selection and sustained consumption (LeDue et al., 2015).

These results set the stage for a comprehensive study of chemosensory receptor expression in the pharynx, which showed that pharyngeal taste neurons and their central projections in the taste center, the subesophageal zone, are intact in Poxn mutants (Chen and Dahanukar, 2017). Thus, although Poxn mutants have lost all external taste bristles, they are not rendered taste-blind by virtue of taste sensory neurons preserved in the pharynx-the role of pharyngeal taste in driving Poxn behaviors that were thought to be taste-independent should therefore be evaluated. For example, previous studies suggested that, in addition to sweetness, the caloric content of sugar can also drive food preference. Taste-independent detection of the caloric content of sugar was evaluated using Poxn mutants, which were insensitive to the taste of sugar in proboscis extension assays but exhibited preference for the nutritive sugar in feeding assays (Dus et al., 2011, 2013). Given that pharyngeal Gr43a taste neurons are still functional and drive selection of both nutritive and non-nutritive sugars in Poxn mutants (LeDue et al., 2015), the possibility of their functional interactions with neurons identified as having internal nutrient-sensing capabilities, such as SLC5A11 - (Dus et al., 2011, 2013) or DH44-neurons (Dus et al., 2015), cannot be ruled out. In addition, a recent study showed that another group of pharyngeal taste neurons expressing Ir60 b responds strongly to sucrose but weakly to glucose (Joseph et al., 2017). Interestingly, Ir60b mutants have specific defects in sensing sucrose but not in detecting other nutritive or nonnutritive sugars, suggesting that there are distinct pharyngeal sugar-sensing mechanisms that allow discrimination between various sugars. It will be of interest to evaluate functional intersections of pharyngeal taste and nutrient sensing in the future. 
Nevertheless, Poxn mutants present a useful vehicle for dissecting the role of external taste input in many behaviors of interests (Table 2). In many behavioral assays, Poxn mutants have shown a degree of deficit as compared to wild-type controls, indicating contributions of information from external taste organs in oviposition site selection (Joseph et al., 2009; Hussain et al., 2016), the effect of pheromones on life span and physiology (Gendron et al., 2014), trehalose consumption (Usui-Aoki et al., 2005), and the effect of food $\mathrm{pH}$ on palatability (Deshpande et al., 2015).

\section{PHARYNGEAL TASTE PRESENTS A MISSING LINK BETWEEN TASTE INPUT AND FEEDING BEHAVIORAL OUTPUT}

Given the anatomical location of pharyngeal taste organs, it has long been assumed that they act as gatekeepers for monitoring food quality and controlling ingestion, but there is little direct knowledge of the functional roles of sensory neurons that reside within. Poxn mutants offer a minimal taste model for probing the roles of pharyngeal taste neurons in feeding behaviors. In the context of Poxn mutants, pharyngeal sensitivity to tastants other than sweet compounds has not been explored in depth, and there are recent studies hinting at the function of pharyngeal taste neurons in detecting bacterial lipopolysaccharides (Soldano et al., 2016) and high concentrations of salt ( $\mathrm{NaCl}$ ) (Kim et al., 2017). A comprehensive examination of pharyngeal taste receptivity has not yet been done, but pharyngeal expression of chemosensory receptors involved in sensing water (Cameron et al., 2010), bitter (Weiss et al., 2011), salt (Zhang et al., 2013), and electrophiles (Kang et al., 2010) implies that the potential for detecting other categories of tastants exists. Indeed, Poxn mutants are capable of selecting appetitive tastants such as sugars and amino acids, and rejecting aversive tastants such as bitter compounds, high salt concentration, and very low $\mathrm{pH}$, suggesting that pharyngeal taste organs pose an important link between taste sensory input and feeding behavioral output. With the pharyngeal receptor-toneuron maps established recently (Chen and Dahanukar, 2017), it is now possible to use genetic dissection strategies to interrogate the function of different neuronal subsets in driving behavioral responses to various tastants. We expect that such experiments will be of value, not only to demonstrate the contributions of pharyngeal taste neurons in controlling food intake, but also to probe the sensory functions of the many remaining orphan neurons.

\section{THE FUNCTION OF POXn IN THE DEVELOPING NERVOUS SYSTEM}

Poxn mutants have been described as having defects in p-es organs but not in m-es organs. The mechanisms underlying the specificity of this defect are not yet clear, but it is conceivable that internal pharyngeal taste organs rely on other transcription factors and signaling networks. In fact, there is a difference in developmental timing between adult pharyngeal taste neurons, which are born during embryogenesis and persist through metamorphosis (Gendre et al., 2004), and external taste neurons that originate only during metamorphosis. Notably, many olfactory sensilla in the antennae and maxillary palps also house multiple olfactory receptor neurons (1-4 ORNs). However, olfactory sensilla do not appear to be affected in Poxn mutants, since ORN projections remain intact in the antennal lobes (Chen and Dahanukar, 2017). Thus, Poxn mutants have specific defects in gustatory but not olfactory bristles.

In addition to the peripheral nervous system, Poxn is also expressed in various postmitotic neurons in the developing brain, including a protocerebral dorsal cluster and a deutocerebral ventral cluster (Minocha et al., 2017). The former is crucial for connections of the bulb with the ellipsoid body, while the latter is important for connections between the antennal lobe and lateral horn. In Poxn mutants, the Poxn-expressing brain neurons cannot establish proper connections with their targets. The behavioral consequences of the central nervous system defects are not clear and await further characterization. Although the wiring defects were observed in Poxn mutants homozygous for the $\Delta M 22-B 5$ allele, created by an imprecise excision spanning over $17 \mathrm{~kb}$ that removes part of the Poxn gene and promoter as well as an adjacent gene encoding a sugar transporter homolog, CG8249 (Boll and Noll, 2002), a recent study pinpoints a $1442 \mathrm{~kb}$ upstream fragment as an important enhancer for brain function (Minocha et al., 2017). In addition to the defects in the central nervous system, mutants homozygous for the $\Delta M 22-B 5$ allele also have defects in leg/antenna segmentation, male courtship, male fertility, and flight (Boll and Noll, 2002). Although defined enhancer regions have been implicated for specific functions (Boll and Noll, 2002), little is known about involvement of the adjacent gene that is removed in the $\Delta M 22-B 5$ allele in fly behavior. An ethyl methanesulfonate (EMS)-generated allele, Poxn ${ }^{70}$, has been reported to be an amorphic allele (Awasaki and Kimura, 1997) with adjacent genes likely intact. Thus, the transheterozygous allelic combination of Poxn ${ }^{\Delta M 22-B 5} / P_{o x n}{ }^{70}$ in recent studies might circumvent some of the defects described in flies homozygous for the $\Delta M 22-B 5$ allele (LeDue et al., 2015; Chen and Dahanukar, 2017), although this remains unconfirmed.

\section{CONSIDERATIONS IN USING Poxn MUTANTS FOR GUSTATION RESEARCH}

The proboscis extension reflex (PER) assay has been tremendously valuable as a measure of taste behavior response (Shiraiwa and Carlson, 2007). However, while Poxn mutants lack functional external taste bristles and are insensitive to labellar or tarsal stimulations with appetitive sugar solutions, they are indeed able to identify and consume food, enabling the use of the many food intake assays available for assessing contributions of pharyngeal taste neurons to feeding behaviors. Given that Poxn mutants are defective in external taste sensing, care must be taken in selecting appropriate assays for quantifying food 
intake. An increasing number of assays have been developed for measuring food intake in Drosophila, including the quantification of food labeled with radiotracers or colorimetric dyes, or direct monitoring of consumed volume of liquid diet (solutions of yeast or sugar) in the Capillary Feeder (CAFE) assay (Deshpande et al., 2014). Poxn mutants have been shown to ingest food as either liquid (i.e., in the CAFE assay) (Devineni and Heberlein, 2009) or solid (i.e., radiolabeling or colorimetric dyes in agar-based medium) (Deshpande et al., 2015; LeDue et al., 2015; Chen and Dahanukar, 2017). However, a recent report showed that Poxn mutant flies have difficulty in finding food sources with increased distance between them in binary choice assays (Abu et al., 2018), suggesting a context-dependent foraging deficiency in Poxn flies. Recently developed tools such as FlyPAD, which can be used for high resolution quantification of contacts with food, showed normal yeast feeding behavior in Poxn mutants (Steck et al., 2018), and thus offer alternatives for testing Poxn mutants.

In addition to the effects of different Poxn mutant alleles on the development of the central nervous system, another precaution in using Poxn mutants in gustatory research is that there has been no assessment of whether the supernumerary mechanosensory bristles have any function in mechanosensing and thus impart hypersensitivity to mechanical stimuli. Indeed, recent studies have identified at least two different neuronal populations that mediate feeding preference on the basis of texture. One class is the mechanosensory neurons in labellar taste sensilla, which express a mechanosensory receptor, NOMPC (Sanchez-Alcaniz et al., 2017). The second is multidendritic neurons in the labellum (md-L), expressing the transmembrane channel-like (TMC) protein (Zhang et al., 2016). It is not clear how these two mechanosensing mechanisms interact, but the contribution of mechanosensation in feeding behaviors cannot be ignored. Ensuring that all genetic manipulations and comparisons use the same Poxn mutant background will help minimize or rule out hypersensitivity in mechanosensing, as well as other potential defects, as confounds in interpreting results.

\section{REFERENCES}

Abu, F., Wang, J. G., Oh, Y., Deng, J., Neubert, T. A., and Suh, G. S. B. (2018). Communicating the nutritional value of sugar in Drosophila. Proc. Natl. Acad. Sci. U.S.A. 115, E2829-E2838. doi: 10.1073/pnas.171982 7115

Awasaki, T., and Kimura, K. (1997). pox-neuro is required for development of chemosensory bristles in Drosophila. J. Neurobiol. 32, 707-721. doi: 10.1002/ (SICI) 1097-4695(19970620)32:7<707::AID-NEU6>3.0.CO;2-8

Bhandari, P., Gargano, J. W., Goddeeris, M. M., and Grotewiel, M. S. (2006). Behavioral responses to odorants in drosophila require nervous system expression of the beta integrin gene myospheroid. Chem. Senses 31, 627-639. doi: 10.1093/chemse/bjl002

Bharucha, K. N., Tarr, P., and Zipursky, S. L. (2008). A glucagon-like endocrine pathway in Drosophila modulates both lipid and carbohydrate homeostasis. J. Exp. Biol. 211, 3103-3110. doi: 10.1242/jeb.016451

Boll, W., and Noll, M. (2002). The Drosophila pox neuro gene: control of male courtship behavior and fertility as revealed by a complete dissection of all enhancers. Development 129, 5667-5681. doi: 10.1242/dev. 00157

\section{CONCLUSION AND PERSPECTIVE}

Over the last couple of decades, the Poxn mutation, which specifically affects the developmental fate of gustatory bristles, has presented unique opportunities for investigating molecular and cellular principles of taste system function. The Poxn mutant has been subjected to a diverse range of approaches, spanning differential gene analysis for identifying taste-related genes, to behavioral analysis for identifying the contribution of specific gustatory inputs. Importantly, recent studies have shown that all internal pharyngeal taste organs remain intact in Poxn mutants, which brings immediate attention to the research community that Poxn mutants are not taste-blind and warrants revisiting taste-independent nutrient-sensing mechanisms established through their use. Instead, the Poxn mutant provides a model with a minimal pharyngeal taste system with which to dissect the function of pharyngeal taste neurons. Combined with the genetic toolkit derived from the recently described map of pharyngeal taste neurons, we now have the means to evaluate the sensory function of a taste organ that has often been overlooked while interpreting results of feeding behavior experiments.

\section{AUTHOR CONTRIBUTIONS}

Y-CC, WJ, and AD conceptualized the study. Y-CC wrote the original draft. Y-CC, SP, WJ, and AD wrote, reviewed, and edited the manuscript. WJ and AD supervised the study. WJ and AD acquired funding.

\section{FUNDING}

This work was funded by grants from the NIH (R01DC013587 to AD and R01AG045036 to WJ). Y-CC is a Howard Hughes Medical Institute International Student Research Fellow.

Brand, A. H., and Perrimon, N. (1993). Targeted gene expression as a means of altering cell fates and generating dominant phenotypes. Development 118 , 401-415.

Cameron, P., Hiroi, M., Ngai, J., and Scott, K. (2010). The molecular basis for water taste in Drosophila. Nature 465, 91-95. doi: 10.1038/nature09011

Chen, Y. D., and Dahanukar, A. (2017). Molecular and cellular organization of taste neurons in adult Drosophila pharynx. Cell Rep. 21, 2978-2991. doi: 10.1016/j. celrep.2017.11.041

Chen, Z., Wang, Q., and Wang, Z. (2010). The amiloride-sensitive epithelial $\mathrm{Na}+$ channel PPK28 is essential for drosophila gustatory water reception. J. Neurosci. 30, 6247-6252. doi: 10.1523/JNEUROSCI.0627-10.2010

Chowdhury, Z. S., Sato, K., and Yamamoto, D. (2017). The core-promoter factor TRF2 mediates a fruitless action to masculinize neurobehavioral traits in Drosophila. Nat. Commun. 8:1480. doi: 10.1038/s41467-017-01623-z

Clowney, E. J., Iguchi, S., Bussell, J. J., Scheer, E., and Ruta, V. (2015). Multimodal chemosensory circuits controlling male courtship in Drosophila. Neuron 87, 1036-1049. doi: 10.1016/j.neuron.2015.07.025

Clyne, P. J., Warr, C. G., and Carlson, J. R. (2000). Candidate taste receptors in Drosophila. Science 287, 1830-1834. doi: 10.1126/science.287.5459. 1830 
Dambly-Chaudiere, C., Jamet, E., Burri, M., Bopp, D., Basler, K., Hafen, E., et al. (1992). The paired box gene pox neuro: a determinant of poly-innervated sense organs in Drosophila. Cell 69, 159-172. doi: 10.1016/0092-8674(92)90127-X

Deshpande, S. A., Carvalho, G. B., Amador, A., Phillips, A. M., Hoxha, S., Lizotte, K. J., et al. (2014). Quantifying Drosophila food intake: comparative analysis of current methodology. Nat. Methods 11, 535-540. doi: 10.1038/nmeth.2899

Deshpande, S. A., Yamada, R., Mak, C. M., Hunter, B., Soto Obando, A., Hoxha, S., et al. (2015). Acidic food $\mathrm{Ph}$ increases palatability and consumption and extends Drosophila lifespan. J. Nutr. 145, 2789-2796. doi: 10.3945/jn.115.222380

Dethier, V. G. (1976). The Hungry Fly :A Physiological Study of the Behavior Associated with Feeding. Cambridge, MA: Harvard University Press.

Devineni, A. V., and Heberlein, U. (2009). Preferential ethanol consumption in Drosophila models features of addiction. Curr. Biol. 19, 2126-2132. doi: 10.1016/j.cub.2009.10.070

Diaper, D. C., Adachi, Y., Sutcliffe, B., Humphrey, D. M., Elliott, C. J., Stepto, A., et al. (2013). Loss and gain of Drosophila TDP-43 impair synaptic efficacy and motor control leading to age-related neurodegeneration by loss-of-function phenotypes. Hum. Mol. Genet. 22, 1539-1557. doi: 10.1093/hmg/ddt005

Dus, M., Ai, M., and Suh, G. S. (2013). Taste-independent nutrient selection is mediated by a brain-specific $\mathrm{Na}+$ /solute co-transporter in Drosophila. Nat. Neurosci. 16, 526-528. doi: 10.1038/nn.3372

Dus, M., Lai, J. S., Gunapala, K. M., Min, S., Tayler, T. D., Hergarden, A. C., et al. (2015). Nutrient Sensor in the brain directs the action of the brain-gut axis in Drosophila. Neuron 87, 139-151. doi: 10.1016/j.neuron.2015.05.032

Dus, M., Min, S., Keene, A. C., Lee, G. Y., and Suh, G. S. (2011). Taste-independent detection of the caloric content of sugar in Drosophila. Proc. Natl. Acad. Sci. U.S.A. 108, 11644-11649. doi: 10.1073/pnas.1017096108

Falk, R., Bleiseravivi, N., and Atidia, J. (1976). Labellar taste organs of Drosophila melanogaster. J. Morphol. 150, 327-341. doi: 10.1002/jmor.1051500206

Freeman, E. G., and Dahanukar, A. (2015). Molecular neurobiology of Drosophila taste. Curr. Opin. Neurobiol. 34, 140-148. doi: 10.1016/j.conb.2015.06.001

Galindo, K., and Smith, D. P. (2001). A large family of divergent Drosophila odorant-binding proteins expressed in gustatory and olfactory sensilla. Genetics 159, 1059-1072.

Gendre, N., Luer, K., Friche, S., Grillenzoni, N., Ramaekers, A., Technau, G. M., et al. (2004). Integration of complex larval chemosensory organs into the adult nervous system of Drosophila. Development 131, 83-92. doi: 10.1242/dev.00879

Gendron, C. M., Kuo, T. H., Harvanek, Z. M., Chung, B. Y., Yew, J. Y., Dierick, H. A., et al. (2014). Drosophila life span and physiology are modulated by sexual perception and reward. Science 343, 544-548. doi: 10.1126/science.1243339

Ghysen, A., and Dambly-Chaudiere, C. (2000). A genetic programme for neuronal connectivity. Trends Genet. 16, 221-226. doi: 10.1016/S0168-9525(99)01969-1

Houot, B., Gigot, V., Robichon, A., and Ferveur, J. F. (2017). Free flight odor tracking in Drosophila: effect of wing chemosensors, sex and pheromonal gene regulation. Sci. Rep. 7:40221. doi: 10.1038/srep40221

Hussain, A., Zhang, M., Ucpunar, H. K., Svensson, T., Quillery, E., Gompel, N., et al. (2016). Ionotropic chemosensory receptors mediate the taste and smell of polyamines. PLoS Biol. 14:e1002454. doi: 10.1371/journal.pbio.1002454

Jeong, Y. T., Shim, J., Oh, S. R., Yoon, H. I., Kim, C. H., Moon, S. J., et al. (2013). An odorant-binding protein required for suppression of sweet taste by bitter chemicals. Neuron 79, 725-737. doi: 10.1016/j.neuron.2013.06.025

Joseph, R. M., Devineni, A. V., King, I. F., and Heberlein, U. (2009). Oviposition preference for and positional avoidance of acetic acid provide a model for competing behavioral drives in Drosophila. Proc. Natl. Acad. Sci. U.S.A. 106, 11352-11357. doi: 10.1073/pnas.0901419106

Joseph, R. M., and Heberlein, U. (2012). Tissue-specific activation of a single gustatory receptor produces opposing behavioral responses in Drosophila. Genetics 192, 521-532. doi: 10.1534/genetics.112.142455

Joseph, R. M., Sun, J. S., Tam, E., and Carlson, J. R. (2017). A receptor and neuron that activate a circuit limiting sucrose consumption. eLife 6:e24992. doi: 10.7554/eLife.24992

Kang, K., Pulver, S. R., Panzano, V. C., Chang, E. C., Griffith, L. C., Theobald, D. L., et al. (2010). Analysis of Drosophila TRPA1 reveals an ancient origin for human chemical nociception. Nature 464, 597-600. doi: 10.1038/nature08848

Kim, H., Jeong, Y. T., Choi, M. S., Choi, J., Moon, S. J., and Kwon, J. Y. (2017). Involvement of a Gr2a-expressing Drosophila pharyngeal gustatory receptor neuron in regulation of aversion to high-salt foods. Mol. Cells 40, 331-338. doi: $10.14348 /$ molcells.2017.0028
Koganezawa, M., and Shimada, I. (2002). Novel odorant-binding proteins expressed in the taste tissue of the fly. Chem. Senses 27, 319-332. doi: 10.1093/ chemse/27.4.319

Koh, T. W., He, Z., Gorur-Shandilya, S., Menuz, K., Larter, N. K., Stewart, S., et al. (2014). The Drosophila IR20a clade of ionotropic receptors are candidate taste and pheromone receptors. Neuron 83, 850-865. doi: 10.1016/j.neuron.2014.07. 012

Kojima, T., Furuyama, A., Isono, K., Hamada, T., Ohsuga, K., and Takada, S. (2018). Effects of salt taste disorder on behavior and lifespan in Drosophila melanogaster. J. Oral Biosci. 60, 15-20. doi: 10.1016/j.job.2018.01.001

Krstic, D., Boll, W., and Noll, M. (2009). Sensory integration regulating male courtship behavior in Drosophila. PLoS One 4:e4457. doi: 10.1371/journal.pone. 0004457

LeDue, E. E., Chen, Y. C., Jung, A. Y., Dahanukar, A., and Gordon, M. D. (2015). Pharyngeal sense organs drive robust sugar consumption in Drosophila. Nat. Commun. 6:6667. doi: 10.1038/ncomms7667

Liman, E. R., Zhang, Y. V., and Montell, C. (2014). Peripheral coding of taste. Neuron 81, 984-1000. doi: 10.1016/j.neuron.2014.02.022

Lin, C. C., Prokop-Prigge, K. A., Preti, G., and Potter, C. J. (2015). Food odors trigger Drosophila males to deposit a pheromone that guides aggregation and female oviposition decisions. eLife 4:e08688. doi: 10.7554/eLife. 08688

Liu, C., Bai, X. B., Sun, J. H., Zhang, X. F., and Li, Y. (2015). Behavioral switch of food preference upon sugar deficiency is regulated by GPCRs in Drosophila. J. Genet. Genom. 42, 409-412. doi: 10.1016/j.jgg.2015.05.004

Liu, T., Starostina, E., Vijayan, V., and Pikielny, C. W. (2012). Two Drosophila $\mathrm{DEG} / \mathrm{ENaC}$ channel subunits have distinct functions in gustatory neurons that activate male courtship. J. Neurosci. 32, 11879-11889. doi: 10.1523/ JNEUROSCI.1376-12.2012

Lu, B., Lamora, A., Sun, Y., Welsh, M. J., and Ben-Shahar, Y. (2012). ppk23Dependent chemosensory functions contribute to courtship behavior in Drosophila melanogaster. PLoS Genet. 8:e1002587. doi: 10.1371/journal.pgen. 1002587

Lu, B., Zelle, K. M., Seltzer, R., Hefetz, A., and Ben-Shahar, Y. (2014). Feminization of pheromone-sensing neurons affects mating decisions in Drosophila males. Biol. Open 3, 152-160. doi: 10.1242/bio.20147369

Masek, P., and Keene, A. C. (2013). Drosophila fatty acid taste signals through the PLC pathway in sugar-sensing neurons. PLoS Genet. 9:e1003710. doi: 10.1371/ journal.pgen.1003710

Mellert, D. J., Knapp, J. M., Manoli, D. S., Meissner, G. W., and Baker, B. S. (2010). Midline crossing by gustatory receptor neuron axons is regulated by fruitless, doublesex and the Roundabout receptors. Development 137, 323-332. doi: 10.1242/dev.045047

Minocha, S., Boll, W., and Noll, M. (2017). Crucial roles of pox neuro in the developing ellipsoid body and antennal lobes of the Drosophila brain. PLoS One 12:e0176002. doi: 10.1371/journal.pone.0176002

Mitri, C., Soustelle, L., Framery, B., Bockaert, J., Parmentier, M. L., and Grau, Y. (2009). Plant insecticide L-canavanine repels Drosophila via the insect orphan GPCR DmX. PLoS Biol. 7:e1000147. doi: 10.1371/journal.pbio.1000147

Moon, S. J., Lee, Y., Jiao, Y., and Montell, C. (2009). A Drosophila gustatory receptor essential for aversive taste and inhibiting male-to-male courtship. Curr. Biol. 19, 1623-1627. doi: 10.1016/j.cub.2009.07.061

Murata, S., Brockmann, A., and Tanimura, T. (2017). Pharyngeal stimulation with sugar triggers local searching behavior in Drosophila. J. Exp. Biol. 220, 3231-3237. doi: 10.1242/jeb.161646

Park, J., Lee, J., Shim, J., Han, W., Lee, J., Bae, Y. C., et al. (2013). dTULP, the Drosophila melanogaster homolog of tubby, regulates transient receptor potential channel localization in cilia. PLoS Genet. 9:e1003814. doi: 10.1371/ journal.pgen.1003814

Raad, H., Ferveur, J. F., Ledger, N., Capovilla, M., and Robichon, A. (2016). Functional gustatory role of chemoreceptors in Drosophila wings. Cell Rep. 15, 1442-1454. doi: 10.1016/j.celrep.2016.04.040

Sanchez-Alcaniz, J. A., Zappia, G., Marion-Poll, F., and Benton, R. (2017). A mechanosensory receptor required for food texture detection in Drosophila. Nat. Commun. 8:14192. doi: 10.1038/ncomms 14192

Schneider, J., Dickinson, M. H., and Levine, J. D. (2012). Social structures depend on innate determinants and chemosensory processing in Drosophila. Proc. Natl. Acad. Sci. U.S.A. 109(Suppl. 2), 17174-17179. doi: 10.1073/pnas.1121252109 
Schneider, J., and Levine, J. D. (2014). Automated identification of social interaction criteria in Drosophila melanogaster. Biol. Lett. 10:20140749. doi: $10.1098 / \mathrm{rsbl} .2014 .0749$

Scott, K. (2018). Gustatory processing in Drosophila melanogaster. Annu. Rev. Entomol. 63, 15-30. doi: 10.1146/annurev-ento-020117-043331

Scott, K., Brady, R. Jr., Cravchik, A., Morozov, P., Rzhetsky, A., Zuker, C., et al. (2001). A chemosensory gene family encoding candidate gustatory and olfactory receptors in Drosophila. Cell 104, 661-673. doi: 10.1016/S00928674(01)00263-X

Shiraiwa, T., and Carlson, J. R. (2007). Proboscis extension response (PER) assay in Drosophila. J. Vis. Exp. 3:e193. doi: 10.3791/193

Soldano, A., Alpizar, Y. A., Boonen, B., Franco, L., Lopez-Requena, A., Liu, G., et al. (2016). Gustatory-mediated avoidance of bacterial lipopolysaccharides via TRPA1 activation in Drosophila. eLife 5:e13133. doi: 10.7554/eLife.13133

Sovik, E., Lamora, A., Seehra, G., Barron, A. B., Duncan, J. G., and Ben-Shahar, Y. (2017). Drosophila divalent metal ion transporter Malvolio is required in dopaminergic neurons for feeding decisions. Genes Brain Behav. 16, 506-514. doi: $10.1111 / \mathrm{gbb} .12375$

Starostina, E., Liu, T., Vijayan, V., Zheng, Z., Siwicki, K. K., and Pikielny, C. W. (2012). A Drosophila DEG/ENaC subunit functions specifically in gustatory neurons required for male courtship behavior. J. Neurosci. 32, 4665-4674. doi: 10.1523/JNEUROSCI.6178-11.2012

Steck, K., Walker, S. J., Itskov, P. M., Baltazar, C., Moreira, J. M., and Ribeiro, C. (2018). Internal amino acid state modulates yeast taste neurons to support protein homeostasis in Drosophila. eLife 7:e31625. doi: 10.7554/eLife.31625

Stocker, R. F. (1994). The organization of the chemosensory system in Drosophila melanogaster: a review. Cell Tissue Res. 275, 3-26. doi: 10.1007/BF00305372

Sun, F., Wang, Y., Zhou, Y., Van Swinderen, B., Gong, Z., and Liu, L. (2014). Identification of neurons responsible for feeding behavior in the Drosophila brain. Sci. Chin. Life Sci. 57, 391-402. doi: 10.1007/s11427-014-4641-2

Ueno, K., Ohta, M., Morita, H., Mikuni, Y., Nakajima, S., Yamamoto, K., et al. (2001). Trehalose sensitivity in Drosophila correlates with mutations in and expression of the gustatory receptor gene Gr5a. Curr. Biol. 11, 1451-1455. doi: 10.1016/S0960-9822(01)00450-X

Usui-Aoki, K., Matsumoto, K., Koganezawa, M., Kohatsu, S., Isono, K., Matsubayashi, H., et al. (2005). Targeted expression of Ip3 sponge and Ip3 dsRNA impaires sugar taste sensation in Drosophila. J. Neurogenet. 19, 123-141. doi: 10.1080/01677060600569713

Verschut, T. A., Carlsson, M. A., Anderson, P., and Hamback, P. A. (2017). Sensory mutations in Drosophila melanogaster influence associational effects between resources during oviposition. Sci. Rep. 7:9352. doi: 10.1038/s41598-017-09 728-7
Vijayan, V., Thistle, R., Liu, T., Starostina, E., and Pikielny, C. W. (2014). Drosophila pheromone-sensing neurons expressing the ppk25 ion channel subunit stimulate male courtship and female receptivity. PLoS Genet. 10:e1004238. doi: 10.1371/journal.pgen.1004238

Wang, K. Y., Guo, Y. M., Wang, F., and Wang, Z. R. (2011). Drosophila TRPA channel painless inhibits male-male courtship behavior through modulating olfactory sensation. PLoS One 6:e25890. doi: 10.1371/journal.pone.002 5890

Weiss, L. A., Dahanukar, A., Kwon, J. Y., Banerjee, D., and Carlson, J. R. (2011). The molecular and cellular basis of bitter taste in Drosophila. Neuron 69, 258-272. doi: 10.1016/j.neuron.2011.01.001

Yanagawa, A., Chabaud, M. A., Imai, T., and Marion-Poll, F. (2018). Olfactory cues play a significant role in removing fungus from the body surface of Drosophila melanogaster. J. Invertebr. Pathol. 151, 144-150. doi: 10.1016/j.jip.2017. 11.011

Yanagawa, A., Guigue, A. M., and Marion-Poll, F. (2014). Hygienic grooming is induced by contact chemicals in Drosophila melanogaster. Front. Behav. Neurosci. 8:254. doi: 10.3389/fnbeh.2014.00254

Yang, Z., Yu, Y., Zhang, V., Tian, Y., Qi, W., and Wang, L. (2015). Octopamine mediates starvation-induced hyperactivity in adult Drosophila. Proc. Natl. Acad. Sci. U.S.A. 112, 5219-5224. doi: 10.1073/pnas.1417838112

Yilmazer, Y. B., Koganezawa, M., Sato, K., Xu, J., and Yamamoto, D. (2016). Serotonergic neuronal death and concomitant serotonin deficiency curb copulation ability of Drosophila platonic mutants. Nat. Commun. 7:13792. doi: $10.1038 /$ ncomms13792

Zhang, Y. V., Aikin, T. J., Li, Z., and Montell, C. (2016). The basis of food texture sensation in Drosophila. Neuron 91, 863-877. doi: 10.1016/j.neuron.2016.07.013

Zhang, Y. V., Ni, J., and Montell, C. (2013). The molecular basis for attractive salt-taste coding in Drosophila. Science 340, 1334-1338. doi: 10.1126/science. 1234133

Conflict of Interest Statement: The authors declare that the research was conducted in the absence of any commercial or financial relationships that could be construed as a potential conflict of interest.

Copyright (C) 2018 Chen, Park, Ja and Dahanukar. This is an open-access article distributed under the terms of the Creative Commons Attribution License (CC BY). The use, distribution or reproduction in other forums is permitted, provided the original author(s) and the copyright owner(s) are credited and that the original publication in this journal is cited, in accordance with accepted academic practice. No use, distribution or reproduction is permitted which does not comply with these terms. 


\section{OPEN ACCESS}

Edited by:

Francesco Moccia,

University of Pavia, Italy

Reviewed by:

Nancy E. Rawson,

Monell Chemical Senses Center,

United States

Andre Fiala,

Georg-August-Universität Göttingen,

Germany

*Correspondence: Dieter Wicher

dwicher@ice.mpg.de

Received: 27 February 2018 Accepted: 11 June 2018

Published: 03 July 2018

Citation:

Halty-deLeon L, Hansson BS and Wicher D (2018) The Drosophila melanogaster $\mathrm{Na}^{+} / \mathrm{Ca}^{2+}$ Exchanger CALX Controls the $\mathrm{Ca}^{2+}$ Level in Olfactory Sensory Neurons at Rest and After Odorant Receptor Activation.

Front. Cell. Neurosci. 12:186. doi: 10.3389/fncel.2018.00186

\section{The Drosophila melanogaster $\mathrm{Na}^{+} / \mathrm{Ca}^{2+}$ Exchanger CALX Controls the $\mathrm{Ca}^{2+}$ Level in Olfactory Sensory Neurons at Rest and After Odorant Receptor Activation}

\author{
Lorena Halty-deLeon, Bill S. Hansson and Dieter Wicher* \\ Department of Evolutionary Neuroethology, Max Planck Institute for Chemical Ecology, Jena, Germany
}

CALX, the $\mathrm{Na}^{+} / \mathrm{Ca}^{2+}$ exchanger in Drosophila, is highly expressed in the outer dendrites of olfactory sensory neurons (OSNs) which are equipped with the odorant receptors (ORs). Insect OR/Orco dimers are nonselective cation channels that pass also calcium which leads to elevated calcium levels after OR activation. CALX exhibits an anomalous regulation in comparison to its homolog in mammals sodium/calcium exchanger, NCX: it is inhibited by increasing intracellular calcium concentration $\left[\mathrm{Ca}^{2+}\right]_{\mathrm{i}}$. Thus, CALX mediates only $\mathrm{Ca}^{2+}$ efflux, not influx. The main goal of this study was to elucidate a possible role of this protein in the olfactory response. We first asked whether already described NCX inhibitors were capable of blocking CALX. By means of calcium imaging techniques in ex-vivo preparations and heterologous expression systems, we determined ORM-10962 as a potent CALX inhibitor. CALX inhibition did not affect the odor response but it affected the recovery of the calcium level after this response. In addition, CALX controls the calcium level of OSNs at rest.

Keywords: insect olfaction, Drosophila melanogaster, $\mathrm{Na}^{+} / \mathrm{Ca}^{2+}$ exchanger, $\mathrm{CALX}$, Orco, calcium imaging

\section{INTRODUCTION}

Odorant receptor (ORs), a special class of olfactory receptors, have evolved in winged insects probably as a response to the challenge of detecting airborne odors (Missbach et al., 2014). These are composed by a specific ligand-binding subunit (OrX) and Orco, a highly conserved co-receptor protein (Larsson et al., 2004; Benton et al., 2006), which together form ligand-gated ion channels (Sato et al., 2008; Wicher et al., 2008). ORs are expressed in the dendrites of olfactory sensory neurons (OSNs) in the antennae and maxillary palps (Joseph and Carlson, 2015). Stimulation of ORs leads to ions fluxes across the OSN plasma membrane, including an influx of the ubiquitary second messenger $\mathrm{Ca}^{2+}$. Taking into account that the $\mathrm{Ca}^{2+}$ concentration outside the cells is around 10,000-fold higher than in the interior (Guerini et al., 2005) it is of vital importance to tightly regulate the free intracellular $\mathrm{Ca}^{2+}$ concentration $\left[\mathrm{Ca}^{2+}\right]_{\mathrm{i}}$. Thus, during and after agonist stimulation, the $\mathrm{Ca}^{2+}$ influx and efflux must be balanced in order to restore basal calcium levels.

There are two main $\mathrm{Ca}^{2+}$ extrusion systems working in parallel to maintain $\mathrm{Ca}^{2+}$ homeostasis (Guerini et al., 2005). These are the plasma membrane $\mathrm{Ca}^{2+}$-ATPase (PMCA) and the sodium/calcium exchanger (NCX). ATP-driven calcium pumps bind $\mathrm{Ca}^{2+}$ with high 
affinity but display a low turnover rate, NCX extrudes $\mathrm{Ca}^{2+}$ with a >10-fold higher turnover rate compared to PMCA pumps (Blaustein and Lederer, 1999). Consequently, at elevated intracellular calcium concentration $\left(\left[\mathrm{Ca}^{2+}\right]_{\mathrm{i}}\right)$, e.g., during an action potential, the NCX is likely to be more efficient in extruding calcium than the PMCA.

In mammals, NCX acts as a bidirectional transporter. In its efflux (forward) mode, it exports one $\mathrm{Ca}^{2+}$ ion for the uptake of three $\mathrm{Na}^{+}$first described in Nicoll et al. (1990). During pathological conditions or after agonist receptor stimulation and membrane depolarization, the reversed mode is triggered resulting in the uptake of $\mathrm{Ca}^{2+}$ and extrusion of $\mathrm{Na}^{+}$(Blaustein and Lederer, 1999; Guerini et al., 2005; Lytton, 2007). Interestingly CALX, the NCX homolog in Drosophila, exhibits an anomalous regulation in that it is inhibited by increasing $\left[\mathrm{Ca}^{2+}\right]_{\mathrm{i}}$ (Hryshko et al., 1996). CALX shares $52 \%$ homology and a conserved structure with NCX. It is formed by 10 transmembrane helices with two intracellular calcium binding domains, CBD1 and CBD2 (Schwarz and Benzer, 1997). In contrast to NCX, CBD1 in CALX is the only calcium binding domain (Wu et al., 2010). Conformational changes as a result of $\mathrm{Ca}^{2+}$ binding to CBD1 are likely to be responsible for the $\mathrm{Ca}^{2+}$ inhibition of CALX (Wu et al., 2011). The physiological significance of this differential response to cytosolic $\mathrm{Ca}^{2+}$ remains to be elucidated. Since CALX is active only in its forward mode, little attention has been paid to its possible role in the sensory response even though it is the major $\mathrm{Ca}^{2+}$ extrusion system in Drosophila sensory neurons (Zheng et al., 2013). However, in Drosophila photoreceptor cells, previous studies have shown a role of CALX in amplification, adaptation and termination of the visual response (Wang et al., 2005; Wang and Montell, 2007). Its effect in other sensory modalities remains to be elucidated.

Due to the bimodal function of NCX, an inhibitor of the reversed mode could protect against $\mathrm{Ca}^{2+}$ overload in a situation of ischemia/reperfusion injury (Iwamoto, 2004). As a consequence, NCX inhibitors have attracted attention as potential $\mathrm{Ca}^{2+}$ regulators. The first NCX inhibitor described to block the reversed mode was KB-R973 (Iwamoto et al., 1996). Later on, SEA 0400 was developed as a more selective inhibitor by Matsuda et al. (2001). However, both compounds were seen to be not completely NCX-specific (Reuter et al., 2002). Recently, ORM-10962, a new selective inhibitor of the forward and reversed mode of NCX has been described (Kohajda et al., 2016).

Intracellular $\mathrm{Ca}^{2+}$ signaling modulates the signal amplification (Ignatious Raja et al., 2014) and the response profile (Fluegge et al., 2012) of the olfactory response. Bobkov et al. (2014) showed that the NCX inhibitor, KB-R7943, blocks odor-evoked activation in mosquito ORs expressed in heterologous expression system. Their results suggested that Orco could be a target for the drug action, raising the question of whether or not ORs could be linked directly or indirectly to a $\mathrm{Na}^{+} / \mathrm{Ca}^{2+}$ exchanger. Therefore, in the present study we asked whether CALX, as the major $\mathrm{Ca}^{2+}$ extrusion mechanism, could have a role in the odor response of OSNs in Drosophila melanogaster. We stimulated the OR expressing OSNs with the synthetic Orco agonist VUAA1 (Jones et al., 2011). In addition, it was crucial to use a selective CALX inhibitor. Using calcium imaging in ex-vivo preparations of fly antennae and a heterologous expression system, we tested three candidates for CALX inhibition: KB-R7943, SEA 0400 and ORM-10962. Among these, ORM-10962 was identified as a potent CALX inhibitor. Furthermore, we confirmed that CALX acts as the primary $\mathrm{Ca}^{2+}$ extrusion mechanism in D. melanogaster OSNs. Its major contribution to the odor response is restoring the basal calcium level after stimulation, with no significant further role in modulating the response.

\section{MATERIALS AND METHODS}

\section{Cell Culture and Transfection}

Drosophila melanogaster Orco was cloned into pcDNA3.1(-) expression vector as previously described (Mukunda et al., 2014). HEK cells (DSMZ no. ACC 305) were purchased from the Leibniz Institute DSMZ GmbH (Braunschweig, Germany) and grown in DMEM/F12 1:1 medium (Gibco, Life Technologies, Grand Island, NY, USA) supplied with $10 \%$ Fetal Bovine Serum at $37^{\circ} \mathrm{C}$ and $5 \% \mathrm{CO}_{2}$. HEK293 cells were electroporated with $1.6 \mu \mathrm{g}$ Or83b-pcDNA3.1(-) using an Amaxa 4D-Nucleofector (Lonza GmbH, Cologne, Germany) with the SF Cell Line 4D-Nucleoefector X Kit (Lonza GmbH, Cologne, Germany). After electroporation, cells were cultured on poly-L-lysine (0.01\%, Sigma-Aldrich, Steinheim, Germany) coated coverslips at a density of $\sim 3 \times 10^{5}$ cells per well ( 24 well plates). For experiments cells were exposed to normal bath solution (in $\mathrm{mM}$ : $\mathrm{NaCl}, 135 ; \mathrm{KCl}, 5 ; \mathrm{MgCl}_{2}, 1 ; \mathrm{CaCl}_{2}$, 1; HEPES, 10; d-glucose, 10; $\mathrm{pH}=7.4$; osmolarity $295 \mathrm{mOsmol} / \mathrm{l})$.

\section{Fly Rearing and Antennal Preparation}

Drosophila melanogaster flies with genotype w;UASGCaMP6f/Cyo;Orco-Gal4/TM6B were reared under a $12 \mathrm{~h}$ light: $12 \mathrm{~h}$ dark cycle at $25^{\circ} \mathrm{C}$ on conventional agar medium. For experiments, antennae of 4-8 days old females were excised and prepared as described in Mukunda et al. (2014). Briefly, flies were anesthetized on ice. Antennae were excised and fixed in vertical position with a two-component silicone and immersed in Drosophila Ringer solution (in mM: HEPES, 5; $\mathrm{NaCl}, 130$; $\mathrm{KCl}, 5 ; \mathrm{MgCl}_{2}, 2 ; \mathrm{CaCl}_{2}, 2$; and sucrose, $36 ; \mathrm{pH}=7.3$ ) or $\mathrm{Na}^{+}$ free Ringer solution (in mM: HEPES, 5; N-Methyl-D-glucamine (NMDG), 130; $\mathrm{HCl}, 10 ; \mathrm{KCl}, 5 ; \mathrm{MgCl}_{2} \cdot 6 \mathrm{H}_{2} \mathrm{O}, 2 ; \mathrm{Ca}, 2$; and sucrose, $30 ; \mathrm{pH}=7.3$. Thereafter the funiculus was cut allowing access to the OSNs for experiments. Antennae were immersed in solution during the experiments.

\section{Calcium Imaging}

Imaging was performed employing a monochromator (Polychrome V, Till Photonics, Munich, Germany), coupled to an epifluorescence microscope (Axioskop FS, Zeiss, Jena, Germany). A water immersion objective (LUMPFL $40 \times$ W/IR/0.8; Olympus, Hamburg, Germany) was used controlled by an imaging control unit (ICU, Till Photonics). Fluorescence images were acquired using a cooled CCD camera controlled by TILLVision 4.5 software (TILL Photonics). Experiments lasted 
20 min with a sampling interval of 5 s. One-hundred microliter of the different chemicals were applied via pipette in proximity of the objective. VUAA1 was applied at a concentration of $25 \mu \mathrm{M}$, KB-R7943 at $100 \mu \mathrm{M}$, SEA 0400 at $0.1 \mu \mathrm{M}$ and ORM-10962 at $1 \mu \mathrm{M}$. Samples were continuously perfused with bath solution in the perfusion/recording chamber (RC-27, Warner Instruments Inc., Hamden, CT, USA) during the experiments. TillVision software (Version 4.5, Till Photonics) was used to subtract background fluorescence and to define regions of interest (ROI) characterized by a change in the $\left[\mathrm{Ca}^{2+}\right]_{\mathrm{i}}$. Imaging experiments of cells were conducted $24 \mathrm{~h}$ after electroporation. Cells were incubated in Opti-MEM medium (Gibco) and loaded with $5 \mu \mathrm{M}$ Fura-2 acetoxymethyl ester (Molecular Probes, Invitrogen) for $30 \mathrm{~min}$ at room temperature. After wash, cells were kept during the experiment in bath solution. Free intracellular $\mathrm{Ca}^{2+}$ concentration $\left(\left[\mathrm{Ca}^{2+}\right]_{\mathrm{i}}\right)$ was calculated according to $\left[\mathrm{Ca}^{2+}\right]_{\mathrm{i}}=\mathrm{K}_{\mathrm{eff}}\left(\mathrm{R}-\mathrm{R}_{\min }\right) /\left(\mathrm{R}_{\max }-\mathrm{R}\right)$, where $\mathrm{R}_{\min }$ and $\mathrm{R}_{\max }$ were determined as described in Mukunda et al. (2014). Emitted light was separated by a $400 \mathrm{~nm}$ dichroic mirror and filtered with a $420 \mathrm{~nm}$ long-pass filter. Image pairs were obtained by excitation at $340 \mathrm{~nm}$ and $380 \mathrm{~nm}$ for $150 \mathrm{~ms}$, background fluorescence was subtracted. The final resolution was $640 \times 480$ pixel in a frame of $175 \mu \mathrm{m} \times 130 \mu \mathrm{m}$. In antenna preparations, GCaMP6f was exited with $475 \mathrm{~nm}$ light at $0.2 \mathrm{~Hz}$ frequency with an exposition time of $50 \mathrm{~ms}$. Emitted light was separated by a $490 \mathrm{~nm}$ dichroic mirror and filtered with a $515 \mathrm{~nm}$ long-pass filter. The response magnitude was calculated as the average $\Delta F / F_{0}$ in percentage following Mukunda et al. (2014).

\section{Chemicals}

VUAA1 (N-(4-ethylphenyl)-2-((4-ethyl-5-(3-pyridinyl)-4H-1,2, 4-triazol-3-yl)thio)acetamide) was synthesized by the group "Mass Spectrometry/Proteomics" of the Max-Planck Institute for Chemical Ecology (Jena, Germany). KB-R7943 (2-[4-[(4-nitrophenyl)methoxy]phenyl]ethyl ester carbamimidothiotic acid, monomethanesulfonate) from Cayman Chemical (Ann Arbor, MI, USA). SEA-0400 (2-[4-[(2,5-difluorophenyl)methoxy]phenoxy]-5-ethoxyaniline) was purchased from ApexBio Tech LLC (Houston, TX, USA). ORM-10962 ([2-(4-hydroxy-piperidin-1-yl)-N-(6-((2phenylchroman-6yl)oxy)pyridin-3-yl)acetamide]) was kindly provided by Orion Corporation (Orion Pharma, Espoo, Finland). All chemicals were dissolved in DMSO to yield a stock solution. When used as control DMSO was dissolved 1:1000.

\section{Immunolocalization}

\section{Antenna}

Female flies between 4 days and 8 days old were collected. First, the head was removed and prefixed in $4 \%$ PFA (paraformaldehyde) $+0.1 \%$ Triton for $10 \mathrm{~min}$ on ice. For preparation of the antenna, the 3rd segment was excised and fixed with $4 \%$ PFA (paraformaldehyde) $+0.1 \%$ Triton for $2 \mathrm{~h}$ on ice. Antenna were washed with phosphate-buffered saline solution containing $0.1 \%$ Triton X-100 (PBST) for $3 \times 20 \mathrm{~min}$. Antennae were blocked in normal goat serum (NGS) in PBST (PBST-NGS) for $60 \mathrm{~min}$ at room temperature and then incubated with the primary antibodies in PBST-NGS for 2 days at $4^{\circ} \mathrm{C}$. After $4 \times 15$ min wash at room temperature, antennae were incubated with secondary antibodies in PBST-NGS for 2 days at $4^{\circ} \mathrm{C}$. Finally, antennae were washed again for $3 \times 20 \mathrm{~min}$ and mounted in Vectashield (Vector, Burlingame, CA, USA). Primary antibodies used: mouse anti-GFP (A-11001, Invitrogen, Carlsbad, CA, USA), rabbit anti-CALX (provided by Dr. Craig Montell, University of California, USA). Secondary antibodies used: goat anti mouse Alexa 488- and goat anti rabbit Alexa 546 (A-11120, Invitrogen, Carlsbad, CA, USA).

\section{Eyes}

We prepared female flies (4 and 8 days old) according to Hsiao et al. (2012). Briefly, flies were anesthetized on ice, the head was removed and the retinas were dissected and fixed with 4\% PFA (paraformaldehyde) for $15 \mathrm{~min}$ at room temperature. Afterwards, samples were washed with $1 \times$ phosphate-buffered saline solution (PBS) for $1 \mathrm{~h}$. Retinas were blocked in 5\% NGS in PBST for $20 \mathrm{~min}$ at room temperature followed by the incubation with the primary antibody overnight. After $3 \times 15$ min wash at with PBST, retinas were incubated with secondary antibodies in PBST-NGS overnight at room temperature. At last, retinas were washed with PBST for $1 \mathrm{~h}$ and mounted in Vectashield (Vector, Burlingame, CA, USA).

\section{Confocal Microscope}

Images were acquired on a cLSM 880 (Carl Zeiss, Oberkochen, Germany) using a $40 \times$ water immersion objective (C-Apochromat, NA: 1.2, Carl Zeiss). Images were obtained at $0.10-0.10 \mu \mathrm{m}$ intervals at $1592 \times 1592$ pixel resolution for the antennae overview and at $0.12-0.12 \mu \mathrm{m}$ intervals at $512 \times 512$ pixel resolution for the detailed section. Confocal images were adjusted for contrast and brightness by using LSM Image Browser 4.0 (Carl Zeiss) and Adobe Photoshop CS.

\section{Data Analysis}

Statistical analyses were performed using Prism 4 (GraphPad Software Inc., La Jolla, CA, USA). Data are given as mean \pm SEM (standard error of the mean) and were analyzed using Paired $t$-test or Unpaired $t$-test. The decay was calculated and normalized in percentage during washing periods. Each washing period entails $150 \mathrm{~s}$.

\section{RESULTS}

\section{Localization of CALX in OSN}

As ORs are $\mathrm{Ca}^{2+}$-permeable, any receptor activation causes a $\mathrm{Ca}^{2+}$ influx. To ensure a fast and reliable $\mathrm{Ca}^{2+}$ handling after OR activation, we expected a high expression of CALX in the outer dendrites of OSNs, where ORs are expressed. To demonstrate this, we performed CALX immunostaining in the Drosophila antenna. Figure $\mathbf{1}$ shows that CALX indeed is mainly expressed in the outer dendrites whereas it is almost absent in the somata. To control for the specificity of anti-CALX we performed an immunostaining in the rhabdomeres of Drosophila eyes. The immunolocalization of CALX in photoreceptors cells had been previously shown by Wang et al. (2005), and our results 

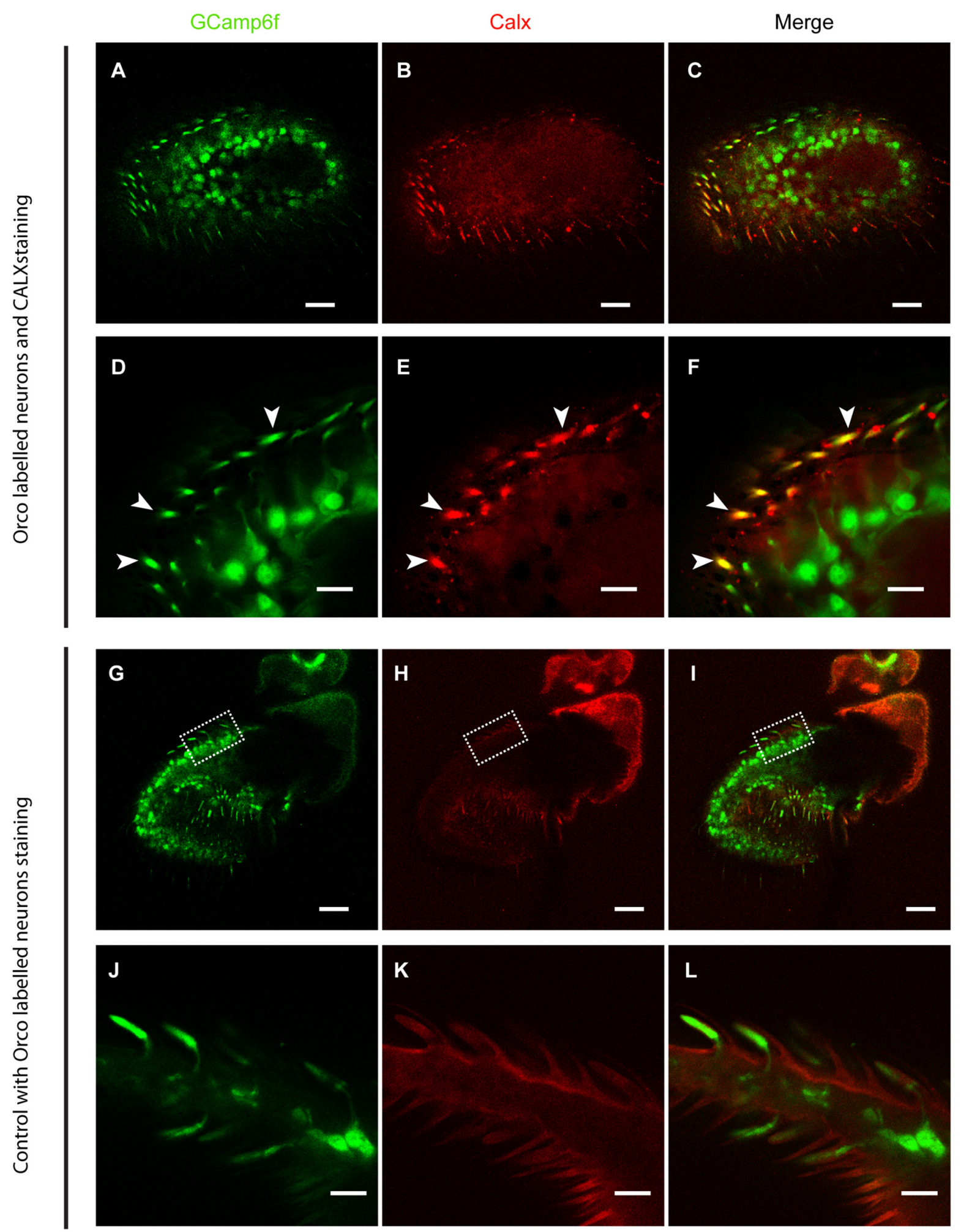

FIGURE 1 | CALX is highly expressed in the outer dendrites of olfactory sensory neurons (OSNs). Superior panels: (A,D) GCaMP6f staining with Alexa 488. (B,E) CALX staining with Alexa 546. (C,F) Merged stainings. (A-C) General overview of female Drosophila antennae. Bars, $20 \mu \mathrm{m}$. (D-F) Details of a section at higher magnification, Bars, $5 \mu \mathrm{m}$. White arrows indicate localization of CALX in the dendrites. Lower panels: (G,J) GCaMP6f staining with Alexa 488. (H,K) Control staining with only Alexa 546. (I,L) Merged stainings. (G-I) General overview of female Drosophila antennae. Bars, 20 m. (J-L) Details of a section at higher magnification, Bars, $5 \mu \mathrm{m}$. 
are in agreement with their findings (for detail see Figure 1E in Wang et al., 2005 and Supplementary Figure S1 in this publication).

\section{KB-R7943 Attenuates Receptor Activation in Drosophila melanogaster OSNs}

In order to elucidate a possible role of CALX in the odor response, it was first necessary to determine a selective inhibitor that did not affect OR function. To determine if the previously described NCX inhibitors may act on ORs, we performed experiments using an ex-vivo preparation of Drosophila antennae. Two applications of the Orco agonist VUAA1 at $25 \mu \mathrm{M}$, one as control and one in the presence of the different compounds, were applied during the experiments. As a negative control, we performed the experiments only with applications of VUAA1. In control experiments there was no significant difference between the calcium fluorescence intensity of the first response $(155 \pm 22)$ compared to that of the second response (133.6 \pm 28.7 ; Figures 2A,B; Paired $t$-test, ns $>0.05 ; n=8)$. Our first candidate for CALX inhibition, KB-R7943, was already reported to block activation of ORs in the mosquito, and our results further supported this finding in Drosophila OSNs. The intensity of the response decreased from $114.2 \pm 16.3$ to $25.51 \pm 9$ after VUAA1 $25 \mu \mathrm{M}$ in the presence of KB-R7943 (100 $\mu \mathrm{M}$; Figures 2C,D; Paired $t$-test, ${ }^{* *} p<0.01$; $n=7)$.

To confirm the blocking of Orco by KB-R7943, we then performed experiments in heterologous expression system. Drosophila Orco was expressed in Human Embryonic Kidney cells (HEK293) and the change in $\left[\mathrm{Ca}^{2+}\right]_{i}$ was monitored with fura 2. The average increase in $\left[\mathrm{Ca}^{2+}\right]_{\mathrm{i}}$ decreased from $418.66 \pm 47$ to $146.67 \pm 13.46 \mathrm{nM}$ under VUAA1 $100 \mu \mathrm{M}$ conditions (Figures 3A,B; Paired $t$-test ${ }^{* * *} p<0.001 ; n=6$ ). To exclude the possibility of Orco adaptation after a robust first response at VUAA1 $100 \mu \mathrm{M}$, we repeated the experiments using a moderate stimulation of VUAA1 $50 \mu \mathrm{M}$. Under this condition, the average increase in $\left[\mathrm{Ca}^{2+}\right]_{\mathrm{i}}$ also decreased significantly from $214.02 \pm 16.94$ to $135.03 \pm 4.11 \mathrm{nM}$ (Figures 3C,D; Paired $t$-test; ${ }^{*} p<0.05 ; n=5$ ). Hereby we confirmed that KB-R7943 is blocking Orco and therefore is not suitable to investigate a possible role of CALX in the odor response.

Our second candidate, SEA 0400, is a benzyloxyphenyl derivative, as KB-R7943, but was reported to be a much more potent NCX inhibitor (Matsuda et al., 2001). Due to the similarity to KB-R7943, we asked whether SEA 0400 could also block insect ORs. Wu et al. (2008) demonstrated that at a high concentration of $10 \mu \mathrm{M}$ SEA 0400 was able to block the reverse mode of NCX in cultured rat embryonic cortical neurons. Therefore, we challenged Drosophila OSNs with SEA 0400 at $10 \mu \mathrm{M}$ to test for a negative effect on Orco. In presence of SEA $0400(10 \mu \mathrm{M})$, there was no significant difference between the intensity of the first response $(140.6 \pm 22.9)$ and the intensity of the second (98.34 \pm 18.78; Figures 2E,F; Paired $t$-test, ns $>0.05 ; n=7$ ). Hence, SEA 0400 has no blocking effect on ORs and is a putative candidate for investigating the role of CALX in the odor response.
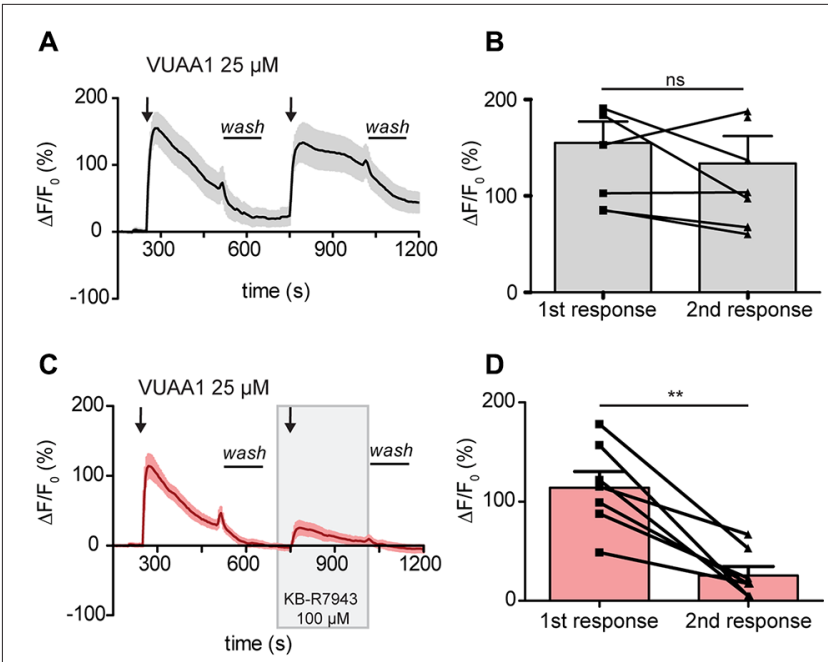

E
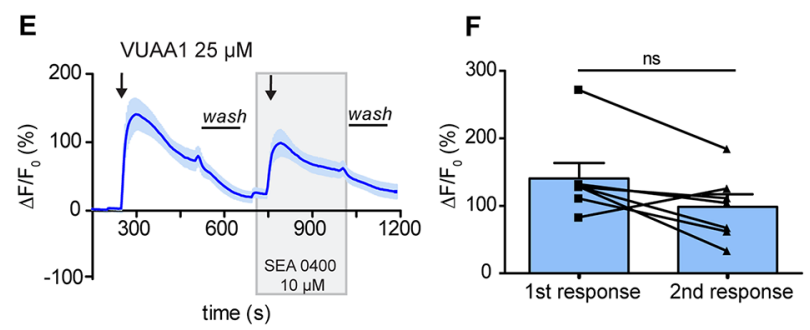

G
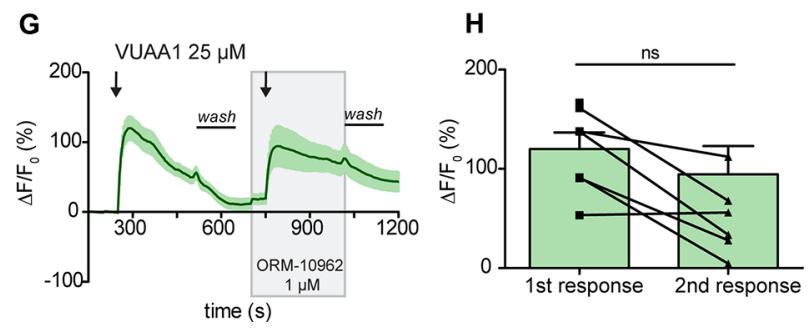

FIGURE 2 | Activation of Drosophila melanogaster odorant receptors (ORs) is attenuated by KB-R7943. Left panels: averaged time course of the change in fluorescence intensity $\Delta F / F_{0}$ in Drosophila OSNs upon two stimulations with VUAA1 under control conditions $(\mathbf{A}, n=8)$ and in the presence of KB-R7943 (C, $n=7)$, SEA $0400(\mathbf{E}, n=7)$ and ORM-10962 (G, $n=7)$. Black arrows indicate application of $100 \mu \mathrm{l}$ of VUAA1. Frames indicate the presence of sodium/calcium exchanger (NCX) inhibitors. Wash indicates the period of washing with Ringer solution between stimulations. Right panels (B,D,F,H): maximum increase in $\Delta F / F_{0}$ after VUAA1 applications as in left panels. Data represent mean \pm SEM, Paired $t$-test, ns, not significant, ${ }^{* *} p<0.01$.

We then focused our attention in the last and most recently described NCX inhibitor: ORM-10962. This compound has been reported to be a new selective inhibitor of NCX in its reverse and forward mode at $1 \mu \mathrm{M}$ (Kohajda et al., 2016). This fact made it particularly interesting since CALX is only functional in a forward mode. Our results show that this compound did not affect OR function. There was no significant difference in the OSNs response between control conditions (120 \pm 16.6$)$ and in the presence of ORM-10962 (94.62 \pm 28.42 ; Figures 2G,H; Paired $t$-test; ns, $p>0.05 ; n=7)$.

The lack of effect of SEA 0400 and ORM-10962 in the maximum of the odor response could indicate that: (i) these 

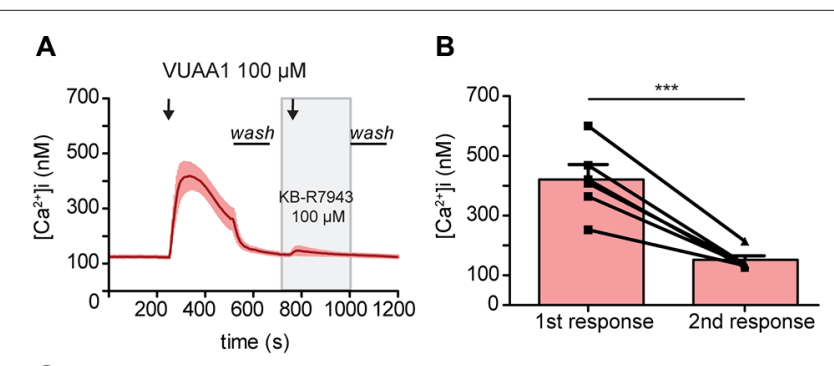

C

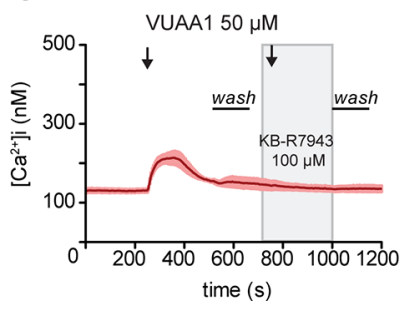

D

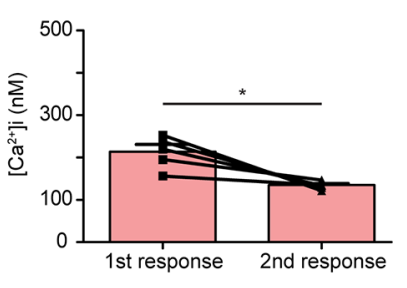

FIGURE 3 | Activation of the odorant co-receptor Orco expressed in HEK293 cells is attenuated by KB-R7943. (A,C) Averaged time course of $\left[\mathrm{Ca}^{2+}\right]_{i}$ upon stimulation with VUAA1 at different concentrations. $(\mathbf{A}, n=6$; $\mathbf{C}, n=5)$. (B,D) Maximum increase in $\left[\mathrm{Ca}^{2+}\right]_{\mathrm{i}}$ as in $\mathbf{( A , C )}$. Data represent mean \pm SEM, Paired $t$-test, ${ }^{*} p<0.05,{ }^{* * *} p<0.001$.

compounds do not act on CALX; or (ii) CALX does not affect the maximum of the response. However, as shown in Figures 2E,G, inhibition of CALX seems to affect the recovery of the $\mathrm{Ca}^{2+}$ level after washing off the OR ligand.

\section{CALX Shapes the Decay of the $\mathrm{Ca}^{2+}$ Level After an Odor Response}

We thus focused our attention on the decay of the response. To evaluate the contribution of CALX to $\mathrm{Ca}^{2+}$ level recovery, the decay of the response was calculated and normalized in percentage during washing periods (see Figure 2 for washing period). There was no significant difference between the decay after the first and the second response upon application of VUAA1 $(25 \mu \mathrm{M})$ nor in the presence of SEA $0400(10 \mu \mathrm{M}$; Figure 4A; Paired $t$-test; $n s, p>0.05 ; n_{(V U A A 1)}=8$; Figure 4B; Paired $t$-test; ns, $p>0.05 ; n_{\text {(SEA) }}=8$ ). However, we observed a significantly lower decay, $45 \%$ compared to $74 \%$ under control conditions, after application of ORM-10962 (Figure 4C, Paired $t$-test; $\left.{ }^{* *} p<0.05 ; n_{(\mathrm{ORM})}=7\right)$. To further test the influence of CALX in the decay of the $\mathrm{Ca}^{2+}$ levels, we performed the control experiments under $\mathrm{Na}^{+}$free conditions (Figure 4D). Under the assumption that CALX function is impaired in the absence of $\mathrm{Na}^{+}$, we expected a reduced decay compared to the control experiment in normal conditions. This was indeed the case. The first (29\%) and the second (29\%) decay in $\mathrm{Na}^{+}$ free conditions (Figure 4E) are significantly lower compared to the first $(71 \%)$ and the second (58\%) decay under normal conditions ( $t$-test 1 st decay; ${ }^{*} p<0.05$; $t$-test 2 nd decay; ${ }^{*} p<0.05$; $\left.n_{\text {(VUAAl })}=8, n_{\left(\mathrm{VUAA}_{\mathrm{Na}}+\text { free }\right.}=8\right)$ ). Furthermore, the decay in $\mathrm{Na}^{+}$free conditions is comparable to the decay in presence of ORM ( $t$-test; ns, $p>0.05$; ns, not significant). These results point out that CALX plays a major role as a calcium extrusion

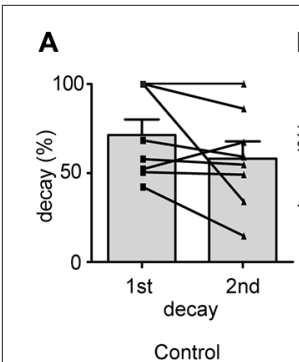

D

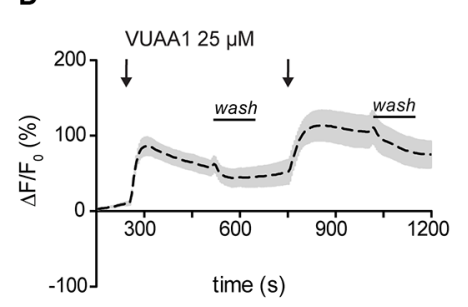

C

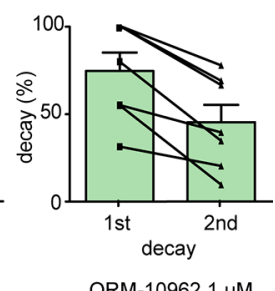

E

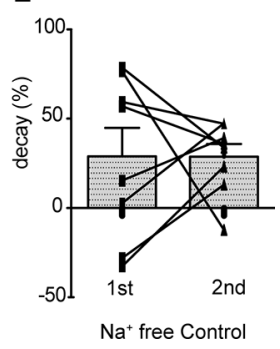

FIGURE 4 | CALX function shapes the recovery of $\mathrm{Ca}^{2+}$ level after OR stimulation. Normalized decay in percentage during washing periods in the presence of VUAA1 under control conditions (A), in presence of SEA0400 (B) and ORM-10962 (C). In (D) averaged time course of the change in fluorescence intensity $\Delta F / F_{0}$ in Drosophila OSNs upon two stimulations with VUAA1 under $\mathrm{Na}^{+}$free conditions $(n=8)$ and the correspondent normalized decay $(\mathbf{E})$. Data represent mean \pm SEM.

mechanism and that it is involved in restoring $\mathrm{Ca}^{2+}$ levels after an odor response. It also provides a strong hint about the effectiveness of ORM-10962 as CALX inhibitor.

\section{ORM-10962 as CALX Inhibitor}

To confirm ORM-10962 as a putative CALX inhibition, we performed experiments under resting conditions in the antenna preparation. In case of blocking the main $\mathrm{Ca}^{2+}$ extrusion mechanism, we would expect an increase in the $\left[\mathrm{Ca}^{2+}\right]_{\mathrm{i}}$ over time. For analysis, we compared the calcium levels at the end of the recording (900 s) between treatments and control (DMSO 0.1\%; Figure 5A). In the presence of SEA 0400 there was no significant increase in the $\left[\mathrm{Ca}^{2+}\right]_{\mathrm{i}}$ compared to control conditions $\left(t\right.$-test ns, $p>0.05 ; n_{(\mathrm{DMSO})}=9, n_{(\mathrm{SEA})}=9$; Figure 5B). We hereby conclude that even though SEA 0400 has no negative effect on Orco channels it does not inhibit CALX. On the other hand, when ORM-10962 was applied, we observed a significant increase in $\left[\mathrm{Ca}^{2+}\right]_{\mathrm{i}}$ at $900 \mathrm{~s}(44.9 \pm 8.8)$ compared to control conditions $\left(7.8 \pm 5.9\right.$; Figure $5 \mathrm{C}$; $t$-test; ${ }^{* *} p<0.01$, $\left.n_{\text {(DMSO) }}=9, n_{\text {(ORM) }}=8\right)$. When we performed the control experiments under $\mathrm{Na}^{+}$free conditions we observed an increase in the $\left[\mathrm{Ca}^{2+}\right]_{\mathrm{i}}$ over time (Figure 5D). This is in line with the assumption that under $\mathrm{Na}^{+}$free conditions there is an impairment of CALX and therefore an accumulation of calcium is expected. Indeed, at $900 \mathrm{~s}$ we observed no significant difference between control in $\mathrm{Na}^{+}$free $(45.7 \pm 13.3)$ and in the presence of ORM $1 \mu \mathrm{M}(44.9 \pm 8.8)$, $t$-test; $p>0.05$; ns, not significant, $\left.\left.n_{\left(\mathrm{DMSO}_{\mathrm{Na}}+\text { free }\right.}\right)=7, n_{(\mathrm{ORM})}=8\right)$. However, there is a significant difference between the quantified $\left[\mathrm{Ca}^{2+}\right]_{\mathrm{i}}$ between control and 


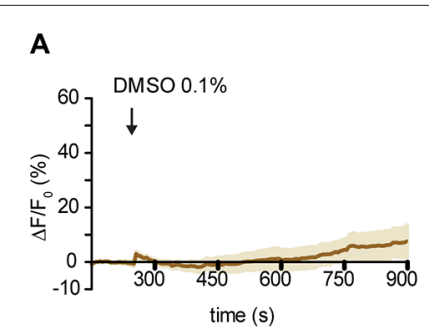

B

C
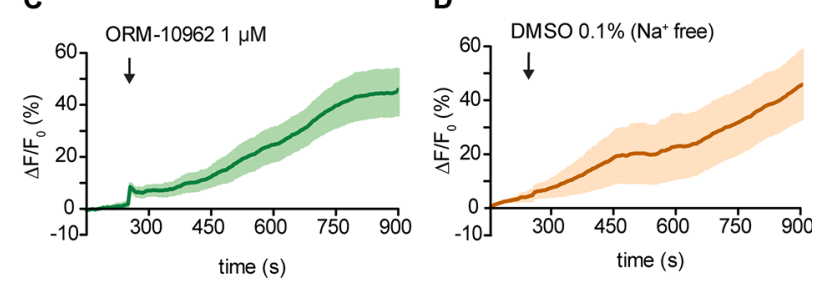

FIGURE 5 | Effect of NCX inhibitors on resting $\mathrm{Ca}^{2+}$ fluorescence in OSNs. (A-D) Averaged fluorescence intensity over time in Drosophila OSNs after application of the chemicals (black arrows) under resting conditions. Control application of DMSO solution at 1\% in Drosophila ringer (A) and in $\mathrm{Na}^{+}$free ringer (D). In (B) and (C) time course of the response to the NCX inhibitor SEA 0400 and ORM 10962 respectively. $\left.n_{(\mathrm{DMSO})}=9, n_{\left(\mathrm{DMSO}_{\mathrm{Na}}+\text { free }\right.}\right)=6$, $n_{(\mathrm{SEA})}=9, n_{(\mathrm{ORM})}=8$.

$\mathrm{Na}^{+}$free conditions at $900 \mathrm{~s}$ ( $t$-test with Welch's correction, $\left.\left.{ }^{*} p<0.05, n_{\left(\mathrm{DMSO}_{\mathrm{Na}}+\text { free }\right.}\right)=7, n_{(\mathrm{DMSO})}=9\right)$.

Taken together, our results point out that ORM-10962 is capable of blocking the Drosophila $\mathrm{Na}^{+} / \mathrm{Ca}^{2+}$ exchanger CALX without side effects on the co-receptor Orco and that CALX seems to play no role in the modulation of the odor response. However, according to our $\mathrm{Na}^{+}$free and resting conditions experiments, CALX controls the $\mathrm{Ca}^{2+}$ level of OSNs at rest and is involved in shaping the recovery after odor stimulation.

\section{DISCUSSION}

Calcium entry following receptor activation in OSNs needs to be balanced to restore resting calcium levels in preparation for new stimuli. Calcium can be taken up by intracellular stores such as mitochondria and endoplasmic reticulum or extruded from the cell by $\mathrm{Ca}^{2+}$ pumps or exchangers. Sensory cascades operating through rapid $\mathrm{Ca}^{2+}$-mediated signaling seem to rely on $\mathrm{Na}^{+} / \mathrm{Ca}^{2+}$ exchange mechanisms. For example, Drosophila photoreceptor cells are very sensitive to perturbations in the $\mathrm{Na}^{+} / \mathrm{Ca}^{2+}$ exchange activity mediated by CALX (Wang et al., 2005). Furthermore, NCX was reported to be responsible for returning the concentration of intracellular $\mathrm{Ca}^{2+}$ to its basal level after odor stimulation in frog olfactory neurons (Reisert and Matthews, 1998). However, the involvement of CALX in the Drosophila odor response was so far unknown. The aim of the present study was to investigate this process.

Our immunohistochemistry results are in good agreement with previous studies where NCX was observed to be expressed in olfactory cilia (Noé et al., 1997; Danaceau and Lucero, 2000; Castillo et al., 2007) and dendrites (Jung et al., 1994). By measuring the change in calcium within the different neuronal compartments in Xenopus, Jung et al. (1994) observed an increase in calcium first in the dendritic compartments, whereas the increase in the soma and dendritic knob was delayed and less pronounced. Due to the fact that ATPase has a lower transport capacity for calcium than CALX, it seems plausible that CALX in the dendrites would be as a sink for calcium under conditions of elevated intracellular calcium concentration, such as after a receptor activation event, transporting calcium from the dendritic cytosol into the sensillum lymph.

In mammals, NCX is particularly important in cardiac myocytes. It has a key role in removing $\mathrm{Ca}^{2+}$ after excitation and contraction under normal conditions. However, it is also known to play an important role under pathological situations (Blaustein and Lederer, 1999; Iwamoto et al., 2004). In the case of arrhythmias, the reversed mode of NCX could lead to a $\mathrm{Ca}^{2+}$ overload (Sipido et al., 2007). The development of NCX inhibitors has therefore been targeted as a strategy to study regulatory calcium mechanisms. In contrast to KB-R7943 and SEA 0400, where both compounds preferentially block the reverse mode of NCX (Iwamoto et al., 2004), ORM-10962 acts on the two opposite NCX operational modes (Kohajda et al., 2016). Yet, no inhibitor of the Drosophila $\mathrm{Na}^{+} / \mathrm{Ca}^{2+}$ exchanger CALX had been described. To understand a possible role of CALX in Drosophila olfactory transduction, it was crucial to selectively block it independently of other elements in the transduction cascade. Given evidence of three NCX inhibitors, we studied these compounds as potential blockers of CALX.

The first two compounds, KB-R7943 and SEA 0400, are amiloride derivatives. Besides mainly blocking the reverse mode of NCX, amiloride derivatives have been shown to block odorant-evoked activity in lobster olfactory receptor neurons (Bobkov and Ache, 2006). Specifically, KB-R7943 blocked the olfactory response in lobster (Pezier et al., 2009) and mosquito (Bobkov et al., 2014). In both studies, inhibition of the olfactory response was almost total between $50 \mu \mathrm{M}$ and $100 \mu \mathrm{M}$ of KB-R7943. Accordingly, our experiments confirmed that KB-R7943 attenuated the activation of Drosophila ORs significantly (Figure 2). In addition, our results in HEK cells strongly suggest that KB-R7943 acts on the co-receptor Orco directly (Figure 3). This is further supported by the fact that-when testing the other putative inhibitors, namely SEA 0400 or ORM-10962-we observed no attenuation in the Orco response (Figure 2). Our data indicate that KB-R7943 blocks the co-receptor Orco, and hence cannot be used to study the role of CALX in olfaction.

In contrast to KB-R7943, SEA 0400 appear to have no side effect on Orco (Figures 2E,F). The minor, insignificant inhibition could be due to a weak specificity for NCX as stated by Reuter et al. (2002). This result, together with the fact that Bouchard et al. (2004) postulated a state-dependent inhibition of NCX by SEA 0400, made this compound a putative CALX inhibitor. However, although SEA had been reported to be more selective for NCX (Matsuda et al., 2001) and being 30 times more potent than KB-R7943 (Iwamoto et al., 2004), our results indicate that it is not potently acting on CALX. Even at a high concentration of $10 \mu \mathrm{M}$ SEA 0400 failed to inhibit the forward mode of the exchanger (Figure 5B). Iwamoto et al. (2004) 
showed that SEA 0400 preferentially inhibits the reverse mode of mainly NCX1 but not the other NCX isoforms (NCX2 and NCX3) at concentrations between $10 \mathrm{nM}$ and $1 \mu \mathrm{M}$. Such isoform specificity could be the reason for the lack of effect on CALX. Nonetheless, the calcium binding domain (CBD1) in CALX and NCX share 60\% sequence identity (Wu et al., 2010). Therefore, the lack of effect on CALX could be attributable to the absence of a reverse mode in CALX or the reduced specificity mentioned before.

The experiments with the last candidate for inhibition of CALX, ORM-10962, indicated that there was no negative effect in the Orco-response (Figures 2G,H). By contrast, the decay of the $\mathrm{Ca}^{2+}$ signal back to baseline was significantly altered (Figure 4C). This indicates that the restitution of the $\mathrm{Ca}^{2+}$ levels in the presence of ORM-10962 was impaired, which is confirmed by our experiments in resting conditions (Figure 5C). The importance of CALX in restoring calcium levels is also highlighted by our results under $\mathrm{Na}^{+}$free conditions (Figures 4D,E). Under this circumstance, CALX function is impaired and thus the decay of the first and the second response is comparable to the decay in presence of ORM10926. Elevated calcium levels could be reduced by efflux through the plasma membrane by $\mathrm{Na}^{+} / \mathrm{Ca}^{2+}$ exchange and/or the PMCAs. Previous studies reported that NCX acts as the major $\mathrm{Ca}^{2+}$ extrusion mechanism in frogs (Jung et al., 1994; Reisert and Matthews, 1998) and mouse (Noé et al., 1997) olfactory response. However, Castillo et al. (2007) suggested that PMCA could also play an important role in restoring calcium basal levels in rat (Sprague-Dawley) and toad (Caudiverbera caudiverbera) olfactory neurons. They argued that because of its lower affinity to calcium and its voltage dependent properties, NCX's efficiency will decline with depolarization of the neurons during an odor response. Their evidence suggests that both $\mathrm{Ca}^{2+}$ transporters contribute to re-establish resting $\mathrm{Ca}^{2+}$ levels in the cilia following olfactory responses. However, our results suggest that in Drosophila, CALX plays a more important role in maintaining calcium homeostasis (Figure 5). Calmodulin, a $\mathrm{Ca}^{2+}$ binding protein, modulates Drosophila odorant receptor function through Orco (Mukunda et al., 2014) and is able to potentiate the action of PMCA in olfactory cilia (Castillo et al., 2007). Hence, the slower decay observed in the presence of ORM after stimulation of Orco could be due to the action of PMCA (Figure 4). Further experiments to investigate these processes will be important to shed more light into $\mathrm{Ca}^{2+}$ regulatory mechanisms in Drosophila olfactory transduction.

\section{REFERENCES}

Benton, R., Sachse, S., Michnick, S. W., and Vosshall, L. B. (2006). Atypical membrane topology and heteromeric function of Drosophila odorant receptors in vivo. PLoS Biol. 4:e20. doi: 10.1371/journal.pbio.0040020

Blaustein, M. P., and Lederer, W. J. (1999). Sodium/calcium exchange: its physiological implications. Physiol. Rev. 79, 763-854. doi: 10.1152/physrev. 1999.79.3.763

Bobkov, Y., and Ache, B. (2006). Block by amiloride derivatives of odor-evoked discharge in lobster olfactory receptor neurons through action on a presumptive TRP channel. Chem. Senses 32, 149-159. doi: 10.1093/ chemse/bj1041
In conclusion, in the current study we identified ORM-10962 as potent CALX inhibitor. As in other organisms, where $\mathrm{Na}^{+} / \mathrm{Ca}^{2+}$ exchangers are important for the dynamics of the olfactory response (Jung et al., 1994; Noé et al., 1997; Reisert and Matthews, 1998; Danaceau and Lucero, 2000; Castillo et al., 2007), CALX appears to function as the major calcium extrusion mechanisms in Drosophila olfactory neurons both under resting conditions and after enhanced activity.

\section{ETHICS STATEMENT}

This study on the vinegar fly Drosophila melanogaster was performed in Germany where the research on invertebrates does not require a permit from a committee that approves animal research.

\section{AUTHOR CONTRIBUTIONS}

DW and LH-L designed the experiments. LH-L conducted the experiments and the analysis. LH-L, DW and $\mathrm{BH}$ wrote the article.

\section{FUNDING}

This study was supported by the Max Planck Society (LH-L, $\mathrm{BH}$ and $\mathrm{DW}$ ) and the Deutsche Forschungsgemeinschaft (SPP 1392, DW).

\section{ACKNOWLEDGMENTS}

We thank Sabine Kaltofen, Regina Stieber, Dr. Fabio Miazzi and Dr. Veit Grabe for technical support. We thank Dr. Craig Montell for kindly supplying the CALX antibody, the Orion Corporation Orion Pharma (Espoo, Finland) for supplying ORM-10962, and Drs. Jerrit Weißflog and Aleš Svatoš for VUAA1 synthesis.

\section{SUPPLEMENTARY MATERIAL}

The Supplementary Material for this article can be found online at: https://www.frontiersin.org/articles/10.3389/fncel. 2018.00186/full\#supplementary-material

FIGURE S1 | CALX staining in the eye of Drosophila melanogaster. Staining of CALX in the rhabdomeres with Alexa 546. Bar, $5 \mu \mathrm{m}$.

Bobkov, Y., Corey, E., and Ache, B. (2014). An inhibitor of $\mathrm{Na}^{+} / \mathrm{Ca}^{2+}$ exchange blocks activation of insect olfactory receptors. Biochem. Biophys. Res. Commun. 450, 1104-1109. doi: 10.1016/j.bbrc.2014. 06.120

Bouchard, R., Omelchenko, A., Le, H. D., Choptiany, P., Matsuda, T., Baba, A., et al. (2004). Effects of SEA0400 on mutant NCX1.1 $\mathrm{Na}^{+}-\mathrm{Ca}_{2+}$ exchangers with altered ionic regulation. Mol. Pharmacol. 65, 802-810. doi: 10.1124/mol.65.3.802

Castillo, K., Delgado, R., and Bacigalupo, J. (2007). Plasma membrane $\mathrm{Ca}^{2+}$ ATPase in the cilia of olfactory receptor neurons: possible role in $\mathrm{Ca}^{2+}$ clearance. Eur. J. Neurosci. 26, 2524-2531. doi: 10.1111/j.1460-9568.2007. 05863.x 
Danaceau, J. P., and Lucero, M. T. (2000). Electrogenic $\mathrm{Na}^{+} / \mathrm{Ca}^{2+}$ exchange. A novel amplification step in squid olfactory transduction. J. Gen. Physiol. 115, 759-768. doi: 10.1085/jgp.115.6.759

Fluegge, D., Moeller, L. M., Cichy, A., Gorin, M., Weth, A., Veitinger, S., et al. (2012). Mitochondrial $\mathrm{Ca}^{2+}$ mobilization is a key element in olfactory signaling. Nat. Neurosci. 15, 754-762. doi: 10.1038/nn.3074

Guerini, D., Coletto, L., and Carafoli, E. (2005). Exporting calcium from cells. Cell Calcium 38, 281-289. doi: 10.1016/j.ceca.2005.06.032

Hryshko, L. V., Matsuoka, S., Nicoll, D. A., Weiss, J. N., Schwarz, E. M., Benzer, S., et al. (1996). Anomalous regulation of the Drosophila $\mathrm{Na}^{+}-\mathrm{Ca}^{2+}$ exchanger by $\mathrm{Ca}^{2+}$. J. Gen. Physiol. 108, 67-74. doi: 10.1085/jgp.108.1.67

Hsiao, H.-Y., Johnston, R. J., Jukam, D., Vasiliauskas, D., Desplan, C., and Rister, J. (2012). Dissection and immunohistochemistry of larval, pupal and adult Drosophila retinas. J. Vis. Exp. 69:4347. doi: 10.3791/4347

Ignatious Raja, J. S., Katanayeva, N., Katanaev, V. L., and Galizia, C. G. (2014). Role of Go/i subgroup of $G$ proteins in olfactory signaling of Drosophila melanogaster. Eur. J. Neurosci. 39, 1245-1255. doi: 10.1111/ ejn. 12481

Iwamoto, T. (2004). Forefront of $\mathrm{Na}^{+} / \mathrm{Ca}^{2+}$ exchanger studies: molecular pharmacology of $\mathrm{Na}^{+} / \mathrm{Ca}^{2+}$ exchange inhibitors. J. Pharmacol. Sci. 96, 27-32. doi: 10.1254/jphs.fmj04002x6

Iwamoto, T., Kita, S., Uehara, A., Imanaga, I., Matsuda, T., Baba, A., et al. (2004). Molecular determinants of $\mathrm{Na}^{+} / \mathrm{Ca}^{2+}$ exchange (NCX1) inhibition by SEA0400. J. Biol. Chem. 279, 7544-7553. doi: 10.1074/jbc.M3104 91200

Iwamoto, T., Watano, T., and Shigekawa, M. (1996). A novel isothiourea derivative selectively inhibits the reverse mode of $\mathrm{Na}^{+} / \mathrm{Ca}^{2+}$ exchange in cells expressing NCX1. J. Biol. Chem. 271, 22391-22397. doi: 10.1074/jbc.271. 37.22391

Jones, P. L., Pask, G. M., Rinker, D. C., Zwiebel, L. J., and John Hildebrand, G. (2011). Functional agonism of insect odorant receptor ion channels. Proc. Natl. Acad. Sci. U S A 108, 8821-8825. doi: 10.1073/pnas.1102425108

Joseph, R. M., and Carlson, J. R. (2015). Drosophila chemoreceptors: a molecular interface between the chemical world and the brain. Trends Genet. 31, 683-695. doi: 10.1016/j.tig.2015.09.005

Jung, A., Lischka, F. W., Engel, J., and Schild, D. (1994). Sodium/calcium exchanger in olfactory receptor neurons of Xenopus laevis. Neuroreport 5, 1741-1744. doi: 10.1097/00001756-199409080-00013

Kohajda, Z., Farkas-Morvay, N., Jost, N., Nagy, N., Geramipour, A., Horváth, A., et al. (2016). The effect of a novel highly selective inhibitor of the sodium/calcium exchanger (NCX) on cardiac arrhythmias in in vitro and in vivo experiments. PLoS One 11:e0166041. doi: 10.1371/journal.pone. 0166041

Larsson, M. C., Domingos, A. I., Jones, W. D., Chiappe, M. E., Amrein, H., and Vosshall, L. B. (2004). Or83b encodes a broadly expressed odorant receptor essential for Drosophila olfaction. Neuron 43, 703-714. doi: 10.1016/j.neuron. 2004.08.019

Lytton, J. (2007). $\mathrm{Na}^{+} / \mathrm{Ca}^{2+}$ exchangers: three mammalian gene families control $\mathrm{Ca}^{2+}$ transport. Biochem. J. 406, 365-382. doi: 10.1042/BJ20070619

Matsuda, T., Arakawa, N., Takuma, K., Kishida, Y., Kawasaki, Y., Sakaue, M., et al. (2001). SEA0400, a novel and selective inhibitor of the $\mathrm{Na}^{+}-\mathrm{Ca}^{2+}$ exchanger, attenuates reperfusion injury in the in vitro and in vivo cerebral ischemic models. J. Pharmacol. Exp. Ther. 298, 249-256.

Missbach, C., Dweck, H. K. M., Vogel, H., Vilcinskas, A., Stensmyr, M. C., Hansson, B. S., et al. (2014). Evolution of insect olfactory receptors. Elife 3:e02115. doi: 10.7554/eLife.02115

Mukunda, L., Miazzi, F., Kaltofen, S., Hansson, B. S., and Wicher, D. (2014). Calmodulin modulates insect odorant receptor function. Cell Calcium 55, 191-199. doi: 10.1016/j.ceca.2014.02.013

Nicoll, D. A., Longoni, S., and Philipson, K. D. (1990). Molecular cloning and functional expression of the cardiac sarcolemmal Na${ }^{+}-\mathrm{Ca}^{2+}$ exchanger. Science 250, 562-565. doi: 10.1126/science. 1700476
Noé, J., Tareilus, E., Boekhoff, I., and Breer, H. (1997). Sodium/calcium exchanger in rat olfactory neurons. Neurochem. Int. 30, 523-531. doi: 10.1016/s01970186(96)00090-3

Pezier, A., Bobkov, Y. V., and Ache, B. W. (2009). The $\mathrm{Na}^{+} / \mathrm{Ca}^{2+}$ exchanger inhibitor, KB-R7943, blocks a nonselective cation channel implicated in chemosensory transduction. J. Neurophysiol. 101, 1151-1159. doi: 10.1152/jn. 90903.2008

Reisert, J., and Matthews, H. R. (1998). $\mathrm{Na}^{+}$-dependent $\mathrm{Ca}^{2+}$ extrusion governs response recovery in frog olfactory receptor cells. J. Gen. Physiol. 112, 529-535. doi: 10.1085/jgp.112.5.529

Reuter, H., Henderson, S. A., Han, T., Matsuda, T., Baba, A., Ross, R. S., et al. (2002). Knockout mice for pharmacological screening: testing the specificity of $\mathrm{Na}^{+}-\mathrm{Ca}^{2+}$ exchange inhibitors. Circ. Res. 91, 90-92. doi: 10.1161/01.res. 0000027529.37429 .38

Sato, K., Pellegrino, M., Nakagawa, T., Nakagawa, T., Vosshall, L. B., and Touhara, K. (2008). Insect olfactory receptors are heteromeric ligand-gated ion channels. Nature 452, 1002-1006. doi: 10.1038/nature06850

Schwarz, E. M., and Benzer, S. (1997). Calx, a Na ${ }^{+}-\mathrm{Ca}^{2+}$ exchanger gene of Drosophila melanogaster. Genetics 94, 10249-10254. doi: 10.1073/pnas.94.19. 10249

Sipido, K. R., Bito, V., Antoons, G., Volders, P. G., and Vos, M. A. (2007). $\mathrm{Na}^{+} / \mathrm{Ca}^{2+}$ exchange and cardiac ventricular arrhythmias. Ann. $N$ Y Acad. Sci. 1099, 339-348. doi: 10.1196/annals.1387.066

Wang, T., and Montell, C. (2007). Phototransduction and retinal degeneration in Drosophila. Eur. J. Physiol. 454, 821-847. doi: 10.1007/s00424-007-0251-1

Wang, T., Xu, H., Oberwinkler, J., Gu, Y., Hardie, R. C., and Montell, C. (2005). Light activation, adaptation, and cell survival functions of the $\mathrm{Na}^{+} / \mathrm{Ca}^{2+}$ exchanger CalX. Neuron 45, 367-378. doi: 10.1016/j.neuron.2004.12.046

Wicher, D., Schäfer, R., Bauernfeind, R., Stensmyr, M. C., Heller, R. Heinemann, S. H., et al. (2008). Drosophila odorant receptors are both ligandgated and cyclic-nucleotide-activated cation channels. Nature 452, 1007-1011. doi: 10.1038/nature06861

Wu, M.-P., Kao, L.-S., Liao, H.-T., and Pan, C.-Y. (2008). Reverse mode $\mathrm{Na}^{+} / \mathrm{Ca}^{2+}$ exchangers trigger the release of $\mathrm{Ca}^{2+}$ from intracellular $\mathrm{Ca}^{2+}$ stores in cultured rat embryonic cortical neurons. Brain Res. 1201, 41-51. doi: 10.1016/j. brainres.2008.01.042

Wu, M., Le, H. D., Wang, M., Yurkov, V., Omelchenko, A., Hnatowich, M., et al. (2010). Crystal structures of progressive $\mathrm{Ca}^{2+}$ binding states of the $\mathrm{Ca}^{2+}$ sensor $\mathrm{Ca}^{2+}$ binding domain 1 (CBD1) from the CALX Na${ }^{+} / \mathrm{Ca}^{2+}$ exchanger reveal incremental conformational transitions. J. Biol. Chem. 285, 2554-2561. doi: 10.1074/jbc.M109.059162

Wu, M., Tong, S., Gonzalez, J., Jayaraman, V., Spudich, J. L., and Zheng, L. (2011). Structural basis of the $\mathrm{Ca}^{2+}$ inhibitory mechanism of Drosophila $\mathrm{Na}^{+} / \mathrm{Ca}^{2+}$ exchanger CALX and its modification by alternative splicing. Structure 19 , 1509-1517. doi: 10.1016/j.str.2011.07.008

Zheng, L., Wu, M., and Tong, S. (2013). "Structural studies of the $\mathrm{Ca}^{2+}$ regulatory domain of Drosophila $\mathrm{Na}^{+} / \mathrm{Ca}^{2+}$ exchanger CALX," in Sodium Calcium Exchange: A Growing Spectrum of Pathophysiological Implications, ed. L. Annunziato (Boston, MA: Springer), 419-431.

Conflict of Interest Statement: The authors declare that the research was conducted in the absence of any commercial or financial relationships that could be construed as a potential conflict of interest.

Copyright (C) 2018 Halty-deLeon, Hansson and Wicher. This is an open-access article distributed under the terms of the Creative Commons Attribution License (CC BY). The use, distribution or reproduction in other forums is permitted, provided the original author(s) and the copyright owner(s) are credited and that the original publication in this journal is cited, in accordance with accepted academic practice. No use, distribution or reproduction is permitted which does not comply with these terms. 


\section{OPEN ACCESS}

Edited by:

Frédéric Marion-Poll, AgroParisTech Institut des Sciences et Industries du Vivant et de

L'environnement, France

Reviewed by:

Shannon Bryn Olsson, National Centre for Biological

Sciences, India

Thomas Heinbockel, Howard University, United States

*Correspondence: Monika Steng stengl@uni-kassel.de

Received: 25 March 2018 Accepted: 03 July 2018 Published: 24 July 2018

Citation:

Gawalek $P$ and Stengl M (2018) The Diacylglycerol Analogs OAG and DOG Differentially Affect Primary Events of

Pheromone Transduction in the Hawkmoth Manduca sexta in a Zeitgebertime-Dependent Manner Apparently Targeting TRP Channels.

Front. Cell. Neurosci. 12:218 doi: 10.3389/fncel.2018.00218

\section{The Diacylglycerol Analogs OAG and DOG Differentially Affect Primary Events of Pheromone Transduction in the Hawkmoth Manduca sexta in a Zeitgebertime-Dependent Manner Apparently Targeting TRP Channels}

\author{
Petra Gawalek and Monika Stengl* \\ Animal Physiology, FB 10, Biology, University of Kassel, Kassel, Germany
}

For the hawkmoth Manduca sexta accumulating evidence suggests that pheromone transduction acts via a metabotropic signal transduction cascade, with G-protein-dependent phospholipase C (PLC) activations generating diacylglycerol (DAG) and inositol trisphosphate as the primary events in hawkmoth pheromone transduction. In contrast, ionotropic olfactory receptor (OR) coreceptor (Orco)dependent mechanisms do not appear to be involved. In hawkmoths pheromones activated a specific sequence of PLC-dependent ion channels of unknown identity. In several sensory systems transient receptor potential (TRP) ion channels were found downstream of PLC as primary transduction channels. Also in the mammalian vomeronasal organ, DAG-dependent TRP channels are employed. Therefore, we hypothesized that TRPs may be downstream targets for DAG also in the hawkmoth pheromone signal transduction pathway. To test this, we employed two DAG analogs, OAG and DOG for in vivo single-sensillum tip-recordings of pheromone-sensitive sensilla. Since olfactory receptor neurons (ORNs) expressed circadian changes in sensitivity throughout the day, we recorded at two different Zeitgebertimes (ZTs), the hawkmoths activity phase at ZT 1 and its resting phase at ZT 9. We found that the DAG analogs targeted at least two different TRP-like channels that underlie the primary events of hawkmoth pheromone transduction daytime-dependently. At both ZTs OAG sped up and increased the Orco-independent phasic action potential response without affecting the Orco-dependent late, long-lasting pheromone response. Thus, OAG most likely opened a transient $\mathrm{Ca}^{2+}$ permeable TRP channel that was available at both ZTs and that opened pheromone-dependently before Orco. In contrast, DOG slowed down and decreased the sensillum potential, the phasic-, and the late, long-lasting pheromone response. Therefore, DOG appeared to activate a protein kinase $\mathrm{C}(\mathrm{PKC})$ that closed TRP-like $\mathrm{Ca}^{2+}$ permeable channels and opened $\mathrm{Ca}^{2+}$ impermeable cation channels, which have been previously described and are most abundant at ZT 9. These data support our hypothesis that hawkmoth pheromone 
transduction is mediated by metabotropic PLC-dependent mechanisms that activate TRP-like channels as the primary event of pheromone transduction. In addition, our data indicate that at different times of the day different second messenger-dependent ion channels are available for pheromone transduction cascades.

Keywords: transient receptor potential ion channels, insect olfaction, olfactory transduction, diacylglycerol, tip recordings, olfactory sensilla

\section{INTRODUCTION}

The circadian release of pheromones synchronizes hawkmoths physiology and behavior (Riffell et al., 2008; Schendzielorz et al., 2015). Females of the nocturnal hawkmoth Manduca sexta attract their mates at night. They release their species-specific sex-pheromone blend in a strictly circadian rhythm (Sasaki and Riddiford, 1984; Tumlinson et al., 1989). Also the male's sensitivity to detect these pheromones expresses a circadian rhythm, governed by fluctuating hormone levels (review: Stengl, 2010). With long trichoid sensilla on their antennae male hawkmoths detect the sex-pheromone blend over about $8 \log$ units of concentrations during their activity phase at night, while they are considerably less sensitive during the day when moths are at rest (Dolzer et al., 2003; Flecke and Stengl, 2009; Flecke et al., 2010). Each trichoid sensillum is innervated by two olfactory receptor neurons (ORNs) that extend their dendritic cilia into the long hair shaft (Sanes and Hildebrand, 1976; Keil and Steinbrecht, 1984; Keil, 1989). One of the ORNs responds to bombykal, the main sex pheromone component, while the other is sensitive to other components of the pheromone blend (Kaissling et al., 1989). The pheromones are detected via specified pheromone receptors on the cilia of the ORNs that were cloned before, but their functions were still not understood (GroßeWilde et al., 2010; Wicher et al., 2017; reviews: Nakagawa and Vosshall, 2009; Stengl, 2010, 2017). The goal of this study is to delineate the signaling pathway by which pheromones activate hawkmoth ORNs. The large hawkmoths are an established model system for olfaction and are better suited to physiological studies as compared to the tiny fruitflies that instead are excellent genetic model organisms.

\section{Insect Odor Transduction Is Still Under Debate}

Drosophila melanogaster ORs are inverse 7-transmembrane receptors with an intracellular N-terminus (Benton et al., 2006; Lundin et al., 2007) that heteromerize with the conserved olfactory receptor coreceptor (Orco) (Vosshall and Hansson, 2011). Orco is necessary for the localization and maintenance of ORs in the ciliary membranes of Drosophila ORNs (Larsson et al., 2004; Benton et al., 2006). Next to this "chaperon function" of Orco it forms a spontaneously opening ion channel controlling the spontaneous activity of ORNs in different species (Larsson et al., 2004; Benton et al., 2007; Sato et al., 2008; Wicher et al., 2008; Deng et al., 2011; Jones et al., 2011; Sargsyan et al., 2011; Nolte et al., 2013). Despite of extensive molecular genetic studies, it is still not agreed upon whether or how Orco is directly involved in the primary events of insect odor transduction. Thus, it is still not resolved which ion channels underlie the receptor potential that triggers pheromone-dependently action potentials (APs) in insect ORNs (Jones et al., 2011; Sargsyan et al., 2011; Nolte et al., 2013, 2016; reviews: Nakagawa and Vosshall, 2009; Stengl, 2010, 2017).

\section{Hawkmoth Pheromone Transduction Involves G-Protein-Dependent Activation of Phospholipase $\mathbf{C} \beta$}

Patch clamp studies of $M$. sexta ORNs in primary cell culture combined with pharmacology characterized several bombykal- and second messenger-dependent ion channels in ORNs with properties of transient receptor potential (TRP)like ion channels (Minke et al., 1975; Montell et al., 1985; Stengl and Hildebrand, 1990; Stengl et al., 1992; Stengl, 2010). These studies showed that pheromone activates phospholipase $\mathrm{C}$ (PLC) in a G-protein dependent manner. This generated diacylglycerol (DAG) and inositol-trisphosphate $\left(\mathrm{IP}_{3}\right)$ as a function of pheromone concentration as measured in different insect antennae (Boekhoff et al., 1990, 1993; Breer et al., 1990). Infusion of GTP $\gamma \mathrm{S}$ or $\mathrm{IP}_{3}$ opened the same sequence of TRP/TRPL-like ion channels as does application of pheromone (Stengl, 1993, 1994; Dolzer et al., 2001; review: Stengl, 2010). In the presence of adapting concentrations of pheromone DAG and elevated $\mathrm{Ca}^{2+}$-levels activated protein kinase $\mathrm{C}$ (PKC) that closed the previously opened pheromone-dependent channels (Stengl, 1993, 1994; Dolzer et al., 2001; review: Stengl, 2010). Thus, depending on pheromone concentration, different second messenger cascades become active. It has been established previously that DAG directly opens odordependent $\mathrm{Ca}^{2+}$-permeable TRPC2- channels in vertebrate ORNs (Lucas et al., 2003). Also TRP channels are closed via PKCdependent phosphorylation (Huang, 1989; Liu and Heckman, 1998; Venkatachalam et al., 2003). Therefore, DAG has at least two different antagonistic functions. It can directly open TRP channels causing membrane potential depolarizations and it can directly activate PKC which is involved in negative feedback regulation, closing TRP channels and curtailing membrane potential depolarizations.

\section{Directly- or Indirectly DAG-Dependent Ion Channels Belong to the Transient Receptor Potential (TRP) Superfamily of Ion Channels}

The superfamily of TRP ion channels comprises 7 subfamilies of evolutionary highly conserved cation channels first detected in fly vision (Minke et al., 1975; Montell et al., 1985; Hofmann 
et al., 1999; reviews: Ramsey et al., 2006; Fowler and Montell, 2013). The subfamily of classical or canonical TRPs (TRPCs) are the closest mammalian homologs of Drosophila melanogaster TRP/TRP-like $\mathrm{Ca}^{2+}$ permeable cation channels. They comprise 7 members, TRPC1-7, that all depend on phospholipidhydrolysis (Storch et al., 2017). The TRPCs are activated by membrane receptors that couple via $G_{q / 11}$ and/or via $G_{i / o}$ to isoforms of PLC. It was shown previously that TRPC1 plays an important role in store-operated calcium entry pathways, being activated by DAG and being inactivated by PKC-dependent phosphorylation (review: Ambudkar et al., 2017). Furthermore, DAG, as well as the membrane permeant DAG analogs OAG and DOG (1,2-dioctanoyl-cn-glycerol = DOG; 1-oleoyl-2-acetyl-snglycerol = OAG) activated TRPC2, $-3,-6$, and -7 ion channels apparently directly. The TRPC3, but not TRPC5 channels were directly opened by OAG, independent of PKC activation. Instead, OAG and DOG prevented TRPC5 and TRPC4 activation PKCdependently (Venkatachalam et al., 2003). More recent studies (Storch et al., 2017) showed that TRPC4 and TRPC5 differ from the other TRPC channels, since they express a PDZ-binding motif in the $\mathrm{C}$ termini that also harbor a PKC phosphorylation site crucial for TRPC5 desensitization upon receptor activation (Zhu et al., 2005). Only if PKC-dependent phosphorylation of TRPC4/5 is prevented OAG or DOG were able to activate TRPC4/5 channels directly (Storch et al., 2017). Furthermore, while $\mathrm{PIP}_{2}$ depletion activated TRPC4 and -5 channels, it inhibited TRPC6 and -7 channels. In contrast to the directly DAG-dependently activated heteromultimers of TRPC3, TRPC6, and TRPC7 channel subunits, TRPC7 homomultimers show distinct properties such as constitutive activity and inhibition via PKC (Zhang and Trebak, 2014). Therefore, the DAG analogs OAG and DOG antagonistically affect various TRPC channels in mammals, either activating them directly, or inactivating them via $\mathrm{PKC}$-dependent phosphorylation. We thus hypothesize that also in the hawkmoth DAG analogs antagonistically target TRPCrelated ion channels that underlie the pheromone transduction cascade, since PLC and $\mathrm{G}_{\mathrm{i} / \mathrm{o}}$ were located to moth ORNs (review: Stengl, 2010).

So far, only cDNA encoding a TRP $\gamma$ channel was identified in antennae of Spodoptera littoralis. Using in situ hybridization the transcript of this channel could be localized at the base and the shaft of pheromone-sensitive sensilla trichoidea hinting a role in olfactory transduction (Chouquet et al., 2009). Furthermore, a TRP channel was cloned and heterologously expressed from hawkmoth antennae (Ackermann, 2008) and DAG-dependent TRP-like channels were physiologically characterized from the hawkmoth (Krannich, 2008). How many TRP/TRPL-like ion channels are involved in the primary events of hawkmoth pheromone transduction and whether they are closed PKCdependently as a form of short-term adaptation, however, is not known.

\section{We Want to Examine Whether DAG-Dependent Ion Channels Underlie the Phasic Pheromone Response}

Since not much is known about DAG-dependent TRP channels in insect odor transduction (Zufall and Hatt, 1991; reviews:
Fowler and Montell, 2013; Thiel et al., 2018) we wanted to examine whether DAG affects the primary events of pheromone transduction in the hawkmoth. Unfortunately, very specific ion channel antagonists for the different types of insect TRP channels are not known. Thus, to search for directly and indirectly DAG-dependent TRP channels in hawkmoths we employed the two membrane-permeable DAG analogs DOG and OAG that were shown before to affect TRP channels. In vivo in intact hawkmoths tip-recordings were performed. A brief pulse of bombykal at physiological concentrations elicits a sequence of potential changes in the bombykal-sensitive ORN that can be measured extracellularly (Nolte et al., 2016). Opening of bombykal-dependent ion channels in the cilium result in the depolarizing sensillum potential (Kaissling et al., 1987; review: Stengl, 2010). The depolarization elicits an action potential response in the axon with different kinetics. Only the first phasic action potential response within the first $\sim 100$ ms encodes bombykal concentration changes. About a second later, it is followed by the Orco-dependent late, long-lasting pheromone response that can persist over minutes or even hours after stimulation, depending on the strength of bombykal stimulation (Dolzer et al., 2003; Nolte et al., 2016). Thus, we examined whether perfusion of the two DAG analogs into the sensillum lymph affects the different parameters of the bombykal response. Since $M$. sexta ORNs are peripheral circadian pacemakers that modulate their pheromone sensitivity over the course of the day we performed our experiments either during the late activity phase at Zeitgebertime 1 (ZT 1), or during rest at ZT 9 (Schuckel et al., 2007; Flecke and Stengl, 2009; Flecke et al., 2010; Schendzielorz et al., 2012).

\section{MATERIALS AND METHODS}

\section{Animals and Preparation}

For all experiments male M. sexta (Johannson) (Lepidoptera; Sphingidae) hawkmoths were used that were raised from egg at the University of Kassel. Larvae were fed on an artificial diet (modified after Bell and Joachim, 1976), adult moths could feed on sugar solution ad libitum. Animals were kept under long-day conditions ( $17 \mathrm{~h}: 7 \mathrm{~h}, \mathrm{~L}: \mathrm{D})$ at $24-26^{\circ} \mathrm{C}$ and a relative humidity of $40-60 \%$. Male pupae were isolated to avoid contact with female pheromone. For the recordings only adult male $M$. sexta that previously were kept at two dark-phases were caught $30 \mathrm{~min}$ before each experiment. Animals were fixed in a custombuilt Teflon ${ }^{\mathrm{TM}}$ holder and the flagellum of the right antenna was immobilized with dental wax (Boxing wax, Sybron/Kerr, Romulus, Michigan, USA). The upmost $\sim 15$ segments of the apical end of the antenna were cut off with micro-scissors. A glass electrode filled with hemolymph Ringer solution (6.4 $\mathrm{mmol} / \mathrm{L} \mathrm{KCl}, 12.0 \mathrm{mmol} / \mathrm{L} \mathrm{MgCl}_{2}, 1.0 \mathrm{mmol} / \mathrm{L} \mathrm{CaCl}_{2}, 12.0$ $\mathrm{mmol} / \mathrm{L} \mathrm{NaCl}, 10 \mathrm{mmol} / \mathrm{L}$ HEPES, $354.0 \mathrm{mmol} / \mathrm{L}$ glucosemonohydrate) (Kaissling, 1995) was inserted into the lumen of the flagellum as indifferent electrode. To avoid desiccation the cut was sealed with electrode gel (electrode gel, GE Medical Systems Information Technologies, Freiburg, Germany). To access the sensillum lymph with forceps the upper quarter of the long trichoid sensilla from the apical row of the second remaining annulus were cut. The recording electrode filled with sensillum 
lymph Ringer solution (171.9 mmol/L KCl, $3.0 \mathrm{mmol} / \mathrm{L} \mathrm{MgCl}_{2}$, $1.0 \mathrm{mmol} / \mathrm{L} \mathrm{CaCl} 2,25 \mathrm{mmol} / \mathrm{L} \mathrm{NaCl}, 10 \mathrm{mmol} / \mathrm{L}$ HEPES, 22.5 $\mathrm{mmol} / \mathrm{L}$ glucose-monohydrate) (Kaissling, 1995) was pulled over one of the cut sensilla. To record the potentials between both electrodes $\mathrm{Ag} / \mathrm{AgCl}$ wires were inserted into the Ringer solutions. The electrodes were connected to a custom-built amplifier $(0$ $\mathrm{Hz}-2 \mathrm{kHz}$, input impedance $10^{12} \Omega$ ) where the recorded signals were amplified 200-fold. Subsequently, for data acquisition the signals were digitized with a Digidata 1200B (Molecular Devices, Sunnyvale, California, USA) and recorded with pCLAMP 8 software. All experiments were performed in the photophase of the animals. To investigate time-dependent differences in the pheromone responses one part of the recordings started at Zeitgebertime (ZT) 1, $1 \mathrm{~h}$ after lights on (ZT0), during the end of the activity phase of the animals. The other experiments started at ZT 9, during the middle of the resting phase. The recordings lasted for $2 \mathrm{~h}$ and all experiments were performed with room lights switched on.

\section{Application of Drugs}

Two membrane permeable DAG-analogs, 1,2-dioctanoyl-snglycerol (DOG) and 1-oleoyl-2-acetyl-sn-glycerol (OAG), were dissolved in dimethylsulfoxid (DMSO) and subsequently diluted in the sensillum lymph Ringer solution. For applications Ringer solutions containing concentrations of 1,100 or $200 \mu \mathrm{mol}$ $1^{-1}$ DOG and OAG in $0.1 \%$ DMSO, were employed. Agents were applied passively via diffusion into the sensillum lymph (Kaissling et al., 1991) during the tip-recordings. All Ringer solutions were adjusted to $\mathrm{pH}$ 6.5. With mannitol osmolarity was adjusted to 475 mosmol $1^{-1}$ for the hemolymph Ringer, and to 450 mosmol $^{-1}$ for the sensillum lymph Ringer.

\section{Pheromone Stimulation}

Pheromone was presented to the recording site as an airborne plume within a constant stream of air. The charcoal-filtered and moistened air was constantly blown over the preparation through a glass cartridge to ensure constant conditions throughout the recording. A second cartridge containing a filter paper (about $1 \mathrm{~cm}^{2}$ ) loaded with synthetic bombykal (E,Z-10,12hexadecadienal) was installed parallel to the constant air stream. Stock solutions used for the experiments contained $10^{-1} \mathrm{mg} / \mathrm{ml}$ bombykal [generously provided by T. Christensen (University of Arizona, Tucson, Arizona, USA) and J. Krieger (University of Hohenheim, Stuttgart, Germany)] dissolved in $n$-hexane (Roth, Karlsruhe, Germany). The filter paper was loaded with $10 \mu \mathrm{l}$ of the stock solutions resulting in final concentrations of $1 \mu \mathrm{g}$ bombykal. For stimulation the air stream was re-directed to the second cartridge via a computer controlled valve (JFMH-5PK-3, Festo, Esslingen, Germany). To avoid evaporation of the pheromone between stimulations a second valve (PA 202-004 P, Staiger, Erligheim, Germany) was positioned in front of the cartridge containing the bombykal. The outlets of both cartridges were placed at a distance of about $5 \mathrm{~cm}$ from the recording site. Every $5 \mathrm{~min}$ over a total recording time of $120 \mathrm{~min} 50 \mathrm{~ms}$ pheromone stimuli were applied, resulting in 24 stimulations per tip-recording. An interstimulus interval (ISTI) of 5 min was necessary to avoid adaptation of the bombykal response, as assessed by a decrease of the phasic pheromone response and a decline in the sensillum potential amplitude. Between recordings
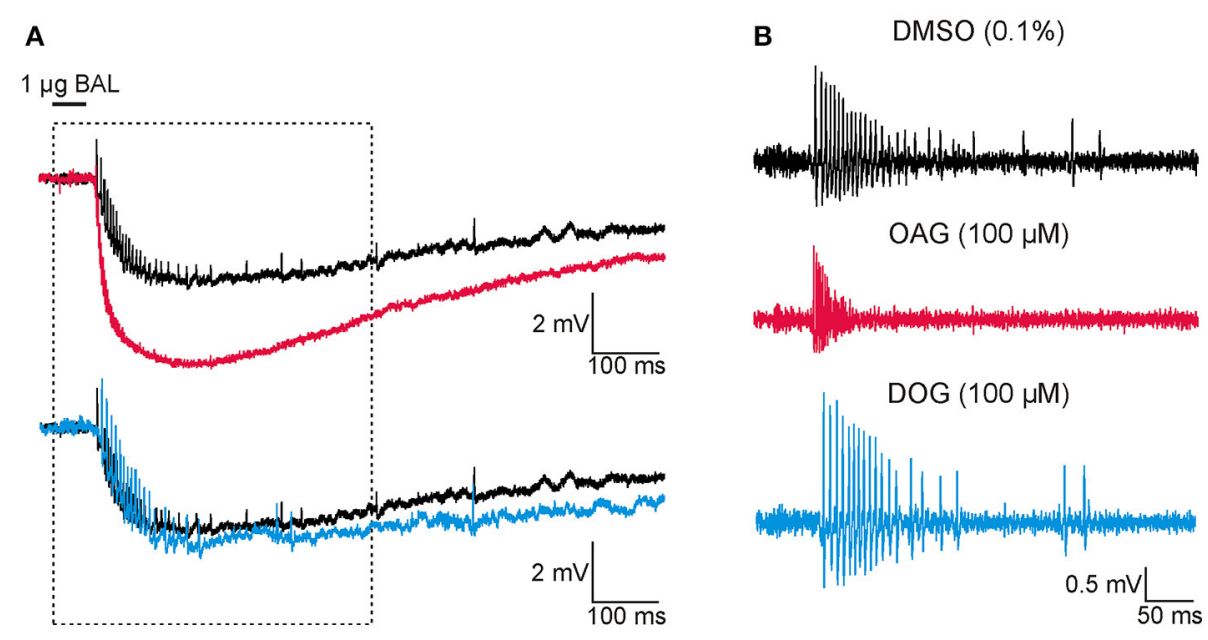

FIGURE 1 | At Zeitgeberzeit 1 (ZT 1) the diacylglycerol-analog OAG increased the response to bombykal (BAL, $1 \mu \mathrm{g})$ while the diacylglycerol-analog DOG reduced it. Thus, both analogs target different DAG-dependent components of the BAL transduction cascade. Original tip-recordings from pheromone-sensitive trichoid sensilla on the antenna of the hawkmoth Manduca sexta show responses to 50 ms pulses of BAL ( $1 \mu \mathrm{g}$ ). Infusion of the DAG-analogs OAG (magenta, $100 \mu \mathrm{M}$ ) or DOG (cyan, $100 \mu \mathrm{M}$ ) via the tip-recording electrode were compared to control infusions with vehicle only (Ringer containing $0.1 \%$ DMSO, black) at the late activity phase at ZT 1. BAL-responses shown occurred about 30 min after the beginning of the recordings. (A) Unfiltered recordings. Infusion of OAG (magenta), but not of DOG (cyan) accelerated and increased the pheromone-dependent sensillum potential amplitude and action potential response. In contrast, DOG rather slowed down the repolarization of the pheromone-response. Recordings in boxed area were enlarged in (B). (B) High-pass filtered BAL responses illustrated that OAG (magenta) increased the frequency of the BAL-dependent action potential response and rendered it more phasic, as compared to the control (black). In contrast, DOG (cyan) slightly decreased and slowed down the BAL-response. 
the cartridges containing bombykal were stored in scintillation vials at $-20^{\circ} \mathrm{C}$. They were used for about 10 recordings before replacing the filter paper.

\section{Acquisition Protocols}

Tip-recordings of spontaneous activity detected APs of two different amplitudes generated by the two ORNs innervating a trichoid sensillum while bombykal responses only activated one cell. Since AP amplitudes of the bombykal -sensitive ORN were always higher both ORNs could be distinguished easily (Dolzer et al., 2001). During stimulations not only the APs but also slower changes in the transepithelial potential (TEP) were recorded with glass electrodes. The TEP represents the sum of the membrane potentials of all cells located between the electrodes and does not only reflect the bombykal-dependent receptor potential generated. Thus, to prevent confusion with the receptor potential, it was termed sensillum potential (SP). The recorded pheromone responses covered a time frame of $5,161 \mathrm{~ms}$ with a pre-trigger part of $180 \mathrm{~ms}$ and a post-trigger part of $4,931 \mathrm{~ms}$ at a continuous sampling rate of $20 \mathrm{kHz}$ (Clampex 8, episodic stimulation mode; Molecular Devices, Sunnyvale, California, USA).

\section{Data Analysis and Statistics}

The pheromone responses were analyzed using Spike 2 version 7.01 (Cambridge Electronic Design, Cambridge, UK; script written by A. Nolte) and Microsoft Excel. To analyze the SP the response was low-pass filtered at a cut-off frequency of $50 \mathrm{~Hz}$. Evaluated was the maximal SP amplitude, that is the negative deflection of the TEP (Figure 1A) for each stimulation. Because
A

late activity (ZT 1)

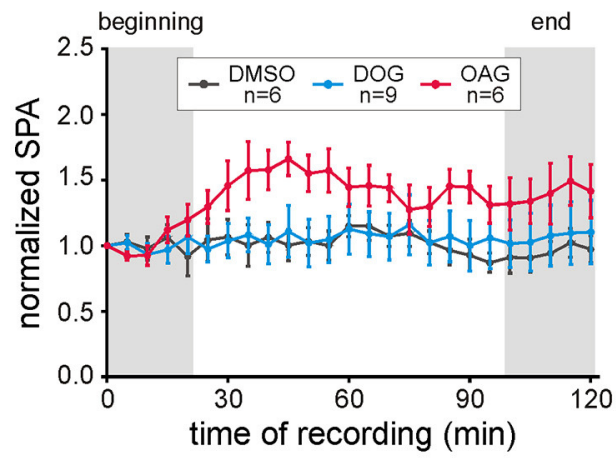

C

rest (ZT 9)

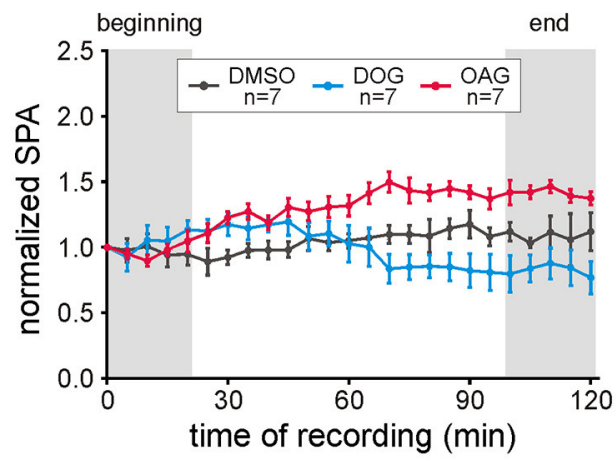

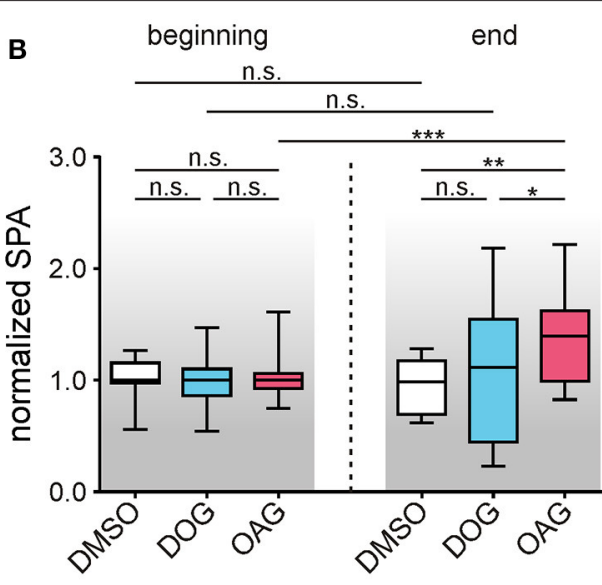

D

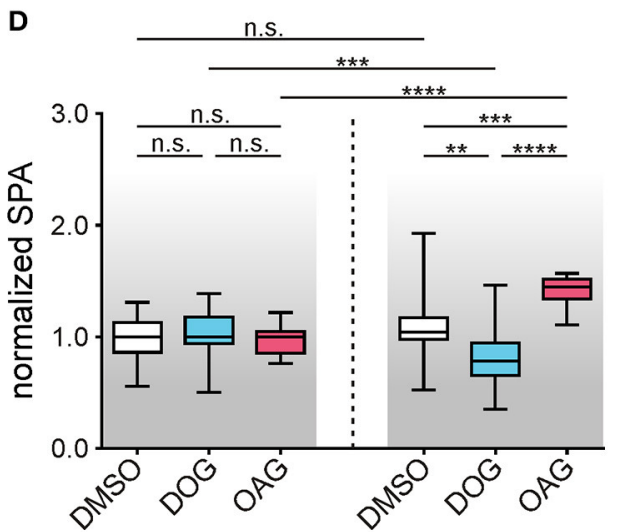

FIGURE 2 | The DAG analog OAG (magenta) increased the bombykal (BAL)-dependent sensillum potential amplitude (SPA) at ZT 1 and ZT 9, while the DAG analog DOG (cyan) decreased it at ZT 9 only. Long-term tip-recordings over 120 min were performed from pheromone-sensitive trichoid sensilla on the antenna of the hawkmoth Manduca sexta either during the late activity phase at Zeitgebertime 1 (ZT 1) (A,B), or during the resting phase at ZT 9 (C,D). Every 5 min 50 ms pulses of BAL $(1 \mu \mathrm{g})$ were applied. Via the tip-recording electrode either the DAG-analogs OAG (magenta, $100 \mu \mathrm{M}$ ) or DOG (cyan, $100 \mu \mathrm{M}$ ) were applied, in comparison to control infusions with vehicle only (Ringer containing $0.1 \%$ DMSO, black). The SPA in response to stimulation with BAL was normalized to the value of the first stimulation. Relative changes in the SPA were shown. Statistical analysis was performed during the first and last 20 min of the recordings (Table 1; gray areas A,C). During the late activity phase (ZT 1-3) the normalized SPA was stable for control- and DOG recordings (A,B). Application of $100 \mu$ M OAG increased the SPA over the course of the recording resulting in significant differences to controls and DOG. (C,D) Also, at ZT 9-11 the SPA remained stable in controls, while DOG decreased-, and OAG significantly increased it over time (D, exact $P$-values: Table 1). n.s. $=$ not significant; ${ }^{*} P<0.05 ;{ }^{* \star} P<0.01 ;{ }^{* \star *} P<0.001 ;{ }^{* \star \star *} P<0.0001$; Mann-Whitney test or Kruskal-Wallis test with Dunn's post-hoc test for multiple comparison. For mean values \pm SEM see $S 1$. 
TABLE 1 | Statistical analysis of the normalized SPA in the presence or absence of $100 \mu \mathrm{M}$ DOG or OAG during the late activity phase and at rest (Figure 2).

\begin{tabular}{|c|c|c|c|c|}
\hline \multicolumn{2}{|c|}{ Compared groups } & \multicolumn{2}{|c|}{$P$-value } & \multirow[t]{2}{*}{ Test } \\
\hline & & Beginning (0-20 min) & End (100-120 min) & \\
\hline \multicolumn{5}{|c|}{ LATE ACTIVITY (ZT 1) } \\
\hline DMSO & $100 \mu \mathrm{M}$ DOG & n.s. $P>0.9999$ & n.s. $P>0.9999$ & $\begin{array}{l}\text { Kruskal-Wallis test with } \\
\text { Dunn's post hoc test }\end{array}$ \\
\hline DMSO & $100 \mu \mathrm{M} O A G$ & n.s. $P>0.9999$ & ${ }^{* \star} P=0.0042$ & \\
\hline $100 \mu \mathrm{M}$ DOG & $100 \mu \mathrm{M} O A G$ & n.s. $P>0.9999$ & ${ }^{\star} P=0.0210$ & \\
\hline \multicolumn{5}{|c|}{ Beginning vs. End } \\
\hline DMSO & DMSO & n.s. $P=0.8139$ & & Mann-Whitney test \\
\hline $100 \mu \mathrm{M}$ DOG & $100 \mu \mathrm{M} D O G$ & n.s. $P=0.6816$ & & Mann-Whitney test \\
\hline $100 \mu \mathrm{M}$ OAG & $100 \mu \mathrm{M} O A G$ & ${ }^{* \star \star} P=0.0008$ & & Mann-Whitney test \\
\hline \multicolumn{5}{|l|}{ REST (ZT 9) } \\
\hline DMSO & $100 \mu \mathrm{M}$ DOG & n.s. $P=0.3910$ & ${ }^{\star \star} P=0.0097$ & $\begin{array}{l}\text { Kruskal-Wallis test with } \\
\text { Dunn's post hoc test }\end{array}$ \\
\hline DMSO & $100 \mu \mathrm{M} O A G$ & n.s. $P>0.9999$ & ${ }^{\star \star \star \star} P=0.0002$ & \\
\hline $100 \mu \mathrm{M}$ DOG & $100 \mu \mathrm{M} O A G$ & n.s. $P=0.2173$ & ${ }^{\star \star \star \star} P<0.0001$ & \\
\hline \multicolumn{5}{|c|}{ Beginning vs. End } \\
\hline DMSO & DMSO & n.s. $P=0.2346$ & & Mann-Whitney test \\
\hline $100 \mu \mathrm{M}$ DOG & $100 \mu \mathrm{M}$ DOG & ${ }^{\star * \star} P=0.0002$ & & Mann-Whitney test \\
\hline $100 \mu \mathrm{M} O A G$ & $100 \mu \mathrm{M} O A G$ & ${ }^{\star \star \star \star} P<0.0001$ & & Mann-Whitney test \\
\hline
\end{tabular}

Level of significance: $\alpha=0.05$; n.s. $=$ not significant; ${ }^{\star} P<0.05 ;{ }^{* \star} P<0.01 ;{ }^{* \star *} P<0.001 ;{ }^{* \star * *} P<0.0001$.

of high variability between animals the SP amplitude of each recording was normalized to the first response. By comparing the normalized SP amplitude of the first 5 stimulations (beginning) and the amplitude of the last 5 stimulations (end) relative changes within recordings were analyzed. Additionally, the response was high-pass filtered with a cut-off frequency of $150 \mathrm{~Hz}$ to evaluate different parameters of the AP response. The peak frequency of the first 5 interspike intervals (AP frequency) evaluates the phasic pheromone response (Nolte et al., 2016). With post-stimulus time histograms (PSTHs; APs binned in intervals of $10 \mathrm{~ms}$ ) the distribution of APs within the first 1,000 ms was analyzed, showing the phasic and tonic pheromone responses. For analysis of the late, long-lasting pheromone response the spikes occurring between 5 and $295 \mathrm{~s}$ after the bombykal stimulus were evaluated. Analysis of the spontaneous activity of ORNs without bombykal stimulation was performed equally. For statistical analysis results were tested for Gaussian distribution using the KolmogorovSmirnov test. If at least one data set failed the normalitytest the Kruskal-Wallis test followed by Dunn's post-hoc-test was used for multiple comparisons. The Mann-Whitney test was applied to analyse the effects in relation to the recording time.

\section{RESULTS}

In tip-recordings of bombykal-sensitive trichoid sensilla of the hawkmoth Manduca sexta we examined whether two membrane-permeable DAG-analogs, DOG and OAG that target TRP channels and PKC, affected different parameters of the bombykal responses $(n=91)$. Parameters examined were the bombykal-dependent sensillum potential amplitude (Figures 1A, 2, 3), the phasic bombykal-dependent action potential response (Figures 1B, 4-7), the Orco-dependent late, long-lasting pheromone response (Figure 8), and the spontaneous activity (Figure 9). The two DAG analogs were expected to either activate TRP channels directly and/or affect them indirectly/antagonistically via PKC activation. Activation of $\mathrm{Ca}^{2+}$-permeable TRP channels would increase the rise time and amplitude of the sensillum potential, as well as the frequency of the phasic pheromone response. In contrast, PKC inactivates TRP channels via negative feedback mechanisms at elevated intracellular $\mathrm{Ca}^{2+}$ concentrations. Thus, the rise time of the sensillum potential and its amplitude, as well as the phasic pheromone response would be decreased by PKC activation. Thus, we expected to observe antagonistic effects of both analogs if one preferentially activates TRP channels and the other preferentially activates PKC (Venkatachalam et al., 2003). The experiments were performed at the end of the activity phase (ZT 1-3) and during the resting phase (ZT 911) to account for circadian clock-dependent modulation of pheromone transduction in accordance with the physiological state of the animal. We wanted to know whether any of the two DAG-analogs directly or indirectly targets bombykal-dependent ion channels that are only available at rest or activity phases of the hawkmoth.

\section{Pheromone-Dependent Sensillum Potential Amplitudes Were Differentially and ZT-Dependently Affected by Both DAG Analogs $(100 \mu \mathrm{M})$ in Long-Term Tip-Recordings}

Two hrs long tip-recordings at ZT 1 and ZT 9 examined how infusion of $100 \mu \mathrm{M}$ DOG $(n=16)$ and $100 \mu \mathrm{M}$ OAG 
A

late activity (ZT 1)

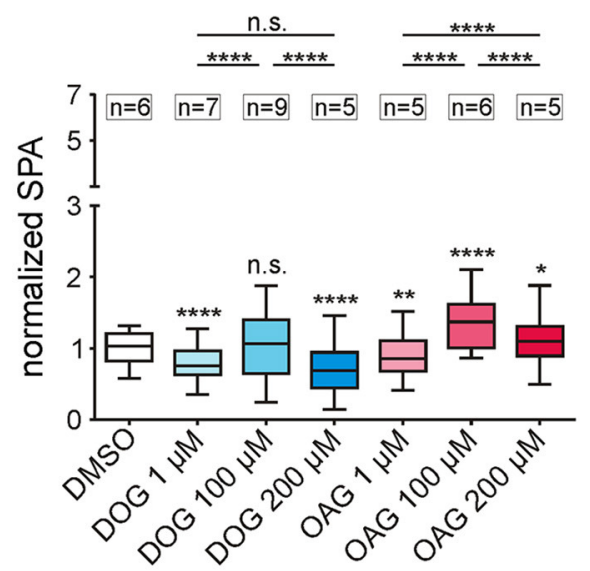

C

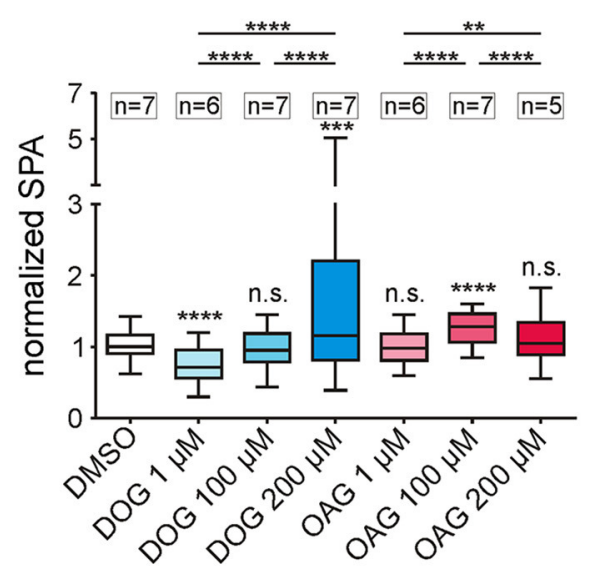

B

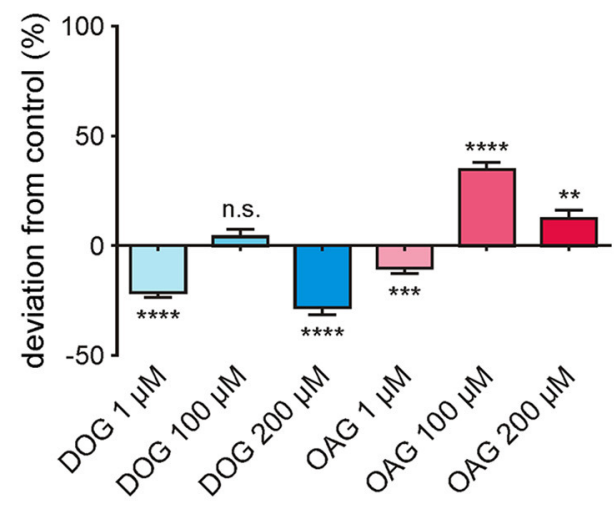

D

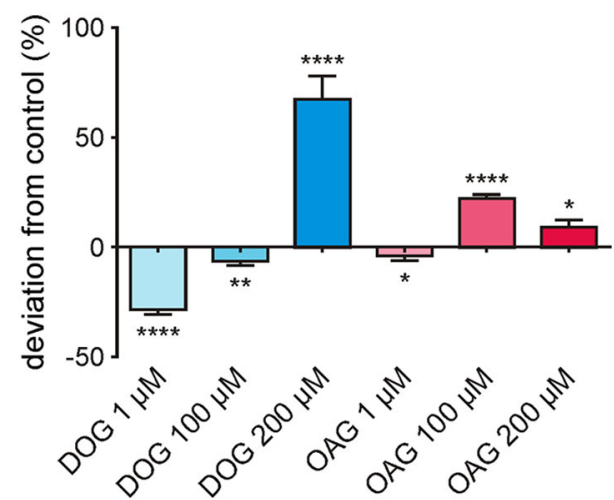

FIGURE 3 | Infusion of different doses of the DAG analogs DOG and OAG showed that both analogs affected different ion channels underlying the bombykal-dependent normalized sensillum potential amplitude (SPA) with different sensitivity. (A,B) 1, 100, and $200 \mu M$ of the DAG-analogs DOG and OAG were applied in tip recordings during the late activity phase (ZT 1). The overall values (A) as well as the percentage changes to the controls (B) show a significant decrease in the normalized SPA in the presence of $1 \mu \mathrm{M}(n=7)$ or $200 \mu \mathrm{M} \mathrm{DOG}(n=5)$, while $100 \mu \mathrm{M} \mathrm{DOG}(n=9)$ had no effect. Application of $1 \mu \mathrm{M}$ OAG reduced the normalized SPA $(n=5)$, while $200 \mu$ M OAG $(n=5)$ moderately- and $100 \mu$ M OAG more strongly increased it $(n=6)$. (C) During rest (ZT 9) the mean values of the normalized SPA were significantly reduced by $1 \mu \mathrm{M} \mathrm{DOG}(n=6)$ and significantly increased by application of $200 \mu \mathrm{M} \mathrm{DOG}(n=7)$ and $100 \mu \mathrm{M} \mathrm{OAG}(n=7)$. All other concentrations showed no significant differences from the controls in their mean values. (D) However, all concentrations applied showed significant differences in the percentage change from the control (for exact $P$-values see Table 2; for mean values \pm SEM see S2. n.s. $=$ not significant; ${ }^{*} P<0.05$; ${ }^{\star \star} P<0.01$; ${ }^{\star \star \star} P<0.001$; ${ }^{* \star \star \star} P<0.0001$; Wilcoxon test, Mann-Whitney test or Kruskal-Wallis test with Dunn's post-hoc test for multiple comparison).

( $n=13)$ affected sensillum potentials in response to pheromone stimulation ( $50 \mathrm{~ms}$ pulses of $1 \mu \mathrm{g}$ bombykal, every $5 \mathrm{~min}$ ) (Figures 1A, 2A-D; Table 1; S1). With OAG present during the pheromone stimulations the sensillum potential amplitude rose faster to a higher amplitude and also declined faster (Figure 1A). The OAG effects were stronger and faster at ZT $1(n=6)$ compared to ZT $9(n=7)$ and became statistically significant during the course of the 2 hrs long recording (Figures 2A-D; Table 1; S1). In contrast, the presence of DOG had no significant effects on any parameter of the bombykaldependent sensillum potential during ZT 1 (Figures 2A,B; Table 1), either because DOG-dependent ion channels were not available at the late activity phase, or, because they were already activated/inactivated. However, at ZT 9 during the course of the tip-recording DOG significantly reduced the sensillum potential amplitude (Figures 2C,D; Table 1; S1) and slowed down its rising- and declining phase (not shown). The amplitude of the control recordings remained unchanged during the course of the recordings at ZT $1(n=6)$ and ZT $9(n=7)$ (Figures 2A-D). Statistical analysis is summarized in Table 1, for mean values \pm SEM (see Supplemental Figure S1). In summary, ZT-dependently OAG and DOG affected at least two different ion channels underlying the rising phase of the bombykaldependent sensillum potential. Apparently, OAG opens a cation channel with fast kinetics that is available at both ZTs, but being more abundant or more available for activation at ZT1. 
TABLE 2 | Statistical analysis of the normalized SPA of long-term recordings in the presence or absence of 1, 100, or 200 $\mu$ M DOG or OAG during the late activity phase and at rest (Figure 3).

\begin{tabular}{|c|c|c|c|c|}
\hline \multicolumn{2}{|c|}{ Compared groups } & \multicolumn{2}{|c|}{$P$-value } & Test \\
\hline LATE ACTIVI & & Absolute values (a) & Deviation from control (\%) (b) & \\
\hline DMSO & $100 \mu \mathrm{M}$ DOG & n.s. $P=0.878$ & n.s. $P=0.3154$ & (b): Mann-Whitney test \\
\hline DMSO & $200 \mu \mathrm{M}$ DOG & ${ }^{* \star \star \star} P<0.0001$ & ${ }^{\star \star \star \star} P<0.0001$ & \\
\hline $1 \mu \mathrm{M} \mathrm{DOG}$ & $200 \mu \mathrm{M}$ DOG & n.s. $P=0.2918$ & & \\
\hline $100 \mu \mathrm{M}$ DOG & $200 \mu \mathrm{M}$ DOG & ${ }^{\star \star \star \star \star} P<0.0001$ & & \\
\hline DMSO & $1 \mu \mathrm{M} \mathrm{OAG}$ & ${ }^{\star \star} P=0.0012$ & ${ }^{* \star \star} P=0.0001$ & $\begin{array}{l}\text { (a): Kruskal-Wallis test with } \\
\text { Dunn's post hoc test; }\end{array}$ \\
\hline DMSO & $100 \mu \mathrm{M} O A G$ & ${ }^{* \star \star \star} P<0.0001$ & ${ }^{\star \star \star \star \star} P<0.0001$ & (b): Mann-Whitney test \\
\hline $100 \mu \mathrm{M} O A G$ & $200 \mu \mathrm{M} O A G$ & ${ }^{\star \star \star \star \star} P<0.0001$ & & \\
\hline \multicolumn{5}{|l|}{ REST (ZT 9) } \\
\hline DMSO & $1 \mu \mathrm{M} \mathrm{DOG}$ & ${ }^{* \star \star \star} P<0.0001$ & ${ }^{* \star \star \star} P<0.0001$ & $\begin{array}{l}\text { (a): Kruskal-Wallis test with } \\
\text { Dunn's post hoc test; }\end{array}$ \\
\hline DMSO & $100 \mu \mathrm{M}$ DOG & n.s. $P=0.3070$ & ${ }^{* \star} P=0.0076$ & (b): Mann-Whitney test \\
\hline DMSO & $200 \mu \mathrm{M}$ DOG & ${ }^{\star \star \star} P=0.0001$ & ${ }^{* \star \star \star} P<0.0001$ & \\
\hline $1 \mu \mathrm{M} \mathrm{DOG}$ & $100 \mu \mathrm{M}$ DOG & ${ }^{* \star * \star} P<0.0001$ & & $\begin{array}{l}\text { Kruskal-Wallis test with } \\
\text { Dunn's post hoc test }\end{array}$ \\
\hline $1 \mu \mathrm{M} \mathrm{DOG}$ & $200 \mu \mathrm{M}$ DOG & ${ }^{* \star \star \star} P<0.0001$ & & \\
\hline $100 \mu \mathrm{M} \mathrm{DOG}$ & $200 \mu \mathrm{M}$ DOG & ${ }^{* \star \star \star} P<0.0001$ & & \\
\hline
\end{tabular}

Level of significance: $\alpha=0.05 ;$ n.s. $=$ not significant; ${ }^{*} P<0.05 ;{ }^{* *} P<0.01 ;{ }^{* * * *} P<0.001 ;{ }^{* *+* *} P<0.0001$.

In contrast DOG-dependent ion channels were not available at ZT 1, but appeared to underlie the pheromone-dependent sensillum potential at ZT 9. Directly or indirectly (via PKC) DOG either activated hyperpolarizing ion channels or inactivated depolarizing ion channels underlying the sensillum potential at ZT 9 but not at ZT 1 .

\section{Different Doses of the DAG Analogs DOG and OAG Revealed That They Each Affected More Than One Ion Channel Underlying the Bombykal-Dependent Sensillum Potential}

To further distinguish respectively targeted ion channels different doses of the two DAG analogs were applied (Figures 3A-D; Table 2; S2). We wanted to determine whether both analogs had dose-dependent effects on ion channels that generated the bombykal-dependent sensillum potential. The rational was that each analog has differential specificity for different TRP channels and for PKC. Thus, we expected that, e.g., OAG activates dose-dependently TRP channels and DOG activates dose-dependently PKC. Surprisingly, at both ZTs tested, none of the two components showed dose-dependent effects. Thus, each component either directly or indirectly affected the same ion channel antagonistically. Alternatively, they antagonistically affected more than one ion channel. Both analogs (OAG: $n=34$; DOG: $n=40$ ) could either increase or decrease the sensillum potential amplitude, depending on the concentrations and depending on the time of day (Figures 3A-D; Table 2; S2). Since the different doses of OAG $(n=34)$ showed the same distinct effects at both ZTs, just being more effective at ZT 1, the OAG-dependent ion channels were present and available at 
late activity (ZT 1)

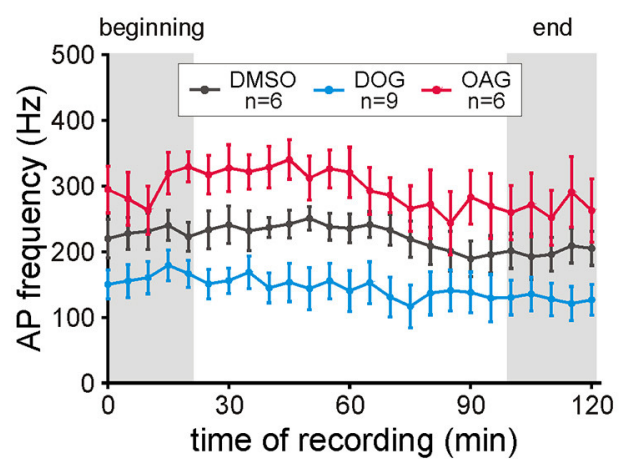

C

rest (ZT 9)

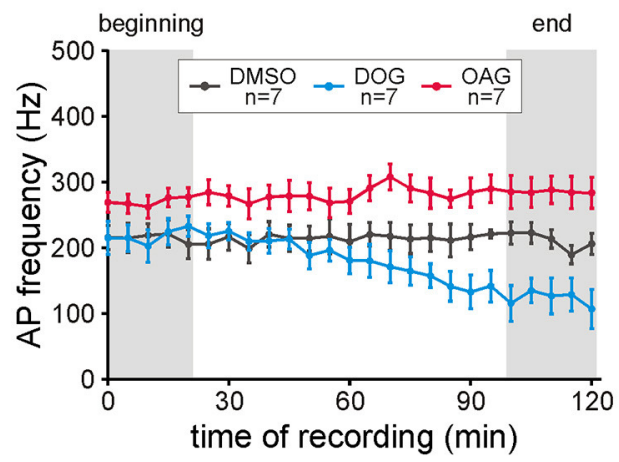

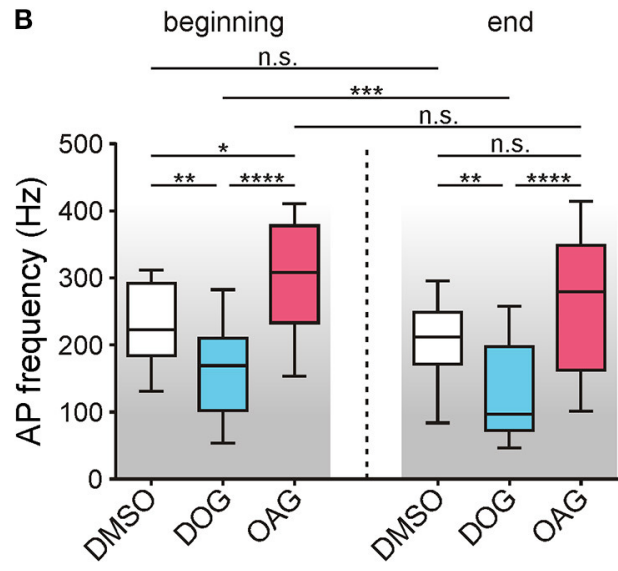

D

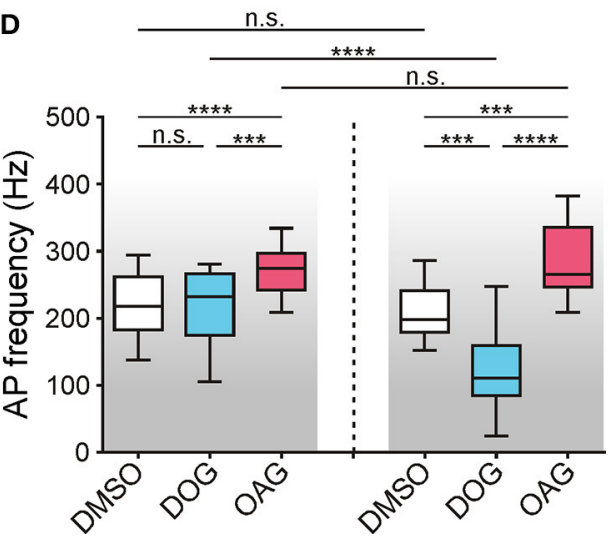

FIGURE 4 | The phasic pheromone response was increased by OAG but decreased by DOG. (A,B) The phasic pheromone response (=frequency of the first 6 bombykal (BAL)-dependent action potentials (APs)) was stable in recordings during the late activity phase (ZT 1) for controls as well as for application of $100 \mu \mathrm{M}$ OAG. However, with OAG phasic pheromone responses were significantly elevated in comparison to the controls at the beginning- and at the end of the recordings. Infusion of $100 \mu \mathrm{M}$ DOG significantly reduced the phasic pheromone response as compared to controls already during the first 20 min and further decreased it toward the end of the recordings. (C,D) In the first hour of recordings during the animals' resting phase (ZT 9) application of $100 \mu \mathrm{M} O A G$ significantly increased the AP frequencies while $100 \mu \mathrm{M}$ DOG had no effect. In the second hour of the recordings DOG continuously decreased the frequency of the first 6 BAL-dependent APs while in controls as well as with $100 \mu \mathrm{M}$ OAG the levels were unchanged (for exact $P$-values see Table 3; for mean values \pm SEM see S3. n.s. $=$ not significant; ${ }^{\star} P<0.05$; ${ }^{\star \star} P<0.01$; ${ }^{\star \star \star} P<0.001 ;{ }^{* \star \star \star} P<0.0001$; Wilcoxon test, Mann-Whitney test or Kruskal-Wallis test with Dunn's post-hoc test for multiple comparison).

both ZTs, with higher abundance at ZT 1 . At both ZTs $1 \mu \mathrm{M}$ OAG decreased the sensillum potential amplitude $(n=11)$, higher concentrations increased it, with $100 \mu \mathrm{M}$ being most effective $(n=13)$. Thus, at least $100 \mu \mathrm{M} \mathrm{OAG}$ are necessary to open a cation channel increasing the depolarization and apparently also the $\mathrm{Ca}^{2+}$ influx of the bombykal-dependent sensillum potential. Since $200 \mu \mathrm{M}(n=10)$ were less effective, an aversive effect appeared to accumulate at higher OAG concentrations, consistent with accumulating concentrations of intracellular $\mathrm{Ca}^{2+}$ or with PKC-dependent ion channel closure. At concentrations of $1 \mu \mathrm{M}$ DOG $(n=13)$ had the same aversive effects as OAG $(n=11)$, but to a larger extent. In addition, DOG was more effective at ZT 9, in contrast to OAG. Since direction of effects and strength of effects were not similar at both ZTs for both agonists, they appeared to not affect the same targets. At $100 \mu \mathrm{M}$ DOG had no effect at ZT $1(n=9)$.
Slightly, but significantly $100 \mu \mathrm{M}$ DOG reduced the bombykaldependent sensillum potential amplitude at ZT $9(n=7)$. In contrast, at $200 \mu \mathrm{M}$ DOG significantly reduced the bombykaldependent sensillum potential amplitude at ZT $1(n=5)$, while it strongly increased it at ZT 9 (Figures 3A-D; Table 2; S2; $n=5$ ). In summary, OAG and DOG targeted not the same ion channels. The OAG-dependent ion channels were more abundant at ZT 1, while the DOG-dependent ion channels were more abundant at ZT 9. Depending on the dose, the two analogs affected at least two different ion channels each. Or, they affected the same analog-dependent ion channel antagonistically at different concentrations. At $100 \mu \mathrm{M}$ concentrations the effects of DOG and OAG differed most strongly. Thus, $100 \mu \mathrm{M}$ concentrations of the analogs were suited best to distinguish different DAG-dependent targets in the hawkmoth's pheromone transduction cascade. 
TABLE 3 | Statistical analysis of the AP frequency in the presence or absence of $100 \mu$ M DOG or OAG during the late activity phase and at rest (Figure 4).

\begin{tabular}{|c|c|c|c|c|}
\hline \multicolumn{2}{|c|}{ Compared groups } & \multicolumn{2}{|c|}{$P$-value } & \multirow[t]{2}{*}{ Test } \\
\hline & & Beginning (0-20 min) & End (100-120 min) & \\
\hline \multicolumn{5}{|c|}{ LATE ACTIVITY (ZT 1) } \\
\hline DMSO & $100 \mu \mathrm{M}$ DOG & ${ }^{\star \star} P=0.0019$ & ${ }^{\star \star} P=0.0018$ & $\begin{array}{l}\text { Kruskal-Wallis test with } \\
\text { Dunn's post hoc test }\end{array}$ \\
\hline DMSO & $100 \mu \mathrm{M} O A G$ & ${ }^{*} P=0.0221$ & n.s. $P=0.1300$ & \\
\hline $100 \mu \mathrm{M}$ DOG & $100 \mu \mathrm{M} O A G$ & ${ }^{\star \star \star \star} P<0.0001$ & ${ }^{\star \star \star \star \star} P<0.0001$ & \\
\hline \multicolumn{5}{|c|}{ Beginning vs. End } \\
\hline DMSO & DMSO & n.s. $P=0.4515$ & & Mann-Whitney test \\
\hline $100 \mu \mathrm{M}$ DOG & $100 \mu \mathrm{M} D O G$ & ${ }^{* \star *} P=0.0005$ & & Mann-Whitney test \\
\hline $100 \mu \mathrm{M}$ OAG & $100 \mu \mathrm{M} O A G$ & n.s. $P=0.1349$ & & Mann-Whitney test \\
\hline \multicolumn{5}{|l|}{ REST (ZT 9) } \\
\hline DMSO & $100 \mu \mathrm{M}$ DOG & n.s. $P>0.9999$ & ${ }^{\star \star \star} P=0.0003$ & $\begin{array}{l}\text { Kruskal-Wallis test with } \\
\text { Dunn's post hoc test }\end{array}$ \\
\hline DMSO & $100 \mu \mathrm{M} O A G$ & ${ }^{\star \star \star \star \star} P<0.0001$ & ${ }^{\star \star \star \star} P=0.0003$ & \\
\hline $100 \mu \mathrm{M}$ DOG & $100 \mu \mathrm{M} O A G$ & ${ }^{\star \star \star} P=0.0002$ & ${ }^{\star \star \star \star} P<0.0001$ & \\
\hline \multicolumn{5}{|c|}{ Beginning vs. End } \\
\hline DMSO & DMSO & n.s. $P=0.5843$ & & Mann-Whitney test \\
\hline $100 \mu \mathrm{M}$ DOG & $100 \mu \mathrm{M}$ DOG & ${ }^{* \star \star \star} P<0.0001$ & & Mann-Whitney test \\
\hline $100 \mu \mathrm{M} O A G$ & $100 \mu \mathrm{M} O A G$ & n.s. $P=0.0708$ & & Mann-Whitney test \\
\hline
\end{tabular}

Level of significance: $\alpha=0.05$; n.s. $=$ not significant; ${ }^{*} P<0.05 ;{ }^{* \star} P<0.01 ;{ }^{\star \star \star *} P<0.001 ;{ }^{* \star \star *} P<0.0001$.

\section{OAG Increased the Phasic Bombykal Response}

The pheromone-dependent depolarizing receptor potentials elicit the different kinetic phases of the action potential response. It has to be kept in mind, though, that the sensillum potential does not just reflect the receptor potentials (Dolzer et al., 2003). We focused on the phasic action potential response (=phasic pheromone response) since only the first few spikes of the pheromone response encode stimulus concentration (Dolzer et al., 2003). The phasic pheromone response in control recordings was stable over the $120 \mathrm{~min}$ long recordings at ZT 1 and ZT 9 (Figures 4A,C; $n=13$ ). The median value of controls at the beginning of recordings during the activity phase (Table 3 ) was significantly higher than at the beginning of the resting phase (Table 3; S3). Application of $100 \mu \mathrm{M}$ DOG significantly decreased the AP frequency compared to the controls during the activity phase, already at the beginning of the recording (Figures 4A,B; Table 3; S3). During rest this DOG-dependent decline occurred only at the end of the recordings (Figures 4C,D; Table 3; S3). In contrast, infusion of $100 \mu \mathrm{M}$ OAG elevated the AP frequency slightly during the activity phase (Figures $4 A, B$ ). During rest (Figures 4C,D) application of OAG resulted in a highly significant increase of the AP frequency compared to the controls. In recordings with OAG $(100 \mu \mathrm{M})$ at ZT 1 (Table 3; S3) and ZT 9 (Table 3; S3) there was no significant difference between the median values at the beginning and at the end of the recordings (Figures $4 \mathrm{~A}-\mathrm{D}$ ). Only during application of DOG $(100 \mu \mathrm{M})$ a significant decline between the first and the last 5 stimulations could be observed at ZT 1 as well as at ZT 9 (Table 3; S3). In summary, at both ZTs $100 \mu \mathrm{M}$ OAG increased the frequency of the phasic pheromone response and also rendered it more phasic (Figure 1B). In contrast, $100 \mu \mathrm{M}$ DOG decreased it faster at ZT 1 as compared to ZT 9. Strongest effects of both analogs occurred at the end of the recording during rest, at ZT 11. Thus, OAG- and DOG-dependent targets were available ZT-dependently.

\section{Only $100 \mu$ M OAG Increased the Phasic Pheromone Response at Both ZTs, While All Other Doses Tested of Both OAG and Dog Decreased It}

At the late activity phase increasing doses of DOG (1-,100-, $200 \mu \mathrm{M})$ dose-dependently and significantly decreased the phasic pheromone response (Figures $\mathbf{5 A}, \mathbf{B}$; S4, 5; $n=21$ ). At rest, however, the decreasing effect of $100 \mu \mathrm{M}$ DOG was significantly smaller as compared to 1 and $200 \mu \mathrm{M}$ (Figures 5C,D; S4, 5; $n=20)$. This indicated that at ZT 9, as compared to ZT 1 an additional target or ion channel was affected antagonistically by DOG. In contrast, at both ZTs $100 \mu \mathrm{M}$ OAG $(n=13)$ increased the phasic pheromone response while at both ZTs all other concentrations tested decreased it (Figures 5A-D; S4, 5; $n=21)$. Thus, OAG had antagonistic effects either on the same or different ion channels. Furthermore, there was no direct correlation between DAG analog-dependent changes in the sensillum potential and the action potential response at both ZTs (Figures 3A-D, 5A-D). In summary, at both ZTs at least two different ion channels with antagonistic effects on the phasic pheromone response were affected by DOG and OAG, each. Furthermore, the sensillum potential clearly does not represent the receptor potential of the ORN since there were no direct 
A

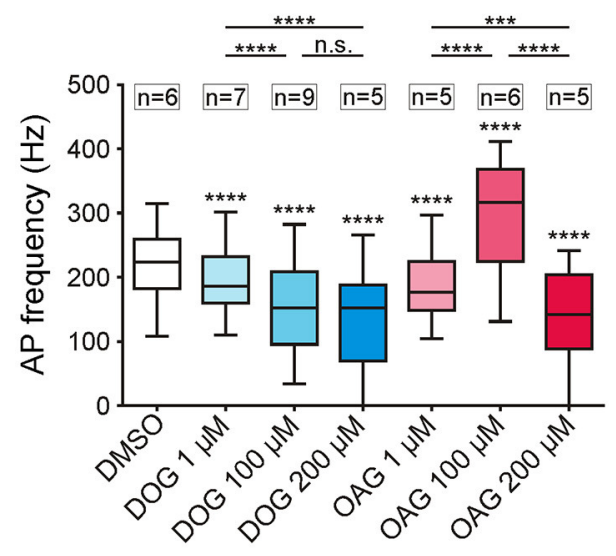

C

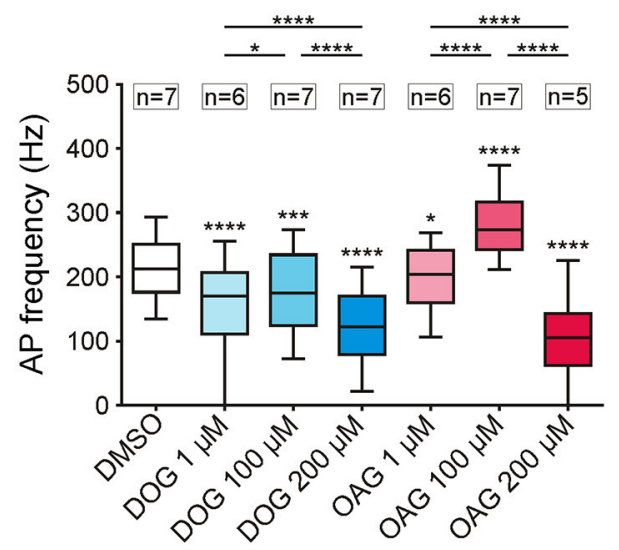

B

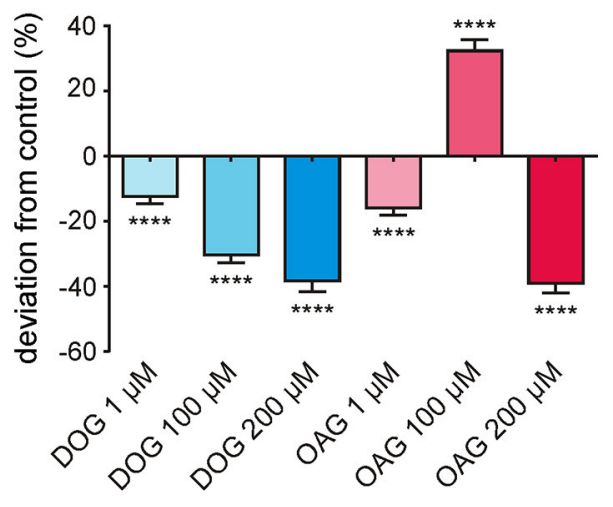

D

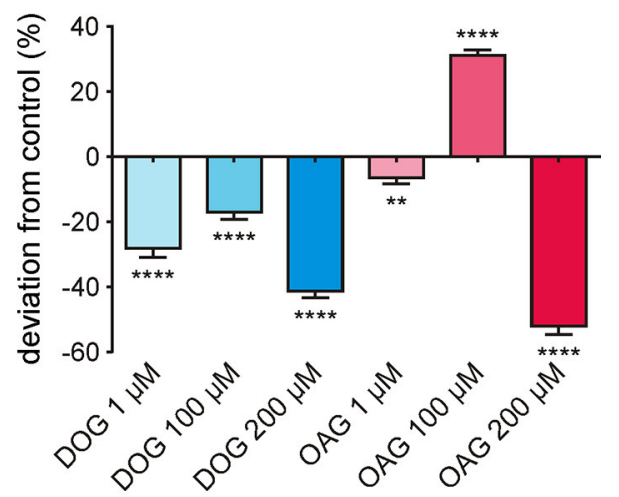

FIGURE 5 | Only $100 \mu$ M OAG increased the phasic pheromone response while all other concentrations of both DAG-analogs caused reductions. (A,C) Box-plots show the phasic pheromone response [=frequency of the first 6 bombykal-dependent action potentials (APs)] over the $2 \mathrm{~h}$ recordings, with or without (control) infusion of different concentrations of DOG or OAG with $0.1 \%$ DMSO. A significant decrease in the phasic pheromone response was found for all concentrations of DOG (1, $100,200 \mu \mathrm{M}$ ) as well as for 1 and $200 \mu \mathrm{M}$ OAG at both Zeitgebertimes. Only application of $100 \mu \mathrm{M}$ OAG increased the phasic pheromone response significantly during the late activity phase and at rest. (B,D) The percentage change illustrates that the highest concentrations of either DAG-analog resulted in the strongest reduction of the AP frequencies in comparison to the controls. Solely $100 \mu \mathrm{M}$ OAG elevated the frequencies by about $30 \%$ in the late activity phase as well as during rest (for mean values \pm SEM see S4; for exact $P$-values see S5; n.s. = not significant; ${ }^{*} P<0.05$; ${ }^{\star \star} P<0.01$; ${ }^{\star \star \star} P<0.001$; ${ }^{\star \star \star \star} P<0.0001$; Wilcoxon test,

Mann-Whitney test or Kruskal-Wallis test with Dunn's post-hoc test for multiple comparison).

correlations between both the bombykal-dependent sensillum potential and the bombykal-dependent action potential response.

\section{The Distribution of Action Potentials Revealed Faster Kinetics With OAG and Slower With DOG}

In post-stimulus time histograms (PSTHs) the distribution of bombykal-dependent APs occurring within the first $1000 \mathrm{~ms}$ after stimulation was analyzed (Figures 6A-D). During the course of the 2 -h recordings in the activity phase (ZT 1) the response pattern of control recordings changed from phasic to more tonic responses (Figures 6A,B). Application of $100 \mu \mathrm{M}$ DOG intensified this trend to tonic responses at the end of recordings during rest (Figures 6C,D) and directly from the beginning of recordings during the activity phase (Figures 6A,B). This became also apparent by the significantly reduced number of APs in the first $150 \mathrm{~ms}$ at the end of the resting phase (Figure 6D; $P<0.0001$ ) and during the first 5 stimulations at the activity phase (Figure 6A; $P<0.0001$ ). During the activity phase the distribution of APs under the influence of $100 \mu \mathrm{M}$ OAG showed a similar pattern as the control recordings resulting in equal numbers of APs during the first $150 \mathrm{~ms}$ of the responses (Figure 6A). When OAG was applied at rest the phasic character of the response was maintained throughout the recordings (Figures 6C,D) and the number of APs during the first $150 \mathrm{~ms}$ was significantly increased compared to controls (Figures 6C,D; beginning: $P<0.0001$, end: $P=0.0018)$. In summary, $\mathrm{OAG}$ 


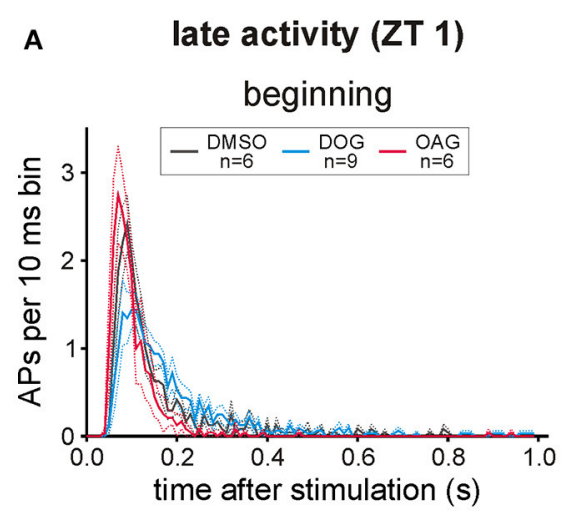

C

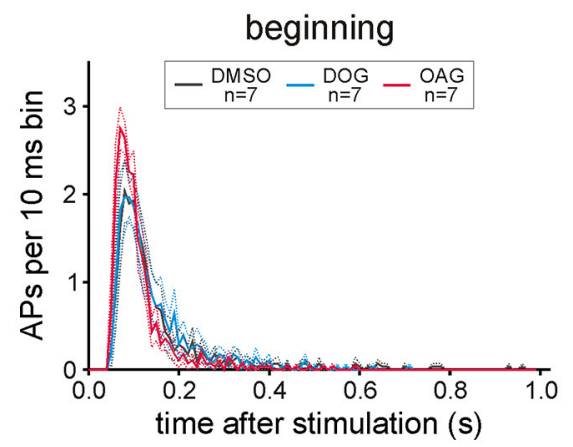

B late activity (ZT 3)

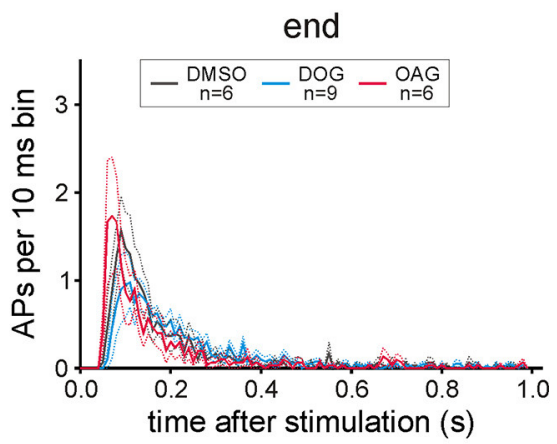

D

rest (ZT 11)

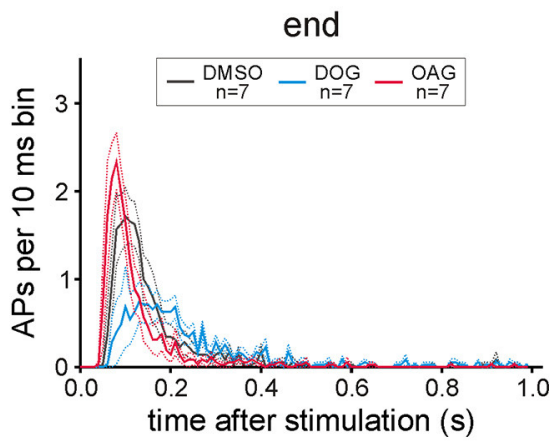

FIGURE 6 | Both diacylglycerol-analogs affected the distribution of bombykal (BAL)- dependent action potentials (APs). In post-stimulus time histograms the mean number of APs in $10 \mathrm{~ms}$ bins was investigated for $1 \mathrm{~s}$ after BAL stimulation in the first- and last $20 \mathrm{~min}$ of each recording. (A) While in the first 20 min of recordings in the late activity phase (ZT 1) application of $100 \mu \mathrm{M}$ OAG (magenta) only slightly increased and sped up the phasic-tonic response pattern, $100 \mu \mathrm{M}$ DOG (cyan) reduced the number of APs per bin and rendered the response more tonic. (B) This shift to slower kinetics and an adapted AP response could be seen at the end of the recordings during the late activity phase (ZT 3), also in controls and with OAG, but strongest with DOG. (C,D) Also at rest (ZT 9) the pheromone responses were stronger and faster in the presence of OAG, as compared to controls and DOG infusion. Instead, application of DOG resulted in a strong reduction of APs per bin, which resulted in a decreased more tonic pheromone response especially at the end of recordings at ZT 11 (solid lines $=$ mean; dotted lines $=$ SEM).

rendered the pheromone-dependent action potential response more phasic increasing it at all ZTs while DOG rendered it less phasic ZT-dependently. DOG slowed it down and decreased it the most at the end of the long-term recordings at ZT 11.

\section{The Latency of the First Bombykal-Dependent Action Potential Was
Delayed by DOG but Not by OAG} For temporal information processing the timing of the first stimulus-dependent action potential is most relevant. Thus, we analyzed how the DAG analogs affect the latency of the first bombykal-dependent action potential with respect to the start of the sensillum potential (Figures 7A-D; Table 4; S6). At all ZTs tested compared to vehicle controls $(n=13)$ OAG application $(100 \mu \mathrm{M})$ did not significantly affect the latency of the pheromone response $(n=13)$ (Figures 7A-D; Table 4; S6). However, comparison of the overall recordings resulted in significantly lower latencies in the presence of $\mathrm{OAG}$, decreasing the scatter of timing $(P<0.0001$; Table 4 ; S6). In contrast, infusion of DOG increased the latency of the first pheromonedependent spike at all ZTs $(n=16)$, but strongest at the end of the recording at rest, around ZT $11(n=7)$. In summary, while DOG increased the latency of the bombykal response ZT-dependently with the strongest effect at the end of the recording during rest, OAG rather decreased it.

\section{Only DOG but Not OAG Decreased the Late, Long-Lasting Pheromone Response}

The late, long-lasting pheromone response occurs seconds to hours after the pheromone stimulus, not encoding stimulus concentration and duration, but rather being an hour-long reminder of the stimulus occurrence (reviews: Stengl, 2010; Stengl and Funk, 2013). Infusion of the DAG analog OAG had no significant effects on the late, long-lasting bombykal response compared to vehicle controls $(n=13)$ at all ZTs tested $(n=13)$ (Figures 8A-D; Table 5; S7). In contrast, application of DOG significantly decreased the late, long-lasting bombykal response at all ZTs tested $(n=16)$ (Figures 8A-D; Table 5; S7). The strongest effect of DOG occurred at the end of the 
A

late activity (ZT 1)

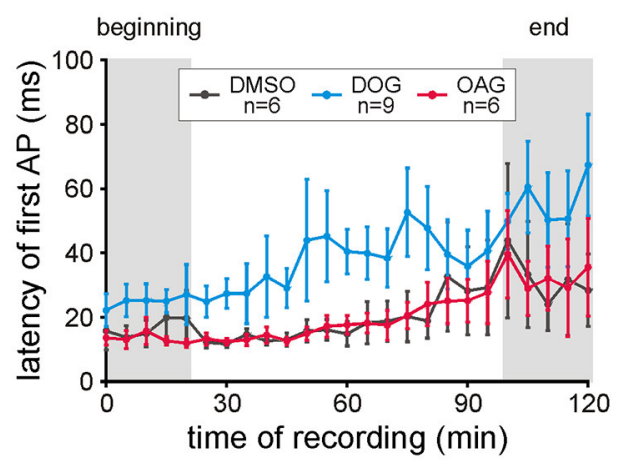

C

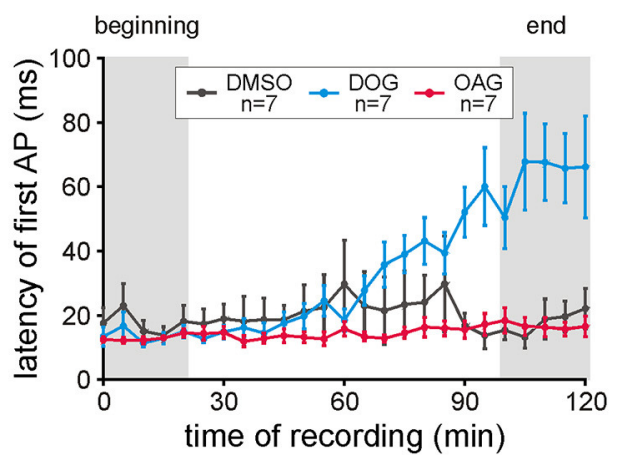

B

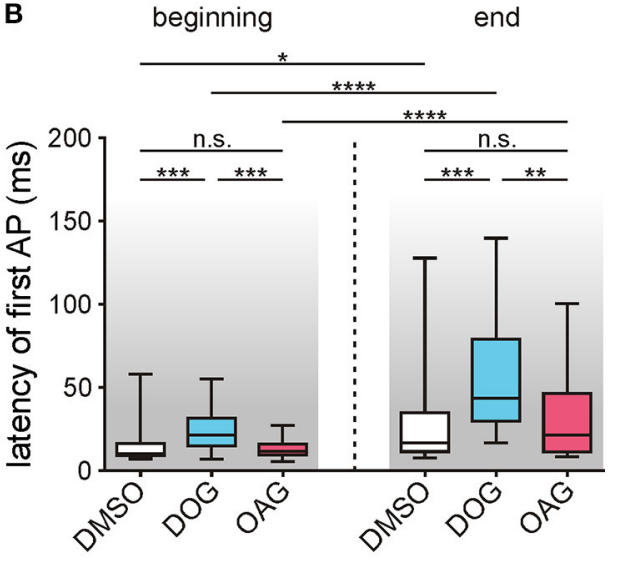

D

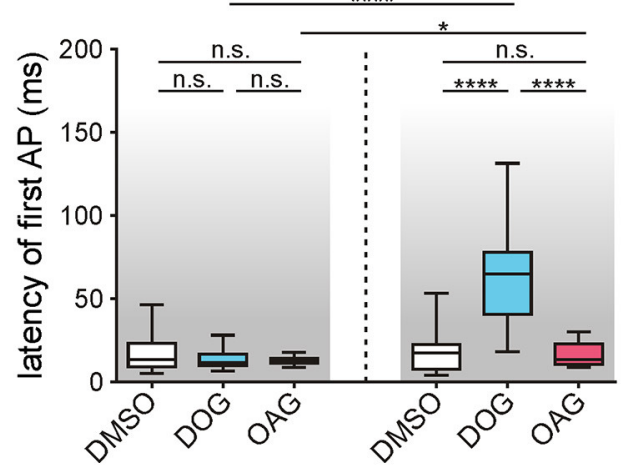

FIGURE 7 | The latency of the first bombykal-dependent action potential (AP) increased only with DOG infusion. (A,B) During the late activity phase the latency of the first pheromone-dependent AP increased during the course of time in control recordings with $0.1 \%$ DMSO $(n=6)$ as well as with application of $100 \mu \mathrm{M}$ DOG $(n=9)$ or $100 \mu \mathrm{M} \mathrm{OAG}(n=6)$. In comparison to the controls only DOG significantly increased the latencies while application of OAG showed no significant differences. (C,D) In control recordings during rest $(n=7)$ no changes in the latency occurred during the course of the recordings and only a small increase was found under the influence of OAG $(n=7)$. However, application of DOG strongly and significantly increased the latency of the first AP in the last 20 min of the recordings (for exact $P$-values see Table 4; for mean values \pm SEM see S6. n.s. $=$ not significant; ${ }^{\star} P<0.05$; ${ }^{\star \star} P<0.01$; ${ }^{\star \star \star} P<0.001$; ${ }^{\star \star \star \star} P<0.0001$; Wilcoxon test, Mann-Whitney test or Kruskal-Wallis test with Dunn's post-hoc test for multiple comparison).

recording at rest (Figures $\mathbf{8 C}, \mathbf{D}$; Table 5; S7), around ZT 11, where DOG almost abolished the late, long-lasting pheromone response $(n=7)$. In summary, while the DAG analog OAG had no effects, DOG significantly decreased the late, long-lasting pheromone response at both ZTs. At the end of the long-term tip-recordings at rest, DOG effects were strongest.

\section{The DAG Analogue OAG Significantly Increased Spontaneous Activity at Rest}

Insect ORNs can express spontaneous APs also in the absence of pheromone stimuli, however with quite strong variability. Thus, due to the wide scattering of values which appeared in all tip-recordings (Figure 9A) spontaneous activity was recorded for 40 min under control conditions with $0.1 \%$ DMSO, without application of pheromone pulses. After control recordings, the recording capillary was switched and the activity of the same sensillum was investigated for another $40 \mathrm{~min}$ in the presence of either $100 \mu \mathrm{M}$ DOG or $100 \mu \mathrm{M}$ OAG. The mean spontaneous activity of untreated ORNs ranged from $19.97 \pm 6.04$ APs at ZT 1 to $72.65 \pm 27.14$ APs in $295 \mathrm{~s}$ during rest (S8). Hence, the activity of ORNs is more than 10 -fold increased after stimulation with $1 \mu \mathrm{g}$ BAL $(\mathrm{S} 7,8)$. Application of $100 \mu \mathrm{M}$ DOG either increased or decreased spontaneous activity (Figure 9A). In most cases only small changes in spontaneous activity were observed in the late activity phase $(n=10)$ as well as during rest $(n=10)$. In some recordings, especially when activity was low in controls during the activity phase an increased activity was measured in the presence of DOG (Figure 9A). With respect to controls DOG expressed non-significant increases at ZT 1 (Table 6) and during rest almost no changes occurred (Figure 9B; Table 6; S8). Infusion of $100 \mu \mathrm{M}$ OAG almost always increased the spontaneous activity in comparison to the previous control (Figure 9B; Table 6; S8). During the late activity phase mean values for spontaneous activity were elevated with OAG application $(n=7)(\mathrm{S} 8)$. Spontaneous activity of some control recordings were higher at rest, nevertheless, here the 
TABLE 4 | Statistical analysis of the latencies of the first bombykal-induced AP in the presence or absence of $100 \mu \mathrm{M}$ DOG or OAG during the late activity phase and at rest (Figure 7).

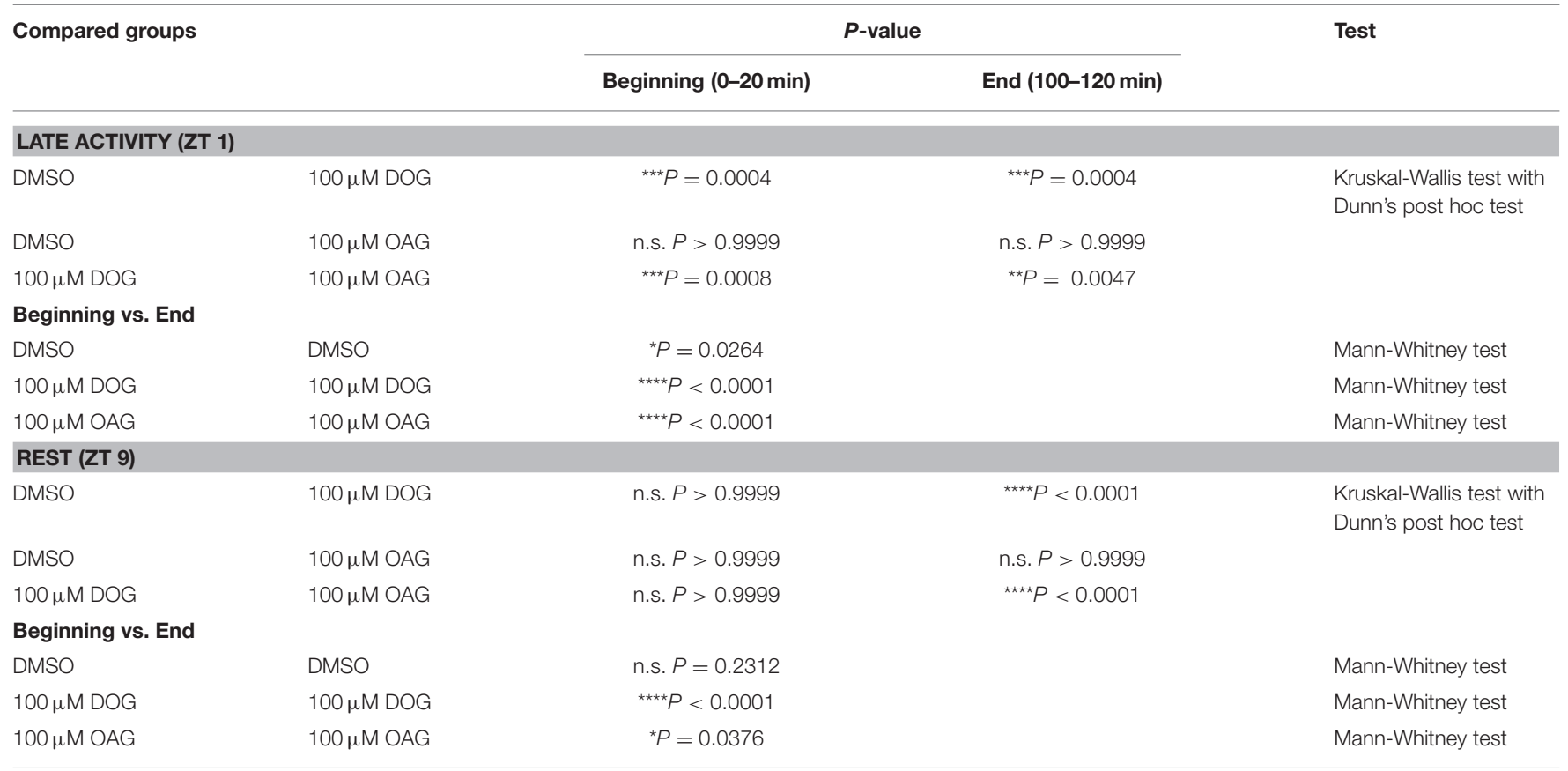

Level of significance: $\alpha=0.05$; n.s. $=$ not significant; ${ }^{*} P<0.05 ;{ }^{* *} P<0.01 ;{ }^{* *+*} P<0.001 ;{ }^{* * *+*} P<0.0001$.

OAG-dependent increases were even stronger $(n=9)$ (Table 6; S8). This increase was significant for the absolute values as well as for the percentage change (Table 6; S8). In summary, there was very strong scatter for absolute values of spontaneous activity at different ZTs recorded and OAG or DOG applications showed strongly variable, sometimes antagonistic responses. Thus, only at rest $\mathrm{OAG}$ significantly increased spontaneous activity, most likely via $\mathrm{Ca}^{2+}$-dependent effects.

\section{DISCUSSION}

With tip-recordings of antennal trichoid sensilla in vivo it was examined whether two DAG analogs, DOG and OAG, modulated parameters of the primary events of bombykal transduction of male $M$. sexta hawkmoths. Recordings were performed at two different Zeitgebertimes (ZTs), because ORNs are circadian pacemaker neurons that regulate odor sensitivity in a daytime-dependent fashion. Since DAG has at least two different antagonistic functions, activation of $\mathrm{Ca}^{2+}$ permeable TRP ion channels and activation of PKC, we hoped to distinguish both functions with specific concentrations of DOG and OAG in hawkmoth pheromone transduction (review: Stengl, 2010). Indeed, at $100 \mu \mathrm{M}$ concentrations the two analogs preferentially recognized different targets in the pheromone transduction cascade, daytime-dependently. While $100 \mu \mathrm{M} \mathrm{OAG}$ likely opened a $\mathrm{Ca}^{2+}$-permeable TRP-like channel underlying the pheromone-dependent receptor potential, DOG potentially closed pheromone-dependent channels very likely via PKCdependent negative feedback. Thus, we hypothesize that OAG opens TRPC-type channels underlying pheromone transduction in $M$. sexta. In contrast, DOG activates PKC, and, thereby potentially closes TRPC-type ion channels which before were opened during the pheromone transduction cascade.

\section{The DAG Analogs OAG and DOG Appear Not to Affect Orco in M. sexta ORNs}

While it was suggested that insect odor transduction is mediated via an ionotropic mechanism based upon odor-gated activation of OR-Orco receptor ion channel complexes (Sato et al., 2008; Wicher et al., 2008), in hawkmoths previously we found no evidence for a role of the 7 transmembrane channel Orco in primary processes of pheromone transduction (Nolte et al., 2013, 2016). Instead, Orco appears to be a voltage-dependent ion channel that opens during the late, long-lasting pheromone response but not during the first few hundred ms of the phasic pheromone response. Since Orco agonists and antagonists were mostly effective during the activity phase of the hawkmoth, more Orco protein appears to be available during the night and early day as compared to the middle of the day. In addition, Orco constitutes a spontaneously opening $\mathrm{Ca}^{2+}$-permeable non-specific cation channel with a reversal potential around $0 \mathrm{mV}$. Thus, Orco depolarizes the membrane potential, thereby controlling spontaneous activity of hawkmoth and fruitfly ORNs. Furthermore, Orco appears to be opened voltage-, and second messenger-dependently (Larsson et al., 2004; Benton et al., 2007; Sato et al., 2008; Wicher et al., 2008; Deng et al., 2011; Jones et al., 2011; Sargsyan et al., 2011; Nolte et al., 2013, 2016). In addition to the primary ionotropic odor transduction, Wicher et al. (2008) suggested an additional slower transduction via OR coupling to $\mathrm{G}_{\alpha \mathrm{s}}$ resulting in elevation of cAMP levels. In $D$. 
A

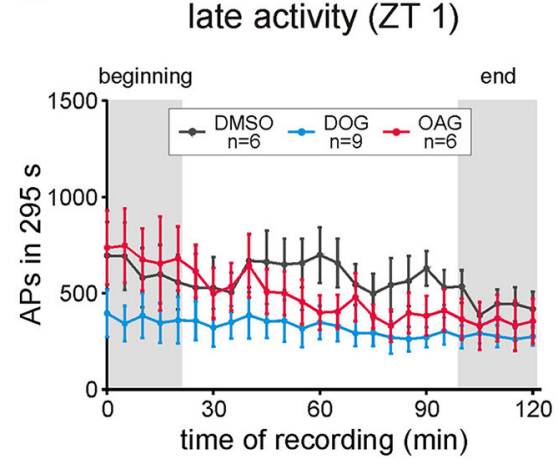

C

rest (ZT 9)

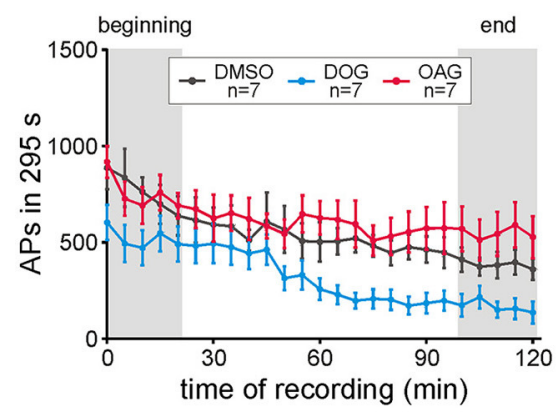

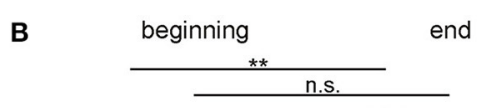

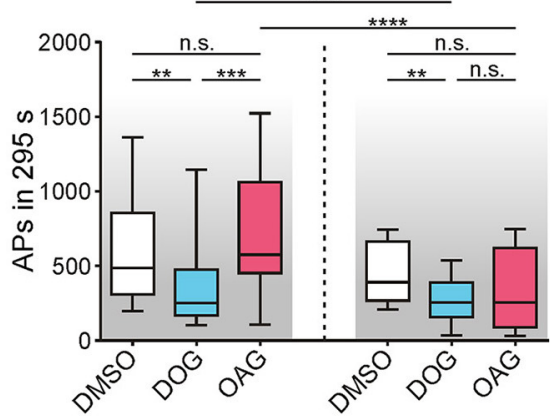

D

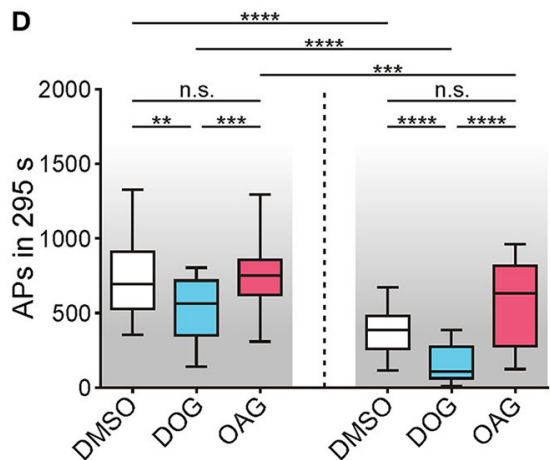

FIGURE 8 | Only the diacylglycerol-analog DOG but not OAG decreased the late, long-lasting pheromone response (LLPR) at both Zeitgebertimes (ZTs). The number of APs between $5 \mathrm{~s}$ and $300 \mathrm{~s}$ after each stimulation with $1 \mu \mathrm{g}$ bombykal was evaluated and statistical analysis was performed for the first and last 20 min of the recordings (gray areas, $\mathbf{A}, \mathbf{C})$. (A,B) During the late activity phase the LLPR decreased in controls $(n=6)$ and during application of $100 \mu M$ OAG $(n=6)$ but no significant differences were found when comparing both groups. However, $100 \mu \mathrm{M} \mathrm{DOG}(n=9)$ highly significantly decreased the LLPR in comparison to the controls but showed no changes over the course of time. $(\mathbf{C}, \mathbf{D})$ In recordings during rest the LLPR continuously decreased in controls $(n=7)$ as well as with DOG $(n=7)$ or OAG $(n=7)$. Again, application of OAG did not change the LLPR in comparison to the controls while with DOG a significant reduction and steady decline occurred in the first and the last 20 min of the recordings (for exact $P$-values see Table 5; for mean values \pm SEM see S7. n.s. $=$ not significant; ${ }^{\star} P<0.05$; ${ }^{\star \star} P<0.01$;

${ }^{\star \star \star} P<0.001 ;{ }^{\star \star \star \star} P<0.0001$; Wilcoxon test, Mann-Whitney test or Kruskal-Wallis test with Dunn's post-hoc test for multiple comparison).

melanogaster Orco needs to be phosphorylated PKC-dependently first, before it can be activated by cGMP and cAMP (Sargsyan et al., 2011). Thus, OAG, as well as phorbol ester (PMA) can activate Orco channels PKC-dependently in fruitflies. However, it is unlikely that in our experiments OAG or DOG activated Orco. Since in the hawkmoth OAG did not affect the late, long-lasting pheromone response at ZT 1 and ZT 9, it can be assumed that OAG does not affect Orco. In addition, since DOG decreased the late, long-lasting pheromone response at both ZTs with strongest effect at ZT 11, it is also unlikely that it mediated its effect via Orco activation or Orco inactivation since more Orco protein is present during the activity phase of the hawkmoth. Furthermore, also the OAG-dependent activation of spontaneous activity which only occurred at ZT 9 cannot predominantly be mediated via Orco, since apparently Orco abundance at ZT 1 is higher as compared to ZT 9 (Nolte et al., 2013, 2016). Therefore, we suggest that additional TRP-like ion channels are activated during the late, long-lasting pheromone response and that $\mathrm{Ca}^{2+}$-dependent ion channels such as TRP-like ion channels are also involved in the generation of spontaneous activity of ORNs, next to Orco. Since our pharmacological studies cannot exclude additional effects on so far unknown targets, molecular identification and manipulation of Orco and TRP-like ion channels in the hawkmoth are necessary to further characterize pheromone transduction cascades. So far, our studies do support a role of Orco as pacemaker channel in hawkmoth ORNs that controls the resting membrane potential, but not as the primary channel of pheromone transduction. Future studies need to separate different possible functions of Orco in different insect species as chaperon that locates and maintains ORs in ciliary membranes, as pacemaker channel affecting membrane potential, and as odor receptor-ion channel complex during the primary events of transduction.

\section{Pheromone Transduction in Hawkmoth ORNs Involves Phospholipase C-Dependent Signaling Cascades Activating Several Ion Channels With Properties of TRP-Type Channels}

Biochemical studies demonstrated that pheromone application to cockroach and moth antennae caused rapid and transient 
TABLE 5 | Statistical analysis of the late, long-lasting pheromone response in the presence or absence of $100 \mu \mathrm{M}$ DOG or OAG during the late activity phase and at rest (Figure 8).

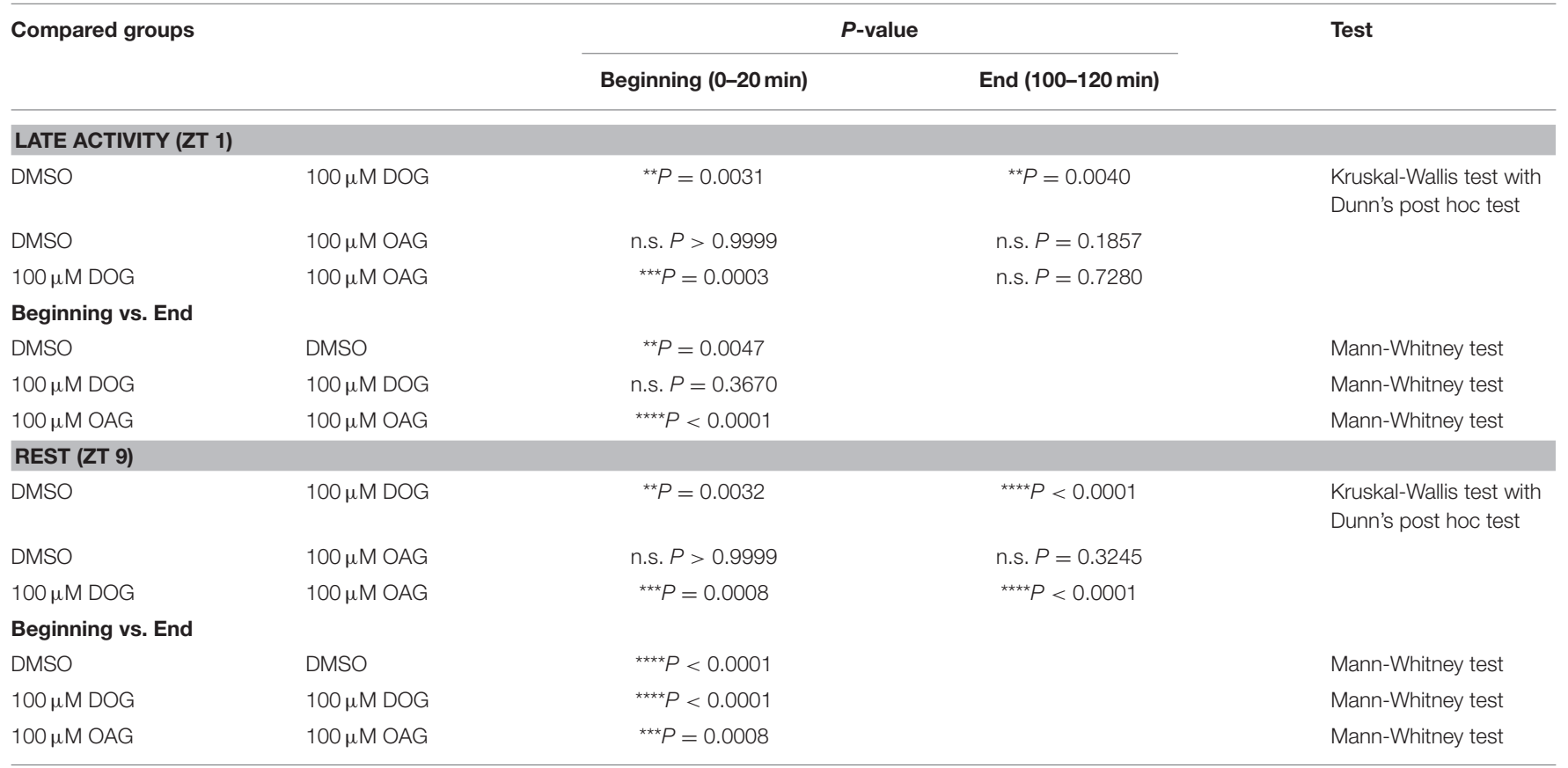

Level of significance: $\alpha=0.05$; n.s. $=$ not significant; ${ }^{*} P<0.05 ;{ }^{* *} P<0.01$; ${ }^{* \star *} P<0.001$; ${ }^{* * \star *} P<0.0001$.

A late activity (ZT 1)

rest (ZT 9)

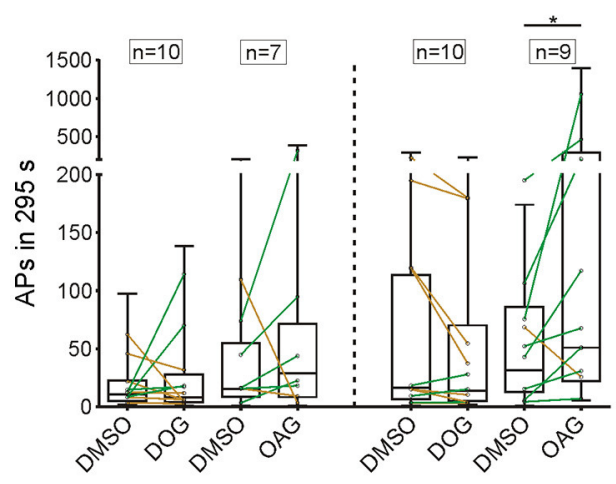

B late activity (ZT 1)

rest (ZT 9)

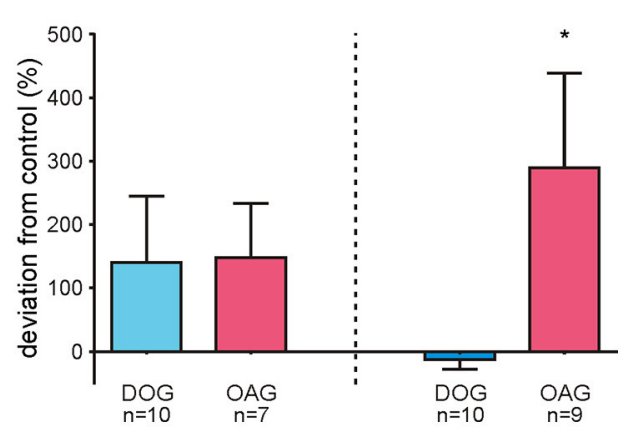

FIGURE 9 | OAG significantly increased the spontaneous activity during rest. (A) The number of APs in $295 \mathrm{~s}$ intervals was measured under control conditions and with $100 \mu \mathrm{M}$ DOG or OAG consecutively from the same sensillum. Mean values of single recordings are shown as connected dots and distribution of all values is represented as box plots. Both DAG-analogs either increased (green lines) or decreased (yellow lines) spontaneous activity in recordings at ZT 1 and ZT 9. Only during the resting phase application of $100 \mu \mathrm{M}$ OAG $(n=9)$ significantly increased the spontaneous activity of olfactory receptor neurons. (B) The deviation from the respective control is shown as percentage change for each dataset. Although both DAG-analogs increased the spontaneous activity in the late activity phase this change was not significant. During rest application of DOG $(n=10)$ had no effect on the number of spontaneous APs while in the presence of OAG $(n=9)$ the deviation from controls was significant and the spontaneous activity increased by almost 300\% (for exact $P$-values see Table 6; for mean values \pm SEM see S8. n.s. = not significant; ${ }^{\star} P<0.05 ;{ }^{* \star} P<0.01 ;{ }^{\star \star \star} P<0.001 ;{ }^{* \star \star *} P<0.0001$; Wilcoxon test).

rises in $\mathrm{IP}_{3}$ levels (Boekhoff et al., 1990, 1993; Breer et al., 1990). Thus, activation of pheromone receptors activates PLC G-protein dependently in insect antennae. Pheromone application to hawkmoth ORNs in primary cell culture elicited a stereotyped sequence of directly and indirectly $\mathrm{Ca}^{2+}$ dependent inward currents. The first pheromone-dependent current promoted $\mathrm{Ca}^{2+}$ influx at positive potentials. This very transient pheromone-dependent $\mathrm{Ca}^{2+}$ channel was blocked by high concentrations of extracellular $\mathrm{Ca}^{2+}$ within ms. Also, inclusion of $\mathrm{IP}_{3}$ in the patch pipette directly or indirectly opened a very transient $\mathrm{Ca}^{2+}$ channel that was blocked by $\mathrm{Ca}^{2+}$ influx within ms in hawkmoth ORNs (Stengl, 1994; review: 
TABLE 6 | Statistical analysis of the spontaneous activity in the presence or absence of $100 \mu$ M DOG or OAG during the late activity phase and at rest (Figure 9).

\begin{tabular}{|c|c|c|c|c|}
\hline \multirow[t]{2}{*}{ Compared groups } & & \multicolumn{2}{|c|}{$P$-value } & \multirow[t]{2}{*}{ Test } \\
\hline & & Absolute value (a) & Deviation from control (\%) (b) & \\
\hline \multicolumn{5}{|c|}{ LATE ACTIVITY (ZT 1) } \\
\hline DMSO (before DOG) & $100 \mu \mathrm{M}$ DOG & n.s. $P>0.9999$ & n.s. $P=0.8652$ & Wilcoxon test \\
\hline DMSO (before OAG) & $100 \mu \mathrm{M} O A G$ & n.s. $P=0.3750$ & n.s. $P=0.1563$ & Wilcoxon test \\
\hline DMSO (before DOG) & DMSO (before OAG) & n.s. $P=0.1609$ & & Mann-Whitney test \\
\hline $100 \mu \mathrm{M}$ DOG & $100 \mu \mathrm{M} O A G$ & n.s. $P=0.3130$ & & Mann-Whitney test \\
\hline \multicolumn{5}{|l|}{ REST (ZT 9) } \\
\hline DMSO (before DOG) & $100 \mu \mathrm{M}$ DOG & n.s. $P=0.0840$ & n.s. $P=0.3750$ & Wilcoxon test \\
\hline DMSO (before OAG) & $100 \mu \mathrm{M} O A G$ & ${ }^{\star} P=0.0273$ & ${ }^{*} P=0.0195$ & Wilcoxon test \\
\hline DMSO (before DOG) & DMSO (before OAG) & n.s. $P=0.8260$ & & Mann-Whitney test \\
\hline $100 \mu \mathrm{M}$ DOG & $100 \mu \mathrm{M}$ OAG & n.s. $P=0.0786$ & & Mann-Whitney test \\
\hline
\end{tabular}

Level of significance: $\alpha=0.05$; n.s. $=$ not significant; ${ }^{\star} P<0.05 ;{ }^{\star \star} P<0.01$; ${ }^{\star \star \star} P<0.001 ;{ }^{\star \star \star \star} P<0.0001$.

Stengl, 2010). Since the $\mathrm{IP}_{3}$-dependent $\mathrm{Ca}^{2+}$ current mimicked the pheromone-dependent $\mathrm{Ca}^{2+}$ current also in hawkmoths bombykal receptors appear to couple to PLC. While it was demonstrated that this first pheromone-dependent $\mathrm{Ca}^{2+}$ channel cannot be identical to the unspecific cation channel Orco, however, its molecular identity remains unknown (Stengl, 1994; Nolte et al., 2013, 2016). Since different TRPC channels were shown to interact with $\mathrm{IP}_{3}$ receptors that are located to the endoplasmic reticulum (review: Ong et al., 2014), it is possible, that this first pheromone-dependent $\mathrm{Ca}^{2+}$ channel belongs to TRPC-like channels. Future studies need to test this hypothesis further and need to examine whether $\mathrm{IP}_{3}$ receptor activation is obligatory for bombykal responses in M. sexta in vivo.

Next, in hawkmoth ORNs the pheromone-dependent influx of $\mathrm{Ca}^{2+}$ elicited a $\mathrm{Ca}^{2+}$-dependent inward current with linear $\mathrm{I} / \mathrm{V}$ curve and reversal potential around $0 \mathrm{mV}$ indicative of an unspecific cation current. It expressed bimodal dependence on extracellular $\mathrm{Ca}^{2+}$. Increases of extracellular $\mathrm{Ca}^{2+}$ first activated, and, then, within seconds blocked this second pheromonedependent inward current (Stengl, 1993). While the molecular identity of its underlying pheromone-dependent ion channel is not known, again its properties resemble properties of TRP-type ion channels (review: Nilius and Flockerzi, 2014). The third pheromone-dependent inward current was also a nonspecific cation current with reversal potential around $0 \mathrm{mV}$. However, it was less $\mathrm{Ca}^{2+}$ selective than the second pheromonedependent current and was activated by PKC. While PKC closed previously opened pheromone-dependent TRP-like ion channels, during this later time window after pheromone application, it opened PKC-dependent cation channels that were less $\mathrm{Ca}^{2+}$. permeable (Stengl, 1993, 1994; Dolzer et al., 2008). Finally, strong or long pheromone stimuli elevate cGMP levels in ORNs that appeared to govern cGMP-dependent ion channel activity and closed other pheromone-dependent ion channels which were opened previously. The cGMP-dependent channels expressed much slower kinetics compared to the pheromoneactivated $\mathrm{Ca}^{2+}$ - or PKC-dependent ion channels and allowed for bombykal responses under conditions of long-term adaptation (Ziegelberger et al., 1990; Boekhoff et al., 1993; Stengl et al.,
2001; Dolzer et al., 2003; Flecke et al., 2006; Krannich and Stengl, 2008). Voltage- and $\mathrm{Ca}^{2+}$ - dependent $\mathrm{K}^{+}$- and $\mathrm{Cl}^{-}$channels then repolarized the ORNs (review: Stengl, 2010).

These different PLC- and pheromone-dependent ion channels in hawkmoth ORNs resembled properties of TRP/TRPL-type channels most closely. Our experiments with the DAG analogs are in accordance with our hypothesis that bombykal receptors couple to PLC and gate TRP-type ion channels. The non-linear, not dose-dependent but ZT-dependent effects of both, OAG and DOG, on the sensillum potential amplitude and the phasic action potential response indicated that several TRP-like ion channels may be involved in bombykal transduction in the hawkmoth $M$. sexta. We hypothesize that $1 \mu \mathrm{M}$ of DOG and OAG inhibited DAG-dependent TRPC ion channels such as channels resembling TRPC4 and TRPC5 (Storch et al., 2017). At $100 \mu \mathrm{M}$ DOG apparently additional DAG-dependent channels resembling TRP2, $-3,-6$, or -7 were activated that were more available at ZT 9 as compared to ZT 1. Also, OAG at $1 \mu \mathrm{M}$ concentrations inhibited channels resembling TRPC4 and TRPC5, but less effectively as compared to DOG. But at $100 \mu \mathrm{M}$ concentrations OAG activated at least one TRPC2, $-3,-6$, or -7 - like channel much more effectively compared to DOG. This OAG-dependent channel was more available at ZT 1 as compared to ZT 9. Further increase of the dose of OAG apparently caused desensitization of the OAG-dependent TRPC-like ion channels similar to the PKC-dependent TRPC7-desensitization (Zhang and Trebak, 2014). We do not know yet which and how many TRP-type ion channels are expressed in ORNs of the hawkmoth. However, since we could elicit a sequence of at least three different bombykal-dependent inward currents upon G-protein activation or after application of $\mathrm{IP}_{3}$ (Stengl, 1993, 1994), we expect at least 3 different TRPC-type ion channels that even may cooperate with $\mathrm{IP}_{3}$ dependent ion channels. The modulation of the different parameters of the bombykal response by $100 \mu \mathrm{M}$ OAG in vivo is most consistently explained via OAG-dependent activation of a strongly $\mathrm{Ca}^{2+}$ permeable TRP-type ion channel. This OAG-dependent TRP channel can be blocked either via PKC or $\mathrm{Ca}^{2+} /$ calmodulin, since increasing concentrations of OAG decreased the sensillum potential amplitude and the 
phasic action potential response. In addition, $100 \mu \mathrm{M}$ DOG effects are consistent with DOG-dependent activation of PKC and PKC-dependent closure of TRP-type ion channels activated by the pheromone transduction cascade. In the mammalian vomeronasal organ the DAG-dependently activated TRPC2 channel is the primary ion channel underlying pheromone transduction (Lucas et al., 2003). In addition, it was shown that $\mathrm{IP}_{3}$ receptor gating appeared not to be involved in TRPC2 activation (Chamero et al., 2017). Whether this is also true for $M$. sexta pheromone transduction and whether/how many TRP channels underly bombykal transduction remains to be examined.

\section{AUTHOR CONTRIBUTIONS}

PG performed all experiments, evaluated the data with statistics, prepared all Figures, and contributed to the final version of the

\section{REFERENCES}

Ackermann, F. (2008). TRP-/TRPL-Like ion Channels in the Antenna of the Hawkmoth Manduca sexta. Diploma thesis University of Marburg.

Ambudkar, I. S., de Souza, L. B., and Ong, H. L. (2017). TRPC1, Orail, and STIM1 in SOCE: friends in tight spaces. Cell Calcium 63, 33-39. doi: 10.1016/j.ceca.2016.12.009

Bell, R. A., and Joachim, F. G. (1976). Techniques for rearing laboratory colonies of tobacco hornworms and pink bollworms. Ann. Ent. Soc. Am. 69, 365-373.

Benton, R., Sachse, S., Michnick, S. W., and Vosshall, L. B. (2006). Atypical membrane topology and heteromeric function of Drosophila odorant receptors in vivo. PLoS Biol. 4:e20. doi: 10.1371/journal.pbio.0040020

Benton, R., Vannice, K. S., and Vosshall, L. B. (2007). An essential role for a CD36related receptor in pheromone detection in Drosophila. Nature 450, 289-293. doi: 10.1038/nature06328

Boekhoff, I., Seifert, E., Goggerle, S., Lindemann, M., Kruger, B. W., and Breer, H. (1993). Pheromone-induced 2nd-messenger signaling in insect antennae. Insect Biochem. Mol. Biol. 23, 757-762. doi: 10.1016/0965-1748(93)90063-X

Boekhoff, I., Strotmann, J., Raming, K., Tareilus, E., and Breer, H. (1990). Odorant-sensitive phospholipase C in insect antennae. Cell. Signal. 2, 49-56. doi: 10.1016/0898-6568(90)90032-6

Breer, H., Boekhoff, I., and Tareilus, E. (1990). Rapid kinetics of second messenger formation in olfactory transduction. Nature 345, 65-68. doi: 10.1038/345 $065 \mathrm{a} 0$

Chamero, P., Weiss, J., Alonso, M. T., Rodríguez-Prados, M., Hisatsune, C., Mikoshiba, K., et al. (2017). Type 3 inositol 1,4,5-trisphosphate receptor is dispensable for sensory activation of the mammalian vomeronasal organ. Sci. Rep. 7:10260. doi: 10.1038/s41598-017-09638-8

Chouquet, B., Debernard, S., Bozzolan, F., Solvar, M., Maïbèche-Coisné M., and Lucas, P. (2009). A TRP channel is expressed in Spodoptera littoralis antennae and is potentially involved in insect olfactory transduction. Insect Mol. Biol. 18, 213-222. doi: 10.1111/j.1365-2583.2008.00857.x

Deng, Y., Zhang, W., Farhat, K., Oberland, S., Gisselmann, G., and Neuhaus, E. M. (2011). The stimulatory Galpha(s) protein is involved in olfactory signal transduction in Drosophila. PLOS ONE 6:e18605. doi: 10.1371/journal.pone.0018605

Dolzer, J., Fischer, K., and Stengl, M. (2003). Adaptation in pheromone-sensitive trichoid sensilla of the hawkmoth Manduca sexta. J. Exp. Biol. 206(Pt 9), 1575-1588. doi: 10.1242/jeb.00302

Dolzer, J., Krannich, S., Fischer, K., and Stengl, M. (2001). Oscillations of the transepithelial potential of moth olfactory sensilla are influenced by octopamine and serotonin. J. Exp. Biol. 204(Pt 16), 2781-2794.

Dolzer, J., Krannich, S., and Stengl, M. (2008). Pharmacological investigation of protein kinase C- and cGMP-dependent ion channels in cultured olfactory manuscript. MS wrote the manuscript and contributed to the interpretation of the data. MS obtained finances for the project with DFG grants.

\section{ACKNOWLEDGMENTS}

We thank the DFG for financing our work with the grants STE 531-19-1, 20-1,2 to MS. We thank Prof. Dr. Sandy Rossie, Perdue University, for considerably improving our writing. In addition, we thank our referees that significantly improved the clarity of our manuscript.

\section{SUPPLEMENTARY MATERIAL}

The Supplementary Material for this article can be found online at: https://www.frontiersin.org/articles/10.3389/fncel. 2018.00218/full\#supplementary-material

receptor neurons of the hawkmoth Manduca sexta. Chem. Senses 33, 803-813. doi: 10.1093/chemse/bjn043

Flecke, C., Dolzer, J., Krannich, S., and Stengl, M. (2006). Perfusion with cGMP analogue adapts the action potential response of pheromonesensitive sensilla trichoidea of the hawkmoth Manduca sexta in a daytimedependent manner. J. Exp. Biol. 209(Pt 19), 3898-3912. doi: 10.1242/ jeb. 02432

Flecke, C., Nolte, A., and Stengl, M. (2010). Perfusion with cAMP analogue affects pheromone-sensitive trichoid sensilla of the hawkmoth Manduca sexta in a time-dependent manner. J. Exp. Biol. 213, 842-852. doi: 10.1242/jeb.032839

Flecke, C., and Stengl, M. (2009). Octopamine and tyramine modulate pheromonesensitive olfactory sensilla of the hawkmoth Manduca sexta in a time-dependent manner. J. Comp. Physiol. A Neuroethol. Sens. Neural Behav. Physiol. 195, 529-545. doi: 10.1007/s00359-009-0429-4

Fowler, M. A., and Montell, C. (2013). Drosophila TRP channels and animal behavior. Life Sci. 92, 394-403. doi: 10.1016/j.lfs.2012.07.029

Große-Wilde, E., Stieber, R., Forstner, M., Krieger, J., Wicher, D., and Hansson, B. S. (2010). Sex-specific odorant receptors of the tobacco hornworm Manduca Sexta. Front. Cell Neurosci. 4:22. doi: 10.3389/fncel.2010. 00022

Hofmann, T., Obukhov, A. G., Schaefer, M., Harteneck, C., Gudermann, T., and Schultz, G. (1999). Direct activation of human TRPC6 and TRPC3 channels by diacylglycerol. Nature 397, 259-263. doi: 10.1038/16711

Huang, K. P. (1989). The mechanism of protein kinase-C activation. Trends Neurosci. 12, 425-432. doi: 10.1016/0166-2236(89)90091-X

Jones, P. L., Pask, G. M., Rinker, D. C., and Zwiebel, L. J. (2011). Functional agonism of insect odorant receptor ion channels. Proc. Natl. Acad. Sci. U.S.A. 108, 8821-8825. doi: 10.1073/pnas.1102425108

Kaissling, K. E. (1995). "Single unit and electroantennogram recordings in insect olfactory organs," in Experimental Cell Biology of Taste and Olfaction: Current Techniques and Protocols, eds A. I. Spielman and J. G. Brand (Boca Raton: CRC Press), 361-386.

Kaissling, K. E., Hildebrand, J. G., and Tumlinson, J. H. (1989). Pheromone receptor-cells in the male moth Manduca-Sexta. Arch. Insect Biochem. Physiol. 10, 273-279. doi: 10.1002/arch.940100403

Kaissling, K. E., Keil, T. A., and Williams, J. L. D. (1991). Pheromone stimulation in perfused sensory hairs of the moth Antheraea-Polyphemus. J. Insect Physiol. 37, 71-78. doi: 10.1016/0022-1910(91)90021-Q

Kaissling, K. E., Zack Strausfeld, C., and Rumbo, E. R. (1987). Adaptation processes in insect olfactory receptors. Mechanisms and behavioral significance. Ann. N Y. Acad. Sci. 510, 104-112. doi: 10.1111/j.1749-6632.1987.tb43475.x

Keil, T. A. (1989). Fine structure of the pheromone-sensitive sensilla on the antenna of the hawkmoth, Manduca sexta. Tissue Cell 21, 139-151. doi: 10.1016/0040-8166(89)90028-1 
Keil, T. A., and Steinbrecht, R. A. (1984). "Mechanosensitive and olfactory sensilla of insects," in Insect Ultrastructure, eds R. C. King and H. Akai. (Boston: Springer), 477-516.

Krannich, S. (2008). Electrophysiological and Pharmacological Characterization of Ion Channels Involved in Moth Olfactory Transduction Cascades. Ph.D. thesis Univ. of Marburg.

Krannich, S., and Stengl, M. (2008). Cyclic nucleotide-activated currents in cultured olfactory receptor neurons of the hawkmoth Manduca sexta. J. Neurophysiol. 100, 2866-2877. doi: 10.1152/jn.01400.2007

Larsson, M. C., Domingos, A. I., Jones, W. D., Chiappe, M. E., Amrein, H., and Vosshall, L. B. (2004). Or83b encodes a broadly expressed odorant receptor essential for Drosophila olfaction. Neuron 43, 703-714. doi: 10.1016/j.neuron.2004.08.019

Liu, W. S., and Heckman, C. A. (1998). The sevenfold way of PKC regulation. Cell. Signal. 10, 529-542. doi: 10.1016/S0898-6568(98)00012-6

Lucas, P., Ukhanov, K., Leinders-Zufall, T., and Zufall, F. (2003). A diacylglycerolgated cation channel in vomeronasal neuron dendrites is impaired in TRPC2 mutant mice: mechanism of pheromone transduction. Neuron 40, 551-561. doi: 10.1016/S0896-6273(03)00675-5

Lundin, C., Kall, L., Kreher, S. A., Kapp, K., Sonnhammer, E. L., Carlson, J. R., et al. (2007). Membrane topology of the Drosophila OR83b odorant receptor. FEBS Lett. 581, 5601-5604. doi: 10.1016/j.febslet.2007.11.007

Minke, B., Wu, C., and Pak, W. L. (1975). Induction of photoreceptor voltage noise in the dark in Drosophila mutant. Nature 258, 84-87. doi: 10.1038/258084a0

Montell, C., Jones, K., Hafen, E., and Rubin, G. (1985). Rescue of the Drosophila phototransduction mutation trp by germline transformation. Science 230, 1040-1043. doi: $10.1126 /$ science. 3933112

Nakagawa, T., and Vosshall, L. B. (2009). Controversy and consensus: noncanonical signaling mechanisms in the insect olfactory system. Curr. Opin. Neurobiol. 19, 284-292. doi: 10.1016/j.conb.2009.07.015

Nilius, B., and Flockerzi, V. (2014). Mammalian transient receptor potential (TRP) cation channels. Vol. 2. Cham: Springer.

Nolte, A., Funk, N. W., Mukunda, L., Gawalek, P., Werckenthin, A., Hansson, B. S., et al. (2013). In situ tip-recordings found no evidence for an Orco-based ionotropic mechanism of pheromone-transduction in Manduca sexta. PLoS ONE 8:e62648. doi: 10.1371/journal.pone.0062648

Nolte, A., Gawalek, P., Koerte, S., Wei, H., Schumann, R., Werckenthin, A., et al. (2016). No Evidence for ionotropic pheromone transduction in the hawkmoth Manduca sexta. PLoS ONE 11:e0166060. doi: 10.1371/journal.pone.0166060

Ong, H. L., de Souza, L. B., Cheng, K. T., and Ambudkar, I. S. (2014). "Physiological functions and regulation of TRPC channels," in Mammalian Transient Receptor Potential (TRP) Cation Channels. Vol 2, eds B. Nilius and V. Flockerzi (Cham: Springer), 1005-1034.

Ramsey, I. S., Delling, M., and Clapham, D. E. (2006). An introduction to TRP channels. Annu. Rev. Physiol. 68, 619-647. doi: 10.1146/annurev.physiol.68.040204.100431

Riffell, J. A., Alarcon, R., Abrell, L., Davidowitz, G., Bronstein, J. L., and Hildebrand, J. G. (2008). Behavioral consequences of innate preferences and olfactory learning in hawkmoth-flower interactions. Proc. Natl. Acad. Sci. U.S.A. 105, 3404-3409. doi: 10.1073/pnas.0709811105

Sanes, J. R., and Hildebrand, J. G. (1976). Structure and development of antennae in a moth, Manduca sexta. Dev. Biol. 51, 280-299. doi: 10.1016/0012-1606(76)90144-5

Sargsyan, V., Getahun, M. N., Llanos, S. L., Olsson, S. B., Hansson, B. S., and Wicher, D. (2011). Phosphorylation via PKC regulates the function of the drosophila odorant co-receptor. Front. Cell. Neurosci. 5:5. doi: 10.3389/fncel.2011.00005

Sasaki, M., and Riddiford, L. M. (1984). Regulation of reproductive-behavior and egg maturation in the tobacco hawk moth, Manduca-Sexta. Physiol. Entomol. 9 , 315-327. doi: 10.1111/j.1365-3032.1984.tb00713.x

Sato, K., Pellegrino, M., Nakagawa, T., Vosshall, L. B., and Touhara, K. (2008). Insect olfactory receptors are heteromeric ligand-gated ion channels. Nature 452, 1002-1006. doi: 10.1038/nature06850

Schendzielorz, T., Peters, W., Boekhoff, I., and Stengl, M. (2012). Time of day changes in cyclic nucleotides are modified via octopamine and pheromone in antennae of the Madeira cockroach. J. Biol. Rhythms 27, 388-397. doi: $10.1177 / 0748730412456265$
Schendzielorz, T., Schirmer, K., Stolte, P., and Stengl, M. (2015). Octopamine regulates antennal sensory neurons via daytime-dependent changes in cAMP and IP3 levels in the hawkmoth Manduca sexta. PLoS ONE 10:e0121230. doi: 10.1371/journal.pone.0121230

Schuckel, J., Siwicki, K. K., and Stengl, M. (2007). Putative circadian pacemaker cells in the antenna of the hawkmoth Manduca sexta. Cell Tissue Res. 330, 271-278. doi: 10.1007/s00441-007-0471-x

Stengl, M. (1993). Intracellular-messenger-mediated cation channels in cultured olfactory receptor neurons. J. Exp. Biol. 178, 125-147.

Stengl, M. (1994). Inositol-trisphosphate-dependent calcium currents precede cation currents in insect olfactory receptor neurons in vitro. J. Comp. Physiol. Sens. Neural Behav. Physiol. 174, 187-194. doi: 10.1007/BF00193785

Stengl, M. (2010). Pheromone transduction in moths. Front. Cell. Neurosci. 4:133. doi: $10.3389 /$ fncel.2010.00133

Stengl, M. (2017). "Chemosensory transduction in arthropods." in Oxford Handbooks Online. The Oxford Handbook of Invertebrate Neurobiology, ed J. H. Byrne (Oxford University Press), 1-42. doi: 10.1093/oxfordhb/9780190456757.013.15

Stengl, M., and Funk, N. W. (2013). The role of the coreceptor Orco in insect olfactory transduction. J. Comp. Physiol. A Neuroethol. Sens. Neural Behav. Physiol. 199, 897-909. doi: 10.1007/s00359-013-0837-3

Stengl, M., and Hildebrand, J. G. (1990). Insect olfactory neurons in vitro: morphological and immunocytochemical characterization of male-specific antennal receptor cells from developing antennae of male Manduca sexta. J. Neurosci. 10, 837-847. doi: 10.1523/JNEUROSCI.10-03-008 37.1990

Stengl, M., Zintl, R., De Vente, J., and Nighorn, A. (2001). Localization of cGMP immunoreactivity and of soluble guanylyl cyclase in antennal sensilla of the hawkmoth Manduca sexta. Cell Tissue Res. 304, 409-421. doi: $10.1007 /$ s004410000336

Stengl, M., Zufall, F., Hatt, H., and Hildebrand, J. G. (1992). Olfactory receptor neurons from antennae of developing male Manduca sexta respond to components of the species-specific sex pheromone in vitro. J. Neurosci. 12, 2523-2531. doi: 10.1523/JNEUROSCI.12-07-02523.1992

Storch, U., Forst, A. L., Pardatscher, F., Erdogmus, S., Philipp, M., Gregoritza, M., et al. (2017). Dynamic NHERF interaction with TRPC4/5 proteins is required for channel gating by diacylglycerol. Proc. Natl. Acad. Sci. U.S.A. 114, E37-E46. doi: $10.1073 /$ pnas.1612263114

Thiel, G., Lesch, A., Rubil, S., Backes, T. M., and Rössler, O. G. (2018). Regulation of gene transcription following stimulation of transient receptor potential (TRP) channels. Int. Rev. Cell Mol. Biol. 335, 167-189. doi: 10.1016/bs.ircmb.2017.07.010

Tumlinson, J. H., Brennan, M. M., Doolittle, R. E., Mitchell, E. R., Brabham, A., Mazomenos, B. E., et al. (1989). Identification of a pheromone blend attractive to Manduca-Sexta (L) males in a wind-tunnel. Arch. Insect Biochem. Physiol. 10, 255-271. doi: 10.1002/arch.940100402

Venkatachalam, K., Zheng, F., and Gill, D. L. (2003). Regulation of canonical transient receptor potential (TRPC) channel function by diacylglycerol and protein kinase C. J. Biol. Chem. 278, 29031-29040. doi: 10.1074/jbc.M302751200

Vosshall, L. B., and Hansson, B. S. (2011). A unified nomenclature system for the insect olfactory coreceptor. Chem. Senses 36, 497-498. doi: 10.1093/chemse/bjr022

Wicher, D., Morinaga, S., Halty-deLeon, L., Funk, N., Hansson, B., Touhara, K., et al. (2017). Identification and characterization of the bombykal receptor in the hawkmoth Manduca sexta. J. Exp. Biol. 220(Pt 10), 1781-1786. doi: $10.1242 /$ jeb. 154260

Wicher, D., Schäfer, R., Bauernfeind, R., Stensmyr, M. C., Heller, R., Heinemann, S. H., et al. (2008). Drosophila odorant receptors are both ligand-gated and cyclic-nucleotide-activated cation channels. Nature 452, 1007-1011. doi: $10.1038 /$ nature 06861

Zhang, X., and Trebak, M. (2014). "Transient receptor potential canonical 7: a diacylglycerol-activated non-selective cation channel," in Mammalian Transient Receptor Potential (TRP) Cation Channels. Vol. 1, eds B. Nilius and V. Flockerzi (Berlin, Heidelberg: Springer), 189-204.

Zhu, M. H., Chae, M., Kim, H. J., Lee, Y. M., Kim, M. J., Jin, N. G., et al. (2005). Desensitization of canonical transient receptor potential channel 
5 by protein kinase C. Am. J. Physiol. Cell Physiol. 289, C591-C600. doi: 10.1152/ajpcell.00440.2004

Ziegelberger, G., van den Berg, M. J., Kaissling, K. E., Klumpp, S., and Schultz, J. E. (1990). Cyclic GMP levels and guanylate cyclase activity in pheromone-sensitive antennae of the silkmoths Antheraea polyphemus and Bombyx mori. J. Neurosci. 10, 1217-1225. doi: 10.1523/JNEUROSCI.10-04-01217.1990

Zufall, F., and Hatt, H. (1991). Dual activation of a sex pheromone-dependent ion channel from insect olfactory dendrites by protein kinase $\mathrm{C}$ activators and cyclic GMP. Proc. Natl. Acad. Sci. U.S.A. 88, 8520-8524. doi: 10.1073/pnas.88.19.8520
Conflict of Interest Statement: The authors declare that the research was conducted in the absence of any commercial or financial relationships that could be construed as a potential conflict of interest.

Copyright (๑) 2018 Gawalek and Stengl. This is an open-access article distributed under the terms of the Creative Commons Attribution License (CC BY). The use, distribution or reproduction in other forums is permitted, provided the original author(s) and the copyright owner(s) are credited and that the original publication in this journal is cited, in accordance with accepted academic practice. No use, distribution or reproduction is permitted which does not comply with these terms. 
OPEN ACCESS

Edited by:

Dieter Wicher

Max Planck Institute for Chemical Ecology (MPG), Germany

Reviewed by: Jeremy C. Mclntyre, University of Florida, United States

Yu-Feng Wang,

Harbin Medical University, China

${ }^{*}$ Correspondence:

Weihong Lin

weihong@umbc.edu

Received: 22 December 2017 Accepted: 27 February 2018 Published: 20 March 2018

Citation:

Fu Z, Ogura T, Luo W and Lin W (2018) ATP and Odor Mixture

Activate TRPM5-Expressing Microvillous Cells and Potentially Induce Acetylcholine Release to Enhance Supporting Cell Endocytosis in Mouse Main Olfactory Epithelium.

Front. Cell. Neurosci. 12:71.

doi: 10.3389/fncel.2018.00071
ATP and Odor Mixture Activate TRPM5-Expressing Microvillous Cells and Potentially Induce Acetylcholine Release to Enhance Supporting Cell Endocytosis in Mouse Main Olfactory Epithelium

\author{
Ziying Fu, Tatsuya Ogura, Wangmei Luo and Weihong Lin * \\ Department of Biological Sciences, University of Maryland, Baltimore County, Baltimore, MD, United States
}

The main olfactory epithelium (MOE) functions to detect odor molecules, provide an epithelial surface barrier, and remove xenobiotics from inhaled air. Mechanisms coordinating the activities of different cell types within the MOE to maintain these functions are poorly understood. Previously, we showed that superficially located microvillous cells (MCs) in the MOE expressing transient receptor potential channel M5 (TRPM5) are cholinergic and chemoresponsive and that they play an important role in maintaining odor responses and olfactory-guided behavior under challenging chemical environment. Here we investigated TRPM5-MC activation and subsequent paracrine regulation. $\mathrm{Ca}^{2+}$ imaging showed that TRPM5-MCs dose-dependently increase their intracellular $\mathrm{Ca}^{2+}$ levels in response to ATP, an important signaling molecule for airway mucociliary movement, and to an odor mixture. Pharmacological examination showed that the ATP responses are primarily mediated by P2X purinergic receptors. Interestingly, using the endocytosis dye pHrodo Red dextran, we found that chemical-activated TRPM5-MCs significantly increase the number of pHrodo-labeled puncta compared to controls without stimulation and compared to cells that do not respond to ATP or to the odor mixture. These results indicate potential vesicle recycling after release of the signaling molecule acetylcholine (ACh). Interestingly, TRPM5 knockout (KO) results in a decrease in ATP-induced pHrodo internalization. We further investigated cholinergic regulation of neighboring supporting cells (SCs). We found that ACh strongly elevates intracellular $\mathrm{Ca}^{2+}$ and potentiates pHrodo endocytosis in SCs. The ACh effects are diminished in the presence of atropine or M3 muscarinic receptor antagonist and in SCs lacking M3 receptors. Collectively, these data suggest that TRPM5-MCs may regulate the MOE's multicellular network activity via cholinergic paracrine signaling for functional maintenance and adaptive plasticity.

Keywords: cholinergic modulation, purinergic receptors, xenobiotics, vesicle release, epithelial maintenance 


\section{INTRODUCTION}

Chemosensory cells expressing transient receptor potential channel M5 (TRPM5) are found in epithelia of various tissue and organs, including the respiratory, gastrointestinal, and urinary tracts. These cells detect chemical stimuli including irritants and toxicants, and play important roles in regulating respiration, protecting vital organs and epithelial defense against bacteria and parasites (Finger et al., 2003; Gulbransen et al., 2008; Lin et al., 2008b; Ogura et al., 2010; Tizzano et al., 2010; Krasteva et al., 2011; Deckmann et al., 2014; Lee et al., 2014; Gerbe et al., 2016; Howitt et al., 2016). While solitary chemosensory cells (SCCs) in the respiratory epithelium and at the entrance of the vomeronasal organ, as well as tuft cells in the gut and brush cells in the urethra, are able to relay their chemosensory activity to innervating nerves and regulate physiological activity via neural circuits (Bezençon et al., 2008; Lin et al., 2008b; Ogura et al., 2010; Krasteva et al., 2011; Deckmann et al., 2014; Saunders et al., 2014), TRPM5-expressing microvillous cells (TRPM5-MCs) in the main olfactory epithelium (MOE) are generally not innervated (Lin et al., 2008a). Actions of TRPM5-MCs following chemical stimulation, as well as mechanisms underlying their potential paracrine effects on the local multicellular epithelial network against chemical insults, are poorly understood.

The TRPM5-MCs identified in the nasal cavity reside superficially throughout the entire MOE, but not in the respiratory epithelium. Their elaborate apical microvilli extend into the mucus layer, enabling detection of xenobiotic chemicals (Hansen and Finger, 2008; Lin et al., 2008a). Using single-cell $\mathrm{Ca}^{2+}$ imaging, we also found that TRPM5-MCs respond to odor molecules at relatively high levels, to bacterial lysates, and to ATP with increases in intercellular $\mathrm{Ca}^{2+}$ levels (Ogura et al., 2011). Interestingly, TRPM5-MCs express cholinergic markers choline acetyltransferase and vesicular acetylcholine (ACh) transporter, indicating that TRPM5-MCs are capable of releasing ACh (Ogura et al., 2011). Similar findings have been found in studies of TRPM5-expressing SCCs and brush cells in the trachea and urogenital tract (Ogura et al., 2010; Krasteva et al., 2011; Deckmann et al., 2014; Saunders et al., 2014). ACh is a potent signaling molecule that can be released by neurons and non-neuronal cells to regulate a wide variety of cellular activities (Kawashima and Fujii, 2008; Wessler and Kirkpatrick, 2008). The release of ACh from urethral brush cells is further shown by Deckmann et al. (2014). There is also pharmacological evidence that SCCs release $\mathrm{ACh}$ as a neurotransmitter to induce nasal inflammation (Saunders et al., 2014). We further demonstrated that, in the MOE, ACh potently increases intracellular $\mathrm{Ca}^{2+}$ levels in supporting cells (SCs) and suppresses evoked $\mathrm{Ca}^{2+}$ increases in approximately half of olfactory sensory neurons (OSNs) tested (Ogura et al., 2011). Thus, it is very likely that TRPM5-MCs exert paracrine regulation to coordinate MOE multicellular activity by releasing ACh. However, the relationship between chemical-induced activation of TRPM5-MCs and ACh release has not been studied.

The MOE is made up of multiple cell types including OSNs, SCs, MCs, basal cells, and cells of Bowman's glands and ducts
(Farbman, 2000). These cells function distinctly in detecting airborne odorants with exquisite sensitivity, providing nasal epithelial lining (Gross et al., 1982) and metabolizing xenobiotics, which protects the lower airway and lungs from chemical insults (Ding and Dahl, 2003; Thiebaud et al., 2010). Because the MOE is vulnerable to random insults by airborne chemical irritants, toxicants and harmful microorganisms, mechanisms coordinating MOE to maintain its structural and functional integrity are important not only for olfaction but also for respiratory health and homeostasis because of airway continuity. Such mechanisms, which currently are poorly understood, are expected to align with activities that remove harmful chemicals in the airway.

We recently showed that TRPM5-MCs are important for maintaining olfactory function and subsequent olfactoryguided behavior in a chemically challenging environment (Lemons et al., 2017) using transcription factor Pou2f3- or Skn1a-knockout (KO; Skn-1a ${ }^{-/-}$) mice lacking TRPM5-MCs (Yamaguchi et al., 2014). Under conventional housing conditions, Skn-1a ${ }^{-/-}$mice exhibit normal odor-evoked electro-olfactogram responses to a panel of odorants tested, as well as normal olfactory-guided behaviors, including finding buried food and preference reactions to socially and sexually relevant odors, that are similar to those of control wild-type mice. However, when housed in a chemically challenging environment for 2 weeks, Skn-1a ${ }^{-/-}$mice, but not control mice, exhibited significant reductions in odor-evoked electro-olfactograms, and their olfactory ability in guiding these behaviors is also impaired. These findings allow us to hypothesize that activated TRPM5-MCs release ACh to modulate OSNs and also to coordinate SC activity in MOE functional maintenance.

The close anatomical proximity between TRPM5-MCs and SCs enables paracrine modulation. SCs are the second most abundant cell type in the MOE (Farbman, 2000), providing a physical and chemical barrier (Rafols and Getchell, 1983), as well as structural and metabolic support for the OSNs (Getchell and Mellert, 1991; Vogalis et al., 2005). The SCs express many xenobiotic-metabolizing enzymes, which can act either intracellularly or extracellularly after being released into the mucus layer (Thornton-Manning and Dahl, 1997; Ding and Dahl, 2003; Hu et al., 2014; Asakawa et al., 2017). SCs possess numerous vesicles and vacuoles in the supranuclear regions of their cell bodies (Getchell and Mellert, 1991), presumably resulting from xenobiotic internalization for enzymatic reactions (Getchell and Getchell, 1992). However, endocytosis in SCs or vesicle recycling following secretion and mechanisms regulating the events are understudied.

In this study, we sought to further understand TRPM5-MC function and cholinergic regulation within the MOE network. Using $\mathrm{Ca}^{2+}$ imaging and the endocytotic dye pHrodo, we first investigated possible vesicle release by monitoring membrane or vesicle recycling in TRPM5-MCs following ATP or odor mixture-induced activation of these cells. ATP released apically from nasal epithelial cells regulates mucociliary movement for xenobiotic removal (Workman et al., 2017). ATP can also be constitutively released from the MOE 
(Hayoz et al., 2012) to modulate olfactory sensitivity (Hegg et al., 2003), intracellular $\mathrm{Ca}^{2+}$ levels in SCs (Hassenklover et al., 2008), and MOE proliferation (Kanekar et al., 2009). Second, we investigated whether ACh increases endocytosis or vesicle recycling in SCs. Finally, we probed intracellular mechanisms mediating ACh effects using pharmacological agents and muscarinic ACh receptor subtype 3 (M3-AChR)KO mice. Our results suggest that activated TRPM5-MCs may release $\mathrm{ACh}$ to potentiate $\mathrm{SC}$-mediated xenobiotic clearance.

\section{MATERIALS AND METHODS}

\section{Animals}

Two- to six-month-old adult male and female C57BL/6background transgenic and $\mathrm{KO}$ mice were used in this study. Both TRPM5-green fluorescent protein (GFP) and TRPM5-KO lines were originally obtained from Robert Margolskee's laboratory. Detailed information on the generation and initial characterization of these mice is published in Clapp et al. (2006) and Damak et al. (2006). In TRPM5-GFP transgenic mice, the TRPM5-promotor drives the expression of GFP, allowing visualization of TRPM5-MCs. The endogenous gene coding for TRPM5 remains unchanged in these mice. In our initial identification of TRPM5-MCs, we had used an anti-TRPM5 antibody to immunolabel MOE sections from TRPM5-GFP mice and showed positive TRPM5 immunoreactivity in GFP-expressing MCs (Lin et al., 2008b). We generated TRPM5-KO GFP mice by cross-mating TRPM5-GFP and TRPM5-KO mice. The M3-AChR KO line was originally obtained from Jürgen Wess (Yamada et al., 2001). All animal care and use procedures were conducted in accordance with the National Institutes of Health Guide for the Care and Use of Laboratory Animals (2006) and approved by the Animal Care and Use Committee of the University of Maryland, Baltimore County, Baltimore, MD, USA.

\section{Solutions and Chemicals}

For single-cell $\mathrm{Ca}^{2+}$ imaging and endocytotic dye imaging, Tyrode's saline was used for the extracellular solution bathing the cells, which contained (in $\mathrm{mM}$ ) $140 \mathrm{NaCl}$, $5 \mathrm{KCl}, 10 \mathrm{HEPES}, 1 \mathrm{MgCl}_{2}, 3 \mathrm{CaCl}_{2}, 10 \mathrm{Na}$-pyruvate, and 10 D-glucose ( $\mathrm{pH} 7.4$ ). $\mathrm{Ca}^{2+} / \mathrm{Mg}^{2+}$-free Tyrode's saline for cell isolation was prepared by omitting $\mathrm{MgCl}_{2}$ and $\mathrm{CaCl}_{2}$ and adding $1 \mathrm{mM}$ BAPTA; $\mathrm{Ca}^{2+}$-free Tyrode's saline was prepared by omitting $\mathrm{CaCl}_{2}$. The odor mixture was prepared as stock solution containing (in mM) 19 ammonium hydroxide, 75 ethyl acetate, 83 propionic acid, and 13 triethylamine in Tyrode's and diluted to $1: 100,1: 50,1: 10$ and 1:5 to determine dose-dependent responses in TRPM5-MCs. We used this mixture because our recent study indicated that TRPM5-MCs play an important role in maintaining olfactory function in mice challenged by 2-week exposure to this odor mixture (Lemons et al., 2017). Detailed justification of using these chemicals can also be found in this article. The following pharmacological agents were dissolved in DMSO and diluted into the bath solution to a final concentration, which include darifenacin $(0.1 \mu \mathrm{M})$, pirenzepine $(0.1 \mu \mathrm{M})$, 4-(4-Butyl-1piperidinyl)-1-(2-methylphenyl)-1-butanone hydrochloride (AC-42, $5 \mu \mathrm{M})$, 1,1-Dimethyl-4-diphenylacetoxypiperidinium iodide (4-DAMP, $0.1 \mu \mathrm{M})$, and 2,4,6-Trimethyl-N-[3(trifluoromethyl)phenyl]benzenesulfonamide (m-3M3FBS $25 \mu \mathrm{M})$. The final concentration of DMSO, which ranged from $0.01 \%$ to $0.1 \%$, did not affect responses when applied alone. ATP, ACh, adenosine, ADP, AMP, UTP, atropine (0.5 $\mu \mathrm{M})$, and pyridoxalphosphate-6-azophenyl-2' $4^{\prime}$-disulfonic acid (PPADS, $5 \mu \mathrm{M}$ ) were dissolved in Tyrode's saline or $\mathrm{Ca}^{2+}$-free Tyrode's saline. All chemicals used in this study were purchased from either Sigma-Aldrich (St. Louis, MO, USA) or Tocris (Minneapolis, MN, USA).

\section{Cell Isolation}

The method of isolating MCs and SCs in the mouse MOE was adapted from our previous study (Ogura et al., 2011). Briefly, mice were euthanized by $\mathrm{CO}_{2}$ asphyxiation followed by cervical dislocation and exsanguination through an open heart. The head skin was removed, and the nose was split from the midline. Then olfactory turbinates were dissected and placed in $\mathrm{Ca}^{2+} / \mathrm{Mg}^{2+}$-free Tyrode's saline containing $\sim 2.5-4 \mathrm{U} / \mathrm{ml}$ activated papain (Worthington, Lakewood, NJ, USA) with $2 \mathrm{mM}$ cysteine for 2.5-3.5 min at room temperature. Gentle pipetting at the end of enzyme incubation facilitated cell dissociation. The supernatant was transferred to an O-ring chamber on a cover slip precoated with concanavalin A (Sigma).

\section{$\mathrm{Ca}^{2+}$ Imaging}

$\mathrm{Ca}^{2+}$ levels in isolated TRPM5-MCs and SCs were monitored as described in our previous studies (Ogura et al., 2011). Our $\mathrm{Ca}^{2+}$ imaging was performed in a well-ventilated room. Stimulus solutions were capped before application and were bath applied. After stimulation, the solutions were removed from the recording chamber by a vacuum pump into a sealed glass waste container. A plastic tube channeled the odorized air from the waste container to the building central exhaust system to keep the room in a low odor environment. For $\mathrm{Ca}^{2+}$ imaging, cells were loaded with the $\mathrm{Ca}^{2+}$-sensitive dye Fura-2 AM $(2 \mu \mathrm{M}$; Molecular Probes) for $20 \mathrm{~min}$. A pair of 340- and 380-nm excitation light images was captured every $3 \mathrm{~s}$ using an epifluorescence microscope equipped with a $40 \times$ oil objective lens (Olympus IX71), a light source/filter changer (Sutter Lambda LS), and a cooled CCD camera (Hamamatsu C9300-221). We measured $\mathrm{Ca}^{2+}$ levels as the ratio of fluorescence values from $340-\mathrm{nm}$ and $380-\mathrm{nm}$ excitation light images. We considered changes in $\mathrm{Ca}^{2+}$ levels as stimulus-induced responses if $\mathrm{Ca}^{2+}$ levels increased $>2 \%$ from stable resting levels within $30 \mathrm{~s}$ after stimulation.

To ensure only healthy isolated cells were imaged, we checked the cell viability in three different ways. First, we examined the cell morphology; only those with a smooth appearance of the cell body (less granulated) with multiple apical microvilli were recorded. Second, we checked their resting $\mathrm{Ca}^{2+}$ level, since we have observed that unhealthy or damaged cells usually show a higher resting $\mathrm{Ca}^{2+}$ level in numerous previous $\mathrm{Ca}^{2+}$ imaging experiments. For the current experiment, we only recorded cells 
that had a resting level below 0.9 (Fura-2 $340 \mathrm{~nm} / 380 \mathrm{~nm}$ ratio). Third, we occasionally stimulated some nonresponding cells with cold saline solution $\left(4^{\circ} \mathrm{C}\right)$, which we previously found to elicit $\mathrm{Ca}^{2+}$ response in TRPM5-MCs (Ogura et al., 2011).

\section{Endocytosis Dye Internalization Measurement}

We used a dextran-conjugated water soluble dye, pHrodo Red dextran $10 \mathrm{~K}$ (Invitrogen), to monitor endocytosis after the cells were stimulated. The dye is $\mathrm{pH}$ sensitive: under natural $\mathrm{pH}$ conditions, such as in extracellular solution, its fluorescence intensity is very weak, but when internalized into intracellular organelles with lower luminal $\mathrm{pH}$, the fluorescence intensity becomes stronger. For the experiment, TRPM5-MCs isolated from TRPM5-GFP and TRPM5-KO GFP mice were first identified by their morphological features, such as apical microvilli (Lin et al., 2008a; Ogura et al., 2011) and GFP fluorescence. The cells were then loaded with the $\mathrm{Ca}^{2+}$-sensitive dye Fura-2 AM (see " $\mathrm{Ca}^{2+}$ Imaging" section) and washed. For an initial control set of images, GFP fluorescence and transluminescence light images of the cells were captured, as well as a prestimulation fluorescence image using the same optical filter setting as for later images with pHrodo dye (530-560 nm excitation, 573-647 $\mathrm{nm}$ emission light). Next, individual cells were stimulated with $100 \mu \mathrm{M}$ ATP or the odor mixture for $10 \mathrm{~s}$ followed by a brief wash. During the stimulation, changes in $\mathrm{Ca}^{2+}$ levels were monitored to determine whether the cell was responsive to the stimulation. Cells were then loaded with pHrodo Red dextran 10K $(25 \mu \mathrm{g} / \mathrm{ml})$ for $20 \mathrm{~min}$. After a brief wash, images of internalized dye were captured at $530-560 \mathrm{~nm}$ excitation and 573-647 nm emission light. All images were taken using an Olympus IX71 epifluorescence microscope equipped with a $40 \times$ oil UV objective lens (N.A. 1.3), a 1.6× intermediate lens, a cooled CCD camera (Hamamatsu C9300-221), and a xenon lamp with filter changer (Sutter Lambda LS) controlled by Axon imaging workbench software. The resolution of image was 7.7 pixels $/ \mu \mathrm{m}$. The number of pHrodo containing puncta in the image of TRPM5-MCs was quantified.

Similarly, we used pHrodo dye for monitoring ACh-stimulated endocytosis or vesicle recycling in SCs. Due to the intense dye labeling within the SCs, we could not distinguish individual puncta. Therefore we measured the average fluorescence intensity level of individual cell body region in the images using ImageJ software (NIH). Endocytic activity was estimated as $\Delta F / F_{0}=[$ (fluorescence level after stimulation) - (prestimulation level)]/prestimulation level.

\section{Immunohistochemistry}

\section{Tissue Preparation}

Our immunolabeling procedure has been described previously (Ogura et al., 2011; Krosnowski et al., 2012; Lemons et al., 2017). Briefly, TRPM5-GFP mice were deeply anesthetized with tribromoethanol (Avertin $250 \mu \mathrm{g} / \mathrm{g}$ body weight), perfusionfixed with a phosphate buffered fixative containing $3 \%$ paraformaldehyde, $19 \mathrm{mM}$ L-lysine monohydrochloride and $0.23 \%$ sodium $\mathrm{m}$-periodate. The nose was harvested, post-fixed for $1.5 \mathrm{~h}$ and then cryoprotected with phosphate buffered $25 \%$ sucrose solution overnight. The surrounding bones were manually removed following our published method (Dunston et al., 2013) and the whole MOE tissue was embedded and cut using a cryostat (Microm International, Walldorf, Germany) into $14 \mu \mathrm{m}$-thick sections and mounted onto charged microscope slides (Globe Scientific, Paramus, NJ, USA).

\section{Immunohistochemistry}

The MOE sections were rinsed and treated with Dako Target Retrieval Solution pH 9 (DAKO Cat\# S2368) for $20 \mathrm{~min}$ at $80^{\circ} \mathrm{C}$ for antigen retrieval. The sections were then incubated in a blocking solution containing $2 \%$ normal donkey serum, $0.3 \%$ Triton X-100 and $1 \%$ bovine serum albumin in $0.1 \mathrm{M}$ phosphate buffered saline for $1.5 \mathrm{~h}$, before immunoreacted for $48 \mathrm{~h}$ at $4^{\circ} \mathrm{C}$ with the primary antibody against early endosome antigen1 (EEA1, 1:250, Sigma Cat\# E4156, RRID:AB_609870). The sections were washed and reacted with a secondary antibody conjugated with Alexa Fluor 555 (1:400; Thermo Fisher Scientific Cat\# A-31572, RRID:AB_162543) for $1 \mathrm{~h}$ at room temperature. Sections were then counterstained with DAPI and coverslipped with Fluoromount-G (Southern Biotech). In control experiments, primary antibodies were omitted, which resulted in negative labeling. Fluorescence images were taken using an Olympus BX 61 epifluorescence microscope equipped with a spinning disc confocal unit and Slidebook 5.0 software (3i, Denver, CO, USA).

\section{Statistical Analysis}

Data are presented as mean \pm SEM. If the $F$ test was not significant and homogeneity of variance was assumed, Student's $t$-test was performed to compare results between two experimental groups. If the $F$ test was significant and homogeneity of variance was not assumed, Welch's $t$-test was used instead. The paired $t$-test was used to compare results from two experimental groups of the same cells. For comparison of data from three or more groups, we performed one-way analysis of variance (ANOVA) and Tukey's post hoc multiple comparison test. To determine significant differences between percentages of cells responsive to two different stimulus conditions, Fisher's exact test was performed using original numbers of cells observed. Prism 6.07 (GraphPad Software, San Diego, CA, USA) was used for statistical analyses. In all tests, $p<0.05$ was considered statistically significant.

\section{RESULTS}

\section{Increase in pHrodo Internalization in TRPM5-MCs Following Activation by ATP and a Odor Mixture}

Dose-dependent activation by ATP and its potential consequences in TRPM5-MCs have not been previously determined. Therefore, we stimulated freshly isolated TRPM5-MCs with various concentrations of ATP and monitored changes in intracellular $\mathrm{Ca}^{2+}$ using $\mathrm{Ca}^{2+}$ imaging. Individual TRPM5-MCs were identified by their GFP expression and by the presence of typical apical microvilli (Lin et al., 2008a) and the 

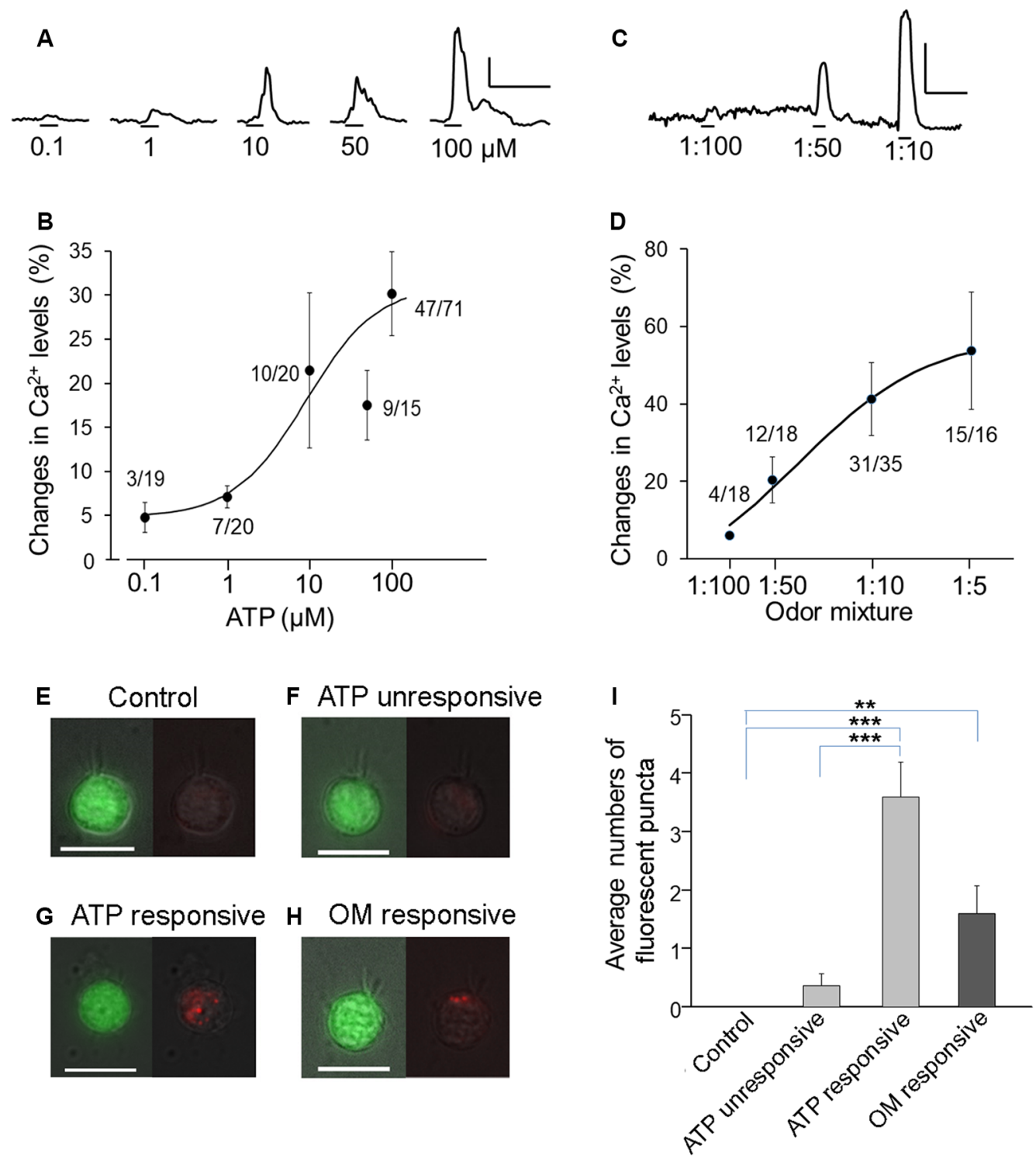

FIGURE 1 | Increases in $\mathrm{Ca}^{2+}$ levels and endocytic dye internalization in transient receptor potential channel M5 (TRPM5)-microvillous cells (MCs) after response to ATP and odor mixture. (A-D) Dose responses to ATP (A,B) and odor mixture (C,D). Traces show Ca ${ }^{2+}$ changes in response to different concentrations (A,C); graphs show dose-peak $\mathrm{Ca}^{2+}$ response relations (B,D). Numbers next to the plots are number of responding cells/number of cells tested. Curve fitting was calculated by the Hill equation with $\mathrm{EC}_{50}=9 \mu \mathrm{M}$ and Hill coefficient $=1.0$ for ATP and $\mathrm{EC}_{50}=3: 100$ dilution and Hill coefficient $=0.93$ for odor mixture. Scale bars in (A,C): 20\% change from resting level and 100 ms. (E-H) Images of TRPM5-MCs. Representative pairs of images of green fluorescent protein (GFP; green, left panels) and internalized $\mathrm{pH}$-sensitive endocytotic pHrodo dye (red, right panels) are shown for each cell. The pHrodo fluorescence images are overlaid onto a weak light image to view the cell shape. (E) Control cell without stimulation. (F) Cell that did not increase Ca ${ }^{2+}$ in response to $100 \mu M$ ATP. (G) Cell that increased Ca ${ }^{2+}$ in response to ATP. (H) Cell that responded to odor mixture (OM, 1:10 dilution). Scale bars: $10 \mu m$. (I) Average number of fluorescent puncta, showing significantly increased numbers of internalized dyed puncta in ATP-responsive and odor mixture (OM)-responsive TRPM5-MCs ${ }^{* *} p<0.01$, ${ }^{* * *} p<0.001$, one-way analysis of variance (ANOVA) followed by Tukey's multiple comparison for ATP data, and $t$-test for odor mixture data $(n=16-35)$.

cell viability was examined (see "Materials and Methods" section for detail). Because the response amplitude to subsequent ATP applications was usually reduced, we applied ATP of a particular concentration only once per cell, except for low concentrations of 0.1 and $1 \mu \mathrm{M}$. To ensure the ATP-nonresponsive cells were viable, we randomly checked and found they could respond to cold saline solution $\left(4^{\circ} \mathrm{C}\right)$ known to elicit $\mathrm{Ca}^{2+}$ response in TRPM5-MCs (Ogura et al., 2011). Figure 1A shows representative traces of ATP-induced $\mathrm{Ca}^{2+}$ increases in TRPM5-MCs cells, which were collected from different cells. At low concentrations, ATP-induced responses were small, and only a few cells responded $(3 / 19$ at $0.1 \mu \mathrm{M})$. With increasing ATP concentrations, the response amplitude generally increased with the exception of the response to $50 \mu \mathrm{M}$ ATP. Also, the percentage of responding cells increased from $35 \%$ at $1 \mu \mathrm{M}$ (20 cells tested) to $66 \%$ at $100 \mu \mathrm{M}$ (71 cells tested; Figure 1B, 
4-14 mice were used for each ATP concentration). These data demonstrate that both the number of responding TRPM5-MCs and their levels of intracellular $\mathrm{Ca}^{2+}$ increase are positively correlated to external ATP concentrations.

In our experiments, cells obtained from both males and females were used. In order to evaluate whether sex is a variable affecting our data, we alternated our daily use of animals to isolate TRPM5-MCs from either males or females and performed a set of experiments under the same experimental conditions and compared the changes in $\mathrm{Ca}^{2+}$ level in responses to ATP $(100 \mu \mathrm{M})$ between males and females. We did not find a statistically significant difference in the response amplitude between males and females ( $t$-test $\left.t_{(17)}=0.200, p=0.843\right)$. We also found a similar percentage of ATP-responding cells, which were $66 \%$ ( 8 out of 12 cells tested) for males and $69 \%$ (11 out of 16 cells tested) for females, respectively. Because these results did not show sex as a variable, we pooled our results obtained from both sexes.

We recently reported that 2 -week exposure to a relatively strong odor mixture significantly impaired olfactory function in Skn-1a $\mathrm{a}^{-/-}$mice but not in control mice (Lemons et al., 2017). Those results imply that TRPM5-MCs respond to the odor mixture and subsequently modulate MOE activity for functional maintenance. We next stimulated TRPM5-GFP with the odor mixture diluted from $1: 100$ to $1: 5$ to determine dose-dependent responses using $\mathrm{Ca}^{2+}$ imaging. The 1:100 odor mixture elicited nearly no response (4/18 cells responded). With increasing concentrations, more TRPM5-MCs responded $(12 / 18,31 / 35$ and $15 / 16$ cells responded at 1:50, $1: 10$ and $1: 5$, respectively), and the peak amplitude of $\mathrm{Ca}^{2+}$ responses also increased, indicating dose-dependent activation. Unlike the response to ATP, odor mixture-induced responses were repeatable (Figure 1C: representative $\mathrm{Ca}^{2+}$ response traces from the same cells. Figure 1D: plot of average dose responses from responding cells. Sixteen to thirty-five cells tested from 14 mice).

TRPM5-MCs express cholinergic markers of ACh synthesis and packaging (Ogura et al., 2011). However, vesicle release of ACh and other signaling molecules from these cells has not been determined. Because the event is commonly followed by endocytotic events to recycle the membrane, we monitored the internalization of an endocytosis dye, pHrodo Red dextran. TRPM5-MCs were first loaded with Fura-2 AM to image evoked responses to either ATP or the odor mixture before pHrodo incubation. Under the control condition without stimulation, we found no dye-labeled puncta inside TRPM5-MCs imaged (Figure 1E, $n=7$ cells). Similarly, no or very low levels of dye internalization were found in cells that did not respond to ATP (100 $\mu \mathrm{M}$; Figure 1F, $n=11$ cells). In contrast, ATP-responsive cells showed more pHrodo-labeled fluorescent puncta in the cytoplasm (Figure 1G, $n=5$ cells). A similar result was obtained when TRPM5-MCs were stimulated with diluted odor mixture (1:10; Figure $\mathbf{1 H}, n=10$ cells). We manually counted the number of fluorescent puncta (Figure 1I, 3-6 mice for each data point). Statistical analysis indicates that significantly more fluorescence puncta were present in cells responsive to ATP than in control or nonresponsive cells (one-way ANOVA: $F_{(2,20)}=38.49, p<0.001$; Tukey's post hoc comparison: $p<0.001)$. Similarly, compared to controls, significantly more fluorescent puncta were found in cells that responded to the odor mixture $\left(t\right.$-test: $t_{(9)}=3.36$, $p=0.008)$. The increased internalization of the endocytotic dye pHrodo after activation suggests that TRPM5-MCs may release signaling molecules after responding to ATP or the odor mixture.

\section{TRPM5-MCs Respond to ATP Mainly Via Activating P2X Receptors}

We next examined whether purinergic receptors $\mathrm{P} 2 \mathrm{X}$ and/or $\mathrm{P} 2 \mathrm{Y}$ were responsible for the ATP-mediated $\mathrm{Ca}^{2+}$ increases in TRPM5-MCs. To date, seven P2X and eight P2Y subunits are identified in other cells and the $\mathrm{P} 2 \mathrm{X}$ subunits can form functional receptors homomerically or triheteromerically (von Kügelgen, 2006; Coddou et al., 2011). We incubated TRPM5-MCs with the nonselective P2 purinergic receptor antagonist PPADS $(5 \mu \mathrm{M})$ for $300 \mathrm{~s}$ and then stimulated them with $100 \mu \mathrm{M}$ ATP. In the presence of PPADS, approximately $22 \%$ of the recorded cells responded with very small increases in $\mathrm{Ca}^{2+}$ levels (Figure 2). Because P2X are ionotropic and mediate $\mathrm{Ca}^{2+}$ increases via $\mathrm{Ca}^{2+}$ influx from the external bath solution, we next monitored ATP-induced responses in $\mathrm{Ca}^{2+}$-free Tyrode's solution. Approximately 11\% of recorded TRPM5-MCs showed $\mathrm{Ca}^{2+}$ increases in response to ATP $(100 \mu \mathrm{M}, 2 / 18$ cells tested), significantly lower than the $66 \%$ responding cells in normal Tyrode's. These results suggest primary involvement of P2X subtypes (Figure 2; Fisher's exact test: ATP vs. PPADS + ATP, $p=0.024 ;$ ATP vs. $\mathrm{Ca}^{2+}$-free ATP, $p<0.001$,

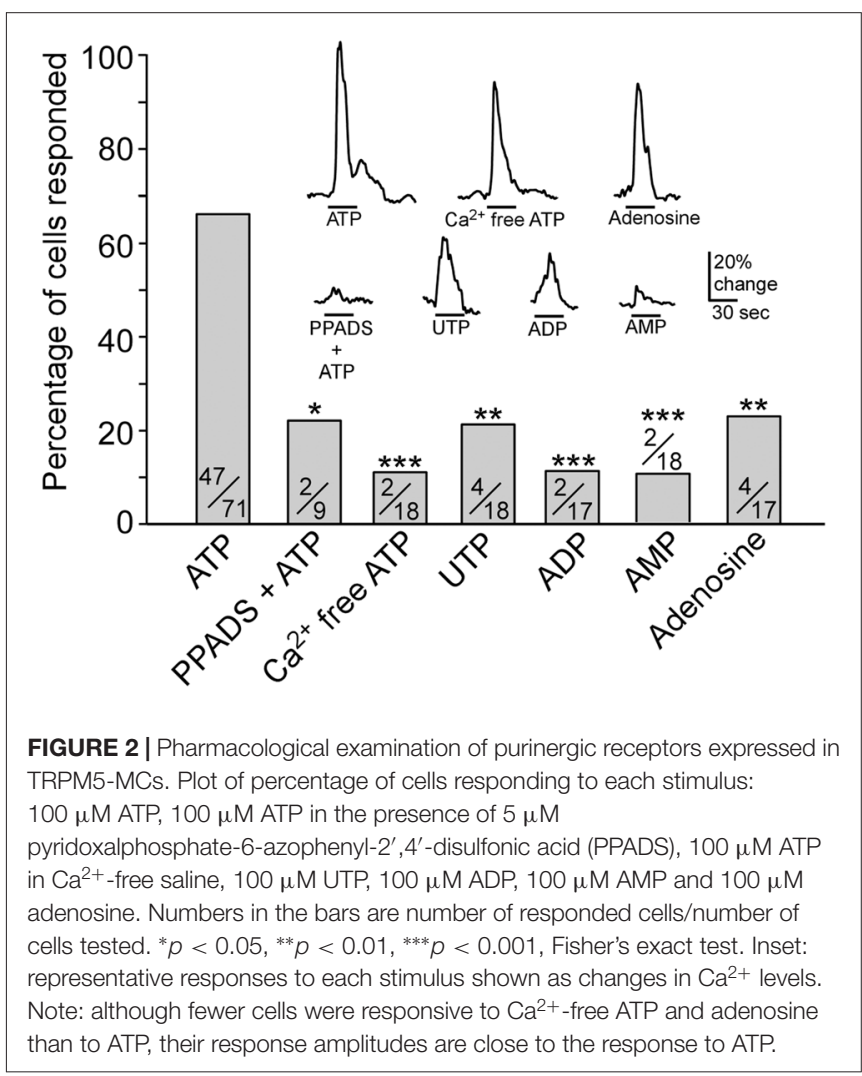



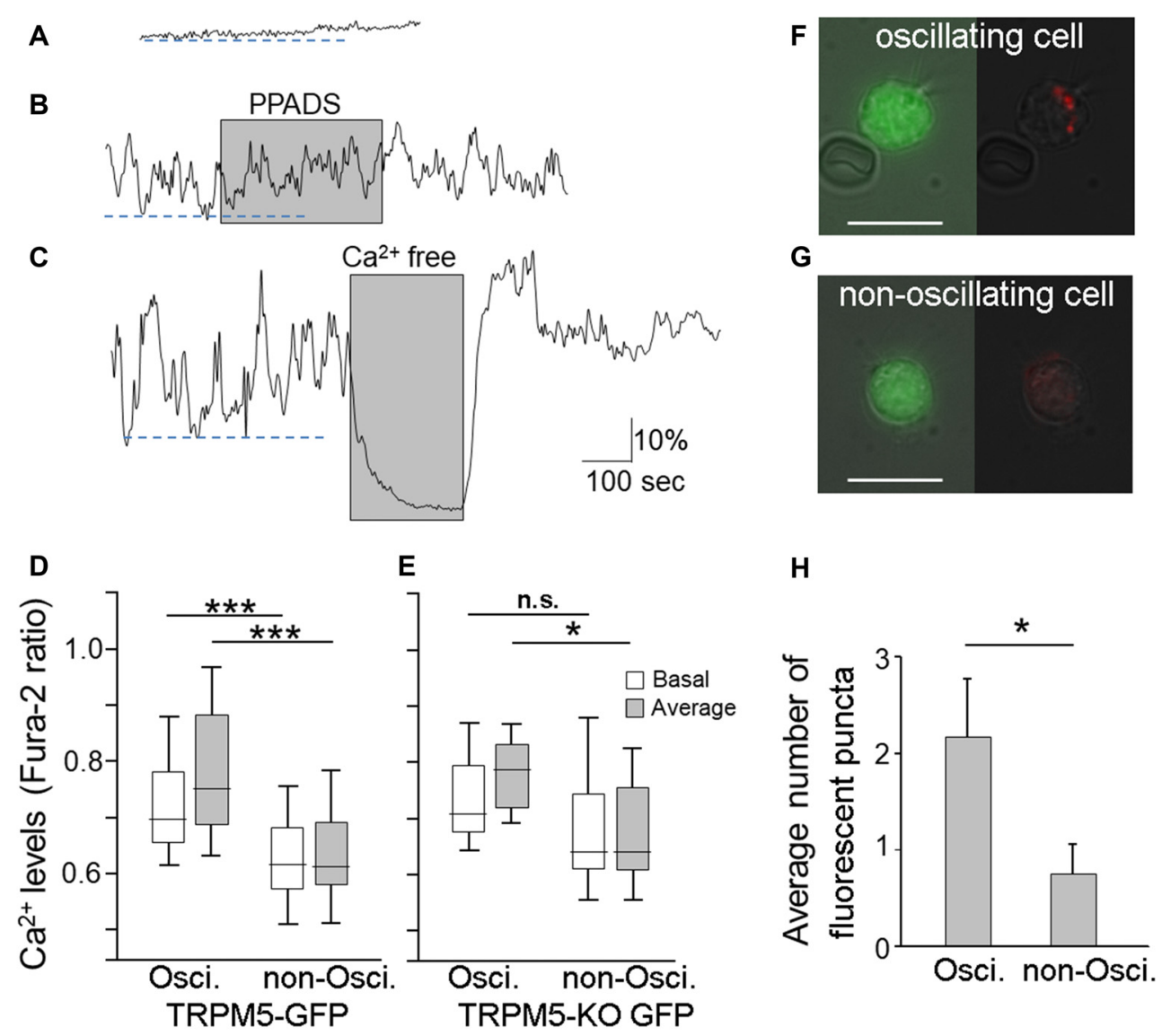

FIGURE 3 | Endocytotic dye internalization in TRPM5-MCs with spontaneous $\mathrm{Ca}^{2+}$ oscillation. (A-C) Traces of changes in Ca ${ }^{2+}$ levels recorded from two TRPM5-MCs. One MC showed a flat baseline $\mathrm{Ca}^{2+}$ level throughout the entire recording time (A); the other exhibited spontaneous $\mathrm{Ca}^{2+}$ oscillation (B,C). The purinergic P2 receptor blocker PPADS $(5 \mu \mathrm{M})$ did not suppress the spontaneous oscillation (B, shaded). Elimination of extracellular $\mathrm{Ca}^{2+}$ using Ca ${ }^{2+}$-free saline diminished the oscillation (C, shaded). Dashed lines indicate basal $\mathrm{Ca}^{2+}$ levels determined by averaging lower peak points of oscillation during the first $300 \mathrm{~s}$ in oscillating cells (B,C) and in non-oscillating cell (A). (D) Basal $\mathrm{Ca}^{2+}$ levels and average $\mathrm{Ca}^{2+}$ levels from oscillating and non-oscillating cells of TRM5-GFP mice $\left({ }^{* * *} p<0.001\right.$, $t$-test, $n=34$ for oscillation cell and 75 for non-oscillating cells, respectively). (E) Basal $\mathrm{Ca}^{2+}$ levels and average Ca ${ }^{2+}$ levels from oscillating and non-oscillating TRPM5knockout (KO)-MCs isolated from TRPM5-KO GFP mice ${ }^{*} p<0.05$, n.s.: not significant, $t$-test, $n=8$ and 8 cells for oscillation cell and for non-oscillating cells, respectively). (F,G) Paired images from an oscillating TRPM5-MC (F) show GFP (green, left panel) and internalized endocytotic dye pHrodo (red) overlaid with weak light image (right panel). A non-oscillating cell (G) shows no internal dye signal. Scale bars: $10 \mu \mathrm{m}$. (H) Average number of dyed puncta inside oscillating and non-oscillating TRPM5-MCs. Oscillating cells have significantly more dyed puncta than do non-oscillating cells $\left({ }^{*} p<0.05, t\right.$-test, $n=6$ for oscillation cell and 8 for non-oscillating cells, respectively).

$n=9-71$ cells from 5 to 14 mice). Because P2X subunits can form functional receptors homomerically or triheteromerically (von Kügelgen, 2006; Coddou et al., 2011), we did not pursue further pharmacological identification of specific P2X subtypes.

In cells that responded to ATP in the $\mathrm{Ca}^{2+}$-free condition, their response amplitude was comparable to those with external $\mathrm{Ca}^{2+}$ (Figure 2, inset). We therefore examined whether TRPM5-MCs express other types of purinergic receptors and the prevalence of these receptors by monitoring the percent of cells responding to the P2Y-specific ligands ADP and UTP $(100 \mu \mathrm{M})$ and the P1 agonist adenosine. Approximately 12 and $22 \%$ of the tested cells responded to ADP and UTP (Figure 2 inset; Fisher's exact test: ATP vs. UTP, $p=0.001$; ATP vs. ADP, $p<0.001$. $n=17-18$ from four mice). These results indicate that a subset of TRPM5-MCs might express G-protein-coupled
P2Y receptors. Further, we tested whether the ATP and ADP metabolite AMP could induce $\mathrm{Ca}^{2+}$ responses in TRPM5MCs. We found that $100 \mu \mathrm{M}$ AMP evoked only a small response in very few cells (2/18 cells from four mice; Figure 2 inset; Fisher's exact test: ATP vs. AMP, $p<0.001)$. Because ectonucleotidase can convert ATP, ADP, and AMP to adenosine, we also examined adenosine $(100 \mu \mathrm{M})$-induced $\mathrm{Ca}^{2+}$ increases in TRPM5-MCs; approximately $24 \%$ of tested cells responded (4/17 from four mice), with response amplitudes comparable to those evoked by ATP (Figure 2 inset; Fisher's exact test: ATP vs. adenosine, $p=0.002$ ). This result suggests potential expression of P1 adenosine receptors in TRPM5-MCs. Taken together, our data indicate that TRPM5-MCs express multiple subtypes of purinergic receptors, with $\mathrm{P} 2 \mathrm{X}$ subtypes being predominant. 

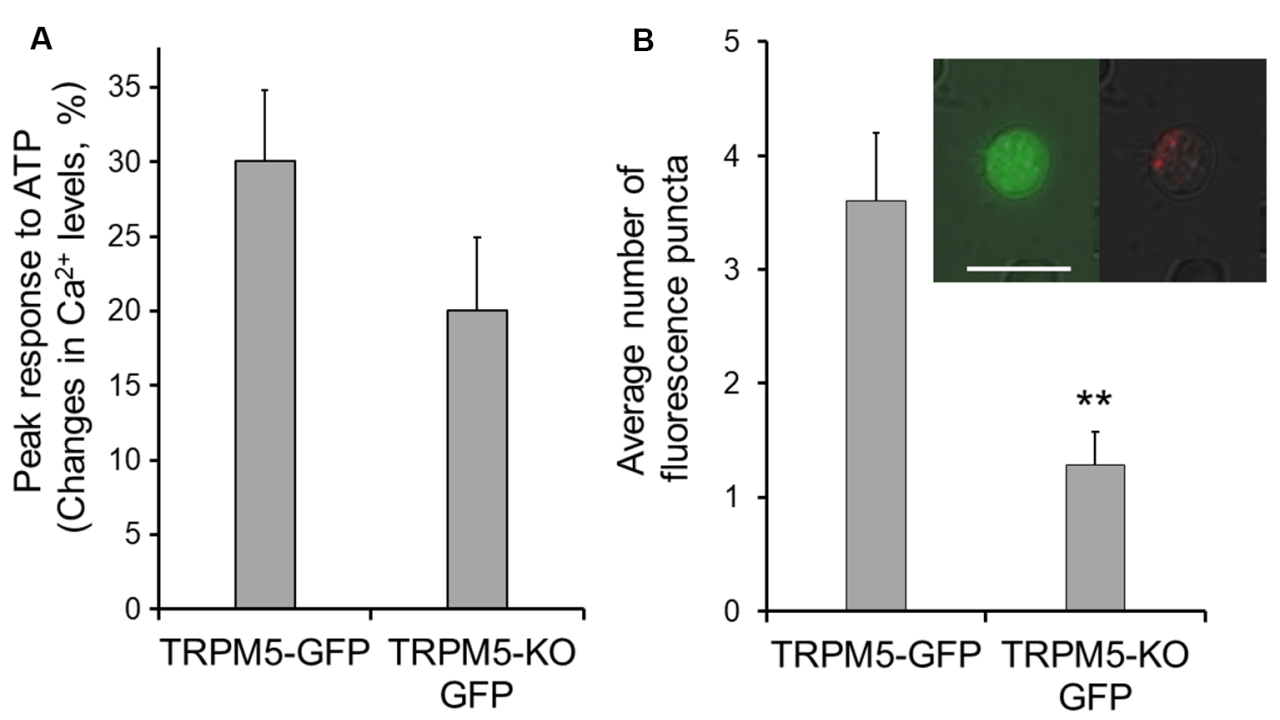

FIGURE 4 | TRPM5-KO MCs of TRPM5-KO GFP mice exhibit lower endocytotic activity. (A) Plot of average peak Ca ${ }^{2+}$ changes in response to 100 $\mu$ M ATP in TRPM5-MCs from TRPM5-GFP mice and TRPM5-KO MCs from TRPM5-KO GFP mice. The average amplitude trends smaller in MCs isolated from TRPM5-KO than in those from TRPM5-GFP mice, although the difference was not significant ( $p=0.152, t$-test, $n=47$ and $n=16$ for control and KO mice, respectively). (B) Average number of internalized pHrodo dye puncta after ATP stimulation. ${ }^{* *} p<0.01, t$-test $(n=5$ and $n=7$ ATP-responsive cells for control and KO mice, respectively). Inset: image of GFP-expressing MCs of TRPM5-KO GFP mice (green, left panel) and of the same cell showing internalized endocytotic dye pHrodo (red, right panel) overlaid with weak light image. Scale bar: $10 \mu \mathrm{m}$.

\section{A Subset of TRPM5-MCs Show Spontaneous Oscillation of $\mathrm{Ca}^{2+}$ Levels and Elevated Endocytotic Dye Internalization}

In our $\mathrm{Ca}^{2+}$ imaging experiments, approximately $70 \%$ of TRPM5-MCs showed stable resting $\mathrm{Ca}^{2+}$ levels (Figure 3A). Our results shown in Figures 1, 2 were obtained in this set of non-oscillating TRPM5-MCs. However, the remaining 30\% of the TRPM5-MCs exhibited spontaneous $\mathrm{Ca}^{2+}$ oscillation (Figures 3B,C). The fluctuating changes ranged between 7.9\% and $100 \%$ from baseline $\mathrm{Ca}^{2+}$ levels (average $30.9 \% \pm 3.8 \%$; $n=34$ cells), and the oscillation frequencies varied from $0.005 \mathrm{~Hz}$ to $0.033 \mathrm{~Hz}(0.015 \pm 0.001 \mathrm{~Hz})$. Application of PPADS $(5 \mu \mathrm{M})$ had no significant effect on the oscillation amplitude or the frequency (Figure 3B, shaded), which eliminated the possible involvement of $\mathrm{P} 2$ receptors. The $\mathrm{Ca}^{2+}$ oscillation disappeared when extracellular $\mathrm{Ca}^{2+}$ was omitted. Interestingly, without external $\mathrm{Ca}^{2+}$, the baseline $\mathrm{Ca}^{2+}$ level was also reduced in the oscillating cells (Figure 3C, shaded). To determine whether the basal $\mathrm{Ca}^{2+}$ level in the oscillating cells was elevated, we compared the basal $\mathrm{Ca}^{2+}$ levels of both the oscillating and non-oscillating cells. We also compared the average values of $\mathrm{Ca}^{2+}$ levels during $300 \mathrm{~s}$ of recording (Figure 3D). Statistical analysis indicated that oscillating cells had significant higher basal $\mathrm{Ca}^{2+}$ levels and average $\mathrm{Ca}^{2+}$ levels than did non-oscillating cells ( $t$ test: $t_{(107)}=4.77$ and 6.24, $p<0.001, n=34$ and $n=75$ from 14 mice, for oscillating and non-oscillating cells, respectively).
We also examined whether TRPM5 influences the oscillation using GFP-expressing MCs dissociated from TRPM5-KO GFP mice. We observed both non-oscillating and oscillating TRPM5-KO MCs. The fluctuations ranged from $7.7 \%$ to $77 \%$ (average $32.73 \% \pm 7.67, n=8$ ) and the oscillation frequencies varied from $0.008 \mathrm{~Hz}$ to $0.027 \mathrm{~Hz}$ (average $0.015 \mathrm{~Hz} \pm 0.002$ ). These values of TRPM5-KO MCs are similar to those of TRPM5-MCs. Additionally, we compared the average values of $\mathrm{Ca}^{2+}$ levels TRPM5-KO MCs (Figure 3E). Statistical analysis indicated that oscillating TRPM5-KO cells had significantly higher average $\mathrm{Ca}^{2+}$ levels than did non-oscillating cells ( $t$-test: $t_{(14)}=2.58, p=0.022, n=8$ and 8 for oscillating and non-oscillating cells, respectively), but there was no significant difference in the basal $\mathrm{Ca}^{2+}$ levels between the two groups ( $t$-test: $\left.t_{(14)}=1.19, p=0.254\right)$. We also examined whether there were differences in basal and average $\mathrm{Ca}^{2+}$ levels between TRPM5-MCs and TRPM5-KO MCs. We found no significant differences both in oscillating and non-oscillating cells $t_{(40)}=0.54$ and 0.26 , $p=0.593$ and 0.799 , for basal and average $\mathrm{Ca}^{2+}$ levels in oscillating cells, and $t_{(81)}=1.66$ and $1.23, p=0.102$ and 0.221 , for basal and average $\mathrm{Ca}^{2+}$ levels in non-oscillating cells, respectively). These data indicate that TRPM5 channels do not significantly influence spontaneous $\mathrm{Ca}^{2+}$ oscillation in these cells.

To test whether the higher levels of intracellular $\mathrm{Ca}^{2+}$ in TRPM5-MCs during oscillation may cause vesicle release and subsequent membrane recycling, we incubated TRPM5-MCs with pHrodo and monitored dye internalization without stimulation (Figure 3F,G). We counted the number of 

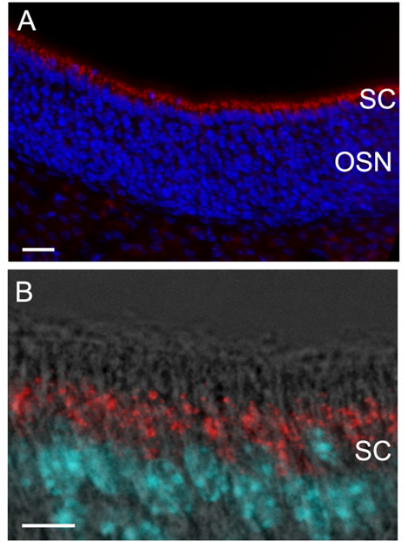
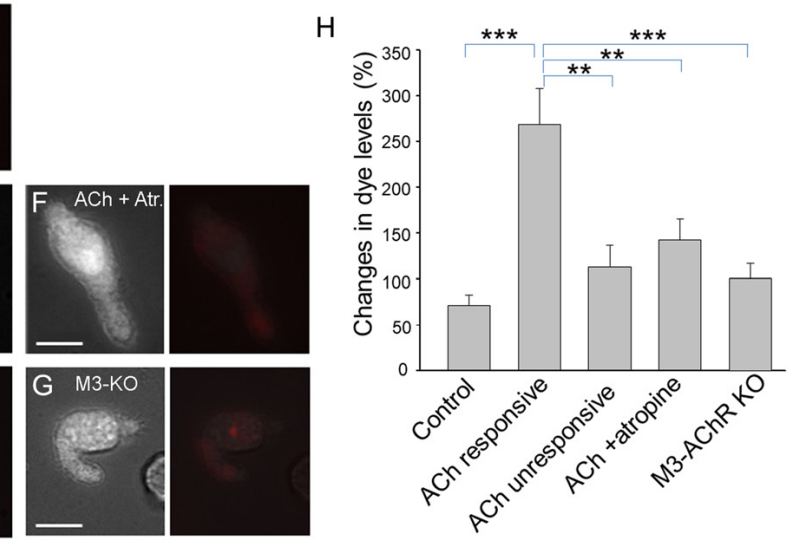

FIGURE 5 | Supporting cells (SCs) of the main olfactory epithelium (MOE) take up endocytotic dye after responses to acetylcholine (ACh). (A,B) Confocal images of EEA1 immunolabeling (red). The MOE section was also stained with nuclear marker DAPI (blue in $\mathbf{A}$ and cyan in $\mathbf{B})$. The higher magnification fluorescence image overlaid with a transmitted light image in (B) shows strong labeling of EEA1 (red) in supranuclear regions of the SCs. (C-G) Paired images of a SC (left panels) and the same cell showing endocytotic dye pHrodo (red) overlaid with weak light image (right panels): cell in control condition without ACh stimulation (C), cell responsive to $100 \mu \mathrm{M}$ ACh (D), ACh-nonresponsive cell (E), ACh stimulation in the presence of $0.5 \mu \mathrm{M}$ atropine (F), and ACh stimulation in a SC isolated from a M3-AChR-KO mouse (G). Scale bars: $10 \mu \mathrm{m}$. (H) Average changes in fluorescence levels of the endocytotic dye pHrodo in the experimental conditions used for (A-E). Averaged fluorescence level was measured from an entire cell body. ${ }^{* *} p<0.01,{ }^{* * *} p<0.001$, one-way ANOVA and Tukey's post hoc test $(n=5-10)$.

dye-labeled puncta and found that oscillating TRPM5-MCs had significantly more puncta than did non-oscillating cells (Figure $3 \mathrm{H}$; t $t$-test: $t_{(12)}=2.25, p=0.044 ; n=6$ and $n=8$ for oscillating and non-oscillating cells, four and three mice, respectively). These results indicate that the oscillating TRPM5-MCs may undergo spontaneous vesicle release after elevated intracellular $\mathrm{Ca}^{2+}$ levels during oscillation.

\section{TRPM5 Knockout Results in a Decrease in ATP-Induced pHrodo Internalization}

In the gut, TRPM5-expressing tuft cells release cytokines to initiate type 2 immunity, in which TRPM5 is indispensable (Gerbe et al., 2016; Howitt et al., 2016). We therefore tested whether TRPM5 is important for vesicle release in TRPM5-MCs of the MOE. We stimulated TRPM5-KO MCs isolated from TRPM5-KO GFP mice with ATP $(100 \mu \mathrm{M})$ and monitored pHrodo internalization following the stimulation. Compared to the average response amplitude obtained from control TRPM5-MCs, the ATP-induced $\mathrm{Ca}^{2+}$ increases in TRPM5-KO MCs trended smaller, but the difference was not statistically significant (Figure 4A; $t$-test: $t_{(43.26)}=1.46, p=0.152$, $n=47$ and $n=16$ for control and KO, 14 and 7 mice, respectively). When examining pHrodo dye internalization following ATP stimulation, we found that ATP-responsive TRPM5 KO MCs had significantly fewer dye-labeled puncta in their cytoplasm than did TRPM5-MCs (Figure 4B and inset; $t$-test: $t_{(10)}=3.83, p=0.003, n=5$ and $n=7$ ATP-responsive cells from three control and seven TRPM5-KO GFP mice, respectively). These data imply that TRPM5 channels might enhance ATP-induced vesicle release and subsequent membrane recycling in TRPM5-MCs.

\section{ACh Stimulates Endocytosis in Supporting Cells (SCs)}

We had previously hypothesized that TRPM5-MCs modulate activity of SCs by releasing $\mathrm{ACh}$, based on the results showing that TRPM5-MCs are cholinergic and that ACh potently increases $\mathrm{Ca}^{2+}$ levels in SCs via muscarinic ACh receptors (AChRs; Ogura et al., 2011). However, it is not known whether ACh-induced increases in $\mathrm{Ca}^{2+}$ levels result in endocytosis in the SCs, or the underlying molecular pathways. Using an antibody against EEA1 in immunolabeling, we first showed the presence of early endosome primary in the supra-nuclear regions, indicating endocytotic activity in SCs (Figures 5A,B). We next monitored ACh-induced changes in $\mathrm{Ca}^{2+}$ levels and pHrodo internalization in SCs using $\mathrm{Ca}^{2+}$ imaging and pHrodo dye. Without ACh stimulation, small amounts of dye were internalized under control conditions, resulting in an average $71 \%$ change in fluorescence intensity (Figures 5C,H). When stimulated with $100 \mu \mathrm{M} A C h$, the ACh-responding SCs were strongly fluorescent, with an average $268 \%$ change in fluorescence intensity, indicating active dye internalization (Figures 5D,H). Because of the substantial amount of dye internalized, we measured the fluorescence intensity of entire SCs and the average changes in the pHrodo dye intensity. SCs that did not respond to ACh showed only low levels of labeling, slightly more than control (Figures 5E,H). Because our previous study showed that ACh-induced $\mathrm{Ca}^{2+}$ increases in SCs are blocked by the muscarinic AChR antagonist atropine (Ogura et al., 2011), we next tested SC endocytosis in the presence of $0.5 \mu \mathrm{M}$ atropine, which significantly attenuated the $100 \mu \mathrm{M}$ ACh-induced pHrodo uptake (Figures 5F,H). Furthermore, we determined the involvement of M3-AChR using SCs isolated from M3AChR-KO mice. pHrodo dye uptake in SCs with a null 

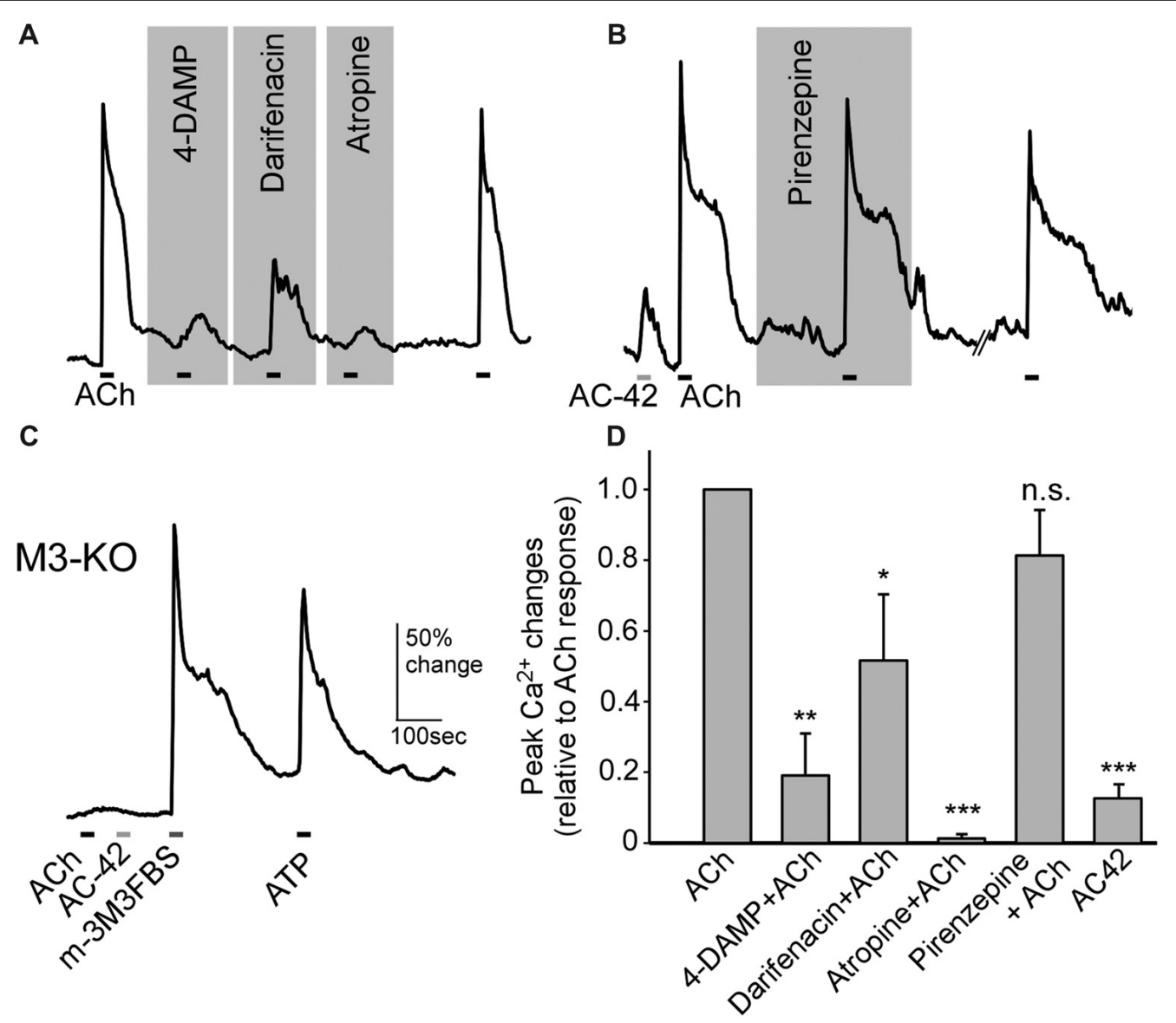

FIGURE 6 | SCs of the MOE increase $\mathrm{Ca}^{2+}$ levels in response to ACh via M3-subtype muscarinic ACh receptors. (A,B) Changes in Ca ${ }^{2+}$ levels in response to $100 \mu \mathrm{M}$ ACh from SCs in the presence of the M3-AChR antagonists 1,1-Dimethyl-4-diphenylacetoxypiperidinium iodide (4-DAMP; 0.1 $\mu$ M), darifenacin (0.1 $\mu$ M) and the pan-muscarinic $\mathrm{AChR}$ antagonist atropine $(0.5 \mu \mathrm{M} ; \mathbf{A})$, and in the presence of the $\mathrm{M} 1$ muscarinic $\mathrm{AChR}$ antagonist pirenzepine $(0.1 \mu \mathrm{M})$ and in response to the M1 muscarinic AChR agonist AC-42 (5 $\mu \mathrm{M}$; B). The ACh response was recoverable after wash. Horizontal bars under the traces indicate application of ACh or AC-42. (C) Recording of changes in $\mathrm{Ca}^{2+}$ levels from a SC of an M3-AChR-KO mouse. The cell failed to respond to ACh and AC-42 but responded to the phospholipase C (PLC) activator m-3M3FBS $(25 \mu \mathrm{M})$ and to ATP (100 $\mu \mathrm{M})$. (D) Average response amplitudes in the presence of the muscarinic receptor antagonists used in (A,B) and to the M1 muscarinic AChR agonist AC-42, as a percentage of average responses to ACh alone in the same cells. Statistical analysis was performed using the original amplitude values between ACh alone and each condition using paired $t$-tests. ${ }^{*} p<0.05,{ }^{* *} p<0.01$, ${ }^{* * *} p<0.001$, n.s.: not significant ( $n=9-11$ for antagonists, $n=43$ for AC-42).

M3-AChR was significantly reduced, to the level similar to that obtained from ACh-nonresponsive SCs or in the presence of atropine in control SCs with a functional M3 (Figures $5 \mathbf{G}, \mathbf{H}$ ). Statistical analysis showed significantly higher levels of dye intensity in ACh-responsive SCs than in those from wild-type mice without stimulation or in the presence of atropine, or in SCs from M3-AChR-KO mice (one-way ANOVA: $F_{(4,33)}=8.639, p<0.001 ;$ Tukey's post hoc comparison: $p<0.001, n=7$ and $n=8$, for control vs. ACh-responsive; $p=0.005, n=8$ and $n=8$ for ACh-unresponsive vs. ACh-responsive; $p=0.007, n=10$ and $n=8$ for ACh + atropine vs. ACh-responsive; $p<0.001, n=5$ and $n=8$ for M3-AChR KO vs. ACh-responsive, three mice were used for each data point). These data strongly suggest that ACh enhances endocytosis by activating the M3-AChR in ACh-responsive SCs.

\section{Supporting Cells Respond to ACh Mainly Via M3-AChR}

Using subtype-specific antibodies, we previously showed strong M3- and also some M1-AChR immunoreactivity in SCs (Ogura et al., 2011). However, pharmacological studies have not been done to confirm the results, and the role of M1 has not been examined. Using $\mathrm{Ca}^{2+}$ imaging, we found that the ACh-induced $\mathrm{Ca}^{2+}$ increases were greatly reduced in the presence of the M3-AChR-selective antagonists 4-DAMP $(0.1 \mu \mathrm{M})$ or darifenacin $(0.1 \mu \mathrm{M})$. As control, we tested the muscarinic nonselective antagonist atropine, which produced similar suppression $(0.5 \mu \mathrm{M}$; Figure 6A). The ACh responses were recovered after the antagonists were washed off with normal saline (Figure 6A). We next examined the effect of the M1AChR-selective agonist AC-42 (5 $\mu \mathrm{M})$ and found it induced a smaller response than did ACh (Figure 6B). Also, an application 
of $\mathrm{ACh}$ in the presence of the M1-AChR-selective antagonist pirenzepine $(0.1 \mu \mathrm{M})$ resulted in only a very small reduction in the response amplitude (Figure 6B). Furthermore, in SCs isolated from M3-AChR-KO mice, both ACh and AC-42 failed to elicit responses (Figure 6C; $n=19$ cells tested). M3-AChR-KO SCs were vital, as the same cells responded to the phospholipase C (PLC) activator m-3M3FBS $(25 \mu \mathrm{M})$ and ATP $(100 \mu \mathrm{M}$; Figure 6C). The data are summarized in Figure 6D, which plots the averaged $\mathrm{ACh}$ responses alone or in the presence of M3 and M1 antagonists, as well as responses to the M1 agonist. The ACh responses were significantly smaller in the presence of antagonists 4-DAMP (paired $t$-test: $t_{(8)}=4.21, p=0.002, n=9$ ), darifenacin (paired $t$-test: $t_{(7)}=2.36, p=0.025, n=8$ ), and atropine $\left(t_{(7)}=5.14, p=0.001, n=8\right)$ but not pirenzepine (paired $t$-test: $t_{(10)}=1.62, p=0.069, n=11$ ). Similar statistical analysis shows that AC- 42 responses are significantly smaller than ACh responses (paired $t$-test: $t_{(42)}=8.12, p<0.001, n=43$ ). Four to 10 mice were used for each data point. Taken together, these pharmacological/physiological results strongly indicate that the $\mathrm{M} 3-\mathrm{AChR}$ is the main excitatory muscarinic receptor mediating ACh modulation of intracellular $\mathrm{Ca}^{2+}$ and endocytosis in SCs.

\section{DISCUSSION}

In this study we investigated chemical responses and potential vesicle release of signaling molecule $\mathrm{ACh}$ in chemoresponsive and cholinergic TRPM5-MCs and ACh-mediated functional modulation of SCs. Our results showed that stimulation of TRPM5-MCs with the signaling molecule ATP and the odor mixture resulted in increases in intracellular $\mathrm{Ca}^{2+}$ and the number of responding cells in a dose-dependent fashion, and ATP responses were primarily mediated by P2X receptors. Importantly, the activated TRPM5-MCs showed a significant increase in the number of pHrodo labeled puncta within their cell bodies, indicating membrane recycling following presumably vesicle release of ACh. TRPM5 KO results in a decrease in ATP-induced pHrodo internalization. Interestingly, spontaneous pHrodo uptake can be found in TRPM5-MCs with elevated and oscillated intracellular $\mathrm{Ca}^{2+}$. Furthermore, we demonstrated that ACh significantly increased intracellular $\mathrm{Ca}^{2+}$ levels and potentiates endocytosis in the SCs. Additionally, we provided evidence for the dominant role of the M3-subtype muscarinic receptors in mediating the ACh effects in SCs. These results are consistent with our previous findings and further demonstrate cholinergic mechanisms in regulating and coordinating MOE multicellular network.

\section{Purinergic Responses and Expression of Purinergic Receptors in TRPM5-MCs}

ATP and its derivatives are known to potently regulate a wide variety of cellular processes in both neuronal and non-neuronal cells via their specific receptors, including exocrine and endocrine secretion, immune responses, inflammation (Burnstock, 2006). Purinergic receptors are divided into P1 type, with adenosine being the endogenous agonist, and P2 type, which can be further divided into ionotropic P2X and metabotropic G-protein coupled P2Y subtypes. The $\mathrm{P} 2 \mathrm{X}$ receptors are non-selective cation channels in general. Activation of the P2X receptors, which we found to be the dominant receptors mediating ATP responses in TRPM5-MCs in this study, is expected to depolarize the cell membrane and increase intracellular $\mathrm{Ca}^{2+}$ levels via $\mathrm{Ca}^{2+}$ influx. The $\mathrm{Ca}^{2+}$ increase may be further enhanced by voltage-gated $\mathrm{Ca}^{2+}$ channels or other $\mathrm{Ca}^{2+}$-activated TRPM5 channels, which are known to potentiate the transduction signal in OSNs (López et al., 2014). TRPM5 is also an essential signaling molecule in taste and other chemosensory receptor cells (Liman, 2007). In our study, we found that ATP response in TRPM5KO-MCs trended smaller and there is a significant reduction in the number of pHrodo-labeled puncta, suggesting that TRPM5 may amplify the ATP signaling in TRPM5-MCs.

While our data obtained from experiments with the nonselective P2 receptor antagonist PPADS (Ralevic and Burnstock, 1998) and $\mathrm{Ca}^{2+}$-free saline demonstrate P2X being the dominant ATP receptors in TRPM5-MCs, the $\mathrm{Ca}^{2+}$ responses induced by UTP and ADP indicate that a small percentage of TRPM5-MCs (approximately 22\% and 12\%) also express P2Y-subtype receptors. Consistently, a few cells responded to ATP in $\mathrm{Ca}^{2+}$-free saline, with the response amplitude similar to that obtained in normal saline. This result is expected because activation of $\mathrm{P}_{2} \mathrm{Y}_{1,2,4,6,11}$ results in $\mathrm{Ca}^{2+}$ release from internal $\mathrm{Ca}^{2+}$ stores via $\mathrm{G}_{\mathrm{q} / 11}$ and the PLC-IP3 pathway (Fredholm et al., 2011). Such $\mathrm{Ca}^{2+}$ increases would persist in $\mathrm{Ca}^{2+}$-free saline.

Our data also indicate the potential presence of P1 receptors since adenosine increased $\mathrm{Ca}^{2+}$ levels in about $24 \%$ cells. The P1 receptors include $A_{1,2 \mathrm{~A}, 2 \mathrm{~B}, 3}$, all of which are G-protein coupled receptors either stimulating $\left(A_{1}\right.$ and $\left.A_{3}\right)$ or inhibiting $\left(\mathrm{A}_{2 \mathrm{~A}}\right.$ and $\left.\mathrm{A}_{2 \mathrm{~B}}\right)$ adenylate cyclase activity (Fredholm et al., 2011). $A_{2 B}$ can also couple through $G_{q / 11}$ to regulate PLC activity (Burnstock, 2007). Therefore depending on P1 subtypes and their downstream signaling, adenosine-mediated purinergic modulation can be diverse. Our result that adenosine application led to an increase in $\mathrm{Ca}^{2+}$ level implies that TRPM5-MCs express stimulatory subtypes and/or $\mathrm{G}_{\mathrm{q} / 11}$ coupled type. The sources of adenosine in the MOE are not determined. Adenosine in other tissues can be generated intracellularly and transported to the extracellular matrix via transporters or extracellularly via ectonucleotidases-mediated metabolizing ATP and its derivatives. A recent study found expression of ectonucleotidases in nasal epithelial cells of zebrafish (Wakisaka et al., 2017). Currently, it is unknown whether TRPM5-MCs express ectonucleotidases on the surface of the cell membrane. In our single-cell recording condition, adenosine generated through ATP metabolism may not be potent enough to evoke responses even if the enzymes are present, due to quick perfusion of the bath solution. However, adenosine generated by surrounding cells in the nose might activate TRPM5-MCs via adenosine receptors under in vivo conditions.

The finding that ATP stimulates TRPM5-MC endocytosis of pHrodo, potentially following vesicle release of $\mathrm{ACh}$, is 
significant because it may provide a mechanism connecting cholinergic and purinergic signaling for the MOE to act in a concerted fashion with the rest of the respiratory mucosa in defense against xenobiotic insults. ATP is an important signaling molecule for airway health and diseases. Nasal epithelial cells release ATP apically via pannexin channels and P2X7 channels to regulate ciliary beating frequency, which plays an important role in airway xenobiotic clearance (Workman et al., 2017). Elevated ATP release can also be caused by hypotonic stress or mechanical stimulation (Seminario-Vidal et al., 2011). ATP can also be released from the $\mathrm{MOE}$ in neonatal slice preparations (Hayoz et al., 2012). The released ATP is postulated to play a role in neuronal homeostasis (Jia et al., 2009), modulating olfactory sensitivity (Hegg et al., 2003), changing intracellular $\mathrm{Ca}^{2+}$ in SCs (Hassenklover et al., 2008), and MOE proliferation (Kanekar et al., 2009). Both P2X and P2Y receptors have been reported in OSNs and SCs based on $\mathrm{Ca}^{2+}$ imaging and immunohistochemistry (Hegg et al., 2003; Gayle and Burnstock, 2005; Hassenklover et al., 2008; Hayoz et al., 2012). However, expression of purinergic receptors in TRPM5-MCs has not been previously reported until this study.

In our $\mathrm{Ca}^{2+}$ imaging study, we found that TRPM5-MCs desensitized in response to repeat ATP stimulation, which prevented us from obtaining ATP dose responses from a single cell. Because the dose response curve was generated by recordings of different cells that were stimulated only once with a particular ATP concentration, with the exception of two lowest concentrations, the variability tended to be large. While more TRPM5-MCs responded to ATP as ATP concentration increased, the average ATP response amplitude obtained with $10 \mu \mathrm{M}$ ATP was larger than that with $50 \mu \mathrm{M}$ ATP although difference was not statistically significant ( $p=0.72$, $t$-test). P2X receptors desensitize and the degrees of which are subunitsdependent. It has been shown that $\mathrm{P} 2 \mathrm{X}_{1-2}$ and $\mathrm{P} 2 \mathrm{X}_{2}$ desensitize rapidly while $\left(\mathrm{P}_{2} \mathrm{X}_{3,4,5,7}\right)$ undergo slow or no desensitization using whole-cell patch recordings from cell lines heterologously expressing homomers of the specific P2X subtypes (North, 2002; Giniatullin and Nistri, 2013; Hausmann et al., 2015). Both fast and slow or non-desensitized P2X are present in nasal epithelia (Hegg et al., 2003; Gayle and Burnstock, 2005). Molecular identity of the $\mathrm{P} 2 \mathrm{X}$ receptors in TRPM5-MCs may provide insight into the desensitization.

\section{Response to Odor Mixture in TRPM5-MCs}

The odor mixture used in this study was the same as that used for the 2-week exposure experiments in our previous study, in which we discovered the role of TRPM5-MCs in maintaining the olfactory responses and guided behaviors (Lemons et al., 2017). The odor components in this mixture were selected because they either are commonly used in manufacturing and regulated for occupational health or are secreted from bacteria known to be present in the nose (Kuwabara et al., 2007; Boase et al., 2013). The new data obtained directly from TRPM5-MC Ca ${ }^{2+}$ imaging in the present study showed that at a 1:50 dilution, which would be a concentration range between 0.3 and $1.7 \mathrm{mM}$ for individual odorants, the odor mixture induced increases in $\mathrm{Ca}^{2+}$ levels in approximately $67 \%$ of cells, supporting our previous hypothesis that TRPM5-MCs are responsive to strong environmental odorants.

\section{Vesicle Release and Membrane Recycling in TRPM5-MCs}

How may TRPM5-MCs exert their protective role to maintain MOE function after activation by chemical stimuli, as revealed in our recent publication (Lemons et al., 2017)? If TRPM5-MCs are to coordinate or modulate activities of the multicellular MOE, paracrine signaling via $\mathrm{ACh}$ may be a major mechanism since ACh has been shown to modulate activities of OSNs and SCs (Jia et al., 2009; Ogura et al., 2011). Further, chemosensory cells in the nose, trachea, gut and urethra are cholinergic (Ogura et al., 2010, 2011; Krasteva et al., 2011; Deckmann et al., 2014; Saunders et al., 2014; Schütz et al., 2015; Hayakawa et al., 2017) and ACh release from the isolated cells was measured in urethra (Deckmann et al., 2014). However, evidence of vesicle release is missing for TRPM5-MCs, as well as for other SCCs. In the gastrointestinal tract, TRPM5-expressng tuft cells release the cytokine interleukin-25 to initiate type 2 immunity against parasite infection (Howitt et al., 2016; von Moltke et al., 2016). Cytokines are commonly packaged in vesicles and released via exocytosis, which would be accompanied by membrane recycling (Stanley and Lacy, 2010). However, cholinergic brush cells in the gut and biliary tract do not express vesicular ACh transporter, which implies non-vesicle release of ACh (Schütz et al., 2015). In our study, we examined vesicle release using the endocytosis dye pHrodo. The significant increases in the number of pHrodo-labeled puncta in TRPM5-MCs following ATP- and odor mixture-induced increases in intracellular $\mathrm{Ca}^{2+}$ suggest that activation of these cells leads to vesicle release and subsequent recycling that internalizes the dye. Because the epi-florescence images of pHrodo-labeled puncta are convoluted and as such, they would most likely appear larger than the actual sizes.

\section{Role of TRPM5 in $\mathrm{Ca}^{2+}$ Signaling and Vesicle Release in TRPM5-MCs}

In the gut, TRPM5 $\mathrm{KO}$ in tuft cells significantly impaired the type 2 immunity against parasite (Howitt et al., 2016), indicating that TRPM5 plays an important role in the function of these chemosensory cells. Consistently, we found that TRPM5 KO significantly reduced the number of pHrodolabeled puncta after responses to ATP. TRPM5 is known to serve as the downstream effector of the PLC pathway in nonneuronal chemosensory cells, including SCCs, brush cells and tuft cells found in various tissues, and taste receptor cells (Finger and Kinnamon, 2011). TRPM5 activation requires a rapid increase of intracellular $\mathrm{Ca}^{2+}$ (Liu and Liman, 2003). Elevated intracellular $\mathrm{Ca}^{2+}$ by ATP may subsequently activate TRPM5 channels, further amplifying the $\mathrm{Ca}^{2+}$ signal for vesicle release. This likely represents a newly identified role of TRPM5 in these cells.

For potential vesicle release in TRPM5-MCs, $\mathrm{Ca}^{2+}$ load may be a key factor, as we found that ATP-responsive TRPM5-MCs showed a higher number of pHrodo-labeled 
puncta than those cells responsive to the odor mixture. We noticed that the $\mathrm{Ca}^{2+}$ responses to ATP lasted longer than the response to odor mixture which presumably increased the total $\mathrm{Ca}^{2+}$ load in these cells. Interestingly, we observed spontaneous intracellular $\mathrm{Ca}^{2+}$ oscillation in a subset of isolated TRPM5-MCs. When examining pHrodo uptake in these $\mathrm{Ca}^{2+}$ oscillating cells, we found that the number of dye-labeled puncta was similar to that found in cells after responses to the odor mixture. These data suggest that the spontaneous oscillation and elevated basal $\mathrm{Ca}^{2+}$ levels in these cells were enough for vesicle release without stimulation. In agreement, a recent report showed that tuft cells release basal levels of cytokines, tuning immunity in the gut (von Moltke et al., 2016).

\section{Endocytosis in the SCs}

Using the endocytosis dye pHrodo, we were able to show that both unstimulated and stimulated endocytosis was occurring in the SCs. Unlike the dye labeling in TRPM5-MCs, where we could discern individual pHrodo-labeled puncta, labeling in SCs, especially after activation, was massive, and labeled vesicles or vacuoles were hard to separate. Our pHrodo labeling result is consistent with the positive immunoreaction of EEA1 that labels early endosome in SCs and also consistent with previous electron microscopy findings that SCs contain numerous vesicle and vacuoles, especially in the supranuclear region (Getchell and Mellert, 1991; Getchell and Getchell, 1992). SCs are the key cell type for xenobiotic removal in the upper airway. SCs express a variety of xenobiotic-metabolizing enzymes. Some of these enzymes function intracellularly, which requires engulfing of xenobiotics. Some other enzymes may be secreted to the mucus layer where they metabolize xenobiotics including odor molecules (Menco and Morrison, 2003; Asakawa et al., 2017). Currently, there is little information how these events are regulated. In our study, we found that ACh strongly potentiates dye uptake, and this modification relied primarily on the functional expression of M3-subtype muscarinic receptors. Because of the close anatomical relation between the TRPM5-MCs and SCs, we consider that ACh most likely is released from the cholinergic TRPM5-MCs (Ogura et al., 2011). Release of ACh has been measured in TRPM5expressing chemosensory cells in the urethra (Deckmann et al., 2014). The cholinergic paracrine regulation would enable TRPM5-MCs to modify and coordinate SC activity with OSNs and potential other MOE cell types in xenobiotic removal and MOE maintenance.

ACh has been found to modulate variety of cellular functions via paracrine pathways. For example, adipose stem cells enhance myoblast proliferation via paracrine secretion of ACh (El-Habta et al., 2018). Granulosa cells and luteal cells in ovary release ACh to promote follicular development and female fertility (Mayerhofer and Fritz, 2002; Urra et al., 2016). In the carotid body, intrinsic release of $\mathrm{ACh}$ is used for intercellular coordinated chemical sensing (Kåhlin et al., 2014). In the trachea, ACh from chemosensory brush cells modulates breath rate and airway clearance (Krasteva et al., 2011). Thus, ACh-mediated modification allows different cell types to work in concerted fashion within multicellular networks.

Although we cannot rule out the possibility that ACh from sources other than TRPM5-MCs activates the SCs, our previous study using both transgenic mice and immunolabeling all showed that within the MOE cells, TRPM5-MCs are the only cell type expressing ChAT and VAChT. Their cholinergic nature was further demonstrated by using transgenic $\mathrm{ChAT}^{(\mathrm{BAC})-} \mathrm{eGFP}$ mice in which GFP expression is strong in both cell bodies and nerve fibers (Ogura et al., 2011; Krosnowski et al., 2012; Marking et al., 2017). We observed cholinergic fibers $(\mathrm{GFP}+)$ in lamina propria innervating submucosal blood vesseles and glandular tissues, but rarely noticed GFP+ nerve fibers penetrating into the cell layers of the MOE. We therefore believe that TRPM5-MCs in the MOE are the primary source for ACh release to regulate SC activity. Future experiments on the release of ACh from TRPM5-MCs will greatly advance our understanding of the MOE cholinergic network and regulation.

\section{Cholinergic Receptors in SCs}

Previously we demonstrated using intracellular $\mathrm{Ca}^{2+}$ imaging and immunolabeling that $\mathrm{ACh}$ induces $\mathrm{Ca}^{2+}$ increases in SCs via muscarinic receptors and expression of M3 and M1 subtypes (Ogura et al., 2011). Our present results obtained using pharmacological agents as well as M3-AChR-KO mice further provide evidence that $\mathrm{M} 3-\mathrm{AChR}$ plays a major role in mediating ACh-induced $\mathrm{Ca}^{2+}$ responses in SCs. However, inconsistent results were obtained from the M1-subtype antagonist and agonist. While the M1-selective antagonist pirenzepine did not significantly reduce responses to $\mathrm{ACh}$ in control mice, the M1-selective agonist AC-42 induced responses in some SCs. Intriguingly, in SCs isolated from M3-AChR-KO mice we did not observe responses to AC-42. One possible explanation is that AC-42 might have activated the M3 subtype. However, AC-42 reportedly is highly selective for the M1 subtype up to 100 $\mu \mathrm{M}$ in heterologous cells expressing human muscarinic subtypes (Spalding et al., 2002; Jacobson et al., 2010). At maximum levels AC-42 induces phosphatidylinositol turnover and $\mathrm{Ca}^{2+}$ mobilization equivalent to $66 \%-85 \%$ of the maximum responses induced by ACh or carbachol (Spalding et al., 2002; Langmead et al., 2006; Jacobson et al., 2010). In our study only a subset of SCs (5 of 43 SCs tested) were responsive to AC-42, and the response amplitude was significantly less than average ACh response amplitude; therefore, M1 receptors, if involved, likely do not play a major role in SCs.

In sum, our results show that TRPM5-MCs dose-dependently respond to ATP and odor mixture and may release ACh to potentiate endocytosis in SCs, possibly promoting xenobiotic removal from the MOE. These results have unveiled cholinergic regulation in the MOE coordinating SC activity important for protecting the epithelium and airway. That TRPM5-MCs are sensitive to ATP and express multiple purinergic receptors also suggests an additional mechanism for the MOE to act in a concerted fashion with the rest of the respiratory mucosa to defend against xenobiotic insults. Taken together, these novel results of cholinergic paracrine 
signaling in the MOE increase our understanding of how the MOE maintains its function and prevents chemical-induced damage.

\section{DATASETS ARE AVAILABLE ON REQUEST}

The raw data supporting the conclusions of this manuscript will be made available by the authors, without undue reservation, to any qualified researcher.

\section{AUTHOR CONTRIBUTIONS}

WLin conceived and supervised the project; wrote the manuscript with drafts and input from the other authors.

\section{REFERENCES}

Asakawa, M., Fukutani, Y., Savangsuksa, A., Noguchi, K., Matsunami, H., and Yohda, M. (2017). Modification of the response of olfactory receptors to acetophenone by CYP1a2. Sci. Rep. 7:10167. doi: 10.1038/s41598-017-10862-5

Bezençon, C., Fürholz, A., Raymond, F., Mansourian, R., Métairon, S., Le Coutre, J., et al. (2008). Murine intestinal cells expressing Trpm5 are mostly brush cells and express markers of neuronal and inflammatory cells. J. Comp. Neurol. 509, 514-525. doi: 10.1002/cne.21768

Boase, S., Foreman, A., Cleland, E., Tan, L., Melton-Kreft, R., Pant, H., et al. (2013). The microbiome of chronic rhinosinusitis: culture, molecular diagnostics and biofilm detection. BMC Infect. Dis. 13:210. doi: 10.1186/1471-2334 $-13-210$

Burnstock, G. (2006). Pathophysiology and therapeutic potential of purinergic signaling. Pharmacol. Rev. 58, 58-86. doi: 10.1124/pr.58.1.5

Burnstock, G. (2007). Purine and pyrimidine receptors. Cell. Mol. Life Sci. 64, 1471-1483. doi: 10.1007/s00018-007-6497-0

Clapp, T. R., Medler, K. F., Damak, S., Margolskee, R. F., and Kinnamon, S. C. (2006). Mouse taste cells with G protein-coupled taste receptors lack voltagegated calcium channels and SNAP-25. BMC Biol. 4:7. doi: 10.1186/17417007-4-7

Coddou, C., Yan, Z., Obsil, T., Huidobro-Toro, J. P., and Stojilkovic, S. S. (2011). Activation and regulation of purinergic $\mathrm{P} 2 \mathrm{X}$ receptor channels. Pharmacol. Rev. 63, 641-683. doi: 10.1124/pr.110.003129

Damak, S., Rong, M., Yasumatsu, K., Kokrashvili, Z., Pérez, C. A., Shigemura, N., et al. (2006). Trpm5 null mice respond to bitter, sweet and umami compounds. Chem. Senses 31, 253-264. doi: 10.1093/chemse/bjj027

Deckmann, K., Filipski, K., Krasteva-Christ, G., Fronius, M., Althaus, M., Rafiq, A., et al. (2014). Bitter triggers acetylcholine release from polymodal urethral chemosensory cells and bladder reflexes. Proc. Natl. Acad. Sci. U S A 111, 8287-8292. doi: 10.1073/pnas.1402436111

Ding, X., and Dahl, A. R. (2003). "Olfactory mucosa: composition, enzymatic localization, and metabolism," in Handbook of Olfaction and Gustation, 2nd Edn. ed. R. L. Doty (New York, NY: Marcel Dekker, Inc.), 51-73.

Dunston, D., Ashby, S., Krosnowski, K., Ogura, T., and Lin, W. (2013). An effective manual deboning method to prepare intact mouse nasal tissue with preserved anatomical organization. J. Vis. Exp. 78:e50538. doi: 10.3791/ 50538

El-Habta, R., Kingham, P. J., and Backman, L. J. (2018). Adipose stem cells enhance myoblast proliferation via acetylcholine and extracellular signalregulated kinase 1/2 signaling. Muscle Nerve 57, 305-311. doi: 10.1002/mus. 25741

Farbman, A. (2000). "Cell biology of olfactory epithelium," in The Neurobiology of Taste and Smell, 2nd Edn. eds T. E. Finger, W. L. Silver and D. Restrepo (New York, NY: Wiley-Liss), 131-158.

Finger, T. E., Böttger, B., Hansen, A., Anderson, K. T., Alimohammadi, H., and Silver, W. L. (2003). Solitary chemoreceptor cells in the nasal cavity serve as sentinels of respiration. Proc. Natl. Acad. Sci. U S A 100, 8981-8986. doi: 10.1073/pnas.1531172100
ZF performed most imaging experiments. TO performed some imaging experiments. WLuo did the immunolabeling. WLin, ZF and TO designed the experiments and analyzed data.

\section{FUNDING}

This work was supported by NIH/National Institute on Deafness and Other Communication Disorders (NIDCD) grant R01DC012831 to WLin.

\section{ACKNOWLEDGMENTS}

We thank Kayla Lemons and Abdullah Almatrouk for critically reading the manuscript.

Finger, T. E., and Kinnamon, S. C. (2011). Taste isn't just for taste buds anymore. Biol. Rep. 3:20. doi: 10.3410/B3-20

Fredholm, B. B., Ap, I. J., Jacobson, K. A., Linden, J., and Müller, C. E. (2011). International union of basic and clinical pharmacology. LXXXI. Nomenclature and classification of adenosine receptors-an update. Pharmacol. Rev. 63, 1-34. doi: 10.1124/pr.110.003285

Gayle, S., and Burnstock, G. (2005). Immunolocalisation of P2X and P2Y nucleotide receptors in the rat nasal mucosa. Cell Tissue Res. 319, 27-36. doi: 10.1007/s00441-004-0979-2

Gerbe, F., Sidot, E., Smyth, D. J., Ohmoto, M., Matsumoto, I., Dardalhon, V., et al. (2016). Intestinal epithelial tuft cells initiate type 2 mucosal immunity to helminth parasites. Nature 529, 226-230. doi: 10.1038/nature16527

Getchell, M. L., and Getchell, T. V. (1992). Fine structural aspects of secretion and extrinsic innervation in the olfactory mucosa. Microsc. Res. Tech. 23, 111-127. doi: 10.1002/jemt.1070230203

Getchell, M. L., and Mellert, T. K. (1991). "Olfactory mucus secretion," in Smell and Taste in Health and Disease, eds T. V. Getchell, R. L. Doty, L. Bartoshuk and J. B. Snow (New York, NY: Raven Press), 83-95.

Giniatullin, R., and Nistri, A. (2013). Desensitization properties of P2X3 receptors shaping pain signaling. Front. Cell. Neurosci. 7:245. doi: 10.3389/fncel.2013. 00245

Gross, E. A., Swenberg, J. A., Fields, S., and Popp, J. A. (1982). Comparative morphometry of the nasal cavity in rats and mice. J. Anat. 135, 83-88.

Gulbransen, B. D., Clapp, T. R., Finger, T. E., and Kinnamon, S. C. (2008). Nasal solitary chemoreceptor cell responses to bitter and trigeminal stimulants in vitro. J. Neurophysiol. 99, 2929-2937. doi: 10.1152/jn.00066.2008

Hansen, A., and Finger, T. E. (2008). Is TrpM5 a reliable marker for chemosensory cells? Multiple types of microvillous cells in the main olfactory epithelium of mice. BMC Neurosci. 9:115. doi: 10.1186/1471-2202-9-115

Hassenklover, T., Kurtanska, S., Bartoszek, I., Junek, S., Schild, D., and Manzini, I. (2008). Nucleotide-induced $\mathrm{Ca}^{2+}$ signaling in sustentacular supporting cells of the olfactory epithelium. Glia 56, 1614-1624. doi: 10.1002/glia.20714

Hausmann, R., Kless, A., and Schmalzing, G. (2015). Key sites for P2X receptor function and multimerization: overview of mutagenesis studies on a structural basis. Curr. Med. Chem. 22, 799-818. doi: $10.2174 / 0929867322666141128163215$

Hayakawa, Y., Sakitani, K., Konishi, M., Asfaha, S., Niikura, R., Tomita, H., et al. (2017). Nerve growth factor promotes gastric tumorigenesis through aberrant cholinergic signaling. Cancer Cell 31, 21-34. doi: 10.1016/j.ccell.2016. 11.005

Hayoz, S., Jia, C., and Hegg, C. (2012). Mechanisms of constitutive and ATP-evoked ATP release in neonatal mouse olfactory epithelium. BMC Neurosci. 13:53. doi: 10.1186/1471-2202-13-53

Hegg, C. C., Greenwood, D., Huang, W., Han, P., and Lucero, M. T. (2003). Activation of purinergic receptor subtypes modulates odor sensitivity. J. Neurosci. 23, 8291-8301.

Howitt, M. R., Lavoie, S., Michaud, M., Blum, A. M., Tran, S. V., Weinstock, J. V., et al. (2016). Tuft cells, taste-chemosensory cells, orchestrate parasite type 2 immunity in the gut. Science 351, 1329-1333. doi: 10.1126/science.aaf1648 
Hu, J., Sheng, L., Li, L., Zhou, X., Xie, F., D'Agostino, J., et al. (2014). Essential role of the cytochrome P450 enzyme CYP2A5 in olfactory mucosal toxicity of naphthalene. Drug Metab. Dispos. 42, 23-27. doi: 10.1124/dmd.113. 054429

Jacobson, M. A., Kreatsoulas, C., Pascarella, D. M., O’Brien, J. A., and Sur, C. (2010). The M1 muscarinic receptor allosteric agonists AC-42 and 1-[1'(2-methylbenzyl)-1,4'-bipiperidin-4-yl]-1,3-dihydro-2H-benzimidazol-2-one bind to a unique site distinct from the acetylcholine orthosteric site. Mol. Pharmacol. 78, 648-657. doi: 10.1124/mol.110.065771

Jia, C., Doherty, J. P., Crudgington, S., and Hegg, C. C. (2009). Activation of purinergic receptors induces proliferation and neuronal differentiation in Swiss Webster mouse olfactory epithelium. Neuroscience 163, 120-128. doi: 10.1016/j.neuroscience.2009.06.040

Kåhlin, J., Mkrtchian, S., Ebberyd, A., Hammarstedt-Nordenvall, L., Nordlander, B., Yoshitake, T., et al. (2014). The human carotid body releases acetylcholine, ATP and cytokines during hypoxia. Exp. Physiol. 99, 1089-1098. doi: 10.1113/expphysiol.2014.078873

Kanekar, S., Jia, C., and Hegg, C. C. (2009). Purinergic receptor activation evokes neurotrophic factor neuropeptide $\mathrm{Y}$ release from neonatal mouse olfactory epithelial slices. J. Neurosci. Res. 87, 1424-1434. doi: 10.1002/jnr. 21954

Kawashima, K., and Fujii, T. (2008). Basic and clinical aspects of non-neuronal acetylcholine: overview of non-neuronal cholinergic systems and their biological significance. J. Pharmacol. Sci. 106, 167-173. doi: 10.1254/jphs. fm0070073

Krasteva, G., Canning, B. J., Hartmann, P., Veres, T. Z., Papadakis, T., Muhlfeld, C., et al. (2011). Cholinergic chemosensory cells in the trachea regulate breathing. Proc. Natl. Acad. Sci. U S A 108, 9478-9483. doi: 10.1073/pnas.1019418108

Krosnowski, K., Ashby, S., Sathyanesan, A., Luo, W., Ogura, T., and Lin, W. (2012). Diverse populations of intrinsic cholinergic interneurons in the mouse olfactory bulb. Neuroscience 213, 161-178. doi: 10.1016/j.neuroscience.2012. 04.024

Kuwabara, Y., Alexeeff, G. V., Broadwin, R., and Salmon, A. G. (2007). Evaluation and application of the RD50 for determining acceptable exposure levels of airborne sensory irritants for the general public. Environ. Health Perspect. 115, 1609-1616. doi: 10.1289/ehp.9848

Langmead, C. J., Fry, V. A., Forbes, I. T., Branch, C. L., Christopoulos, A., Wood, M. D., et al. (2006). Probing the molecular mechanism of interaction between 4- $n$-butyl-1-[4-(2-methylphenyl)-4-oxo-1-butyl]-piperidine (AC42) and the muscarinic $M(1)$ receptor: direct pharmacological evidence that AC-42 is an allosteric agonist. Mol. Pharmacol. 69, 236-246. doi: 10.1124/mol.105.017814

Lee, R. J., Chen, B., Redding, K. M., Margolskee, R. F., and Cohen, N. A. (2014). Mouse nasal epithelial innate immune responses to Pseudomonas aeruginosa quorum-sensing molecules require taste signaling components. Innate Immun. 20, 606-617. doi: 10.1177/1753425913503386

Lemons, K., Fu, Z., Aoudé, I., Ogura, T., Sun, J., Chang, J., et al. (2017). Lack of TRPM5-expressing microvillous cells in mouse main olfactory epithelium leads to impaired odor-evoked responses and olfactory-guided behavior in a challenging chemical environment. eNeuro 4:ENEURO.0135-17.2017. doi: 10.1523/eneuro.0135-17.2017

Liman, E. R. (2007). "TRPM5 and taste transduction," in Transient Receptor Potential (TRP) Channels. Handbook of Experimental Pharmacology, (Vol. 179) eds V. Flockerzi and B. Nilius (Heidelberg, Berlin: Springer), 287-298.

Lin, W., Ezekwe, E. A. Jr., Zhao, Z., Liman, E. R., and Restrepo, D. (2008a). TRPM5-expressing microvillous cells in the main olfactory epithelium. BMC Neurosci. 9:114. doi: 10.1186/1471-2202-9-114

Lin, W., Ogura, T., Margolskee, R. F., Finger, T. E., and Restrepo, D. (2008b). TRPM5-expressing solitary chemosensory cells respond to odorous irritants. J. Neurophysiol. 99, 1451-1460. doi: 10.1152/jn.01195.2007

Liu, D., and Liman, E. R. (2003). Intracellular $\mathrm{Ca}^{2+}$ and the phospholipid PIP2 regulate the taste transduction ion channel TRPM5. Proc. Natl. Acad. Sci. U S A 100, 15160-15165. doi: 10.1073/pnas.2334159100

López, F., Delgado, R., López, R., Bacigalupo, J., and Restrepo, D. (2014). Transduction for pheromones in the main olfactory epithelium is mediated by the $\mathrm{Ca}^{2+}$-activated channel TRPM5. J. Neurosci. 34, 3268-3278. doi: 10.1523/jneurosci.4903-13.2014
Marking, S., Krosnowski, K., Ogura, T., and Lin, W. (2017). Dichotomous distribution of putative cholinergic interneurons in mouse accessory olfactory bulb. Front. Neuroanat. 11:10. doi: 10.3389/fnana.2017. 00010

Mayerhofer, A., and Fritz, S. (2002). Ovarian acetylcholine and muscarinic receptors: hints of a novel intrinsic ovarian regulatory system. Microsc. Res. Tech. 59, 503-508. doi: 10.1002/jemt.10228

Menco, B. P., and Morrison, E. E. (2003). "Morphology of the mammalian olfactory epithelium: form, fine structure, function, and pathology," in Handbook of Olfaction and Gustation, 2nd Edn. ed. R. L. Doty (New York, NY: Marcel Dekker, Inc.), 17-50.

North, R. A. (2002). Molecular physiology of P2X receptors. Physiol. Rev. 82, 1013-1067. doi: 10.1152/physrev.00015.2002

Ogura, T., Krosnowski, K., Zhang, L., Bekkerman, M., and Lin, W. (2010). Chemoreception regulates chemical access to mouse vomeronasal organ: role of solitary chemosensory cells. PLoS One 5:e11924. doi: 10.1371/journal.pone. 0011924

Ogura, T., Szebenyi, S. A., Krosnowski, K., Sathyanesan, A., Jackson, J., and Lin, W. (2011). Cholinergic microvillous cells in the mouse main olfactory epithelium and effect of acetylcholine on olfactory sensory neurons and supporting cells. J. Neurophysiol. 106, 1274-1287. doi: 10.1152/jn. 00186.2011

Rafols, J. A., and Getchell, T. V. (1983). Morphological relations between the receptor neurons, sustentacular cells and Schwann cells in the olfactory mucosa of the salamander. Anat. Rec. 206, 87-101. doi: 10.1002/ar.10920 60111

Ralevic, V., and Burnstock, G. (1998). Receptors for purines and pyrimidines. Pharmacol. Rev. 50, 413-492.

Saunders, C. J., Christensen, M., Finger, T. E., and Tizzano, M. (2014). Cholinergic neurotransmission links solitary chemosensory cells to nasal inflammation. Proc. Natl. Acad. Sci. U S A 111, 6075-6080. doi: 10.1073/pnas.1402 251111

Schütz, B., Jurastow, I., Bader, S., Ringer, C., von Engelhardt, J., Chubanov, V., et al. (2015). Chemical coding and chemosensory properties of cholinergic brush cells in the mouse gastrointestinal and biliary tract. Front. Physiol. 6:87. doi: 10.3389/fphys.2015.00087

Seminario-Vidal, L., Okada, S. F., Sesma, J. I., Kreda, S. M., Van Heusden, C. A., Zhu, Y., et al. (2011). Rho signaling regulates pannexin 1-mediated ATP release from airway epithelia. J. Biol. Chem. 286, 26277-26286. doi: 10.1074/jbc.M111. 260562

Spalding, T. A., Trotter, C., Skjaerbaek, N., Messier, T. L., Currier, E. A., Burstein, E. S., et al. (2002). Discovery of an ectopic activation site on the M(1) muscarinic receptor. Mol. Pharmacol. 61, 1297-1302. doi: 10.1124/mol. 61.6.1297

Stanley, A. C., and Lacy, P. (2010). Pathways for cytokine secretion. Physiology 25, 218-229. doi: 10.1152/physiol.00017.2010

Thiebaud, N., Sigoillot, M., Chevalier, J., Artur, Y., Heydel, J. M., and Le Bon, A. M. (2010). Effects of typical inducers on olfactory xenobioticmetabolizing enzyme, transporter and transcription factor expression in rats. Drug Metab. Dispos. 38, 1865-1875. doi: 10.1124/dmd.110. 035014

Thornton-Manning, J. R., and Dahl, A. R. (1997). Metabolic capacity of nasal tissue interspecies comparisons of xenobiotic-metabolizing enzymes. Mutat. Res. 380, 43-59. doi: 10.1016/S0027-5107(97)00126-7

Tizzano, M., Gulbransen, B. D., Vandenbeuch, A., Clapp, T. R., Herman, J. P., Sibhatu, H. M., et al. (2010). Nasal chemosensory cells use bitter taste signaling to detect irritants and bacterial signals. Proc. Natl. Acad. Sci. U S A 107, 3210-3215. doi: 10.1073/pnas.0911934107

Urra, J., Blohberger, J., Tiszavari, M., Mayerhofer, A., and Lara, H. E. (2016). In vivo blockade of acetylcholinesterase increases intraovarian acetylcholine and enhances follicular development and fertility in the rat. Sci. Rep. 6:30129. doi: 10.1038/srep30129

Vogalis, F., Hegg, C. C., and Lucero, M. T. (2005). Ionic conductances in sustentacular cells of the mouse olfactory epithelium. J. Physiol. 562, 785-799. doi: 10.1113/jphysiol.2004.079228

von Kügelgen, I. (2006). Pharmacological profiles of cloned mammalian P2Y-receptor subtypes. Pharmacol. Ther. 110, 415-432. doi: 10.1016/j. pharmthera.2005.08.014 
von Moltke, J., Ji, M., Liang, H. E., and Locksley, R. M. (2016). Tuft-cell-derived IL-25 regulates an intestinal ILC2-epithelial response circuit. Nature 529, 221-225. doi: 10.1038/nature16161

Wakisaka, N., Miyasaka, N., Koide, T., Masuda, M., Hiraki-Kajiyama, T., and Yoshihara, Y. (2017). An adenosine receptor for olfaction in fish. Curr. Biol. 27, 1437.e4-1447e.4. doi: 10.1016/j.cub.2017.04.014

Wessler, I., and Kirkpatrick, C. J. (2008). Acetylcholine beyond neurons: the non-neuronal cholinergic system in humans. Br. J. Pharmacol. 154, 1558-1571. doi: 10.1038/bjp.2008.185

Workman, A. D., Carey, R. M., Chen, B., Saunders, C. J., Marambaud, P., Mitchell, C. H., et al. (2017). CALHM1-mediated ATP release and ciliary beat frequency modulation in nasal epithelial cells. Sci. Rep. 7:6687. doi: 10.1038/s41598-017-07221-9

Yamada, M., Miyakawa, T., Duttaroy, A., Yamanaka, A., Moriguchi, T., Makita, R., et al. (2001). Mice lacking the M3 muscarinic acetylcholine receptor are hypophagic and lean. Nature 410, 207-212. doi: 10.1038/350 65604
Yamaguchi, T., Yamashita, J., Ohmoto, M., Aoudé, I., Ogura, T., Luo, W., et al. (2014). Skn-1a/Pou2f3 is required for the generation of Trpm5-expressing microvillous cells in the mouse main olfactory epithelium. BMC Neurosci. 15:13. doi: 10.1186/1471-2202-15-13

Conflict of Interest Statement: The authors declare that the research was conducted in the absence of any commercial or financial relationships that could be construed as a potential conflict of interest.

Copyright (C) $2018 \mathrm{Fu}$, Ogura, Luo and Lin. This is an open-access article distributed under the terms of the Creative Commons Attribution License (CC BY). The use, distribution or reproduction in other forums is permitted, provided the original author(s) and the copyright owner are credited and that the original publication in this journal is cited, in accordance with accepted academic practice. No use, distribution or reproduction is permitted which does not comply with these terms. 


\section{OPEN ACCESS}

Edited by:

Dieter Wicher,

Max-Planck-Institut für chemische

Ökologie, Germany

Reviewed by:

Terrance P. Snutch,

The University of British Columbia,

Canada

Jean-François Cloutier,

McGill University, Canada

Daniel W. Wesson,

University of Florida, United States

*Correspondence:

Martina Pyrski

martina.pyrski@uks.eu

†'Present address:

Livio Oboti,

Institute for Biology, Humboldt University of Berlin, Berlin, Germany

Diego J. Rodriguez-Gil,

Department of Biomedical Sciences,

East Tennessee State University,

Johnson City, TN, United States

Received: 28 June 2018

Accepted: 16 August 2018

Published: 04 September 2018

Citation:

Pyrski M, Tusty M, Eckstein E, Oboti L, Rodriguez-Gil DJ, Greer CA and Zufall F (2018) P/Q Type Calcium

Channel Cav2. 1 Defines a Unique Subset of Glomeruli in the Mouse Olfactory Bulb.

Front. Cell. Neurosci. 12:295. doi: 10.3389/fncel.2018.00295

\section{P/Q Type Calcium Channel Cav2.1 Defines a Unique Subset of Glomeruli in the Mouse Olfactory Bulb}

\author{
Martina Pyrski ${ }^{1 *}$, Mahbuba Tusty ${ }^{2}$, Eugenia Eckstein ${ }^{1}$, Livio Obotit ${ }^{1+}$ \\ Diego J. Rodriguez-Gil2t, Charles A. Greer ${ }^{2}$ and Frank Zufall ${ }^{1}$ \\ ${ }^{1}$ Center for Integrative Physiology and Molecular Medicine, Saarland University, Homburg, Germany, ${ }^{2}$ Department \\ of Neuroscience and Department of Neurosurgery, School of Medicine, Yale University, New Haven, CT, United States
}

Voltage-gated calcium (Cav) channels are a prerequisite for signal transmission at the first olfactory sensory neuron (OSN) synapse within the glomeruli of the main olfactory bulb (MOB). We showed previously that the N-type Cav channel subunit Cav2.2 is present in the vast majority of glomeruli and plays a central role in presynaptic transmitter release. Here, we identify a distinct subset of glomeruli in the MOB of adult mice that is characterized by expression of the P/Q-type channel subunit Cav2.1. Immunolocalization shows that Cav2.1+ glomeruli reside predominantly in the medial and dorsal $\mathrm{MOB}$, and in the vicinity of the necklace glomerular region close to the accessory olfactory bulb. Few glomeruli are detected on the ventral and lateral MOB. Cav2.1 labeling in glomeruli colocalizes with the presynaptic marker vGlut2 in the axon terminals of OSNs. Electron microscopy shows that Cav2.1+ presynaptic boutons establish characteristic asymmetrical synapses with the dendrites of second-order neurons in the glomerular neuropil. Cav2.1+ glomeruli receive axonal input from OSNs that express molecules of canonical OSNs: olfactory marker protein, the ion channel Cnga2, and the phosphodiesterase Pde4a. In the main olfactory epithelium, Cav2.1 labels a distinct subpopulation of OSNs whose distribution mirrors the topography of the MOB glomeruli, that shows the same molecular signature, and is already present at birth. Together, these experiments identify a unique Cav2.1+ multiglomerular domain in the MOB that may form a previously unrecognized olfactory subsystem distinct from other groups of necklace glomeruli that rely on cGMP signaling mechanisms.

\footnotetext{
Keywords: olfactory subsystem, olfactory bulb, olfactory epithelium, voltage-gated calcium channel, Cav2.1 $\alpha-1 a$ subunit, Cacna1a, synaptic localization, olfactory glomerulus
}

\section{INTRODUCTION}

The mammalian olfactory system exhibits a highly complex organization that comprises several anatomically segregated chemoreceptive organs including the main olfactory epithelium (MOE), the vomeronasal organ (VNO), and the Grueneberg ganglion (GGN). Each of these organs contains functionally distinct groups of sensory neurons, or olfactory subsystems, that are differentiated 
based on their signal transduction mechanisms, the chemosensory cues they detect, and the axonal connections they establish to target specific regions in the olfactory bulb of the forebrain (Zufall and Munger, 2001; Munger et al., 2009; Bear et al., 2016). Sensory neurons located within the MOE and GGN project their axons to the main olfactory bulb (MOB) whereas vomeronasal sensory neurons (VSNs) project to a specific nucleus located posterior to the dorsal MOB known as the accessory olfactory bulb (AOB). A complete understanding of the organization of the olfactory system is essential for understanding the processing of chemosensory information and the role that each subsystem plays in odor-guided behaviors (Zufall and Munger, 2016).

The MOB of the mouse comprises $\sim 3,600$ glomeruli (Richard et al., 2010) which form a sensory map on the surface of the bulb (Mori and Sakano, 2011). An olfactory glomerulus is a complex neuropil that receives axonal input from a distinct population of olfactory sensory neurons (OSNs) and contains the first synaptic connections of the olfactory pathway formed by presynaptic OSN boutons and post-synaptic dendrites of mitral and tufted cells or local interneurons (Shepherd et al., 2004; Wachowiak and Shipley, 2006). An estimate of the number of OSN synapses per glomerulus is $\sim 260,000$, assuming 26 synapses per axon (Klenoff and Greer, 1998) and an average number of OSNs expressing a given odorant receptor (OR) gene of $\sim 10,000$ (Bressel et al., 2016). The vast majority of $\mathrm{MOB}$ glomeruli receive sensory input from canonical OSNs. These employ a cAMP signaling cascade, express a given OR, and the axons from OSNs expressing the same OR genes coalesce into two or a few glomeruli (Ressler et al., 1994; Vassar et al., 1994; Firestein, 2001; Treloar et al., 2002; Richard et al., 2010; Bressel et al., 2016). The mouse MOB also comprises $>50$ Taar+ glomeruli receiving input from OSNs that express a single receptor of the trace-amine associated receptor (Taar) family (14 members) and seem to rely on classical cAMP signal transduction (Liberles and Buck, 2006; Li and Liberles, 2016). Most Taar+ OSNs project to glomeruli located in the dorsal center of the olfactory bulb (Pacifico et al., 2012). Furthermore, a small number of OSNs rely on cGMP signaling mechanisms (Zufall and Munger, 2010). These include the GCD+ OSNs which express the receptor guanylate cyclase GC-D (Fülle et al., 1995; Juilfs et al., 1997; Meyer et al., 2000) and are involved in the social transmission of food preference (LeindersZufall et al., 2007; Munger et al., 2010). GC-D+ OSNs have their axonal targets in 9-12 glomeruli of the "necklace" region encircling the caudal MOB (Juilfs et al., 1997; Leinders-Zufall et al., 2007). Interestingly, sensory neurons of the Grueneberg ganglion (GGNs), which express the receptor guanylate cyclase GC-G (Fleischer et al., 2009; Liu et al., 2009), also have their axonal targets in the MOB necklace region, although within nine distinct glomeruli (Matsuo et al., 2012; Bumbalo et al., 2017). A recently discovered subpopulation of OSNs (known as type $\mathrm{B}$ cells) that contain the ion channel Trpc2 and the soluble guanylate cyclase Gucy1b2 also use cGMP signaling to detect low environmental oxygen (Omura and Mombaerts, 2014, 2015; Bleymehl et al., 2016). The glomerular targets of these sensory neurons are located on the posteroventral surface of the MOB, in proximity to the necklace glomeruli (Omura and Mombaerts,
2014, 2015). Together, these results have revealed a surprisingly complex spatial and molecular organization of axonal projection targets in the mouse olfactory bulb representing the neural and genetic architecture of vertebrate olfaction (Bear et al., 2016).

Here, we present evidence for the existence of a novel multiglomerular domain in the mouse MOB that may represent a previously unrecognized olfactory subsystem. This subsystem is differentiated by the expression of the voltage-activated calcium (Cav) channel subunit Cav2.1 (also known as $\alpha 1 \mathrm{~A}$, encoded by the gene Cacnala in mice). Cav channels produce locally restricted intracellular $\mathrm{Ca}^{2+}$ transients in response to action potential firing at the active zone of presynaptic terminals (Catterall, 2011; Kamp et al., 2012). Of the three structurally and functionally related families (Cav1-Cav3) identified in the mammalian central nervous system, the Cav2 family members P/Q- (Cav2.1), N- (Cav2.2), and R-type (Cav2.3) conduct $\mathrm{Ca}^{2+}$ currents that initiate synaptic transmission. Neurotransmitter release at central synapses is primarily mediated by the highvoltage activated channels Cav2.1 and Cav2.2 (Catterall, 2011). In the olfactory system, both subunits have been identified in the olfactory mucosa using RNA techniques and Western blot analyses (Shiraiwa et al., 2007; Ibarra-Soria et al., 2014). Presynaptic Cav2.2 protein is present in the vast majority of glomeruli of MOB and AOB (Weiss et al., 2014) and plays a central role in transmitter release of OSNs and VSNs (Isaacson and Strowbridge, 1998; Wachowiak et al., 2005; Weiss et al., 2014). In the present study, we show that Cav2.1 represents a second candidate for olfactory signal transmission within a distinct subset of MOB glomeruli. These results provide important new information for isolating a separate population of glomeruli and their corresponding chemosensory neurons and for understanding the functional significance of this organization for regulating excitability of peripheral afferents in the olfactory system.

\section{MATERIALS AND METHODS}

\section{Mice}

All procedures were approved by the Institutional Animal Care and Use Committee of Saarland University and were in full accordance with the laws for animal experiments of the German government. Experiments were performed on mouse tissues derived from mice at different ages and of both sexes. For the developmental study, we used mice at embryonic day 18 (E18), postnatal day 1 (P1), P7, P14, and $\mathrm{P} 21$. Results from adult mice were obtained at 6-18 weeks of age. We used wild type mice (C57BL/6J, denoted as B6), OMP$\mathrm{GFP}^{+/-}$mice (B6; 129P2-Omp $\left.{ }^{\text {tm } 3 \mathrm{Mom}} / \mathrm{Mom}\right)$, The Jackson Laboratory; stock\# 006667) that were heterozygous for both OMP (olfactory marker protein) and GFP (green fluorescent protein) (Potter et al., 2001) Gucy2d-Mapt-lacZ mice (LeindersZufall et al., 2007), GCG-Cre-GFP mice denoted as GCGGFP (line 52, kindly provided by Ivan Rodriguez, University of Geneva, Switzerland) (Matsuo et al., 2012), Gucy1b2-IREStauGFP, Trpc2-IRES-taumCherry, and Trpc2-IRES-taulacZ mice (Omura and Mombaerts, 2014, 2015). Mice were housed in 
micro-isolator cages on a reverse 12:12-h light/dark cycle with water and food available ad libitum.

\section{Olfactory Tissue Preparation}

Mouse tissue preparation followed previously described methods (Weiss et al., 2011, 2014; Bolz et al., 2017). Mice were anesthetized [165 mg/kg body weight ketamine (Pharmacia GmbH, Berlin, Germany) and $11 \mathrm{mg} / \mathrm{kg}$ body weight xylazine (Bayer Health Care, Leverkusen, Germany)] and transcardially perfused with phosphate-buffered saline (PBS) pH 7.4, followed by 2 or $4 \%$ (w/v) paraformaldehyde in PBS (for Ncam2 antibody, 2\% PFA was used, see below). Mice younger than 2 days were decapitated and fixed by immersion in 2 or $4 \%$ PFA prepared in PBS for $24 \mathrm{~h}$ instead. For cryostat sections, olfactory tissue was incubated in $30 \%$ sucrose in PBS at $4^{\circ} \mathrm{C}$ for 2 days, embedded in O.C.T. (Tissue-Tek), and snap-frozen in a dry ice/2-methylbutane bath. Frozen tissue sections $(12-50 \mu \mathrm{m})$ were collected on a cryostat (HM525; Microm, Walldorf, Germany), thaw-mounted onto glass slides (Superfrost Plus, Polysciences), and stored at $-80^{\circ} \mathrm{C}$.

\section{RNAscope Fluorescence in situ Hybridization}

Coronal MOE cryosections $(14 \mu \mathrm{m})$ of adult B6 mice were subjected to RNAscope in situ hybridization (Wang et al., 2012) using the RNAscope Fluorescent Multiplex Detection Kit (ACD Biotechne) and specific Cacnala probes. The procedure was performed according to the manufacturer's recommendations. Sections were pretreated in $1 \mathrm{x}$ citrate buffer $\mathrm{pH}$ 6.0 (DAKO, Germany) for $5 \mathrm{~min}$ at $98^{\circ} \mathrm{C}$, rinsed in distilled water, dehydrated in $100 \%$ ethanol, air dried and treated with Protease III for $30 \mathrm{~min}$ at $40^{\circ} \mathrm{C}$. Then, sections were rinsed in water and hybridized with the RNA scope target probe generated for mouse Cacnala (ACD Biotechne) that was designed as channel 2 probe containing 20 ZZ structures targeting base pairs 5705-6727 of NM_007578.3 ${ }^{1}$ distributing to exons 37-46. Two negative controls were run in parallel with the sample, the bacterial DapB probe supplied by the company (ACD Biotechne) and a negative control without any probe. Following hybridization $\left(2 \mathrm{~h}, 40^{\circ} \mathrm{C}\right)$, sections were rinsed twice for $2 \mathrm{~min}$ in wash buffer (ACD Biotechne), and sequentially treated with amplification solutions at $40^{\circ} \mathrm{C}(30 \mathrm{~min}$ Amp1-FL, 15 min Amp2-FL, 30 min Amp3-FL) and fluorescence reagent (15 $\mathrm{min}, 40^{\circ} \mathrm{C} \mathrm{Amp} 4$-altB), with intermitting washing steps between reagents at room temperature. Cell nuclei were stained with DAPI solution (ACD Biotechne) for $1 \mathrm{~min}$, and tissue sections were mounted with DAKO fluorescence medium and stored for $12 \mathrm{~h}$ at $4^{\circ} \mathrm{C}$. Fluorescence images were acquired on a Zeiss LSM 880 confocal microscope containing a 32-channel GaAsP-PMT and 2-channel PMT QUASAR detector.

\section{Reverse Transcription Polymerase Chain Reaction (RT-PCR)}

Main olfactory epithelium tissue of two adult B6 mice was dissected, pooled, and then transferred to RNAlater ${ }^{\circledR}$ solution (Ambion). Total RNA extraction and cDNA synthesis were

${ }^{1}$ www.ncbi.nlm.nih.gov/nuccore/NM_007578.3 exactly as described previously (Pyrski et al., 2017). For polymerase chain reactions (PCR), we used $0.5 \mu \mathrm{l}$ of cDNA, Cacnala-specific primers, and the Phusion High Fidelity DNA polymerase (NEB) according to the manufacturer's protocol. Cacnala sequence information was retrieved from the Ensembl database (ENSMUSG00000034656). The Cacnala mRNA contains 47 exons. Primers were chosen to amplify the region between exons 24-43. Forward (F) and reverse (R) primers in $5^{\prime}-3^{\prime}$ orientation were F6-ex24: GGGGCCTATTTCCGTGAC, R3-ex38: GTCCATCCGCAGGAGTCTC, F8-ex37: CCTCATAGGG TTGCTTGCAAG, R7-ex43: CCATTTCTCGCATCTCCACAG, F9-ex34: CCAAATCACGGAGCACAATAAC (Eurofins Genomics, Ebersberg, Germany). Cycling parameters were $15 \mathrm{~s}$ at $98^{\circ} \mathrm{C}$, followed by 39 cycles of $\left(10 \mathrm{~s}\right.$ at $98^{\circ} \mathrm{C}-10 \mathrm{~s}$ at $60^{\circ} \mathrm{C}-30 \mathrm{~s}$ at $\left.72^{\circ} \mathrm{C}\right)$, and a final elongation $\left(5 \mathrm{~min}\right.$ at $\left.72^{\circ} \mathrm{C}\right)$ prior to cooling samples to $4^{\circ} \mathrm{C}$. Amplicon size in base pairs (bp) for each reaction was F6-ex24/R3-ex38: 1,731 bp; F8-ex37/R7-ex43: 607 bp; PCR-products were verified by gel electrophoresis on $1 \%$ agarose gels containing ethidium bromide, and by direct sequencing (Seqlab) of the purified amplicons using the primers listed above.

\section{Immunohistochemistry}

All procedures were conducted at room temperature $\left(20^{\circ} \mathrm{C}\right)$ except for incubations of tissue sections with primary antibodies that was done at $4^{\circ} \mathrm{C}$. Tissue sections were incubated for $1 \mathrm{~h}$ in blocking reagent containing $4 \%$ normal horse serum (NHS, Vector Laboratories) and $0.3 \%$ Triton X-100 prepared in PBS, followed by incubation in primary antibody diluted in blocking reagent for at least $24 \mathrm{~h}$. Primary antibodies and control peptides were Cav2.1 rabbit polyclonal (1:1000, \#152103 Synaptic Systems) or a biotinylated version of that same antibody (1:1000, synthetized on request, Synaptic Systems); control peptide RDPDARRAWPGSPERAPGREGPYGRESEPQQRE (Genscript) corresponding to amino acids 856-888 of the mouse P/Q-type $\alpha$-1a channel subunit Cav2.1, UniProt Id: P97445; $\beta$-galactosidase chicken polyclonal (1:500, cat. \#ab9361, Abcam); Cnga2, rabbit polyclonal (1:500, Alomone cat. \# APC-045); RFP rabbit polyclonal (1:2000, cat. \#ABIN 129578, AK-Online); GFP chicken polyclonal (1:1000, cat. \#ab13970, Abcam), Map2 chicken polyclonal (1:300, cat. \#ab5392, Abcam); Ncam2 goat polyclonal (1:100, cat. \#AF778, R\&D Systems), OMP (1:3000, goat polyclonal; gift of F. Margolis, University of Maryland, College Park, MD, United States); Pde2a goat polyclonal (1:200, cat. \#sc-17227, St. Cruz); Pde4a rabbit polyclonal (1:200, cat. \#PD4-112ab, FabGennix); panTaar goat polyclonal (1:100, cat. \#sc-54398, St. Cruz); vGlut1 mouse monoclonal (1:100, cat. \#135511, Synaptic Systems); vGlut2 rabbit polyclonal (1:2000, cat. \#135403, Synaptic Systems). Sections were washed $3 \times 10 \mathrm{~min}$ in PBS and incubated with fluorescence-labeled secondary antibody or streptavidin conjugates (all Invitrogen) as follows. Alexa Fluor 488 donkey-anti-goat (1:1000, A-11055), Alexa Fluor 488 donkey-anti-chicken (1:1000, A-11039), Alexa Fluor 488 goat-anti-rabbit (1:1000, A-11034), Alexa Fluor 488 goat-anti-mouse (1:1000, A-11029), Alexa Fluor 555 donkey-anti-rabbit (1:1000, A-31572), Alexa Fluor 546 
donkey-anti-goat (1:1000, A-11056); Alexa Fluor 647 donkeyanti-mouse (1:1000, A-31571), biotinylated goat-anti-rabbit (1:100, BA-1000, Vector Laboratories), Alexa 488-conjugated streptavidin (1:400; S-32354), Alexa Fluor 647 goat-antichicken (1:1000, A-21449), Alexa 546-conjugated streptavidin (1:400; S-11225). For the colocalization of Cav2.1 with a second primary antibody made in rabbit, we performed two sequential reactions with the biotinylated Cav2.1 antibody and fluorescence-conjugated streptavidin in the second reaction. Alternatively, we introduced a blocking step between reactions in which unbound rabbit IgG epitopes from the first reaction were blocked for $1 \mathrm{~h}$ with donkey-anti-rabbit Fab-fragments (1:50, Biomol, Rockland, ME, United States). Nuclei were stained with $2 \mu \mathrm{M}$ Hoechst 33342 nuclear dye (Invitrogen) or with $5 \mu \mathrm{M}$ Draq5 (BD Pharmingen) prior to cover slipping sections in fluorescence mounting medium (DAKO). The specificity of the immunostainings was verified by control experiments omitting the primary antibody or by incubation with pre-absorbed Cav2.1 antiserum ( $5 \mu \mathrm{g} / \mathrm{ml}$ blocking peptide).

\section{Whole-Mount Immunohistochemistry}

Following transcardial perfusion with 4\% PFA, olfactory bulbs were dissected and washed twice in PBS for $15 \mathrm{~min}$ prior to dehydration in graded methanol $(25,50,100 \%$ methanol in PBS, 10 min each step). Olfactory bulbs underwent three freeze-thaw cycles for $30 \mathrm{~min}$ at $-80^{\circ} \mathrm{C}$ and $10 \mathrm{~min}$ at room temperature. Following rehydration in graded methanol (50\%, 25\% methanol in PBS) for each $10 \mathrm{~min}$, olfactory bulbs were washed in PBS for 15 min incubated in blocking buffer containing $0.5 \%$ Triton $\mathrm{X}-100$ and 5\% normal horse serum (Vector Laboratories) for $3 \mathrm{~h}$ at room temperature. Tissue was then incubated in primary antibody solution (see above) for 3 days at $4^{\circ} \mathrm{C}$, washed three times in PBS for $1 \mathrm{~h}$ each, incubated in secondary antibody (1:1000) overnight at $4^{\circ} \mathrm{C}$, and washed three times in PBS for $1 \mathrm{~h}$ each.

\section{Fluorescence Microscopy and Image Assembly}

Fluorescence images of stained cryosections were acquired on a BX61 epifluorescence microscope attached to a DP71 camera (Olympus), or on a LSM 880/ConfoCor-3 confocal microscope (Zeiss). Confocal images are $1 \mu \mathrm{m}$ digital images or Z-stacks presented as maximum intensity projections of 10-20 confocal sections, each $0.4 \mu \mathrm{m}$ thick. Fluorescence images of olfactory bulb whole-mount preparations were acquired on a SZX16 epifluorescence binocular (Olympus). Images were assembled and adjusted in contrast and brightness using Photoshop Elements 10 (Adobe Photoshop).

\section{Olfactory Bulb 3D Reconstruction}

The $3 \mathrm{D}$ reconstruction technique was as described previously (Luzzati et al., 2011). For the spatial representation of Cav2.1+ glomeruli in the MOB, serial coronal cryosections $(50 \mu \mathrm{m})$ of adult OMP-GFP mice were subjected to Cav2.1 immunolabeling visualized with Alexa555-conjugated secondary antibody and nuclear staining using DAPI (4', 6-diamidino-2-phenylindole,
Vector Laboratories). Every section was acquired and digitized images were uploaded into Reconstruct software (Fiala, 2005). The 3D morphology of Cav2.1+ glomeruli was captured by $2 \mathrm{D}$ contour delineation that was verified by the presence of endogenous OMP-GFP fluorescence in each glomerulus and by the glomerular shape given by the nuclear staining of periglomerular cells. Import of the $3 \mathrm{D}$ assembly into the open source software Blender (Blender.org) allowed the editing of shading, transparency, and lighting.

\section{Immuno-Electron Microscopy}

Processing for immuno-electron microscopy followed procedures we employ routinely (Weiss et al., 2011; Bartel et al., 2015). Adult (postnatal days 57-58) CD1 mice were euthanized with Euthasol and perfused using a low/high-pH PFA fixation strategy (Treloar et al., 2002). This strategy was adopted after preliminary studies indicated that ultrastructural immunolocalization of the Cav2.1 antibody could be compromised in tissue perfused with fixatives containing glutaraldehyde. Tissue was initially perfused with 4\% PFA in PBS $(0.1 \mathrm{M}$ phosphate buffer and $0.9 \% \mathrm{NaCl})$ at $\mathrm{pH} 6.5$ for $5 \mathrm{~min}$ followed by $4 \% \mathrm{PFA}$ in PBS at $\mathrm{pH}$ 10.5. Brains were removed and post-fixed overnight at $4^{\circ} \mathrm{C}$ in the second perfusate. Coronal vibratome sections of the MOB were freefloating immunostained using the Cav2.1 antibody as described above. Sections were incubated in biotin-conjugated goat antirabbit IgG secondary antibody (1:100, cat. \#BA-1000, Vector Laboratories) for $1 \mathrm{~h}$ at room temperature. Sections were washed and incubated with $\mathrm{ABC}$ reagent (prepared by diluting both solution A and solution B at 1:50 in blocking buffer, Vector Laboratories) for $1 \mathrm{~h}$ at room temperature. Sections were washed again prior to a final rinse in PBS. Peroxidase activity was visualized by incubating tissue in $0.05 \% 3.3^{\prime}$-diaminobenzdine tetra hydrochloride (DAB), $0.005 \% \mathrm{H}_{2} \mathrm{O}_{2}$ in TBS. The reaction was stopped in PBS and the tissue then incubated for $1 \mathrm{~h}$ in $2 \%$ glutaraldehyde in PBS. Sections were washed again (as above) and immediately processed for electron microscopy. Sections containing Cav2.1 immunolabeled glomeruli were re-embedded in EPON and sectioned at 70-90 $\mathrm{nm}$ on a Reichert Ultramicrotome prior to imaging in a JEOL 1200 electron transmission microscope.

\section{RESULTS}

\section{Cav2.1 Immunostaining Identifies a Unique Multiglomerular Domain in the Main Olfactory Bulb}

To assess whether the P/Q-type channel subunit Cav2.1 may represent a second calcium channel involved in synaptic transmission at the first olfactory synapse, we conducted a systematic immunohistochemical analysis of the olfactory bulb of adult B6 mice (Figure 1). We analyzed whole-mount preparations $(N=9$ mice $)$ and tissue sections taken at intervals along anterior-to-posterior and medial-to-lateral axes of the MOB $(N=13$ mice $)$ using a Cav2.1-specific antibody that has 


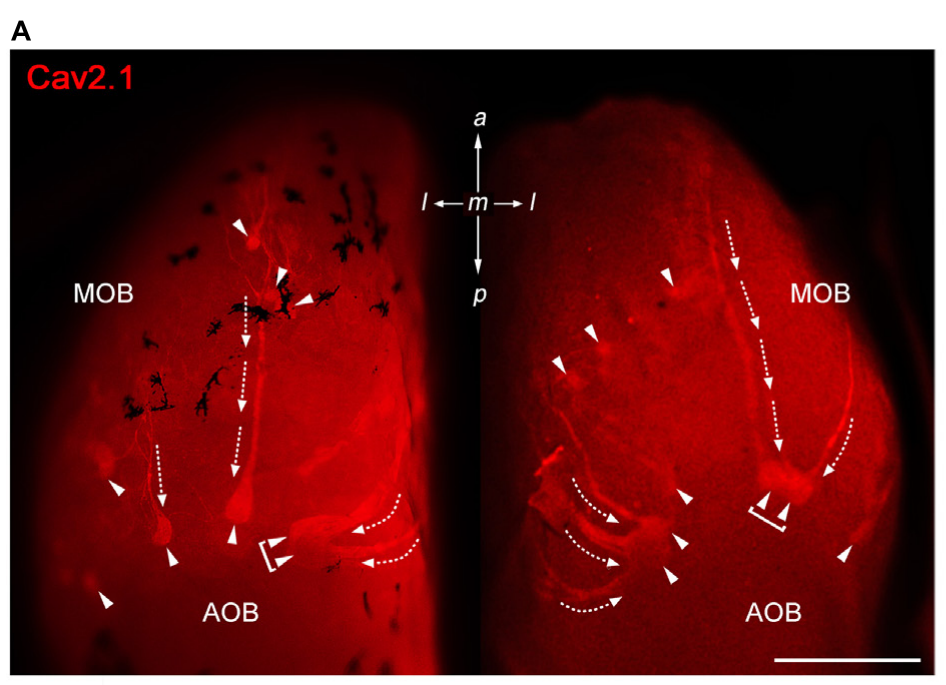

C

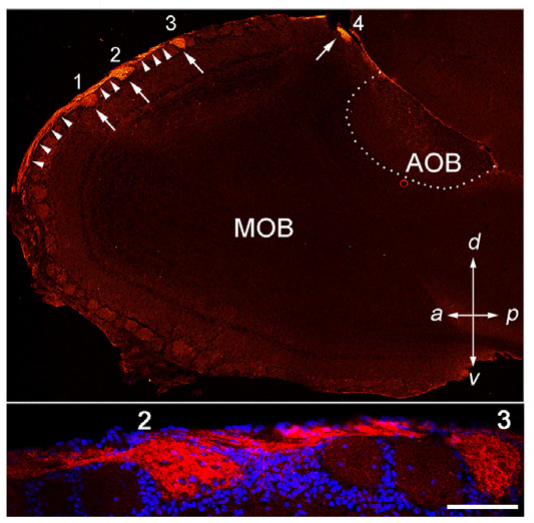

D

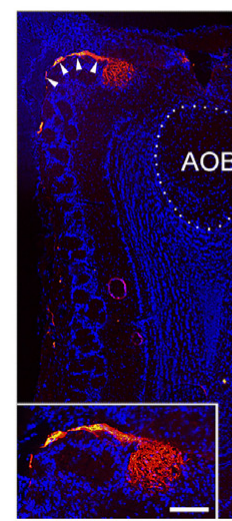

B

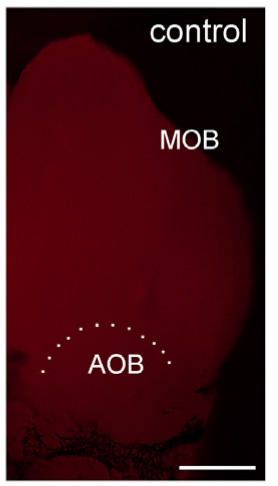

E

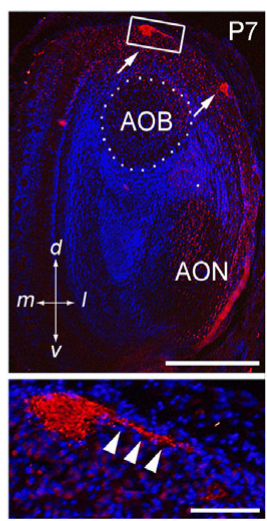

FIGURE 1 | Cav2.1 staining reveals a unique subset of glomeruli in the MOB. (A) Cav2.1 immunoreactivity (red) exemplified in a whole-mount preparation of the left and right MOB (dorso-caudal view) of an adult B6 mouse. Cav2.1+ axon bundles (arrows) terminate into several individual glomeruli (arrowheads). Large Cav2.1+ glomeruli reside in the caudal aspect of the MOB, close to the accessory olfactory bulb (AOB). Frequently, two glomeruli reside next to each other (brackets). Staining is absent in the AOB. (B) Negative control reaction using peptide blocking is devoid of Cav2.1 staining (dorso-caudal view and right bulb). (C) Sagittal section $(14 \mu \mathrm{m})$ at about the MOB midline depicting four individual Cav2.1+ glomeruli (arrows 1-4) and their afferent axonal fibers (arrowheads). The inset (bottom) shows a higher magnification of two glomeruli $(2,3)$ and axonal projections from the nerve layer of the MOB. Nuclei staining with Hoechst dye (blue) verifies glomerular boundaries. (D) Coronal section $(14 \mu \mathrm{m})$ of the posterior MOB showing two large Cav2.1+ glomeruli medial and lateral to the AOB. The inset at the bottom depicts a higher magnification of the medial glomerulus. (E) Cav2.1+ glomeruli (arrows) are already evident at postnatal day 7 (P7) including afferent axons (arrowheads, inset, and bottom). (A-E) Orientations are as depicted by arrows indicating a, anterior; p, posterior; d, dorsal; v, ventral; I, lateral; m, medial; AON, anterior olfactory nucleus. Scale bars (A-E) overviews, $500 \mu \mathrm{m}$; (C,D) insets, $100 \mu \mathrm{m}$; (E) inset, $50 \mu \mathrm{m}$.

been established previously in Cav2.1 knockout mice (Nishimune et al., 2012; Maejima et al., 2013). Whole-mount staining of olfactory bulbs revealed robust Cav2.1 immunoreactivity in a small subset of glomeruli and their afferent axon bundles (Figure 1A) whereas negative control reactions using Cav2.1 antibody that was blocked with its cognate peptide lacked any immunoreactivity (Figure 1B). The Cav2.1+ glomeruli were mainly located in the dorsal and medial aspects of the MOB; ventral and lateral regions only occasionally showed labeled glomeruli. The most prominent Cav2.1+ glomeruli were situated at the dorsal-caudal border of the MOB encompassing 5-7 labeled glomeruli per bulb that surrounded the dorsal AOB in a semi-circular arrangement (Figure 1A). This part of the MOB contains the necklace glomerular region that is innervated by
GC-D OSNs and GGNs both of which employ non-canonical second messenger pathways (Bear et al., 2016). However, Cav2.1+ glomeruli did not exhibit the necklace-typical "beads on a string" connectivity. Cav2.1+ glomeruli in the caudal MOB were rather large $(\geq 100 \mu \mathrm{m}$ diameter) compared to additional smaller ones (40-80 $\mu \mathrm{m}$ diameter) in the more anterior region of the dorsal MOB (Figure 1A). Frequently, we also observed small clusters of 2-3 neighboring Cav2.1 labeled glomeruli in the vicinity to the $\mathrm{AOB}$ (Figure 1A, brackets). However, in contrast to our previous analysis of the expression of N-type calcium channel Cav2.2 (Weiss et al., 2014), we did not detect Cav2.1+ glomeruli within the AOB (Figures 1A,C,D).

Interestingly, afferent OSN axon bundles appeared to coalesce early in the anterior MOB. In some instances, we observed 
thick axon bundles traveling for more than $500 \mu \mathrm{m}$ across the dorsal MOB surface until terminating within posterior glomeruli (Figure 1A). Extending this observation, sagittal MOB sections showed that these axon bundles segregated onto several consecutive glomeruli along the anterior-to-posterior axis (Figure 1C). We also observed axon bundles projecting along the lateral and medial sites of the MOB to reach dorsal glomeruli located in the caudal MOB (Figures 1A,D). Examining the MOB during early postnatal development showed that the subset of Cav2.1+ glomeruli is already detectable as early as postnatal day 7 (Figure 1E).

\section{Quantitative 3D Reconstruction of Cav2.1+ Glomeruli}

To analyze Cav2.1 expression in the MOB in more detail, we generated glomerular maps using $3 \mathrm{D}$ reconstruction of serial MOB sections stained for Cav2.1. Figures 2A,B shows a reconstruction of the bulbs from a 4-month-old OMP-GFP mouse in which we identified 20 glomeruli in the left and 17 glomeruli in the right bulb. Reconstructions from two additional OMP-GFP mice are depicted in Figure 2C. Assembly of sections from these six bulbs revealed average numbers of $18 \pm 1$ Cav2.1+ glomeruli at the left and $21 \pm 4$ Cav2.1+ glomeruli at the right bulb hemisphere (mean \pm SEM). Number and exact positions of labeled glomeruli varied somewhat between the left and right bulbs of individual mice and between individuals as such, but the overall topography of these glomerular domains was comparable between bulbs (Figures 2A,C). The majority of labeled glomeruli occupied the dorsal and medial aspects of the caudal half of the MOB (Figures 2B,C). Occasionally, we also detected labeled glomeruli in the ventral or lateral MOB.

Together, the results of Figures 1, 2 show that Cav2.1 immunolabeling reveals a subset of olfactory glomeruli in the MOB that defines a specific multiglomerular domain.

\section{Cav2.1 Localizes to Presynaptic OSN Axon Terminals}

We next examined whether glomerular Cav2.1 staining solely originates from the OSN presynaptic boutons or also from post-synaptic membranes of mitral and tufted cells that form synapses with OSN axon terminals in the glomerular neuropil. In addition to staining for OMP, we performed double-labeling immunohistochemistry for Cav2.1 in combination with several pre- and post-synaptic markers in MOB tissue sections of adult mice (Figure 3). Our results show that all Cav2.1+ glomeruli are also labeled for OMP indicating that Cav2.1 glomeruli receive axonal input from mature OSNs. Cav2.1 staining also colocalizes with the vesicular glutamate transporter vGlut2 (Figures 3A,B) that is selectively expressed in presynaptic OSN axon terminals (Gabellec et al., 2007; Richard et al., 2010). Thus, Cav2.1+ glomeruli receive axonal input from mature OMP+/vGlut2+ sensory neurons located in the MOE. By contrast, doublelabeling for Cav2.1 and the microtubule-associated protein Map2 that identifies mitral cell dendrites showed no colocalization as illustrated by the separate red and green fluorescence signals, respectively (Figure 3C). Cav2.1 staining was also absent in

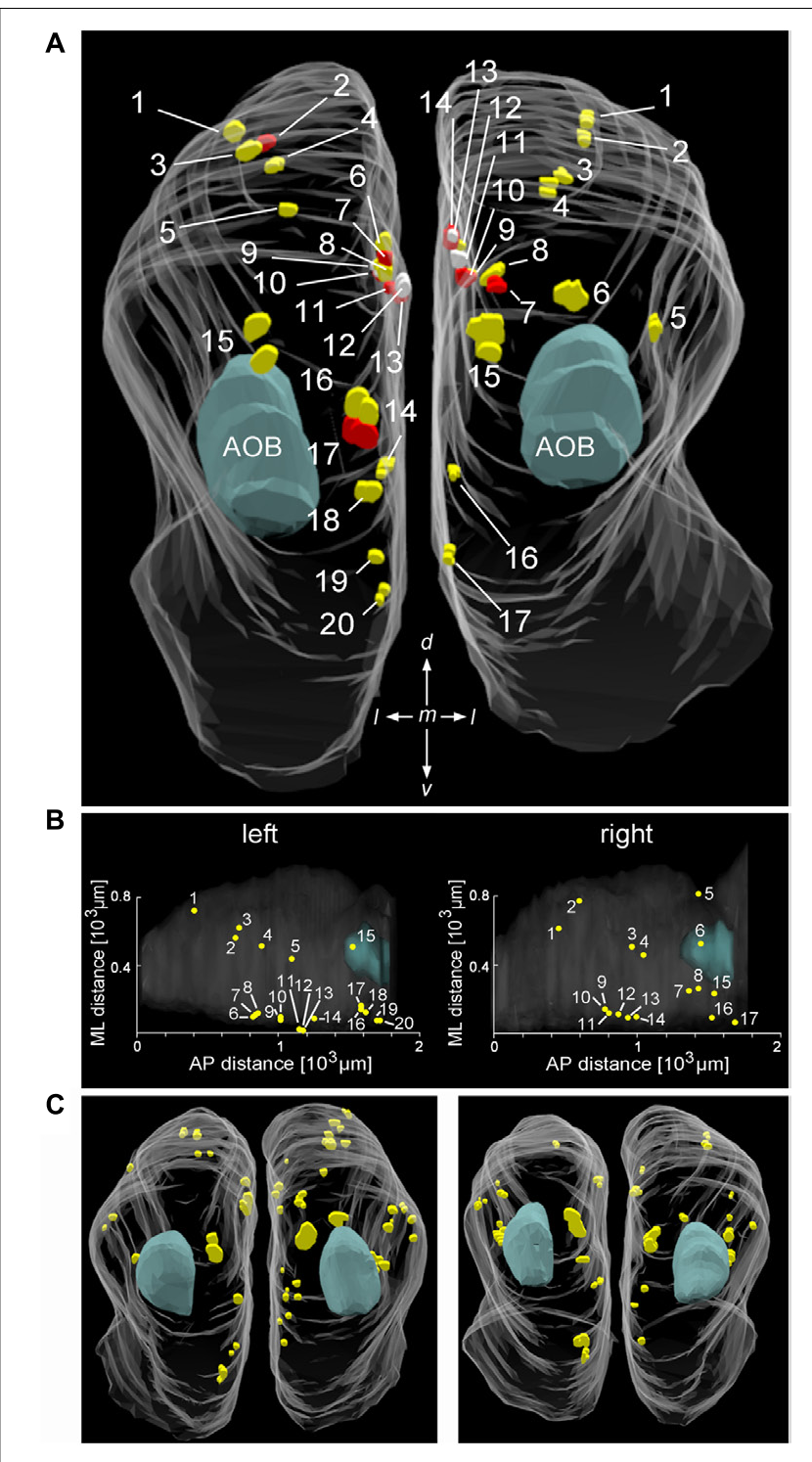

FIGURE 2 | Distribution and position of Cav2.1+ glomeruli in the MOB. (A) Representative bilateral 3D reconstruction of the Cav2.1+ glomeruli in the MOB of an adult mouse (4 month, caudal view). Both left and right olfactory bulbs were collected as serial $50 \mu \mathrm{m}$ sections from the same mouse. Closely adjacent glomeruli are differently colored (yellow, red, and white) to facilitate identification. The majority of Cav2.1+ glomeruli is located on the dorsal and medial MOB surface. The location of the AOB is as indicated in blue. (B) Dorsal view of the two MOBs shown in (A) illustrating the Cartesian localization of Cav2.1+ glomeruli in reference to their position along the medial-to-lateral $(\mathrm{ML})$ axis and their relative position along the anterior-to-posterior (AP) extent of MOBs. (C) 3D reconstruction of the left and right $\mathrm{MOB}$ hemispheres of two additional mice (8 weeks) further illustrates the distribution of Cav2.1+ glomeruli in the dorsal and medial MOB.

dendrodendritic synapses established between mitral/tufted cells and local interneurons, as specified by colocalization with the vesicular glutamate transporter vGlut1 (Figure 3D).

To assess the presynaptic localization of Cav2.1 at a higher level of resolution and to evaluate the morphology of synapses formed by Cav2.1+ presynaptic boutons in 

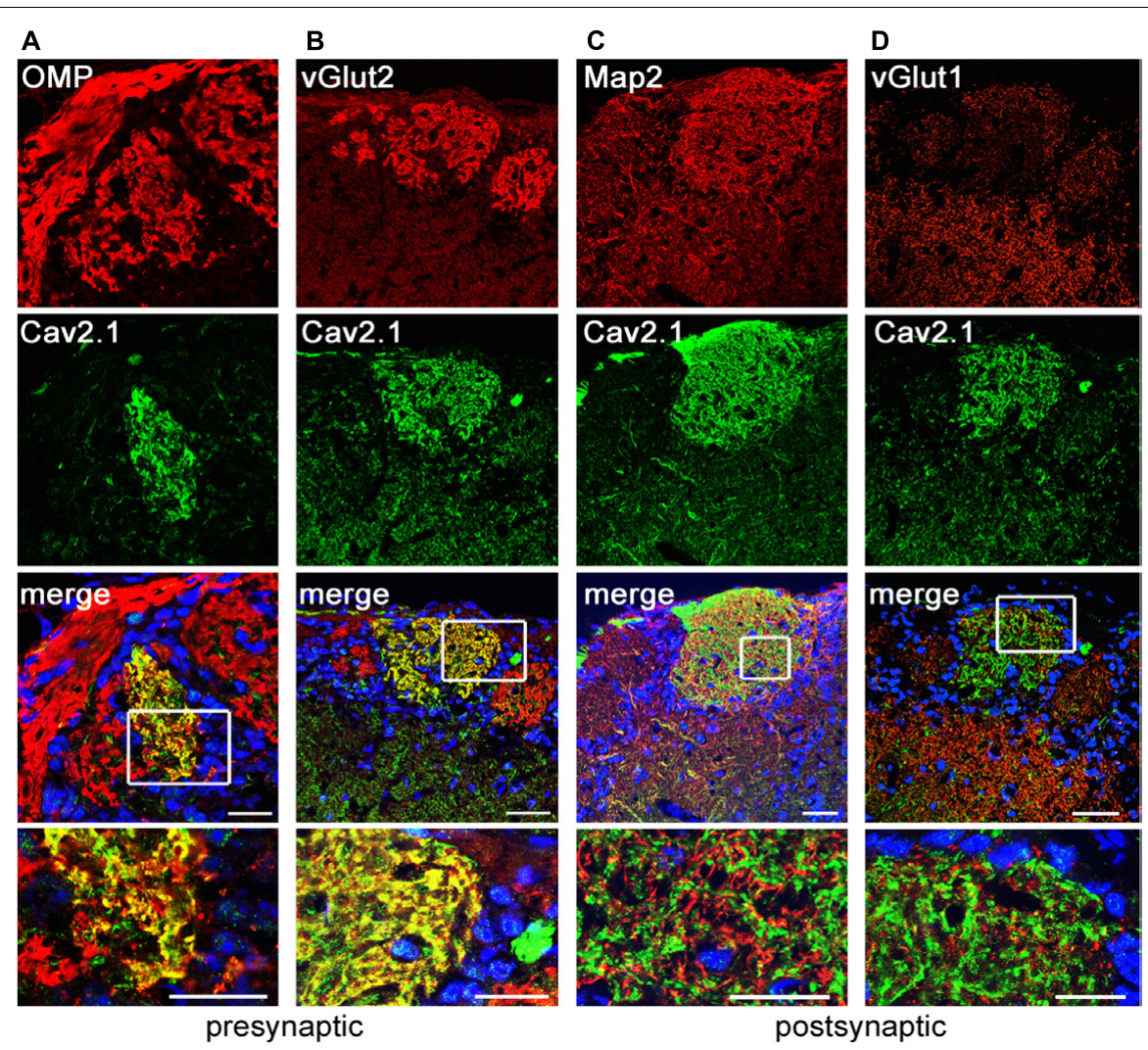

FIGURE 3 | Cav2.1 is localized to presynaptic OSN axon terminals. Confocal images showing immunoreactivity (red) for (A) OMP, (B) vGlut2, (C) Map2, and (D) vGlut1 in combination with Cav2.1 (green) in coronal MOB sections of adult B6 mice. (A,B) The merged images illustrate that the presynaptic markers OMP and vGlut2 colocalize with Cav2.1 yielding the yellow fluorescence signal. (C,D) In contrast, the post-synaptic dendritic markers Map2 and vGlut1 do not colocalize with Cav2.1 in the glomerular neuropil. This is illustrated in the magnified merge at the bottom (boxed area) showing separate red and green fluorescence signals. Scale bars (A-D) $40 \mu \mathrm{m}$, inset magnifications, $20 \mu \mathrm{m}$.

more detail, we conducted immuno-electron microscopy. As illustrated by the different examples shown in Figure 4, OSN axon terminals showed robust immunoreactivity, and formed typical asymmetrical synapses with post-synaptic mitral cell dendrites that were devoid of any staining. Within a glomerulus, there was no evidence of unlabeled OSN terminals proximal to labeled OSN terminals, suggesting that Cav2.1 is homogeneously expressed by the axons innervating these glomeruli. Moreover, ultrastructural features of Cav2.1-labeled and unlabeled OSN terminals in neighboring glomeruli were undistinguishable. Vesicle density, organization of pre- and post-synaptic membrane specializations, and the distribution of mitochondria were altogether unremarkable (Figure 4). Thus, the localization of Cav2.1 to presynaptic axon terminals strongly implies a function of Cav2.1 in the signal transmission and transmitter release at the first olfactory synapse of these glomeruli.

\section{A Small Number of Cav2.1+ Glomeruli Express Ncam2}

The glomerular topography in the MOB is associated with the expression of neural cell adhesion molecules in sensory neurons located in specific zones of the olfactory epithelium. The neural cell adhesion molecule Ncam2 (also known as Ocam) defines the ventral and lateral MOE-to-MOB projections whereas dorsal and medial MOE-to-MOB projections are negative for Ncam2 (Yoshihara et al., 1997; Treloar et al., 2002; Walz et al., 2006). The dorsal and medial distribution of Cav2.1+ glomeruli led us to examine the expression of Ncam 2 in adult B6 mice using double-labeling immunohistochemistry (Figure 5). We found that the majority of dorsal Cav2.1+ glomeruli were negative for Ncam2 whereas Ncam2+ glomeruli of the ventral and lateral MOB were devoid of Cav2.1 staining (Figure 5A,B). However, as an exception to the rule, we detected several Ncam2 + glomeruli in the dorsal MOB. Of these, about 2-3 glomeruli per bulb showed colocalization with Cav2.1 $(N=6$ bulbs from three mice) (Figure 5A). It has been suggested previously that the dorsal glomeruli of the trace amine-associated receptor (Taar) subsystem, in particular those expressing Taar46, typically colocalize with Ncam2 (Johnson et al., 2012). To investigate whether glomeruli of the Cav2.1 and Taar subsystems may partially overlap, we conducted double-labeling experiments in MOB sections using Cav2.1 and a pan-specific antibody that detects a broad range of different Taar family members. However, we found that individual glomeruli in the dorsal MOB were singly 


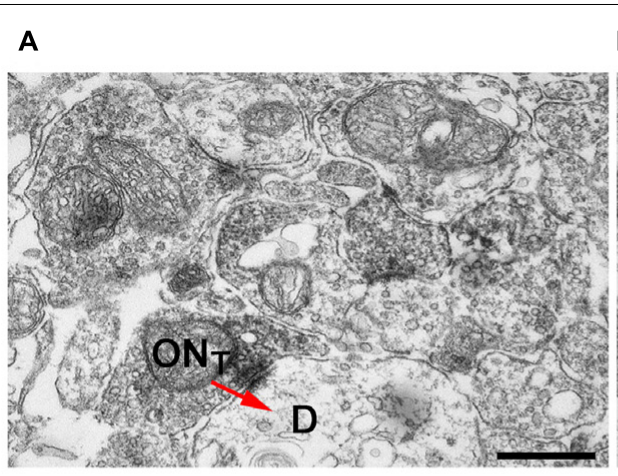

c

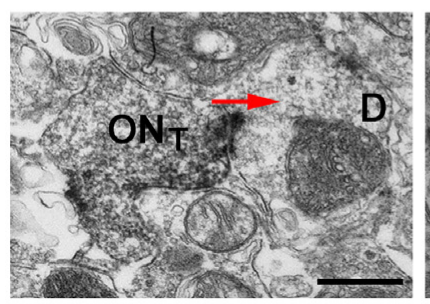

B

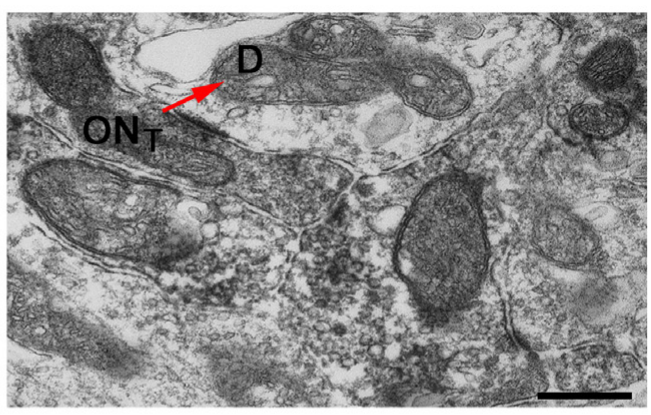

E

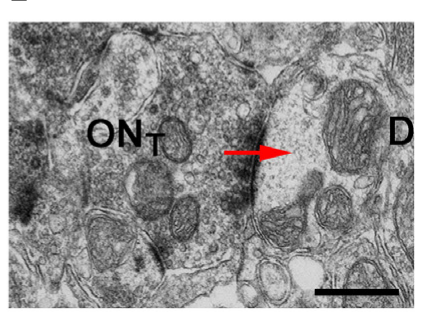

FIGURE 4 | Cav2.1+ OSN axons form typical asymmetrical synapses with post-synaptic dendrites in glomeruli. (A-E) Examples of Cav2.1+ OSN terminals (ONT) establishing asymmetric synapses with unstained and electron lucent dendrites (D) are shown. Arrows (red) indicate the polarity of the synapses. Labeled axonal terminals were evident from the accumulation of the DAB precipitate among the presynaptic vesicles and membrane specializations. There was also a typical darkening of mitochondrial membranes in labeled terminals. In Cav2.1+ glomeruli, only $\mathrm{ON}_{\mathrm{T}}$ are visible suggesting that Cav2.1 is homogeneously expressed by the axons innervating glomeruli. Scale bars: $500 \mathrm{~nm}$.
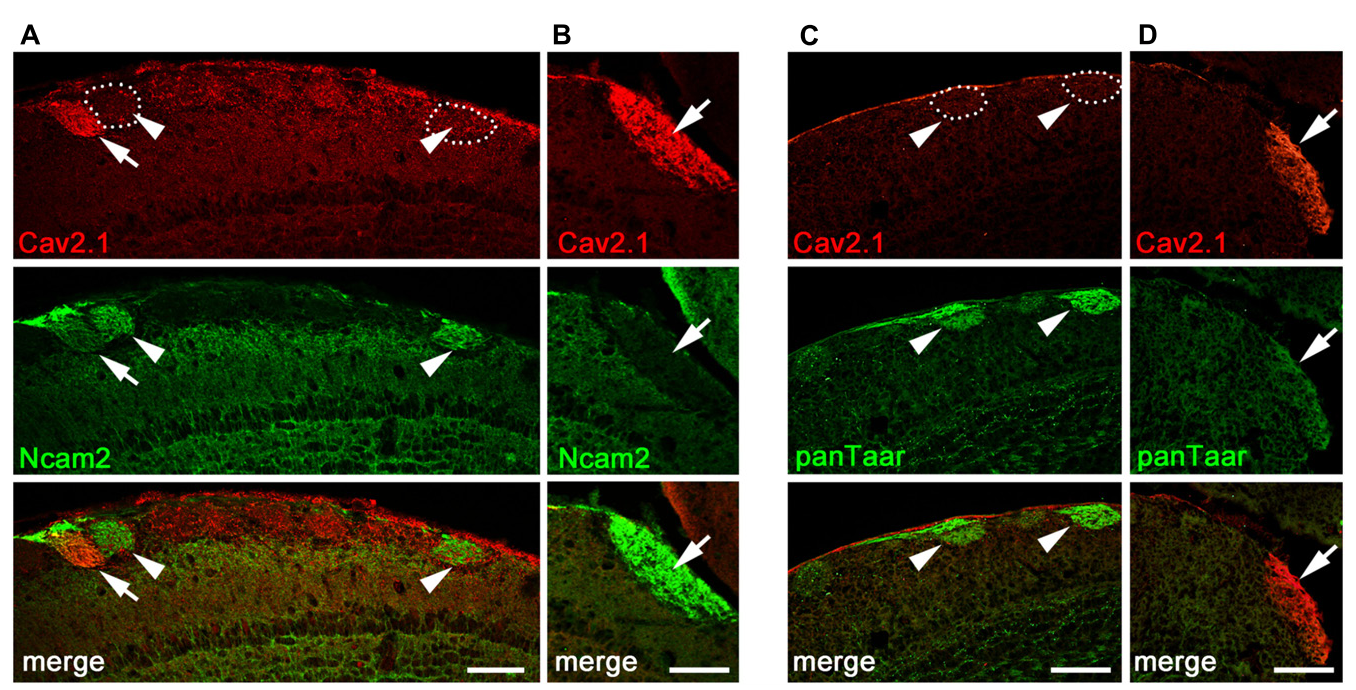

FIGURE 5 | Cav2.1+ glomeruli and expression of neural adhesion molecules. (A,B) Confocal images of the dorsal-caudal MOB (sagittal plane) stained with Cav2.1 (red) and neural cell adhesion molecule Ncam2 (green). (A) The single Cav2.1+ glomerulus in the central-dorsal MOB exhibiting immunoreactivity for Ncam2 (arrow) represents a rare observation. Other Ncam2+ glomeruli in this area are devoid of Cav2.1 immunoreactivity (arrowheads). (B) Cav2.1+ glomeruli in the dorso-caudal MOB are Ncam2- (arrow) as depicted by the large glomerulus. (C,D) Double-labeling immunohistochemistry for Cav2.1 (red) and Taar (green) in the dorsal center (C) and dorso-caudal border (D) of the MOB. Cav2.1+ glomeruli are not labeled by the panTaar antibody used. Images are representatives of $(N=3)$ adult B6 mice, with $N$ representing every second section per mouse. Scale bars: $100 \mu \mathrm{m}$. 
stained for either panTaar (Figure 5C) or for Cav2.1 (Figure 5D) in the mice investigated ( $N=3$ bulbs from three different B6 mice), with individual Cav2.1+ glomeruli receiving axonal input from either Ncam2+ or Ncam2- OSNs.

\section{Cav2.1+ Glomeruli Express Transduction Molecules of Canonical OSNs}

Within the main olfactory system, a number of functionally different subsystems have been identified (Munger et al., 2009; Bear et al., 2016). To determine whether Cav2.1 glomeruli belong to any of the known subsystems, we conducted colocalization experiments with a variety of canonical and non-canonical markers in combination with Cav2.1. Immunostaining of $\mathrm{MOB}$ whole-mounts and tissue sections showed that Cav2.1+ glomeruli colocalize OMP and two typical elements of the canonical signal transduction pathway, the phosphodiesterase Pde4a and the cAMP-activated cation channel Cnga2 (Figures 6A-C). Thus, Cav2.1 glomeruli receive axonal input from mature sensory neurons of the MOE that seem to belong to the group of canonical OSNs in that they express at least two elements of the classical cAMP-mediated signal transduction cascade.

Because of the necklace-like arrangement of some Cav2.1+ glomeruli at the dorsal-caudal border of the MOB, we investigated the colocalization of Cav2.1 with other noncanonical signal transduction molecules. These included phosphodiesterase Pde2a that specifically labels necklace glomeruli targeted by GC-D OSNs of the MOE and the glomeruli targeted by the GGNs (Juilfs et al., 1997; Matsuo et al., 2012); the membrane guanylate cyclase GC-G present in GGNs (Fleischer et al., 2009; Liu et al., 2009); and the soluble guanylate cyclase Gycylb2 and the Trpc2 cation channel that mediate signal transduction in type B cells of the MOE (Bleymehl et al., 2016). We found that Cav2.1+ glomeruli were devoid of Pde2a (Figure 6D). Whole-mount staining revealed that Cav2.1+ glomeruli were located in close proximity but always anteriorly to the Pde2a+ glomeruli (Figure 6D). There was also no colocalization between Cav2.1 and GFP using GCG-GFP mice that exclusively report on glomeruli innervated by GGNs in the MOB necklace region (Matsuo et al., 2012) (Figure 6E). Furthermore, colocalization experiments performed in Trpc2-IRES-taulacZ mice and Gucylb2-IREStaumCherry mice (Omura and Mombaerts, 2014, 2015) showed no colocalization with Cav2.1 glomeruli (Figures 6F,G). Together, these experiments demonstrate that the Cav2.1+ glomeruli do not belong to the olfactory subsystems formed by GC-D+ OSNs, GGNs, or Trpc2+ MOE (type A or type B) cells. Instead, Cav2.1+ glomeruli are characterized by the expression of OMP, Cnga2, and Pde4a.

\section{Cav2.1 Expression Defines a Distinct Subpopulation of OSNs}

Having identified Cav2.1 in a small subset of MOB glomeruli and their afferent axon bundles, we next focused on the corresponding neurons in the MOE. We performed immunohistochemistry for Cav2.1 on sections taken at intervals along the anterior-to-posterior extent of the MOE of adult $\mathrm{B} 6$ mice. As shown in Figure 7, robust Cav2.1 immunoreactivity was detected in a subpopulation of OSNs mainly situated in the medial and dorsal aspects of the posterior two thirds of the MOE. More specifically, Cav2.1+ OSNs occupied the epithelium lining the dorsal roof, the nasal septum, and the dorsomedial tip of endoturbinate II (Figure 7A). Fewer Cav2.1+ OSNs were detected in the dorsal tip of endoturbinate III and the medial aspect of ectoturbinate 2. Interestingly, this pattern is reminiscent to that of ORs. Based on the circumscribed mRNA expression of individual ORs, four dorso-ventral zones have been assigned along the anterior-to-posterior extent of the MOE, with zone 1 representing the most dorsal and medial aspects and zone 4 the most ventral and lateral aspects of the epithelium (Ressler et al., 1994). Referring to these zones, Cav2.1 expression was primarily detected in OSNs of zone 1 and the dorso-medial aspects of zones 2 and 3. Cav2.1 immunoreactivity depicted the typical bipolar OSN morphology and was present in the main subcellular compartments with strongly labeled knobs, dendrites, and somata (Figure 7B). OSN axons in the MOE were also stained but less so than the other subcellular compartments of the OSN (Figure 7B). Cav2.1+ OSNs were scattered throughout the depth of the MOE. The expression pattern was nearly bilaterally symmetrical between left and right nostrils and reproducibly observed among individual mice $(N \geq 3)$. Immunoreactivity was absent when omitting primary antibody and in peptide control reactions, where the Cav2.1 antiserum has been pre-absorbed with the cognate peptide (Figure 7C).

We obtained independent support for these results by RNAscope fluorescence in situ hybridization (Figures 7D-F). We used MOE tissue sections of adult B6 mice taken at anterior, medial and posterior positions of the epithelium and hybridized them with RNAscope probes specific for the $1 \mathrm{~kb}$ region spanning exons 37-46 of the Cacnala mRNA. Our results showed strong hybridization signals in a subpopulation of OSNs that was scattered in the dorsal and medial MOE (Figure 7D). This expression pattern mirrored the pattern obtained by immunohistochemistry, and independently verified the specificity of the Cav2.1 antibody. The specificity of the hybridization signals was verified in control reactions that were devoid of any staining (Figure 7F). In addition, the hybridization region was verified by reverse transcription polymerase chain reaction (RT-PCR) using total RNA prepared from the nasal mucosa of adult B6 mice and gene-specific primers amplifying exons 24-43 of the Cacnala mRNA (Figure 7G). Sequence analysis of the products obtained revealed $100 \%$ identity with the Cacnala mRNA.

Cav channel expression at central nervous system neurons can be subject to developmental changes (Iwasaki et al., 2000; Mark et al., 2011) which may indicate a special requirement during periods of heightened plasticity. We examined Cav2.1 expression in the MOE at different time points during development. We analyzed coronal MOE tissue sections derived from B6 mice at embryonic day 18 (E18), postnatal day 1 (P1), P7, P14, and P21 (Figure 7H). At E18, the MOE was devoid of any Cav2.1 staining. However, at 1 day after birth we detected substantial Cav2.1 immunoreactivity in somata, dendrites, and dendritic knobs of 


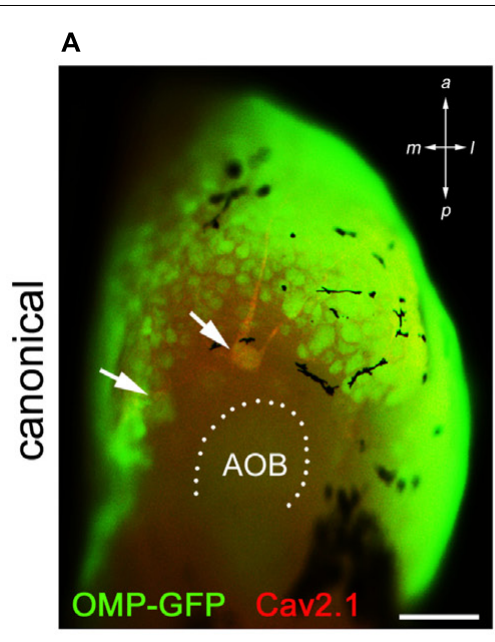

B
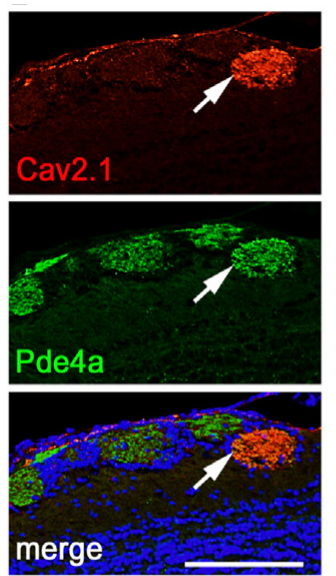

D
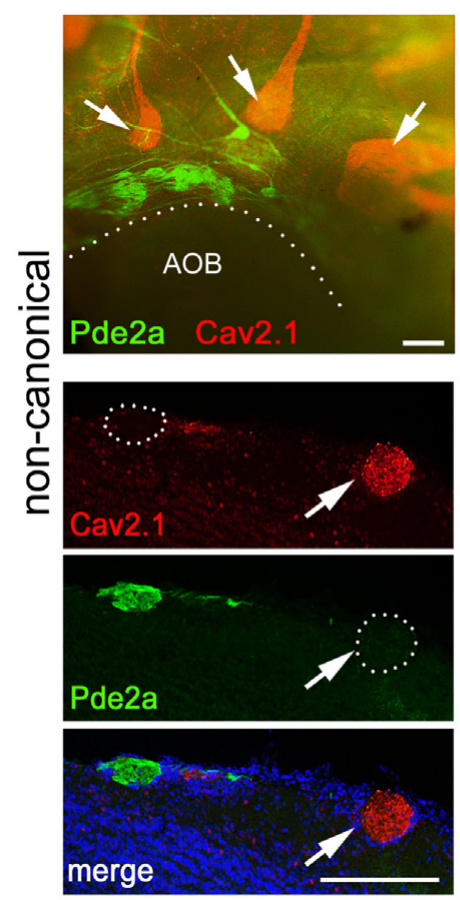

E
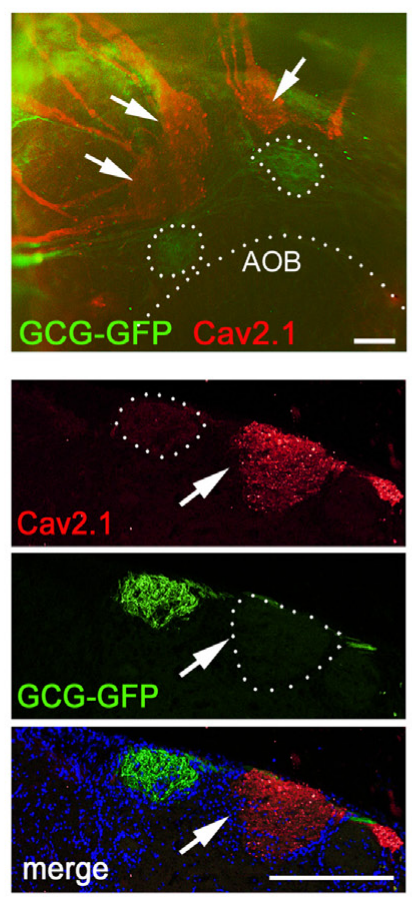

C
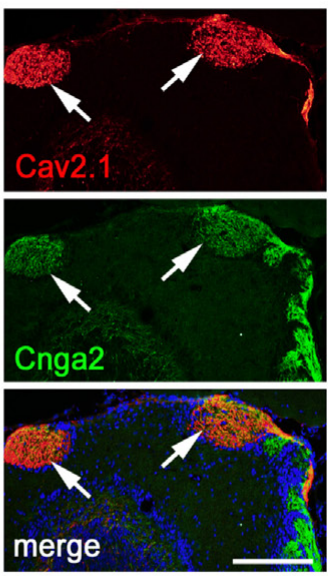

$\mathbf{F}$

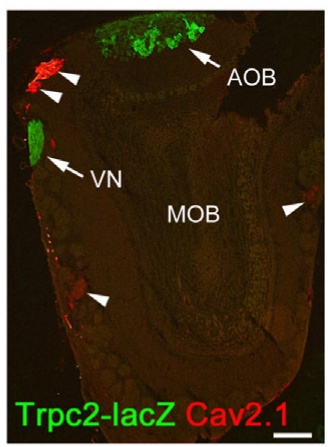

G

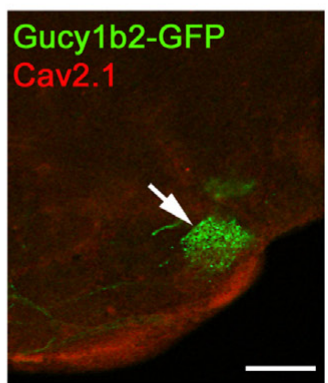

FIGURE 6 | Cav2.1+ glomeruli receive afferent input from a specific subset of OSNs. (A) Cav2.1 immunoreactivity (red) and endogenous GFP fluorescence in a whole-mount MOB preparation (dorsal-caudal view) of an adult OMP-GFP mouse. Cav2.1 and GFP colocalize in individual glomeruli (arrows). Coronal MOB section $(14 \mu \mathrm{m})$ showing that Cav2.1+ glomeruli (red) colocalize with Pde4a (green) (B) and Cnga2 (green) (C). (D,E) Non-canonical markers are absent in Cav2.1+ glomeruli. (D) Whole-mount view (top) showing that Cav2.1+ glomeruli (arrows) reside anterior to the Pde2a+ glomeruli (green) and the AOB. Pde2a and Cav2.1 label separate sets of glomeruli (bottom, $14 \mu \mathrm{m}$ coronal section). (E) Whole-mount view of the MOB (top) of a GCG-GFP mouse. Cav2.1+ glomeruli (red, arrows) reside anterior to the GFP+ glomeruli (green) that receive axonal input from GGNs. GCG-GFP+ and Cav2.1+ glomeruli are separate sets of glomeruli (bottom, $14 \mu \mathrm{m}$ coronal section). (F) Coronal MOB section (14 $\mu \mathrm{m}$ ) from a Trpc2-IRES-taulacZ mouse stained for Cav2.1 (red) and $\beta$-galactosidase (green). Both the AOB and the vomeronasal nerve (VN) but not the Cav2.1+ glomeruli (arrowheads) are positive for $\beta$-galactosidase. (G) Sagittal section (14 $\mu \mathrm{m}$ ) of the caudal-ventral MOB from a Gucy1b2-IRES-tauGFP mouse. The single GFP+ (green) glomerulus shown is devoid of Cav2.1 staining (red). Hoechst nuclear dye (blue) defines glomerular boundaries in the merged images. Images are representatives of $N \geq 2$ mice with $N=$ every second section per mouse. Scale bars (A) $500 \mu \mathrm{m}$, (B-E) $100 \mu \mathrm{m}$, (F) $200 \mu \mathrm{m}$, (G) $50 \mu \mathrm{m}$.

OSNs. The number of labeled OSNs was relatively small at P1 but increased coinciding with age and epithelial thickness over the next 3 weeks of postnatal development. Cav2.1 expression in OSNs distributed in the dorsal and medial MOE was already pronounced at P7 (not shown), but overall we did not observe gross changes in the Cav2.1 expression pattern during the first 3 weeks of postnatal development (Figure 7H).

Together, our results show that Cav2.1 expression identifies a specific subpopulation of OSNs in the dorsal and medial MOB with an expression onset at about birth. This distribution is 

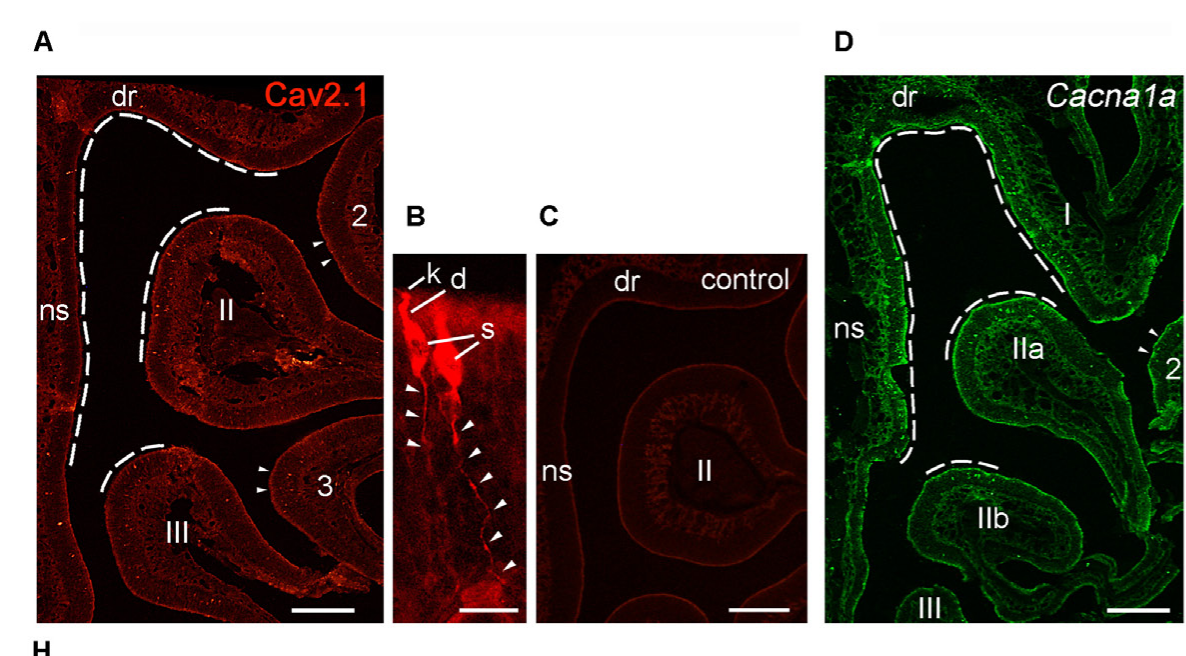

E

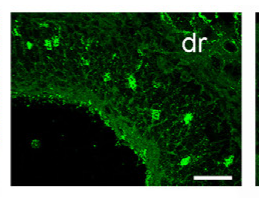

$\mathbf{F}$
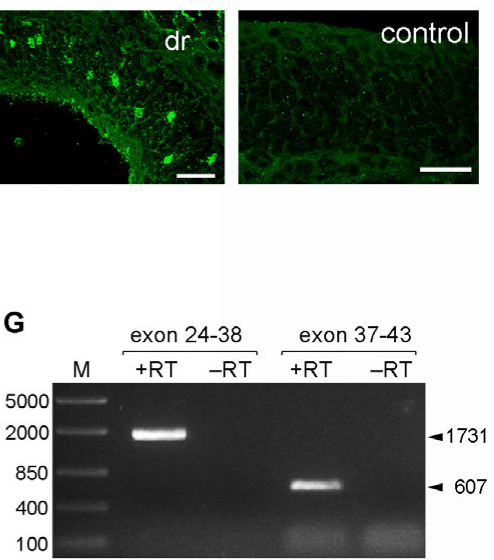

H
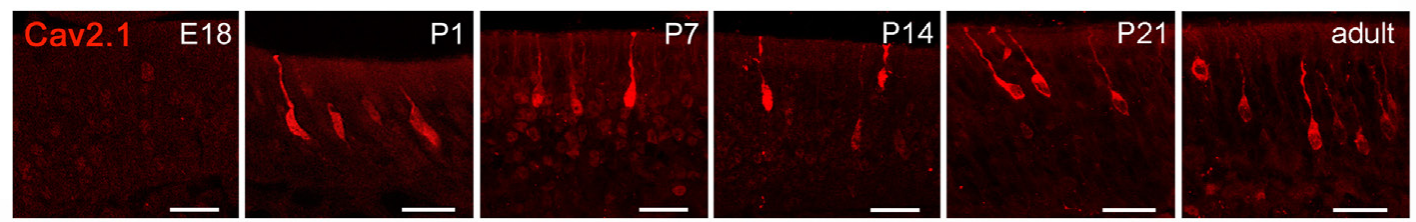

FIGURE 7 | Identification of Cav2.1+ OSNs in the MOE. (A) Coronal MOE sections (14 $\mu \mathrm{m})$ showing the left nasal cavity of an adult B6 mouse stained with Cav2.1. Cav2.1 + OSNs reside mainly in the MOE lining the dorsal roof (dr), nasal septum (ns), the dorsal-medial tip of endoturbinate II, and the tip of endoturbinate III (dashed lines). Few labeled OSNs are detectable at the tips of ectoturbinates 2 and 3. (B) Cav2.1 staining is present in OSN knob (k), dendrite (d), soma (s), and axon (arrowheads). To delineate axonal Cav2.1 staining, contrast and brightness was increased by 50\%. (C) The peptide control reaction is devoid of Cav2.1 staining. (D) RNAscope fluorescence in situ hybridization for Cav2.1 mRNA (Cacna1a, green) in the MOE of an adult B6 mouse (coronal view, left nasal cavity) shows that the distribution of labeled OSNs is closely similar to that obtained by immunohistochemistry (A). The dorsal roof, endoturbinate I, nasal septum, and the dorsal-medial tips of endoturbinates Ila and IIb (dashed lines) show labeled OSNs. Few labeled OSNs reside at the medial tip of ectoturbinate 2 (arrowheads). (E) Higher magnification shows hybridized OSNs at all depths of the epithelial layer. (F) The negative control reaction is devoid of labeling. (G) Agarose gel electrophoresis of the products obtained by RT-PCR using Cacna1a-specific primers and total RNA of adult mouse olfactory tissue. RT-PCR resulted in products (+RT) of the expected sizes using primers amplifying exons 24-38 (1,731 bp, arrowhead) and exons 37-43 (607 bp, arrowhead). Control reactions omitting reverse transcriptase (-RT) yielded no products. M, DNA size marker in base pairs as indicated at the left. $\mathbf{( H )}$ Magnifications of the dorsal MOE derived at different mouse ages stained with Cav2.1. Immunoreactivity for Cav2.1 (red) is absent at embryonic day 18 (E18). Cav2.1+ OSNs become visible at about postnatal day 1 (P1), and expression continues at P7, P14, P21 toward adulthood. Images are representatives of $N \geq 2$ mice per age with $N \geq 10$ sections per mouse. Scale bars (A,C,D) $200 \mu \mathrm{m},(\mathbf{E})$; $50 \mu \mathrm{m} ;(\mathbf{F}, \mathbf{H}) 20 \mu \mathrm{m} ; \mathbf{( B )} 10 \mu \mathrm{m}$.

maintained by the Cav2.1+ glomeruli in a typical MOE-to-MOB topographic projection pattern.

\section{Cav2.1+ OSNs Express Markers of Canonical OSNs}

To gain further insight into the identity of Cav2.1+ OSNs and to verify our results obtained for Cav2.1+ glomeruli in the $\mathrm{MOB}$, we performed immunohistochemical colocalization experiments on MOE tissue sections using several molecular markers. In addition to OMP whose expression coincides with OSN identity and maturation (Keller and Margolis, 1976), we examined expression of Pde4a and Cnga2. Furthermore, we analyzed components that define non-canonical OSNs such as GC-D OSNs, Taar+ OSNs, and Trpc2+ OSNs. These OSN subpopulations are distributed in regions of the MOE that partially overlap with those mapped for Cav2.1+ OSNs. Cav2.1 immunostaining in OMP-GFP reporter mice (Potter et al., 2001) showed that most Cav2.1+ OSNs are mature OSNs (Figure 8A) as indicated by the endogenous GFP fluorescence. Furthermore,
Cav2.1 immunoreactivity clearly colocalized with both Pde4a and with Cnga2 (Figure 8A), demonstrating that Cav2.1+ OSNs express these components of canonical OSNs. By contrast, Cav2.1 showed no colocalization with any of the non-canonical components investigated (Figure 8B). For the colocalization with Trpc2, we analyzed MOE sections of Trpc2-IRES-taumCherry mice (Omura and Mombaerts, 2015) and antibodies directed against the fluorescence reporter. For GC-D, we used olfactory tissue sections from Gucy2d-Mapt-lacZ mice (Leinders-Zufall et al., 2007) and antibodies specific for $\beta$-galactosidase. Cav2.1 staining was also combined with the panTaar antibody. As shown in Figure 8B, Cav2.1+ OSNs were devoid of staining for Trpc2, Taar, and GC-D. Even if OSNs existed in close proximity to those neuron types, they always represented separately labeled OSN populations.

In summary, these results indicate that Cav2.1+ OSNs may represent a specialized subpopulation of canonical OSNs in the MOE that project to a unique subset of Cav2.1+ glomeruli in the MOB. 


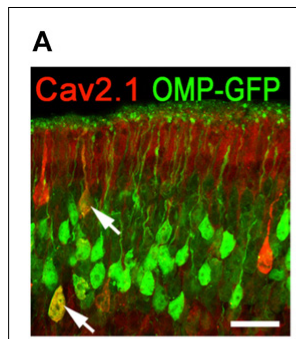

\section{canonical}
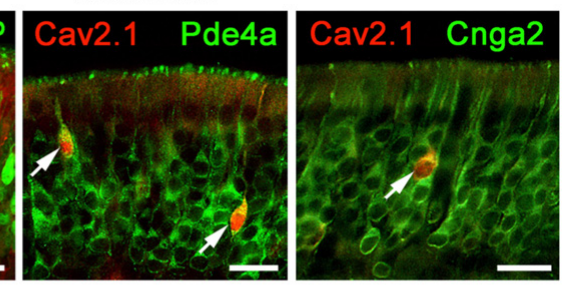

B
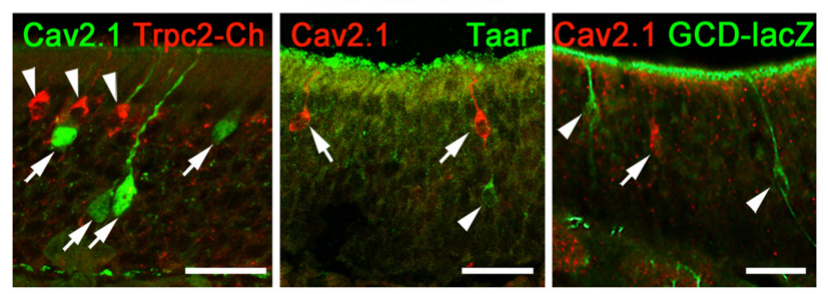

FIGURE 8 | Cav2.1+ OSNs express the canonical markers OMP, Pde4a, and Cnga2. Coronal MOE sections $(14 \mu \mathrm{m})$ of adult mice labeled for Cav2.1 and different canonical (A) and non-canonical (B) signal transduction molecules. (A) As shown at the left, Cav2.1 (red) and GFP (green) colocalize in mature OSNs (arrows) of OMP-GFP mice. Cav2.1+ OSNs (red) express Pde4a (green, middle, arrows) and Cnga2 (green, right, arrow). (B) Cav2.1 immunoreactivity (arrows) is absent in non-canonical OSNs such as those expressing Trpc2 (red, left, arrowheads), Taar (green, middle, arrowhead) or GC-D (green, right, arrowhead). Images are representatives of $(N \geq 2)$ mice with $N=$ every 10 th section along the anterior-to-posterior extent of the MOE in each mouse. Scale bars: $20 \mu \mathrm{m}$.

\section{DISCUSSION}

This study investigated the expression of the P/Q-type voltagegated calcium channel subunit Cav2.1 in the mouse MOB and MOE using immunohistochemistry, immuno-electron microscopy, RT-PCR, and RNA scope in situ hybridization methodology. Our experiments provide several converging lines of evidence indicating that Cav2.1 represents a novel candidate for olfactory signal transmission in a previously unknown subset of MOB glomeruli. The main findings of this study are: (1) Cav2.1 expression in the MOB is limited to a unique subset of glomeruli mainly located in the dorso-caudal and medial aspects of each olfactory bulb. (2) Cav2.1 localizes to the presynaptic axon terminals of OSNs targeting these glomeruli. (3) Both Cav2.1 protein and its corresponding mRNA Cacnala also localize to a defined subpopulation of OSNs in the dorsal and medial MOE, indicating a distinct MOE-to-MOB topography of Cav2.1+ OSNs. (4) Cav2.1 expression demarcates a previously unknown multiglomerular domain in the MOB, perhaps even a novel olfactory subsystem that is characterized by the expression of OMP, Cnga2, and Pde4a. (5) This system is distinct from several cGMP-dependent olfactory subsystems that express type-D and type-G receptor guanylate cyclases or Trpc2 and the soluble guanylate cyclase Gucy1b2.

Our work provides new insight into the architecture, organization, and projection targets of distinct mouse olfactory bulb glomeruli. We showed previously that the vast majority

of glomeruli in the $\mathrm{MOB}$ and $\mathrm{AOB}$ express the N-type calcium channel Cav2.2 and subsequently analyzed its key role in synaptic transmission (Weiss et al., 2014). Here, we identify Cav2.1 as a second candidate for synaptic transmission at the first olfactory synapse within a defined subset of $\mathrm{MOB}$ glomeruli. In adult mice, we detected robust Cav2.1 staining in a distinct set of about 20 glomeruli per bulb hemisphere, predominantly located in the medial and central dorsal MOB, and at the dorso-caudal border (see Figure 9) whereas no staining was detected in the AOB. Remarkably, the position of Cav2.1 glomeruli in the dorsal center and in the dorso-caudal border of the MOB (Figure 9) coincided with two areas harboring known olfactory subsystems that process olfactory cues associated with the detection of threat or danger signals. Notably, 5-7 Cav2.1+ glomeruli resided in juxtaposition to the GC-D+ necklace glomerular subsystem. GC-D+ OSNs use cGMP signaling and are involved in the social transmission of food preference (Leinders-Zufall et al., 2007; Munger et al., 2010). As illustrated in Figure 9, the necklace region also contains the glomeruli targeted by GGNs that mediate the detection of cold stimuli (Mamasuew et al., 2008; Schmid et al., 2010) and responses to predator odors and alarm pheromones (Brechbühl et al., 2008, 2013; Pérez-Gómez et al., 2015; Bumbalo et al., 2017). Our results clearly showed that Cav2.1+ glomeruli in the necklace area are distinct from these two non-canonical olfactory subsystems. Instead, Cav2.1+ glomeruli are characterized by the expression of Cnga2 and Pde4a, both known to be essential signaling components of canonical OSNs (Ferguson and Zhao, 2016). Cav2.1+ glomeruli are also clearly distinct from those glomeruli targeted by a subset of OSNs that use the ion channel Trpc2 and the soluble guanylyl cyclase Gucy1b2 to respond to decreases in environmental oxygen (Omura and Mombaerts, 2015; Bleymehl et al., 2016). The necklace glomerular system exhibits extensive intra-glomerular connections with canonical glomeruli (Cockerham et al., 2009) and functional analyses recently suggested that olfactory input from necklace and juxta-positioned canonical glomeruli could be integrated as part of an interconnected bulbar network (Uytingco et al., 2016). Future experiments will be required to determine whether the Cav2.1+ glomeruli are interconnected with other subsystems of the necklace region.

In the dorsal MOB, we detected Cav2.1+ glomeruli in close proximity to glomeruli of the Taar subsystem (Liberles et al., 2009; Pacifico et al., 2012) known to mediate innate fear to predator odors and aversion to spoiled food odors (Kobayakawa et al., 2007; Matsumoto et al., 2010; Dewan et al., 2013). Dorsal Taar+ glomeruli were previously shown to colocalize with Ncam2 (Johnson et al., 2012), an adhesion molecule usually defining ventro-lateral glomeruli that receive axonal input from OSNs located in zones 2-4 of the MOE (Yoshihara et al., 1997). Most of the Cav2.1+ glomeruli were negative for Ncam 2 which is typical for OSNs of the dorsal zone 1 in the MOE, but we also detected a small number of Cav2.1+/Ncam2+ glomeruli ( $\sim 2-3$ out of 20$)$. However, we found no evidence that these glomeruli are positive for Taar expression. Thus, we conclude that Cav2.1+ glomeruli most likely receive axonal input from a subset of canonical OSNs. We note that we cannot fully exclude the possibility that the sensory neurons targeting the Cav2.1+/Ncam $2+$ glomeruli 

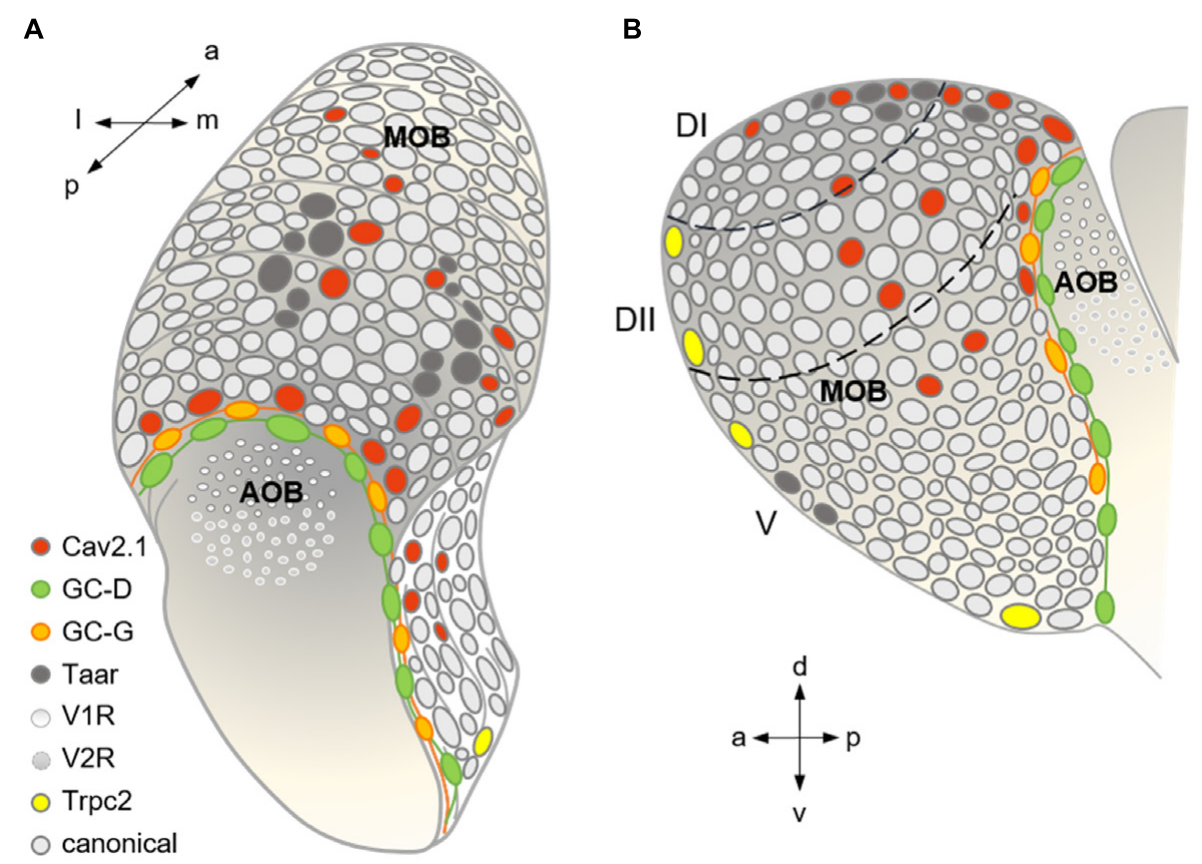

FIGURE 9 | Summary scheme showing the organization of the major olfactory subsystems at the level of a glomerular map. (A) Dorso-caudal view of the olfactory bulb (left side) depicting the different glomerular subsystems as indicated by the color-coded legend. Cav2.1 glomeruli in the caudal MOB reside in juxtaposition to the GC-D necklace glomeruli innervated by GC-D+ OSNs of the MOE and the GC-G glomeruli innervated by GGNs of the Grueneberg ganglion (GGN). Cav2.1 glomeruli in the center of the dorsal MOB intermingle with Taar glomeruli, and are also present in the medial MOB. (B) Medial view of the right olfactory bulb visualizing the necklace glomerular tracks for GC-D and GC-G, the dorsal position of Cav2.1 and Taar glomeruli, the medial Cav2.1 glomeruli, and the rostral and ventral position of Trpc2+ glomeruli in the MOB. Arrows denote orientations a (anterior), p (posterior), d (dorsal), v (ventral), I (lateral), and $m$ (medial); dashed lines indicate dorsal zones DI and DII, and the ventral zone V. Adapted and extended from Bear et al. (2016).

express a particular type of Taar that could exhibit low affinity to the panTaar antibody used here and thus escaped detection.

Consistent with our results in the MOB, we also found Cav2.1 expression in a defined subpopulation of OSNs of the MOE. In agreement with the positions of Cav2.1+ glomeruli in the MOB, Cav2.1+ OSNs were present in the medial and dorsal aspects of the MOE as verified by two independent techniques, immunohistochemistry and RNAscope in situ hybridization. This result is in line with the typical MOE-to-MOB topographic projection patterns observed with OR gene expression (Mori et al., 2006). The scattered distribution of Cav2.1+ OSNs resembled the stochastic patterns known for individual ORs. However, different from the zonal restriction shown for the expression of single OR types to one out of four dorso-ventral zones (Ressler et al., 1994), Cav2.1+ OSNs were present across zones $1-3$. This is consistent with the observation that Cav2.1+ glomeruli seem to receive input from both Ncam2- (zone 1 ORs) and Ncam2+ (zones 2-4 ORs) OSNs (Yoshihara et al., 1997). Detailed future molecular analyses will be required to determine whether Cav2.1+ OSNs express one of the known OR genes, or whether they express yet unknown receptor candidates. Such investigations should provide insight into the specific functions that Cav2.1+ OSNs and the corresponding glomeruli could play in olfaction.

At the level of individual Cav2.1+ OSNs, we detected Cav2.1 immunoreactivity in several OSN compartments. Besides presynaptic axon terminals within a given glomerulus, Cav2.1 was also localized to the OSN dendrite, dendritic knob, somata, and proximal axon. This observation may relate to the multiple functions Cav channels exert at various cellular sites (Catterall, 2011). On the basis of this expression pattern, depolarizationinduced $\mathrm{Ca}^{2+}$ entry through Cav2.1 could influence multiple cellular mechanisms including synaptic release, action potential discharge patterns, activation or regulation of other $\mathrm{Ca}^{2+}$ dependent ion channels, regulation of enzymes, and gene expression. Consistent with an essential role in OSN signaling, we found that Cav2.1 staining of OSN somata, dendrites, and dendritic knobs was already evident shortly after birth but absent at embryonic day 18 , implying that the somatic function of Cav2.1 establishes with birth and is required throughout life.

In summary, our experiments have identified a previously unrecognized multiglomerular domain in the MOB that is defined by the expression of Cav2.1. These glomeruli and their corresponding sensory neurons may form a novel olfactory subsystem. Important questions that remain to be answered in future experiments are the following: (1) Which receptor candidates are expressed by the Cav2.1+ OSNs? (2) Is there any functional relationship of the Cav2.1 glomeruli to other olfactory subsystems present in the necklace region of MOB? (3) What is the overall role of this presumed subsystem for olfactory processing? (4) And does this novel subdomain also exist in 
other mammalian species including humans that normally carry a functional CACNA1A gene?

In this context, it is important to note that the Cav2.1 channel seems to be more efficient in transmitter release than Cav2.2 (Millan and Sanchez-Prieto, 2002; Ladera et al., 2009). This raises the intriguing question whether the Cav2.1 subsystem could be specialized to detect odor cues that require high-speed processing at the first olfactory synapse. Alternatively, Cav2.1 may also mediate specific forms of synaptic plasticity and contribute to the regulation of synaptic transmission (Mochida, 2018; Nanou and Catterall, 2018). Double-labeling experiments for Cav2.2 and Cav2.1 will have to be performed in the future.

\section{AUTHOR CONTRIBUTIONS}

MP: study concept and drafting of the manuscript. MP, MT, LO, and EE: data acquisition (immunohistochemistry, confocal microscopy, 3D reconstruction, electron microscopy, RT-PCR). MP, EE, DR-G, CG, and FZ: data interpretation. MP, MT, LO, and

\section{REFERENCES}

Bartel, D. L., Rela, L., Hsieh, L., and Greer, C. A. (2015). Dendrodendritic synapses in the mouse olfactory bulb external plexiform layer. J. Comp. Neurol 523, 1145-1161. doi: 10.1002/cne.23714

Bear, D. M., Lassance, J. M., Hoekstra, H. E., and Datta, S. R. (2016). The evolving neural and genetic architecture of vertebrate olfaction. Curr. Biol. 26, R1039-R1049. doi: 10.1016/j.cub.2016.09.011

Bleymehl, K., Perez-Gomez, A., Omura, M., Moreno-Perez, A., Macias, D., Bai, Z., et al. (2016). A sensor for low environmental oxygen in the mouse main olfactory epithelium. Neuron 92, 1196-1203. doi: 10.1016/j.neuron.2016.11.001

Bolz, F., Kasper, S., Bufe, B., Zufall, F., and Pyrski, M. (2017). Organization and plasticity of sodium channel expression in the mouse olfactory and vomeronasal epithelia. Front. Neuroanat. 11:28. doi: 10.3389/fnana.2017. 00028

Brechbühl, J., Klaey, M., and Broillet, M. C. (2008). Grueneberg ganglion cells mediate alarm pheromone detection in mice. Science 321, 1092-1095. doi: 10.1126/science. 1160770

Brechbühl, J., Moine, F., Klaey, M., Nenniger-Tosato, M., Hurni, N., Sporkert, F., et al. (2013). Mouse alarm pheromone shares structural similarity with predator scents. Proc. Natl. Acad. Sci. U.S.A. 110, 4762-4767. doi: 10.1073/pnas. 1214249110

Bressel, O. C., Khan, M., and Mombaerts, P. (2016). Linear correlation between the number of olfactory sensory neurons expressing a given mouse odorant receptor gene and the total volume of the corresponding glomeruli in the olfactory bulb. J. Comp. Neurol. 524, 199-209. doi: 10.1002/cne.23835

Bumbalo, R., Lieber, M., Schroeder, L., Polat, Y., Breer, H., and Fleischer, J. (2017). Grueneberg glomeruli in the olfactory bulb are activated by odorants and cool temperature. Cell Mol. Neurobiol. 37, 729-742. doi: 10.1007/s10571-016-0408-6

Catterall, W. A. (2011). Voltage-gated calcium channels. Cold Spring Harb. Perspect. Biol. 3:a003947. doi: 10.1101/cshperspect.a003947

Cockerham, R. E., Puche, A. C., and Munger, S. D. (2009). Heterogeneous sensory innervation and extensive intrabulbar connections of olfactory necklace glomeruli. PLoS One 4:e4657. doi: 10.1371/journal.pone.0004657

Dewan, A., Pacifico, R., Zhan, R., Rinberg, D., and Bozza, T. (2013). Nonredundant coding of aversive odours in the main olfactory pathway. Nature 497, 486-489. doi: 10.1038/nature12114

Ferguson, C. H., and Zhao, H. (2016). "Cyclic AMP signaling in the main olfactory epithelium," in Chemosensory Transduction: The Detection of Odors, Tastes, and Other Chemostimuli, eds F. Zufall and S. D. Munger (Cambridge, MA: Academic Press), 123-140.
D-RG: figure preparation. LO, DR-G, CG, and FZ: revision of the manuscript. All authors take responsibility for data integrity and data analysis accuracy.

\section{FUNDING}

This work was supported by Deutsche Forschungsgemeinschaft (DFG) (Grant No. SFB 894/A17 to FZ) and (Grant No. PY 90/1-1 to MP) and NIH (Grant Nos. DC013791, DC015438, and DC012441 to CG).

\section{ACKNOWLEDGMENTS}

We thank Jan Weiss for valuable discussion of this project, and Petra Hammes and Christine Kaliszewki for technical assistance. Thanks are also due to Ivan Rodriguez (University of Geneva, Switzerland) for providing fixed tissue obtained from GC-G reporter mice.

Fiala, J. C. (2005). Reconstruct: a free editor for serial section microscopy. J. Microsc. 218, 52-61. doi: 10.1111/j.1365-2818.2005.01466.x

Firestein, S. (2001). How the olfactory system makes sense of scents. Nature 413, 211-218. doi: $10.1038 / 35093026$

Fleischer, J., Mamasuew, K., and Breer, H. (2009). Expression of cGMP signaling elements in the Grueneberg ganglion. Histochem. Cell Biol. 131, 75-88. doi: 10.1007/s00418-008-0514-8

Fülle, H. J., Vassar, R., Foster, D. C., Yang, R. B., Axel, R., and Garbers, D. L. (1995). A receptor guanylyl cyclase expressed specifically in olfactory sensory neurons. Proc. Natl. Acad. Sci. U.S.A. 92, 3571-3575. doi: 10.1073/pnas.92.8.3571

Gabellec, M. M., Panzanelli, P., Sassoe-Pognetto, M., and Lledo, P. M. (2007). Synapse-specific localization of vesicular glutamate transporters in the rat olfactory bulb. Eur. J. Neurosci. 25, 1373-1383. doi: 10.1111/j.1460-9568.2007. 05400.x

Ibarra-Soria, X., Levitin, M. O., Saraiva, L. R., and Logan, D. W. (2014). The olfactory transcriptomes of mice. PLoS Genet. 10:e1004593. doi: 10.1371/ journal.pgen. 1004593

Isaacson, J. S., and Strowbridge, B. W. (1998). Olfactory reciprocal synapses: dendritic signaling in the CNS. Neuron 20, 749-761. doi: 10.1016/ S0896-6273(00)81013-2

Iwasaki, S., Momiyama, A., Uchitel, O. D., and Takahashi, T. (2000). Developmental changes in calcium channel types mediating central synaptic transmission. J. Neurosci. 20, 59-65. doi: 10.1523/JNEUROSCI.20-01-00059. 2000

Johnson, M. A., Tsai, L., Roy, D. S., Valenzuela, D. H., Mosley, C., Magklara, A., et al. (2012). Neurons expressing trace amine-associated receptors project to discrete glomeruli and constitute an olfactory subsystem. Proc. Natl. Acad. Sci. U.S.A. 109, 13410-13415. doi: 10.1073/pnas. 1206724109

Juilfs, D. M., Fulle, H. J., Zhao, A. Z., Houslay, M. D., Garbers, D. L., and Beavo, J. A. (1997). A subset of olfactory neurons that selectively express cGMP-stimulated phosphodiesterase (PDE2) and guanylyl cyclase-D define a unique olfactory signal transduction pathway. Proc. Natl. Acad. Sci. U.S.A. 94, 3388-3395. doi: 10.1073/pnas.94.7.3388

Kamp, M. A., Hanggi, D., Steiger, H. J., and Schneider, T. (2012). Diversity of presynaptic calcium channels displaying different synaptic properties. Rev. Neurosci. 23, 179-190. doi: 10.1515/revneuro-2011-0070

Keller, A., and Margolis, F. L. (1976). Isolation and characterization of rat olfactory marker protein. J. Biol. Chem. 251, 6232-6237.

Klenoff, J. R., and Greer, C. A. (1998). Postnatal development of olfactory receptor cell axonal arbors. J. Comp. Neurol. 390, 256-267. doi: 10.1002/(SICI)1096-9861(19980112)390:2<256::AID-CNE8>3.0.CO;2-0 
Kobayakawa, K., Kobayakawa, R., Matsumoto, H., Oka, Y., Imai, T., Ikawa, M., et al. (2007). Innate versus learned odour processing in the mouse olfactory bulb. Nature 450, 503-508. doi: 10.1038/nature06281

Ladera, C., Martin, R., Bartolome-Martin, D., Torres, M., and Sanchez-Prieto, J. (2009). Partial compensation for N-type $\mathrm{Ca}^{2+}$ channel loss by P/Q-type $\mathrm{Ca}^{2+}$ channels underlines the differential release properties supported by these channels at cerebrocortical nerve terminals. Eur. J. Neurosci. 29, 1131-1140. doi: 10.1111/j.1460-9568.2009.06675.x

Leinders-Zufall, T., Cockerham, R. E., Michalakis, S., Biel, M., Garbers, D. L., Reed, R. R., et al. (2007). Contribution of the receptor guanylyl cyclase GC-D to chemosensory function in the olfactory epithelium. Proc. Natl. Acad. Sci. U.S.A. 104, 14507-14512. doi: 10.1073/pnas.0704965104

Li, Q., and Liberles, D. A. (2016). "Odor sensing by trace amine-associated receptors," in Chemosensory Transduction: The Detection of Odors, Tastes, and Other Chemostimuli, eds F. Zufall and S. D. Munger (Cambridge, MA: Academic Press), 67-80.

Liberles, S. D., and Buck, L. B. (2006). A second class of chemosensory receptors in the olfactory epithelium. Nature 442, 645-650. doi: 10.1038/nature05066

Liberles, S. D., Horowitz, L. F., Kuang, D., Contos, J. J., Wilson, K. L., SiltbergLiberles, J., et al. (2009). Formyl peptide receptors are candidate chemosensory receptors in the vomeronasal organ. Proc. Natl. Acad. Sci. U.S.A. 106, 9842-9847. doi: 10.1073/pnas.0904464106

Liu, C. Y., Fraser, S. E., and Koos, D. S. (2009). Grueneberg ganglion olfactory subsystem employs a cGMP signaling pathway. J. Comp. Neurol. 516, 36-48. doi: 10.1002/cne.22096

Luzzati, F., Fasolo, A., and Peretto, P. (2011). Combining confocal laser scanning microscopy with serial section reconstruction in the study of adult neurogenesis. Front. Neurosci. 5:70. doi: 10.3389/fnins.2011.00070

Maejima, T., Wollenweber, P., Teusner, L. U., Noebels, J. L., Herlitze, S., and Mark, M. D. (2013). Postnatal loss of P/Q-type channels confined to rhombic-lip-derived neurons alters synaptic transmission at the parallel fiber to purkinje cell synapse and replicates genomic Cacnala mutation phenotype of ataxia and seizures in mice. J. Neurosci. 33, 5162-5174. doi: 10.1523/JNEUROSCI.5442-12.2013

Mamasuew, K., Breer, H., and Fleischer, J. (2008). Grueneberg ganglion neurons respond to cool ambient temperatures. Eur. J. Neurosci. 28, 1775-1785. doi: 10.1111/j.1460-9568.2008.06465.x

Mark, M. D., Maejima, T., Kuckelsberg, D., Yoo, J. W., Hyde, R. A., Shah, V., et al. (2011). Delayed postnatal loss of P/Q-type calcium channels recapitulates the absence epilepsy, dyskinesia, and ataxia phenotypes of genomic Cacnala mutations. J. Neurosci. 31, 4311-4326. doi: 10.1523/JNEUROSCI.5342-10.2011

Matsumoto, H., Kobayakawa, K., Kobayakawa, R., Tashiro, T., Mori, K., Sakano, H., et al. (2010). Spatial arrangement of glomerular molecular-feature clusters in the odorant-receptor class domains of the mouse olfactory bulb. J. Neurophysiol. 103, 3490-3500. doi: 10.1152/jn.00035.2010

Matsuo, T., Rossier, D. A., Kan, C., and Rodriguez, I. (2012). The wiring of Grueneberg ganglion axons is dependent on neuropilin 1. Development 139, 2783-2791. doi: 10.1242/dev.077008

Meyer, M. R., Angele, A., Kremmer, E., Kaupp, U. B., and Muller, F. (2000). A cGMP-signaling pathway in a subset of olfactory sensory neurons. Proc. Natl. Acad. Sci. U.S.A. 97, 10595-10600. doi: 10.1073/pnas.97.19. 10595

Millan, C., and Sanchez-Prieto, J. (2002). Differential coupling of N- and P/Q-type calcium channels to glutamate exocytosis in the rat cerebral cortex. Neurosci. Lett. 330, 29-32. doi: 10.1016/S0304-3940(02)00719-X

Mochida, S. (2018). Presynaptic calcium channels. Neurosci. Res. 127, 33-44. doi: 10.1016/j.neures.2017.09.012

Mori, K., and Sakano, H. (2011). How is the olfactory map formed and interpreted in the mammalian brain? Annu. Rev. Neurosci. 34, 467-499. doi: 10.1146/ annurev-neuro-112210-112917

Mori, K., Takahashi, Y. K., Igarashi, K. M., and Yamaguchi, M. (2006). Maps of odorant molecular features in the mammalian olfactory bulb. Physiological Review 86, 409-433. doi: 10.1152/physrev.00021.2005

Munger, S. D., Leinders-Zufall, T., Mcdougall, L. M., Cockerham, R. E., Schmid, A., Wandernoth, P., et al. (2010). An olfactory subsystem that detects carbon disulfide and mediates food-related social learning. Curr. Biol. 20, 1438-1444. doi: 10.1016/j.cub.2010.06.021
Munger, S. D., Leinders-Zufall, T., and Zufall, F. (2009). Subsystem organization of the mammalian sense of smell. Annu. Rev. Physiol. 71, 115-140. doi: 10.1146/ annurev.physiol.70.113006.100608

Nanou, E., and Catterall, W. A. (2018). Calcium channels, synaptic plasticity, and neuropsychiatric disease. Neuron 98, 466-481. doi: 10.1016/j.neuron.2018. 03.017

Nishimune, H., Numata, T., Chen, J., Aoki, Y., Wang, Y., Starr, M. P., et al. (2012). Active zone protein Bassoon co-localizes with presynaptic calcium channel, modifies channel function, and recovers from aging related loss by exercise. PLoS One 7:e38029. doi: 10.1371/journal.pone.0038029

Omura, M., and Mombaerts, P. (2014). Trpc2-expressing sensory neurons in the main olfactory epithelium of the mouse. Cell Rep. 8, 583-595. doi: 10.1016/j. celrep.2014.06.010

Omura, M., and Mombaerts, P. (2015). Trpc2-expressing sensory neurons in the mouse main olfactory epithelium of type B express the soluble guanylate cyclase Gucy1b2. Mol. Cell. Neurosci. 65, 114-124. doi: 10.1016/j.mcn.2015.02.012

Pacifico, R., Dewan, A., Cawley, D., Guo, C., and Bozza, T. (2012). An olfactory subsystem that mediates high-sensitivity detection of volatile amines. Cell Rep. 2, 76-88. doi: 10.1016/j.celrep.2012.06.006

Pérez-Gómez, A., Bleymehl, K., Stein, B., Pyrski, M., Birnbaumer, L., Munger, S. D., et al. (2015). Innate predator odor aversion driven by parallel olfactory subsystems that converge in the ventromedial hypothalamus. Curr. Biol. 25, 1340-1346. doi: 10.1016/j.cub.2015.03.026

Potter, S. M., Zheng, C., Koos, D. S., Feinstein, P., Fraser, S. E., and Mombaerts, P. (2001). Structure and emergence of specific olfactory glomeruli in the mouse. J. Neurosci. 21, 9713-9723. doi: 10.1523/JNEUROSCI.21-24-09713. 2001

Pyrski, M., Eckstein, E., Schmid, A., Bufe, B., Weiss, J., Chubanov, V., et al. (2017). Trpm5 expression in the olfactory epithelium. Mol. Cell. Neurosci. 80, 75-88. doi: 10.1016/j.mcn.2017.02.002

Ressler, K. J., Sullivan, S. L., and Buck, L. B. (1994). Information coding in the olfactory system: evidence for a stereotyped and highly organized epitope map in the olfactory bulb. Cell 79, 1245-1255. doi: 10.1016/0092-8674(94)90015-9

Richard, M. B., Taylor, S. R., and Greer, C. A. (2010). Age-induced disruption of selective olfactory bulb synaptic circuits. Proc. Natl. Acad. Sci. U.S.A. 107, 15613-15618. doi: 10.1073/pnas.1007931107

Schmid, A., Pyrski, M., Biel, M., Leinders-Zufall, T., and Zufall, F. (2010). Grueneberg ganglion neurons are finely tuned cold sensors. J. Neurosci. 30, 7563-7568. doi: 10.1523/JNEUROSCI.0608-10.2010

Shepherd, G. M., Chen, W. R., and Greer, C. A. (2004). "Olfactory Bulb," in The Synaptic Organization of the Brain, 5th Edn, ed. G. M. Shepherd (Oxford: Oxford University Press), 165-216. doi: 10.1093/acprof:oso/9780195159561. 003.0005

Shiraiwa, T., Kashiwayanagi, M., Iijima, T., and Murakami, M. (2007). Involvement of the calcium channel $\beta 3$ subunit in olfactory signal transduction. Biochem. Biophys. Res. Commun. 355, 1019-1024. doi: 10.1016/j.bbrc.2007. 02.063

Treloar, H. B., Feinstein, P., Mombaerts, P., and Greer, C. A. (2002). Specificity of glomerular targeting by olfactory sensory axons. J. Neurosci. 22, 2469-2477. doi: 10.1523/JNEUROSCI.22-07-02469.2002

Uytingco, C. R., Puche, A. C., and Munger, S. D. (2016). Interglomerular connectivity within the canonical and GC-D/necklace olfactory subsystems. PLoS One 11:e0165343. doi: 10.1371/journal.pone.0165343

Vassar, R., Chao, S. K., Sitcheran, R., Nunez, J. M., Vosshall, L. B., and Axel, R. (1994). Topographic organization of sensory projections to the olfactory bulb. Cell 79, 981-991. doi: 10.1016/0092-8674(94)90029-9

Wachowiak, M., Mcgann, J. P., Heyward, P. M., Shao, Z., Puche, A. C., and Shipley, M. T. (2005). Inhibition [corrected] of olfactory receptor neuron input to olfactory bulb glomeruli mediated by suppression of presynaptic calcium influx. J. Neurophysiol. 94, 2700-2712. doi: 10.1152/jn.00286.2005

Wachowiak, M., and Shipley, M. T. (2006). Coding and synaptic processing of sensory information in the glomerular layer of the olfactory bulb. Semin. Cell Dev. Biol. 17, 411-423. doi: 10.1016/j.semcdb.2006.04.007

Walz, A., Mombaerts, P., Greer, C. A., and Treloar, H. B. (2006). Disrupted compartmental organization of axons and dendrites within olfactory glomeruli of mice deficient in the olfactory cell adhesion molecule. OCAM Mol. Cell. Neurosci. 32, 1-14. doi: 10.1016/j.mcn.2006.01.013 
Wang, F., Flanagan, J., Su, N., Wang, L. C., Bui, S., Nielson, A., et al. (2012). RNAscope: a novel in situ RNA analysis platform for formalin-fixed, paraffinembedded tissues. J. Mol. Diag. 14, 22-29. doi: 10.1016/j.jmoldx.2011.08.002

Weiss, J., Pyrski, M., Jacobi, E., Bufe, B., Willnecker, V., Schick, B., et al. (2011). Loss-of-function mutations in sodium channel $\mathrm{Na}_{\mathrm{v}} 1.7$ cause anosmia. Nature 472, 186-190. doi: 10.1038/nature0 9975

Weiss, J., Pyrski, M., Weissgerber, P., and Zufall, F. (2014). Altered synaptic transmission at olfactory and vomeronasal nerve terminals in mice lacking N-type calcium channel Cav2.2. Eur. J. Neurosci. 40, 3422-3435. doi: 10.1111/ ejn. 12713

Yoshihara, Y., Kawasaki, M., Tamada, A., Fujita, H., Hayashi, H., Kagamiyama, H., et al. (1997). OCAM: a new member of the neural cell adhesion molecule family related to zone-to-zone projection of olfactory and vomeronasal axons. J. Neurosci. 17, 5830-5842. doi: 10.1523/JNEUROSCI.17-15-05830.1997

Zufall, F., and Munger, S. D. (eds) (2016). Chemosensory Transduction: The Detection of Odors, Tastes, and Other Chemostimuli. Cambridge, MA: Academic Press.
Zufall, F., and Munger, S. D. (2001). From odor and pheromone transduction to the organization of the sense of smell. Trends Neurosci. 24, 191-193. doi: $10.1016 / \mathrm{S} 0166-2236(00) 01765-3$

Zufall, F., and Munger, S. D. (2010). Receptor guanylyl cyclases in mammalian olfactory function. Mol. Cell. Biochem. 334, 191-197. doi: 10.1007/s110 10-009-0325-9

Conflict of Interest Statement: The authors declare that the research was conducted in the absence of any commercial or financial relationships that could be construed as a potential conflict of interest.

Copyright $\odot 2018$ Pyrski, Tusty, Eckstein, Oboti, Rodriguez-Gil, Greer and Zufall. This is an open-access article distributed under the terms of the Creative Commons Attribution License (CC BY). The use, distribution or reproduction in other forums is permitted, provided the original author(s) and the copyright owner(s) are credited and that the original publication in this journal is cited, in accordance with accepted academic practice. No use, distribution or reproduction is permitted which does not comply with these terms. 


\section{OPEN ACCESS}

Edited by:

Frédéric Marion-Poll,

AgroParisTech Institut des Sciences

et Industries du Vivant et de

l'Environnement, France

Reviewed by:

Ronald Sebastian Petralia,

National Institute on Deafness and Other Communication Disorders $(\mathrm{NIH})$, United States

Michael Fox

Virginia Tech Carilion Research

Institute, United States

${ }^{*}$ Correspondence:

Rafael Cantera

rafael.cantera@zoologi.su.se

Received: 16 January 2018 Accepted: 12 March 2018

Published: 27 March 2018

Citation: Gruber L, Rybak J, Hansson BS and Cantera R (2018) Synaptic Spinules in the Olfactory Circuit of Drosophila melanogaster.

Front. Cell. Neurosci. 12:86. doi: 10.3389/fncel.2018.00086

\section{Synaptic Spinules in the Olfactory Circuit of Drosophila melanogaster}

\author{
Lydia Gruber ${ }^{1}$, Jürgen Rybak ${ }^{1}$, Bill S. Hansson ${ }^{1}$ and Rafael Cantera ${ }^{2,3 *}$ \\ ${ }^{1}$ Department of Evolutionary Neuroethology, Max Planck Institute for Chemical Ecology (MPG), Jena, Germany, \\ ${ }^{2}$ Departamento de Biología del Neurodesarrollo, Instituto de Investigaciones Biológicas Clemente Estable (IIBCE), \\ Montevideo, Uruguay, ${ }^{3}$ Zoology Department, Stockholm University, Stockholm, Sweden
}

Here we report on ultrastructural features of brain synapses in the fly Drosophila melanogaster and outline a perspective for the study of their functional significance. Images taken with the aid of focused ion beam-scanning electron microscopy (EM) at $20 \mathrm{~nm}$ intervals across olfactory glomerulus DA2 revealed that some synaptic boutons are penetrated by protrusions emanating from other neurons. Similar structures in the brain of mammals are known as synaptic spinules. A survey with transmission EM (TEM) disclosed that these structures are frequent throughout the antennal lobe. Detailed neuronal tracings revealed that spinules are formed by all three major types of neurons innervating glomerulus DA2 but the olfactory sensory neurons (OSNs) receive significantly more spinules than other olfactory neurons. Double-membrane vesicles (DMVs) that appear to represent material that has pinched-off from spinules are also most abundant in presynaptic boutons of OSNs. Inside the host neuron, a close association was observed between spinules, the endoplasmic reticulum (ER) and mitochondria. We propose that by releasing material into the host neuron, through a process triggered by synaptic activity and analogous to axonal pruning, synaptic spinules could function as a mechanism for synapse tagging, synaptic remodeling and neural plasticity. Future directions of experimental work to investigate this theory are proposed.

Keywords: olfactory circuitry, Drosophila melanogaster, synaptic spinules, FIB-SEM, synaptic plasticity

\section{INTRODUCTION}

Research conducted in evolutionarily distant animals has contributed to our current understanding of olfactory synaptic circuits (Hildebrand and Shepherd, 1997; Ache and Young, 2005). The olfactory neuronal circuitry of the fly Drosophila melanogaster has been investigated successfully with anatomical, physiological, genetic and behavioral approaches and good models have been proposed to understand how chemosensory information is processed and how olfactory circuits contribute to learning and memory (Davis, 2004; Keene and Waddell, 2005; Fiala, 2007; Wilson, 2013; Guven-Ozkan and Davis, 2014; Hige, 2017).

This bounty of knowledge stood until recently in bright contrast to our insufficient understanding of the synaptic connections formed between the different cellular components of the olfactory neuronal network. Because of the small size of synapses and the need to map them in 3D across relatively large volumes of brain tissue, electron microscopy (EM) is necessary to map all synapses of the olfactory circuit. Progress in volume-based EM, image analysis, and automatic 3D reconstruction facilitates this challenging task and makes it possible to image 
and analyze all synaptic sites in the volume spanning the region of interest (Helmstaedter, 2013; Schneider-Mizell et al., 2016; Zheng et al., 2017). These recent advances have already resulted in several publications reporting detailed information on olfactory microcircuits in Drosophila (Berck et al., 2016; Rybak et al., 2016; Takemura et al., 2017; Tobin et al., 2017).

We used focused ion beam-scanning EM (FIB-SEM; Knott et al., 2008) to acquire complete series of images taken at $20 \mathrm{~nm}$ intervals across the entire olfactory DA2 glomerulus in adult Drosophila females (Gruber et al., unpublished data). The ultimate goal is to obtain a complete connectome of this glomerulus, which plays an important ecological role since it senses the odorant geosmin, emitted by mold growing in rotten fruits, and mediates a life-saving escape in the fly (Stensmyr et al., 2012). In the course of our studies we observed that olfactory neurons form deep invaginations of their plasma membrane nearby synaptic sites, occupied by protrusions from other neurons, similar to what has been referred to as synaptic spinules in the mammalian brain and that had yet not been reported for Drosophila. Synaptic spinules are invaginating protrusions of variable size and morphology that penetrate presynaptic terminals and, less frequently, postsynaptic profiles, axons and even glia in the brain of mammals and other vertebrates (reviewed in Petralia et al., 2015). Synaptic spinules are dynamic structures that grow and proliferate following synaptic activity (Richards et al., 2005; Tao-Cheng et al., 2009) and have been suggested to contribute to membrane plasticity as well as to cellto-cell communication and material exchange between neurons in an activity-dependent fashion (Petralia et al., 2015).

Our knowledge of these synapse-associated structures is still very limited. Here we present a viewpoint on this subject. We predict that spinules mediate localized synaptic plasticity mainly among olfactory sensory neurons (OSNs). Thus the finding of synaptic spinules in Drosophila melanogaster opens an avenue for an experimental investigation of their contribution and relevance for synapse plasticity, benefiting from the exceptional advantages offered by this organism.

\section{RESULTS AND DISCUSSION}

The observations reported here were done in the antennal lobe of female adults of Drosophila melanogaster studied with transmission electron microscopy (TEM, five specimens) and FIB-SEM (two specimens) across the entire DA2 glomerulus (see Supplementary Material). To achieve serial sections of this particular region with FIB-SEM it was marked previously by fiducial laser marks (see Supplementary Material). Images revealed that olfactory neurons make an interdigitating system of invaginating protrusions $20-500 \mathrm{~nm}$ in diameter close to active sites. Protrusions, emanating from one synaptic partner (the "protruding cell, PC"), penetrate the narrow funnels formed by deep invaginations of the plasma membrane of another synaptic partner (the "host cell, HC"; Figures 1A,B). The protrusions are therefore covered by two membranes: the evaginated membrane of the PC tightly covered by the invaginated membrane of the $\mathrm{HC}$, which receives the protrusion (Figure 1A). FIB-SEM-based dense reconstructions (done with the TrakEM2 plugin for ImageJ
Fiji $^{1}$; see Supplementary Material) make it possible to study invaginating protrusions in different types of olfactory neurons, which were distinguished according to their morphology (branching pattern and diameter of single branches), their total volume inside one glomerulus and ultrastructural details (as for example their synaptic inventory of input and output synapses) and other criteria described previously (Rybak et al., 2016; Tobin et al., 2017). These criteria allow a clear identification of uniglomerular projection neurons (PNs) and olfactory receptor neurons (OSNs) whereas the remaining cell types were more difficult to distinguish and are described here with the generic term "multigomerular neurons" (MGs). Individual presynaptic boutons of olfactory neurons might receive protrusions from more than one neuron or cell type, most prominently seen in OSNs (Figure 1B), and mutually invaginating protrusions between two neurons were also observed (not shown) as reported previously for other olfactory glomeruli (Rybak et al., 2016; in Figures 5C,D). Many of invaginating protrusions traced to their fiber of origin were found to originate from other OSNs, whereas the remaining ones emanated either from MGs, which includes local interneurons and multiglomerular PNs (Figure 1B), or PNs (see Figures 5C,D in Rybak et al., 2016). The synaptic boutons of PNs were mostly devoid of protrusions (Figures 1C,E).

By size, shape and location these invaginating protrusions are interpreted here to be the type of structures which in mammalian brain have been designated as synaptic spinules (Petralia et al., 2015). They appear to be identical or very similar to invaginated profiles illustrated in images of Drosophila synapses in other brain neurons published by other authors, who did not name them explicitly (see for example Figures 4A,B in Leiss et al., 2009; Figure 3 in Butcher et al., 2012; Figure 1 in Berck et al., 2016; Figures 6D and Supplementary Figure S1A in Zheng et al., 2017). Our survey of several Drosophila brains with the aid of TEM confirmed that spinules are frequent throughout the antennal lobe (data not shown).

The spinules reported here contained cytoplasm and in many cases also clear and dark vesicles (Figures 1A,D, 2A,B). The size of the spinules and that of their host boutons imply that spinules are in close vicinity with other organelles. Practically all spinules were observed in the proximity of presynaptic sites (Figures 1, 2), mitochondria and what appeared to be cisternae of the endoplasmic reticulum (ER) of the HC (Figures 1A,C,D). In many cases spinules appeared to be in physical contact with mitochondria and ER. Therefore, spinules might be part of a recently well described neuronal ER network that includes contacts with the plasma membrane, mitochondria as well as lysosomes and multivesicular bodies (Wu et al., 2017). Similar connections between ER tubules and synaptic invaginations have been observed previously in presynaptic regions of visual receptor cells (Lovas, 1971). The close association between spinules, active sites and two major sources of $\mathrm{Ca}^{2+}$ might have functional consequences.

A quantification of every single spinule penetrating each randomly selected $\mathrm{HC}$ of each neuronal type (in one brain) inside

\footnotetext{
${ }^{1}$ https://imagej.net/TrakEM2
} 


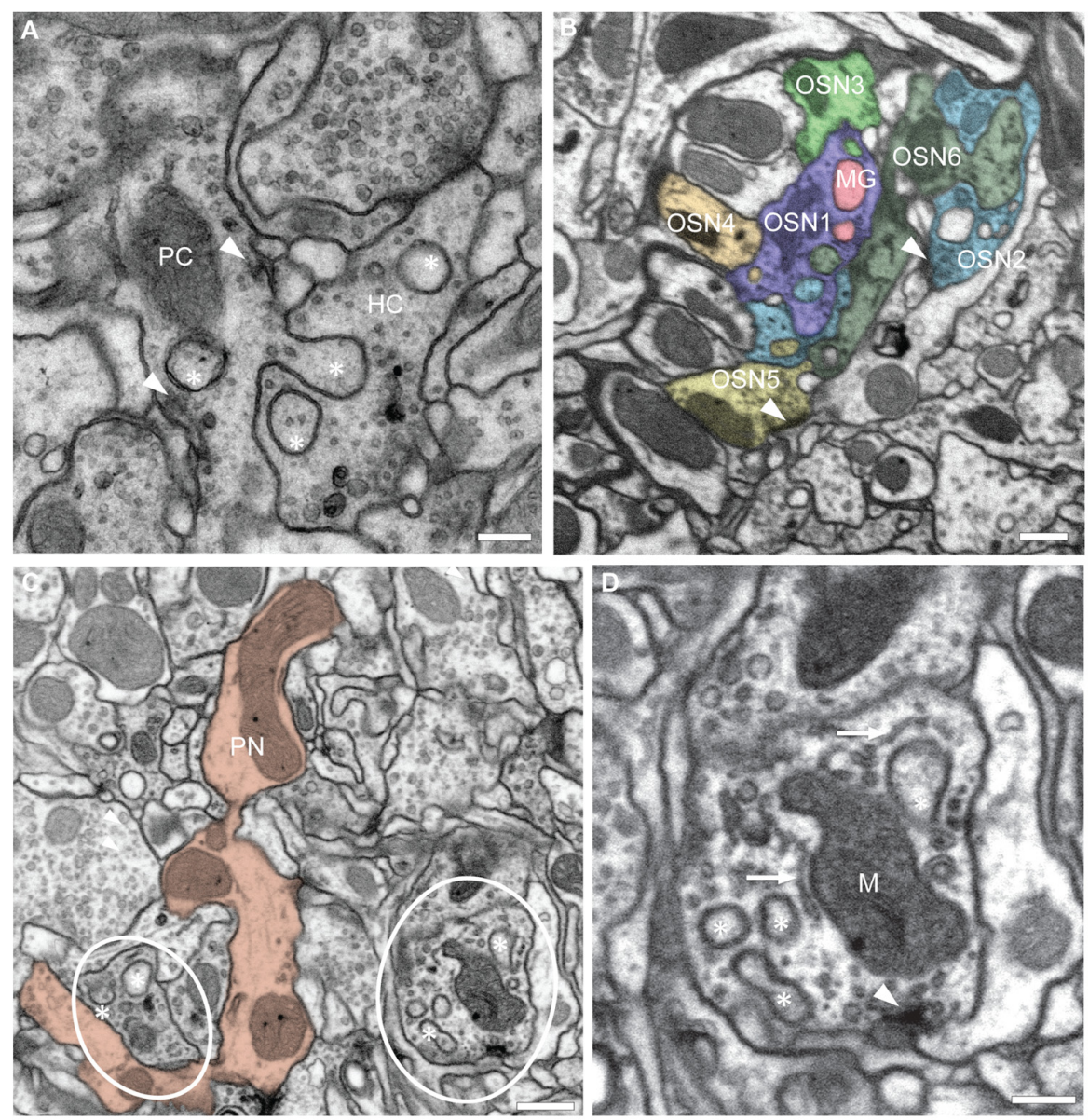

E

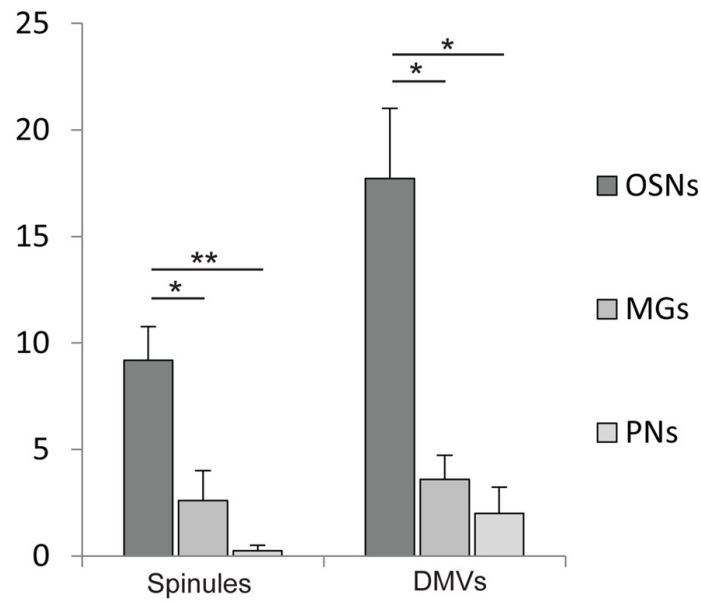

FIGURE 1 | Olfactory neurons in glomerulus DA2 contain invaginating protrusions. (A) Transmission electron microscopy (TEM) image of a $50 \mathrm{~nm}$ section showing examples of invaginating protrusions, or spinules (asterisks), enclosed by two plasma membranes and close to presynaptic sites (arrowheads). Notice that the evaginating membrane of the protruding cell (PC) is tightly adjoined by the invaginating membrane of the host cell (HC). Scale bar $=200 \mathrm{~nm}$. (B) A synaptic bouton can receive invaginating protrusions from more than one neuron. This image from a focused ion beam-scanning EM (FIB-SEM) serial reconstruction of glomerulus DA2 depicts invaginating protrusions in presynaptic boutons of two different olfactory sensory neurons (OSN1 and OSN2) penetrated by protrusions from several neighboring cells. The PCs in this particular example are either a multiglomerular neuron (MG) or other OSNs (OSN3, 4, 5 and 6). Synaptic sites are indicated by an arrowhead and the reconstructed neurons are color-coded to assign the origin of the invaginated protrusions inside HCs. Scale bar $=500 \mathrm{~nm}$. (C) Invaginating protrusions are not equally abundant among different types of olfactory neurons. This image (FIB-SEM) shows for example several boutons (red) of a uniglomerular projection neuron (PN), devoid of protrusions. In contrast, nearby OSN boutons (encircled) contain several protrusions (asterisks; see quantification in E). 


\section{FIGURE 1 | Continued}

Scale bar $=500 \mathrm{~nm}$. (D) FIB-SEM image showing invaginating protrusions (asterisks) close to mitochondria (M), putative endoplasmic reticulum (ER) cisternae (arrow) and a presynaptic site (arrowhead). For 3D surface view of spinules see Figure 2B. Scale bar $=200 \mathrm{~nm}$. (E) Quantification of spinules and double-membrane vesicles (DMVs) found inside reconstructed OSNs $(n=11)$, Projection neurons (PNs) $(n=4)$ and MGs $(n=5)$. OSNs receive a larger number of spinules and DMVs compared to MGs and PNs.

Quantification was done in one brain. Mean values with standard error of the mean are depicted. ${ }^{*} p<0.1$; ${ }^{* *} p<0.01$, one-way ANOVA, Tukey post hoc test.

glomerulus DA2 indicated that OSNs receive spinules most frequently, MGs less frequently and PNs only rarely (Figure 1E). On the other hand, based on EM images published by others (Leiss et al., 2009; Butcher et al., 2012) we propose that PN presynaptic boutons, located in the calyx of the mushroom body, host abundant spinules protruding from their postsynaptic partners, the Kenyon cells.

The shape of spinules appeared to be variable. They were often relatively short and bulbous (Figure $2 \mathbf{A}$ ) but sometimes more elongated, filopodium-like (Figure 2B) or varicose (Figure 2C) and even branched (Figure 2C). Inside their HCs synaptic spinules were closely associated with cellular entrapments of similar appearance and size, but not connected to other neurons and thus entirely embedded in the cytoplasm of the HC (Figure 2A). Similar to what is reported above for spinules, the two membranes in these "disconnected" profiles enclosed a cytosolic content with vesicles (Figure 2A). At synapses in the vertebrate brain, profiles of this type are called "doublemembrane vesicles" (DMVs) and are considered to pinch-off from spinules (see for example Spacek and Harris, 2004; reviewed in Petralia et al., 2015). A quantification of DMVs in randomly selected host neurons (same as for spinule quantification) among the DA2 in one Drosophila brain (see Supplementary Material) revealed that, just like spinules, these structures are most abundant inside OSNs (Figure 1E), thus reinforcing the idea that they are derived from spinules. These vesicles appear to us to be clearly distinct from exosomes and other types of extracellular vesicles used by a variety of cell types and tissues to communicate at a distance through exchange of protein and RNA (Cocucci and Meldolesi, 2015; Budnik et al., 2016) secreted into the extracellular space with consequences for synaptic maintenance, plasticity and homeostasis (Korkut et al., 2009; Budnik et al., 2016; Ashley et al., 2018). A major difference between exosomes and the DMVs reported here is that the latter are delivered directly into the cytoplasm of the $\mathrm{HC}$, enabling modification of the function of individual synapses, without affecting the function of other synapses of the same neuron.

The observation that some of the spinules observed in our reconstructed volume of glomerulus DA2 had a varicose shape might be relevant for a speculative interpretation of their functions. In Drosophila, during its metamorphosis from larva to adult, axonal and dendritic fibers become first varicose and subsequently subdivide into fragments in a process known as pruning, which is controlled by the steroid hormone ecdysone and triggered by $\mathrm{Ca}^{2+}$ (Yaniv and Schuldiner, 2016). We propose that in adult olfactory circuits synaptic-activity induced release of $\mathrm{Ca}^{2+}$ from mitochondria and ER, observed here to be in close proximity and contact to spinules at synaptic sites, could induce not only spinule growth and proliferation as previously proposed (Richards et al., 2005; Tao-Cheng et al., 2009; Ueda and Hayashi, 2013) but also spinule fragmentation inside the host neuron through a process analogous to the pruning of axonal terminals and dendritic branches during metamorphosis, with the difference that in this case the fragments are generated intracellularly and become DMVs in the HC.

It has been suggested that synaptic spinules mediate transsynaptic exchange of material (reviewed in Petralia et al., 2015). Hence, activity-triggered spinule fragmentation mainly in OSNs could be the basis for localized synaptic plasticity, mediated by transference between synaptic partners of microRNA, proteins or other material (Edelstein and Smythies, 2014; Smalheiser, 2014; Busto et al., 2017) and affecting only one synaptic bouton of dozens present among the branches of a given neuron. This localized transference of material between OSNs and other neurons, at individual synaptic boutons that receive spinules and DMVs, could also mediate propagation of epigenetic changes and other modifications. It has been shown that spinules formation is induced by artificial generation of LTP (Toni et al., 1999; Stewart et al., 2005; Ueda and Hayashi, 2013). Concurrent synaptic activity dependent fragmentation of spinules could therefore be involved in synapse tagging and capture (Frey and Morris, 1997; Redondo and Morris, 2011) and would have functional consequences for future synaptic activity, including olfactory learning and memory processes.

Drosophila melanogaster, as a model organism, opens an avenue for future experimental investigations of the ideas outlined here. In a short perspective, experiments should be designed to demonstrate in a more conclusive way that the DMVs reported here are derived from the spinules and that this involves fragmentation of the spinules. Appropriate combinations of genetic labeling of pre- and postsynaptic neurons with different fluorophores and super resolution microscopy can be used for this aim. Screens of genetically tagged marker proteins or RNA, synthetized exclusively by one neuronal type and that ends up inside neurons which do not express the marker, would prove the exchange of material. Furthermore, decrease in activitydependent spinule formation and fragmentation after blockage of mitochondrial $\mathrm{Ca}^{2+}$ release would prove our suggestion of this interplay.

Exchange of material via DMVs might serve synaptic tagging, which is a prerequisite for remodeling and plasticity of individual synapses within a dendritic tree. In the fly visual system it was shown that synaptogenesis correlates with the appearance of mutual invaginations in photoreceptor terminals within a short time window (Rybak and Meinertzhagen, 1997). Using fluorescent markers for pre- and postsynaptic partners in a genetically controlled system (Chen et al., 2014), in combination with the visualization of spinules, correlated cellular activity of spinules and synaptic turnover could be demonstrated. In a longer perspective, using transgenic flies to block spinule fragmentation after synaptic activity, complemented by behavioral assays, will help us understand 


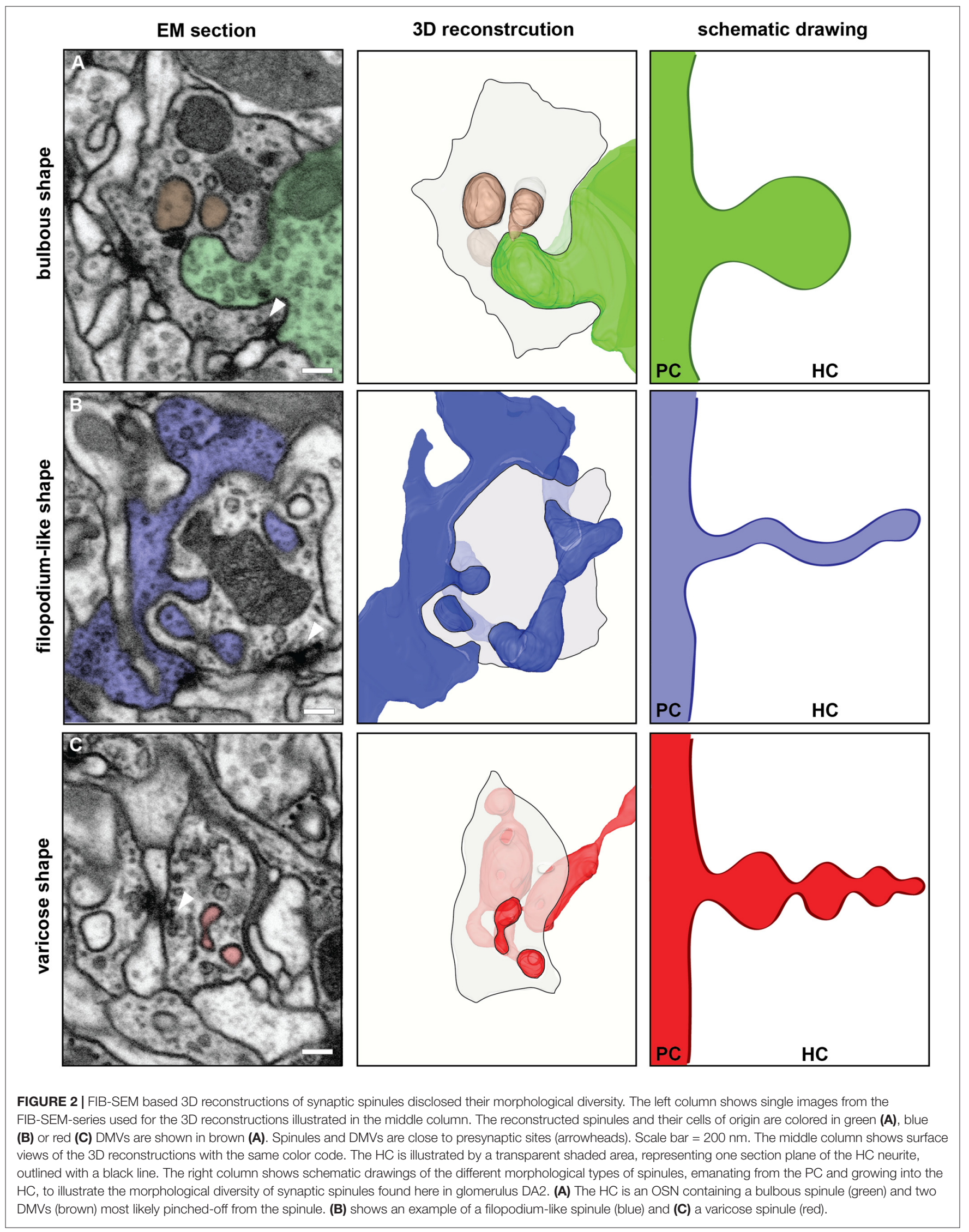


whether the trans-synaptic exchange of material through this novel mechanism has consequences for learning and memory.

\section{AUTHOR CONTRIBUTIONS}

RC, LG and JR conceived and designed the study and the outline for this perspective. Experiments and analyses were planned by JR and LG and performed by LG. LG, JR and RC interpreted and evaluated the data. Figures of this article were prepared by LG. LG, RC, JR and BSH wrote and discussed the manuscript. All authors critically revised the article.

\section{FUNDING}

This project was funded by the Max-Planck-Society, Aura Optik (Jena) and the Agencia Nacional de Investigación e Innovación (Uruguay).

\section{REFERENCES}

Ache, B. W., and Young, J. M. (2005). Olfaction: diverse species, conserved principles. Neuron 48, 417-430. doi: 10.1016/j.neuron.2005. 10.022

Ashley, J., Cordy, B., Lucia, D., Fradkin, L. G., Budnik, V., and Thomson, T. (2018). Retrovirus-like gag protein Arcl binds RNA and traffics across synaptic boutons. Cell 172, 262.e11-274.e11. doi: 10.1016/j.cell.2017. 12.022

Berck, M. E., Khandelwal, A., Claus, L., Hernandez-Nunez, L., Si, G., Tabone, C. J., et al. (2016). The wiring diagram of a glomerular olfactory system. Elife 5:e14859. doi: 10.7554/eLife.14859

Budnik, V., Ruiz-Cañada, C., and Wendler, F. (2016). Extracellular vesicles round off communication in the nervous system. Nat. Rev. Neurosci. 17, 160-172. doi: $10.1038 /$ nrn.2015.29

Busto, G. U., Guven-Ozkan, T., and Davis, R. L. (2017). MicroRNA function in Drosophila memory formation. Curr. Opin. Neurobiol. 43, 15-24. doi: 10.1016/j.conb.2016.10.002

Butcher, N. J., Friedrich, A. B., Lu, Z., Tanimoto, H., and Meinertzhagen, I. A. (2012). Different classes of input and output neurons reveal new features in microglomeruli of the adult Drosophila mushroom body calyx. J. Comp. Neurol. 520, 2185-2201. doi: 10.1002/cne.23037

Chen, Y., Akin, O., Nern, A., Tsui, C. Y. K., Pecot, M. Y., and Zipursky, S. L. (2014). Cell-type-specific labeling of synapses in vivo through synaptic tagging with recombination. Neuron 81, 280-293. doi: 10.1016/j.neuron.2013.12.021

Cocucci, E., and Meldolesi, J. (2015). Ectosomes and exosomes: shedding the confusion between extracellular vesicles. Trends Cell Biol. 25, 364-372. doi: 10.1016/j.tcb.2015.01.004

Davis, R. L. (2004). Olfactory learning. Neuron 44, 31-48. doi: 10.1016/j.neuron. 2004.09.008

Edelstein, L., and Smythies, J. (2014). The role of epigenetic-related codes in neurocomputation: dynamic hardware in the brain. Philos. Trans. R. Soc. Lond. B Biol. Sci. 369:20130519. doi: 10.1098/rstb.2013.0519

Fiala, A. (2007). Olfaction and olfactory learning in Drosophila: recent progress. Curr. Opin. Neurobiol. 17, 720-726. doi: 10.1016/j.conb.2007.11.009

Frey, U., and Morris, R. G. (1997). Synaptic tagging and long-term potentiation. Nature 385, 533-536. doi: 10.1038/385533a0

Guven-Ozkan, T., and Davis, R. L. (2014). Functional neuroanatomy of Drosophila olfactory memory formation. Learn. Mem. 21, 519-526. doi: 10.1101/lm. 034363.114

Helmstaedter, M. (2013). Cellular-resolution connectomics: challenges of dense neural circuit reconstruction. Nat. Methods 10, 501-507. doi: 10.1038/ nmeth. 2476

\section{ACKNOWLEDGMENTS}

We would like to thank Michael Steinert and Thomas Pertsch from the Abbe Center of Photonics, Jena, for their expert assistance with FIB-SEM. We are grateful to Katrin Buder (Leibniz Institute of Aging, Fritz-Lipmann Institute, Jena) for cooperative work in developing the EM protocol. For support and fruitful discussions we are grateful to Roland Kilper (Aura Optik, Jena), Sandor Nietzsche (Electron Microscopic Centrum, Jena), Katrin Buder (Leibniz Institute of Aging, Fritz-Lipmann Institute, Jena) and Veit Grabe (Max Planck Institute for Chemical Ecology).

\section{SUPPLEMENTARY MATERIAL}

The Supplementary Material for this article can be found online at: https://www.frontiersin.org/articles/10.3389/fncel.2018.000 86/full\#supplementary-material

Hige, T. (2017). What can tiny mushrooms in fruit flies tell us about learning and memory? Neurosci. Res. doi: 10.1016/j.neures.2017.05.002 [Epub ahead of print].

Hildebrand, J. G., and Shepherd, G. M. (1997). Mechanisms of olfactory discrimination: converging evidence for common principles across phyla. Annu. Rev. Neurosci. 20, 595-631. doi: 10.1146/annurev.neuro.20.1.595

Keene, A. C., and Waddell, S. (2005). Drosophila memory: dopamine signals punishment? Curr. Biol. 15, R932-R934. doi: 10.1016/j.cub.2005.10.058

Knott, G., Marchman, H., Wall, D., and Lich, B. (2008). Serial section scanning electron microscopy of adult brain tissue using focused ion beam milling. J. Neurosci. 28, 2959-2964. doi: 10.1523/JNEUROSCI.3189-07.2008

Korkut, C., Ataman, B., Ramachandran, P., Ashley, J., Barria, R., Gherbesi, N., et al. (2009). Trans-synaptic transmission of vesicular Wnt signals through Evi/Wntless. Cell 139, 393-404. doi: 10.1016/j.cell.2009.07.051

Leiss, F., Groh, C., Butcher, N. J., Meinertzhagen, I. A., and Tavosanis, G. (2009). Synaptic organization in the adult Drosophila mushroom body calyx. J. Comp. Neurol. 517, 808-824. doi: 10.1002/cne.22184

Lovas, B. (1971). Tubular networks in the terminal endings of the visual receptor cells in the human, the monkey, the cat and the dog. Z. Zellforsch. Mikrosk. Anat. 121, 341-357. doi: 10.1007/bf00337638

Petralia, R. S., Wang, Y. X., Mattson, M. P., and Yao, P. J. (2015). Structure, distribution, and function of neuronal/synaptic spinules and related invaginating projections. Neuromolecular Med. 17, 211-240. doi: 10.1007/s12017-015-8358-6

Redondo, R. L., and Morris, R. G. (2011). Making memories last: the synaptic tagging and capture hypothesis. Nat. Rev. Neurosci. 12, 17-30. doi: $10.1038 /$ nrn2963

Richards, D. A., Mateos, J. M., Hugel, S., de Paola, V., Caroni, P., Gähwiler, B. H., et al. (2005). Glutamate induces the rapid formation of spine head protrusions in hippocampal slice cultures. Proc. Natl. Acad. Sci. U S A 102, 6166-6171. doi: 10.1073/pnas.0501881102

Rybak, J., and Meinertzhagen, I. A. (1997). The effects of light reversals on photoreceptor synaptogenesis in the fly Musca domestica. Eur. J. Neurosci. 9, 319-333. doi: 10.1111/j.1460-9568.1997.tb01402.x

Rybak, J., Talarico, G., Ruiz, S., Arnold, C., Cantera, R., and Hansson, B. S. (2016). Synaptic circuitry of identified neurons in the antennal lobe of Drosophila melanogaster. J. Comp. Neurol. 524, 1920-1956. doi: 10.1002/cne.23966

Schneider-Mizell, C. M., Gerhard, S., Longair, M., Kazimiers, T., Li, F., Zwart, M. F., et al. (2016). Quantitative neuroanatomy for connectomics in Drosophila. Elife 5:e12059. doi: 10.7554/eLife.12059

Smalheiser, N. R. (2014). The RNA-centred view of the synapse: non-coding RNAs and synaptic plasticity. Philos. Trans. R. Soc. Lond. B Biol. Sci. 369:20130504. doi: $10.1098 /$ rstb.2013.0504 
Spacek, J., and Harris, K. M. (2004). Trans-endocytosis via spinules in adult rat hippocampus. J. Neurosci. 24, 4233-4241. doi: 10.1523/JNEUROSCI.0287 $-04.2004$

Stensmyr, M. C., Dweck, H. K., Farhan, A., Ibba, I., Strutz, A., Mukunda, L., et al. (2012). A conserved dedicated olfactory circuit for detecting harmful microbes in Drosophila. Cell 151, 1345-1357. doi: 10.1016/j.cell.2012.09.046

Stewart, M. G., Medvedev, N. I., Popov, V. I., Schoepfer, R., Davies, H. A., Murphy, K., et al. (2005). Chemically induced long-term potentiation increases the number of perforated and complex postsynaptic densities but does not alter dendritic spine volume in CA1 of adult mouse hippocampal slices. Eur. J. Neurosci. 21, 3368-3378. doi: 10.1111/j.1460-9568.2005.04174.x

Takemura, S. Y., Aso, Y., Hige, T., Wong, A., Lu, Z., Xu, C. S., et al. (2017). A connectome of a learning and memory center in the adult Drosophila brain. Elife 6:e26975. doi: 10.7554/eLife.26975

Tao-Cheng, J. H., Dosemeci, A., Gallant, P. E., Miller, S., Galbraith, J. A., Winters, C. A., et al. (2009). Rapid turnover of spinules at synaptic terminals. Neuroscience 160, 42-50. doi: 10.1016/j.neuroscience.2009.02.031

Tobin, W. F., Wilson, R. I., and Lee, W. A. (2017). Wiring variations that enable and constrain neural computation in a sensory microcircuit. Elife 6:e24838. doi: 10.7554/eLife.24838

Toni, N., Buchs, P. A., Nikonenko, I., Bron, C. R., and Muller, D. (1999). LTP promotes formation of multiple spine synapses between a single axon terminal and a dendrite. Nature 402, 421-425. doi: 10.1038/46574

Ueda, Y., and Hayashi, Y. (2013). PIP $_{3}$ regulates spinule formation in dendritic spines during structural long-term potentiation. J. Neurosci. 33, 11040-11047. doi: 10.1523/JNEUROSCI.3122-12.2013
Wilson, R. I. (2013). Early olfactory processing in Drosophila: mechanisms and principles. Annu. Rev. Neurosci. 36, 217-241. doi: 10.1146/annurev-neuro062111-150533

Wu, Y., Whiteus, C., Xu, C. S., Hayworth, K. J., Weinberg, R. J., Hess, H. F., et al. (2017). Contacts between the endoplasmic reticulum and other membranes in neurons. Proc. Natl. Acad. Sci. U S A 114, E4859-E4867. doi: 10.1073/pnas. 1701078114

Yaniv, S. P., and Schuldiner, O. (2016). A fly's view of neuronal remodeling. Wiley Interdiscip. Rev. Dev. Biol. 5, 618-635. doi: 10.1002/w dev. 241

Zheng, Z., Lauritzen, J. S., Perlman, E., Robinson, C. G., Nichols, M., Milkie, D., et al. (2017). A complete electron microscopy volume of the brain of adult Drosophila Melanogaster. bioRxiv 140905. doi: 10.1101/1 40905

Conflict of Interest Statement: The authors declare that the research was conducted in the absence of any commercial or financial relationships that could be construed as a potential conflict of interest.

Copyright (c) 2018 Gruber, Rybak, Hansson and Cantera. This is an open-access article distributed under the terms of the Creative Commons Attribution License (CC BY). The use, distribution or reproduction in other forums is permitted, provided the original author(s) and the copyright owner are credited and that the original publication in this journal is cited, in accordance with accepted academic practice. No use, distribution or reproduction is permitted which does not comply with these terms. 


\section{OPEN ACCESS}

Edited by:

Frédéric Marion-Poll,

AgroParisTech Institut des Sciences et Industries du Vivant et de L'environnement, France

Reviewed by:

Ingrid Martha Skerrett,

Buffalo State College, United States Ashwin Miriyala Newcastle University, United Kingdom Antoine Couto,

University of Cambridge, United Kingdom

${ }^{*}$ Correspondence: Mamiko Ozaki mamiko@port.kobe-u.ac.jp

Received: 01 June 2018 Accepted: 27 August 2018 Published: 19 September 2018

Citation Takeichi Y, Uebi T, Miyazaki N, Murata $K$, Yasuyama $K$, Inoue $K$, Suzaki T, Kubo H, Kajimura N, Takano J, Omori T, Yoshimura $R$

Endo Y, Hojo MK, Takaya E, Kurihara S, Tatsuta K, Ozaki K and Ozaki M (2018) Putative Neural Network Within an Olfactory Sensory Unit for Nestmate and Non-nestmate

Discrimination in the Japanese

Carpenter Ant: The Ultra-structures and Mathematical Simulation. Front. Cell. Neurosci. 12:310. doi: 10.3389/fncel.2018.00310
Putative Neural Network Within an Olfactory Sensory Unit for Nestmate and Non-nestmate Discrimination in the Japanese Carpenter Ant: The Ultra-structures and Mathematical Simulation

Yusuke Takeichi ${ }^{1}$, Tatsuya Uebi ${ }^{1}$, Naoyuki Miyazaki ${ }^{2}$, Kazuyoshi Murata ${ }^{2}$, Kouji Yasuyama ${ }^{3}$, Kanako Inoue ${ }^{4}$, Toshinobu Suzaki ${ }^{1}$, Hideo Kubo ${ }^{5}$, Naoko Kajimura ${ }^{6}$, Jo Takano ${ }^{7}$, Toshiaki Omori ${ }^{7}$, Ryoichi Yoshimura ${ }^{8}$, Yasuhisa Endo ${ }^{8}$, Masaru K. Hojo ${ }^{1}$, Eichi Takaya ${ }^{9}$, Satoshi Kurihara ${ }^{9}$, Kenta Tatsuta ${ }^{10}$, Koichi Ozaki $^{10}$ and Mamiko Ozaki ${ }^{1 *}$

${ }^{1}$ Department of Biology, Graduate School of Science, Kobe University, Kobe, Japan, ${ }^{2}$ National Institute for Physiological Sciences, Okazaki, Japan, ${ }^{3}$ Division of Biology, Department of Natural Sciences, Kawasaki Medical School, Kurashiki, Japan, ${ }^{4}$ Research Center for Ultra-High Voltage Electron Microscopy, Osaka University, Ibaraki, Japan, ${ }^{5}$ Department of Mathematics, Faculty of Sciences, Hokkaido University, Sapporo, Japan, ${ }^{6}$ Graduate School of Frontier Biosciences, Osaka University, Suita, Japan, ${ }^{7}$ Department of Electrical and Electronic Engineering, Graduate School of Engineering, Kobe University, Kobe, Japan, ${ }^{8}$ Department of Applied Biology, Kyoto Institute of Technology, Kyoto, Japan, ${ }^{9}$ Graduate School of Information Systems, The University of Electro-Communications, Chofu, Japan, ${ }^{10}$ Department of Biological Science, Faculty of Life and Environmental Science, Shimane University, Matsue, Japan

Ants are known to use a colony-specific blend of cuticular hydrocarbons (CHCs) as a pheromone to discriminate between nestmates and non-nestmates and the $\mathrm{CHCs}$ were sensed in the basiconic type of antennal sensilla (S. basiconica). To investigate the functional design of this type of antennal sensilla, we observed the ultra-structures at 2D and $3 \mathrm{D}$ in the Japanese carpenter ant, Camponotus japonicus, using a serial block-face scanning electron microscope (SBF-SEM), and conventional and high-voltage transmission electron microscopes. Based on the serial images of 352 cross sections of SBF-SEM, we reconstructed a 3D model of the sensillum revealing that each $S$. basiconica houses > 100 unbranched dendritic processes, which extend from the same number of olfactory receptor neurons (ORNs). The dendritic processes had characteristic beaded-structures and formed a twisted bundle within the sensillum. At the "beads," the cell membranes of the processes were closely adjacent in the interdigitated profiles, suggesting functional interactions via gap junctions (GJs). Immunohistochemistry with anti-innexin (invertebrate GJ protein) antisera revealed positive labeling in the antennae of $C$. japonicus. Innexin 3, one of the five antennal innexin subtypes, was detected as a dotted signal within the $S$. basiconica as a sensory organ for nestmate recognition. These morphological results suggest that ORNs form an electrical network via GJs between dendritic processes. We were unable to functionally certify the electric connections in an olfactory sensory unit comprising such multiple ORNs; however, with the aid 
of simulation of a mathematical model, we examined the putative function of this novel chemosensory information network, which possibly contributes to the distinct discrimination of colony-specific blends of $\mathrm{CHCs}$ or other odor detection.

Keywords: olfactory receptor, chemosensillum, chemical communication, innexin, ant, ultra-structures, mathematical simulation

\section{INTRODUCTION}

The natural environment surrounding living organisms is filled with chemical information, and animals have developed adaptive chemosensory systems to utilize this environmental information for purposes such as food source or mate recognition or individual identification especially in social animals. Insects have characteristic olfactory organs called sensilla, which are involved in general odor or pheromone sensing (Hallberg and Hansson, 1999; Steinbrecht, 1999; Hansson and Stensmyr, 2011; de Fouchier et al., 2017). In some cases, each type of sensilla housing multiple receptor neurons works as a sensory unit for specific biological purpose. For example, many insect species use sex pheromones for attracting mates, and their sex pheromonesensitive sensilla, which house a few olfactory receptor neurons (ORNs), have been enthusiastically studied as simple odor sensory units (Kaissling, 1987; Haupt et al., 2010).

Social insects have evolved sophisticated chemical communication ability by means of various pheromones (Hölldobler, 1995; Vander Meer, 1998; Ozaki et al., 2005; Mizunami et al., 2010; Nick and d'Ettorre, 2012; Ozaki and Hefetz, 2014; Sharma et al., 2015; Leonhardt et al., 2016). In an ant a colony, worker ants use antennation, a typical behavior of contact investigation with antennae, to accept nestmates but reject conspecific non-nestmates and hetero-specific worker ants. In many ant species, worker ants utilize a colony-specific blend of cuticular hydrocarbons (CHCs) as a social pheromone for nestmate recognition (Vander Meer, 1998; Lahav et al., 1999; Ozaki et al., 2005; Brandstaetter et al., 2008; Guerrieri and d'Ettorre, 2008; Guerrieri et al., 2009; Nick and d'Ettorre, 2012; Ozaki and Hefetz, 2014). Worker ants have species-specific composition of different CHC combinations with different components among species, but they have common colonyspecific $\mathrm{CHC}$ blends with the same components within a species. In C. japonicus, worker ants from different colonies have the colony-specific $\mathrm{CHC}$ blends comprising 18 species-specific CHCs (Ozaki et al., 2005). Ozaki et al. (2005) were the first to study the chemosensory system for nestmate vs. non-nestmate discrimination in C. japonicus and proved that the Sensilla basiconica (S. basiconica) function as $\mathrm{CHC}$ sensilla. Within each S. basiconica of C. japonicus, which were later discovered to be female-specific (Nakanishi et al., 2009), more than 100 ORNs extend the dendritic processes (Ozaki et al., 2005). The receptor membranes of ORNs are surrounded by chemosensory protein (CSP)-containing sensillar lymph, allowing lipophilic CHCs to be transported by CSP to the receptor membranes of ORNs (Ozaki et al., 2005; Hojo et al., 2015). In the early electrophysiological recordings in C. japonicus, S. basiconica were stimulated by contact with CHCs that were scattered in a CSP-containing aqueous solution (Ozaki et al., 2005). It was reported that the number of $S$. basiconica responding to nestmate $\mathrm{CHCs}$ was significantly smaller than that responding to non-nestmate $\mathrm{CHCs}$, and similar results were found in other ant species (Kidokoro-Kobayashi et al., 2012). In a later study, when stimulated by the vapor of heated CHCs, S. basiconica of Camponotus floridanus, for example, responded to not only non-nestmate $\mathrm{CHCs}$ but also nestmate $\mathrm{CHCs}$ (Sharma et al., 2015), and it was suggested that there are morphologically similar but functionally different subtypes of S. basiconica on the antennae of $C$. floridanus. Therefore, it is considered that the $S$. basiconica of ant probably classified into subtypes would be multifunctional olfactory organ not only for nestmate and non-nestmate discrimination but also for other hydrocarbon or general odor sensing as in other insects (Kropf et al., 2014; Couto et al., 2017).

In several species, $S$. basiconica ORNs project into a distinct antennal lobe region consisting of a cluster of the same number of glomeruli (Kelber et al., 2010; Nishikawa et al., 2012; McKenzie et al., 2016; Couto et al., 2017). While, the functional properties and sensory mechanism of $S$. basiconica involved in nestmate recognition in ants have been studied, they are not yet fully understood because of its complexity with many ORNs, expressing specific olfactory receptor (OR) genes, respectively. Because of this complexity we suspect that there are some information filtration or modification systems within S. basicomica. Presumably, those ORNs do not behave like independent parallel cables but may functionally connect and influence each other.

In the present paper, we showed the beaded-structures along the dendritic processes and documented in detail about their number and localization. The beaded-structure looked to provide a platform for functional connection among ORNs via close apposition of membranes. However, it was difficult to conduct proper experiments to prove functional modification in the response of a S. basiconica, which was expected by hypothesizing functional connection among ORNs. Thus, we examined a simplified mathematical simulation for the inter-dendritic neural network based on the cable theory and proposed possible modification of its responsiveness to virtual stimulation.

\section{MATERIALS AND METHODS}

\section{Ants}

Worker ants of the Japanese carpenter ant, C. japonicus, were collected from around the nests of several colonies on the Kobe University campus. Nestmates from each colony were transferred 
into a plastic box $\left(23 \times 16 \times 8 \mathrm{~cm}^{3}\right)$ with a small artificial nest box $\left(5 \times 7.5 \times 1.8 \mathrm{~cm}^{3}\right)$ covered with a red plastic sheet and maintained at room temperature in our laboratory for several days or weeks until use. Ants were fed a synthetic diet (Dussutour and Simpson, 2008) and had freely access to water. We also obtained a whole nest with a queen, hundreds of workers, and some virgin queens and males; the nest was transferred to Kawasaki Medical School, where we prepared sample blocks for electron microscopy. Thus, the collected ants were reared in an artificial field comprising a foraging yard $\left(23 \times 16 \mathrm{~cm}^{2}\right)$ and an artificial nest with several plastic chambers $\left(5 \times 7.5 \times 1.8 \mathrm{~cm}^{3}\right)$ connected by plastic tubes ( $8 \mathrm{~mm}$ inner diameter) in Kawasaki Medical School. Ants were fed a diet of diluted maple syrup and mealworms with water.

\section{Electron Microscopy}

The protocol for serial block-face scanning electron microscopy (SBF-SEM) was adopted from Deerinck et al. (SBEM Protocol v7_01_2010; https://ncmir.ucsd.edu/sbem-protocol). C. japonicus workers were anesthetized on ice for $10 \mathrm{~min}$, and the antennae were detached from the head and cut into pieces. The specimens were fixed in $2.5 \%$ glutaraldehyde and $2 \%$ paraformaldehyde in $2 \mathrm{mM} \mathrm{CaCl}_{2}$-containing $0.1 \mathrm{M}$ cacodylate buffer ( $\mathrm{pH} 7.4$ ) for $18 \mathrm{~h}$ at $4^{\circ} \mathrm{C}$. After washing in the same buffer containing $\mathrm{CaCl}_{2}$, specimens were osmicated in $2 \%$ osmium tetroxide in $0.1 \mathrm{M}$ cacodylate buffer $(\mathrm{pH}$ 7.4) containing $1.5 \%$ potassium ferrocyanide for $2 \mathrm{~h}$ at $4^{\circ} \mathrm{C}$. The specimens were then washed with distilled water and placed in Millipore filtered thiocarbohydrazide (TCH) solution for $20 \mathrm{~min}$. After that, the specimens were fixed in $2 \%$ osmium tetroxide in distilled water for $1 \mathrm{~h}$ at room temperature. They were re-washed with distilled water and then incubated in $1 \%$ uranyl acetate (aqueous) for $18 \mathrm{~h}$ at $4^{\circ} \mathrm{C}$. After washing with distilled water, the specimens were treated with Walton's lead aspartate staining for $60 \mathrm{~min}$ at $60^{\circ} \mathrm{C}$. Next, 15 -min-wash in distilled water was three times repeated, and dehydration was performed with a series of 50 , $70,90,100 \%$, and again $100 \%$ ethanol for $15 \mathrm{~min}$, respectively. Specimen were then soaked in propylene oxide for $20 \mathrm{~min}$ at room temperature. Specimens were then transferred into a 1:1 mixture of epoxy resin (49.6\% LUVEAK812; 21.8\% DDSA; 26.7\% MNA; $2.0 \%$ DMP30, nacalai Tesque, Kyoto, Japan) and propylene oxide and maintained on a slow speed rotator in a draft chamber at room temperature overnight. The next day, the antenna pieces were gently rotated in $100 \%$ epoxy resin for $3 \mathrm{~h}$ at room temperature and embedded in $100 \%$ epoxy resin and incubated at $60^{\circ} \mathrm{C}$ for $48 \mathrm{~h}$. The sample block in the epoxy resin was carefully trimmed to obtain a single S. basiconica and was used for three types of electron microscopy: SBF-SEM, conventional transmission electron microscopy (TEM), and ultra-high-voltage electron microscopy (UHV-EM). It was not difficult to discriminately find S. basiconica on the C. japonicus antenna under a stereomicroscope (SZX9 Olympus) by its characteristic outer cuticular structure. Since it is known that there are no other types of antennal sensilla but only S. basiconica houses more than 100 ORNs (see Nakanishi et al., 2009), every S. basiconica chosen as the electron microscopic specimen had been confirmed by counting the number of ORNs within the sensillum. However, in C. japonicus, it is not clear whether there are sub-types of $S$. basiconica on the antenna and either morphological or functional discrimination among sub-types. Hence we did not discriminately choose our specimen among putative subtypes of S. basiconica.

Using SBF-SEM Gatan 3view (Gatan, Inc., CA, USA)-Zeiss IIGMA/VP \& MARLIN (Carl Zeiss Microscopy GmbH, Jena, Germany), serial block-face images of an S. basiconica were obtained at $1.2 \mathrm{kV}$ accelerating voltage. We aligned the serial images and loaded the digital data onto the image processing Amira software program (Indeed Visual Concepts GmbH, Berlin, Germany; TGS Inc.). The dendritic process areas selected on each cross image were manually segmented. Using the surface rendering method, the data were reconstructed into a $3 \mathrm{D}$ structural model of a unit of the dendritic process within a sensillum.

We also obtained ultra-thin sections of $S$. basiconica for conventional TEM observation using JEM-1400 (JEOL Ltd., Tokyo, Japan) at an accelerating voltage of $80 \mathrm{kV}$ and $2 \mu \mathrm{m}$ thick sections for UHVEM observation using H-3000 (Hitachi Co., Tokyo, Japan) at an accelerating voltage of 2,000 kV. For observation by UHVEM, the $2 \mu \mathrm{m}$-thick sections were mounted on Formvar-coated slot grids, immersed in 3\% uranyl acetate in $70 \%$ methanol, heated in a microwave oven for $30 \mathrm{~s}$, incubated for $10 \mathrm{~min}$ at room temperature, and rinsed with distilled water. The sections were then immersed in SATO lead stain solution, heated in a microwave oven for $30 \mathrm{~s}$, incubated for $10 \mathrm{~min}$ at room temperature, and rinsed with distilled water. The sections were covered using an additional Formvar membrane and coated on both sides with evaporated carbon, followed by studding with $20 \mathrm{~nm}$ gold particles. Images were taken at 20,000 $\times$ from $-60^{\circ}$ to $+54^{\circ} \mathrm{C}$ at $2^{\circ} \mathrm{C}$ intervals around a single axis and captured a resolution of $4096 \times 4096$ pixels at a pixel size of $0.85 \mathrm{~nm}$ using 486BK CCD camera (Hitachi Co., Tokyo, Japan). Each set of tilted images were aligned using gold particles as fiducial markers and reconstructed using Simultaneous Iterative Reconstruction Technique (SIRT) (Gilbert, 1972). All tomograms were analyzed using IMOD software (Kremer et al., 1996). 3D models were drawn by tracing membranous structures using "3DMOD," which is the graphics component of IMOD.

\section{Blast and HMM Search for Innexin Family}

We used BLASTp to search the protein CDS references of C. japonicus (Hojo et al., 2015) for innexin candidates using protein sequences of Drosophila melanogaster innexin (inx1 to inx8) with an e-value cutoff of $1.0 \mathrm{E}-15$. We also performed a HMM search using the innexin superfamily (pf00876) as a query.

\section{Western Blot of Antennal Proteins for CjapInx3}

Antennae were detached from $50 \mathrm{C}$. japonicus worker ants that were anesthetized on ice and were immediately frozen in liquid nitrogen in a hand mortar. The samples were then homogenized with $200 \mu \mathrm{l}$ of SDS-polyacrylamide gel electrophoresis (PAGE) sample buffer and used for western blot analysis with the anti-CjapInx3 antiserum raised against a specific epitope (LGIDEGERRYHS) of innexin 3 of C. japonicus (see Figure 4). 

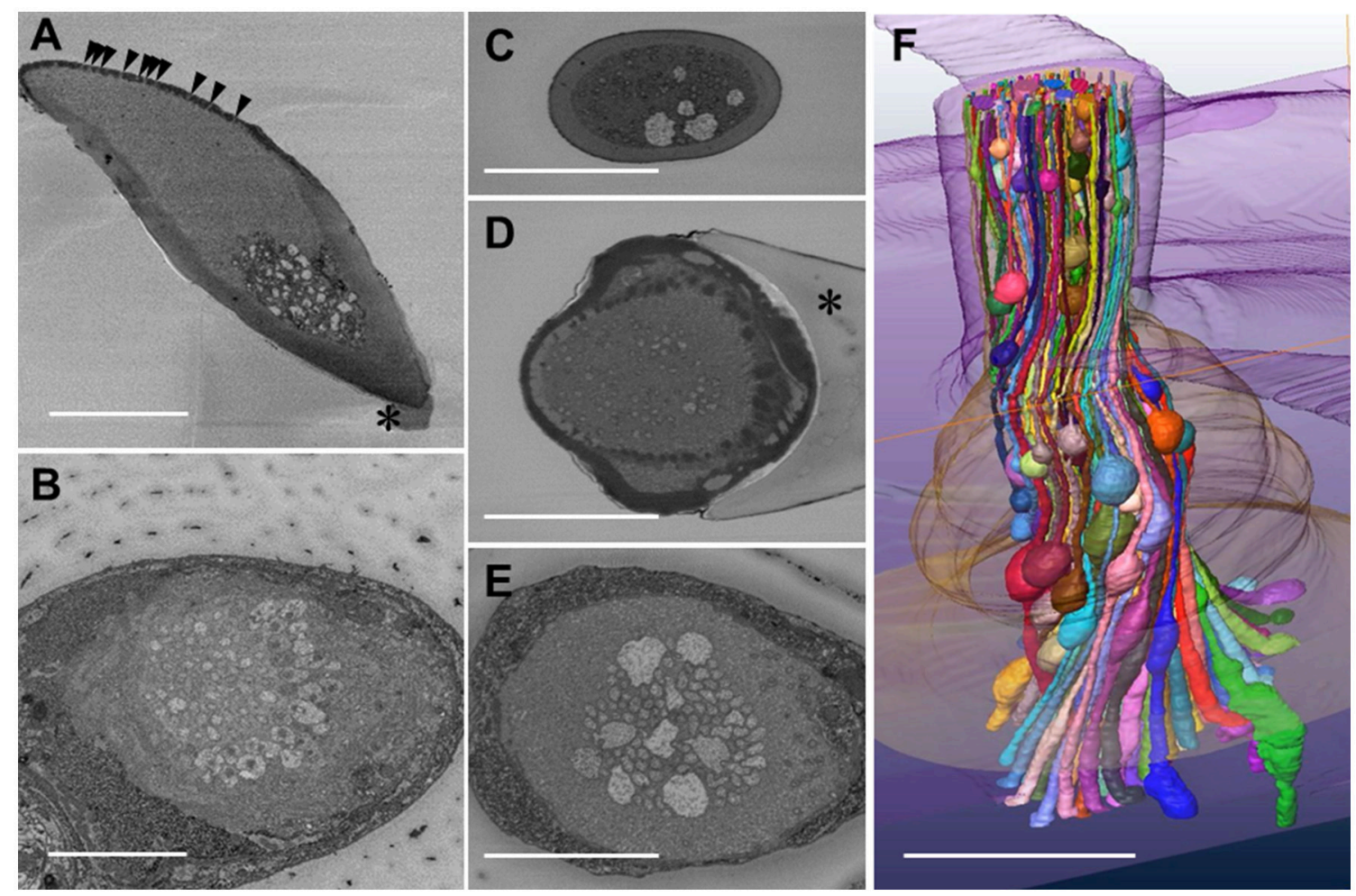

FIGURE 1 | Serial block face scanning electron microscope images and reconstruction of a three-dimensional structure of olfactory receptor neurons' dendritic processes in a Sensilla basiconica of Camponotus japonicus worker ant. (A) A longitudinal oblique section of the S. basiconica. Arrowheads and asterisk indicate openings of olfactory pores and a part of the socket cuticle at the basement of the sensillar shaft, respectively. (B) A cross image of the S. basocinica with 102 dendritic processes at the junction with the inner segments of ORNs. (C-E) The cross images of $S$. basiconica at 6 and $1 \mu \mathrm{m}$ distal and $4 \mu \mathrm{m}$ proximal from the outer surface of the antennal cuticle (see arrowheads of Figure 6A). (F) 3D-structural model of a bundle of color-coded dendritic processes within a sensillum reconstructed by 352 cross images at $70 \mathrm{~nm}$ interval $(24.6 \mu \mathrm{M}$ in total length). Bars indicate $5 \mu \mathrm{m}$.

Five ants equivalent extract in $20 \mu \mathrm{l}$ of sample buffer was loaded per lane for SDS-PAGE. After electrophoresis, proteins were transferred from the gel to polyvinylidene difluoride (PVDF) membranes (immobilon-P; Merck KGaA, Darmstadt, Germany). PVDF membranes were blocked with Blocking One (NACALAI TESQUE, INC., Kyoto, Japan) at room temperature for $2 \mathrm{~h}$, incubated with anti-CjapInx3 antiserum (1:100 dilution with Can Get Signal; TOYOBO CO., LTD., Osaka, Japan) at room temperature for $1 \mathrm{~h}$, and processed using the Vectastain $\mathrm{ABC}$ kit (Vector Laboratories, Burlingame, CA, USA) according to manufacturer's instructions. The CjapInx3 signal was detected using Chemi-Lumi One Super (NACALAI TESQUE, INC., Kyoto, Japan).

\section{Immunohistostaining of Antennal Sections With Anti-CjapInx3 Antibody}

Antennae detached from cold anesthetized C. japonicus worker ants were cut into small pieces and immediately fixed in a solution of $1 \%$ paraformaldehyde, $0.25 \% \mathrm{ZnCl}_{2}, 127 \mathrm{mM}$ $\mathrm{NaCl}$, and $3.5 \mathrm{mM}$ sucrose at $4^{\circ} \mathrm{C}$ overnight. After fixation, the antennae were twice incubated in ant ringer solution $(4.8 \mathrm{mM}$ TES, $127 \mathrm{mM} \mathrm{NaCl}, 6.7 \mathrm{mM} \mathrm{KCl}, 2 \mathrm{mM} \mathrm{CaCl}_{2}$, and $3.5 \mathrm{mM}$ Sucrose) with $30 \%$ sucrose at $4^{\circ} \mathrm{C}$ twice for $1 \mathrm{~h}$ and incubated overnight at $4^{\circ} \mathrm{C}$. Subsequently the antennae were embedded in O.C.T. compound (Sakura Finetek Japan Co., Ltd., Tokyo, Japan) and frozen in a cryostat (CM1850; Leica Biosystems Nussloch $\mathrm{GmbH}$, Wetzlar, Germany) to prepare $8 \mu \mathrm{m}$-thick vertical sections. The obtained sections were mounted on glass slides. The slides were washed in acetone at $-20^{\circ} \mathrm{C}$ for $30 \mathrm{~min}$, dried for $1 \mathrm{~h}$, washed 3 times in ART (ant ringer solution with $0.05 \%$ Triton-X100, $5 \mathrm{~min}$ per time), and then activated by incubation in HistoVT One (NACALAI TESQUE, INC., Kyoto, Japan) at $70^{\circ} \mathrm{C}$ for $20 \mathrm{~min}$. After being washed 3 times in ART, the slides were treated with Blocking One (NACALAI TESQUE, INC., Kyoto, Japan) at room temperature for $2 \mathrm{~h}$ and incubated either with rabbit antisera against CjapInx3 (for test) or with pre-immune rabbit sera (for control) (1:300 diluted with Can Get Signal solution B; TOYOBO CO., LTD., Osaka, Japan) overnight at $4^{\circ} \mathrm{C}$. The next day, the sections were washed 4 times in ART ( 5 min each time) and incubated with Alexa 594-conjugated goat anti-rabbit IgG (A11012; Thermo Fisher Scientific, Wilmington, DE, USA, 1:800 diluted with Can Get Signal solution B) overnight at $4^{\circ} \mathrm{C}$. Subsequently the slides were washed four times in ART and mounted with Fluoromount (K024; DIAGNOSTIC BIOSYSTEMS, Pleasanton, $\mathrm{CA}$, USA). The slides were stored in the dark at $4^{\circ} \mathrm{C}$ 


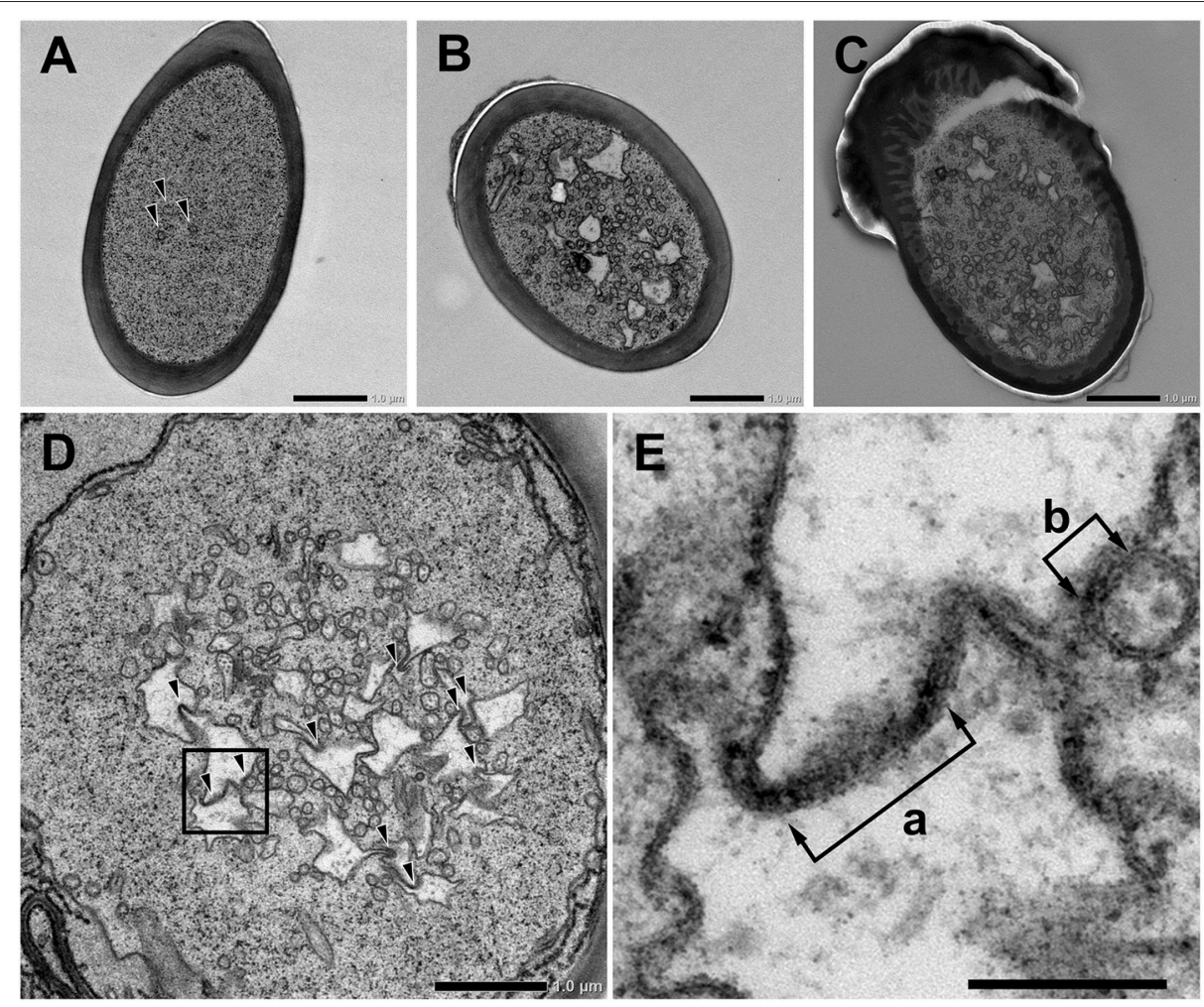

FIGURE 2 | Transmission electron microscope images of Sensilla basiconica and adhesion between olfactory receptor membranes. (A-C) Cross images of ultra-thin sections of an S. basiconica of $C$. japonicus worker ant. Three sections are at the middle of the sensillar shaft, where few dendritic processes are seen (A), more proximal (B) and the level of basal socket, where many dendritic processes are seen (C). (D) A cross section beneath the antennal cuticular surface. (E) High magnification image of a square of (D), showing adhesion between adjacent membranes (double-headed arrows). Bars indicate $1 \mu \mathrm{m}$ in (A-D) and $200 \mathrm{~nm}$ in (E).

until microscopic observation. We acquired the fluorescence images of $S$. basiconica, using a super-resolution microscope system (N-SIM; Nikon Corporation, Tokyo, Japan) coupled to an ECLIPSE Ti2-E inverted microscope (Nikon Corporation, Tokyo, Japan) with a $60 \times$ water immersion objective lens (SR Plan Apo IR $60 \times$, NA1.27; Nikon Corporation, Tokyo, Japan), LU-N3-SIM laser unit (Nikon Corporation, Tokyo, Japan), and ORCA-Flash 4.0 sCMOS camera (Hamamatsu Photonics K.K., Hamamatsu, Japan). The excitation wavelength was $561 \mathrm{~nm}$, and the emission was filtered using a $605 \mathrm{~nm}$ filter. Fluorescence images of the longitudinal ( $0.64 \mu \mathrm{m}$ thickness) and cross sensillar sections ( $6 \mu \mathrm{m}$ thickness) were reconstructed with NIS elements AR software (Nikon Corporation, Tokyo, Japan). Differential interference images of the longitudinal sensillar sections were observed using a FV1000 confocal microscope (Olympus Corporation, Tokyo, Japan) coupled to a BX61W1 microscope (Olympus Corporation, Tokyo, Japan) with a $60 \times$ water immersion objective lens (UPLSAPO60XW, NA 1.20; Olympus Corporation, Tokyo, Japan).

In order to reduce non-specific signals, we tried to examine this immunohistostaining experiment under various conditions in blocking, washing and anti-serum treatment, but it was difficult to completely avoid the non-specific signals. If we sonicated the specimen in the fixative, non-specific staining tended to be reduced. However, thus the sonication treatment increased in risk of breaking sensilla, hence we stopped the sonication treatment. Observation with different excitation wavelengths was not helpful to clarify the outline shape of sensillaum, but we could recognize the outline of the cuticular shaft of the sensillum in the $3 \mathrm{D}$ reconstructed fluorescent images by rotating it using NIS elements AR software (Nikon Corporation, Tokyo, Japan), which is useful to get all directional optical views. Moreover, using this software, fluorescent signals within a sensillum were discriminately seen from those on the outer surface of the sensillum.

\section{Mathematical Simulation}

All simulations were performed using the Julia environment (version 0.6 .1 ). A mathematical model of 10 or 20 cables with mutual connections via GJs, which is based on cable theory (Rall, 1959; Segev et al., 1995; Koch, 1999), was used in the simulations (detailed mathematical expressions are given in the Appendix). Each cable consists of nine serially-connected passive compartments in the distal part of dendritic process and one active compartment as an approximal part with a spiking (impulse generating) mechanism (Morris and Lecar, 1981). GJs are assumed to connect passive compartments among different cables. Hypothesizing that the current influx is generated at 


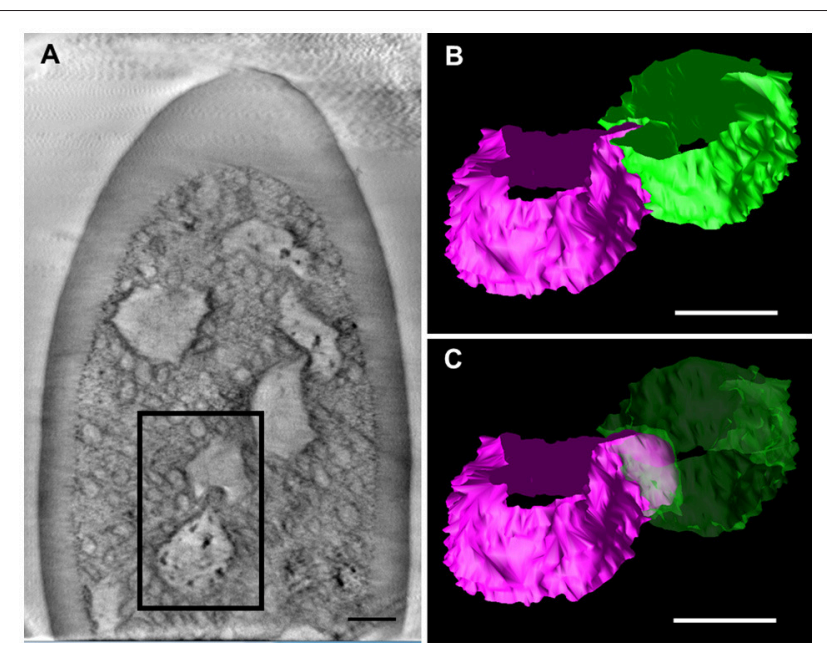

FIGURE 3 | Tomographic analysis of beaded-structures in a Sensilla basiconica. (A) A tomographic slice image of $3.4 \mathrm{~nm}$-thick in an S. Basiconica of $C$. japonicus worker. (B) 3D tomographic models of two adjacent beads, surface of which are colored in magenta and green, in a square of (A). (C) Same as (B), but one bead surface is shown as translucent green. Bars indicate $50 \mathrm{~nm}$.

the most distal parts of the dendritic processes of the ORNs, an external input current is given to one end of the passive compartments (the very top compartments corresponding to the distal part of the dendritic processes) in each cable, and an active compartment with an impulse generating mechanism is connected to the other end of the passive compartments.

\section{RESULTS}

\section{Serial Block-Face Scanning Electron Microscope Observations: Fine Interior Structure of Sensilla basiconica}

We got the SBF-SEM images from nine specimens of S. basiconica, and the dendritic processes with beaded-structures were seen in all of them, although the beaded-structures could not precisely be compared in their number or localization among those specimens. Thus, we show the representative data in Figure 1. Figure 1A presents a longitudinal oblique section of $S$. basiconica, which includes the sensillar top with olfactory pores (arrowheads) and a small piece of the socket cuticle at the basement of the sensillar shaft (asterisk). The thickness of the cuticular wall is not uniform around the perimeter. Around the sensillar top, one side, where the multiple openings of the olfactory pores are seen, is thinner than the other side. Beneath those openings, there is a cell-free space that no dendritic processes can reach; by contrast, many dendritic processes are seen from the middle to the basement of the sensillum (Figures 1B-E).

Using the best prepared specimen, we obtained fine serial cross images of S. basiconica under SBF-SEM, starting at $12 \mu \mathrm{m}$ from the top of the sensillum where we could sufficiently adjust the focus on the membranes of the dendritic processes. In total, 596 cross images were serially obtained from the distal to proximal region at $70 \mathrm{~nm}$ intervals until all the dendritic processes were outside the visual field. Figure 1B shows an example cross image with 102 dendritic processes, some of which show connective cilia at the junctions with the inner segments of ORNs' dendritic processes. Figures 1C,D are cross sections of a sensillum at 6 and $1 \mu \mathrm{m}$ distal and Figure 1E at $4 \mu \mathrm{m}$ proximal from the outer surface of the antennal cuticle, respectively, (see arrowheads in Figure 6A where the outer surface of the antennal cuticle is at zero level). Despite variations among cross images, all sections along this S. basiconica constantly revealed 102 dendritic processes. Almost all cross sections revealed thin dendritic processes; however, Figures 1C,E show 5 and 9 dendritic processes with large cross-sectional areas, respectively. Using 352 images of all 596 serial sections obtained in the representative sensillum, we reconstructed the 3D structure model over $24.6 \mu \mathrm{m}$ along the sensillar shaft (Figure 1F). As expected, the 102 dendritic processes, which form a moderately twisted bundle and are housed in a sensillum, had no branches but had characteristic beaded-structures, which could result in the large cross-sectional areas shown in Figures 1C-E.

\section{Transmission Electron Microscope Observations: Morphological Evidence of Adhesion Between Olfactory Receptor Membranes}

We further observed the cross sections of S. basiconica using TEM. As shown in Figure 1A, there is a cell-free space at the top of the sensillum. Figure $\mathbf{2 A}$ is a cross section of the middle of the sensillum, where only a few small cross sections of the dendritic processes are observed (arrowheads), Figure 2B is more proximal, and Figure $\mathbf{2} \mathrm{C}$ is at the basal socket. The cross images of TEM in Figure 2 are consistent with those of SBF-SEM in Figure 1. At the beads of the dendritic processes, where large cross-sectional areas were observed, we frequently found the closely adjacent cell membranes of dendritic processes (arrowheads in Figure 2D). Figure 2E is a high magnification image of the square area in Figure 2D. At a bead a dendritic process closely adheres to the adjacent membranes of the beady (double-headed arrow a) and non-beady parts of the neighboring dendritic processes (double-headed arrow b) in Figure 2E. In addition, we show UHV-EM image focusing on an adhesion region between beads of adjacent dendritic processes (square area in Figure 3A), in which the membrane of one bead (magenta in Figures 3B,C) is invaginated into the other (green in Figures 3B,C).

\section{Super-Resolution Fluorescent Microscopy Observations: Localization of Cjaplnx3}

Based on the results of RNA sequencing analysis (Hojo et al., 2015), we found that $C$. japonicus expresses five innexin subtypes, namely CjapInx1, 2, 3, 7, and 8, as putative GJ proteins in the antennae (Figure 4). We prepared anti-CjapInx3 antiserum against an amino acid sequence in an extracellular loop of CjapInx3, which was chosen as the specific epitope (white characters in Figure 4). 
CjapInx1

BmorInx1

CjapInx2

CjapInx3

BmorInx 3

BmorInx4

CjapInx7

BmorInx7-like

BmorInx7-like2

CjapInx8

BmorInx8

CjapInx1

BmorInx1

CjapInx2

BmorInx2

CjapInx 3

BmorInx 3

BmorInx4

CjapInx 7

BmorInx7-like1

BmorInx7-like2

CjapInx8

BmorInx 8

CjapInx1

BmorInx1

CjapInx2

BmorInx2

CjapInx 3

BmorInx3

BmorInx4

CjapInx 7

BmorInx7-like2

CjapInx8

BmorInx8

CjapInx1
BmorInx1

CjapInx2

BmorInx2

CjapInx3

BmorInx 3

BmorInx4

CjapInx7

BmorInx7-like

CjapInx8

BmorInx8

CjapInx1

BmorInx1

CjapInx2

BmorInx2

CjapInx3

BmorInx 3

CjapInx4

CjapInx7

BmorInx7-1ike1

CjapInx8

BmorInx 8
BmorInx2

BmorInx7-like1

BmorInx7-like2

BmorInx7-like2

M----YKLLGDLSGYFK----RQEI ITDSMVFRMHNHFTTVLLFTCSILITATQYVGNPISCIT---QGLPTHAINTYCWITSTFTMPDA M----FQLLGGLRVYFQ----YQPIRTDNAVFRLHNVFTTVLLLTCSMIITASQYVGKPIMCIV---NGIPANVVNTFCWITSTFTMPDA M----FEVFGSVKGLLK----LDSVCIDNNVFRLHYKATVIGLI IFSLLVTSRQYIGDPIDCIV---DEIPLHVMDTYCWIYSTFTIPDR MF----DVFGSVKGLLK----LDSVCIDNNVFRLHYKATVIILIAFSLLVTSRQYIGDPIDCIV---DEI PLAVMDTYCWIYSTFTI PNR MV--VFGLVSAVAGEVKVRYLIDKAVIDNMVFRMHYRITSAMLFLCCIIVSANNLIGDPINCIS--ELAGAGHVINTFCWITYTFTLPAN MA--VFGLVSSVAGFVKVRYLIDKAVIDNMVFRMHYRITSAILFLCCILVTANNLIGEPIACIS--DGANPGHVINTFCWITYTFTMLNT M----IDLFMPFRSFLK----FENVCTDNNIFRMHYKLTVIILLVFTLLVTSKQFFGEPIHCMSGNDKGNDKDAVNSYCWIYGTYTLKSQ MAANVLAAFSVLKNHVNFKVNQNAVAIDNLVFRLHYRITFMLLLIGSILVSSRQFIGEHIRCIT--DIAIPAHVVETFCFFMSTYTVVKH

M---LVASLNSLSPRLRFQ--FSKPKIENVAFRLHYQLTVTILLAFVILVCAREYFGDHIKCLS-DQGVPDHVIQTYCFFMATFTIVRH M----LDIFRSLKSLIK----ISHIHTDSAVFRLHYSLTVILLTAFSLIVTTRQYVGNPIDCIH--SKDLPEDVLNTYCWIHSTYTITAA M-- - LDVERGLKNIIK---VIYVHIDSPVFRL

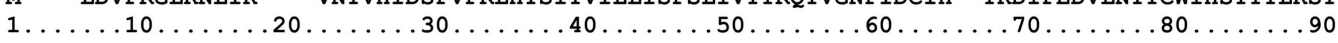

FNRQ--VGLEVAHPGVANDFGDV----DARKYYTYYQWVCFVLFFQAILCYIPQWLWSLWEGGLMNALVMGMNHG--------------FARE--VGKEVAHPGVGPEFEG-----TEKKYYTYYQWVCFVLFFQAIMCYTPKFLWDAFEGGLLRTIVMGLNIG-------------TGI--VGKDLVQPGVAAHVEGE----DEVKYHKYYQWVCFTLFFQAILFYI PRYLWKTWEGGRIKMLVLDLNCP------------LIGR--VGKDYVQPGVGPHVEGQ----DEVKYHKYYQWVCFVLFFQAILFYVPRYLWKTWEGGRIKMLVLDLNCP------------NAKP--VSTHVAHPGLGIDE-------GERRYHSYYQWVPFVLFFQGVLFYI PHWMWKQWEEGKIRI ISEGMRGS-------------TSKT------AAHPGLGDDN-------DEKRIHSYYQWVPFMLFFQGLLFYI PHWIWKNWEEGKVRLISEGMRGT------------LLGV--EGRHMAYVGVGPAKSDD----DEQIKHTYYQWVCFVLLGQATMFYAPRYLWKMWEGGRLKALAADLSSP------------LNATAVLQGELPHPGVGPAAKG-----DPVIHHAYYQWVPFVLFFQALLFYLPHYIWRKMEGNRLSMLVSGLHMASLLLSETELNVNDVK YNESLLQGEFLPHPGVGPILAT----DETIHHTYYQWVPFVLFIQSICFYLPHYIWKTKEGGRIKALVDGLQYAGLALHDDDITVNGTT EGGKIHALMMDLDIG-----------YYRK--EGYEVPFPGIDNSKSYPE---SERKEYRYYQWVCFMLFLQAILFYTPRWLWKGW EGGKIRALMMDLDVA-------------FEKT--VGVEVPYPGIGNSREKGKEDMSDRKVYKYYQWVCFCLFLQAMLFYAPRWLWKSW $\ldots \ldots 100 \ldots \ldots 110 \ldots \ldots 120 \ldots \ldots 130 \ldots \ldots 140 \ldots \ldots 150 \ldots \ldots 160 \ldots \ldots 170 \ldots \ldots 180$

MDT-EENITKKKSALMDYL-IQHIRSHNTYVYRYFACEALCLINIFGQLYLMNRFFDGEFFSYGLRVLQFSDTPQ-------EERVDPMV VCH-QDEKEKKKDVI IDYL-LRHERTHKLYAFRYWGCELLCLVNI ILQMWMMDNFFNGEFMSYGTRVLGYSEVPQ-------EERYDPMI VVS-EDCKSDRRKLLVDYF-TSNLHSQNFYAYRFFLCELLNLINVIGQIFFIDFFLDGEFTTYGSDVLRFTEMEP-------EQRMDPMA IVE-DECKSGRKKLLVDYF-HTNLHTONFYAFRFFICEVLNFINVVROIFFMDFFLDGEFSTYGSDVVSFTEMEP-------EERVDPMA MIEPKQERQAKVERLVQYL-VETMHLHNSYAAAYFFCEALNFVNTICNIFFVDAFLGGAFLTYGTEVLKFSNMNQ-------EQRTDPMI MASIADDKNNRQNRLVQYL-LDTSHMHNTYSFGYFFCEVLNFANVVGNIFFLDTFLGGAFLTYGTDVVRFSNMNQ--------EQRTDPMI MVS-KDWSEFRRKELVSYFNYTNMYTHNMYALRYAFCELLNLVNVVGQIFILDLFLGGSFRNYGAAVAAFTHTPRI PNDFTNFTSVNPMD VPS-KKDRDERIQQIRTGF-INRLHLNRPWAYGLTFCEILNLINVVMQIFLTDWFLGGAFLGLGRSI---SQPRS-------KDEVDPLD ----------------------------EVANLLHVMFQVWVINKFLNGAFMSLGPRVLETKDW---------SHIVDPLE VPS-KKTLENKLDSIRKDIILRYEIE---------CNLYNL---LCS-EVEKKQKKKMLLDYL-WENLRYHNWWAYRYYLCEVLALLNVIGQMFLMNRFFDGAFLTFGIDVLRFLESDQ-------EDRVDPMI VCT-EIEKKTKKKLILDYL-WENLRYHNWWAYRYYLCEALALVNVIGQMFLMNRFFDGEFMTFGLKVIKYMESDQ-------EIRIDPMI $\ldots \ldots 190 \ldots \ldots 200 \ldots \ldots 210 \ldots \ldots 220 \ldots \ldots 230 \ldots \ldots 240 \ldots \ldots 250 \ldots \ldots 260 \ldots \ldots 270 \ldots \ldots$

YVFPRVTKCI-FHKYGASGTIQQHDSLCILPLNIVNEKTYIFIWFWFFILALMLFGLLVYRVVIIFAPMVRPRLLHLSTRLLPIE----YVFPRMTKCT-FHKFGASGSIQTHDSLCVLPLNIVNEKTYIFLWFWYIILAVVLTLLVMYRLVI IFMPAVRPRLLHARSRTLSLE----RVFPKVTKCT-FHKYGASGTVOKFDGLCVLPLNIVNEKIYVFLWFWFIILAALSGLSLFYRVAVLIGPKLRMVLLRARSRLSPOD----RVFPKVTKCT-FHKYGPSGTVQKFDGLCVLPLNIVNEKIYVFLWFWFMILSILSGISLLYRMAVVAGPRVRLYLLRARSRLAPQE----EVFPRVTKCT-FHKFGASGSIQKLDALCVLALNILNEKIYIFLWFWLIFVALITGLSMLYSMAVVLMPSTREAILKKRFKFGTPA---- EVFPRINKCT-FHKFGASGTIQKHDALCVLALNILNEKIFIFLWFWFIILSVVSGLALVYSAAVCLLPSTRETILKRRFRFGTPN----EFFPKLTKCW-LRNYGPSGSLELKDRLCVLPLNIVNEKIFVILWFWLIILTAFSILAVVFRFLLLILYPLRTVMIRGQIRYVKRS---- IVFPKVTKCI-FHKYGPSGGIQKHDALCVMALNIVNEKIYTVLWFWFVVLAI ITGLGLVWRVLTMILHARSTSFNKFVFSMACPGKYNPW LVFPKVTKCI-FHKYGPSGSIQQHDALCVMALNI IHEKIYTVLWFWLLFLFIVSLLAVIWRVISFFLYRRSLRFNEMMFRHVSNAKFNPY ATFPR-YIFPRMKC

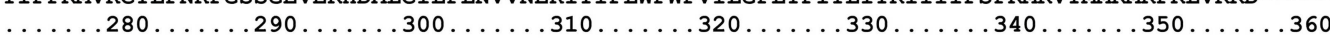

TCHSVSNKVDLGDWWILYILSANMDSLLYKDFLMEFTKKMANTNS-TAVAVSRRTDLGDWWLLYMLARNMD PLIYRELMIDLVKRMGEK-QVKTISDKCQIGDWFILYQLGKNIDPLVYKQLIADLAIKLQGK--OIEAVARKLOIGDWFVLYOLGKNIDPLIYKELMTELAEKFEGN-_TVNALIRKTQVGDFLILHLLGQNMNLMMFNETLEELCRRLQFGSGSGASPTSVPSAPSTLE---MSPIY---PEIEKYA-----KDTEI GVEALVRKTQVGDFLLLHLLGQNMSLRVFGEVLDELSRRLNLGSH-------APSAPSTLE---MAPIY---PDIDKYS-----KETET VVSRIVKRFGFGDWFILHLLGKNMNPI IFKDLVLELAKEIEHN-_-_-_-_NVLKVTHEYYFGDWLFLYYIAKNLDNYVFKELLQKLAEDLENKHHAR--FKILPPFEQSMLKNQWDDI----------- KPSVN NVIRVVNGCEFADWLFLYYLAKNMQGFVFQALFVRLAEELEKRE-------IPYDDQGTEEKGAEPILVGKVDIDDETMPLKRDKKSS

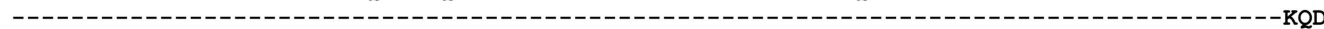
AVETIVRRSKVGDWFLLYMLGENLDTVIYRDVMHELANKLASRHHHG-----VPGVKG----------------------ELQEA NIDTIVRRSKMGDWYLLYILGENLDSI IFRDIMQEFANKLNHNYQHH-----IHGV--- - $\ldots \ldots 370 \ldots \ldots 380 \ldots \ldots 390 \ldots \ldots 400 \ldots \ldots 410 \ldots \ldots 420 \ldots \ldots 40 \ldots \ldots$

FIGURE 4 | Amino acid sequence alignment of innexin molecules expressing in the antenna of Camponotus japonicus. Subtypes of innexin expressing in $C$. japonicus antennae (Cjaplnx1, Cjaplnx2, Cjaplnx3, Cjaplnx7, Cjaplnx8) are compared with each other and with Bombyx innexins in amino acid sequence. A sequence used as an epitope for immunohistostaining is highlighted.

\section{0 150 150 150 150 150 150 150 150 150} 150 150 150 150 150 150 150 150 150 150 150
150 300 300 300 300 300 300 300 300 300 300 300 300

360 360 360 360 360 360 360 360 360 360 360 360 
Using anti-CjapInx3 antiserum, we localized this innexin subtype in the antennae of C. japonicus. Totally, 19 sensilla were examined, however 10 of them were useless; 6 were inconveniently oriented and 4 had no dendritic processes in empty sensillar shafts. Consequently, 9 sensilla, which were precisely observed, exhibited inside staining with anti-CjapInx3 antiserum. Three of them and one control are shown as the supplementary material (Supplementary Figures 1A-C), and the representative images of test and control are shown in Figure 5. Figure 5A shows the representative high resolution immunohistostaining images of $S$. basiconica using antiCjapInx3 antiserum; Left, differential interference image; Right, fluorescence image. Figure 5B shows the representative high resolution immunohistostaining images using preimmune serum; Left, differential interference image; Right, fluorescence image. In Figure 5A Right, fine fluorescent spots were distributed in the proximal half of the sensillar shaft but not in the distal half. Figure 5C shows a $6-\mu \mathrm{m}$ thick cross-sectional view of the $S$. basiconica. The dotted staining is found only inside of the sensillum (the inner perimeter of the sensillar cuticle wall is traced with a broken line), which implies that CjapInx3 is localized inside the proximal half of the S. basiconica. Conversely, Figure 5B Right, a control image hardly exhibits such fine fluorescent dotted staining as Figure 5A Right. Relatively strong fluorescent signals (arrowheads in Figure 5A Right) were sometimes seen on the outer surface of the sensillum even in the distal half (see Supplementary Figures 1A-C). Those signals are presumed to be unavoidable and non-specific (see section Materials and Methods). Nevertheless, by investigating every fluorescent image data with NIS elements AR software (Nikon Corporation, Tokyo, Japan) to get all directional optical view, we could suggest that specific signals exist in the sensillar lumen of all nine test sensilla. Such inside signals appeared more proximal than $11.84 \pm 0.99 \mu \mathrm{m}$ (average \pm standard error, $n=9$ ) from the top of the sensillum. In the representative immunohistostaining data shown in Figure 5A Right, we succeeded to measure fluorescence intensity distribution of the inside signal along the longitudinal axis of the sensillar shaft (see Supplementary Figure 1).

We also confirmed that the anti-CjapInx3 antiserum used for immunohistostaining labeled a band of $37 \mathrm{kDa}$ apparent molecular mass in the western blot of antennal proteins (arrowhead in Figure 5D). Another labeled band of a higher molecular mass may correspond to the dimeric CjapInx3 (double arrowheads in Figure 5D). The pre-immune serum yielded no labeling. Given the above immunohistostaining results and this western blotting data (Figures 5A Right, 5D), we estimated that CjapInx3 is localized inside of the S. basiconica, overlapping the area occupied by the dendritic processes.

\section{Distribution of "Beads" of Dendritic Processes}

Using the morphological data obtained via SBF-SEM (Figure 1), we enumerated all the beads in the beaded-structures of the 102 dendritic processes, identifying every adhesion region between cell membranes. In the observed range, 388 beads and 696

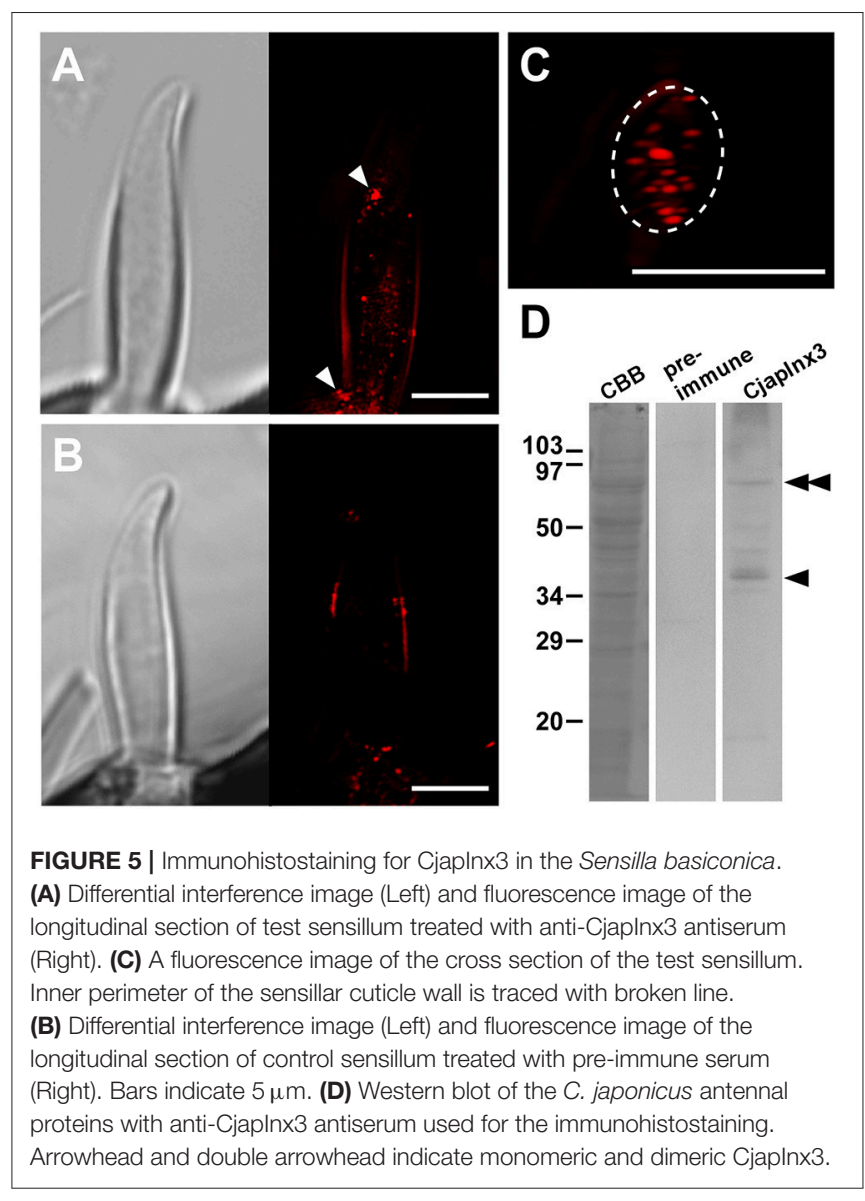

adhesion regions were enumerated. In Figure 6A, the 102 vertical lines with dots indicate the dendritic processes with beads. The number by the dot indicates how many adhesion regions occur at the corresponding bead. The axis of the ordinates in Figures 6A and $6 \mathrm{~B}$ indicates the regional level along the sensillar shaft as the distance from the outer surface level of the antennal cuticle. In Figure 6B, each dot corresponds to a bead distributed on the dendritic process, which is represented by the axis of the ordinate, while the axis of the abscissa indicates the number of adhesion regions at each bead. Thus, the distribution of the number of adhesion regions has two peaks, distal, and proximal, from the outer surface of the antennal cuticle. However, around the level of the basal socket, at a slightly distal level from the outer surface of the antennal cuticle, there are a small number of beads having no or a few adhesion regions. The distal and proximal peaks include 191 and 505 adhesion regions, respectively. When the fluorescence intensity distribution plot based on an immunohistostaining data using anti-CjapInx antiserum (Figure 5A Right) is superimposed on Figure 6B, both distributions indicating localization of CjapInx3 and appearance of beaded-structure are similar to each other, showing the distal peak, middle trough, and rising to the proximal peak (Supplementary Figure 1F). Figure 6C shows that every dendritic process has 1-7 beads, and the mean number of beads is four. Ninety-five of the 388 beads had no adhesion 
regions, but the remaining had 1-12 adhesion regions, as shown in Figure 6D. Every dendritic process is adjacent to 3-17 other dendritic processes. as shown in Figure 6E.

\section{Simplified Mathematical Model for Electric Connections Among Dendritic Processes}

As a simplified model, we first hypothesized that a set of cables (\#1-10) corresponding to 10 dendritic processes existing in parallel, not three dimensionally like inside a sensillum, but two-dimensionally (see left columns in Figures 7 and 8). The cables were hypothesized to have lateral connections to neighboring cable(s) via GJs. Using a system of ordinary differential equations (A1)-(A3) based on cable theory (see Appendix), we computed the direction of propagation of the current in our compartment model described in the Materials and Methods section. When we give input current to the most distal compartments of a limited number of cables, the inward current will passively propagate along the stimulated cables and the electrically connected neighboring cables. The most proximal compartment can generate impulses, only when the inward current is larger than a threshold at the impulse generating site. Such a large inward current, even if it propagates also to the neighboring cables via electric connections, evokes impulse generating activity. In cases where no impulse appears, the inward current decreases to less than the threshold during passive propagation. Whereas in cases where impulses are generated at the most proximal compartment, back propagating firing of impulse discharge from the most proximal compartment occurs at different passive compartments along the concerned cables.

In Figure 7, we show different results in accordance with the location of the connections among cables via electric connections. More precisely, if there is no electric connection at all (see control of Figures 7,8), the external input current given in 7 cables independently contributes to the appearance of impulses in each of the 7 cables, as shown in the top row of Figure 7. However, if there are electric connections at the distal and middle levels in the second and third rows of Figure 7 (electric connections at the distal and middle levels, respectively), the impulses completely disappear, even if the amplitude of the input current is exactly the same as that of the control and the input current should evokes the same change in membrane potential as the control, because the inward current reaching the impulse generating site of each concerned cable is under the threshold level. Moreover, if the cables are connected at the proximal level (close to the impulse generation site at the basement of the dendritic processes or near soma, i.e., active compartment), then the number of cables generating impulses at their active compartments increases to 9. We also examined a different situation by decreasing the amplitude of the input current. Figure 8 shows such an amplitude that no impulse appears even if connections do exist among cables, whereas if there are no connections among cables in control, impulses are still observed in the 7 cables with the input current.

We further examined the similar 2D simulation to Figure 7 but with 20 cables $(\# 1-20)$, seven of which are stimulated (Figure 9). Then, impulse generation is depressed with electric connections at the distal (two of 20 cables generate impulses) or middle level (three of 20 cables generate impulses). If the cables are connected at the proximal level, however, the number of cables generating impulses at their active compartments increases to 8 including a cable that has no direct input. Given these simulations hypothesizing ORN clusters formed by the dendritic network, we suggest in such ORN clusters that electric connections at distal or middle level will decrease, and that those at proximal will increase the number of cables generating impulses at their active compartments. Thus, the ORN clustering by electric connection along dendritic processes can differently modulate the total responsiveness of a sensillum possessing multiple ORNs.

\section{DISCUSSION}

\section{Filtration or Modification of Olfactory Information}

The S. basiconica on the antenna of ants is a small but complicated chemosensory unit. Its complexity is dependent on the repertoire of related OR genes belonging to the 9-exon subfamily (Engsontia et al., 2015; Zhou et al., 2015; McKenzie et al., 2016; Pask et al., 2017; Slone et al., 2017), which are expressed in the S. basiconica ORNs projecting the same number of glomeruli in a specific antennal lobe region called T6 (Zube et al., 2008; Kelber et al., 2010; Nakanishi et al., 2010; Nishikawa et al., 2012; Kropf et al., 2014; McKenzie et al., 2016; Couto et al., 2017). Olfactory information from S. basiconica, when sent to the higher brain, is further processed or memorized and contribute to regulation of behaviors, some of which may be cast- or sex-specific (Zube and Rosslea, 2008; Brandstaetter and Kleineidam, 2011; Brandstaetter et al., 2011; Nishikawa et al., 2012). In this stream, olfactory information of nestmate CHCs as well as non-nestmate CHCs, when sent to the higher brain via antennal lobe, would be memorized. Then the memory of the nestmate odor could be updated as the nestmate $\mathrm{CHC}$ pattern is gradually changed and thereby used as a template for the nestmate recognition (Brandstaetter and Kleineidam, 2011; Brandstaetter et al., 2011; Nishikawa et al., 2012; Ozaki and Hefetz, 2014).

In addition, a possibility of filtration or modification of olfactory information at the peripheral level is considered in the present paper. In the peripheral system of $S$. basiconica in C. japonicus, indeed, more than 100 dendritic processes extended into the cuticular shaft (Ozaki et al., 2005). One of our unexpected findings regarding the fine structure of the sensillum was the cell-free space occupying the distal half of the inside cavity beneath the olfactory pores (Figure $\mathbf{1} \mathbf{A}$ ). This space is filled with sensillar lymph dissolving CjapCSP (Ozaki et al., 2005; Hojo et al., 2015). Although this space is open to outer environmental chemicals through olfactory pores, the aquatic sensillar lymph surrounding the receptor membranes prevents lipophilic chemicals from freely diffusing to the receptor membranes. However, CjapCSP, a carrier protein for lipophilic compounds like CHCs, allows them to reach the receptor membranes. Thus, this space may function as the first filter for 
A
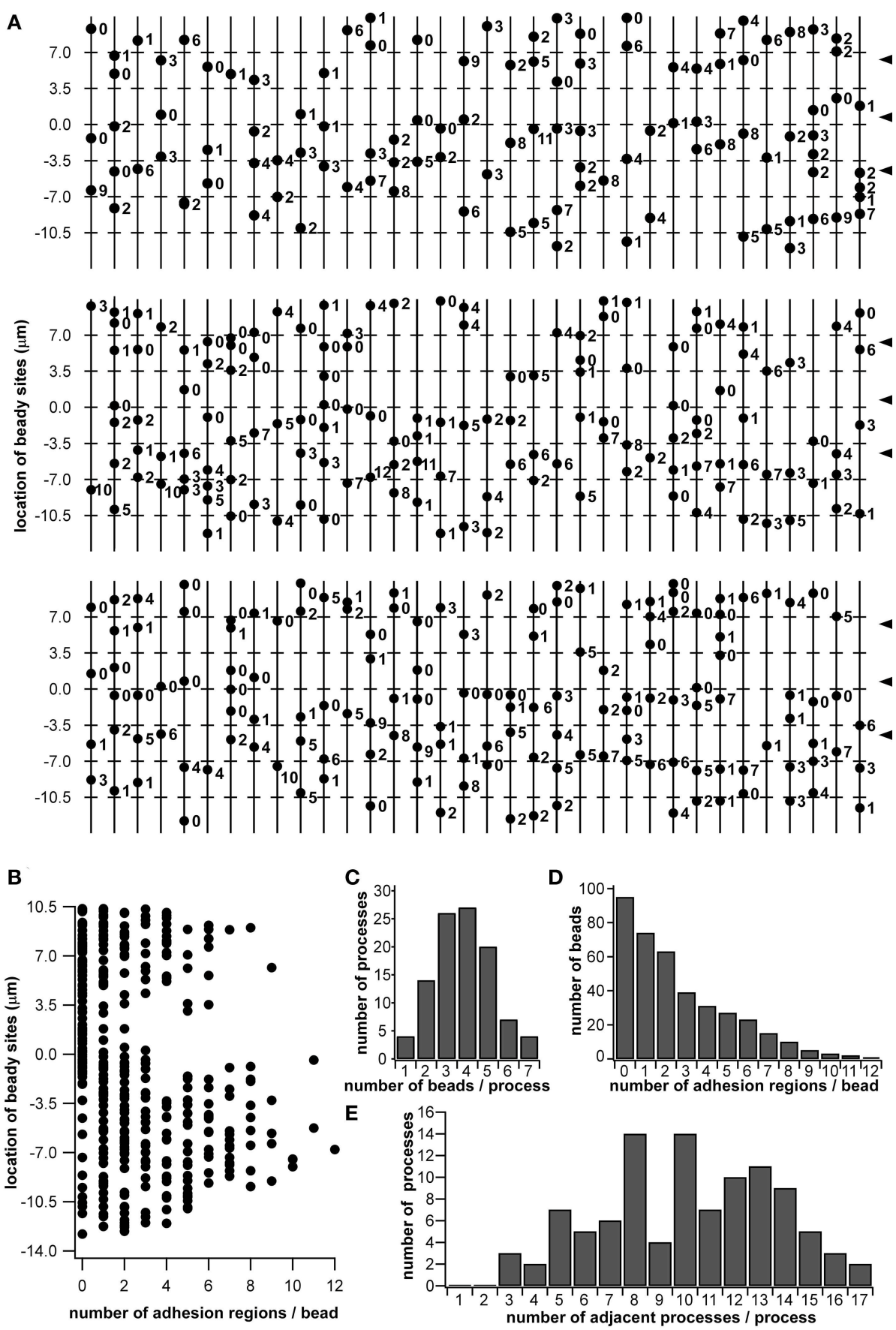

FIGURE 6 | Schematic diagram of beaded-structures in the dendritic processes of ORNs and the bead distribution within a Sensilla basiconica. Based on the SBF-SEM images in Figure 1, beady sites and putative GJ regions on them were counted by hands. (A) Schematic image of beaded-structures on all of 102 dendritic processes of the ORNs within an s. basiconica. (B) Distribution of beady sites along the dendritic processes with the number of adhesion regions as putative GJs at every bead. (C) Distribution of the dendritic processes in the number of beads per process. (D) Distribution of the beads in the number of adhesion regions per bead. (E) Distribution of the dendritic processes in the number of adjacent processes per process.

stimulus chemicals. There were no structural passages crossing the sensillar lymph to the receptor membranes, like the pore tubules reported in sex pheromone-sensitive sensilla of moths (Steinbrecht, 1999). Hence, CHC-CjapCSP complexes can only reach the receptor membranes by diffusion migration. The body surfaces of worker ants, including the antennae, are covered with colony-specific $\mathrm{CHC}$ blends that play a role in nestmate recognition (Wang et al., 2016). Hojo et al. (2015) in their 

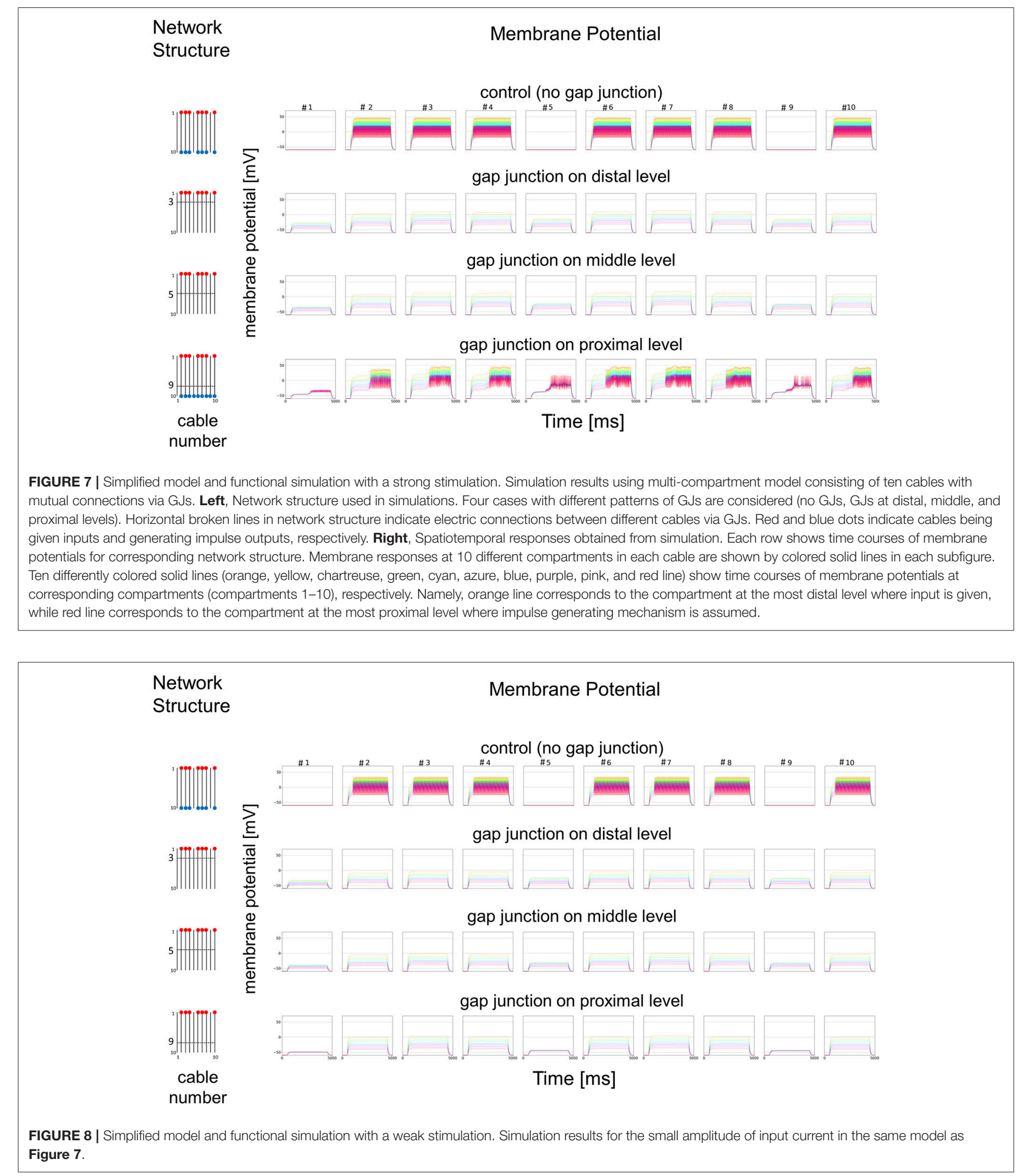

paper on RNAseq analysis in the antenna of C. japonicus showed that there are at least two different CSPs in S. basiconica and suggested that they have different function for carrying different ligands within a sensillum. This may be concerned with subtype formation and complex or multifunctional sensory mechanism in the S. basiconica. 


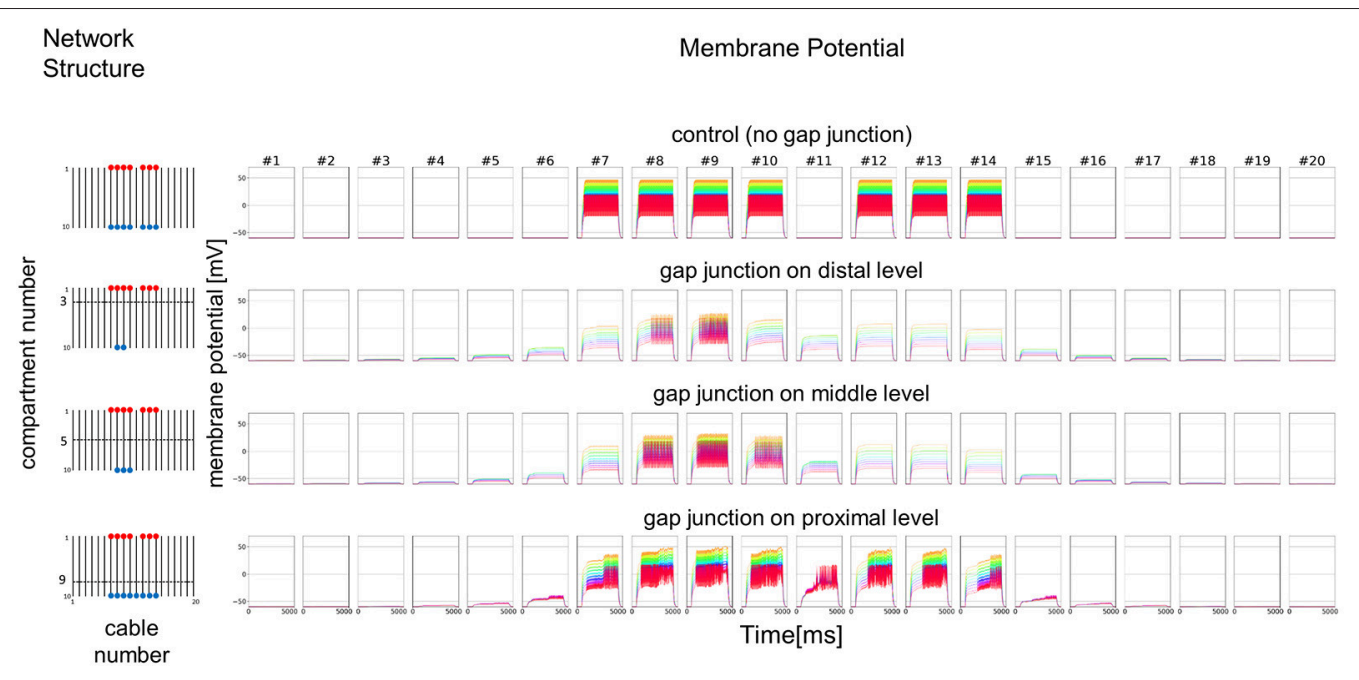

FIGURE 9 | Simplified model with double number of cables and functional simulation with a strong stimulation. Simulation results using multi-compartment model consisting of 20 cables with mutual connections via GJs. Four cases with different patterns of GJs are considered in the same model as Figure 7. The stimulation with the same amplitude as in Figure $\mathbf{7}$ is applied.

Since CHCs can penetrate the cell-free space of S. basiconica through olfactory pores, self-CHCs in complex forms with CjapCSP should always be present in the same ratio as that on the antennae (see Supplemental Material of Ozaki et al., 2005), which could desensitize a portion of ORNs or their receptor membranes to self- $\mathrm{CHC}$ components in a $\mathrm{CHC}$ composition ratio dependent manner. According to a putative explanation by Ozaki et al. (2005) and Ozaki and Hefetz (2014), the ORNs responsible for reception of self-CHC components are desensitized more strongly than other ORNs within S. basiconica. Thus, the cellfree space may function as a second filter, allowing the passage of hetero-specific or non-nestmate CHC information by olfactory signal transduction more efficiently than that of nestmate $\mathrm{CHCs}$ or self-CHCs.

Moreover, we proposed electric connections among dendritic processes probably via GJs in the S. basiconica of C. japonicus, which may be involved in olfactory information modification in this type of sensillum. In fact, we lack direct evidence by electrophysiological experiments or dye coupling between neighboring ORNs because of technical difficulty in handling ORNs in the small cuticular apparatus of insect sensillum. However, our collected morphological data (Figures 1-3, 5) support the existence of the micro-network within the $S$. basiconica; Figure 1 shows the overall shape of the 102 dendritic processes in a bundle of characteristic beaded strings; Figures 2 and 3 indicate a $2 \mathrm{D}$ image and $3 \mathrm{D}$ surface model of adhesion between membranes of dendritic processes at the beads, respectively, and Figure 5 together with Supplementary Figure 1 suggests that CjapInx 3 localizes in the proximal half of the sensillar cavity of $S$. basiconica, showing the consistent distribution with the beady site localization overlapping the dendritic process extensions (Figure 6B).

As for the innexin expression in the C. japonicus antennae, Hojo et al. (2015) reported that there are 4 other types of innexin than CjapInx3 (Figure 4). We have not successfully constructed specific antibodies against all of them, but against CjapInx2, CjapInx3 and CjapInx8, and have got some immuno-cytological data that can preliminarily suggest that CjapInx2 and CjapInx8 localize to other regions than the inside of the sensillar shaft in the S. basiconica, to which CjapInx3 seems to localize (Figure 5 and Supplementary Figure 1). As the cellular components in the inside cavity of the sensillar shaft, there are only dendritic processes of the ORNs without any supportive cells, which surround the cell bodies of ORNs at the basement of the sensillum under the antennal cuticular layer (Figures 1-3). Moreover, there are few intracellular membranous structures except for invaginations of cell membranes of the dendritic processes. As the visible intracellular structures, there are many microtubules along the dendritic processes and connective cilia at the basement (Figure 1). There might be vesicles for membrane turnover, but they were not frequently seen. Therefore, we suppose that membranous proteins like innexin, if immunohistologically detected inside of the sensillar shaft, probably locate on the cell membranes of the dendritic processes.

To convince that CjapInx3 is localized to the beadedstructures on the dendritic processes within a S. basiconica of C. japonicus and that it forms GJs, further immunohistological investigation at the electron microscopic level and functional studies of electrophysiology are required. However, given the structural evidence for apposed membranes, one could say that structural data suggests GJs could play a role and modeling demonstrates some potential implications of coupling. A simple but essential mathematical model (Figures 7, 8) suggested that such a micro-network of connecting multiple cables mimicking dendritic processes might function as a stronger-input-spread (Figure 7) or weaker-input-cut filter (Figure 8) under particular conditions. 


\section{Putative Function of Micro-Network in S. basiconica Suggested by Mathematical Simulation}

The beading of dendritic processes itself is a phenomenon also reported in the sex pheromone-sensitive sensilla of moths (Keil, 1984a,b, 1989). Furthermore, it has been directly observed under a light microscope that beads occur and even move on living dendritic process (Williams, 1988). Therefore, the possibility of beading being a fixation artifact has already been ruled out. However, the biological function of beads remains elusive. No one has proposed its potential role for network communication among ORNs in any species. Nevertheless, our study animal was different and the investigated sensillum, namely $S$. basiconica of ants, is a much more complicated olfactory sensory unit than a sex pheromone-sensitive sensillum of moths. Previous findings of beaded-structures along the dendritic processes were not on such a large scale as the S. basiconica in C. japonicus. Thus, the present paper is the first report taking up the beaded-structure that may be involved in modification of olfactory dependent neuronal/behavioral responses.

Considering putative role of the beaded structures on the nestmate and non-nestmate discrimination of ant, mathematical simulation mimicking dendritic processes, which can function as a stronger-input-spread (Figure 7) or weaker-input-cut filter (Figure 8), is suggestive. As mentioned above, it could be supposed that ORNs in the S. basiconica are exposed to selfCHCs continuously secreted on the antennal cuticle surface, and thus the ORNs might be more strongly desensitized to nestmate $\mathrm{CHCs}$ than to non-nestmate CHCs. Because of the difference in desensitization effect, input current evoked by nestmate $\mathrm{CHCs}$ was expected be weaker than that by non-nestmate CHCs. Moreover, in our micro-network structure simulation, weaker input current like a desensitized input current evoked by nestmate CHCs tends to result in decreased chance of impulse generation in any concerned dendritic processes as shown in Figure 8, whereas less-desensitized or stronger input current tends to result in increased chance of impulse generation as shown in Figure 7. Our mathematical model can result thus the function, only when the parallel cables mimicking the dendritic processes are connected at the proximal level close to the impulse generation site. Less-desensitized or stronger input current like receptor currents generated by the non-nestmate $\mathrm{CHCs}$ can still trigger impulses not only in directly stimulated ORNs but also in neighboring ORNs (Figure 7). By contrast, weaker input in previously desensitized ORNs, after being divided into neighboring ORNs, is reduced under the threshold in all concerning ORNs. However, as was reported by Brandstaetter and Kleineidam (2011), nestmate CHCs still activated the AL glomeruli, this kind of peripheral filtration would not completely function, when the nestmate $\mathrm{CHC}$ stimulation quantitatively overcame desensitization to the self-CHCs. However, such a stronger-input-spread or weaker-input-cut filter, when combined with the desensitization mechanism in sensory system, could be useful for sensitive detection of unusual or novel odors.

In the present study, we precisely enumerated the number of putative adhesion regions between dendritic processes in an
S. basiconica and showed their distribution along all dendritic processes (Figure 6). In that sample, there were indeed a small number of adhesion regions at the level of the outer surface of the antennal cuticle. In contrast, a larger number of adhesion regions, as many as 505, were enumerated in the proximal part and as many as 191 in the distal part. Moreover, as shown in Supplementary Figure 1F, the distribution pattern of adhesion regions (Figure 6B) is similar to the distribution pattern of fluorescence intensity along the sensillar shaft in an example of immunohistological staining using anti-CjapInx3 antisera (Figure 5A Right). This supplementary data suggests involvement of GJs in the dendritic network formation in S. basiconica of C. japonicus.

Besides the electric connection by GJ forming electric synapse, in fact, ephaptic coupling has been known to be able to explain inhibitory and enhancing effects on neighboring ORNs housed in a insulated narrow space like a sensillum as a significant determinant of the olfactory code (White et al., 1990; Su et al., 2012; Van der Goes van Naters, 2013; Chen, 2015; Miriyala et al., 2018). Therefore, also in the case of S. basiconica of ant, ephaptic coupling effect should not be ignored. Nonetheless, in comparison with ephaptic coupling previously reported in chemosensilla of other insect species, there might be some advantage to GJ connection in its potential of flexible change of connective site. The GJ network would be flexible and such a plastic state is difficult to experimentally follow, but in the $S$. basiconica, functional connective site might properly be appeared or disappeared to form different shapes of dendritic networks within an appropriate set of ORNs, depending on age, sex, cast or social task. Based on electrophysiological data, Sharma et al. (2015) suggested that there are different types of $S$. basiconica on the antennae of $C$. floridanus, and that different combination of OR genes are expressed in different subtypes. We presume that those different $S$. basiconica subtypes could also have different shapes of dendritic networks depending on their roles, respectively.

In the present study, we demonstrated ultrastructure in S. basiconica of C. japonicus. It included characteristic beaded structure, which looked like a platform for Cell-Cell interaction. Our morphological study was limited to step into functional insight on that structure. To compensate experimental limitation, we examined mathematical simulation and found putative function as an olfactory information modifier. Yet, we need more convincing morphological data by immunoelectron microscopy and electrophysiology to certify olfactory information modification, which was suggested by mathematical simulation.

\section{AUTHOR CONTRIBUTIONS}

YT: SBF-SEM, TEM, UHV-EM; TU: High resolution microscope, Innexin work; NM, KM: SBF-SEM, 3D model; KY: SBF-SEM, TEM; KI: UHV-EM; NK: Tomography analyses; TS: TEM; HK, JT, and TO: Mathematical model; RY and YE: Innexin work; $\mathrm{MH}$ : Innexin gene analysis; ET and SK: EM Image analysis; KT and KO: Western blot; MO: Supervising. 


\section{FUNDING}

This work was supported by JSPS KAKENHI Grant Number JP24120006 in Scientific Research on Innovative Areas Innovative Materials Engineering Based on Biological Diversity to MO and partially by JSPS KAKENHI Grant Number 16KT0015 to HK, JSPS KAKENHI Grant Number JP15KK0010 in a Fund for the Promotion of Joint International Research (Fostering Joint International Research) to TO, and JSPS KAKENHI Grant Number JP16K00330 in Scientific Research to TO.

\section{ACKNOWLEDGMENTS}

We thank Drs Tadashi Nakamura, Tatsuo Iwasa for their helpful advice and discussion. Our electron microscopic experiment

\section{REFERENCES}

Brandstaetter, A. S., Endler, A., and Kleineidam, C. J. (2008). Nestmate recognition in ants is possible without tactile interaction. Naturwissenschaften 95, 601-608. doi: 10.1007/s00114-008-0360-5

Brandstaetter, A. S., and Kleineidam, C. J. (2011). Distributed representation of social odors indicates parallel processing in the antennal lobe of ants. J. Neurophysiol. 106, 2437-2449. doi: 10.1152/jn.01106.2010

Brandstaetter, A. S., Rössler, W., and Kleineidam, C. J. (2011). Friends and foes from an ant brain's point of view - neuronal correlates of colony odors in a social insect. PLoS ONE 6:e21383. doi: 10.1371/journal.pone. 0021383

Chen, Y.-C. (2015). The interactions between bitter and sweet taste processing in Drosophila. J. Neurosci. 35, 9542-9543. doi: 10.1523/JNEUROSCI.1552-15.2015

Couto, A., Mitra, A., Thiéry, D., Marion-Poll, F., and Sandoz, J. C. (2017). Hornets have it: a conserved olfactory subsystem for social recognition in hymenoptera? Front Neuroanat. 11:48. doi: 10.3389/fnana.2017.00048

de Fouchier, A., Walker, W. B. III, Montagné, N., Steiner, C., Binyameen, M., Schlyter, F., et al. (2017). Functional evolution of Lepidoptera olfactory receptors revealed by deorphanization of a moth repertoire. Nat. Commun. 8:15709. doi: 10.1038/ncomms15709

Dussutour, A., and Simpson, S. J. (2008). Description of a simple synthetic diet for studying nutritional responses in ants. Insectes Sociaux 55, 329-333. doi: 10.1007/s00040-008-1008-3

Engsontia, P., Sangket, U., Robertson, H. M., and Satasook, C. (2015). Diversification of the ant odorant receptor gene family and positive selection on candidate cuticular hydrocarbon receptors. BMC Res. 8:380. doi: 10.1186/s13104-015-1371-x

Gilbert, P. (1972). Iterative methods for the three-dimensional reconstruction of an object from projections. J. Theor. Biol. 36, 105-117.

Guerrieri, F. J., and d'Ettorre, P. (2008). The mandible opening response: quantifying aggression elicited by chemical cues in ants. J. Exp. Biol. 211, 1109-1113. doi: $10.1242 /$ jeb.008508

Guerrieri, F. J., Nehring, V., Jørgensen, C. G., Nielsen, J., Galizia, C. G., and d'Ettorre, P. (2009). Ants recognize foes and not friends. Proc. Biol. Sci. 276, 2461-2468. doi: 10.1098/rspb.2008.1860

Hallberg, E., and Hansson, B. S. (1999). Arthropod sensilla: morphology and phylogenetic considerations. Insect Sens. Struct. 47, 428-439. doi: 10.1002/(SICI)1097-0029(19991215)47:6<428::AID-JEMT6>3.0.CO;2-P

Hansson, B. S., and Stensmyr, M. C. (2011). Evolution of insect olfaction. Neuron 72, 698-711. doi: 10.1016/j.neuron.2011.11.003

Haupt, S. S., Sakurai, T., Namiki, S., Kazawa, T., and Kanzaki, R. (2010). "Chapter 3. Olfactory information processing in moths," in The Neurobiology of Olfaction, ed A. Menini (Boca Raton, FL: CRC Press/Taylor and Francis), 71-112. was supported by the collaborative research program of The National Institute for Physiological Sciences to MO, and by the Advanced Characterization Nanotechnology Platform, Nanotechnology Platform Program of the Ministry of Education, Culture, Sports, Science and Technology (MEXT), Japan at The Research Center for Ultra-High Voltage Electron Microscopy (Nanotechnology Open Failities) in Osaka University. Our super resolution microscopic experiment was technically supported by Nikon Corporation.

\section{SUPPLEMENTARY MATERIAL}

The Supplementary Material for this article can be found online at: https://www.frontiersin.org/articles/10.3389/fncel. 2018.00310/full\#supplementary-material
Hojo, M. K., Ishii, K., Sakura, M., Yamaguchi, K., Shigenobu, S., and Ozaki, M. (2015). Antennal RNA-sequencing analysis reveals evolutionary aspects of chemosensory proteins in the carpenter ant Camponotus japonicus. Sci. Rep. 5:13541. doi: 10.1038/srep13541

Hölldobler, B. (1995). The chemistry of social regulation: multicomponent signals in ant societies. Proc. Natl. Acad. Sci. U.S.A. 92, 19-22. doi: 10.1073/pnas.92.1.19

Kaissling, K.-E. (1987). Wright Lectures on Insect Olfaction. Burnaby, BC: Simon Fraser University.

Keil, T. A. (1984a). Surface coats of pore tubules and olfactory sensory dendrites of a silkmoth revealed by cationic markers. Tissue Cell 16, 705-717.

Keil, T. A. (1984b). Reconstruction and morphometry of silkmoth olfactory hairs: a comparative study of sensilla trichodea on the antennae of male Antheraea polyphemus and Antheraea pernyi (Insecta, Lepidoptera). Zoomorphology 104, 147-156.

Keil, T. A. (1989). Fine structure of the pheromone-sensitive sensilla on the antenna of the hawkmoth, Manduca sexta. Tissue Cell 21, 139-151.

Kelber, C., Rössler, W., and Kleineidam, C. J. (2010). Phenotypic plasticity in number of glomeruli and sensory innervation of the antennal lobe in leaf-cutting ant workers (A. vollenweideri). Dev. Neurobiol. 70, 222-234. doi: 10.1002/dneu.20782

Kidokoro-Kobayashi, M., Iwakura, M., Fujiwara-Tsujii, N., Fujiwara, S., Sakura, M., Sakamoto, H., et al. (2012). Chemical discrimination and aggressiveness via cuticular hydrocarbons in a supercolony-forming ant, Formica yessensis. PLoS ONE 7:e46840. doi: 10.1371/journal.pone.0046840

Koch, C. (1999). Biophysics of Computation. Oxford: Oxford University Press.

Kremer, J. R., Mastronarde, D. N., and McIntosh, J. R. (1996). Computer visualization of three-dimensional image data using IMOD. J. Struct. Biol. 116, 71-76. doi: 10.1006/jsbi.1996.0013

Kropf, J., Kelber, C., Bieringer, K., and Rössler, W. (2014). Olfactory subsystems in the honeybee: sensory supply and sex specificity. Cell Tissue Res. 357, 583-595. doi: 10.1007/s00441-014-1892-y

Lahav, S., Soroker, V., Hefetz, A., and Vander Meer, R. K. (1999). Direct behavioral evidence for hydrocarbons as ant recognition discriminators. Naturwissenschaften 86, 246-249. doi: 10.1007/s001140050609

Leonhardt, S. D., Menzel, F., Nehring, V., and Schmitt, T. (2016). Ecology and evolution of communication in social insects. Cell 164, 2177-1287. doi: 10.1016/j.cell.2016.01.035

McKenzie, S. K., Fetter-Pruneda, I., Ruta, V., and Kronauer, D. J. C. (2016). Transcriptomics and neuroanatomy of the clonal raider ant implicate an expanded clade of odorant receptors in chemical communication. Proc. Natl. Acad. Sci. U.S.A. 113, 14091-14096. doi: 10.1073/pnas.16108 00113

Miriyala, A., Kessler, S., Rind, F. C., and Wright, G. A. (2018). Burst firing in bee gustatory neurons prevents adaptation. Curr. Biol. 28, 1585.e3-1594.e3. doi: 10.1016/j.cub.2018.03.070 
Mizunami, M., Yamagata, N., and Nishino, H. (2010). Alarm pheromone processing in the ant brain: an evolutionary perspective. Front. Behav. Neurosci. 4:28. doi: 10.3389/fnbeh.2010.00028

Morris, C., and Lecar, H. (1981). Voltage oscillations in the barnacle giant muscle fiber. Biophys. J. 35, 193-213. doi: 10.1016/S0006-3495(81)84782-0

Nakanishi, A., Nishino, H., Watanabe, H., Yokohari, F., and Nishikawa, M. (2009). Sex-specific antennal sensory system in the ant Camponotus japonicus: structure and distribution of sensilla on the flagellum. Cell Tissue Res. 338, 79-97. doi: 10.1007/s00441-009-0863-1

Nakanishi, A., Nishino, H., Watanabe, H., Yokohari, F., and Nishikawa, M. (2010). Sex-specific antennal sensory system in the ant Camponotus japonicus: glomerular organizations of antennal lobes. J. Comp. Neurol. 518, 2186-2201. doi: $10.1002 /$ cne.22326

Nick, B., and d'Ettorre, P. (2012). Recognition of social identity in ants. Front. Psychol. 3:83. doi: 10.3389/fpsyg.2012.00083

Nishikawa, M., Watanabe, H., and Yokohari, F. (2012). Higher brain centers for social tasks in worker ants, Camponotus japonicus. J. Comp. Neurol. 520, 1584-1598. doi: 10.1002/cne.23001

Ozaki, M., and Hefetz, A. (2014). Neural mechanisms and information processing in recognition systems. Insects 5, 722-741. doi: 10.3390/insects5040722

Ozaki, M., Wada-Katsumata, A., Fujikawa, K., Iwasaki, M., Yokohari, F., Satoji, Y., et al. (2005). Ant nestmate and non-nestmate discrimination by a chemosensory sensillum. Science 309, 311-314. doi: 10.1126/science.1105244

Pask, G. M., Slone, J. D., Millar, J. G., Das, P., Moreira, J. A., Zhou, X., et al. (2017). Specialized odorant receptors in social insects that detect cuticular hydrocarbon cues and candidate pheromones. Nat. Commun. 8:297. doi: 10.1038/s41467-017-00099-1

Rall, W. (1959). Branching dendritic trees and motoneuron membrane resistivity. Exp. Neurol. 1, 491-527. doi: 10.1016/0014-4886(59)90046-9

Segev, I., Rinzel, J., and Shephard, G. M. (1995). The Theoretical Foundation of Dendritic Function. Cambridge: MIT Press.

Sharma, K. R., Enzmann, B. L., Schmidt, Y., Moore, D., Jones, G. R., Parker, J., et al. (2015). Cuticular hydrocarbon pheromones for social behavior and their coding in the ant antenna. Cell Rep. 12, 1261-1271. doi: 10.1016/j.celrep.2015.07.031

Slone, J. D., Pask, G. M., Ferguson, S. T., Millar, J. G., Berger, S. L., Reinberg, D., et al. (2017). Functional characterization of odorant receptors in the ponerine ant, Harpegnathos saltator. Proc. Natl. Acad. Sci. U.S.A. 114, 8586-8591. doi: 10.1073/pnas.1704647114

Steinbrecht, R. A. (1999). "Olfactory receptors," in Atlas of Arthropod Sensory Receptors -Dynamic Morphology in Relation to Function, eds E. Eguchi and Y. Tominaga (Tokyo: Springer), 155-76.
Su, C.-Y., Menuz, K., Reisert, J., and Carlson, J. R. (2012). Non-synaptic inhibition between grouped neurons in an olfactory circuit. Nature 492, 66-71. doi: $10.1038 /$ nature 11712

Van der Goes van Naters, W. (2013). Inhibition among olfactory receptor neurons. Front. Hum. Neurosci. 7:690. doi: 10.3389/fnhum.2013.00690

Vander Meer, R. K. (1998). Pheromone Communication in Social Insects: Ants, Wasps, Bees, and Termites. Boulder, CO: Westview Press.

Wang, Q., Goodger, J. Q., Woodrow, I. E., and Elgar, M. A. (2016). Locationspecific cuticular hydrocarbon signals in a social insect. Proc. Biol. Sci. 283:20160310. doi: 10.1098/rspb.2016.0310

White, P. R., and Chapman, R. R., Ascoli-Christensen (1990). Interactions between two neurons in contact chemosensilla of the grasshopper, Schistocerca Americana. J. Comp. Physiol. A 167, 431-436. doi: 10.1007/BF001 92578

Williams, J. L. D. (1988). Nodes on the large pheromone-sensitive dendrites of olfactory hairs of the male silkmoth, Antheroeapolyphemus (Cramer) (Lepidoptera: Saturniidae). Int. J. Insect Morphol. Embryol. 17, 145-151. doi: 10.1016/0020-7322(88)90008-6

Zhou, X., Rokas, A., Berger, S. L., Liebig, J., Ray, A., and Zwiebel, L. J. (2015). Chemoreceptor evolution in hymenoptera and its implications for the evolution of eusociality. Genome Biol. Evol. 7, 2407-2416. doi: $10.1093 / \mathrm{gbe} / \mathrm{evv} 149$

Zube, C., Kleineidam, C. J., Kirschner, S., and Nee, f,. J., Rosslea, W. (2008). Organization of the olfactory pathway and odor processing in the antennal lobe of the ant Camponotus floridanus. J. Comp. Neurol. 506, 425-441. doi: $10.1002 /$ cne. 21548

Zube, C., and Rosslea, W. (2008). Caste- and sex-specific adaptations within the olfactory pathway in the brain of the ant Camponotus floridanus. Arthropod Struct. Dev. 37, 469-479. doi: 10.1016/j.asd.2008.05.004

Conflict of Interest Statement: The authors declare that the research was conducted in the absence of any commercial or financial relationships that could be construed as a potential conflict of interest.

Copyright (c) 2018 Takeichi, Uebi, Miyazaki, Murata, Yasuyama, Inoue, Suzaki, Kubo, Kajimura, Takano, Omori, Yoshimura, Endo, Hojo, Takaya, Kurihara, Tatsuta, Ozaki and Ozaki. This is an open-access article distributed under the terms of the Creative Commons Attribution License (CC BY). The use, distribution or reproduction in other forums is permitted, provided the original author $(s)$ and the copyright owner(s) are credited and that the original publication in this journal is cited, in accordance with accepted academic practice. No use, distribution or reproduction is permitted which does not comply with these terms. 


\title{
The Consequences of Preterm Birth and Chorioamnionitis on Brainstem Respiratory Centers: Implications for Neurochemical Development and Altered Functions by Inflammation and Prostaglandins
}

\author{
Vanesa Stojanovska ${ }^{1 *}$, Suzanne L. Miller ${ }^{1,2}$, Stuart B. Hooper ${ }^{1,2}$ \\ and Graeme R. Polglase ${ }^{1,2}$ \\ ${ }^{1}$ The Ritchie Centre, Hudson Institute of Medical Research, Melbourne, VIC, Australia, ${ }^{2}$ Department of Obstetrics and \\ Gynaecology, Monash University and Hudson Institute of Medical Research, Melbourne, VIC, Australia
}

OPEN ACCESS

Edited by: Dieter Wicher, Max Planck Institute for Chemical Ecology (MPG), Germany

Reviewed by: Donald C. Bolser, University of Florida, United States Ana Paula Abdala, University of Bristol, United Kingdom

*Correspondence: Vanesa Stojanovska vanesa.stojanovska@hudson.org.au

Received: 06 November 2017 Accepted: 17 January 2018 Published: 01 February 2018

Citation:

Stojanovska V, Miller SL, Hooper SB and Polglase GR (2018) The Consequences of Preterm Birth and Chorioamnionitis on Brainstem Respiratory Centers: Implications for Neurochemical Development and Altered Functions by Inflammation and Prostaglandins.

Front. Cell. Neurosci. 12:26. doi: 10.3389/fncel.2018.00026
Preterm birth is a major cause for neonatal morbidity and mortality, and is frequently associated with adverse neurological outcomes. The transition from intrauterine to extrauterine life at birth is particularly challenging for preterm infants. The main physiological driver for extrauterine transition is the establishment of spontaneous breathing. However, preterm infants have difficulty clearing lung liquid, have insufficient surfactant levels, and underdeveloped lungs. Further, preterm infants have an underdeveloped brainstem, resulting in reduced respiratory drive. These factors facilitate the increased requirement for respiratory support. A principal cause of preterm birth is intrauterine infection/inflammation (chorioamnionitis), and infants with chorioamnionitis have an increased risk and severity of neurological damage, but also demonstrate impaired autoresuscitation capacity and prevalent apnoeic episodes. The brainstem contains vital respiratory centers which provide the neural drive for breathing, but the impact of preterm birth and/or chorioamnionitis on this brain region is not well understood. The aim of this review is to provide an overview of the role and function of the brainstem respiratory centers, and to highlight the proposed mechanisms of how preterm birth and chorioamnionitis may affect central respiratory functions.

Keywords: chorioamnionitis, brainstem respiratory centers, preBötzinger complex, prostaglandins, preterm birth, apnea

\section{INTRODUCTION}

Preterm birth, defined as childbirth $<37$ weeks gestation, is a leading cause of neonatal morbidity and mortality worldwide (Lawn et al., 2005; Beck et al., 2010; Liu et al., 2012). There are $\sim 15$ million preterm births annually, of which $\sim 1.1$ million neonates die from various complications; the highest cause of neonatal mortality worldwide (WHO, 2012). The causes of preterm birth are multifaceted, ranging from environmental, fetal or maternal abnormalities/compromise (Beck et al., 2010; Haas, 2011). Importantly, more than $60 \%$ of preterm infants $<28$ weeks gestation are exposed to chorioamnionitis (Lahra et al., 2007), making it the most prevalent antecedent of preterm delivery. 
The transition from intrauterine to extrauterine life relies on immediate and complex adaptations to effectively shift from maternal dependence to newborn physiological autonomy. In the first instance, a primary physiological driver for successful extrauterine transition is lung aeration (Hooper et al., 2015). Extensive adaptations such as the clearance of lung liquid, sufficient surfactant production, as well as changes in cardiovascular resistance and flow occur upon birth (Hillman et al., 2012). At this time, the brainstem must be fully functional as it contains vital respiratory centers that generate rhythm and coordinate breathing biomechanics (Garcia et al., 2011; Smith et al., 2013). Thus, the intrauterine to extrauterine transition is critical for neonates, and can have significant health consequences if this process is suboptimal.

Preterm infants frequently present with respiratory distress syndrome upon delivery (Fraser et al., 2004; Gallacher et al., 2016). Respiratory distress syndrome has been attributed to immature lung development, insufficient lung liquid clearance, and surfactant deficiency, which leads to poor respiration, apnoeic episodes and inadequate gas exchange (Fraser et al., 2004; Moss, 2006; Miall and Wallis, 2011; Polglase et al., 2014). As a result, preterm infants often require respiratory support following delivery, and subsequent ventilation upon transfer to the intensive care unit. Further, preterm infants exposed to infection/inflammation (chorioamnionitis) have a greater requirement for respiratory support and increased risk and severity of neurological damage than those not exposed to chorioamnionitis (Grether and Nelson, 1997; Bell and Hallenbeck, 2002; Duncan et al., 2002; Yanowitz et al., 2002; Mallard et al., 2003; Nitsos et al., 2006; Speer, 2006; Shatrov et al., 2010; Polglase et al., 2012; Galinsky et al., 2013). Despite extensive research demonstrating the link between systemic inflammation with intraventricular hemeorrhage, post-hemeorrhagic hydrocephalus and periventricular leukomalacia in the preterm brain (Heep et al., 2003; Hansen-Pupp et al., 2005; Kaukola et al., 2006; Moscuzza et al., 2011; Barton et al., 2014), the effects on the brainstem respiratory centers which regulate breathing remain largely unknown.

\section{NEURAL CONTROL OF RESPIRATION}

The neural circuitry responsible for generating and regulating eupneic respiratory rhythm are located within the brainstem (Rybak et al., 2007; Smith et al., 2009; Garcia et al., 2011). Eupneic breathing occurs in a three-phase rhythm consisting of inspiration, post-inspiration and active expiration (Smith et al., 2013). These respiratory phases are highly conserved in mammals and rely on the optimal functioning of central respiratory centers within the brainstem, which generate breathing rhythm, process and adapt to central and peripheral chemosensory information, as well as receiving afferent information from pulmonary stretch receptors, and provides and coordinates efferent innervation to the motor nerves and muscles supplying the lungs. In addition, these brainstem respiratory centers provide the neural drive for upper airway muscles important for maintaining airway patency. There are several pontomedullary respiratory centers distributed throughout the brainstem, which are critical for eupneic breathing (Figure 1).

\section{Pontine Respiratory Centers}

The dorsolateral pons contains the Kölliker-Fuse (KF) nucleus and the parabrachial (PB) complex (Alheid et al., 2004). Neurons from the $\mathrm{KF}$ nucleus and the $\mathrm{PB}$ complex can modulate respiratory phase alternation (inspiration, post-inspiration, active expiration) through synaptic inputs to medullary nuclei (Cohen and Shaw, 2004; Dutschmann and Herbert, 2006; Ezure and Tanaka, 2006; Martelli et al., 2013; Forster et al., 2014).

\section{Medullary Respiratory Centers}

The medulla oblongata comprises a collection of nuclei that are categorized into ventral and dorsal respiratory groups. The ventral respiratory groups consist of the preBötzinger complex (pBÖTC), BÖTC, retrotrapezoid nucleus $(\mathrm{RTN}) /$ parafacial respiratory group (pFRG), as well as the rostral and caudal ventral respiratory groups (cVRGs; Stornetta, 2008; Smith et al., 2009; Garcia et al., 2011). The dorsal respiratory group is comprised of the nucleus tractus solitarius (NTS)

Several studies have identified the pBÖTC located within the rostral medulla oblongata to be an essential site for respiratory rhythmogenesis (Smith et al., 1991; Ramirez et al., 1998; McKay et al., 2005). Neurons within the pBÖTC are capable of spontaneous, oscillating pace-maker-like activity which is imperative for respiratory rhythmogenesis and inspiratory drive (Smith et al., 1991; Butera et al., 1999; Koshiya and Smith, 1999; Morgado-Valle et al., 2010; Phillips et al., 2016).

The BÖTC is located within the rostral ventrolateral column of the medulla oblongata and predominantly contains inhibitory expiratory neurons (Fortuna et al., 2008). The inhibitory activity of Bötzinger neurons is important for proper phase-switching from inspiratory and expiratory activities (Smith et al., 2007, 2009).

The RTN/pFRG located in the rostral medulla oblongata contains central chemoreceptors that drive respiration in a $\mathrm{CO}_{2}$-dependant manner (Mulkey et al., 2004; Smith et al., 2006; Stornetta et al., 2006; Guyenet and Mulkey, 2010). RTN neurons respond to local tissue acidification (high extracellular $\mathrm{CO}_{2}$, or its proxy, hydrogen ions), paracrine influences (ATP) by $\mathrm{pH}$-sensitive astrocytes, as well as inputs from peripheral chemoreceptors (carotid and aortic bodies; information propagated by the NTS; Mulkey et al., 2004; Takakura et al., 2006; Guyenet et al., 2009; Lazarenko et al., 2009; Gourine et al., 2010; Huckstepp et al., 2010). Furthermore, the caudal raphè contains serotoninergic neurons which have demonstrated chemosensory properties (Smith et al., 2009, 2013). These neurons provide input to the RTN/pFRG, as well as the ventral respiratory column.

The rostral ventral respiratory group (rVRG) is largely comprised of excitatory inspiratory premotor neurons which provide neural input to spinal phrenic and intercostal motorneurons that innervate the diaphragm (Smith et al., 2009, 2013). The cVRG also contains excitatory expiratory premotor neurons which relay information to spinal thoracic 


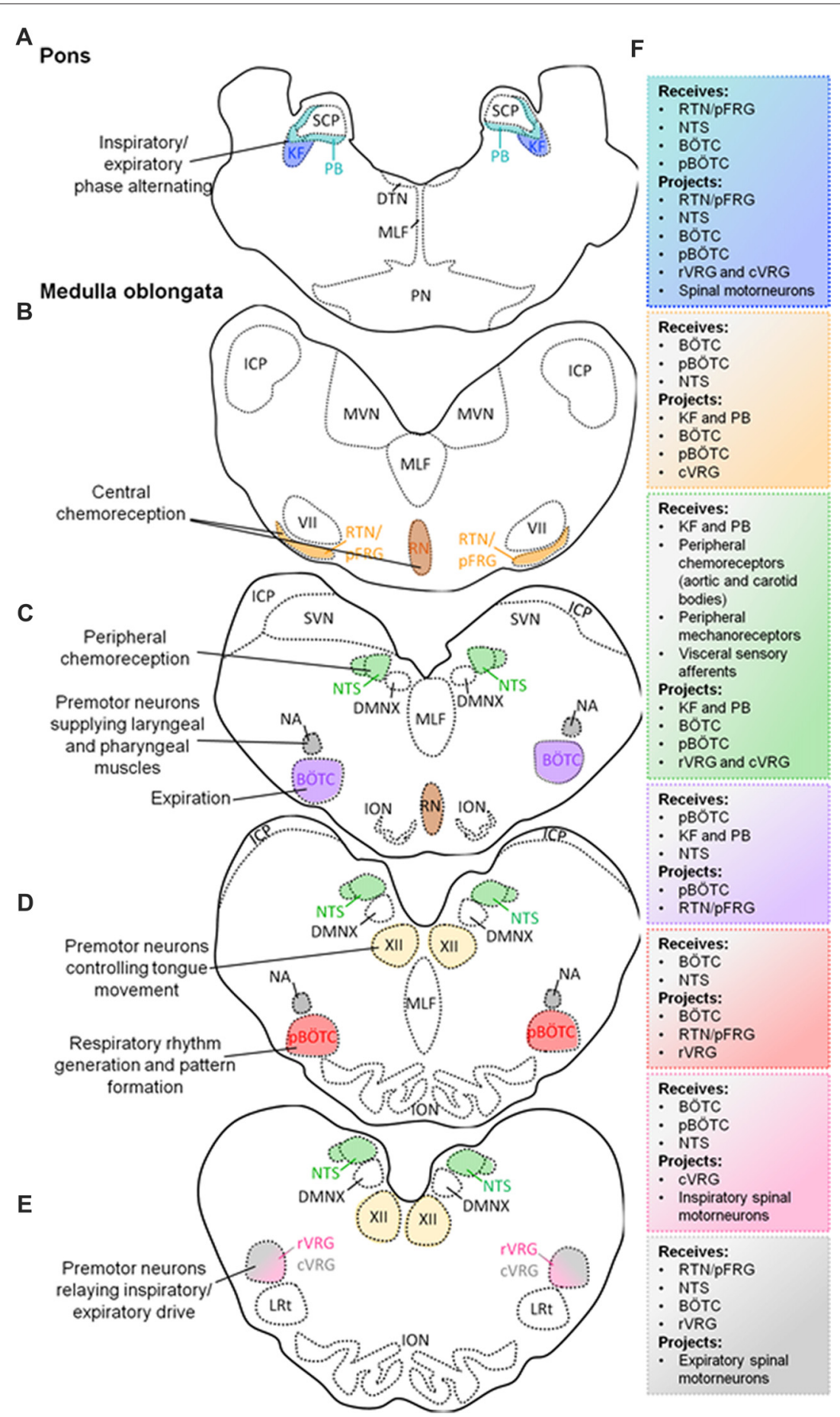

FIGURE 1 | Schematic diagram of transverse sections through the brainstem exposing the main respiratory centers. Localization of the Kölliker-Fuse (KF) and parabrachial (PB) nuclei in the pons, and their simplified functions (A). Localization of medullary respiratory centers: retrotrapezoid nucleus (RTN)/parafacial respiratory group (pFRG) nucleus and raphè nucleus (RN) (B); nucleus tractus solitarius (NTS), Bötzinger complex (BÖTC), nucleus ambiguus (NA; C); pre-BÖTC (pBÖTC), hypoglossal nucleus (XII; D); rostral ventral respiratory group (rVRG), caudal ventral respiratory group (cVRG; E), and their simplified functions. Proposed neural circuitry of the brainstem respiratory centers outlining potential interactions (receiving information from, and projecting to) within, and from the brainstem (F).

and lumbar motorneurons innervating the lungs (Stornetta, 2008; Smith et al., 2013). In addition to the rVRG and cVRG, the medulla oblongata contains other motor nuclei involved in respiratory-related functions. These motor nuclei include the hypoglossal nucleus (XII), and the nucleus ambiguus (NA). The XII nucleus is comprised of motor neurons supplying the 
tongue, and plays an important role in positioning this muscle (Bailey and Fregosi, 2004; Gestreau et al., 2005; Moore et al., 2014). Modulating the position of the tongue is particularly important for maintaining upper airway patency which is critical for acquiring oxygen. Moreover, the NA contains premotor laryngeal and pharyngeal motor neurons. These neurons innervate the larynx and pharynx, through which they are involved in maintaining glottal patency important for the flow of oxygen (Smith et al., 2009; Ludlow, 2015).

The dorsal respiratory group within the medulla oblongata is comprised of the NTS which contains second-order neurons that receive and process visceral sensory information conveyed by vagal afferent nerves (Smith et al., 2009; Zoccal et al., 2014). The NTS receives and integrates chemosensory information from aortic and carotid bodies, sensory information from slow-adapting and rapid-adapting pulmonary stretch receptors, and bronchopulmonary C-fibers (Machado et al., 2000; Kubin et al., 2006). Therefore, the NTS propagates peripheral chemosensory information to all major respiratory centers within the brainstem to evoke appropriate respiratory responses.

These respiratory centers develop early in gestation and continue to mature throughout pregnancy, as evidenced by the maturation of fetal breathing movements (FBMs).

\section{FETAL BREATHING MOVEMENTS}

Although the placenta is the site for respiratory gas exchange in utero, FBMs are detectable at 10 weeks gestational age (Boddy and Mantell, 1973; LoMauro and Aliverti, 2016). Whilst FBMs do not play a role in gas exchange, they do regulate the degree of lung liquid within the developing lungs, which is critical for normal tissue development and maturation, maintenance of intraluminal pressure and lung liquid volume, as well as the priming and entrainment of the respiratory muscles and neural circuitry for effective postnatal breathing (Harding, 1997; BagumaNibasheka et al., 2012; Koos and Rajaee, 2014). Contractions of the diaphragm, intercostal, and laryngeal muscles carry out the physical task of FBMs, however, these muscles are under the control of the brainstem respiratory centers, which generate and coordinate breathing patterns (Dawes, 1984; Harding, 1997; Greer et al., 2006; LoMauro and Aliverti, 2016).

The incidence of FBMs increases and becomes more episodic (Dawes et al., 1970, 1972; Bowes et al., 1981; Dawes, 1984) with gestational age from about $2 \%$ of time at 10 weeks, $6 \%$ at 19 weeks, $13.7 \%$ at $24-26$ weeks (Natale et al., 1988), $14.2 \%$ at 26-28 weeks (Natale et al., 1988) and to $31 \%$ of the time at 30 weeks. Between 30 weeks and 40 weeks, the mean incidence of FBMs remains at about $30 \%-40 \%$, although the differences between individual fetuses can range from $17 \%$ to $65 \%$ (Patrick et al., 1980; Bocking et al., 1982; Harding, 1994). The duration of time spent in apnea also increases, sometimes lasting for up to $2 \mathrm{~h}$ in humans at 38-39 weeks (Patrick et al., 1980). These changes in apnoeic periods correlate with changing/maturing patterns of FBMs which also reduce in frequency in late gestation, but increase their duration (Trudinger and Knight, 1980; Clewlow et al., 1983), mimicking breathing post-partum. The frequency of FBMs is highly variable between individuals. At $24-28$ weeks of gestation in humans, the rate of FBMs is $42-44$ breaths per minute (Natale et al., 1988), increases to 55-62 breaths per minute at 30-31 weeks of gestation (Patrick et al., 1980), and reduces again to $45-48$ breaths per minute at 38-39 weeks of gestation (Patrick et al., 1980).

FBMs are also regulated by a number of physiological factors. As in the adult, increases in the partial pressure of $\mathrm{CO}_{2}\left(\mathrm{P}_{\mathrm{a}} \mathrm{CO}_{2}\right.$; hypercapnia) profoundly stimulate FBMs (Connors et al., 1988), while hypocapnia reduces FBMs (Connors et al., 1988, 1989; Darnall, 2010). Acute hypoxia stimulates respiratory activity in adults, whereas it abolishes FBMs (Koos et al., 1986; LoMauro and Aliverti, 2016). Other factors which can influence FBMs include neurotransmitters, glucose (Natale, 1980), as well as inflammation and prostaglandins (Dong and Feldman, 1995; Herlenius et al., 1997; Olsson et al., 2003; Siljehav et al., 2014).

The changes to the ontogeny of FBMs throughout gestation reflect the maturation of the brainstem respiratory centers. However, being born preterm, particularly extremely preterm ( $<28$ weeks), coincides not only with an immature lung, but also an immature respiratory control center.

\section{PRETERM BIRTH: POTENTIAL EFFECTS ON NEUROCHEMICAL DEVELOPMENT IN THE BRAINSTEM AND RESPIRATORY-RELATED ACTIVITY}

Whilst autonomous neonatal breathing is critical for sustaining life, the brainstem respiratory centers are not completely mature at birth, and instead, continue to develop and refine postnatally (Wong-Riley and Liu, 2008). It is essential that there is sufficient prenatal development of the brainstem respiratory centers to a point of "readiness" at birth in order for the neonate to generate stable and continuous breathing movements (Carroll and Agarwal, 2010).

Preterm infants have demonstrated altered/abnormal ventilatory responses to hypoxic and hypercapnic conditions (Zhao et al., 2011). In hypoxic circumstances, preterm infants display an initial (albeit transient) rise in respiratory rate, alongside an increase in tidal volumes. However, this initial increase in respiration is then followed by a continuous decline in spontaneous breathing ("hypoxic ventilatory depression") which can last for several weeks, and is thought to play a role in delayed respiratory adjustments in postnatal life (Nock et al., 2004; Zhao et al., 2011). Ventilatory responses to hypercapnia by preterm infants are poor. Typical responses by preterm infants include prolonged expiration times, and a lack of increased respiratory rate, and low/insufficient tidal volumes. Consequently, this altered hypercapnic response results in low minute ventilation (Zhao et al., 2011). These insufficient hypoxic and hypercapnic responses likely reflect the immaturity of the brainstem and/or altered neuronal functions.

During the first 2 weeks of postnatal life the brainstem respiratory centers undergo dynamic developmental changes which have been associated with a large degree of neuronal maturation and neurochemical modifications (mostly studied in rodents; Wong-Riley and Liu, 2005). Preterm infants are 
not only forced to sustain continuous breathing movements with immature neural control (neuronal hypoplasia, poor synaptic connections and myelination), but the typical postnatal adjustments of neurotransmitters may not follow the same course as it would in full-term neonates (Stokowski, 2005). There are limited data on the neurochemical changes in the fetal brainstem as most studies have focused on differential expression in the embryonic and postnatal stages of development.

Glutamate is the principal neurotransmitter that drives inspiratory activity and the processing of sensory inputs, and its expression generally increases in rats postnatally, particularly in the pBÖTC and the NTS (Liu and Wong-Riley, 2005; Benarroch, 2007). In addition, studies in rats have shown that the expression of glutaminergic receptors (N-methyl-D-aspartate; NMDA; $\quad \alpha$-amino-3-hydroxy-5-methyl-4-isoxazoleproprionc acid (AMPA); and metabotropic glutaminergic receptors) also increase with age (Liu and Wong-Riley, 2005; Wong-Riley and Liu, 2005). It is unclear how preterm birth can impact the postnatal fluctuations in glutamate expression within the brainstem respiratory centers, and how this is associated with irregular neonatal breathing. But presumably, weak/immature glutaminergic synaptic transmission would alter chemosensory input, and breathing rhythmogenesis and pattern formation.

Gamma-aminobutyric acid (GABA) and glycine are inhibitory neurotransmitters which modulate inspiratory activity to allow for the proper transition to the expiratory phase. The developmental expression of GABA in rats has been shown to steadily decrease in the NTS, but increase in the pBÖTC (Liu and Wong-Riley, 2005), whereas glycine receptor immunoreactivity has been shown to increase with age in the pBÖTC and the NTS (Liu and Wong-Riley, 2005). In late embryonic and early postnatal life, GABAergic and glycinergic neurotransmission can be excitatory until a developmental shift from depolarizing to hyperpolarizing activity occurs. Preterm neonates display an enhanced sensitivity to GABA (amongst other neurotransmitters and neuromodulators including adenosine, serotonin, and prostaglandins) which can lead to respiratory depression (Martin et al., 2004; Zhao et al., 2011). Whether this respiratory depression is due to excessive excitatory or inhibitory activity remains to be determined. The effects of preterm birth on the proper switching of GABAergic and glycinergic neurotransmission (excitatory to inhibitory) in the brainstem respiratory neurons remains to be investigated.

Central chemosensitivity to $\mathrm{CO}_{2}$ within the brainstem is primarily mediated by cholinergic, serotoninergic, and glutaminergic neurotransmission. Acetylcholine is a neurotransmitter acting post-synaptically on inspiratory neurons within the pBÖTC (Burton and Kazemi, 2000; Lai et al., 2001; Shao and Feldman, 2009). It is currently unclear how preterm birth can affect the maturation of cholinergic neurons within brainstem respiratory centers, but this would presumably affect signaling of central chemosensory information. Serotoninergic neurons in the ventral surface of the medulla oblongata and the raphè nucleus $(\mathrm{RN})$ also respond to elevated $\mathrm{CO}_{2}$ and changes in blood pH (Richerson et al., 2001; Hilaire et al., 2010). These chemosensitive neurons continue to develop postnatally (as evidenced in rats; Davis et al., 2006).
Substance $\mathrm{P}$ is a neurotransmitter that can evoke inspiratory activity and propagate chemosensory information through its binding to neurokinin-1 receptors which are strongly expressed on rhythmogenic pre-Bötzinger neurons and central chemoreceptors in the RTN/pFRG (Gray et al., 2001; Nattie and Li, 2002; Shvarev et al., 2002). Research investigating the effects of preterm birth on serotoninergic and substance P-expressing neurons in the brainstem respiratory nuclei are lacking, but impaired development and/or maturation would presumably lead to inadequate breathing adaptations due to poor signaling to the pBÖTC, and other respiratory-related nuclei.

Adenosine is a neurotransmitter which exerts strong inhibitory activity of inspiratory neurons, and ultimately decreases inspiratory drive to phrenic motorneurons innervating the diaphragm (Dong and Feldman, 1995; Herlenius et al., 1997). Postnatal changes of adenosine expression in the brainstem respiratory centers remain unclear, and it is unknown how exactly preterm birth may affect the expression of this neurotransmitter or its respective receptors. However, it is well-established that preterm infants are particularly sensitive to adenosine, and this suggests that adenosine receptors have developed and matured to some degree.

Whilst there are some limited data describing the developmental changes in the brainstem respiratory centers, the effects of preterm birth remain incompletely understood. Additionally, it is well established that inflammation can alter neuronal function, and thus, delineating the effects of preterm birth and chorioamnionitis on the brainstem respiratory centers is challenging.

\section{CHORIOAMNIONITIS}

Infection and inflammation of the chorionic membrane and/or the amnion/amniotic fluid collectively refers to chorioamnionitis. This inflammatory condition is most commonly caused by a maternal ascending polymicrobial infection, and can be contracted by the fetus following exposure to amniotic fluid, or via placental-fetal circulation (Galinsky et al., 2013). A myriad of bacterial, viral and fungal species have been implicated in the pathogenesis of chorioamnionitis, with ureaplasmas being the most common organism isolated (Viscardi, 2010; Sweeney et al., 2016). Given the delayed diagnosis of chorioamnionitis (usually placental pathology days after birth), and insufficient therapeutic intervention to reduce inflammatory insult during gestation, the vital organs of the fetus can be significantly damaged.

Chorioamnionitis is known to induce "fetal inflammatory response syndrome", which is characterized by systemic inflammation, notably injuring the lungs and the brain (Polglase et al., 2012; Galinsky et al., 2013; Kallapur et al., 2014). Research has demonstrated that chorioamnionitis/lipopolysaccharide (LPS)-induced infection is strongly associated with inflammatory and hypoxia-mediated brain injury, observed in both infants and rodents (Yoon et al., 2000; Shalak et al., 2002; Grether et al., 2003; Yang et al., 2005; Dessardo et al., 2012; Ecevit et al., 2014). Chorioamnionitis can lead to neurodevelopmental abnormalities, and poor cognitive, behavioral and neuromotor 
outcomes in infants, and is implicated in $11 \%-22 \%$ of cerebral palsy (Murphy et al., 1995; Wu, 2002; Inder et al., 2003; Wu et al., 2003; Shatrov et al., 2010).

Furthermore, it is well known that infection and inflammation have the capacity to alter autoresuscitation ability and induce apnoeic episodes in neonates, as well as in rodents and piglets (Frøen et al., 2000; Kamaluddeen et al., 2009; Stock et al., 2010; Herlenius, 2011; Lorea-Hernández et al., 2016). Thus, it is unsurprising that chorioamnionitis is linked to sudden infant death syndrome, recurrent apnea in preterm infants, and obstructive sleep apnea syndrome in ex-preterm children (Kaufman and Fairchild, 2004; Weber et al., 2008; Zhao et al., 2011; Simonsen et al., 2014; Tapia et al., 2016).

Antenatal corticosteroids are currently used to accelerate lung maturation in preterm infants (Surbek et al., 2012; Freeman et al., 2015). Corticosteroids have also proven effective in reducing the severity of histological chorioamnionitis and minimizes respiratory distress syndrome (Surbek et al., 2012; Freeman et al., 2015). In addition, antenatal corticosteroids have also shown protective effects against necrotizing enterocolitis, intraventricular hemorrhage, major brain lesions, and periventricular leukomalacia (Surbek et al., 2012; Freeman et al., 2015). Studies investigating the effects of single or repeated corticosteroid use on auditory brainstem responses in preterm infants and animal models with and without chorioamnionitis have revealed conflicting results (Amin et al., 2003; Amin and Guillet, 2007; Church et al., 2012). Few human studies have demonstrated that corticosteroids have no effect on auditory brainstem responses (Amin et al., 2003; Amin and Guillet, 2007). Whereas in rats, repeated courses of corticosteroids negatively affected neural transmission time and auditory brainstem pathways (Church et al., 2012). Furthermore, repeated corticosteroid administration in fetal sheep leads to a reduction in cerebral weight without affecting the cerebellum and brainstem (Huang et al., 1999). Moreover, the effects of corticosteroids on the brainstem respiratory centers remain unknown. The exact mechanisms underlying the changes in respiratory function are not entirely understood, but emerging work highlights key roles for pro-inflammatory cytokines and prostaglandins in depressing respiratory function.

\section{Mechanisms of Chorioamnionitis and Brainstem Function}

Chorioamnionitis can be experimentally induced in animal models through intra-amniotic injections of LPS (cell wall constituent of gram-negative bacteria; Polglase et al., 2012; Barton et al., 2014; Ireland et al., 2015). This bacterial endotoxin gives robust and reproducible inflammatory responses, and as such, is widely used to experimentally induce chorioamnionitis.

LPS is a ligand for toll-like receptors (TLRs, namely TLR4), which stimulate downstream signaling pathways leading to pro-inflammatory cytokine production (Beutler, 2003; Lu et al., 2008). The activated TLR4 pathway results in interferonrelated cytokines, and can potentiate NF-кB gene transcription (Pålsson-McDermott and O'Neill, 2004). Ultimately, gene transcription leads to the production of IL- $1 \beta$, IL-6, IL-8, TNF- $\alpha$,

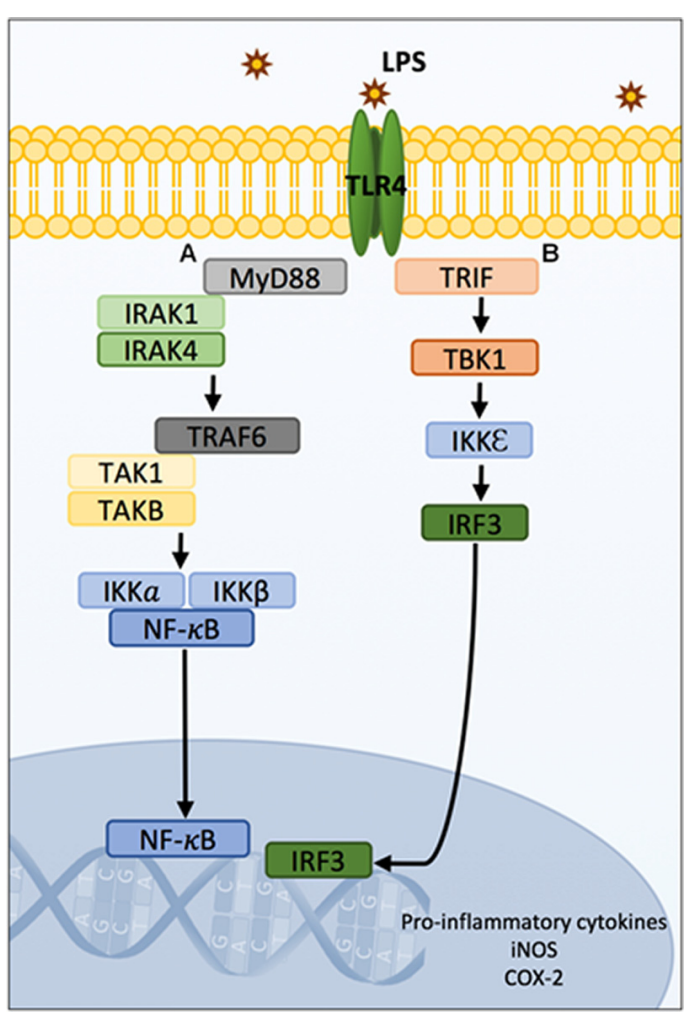

FIGURE 2 | Schematic diagram of TLR4 signaling pathways. MyD88 and TRIF-mediated LPS/TLR4 downstream signaling pathways leading to gene transcription of pro-inflammatory cytokines, iNOS and COX-2. The LPS/TLR4 signal transduction pathways are typically divided into MyD88-dependent and independent cascades. Following LPS stimulation, IRAK1 and IRAK4 are recruited to the MyD88-dependant pathway $(\mathbf{A})$ and interact with TRAF6 proteins. TRAF6 recruits TAK1 and TABs which activate the NF-кB and/or MAPK. In resting states, NF- $\mathrm{BB}$ is sequestered in the cytosol by IKK $\alpha$ and IKK $\beta$. Phosphorylation of the IKK complexes by TAK1 results in their proteasomal degradation and liberation of NF- $\mathrm{kB}$ which subsequently translocates from the cytosol to the nucleus where it can induce gene expression. Concurrently, TAK1 activates the MAPK pathway resulting in the phosphorylation and AP-1 which translocates to the nucleus and binds to DNA. Additionally, the LPS/TLR4 MyD88-independent signaling pathway involves the activation of TRIF (B) and signaling to TBK1, IKK and IRF3. This pathway results in interferon-related cytokines, and can potentiate NF-кB gene transcription. Ultimately, gene transcription leads to the production of IL-1 $\beta$, IL-6, IL-8, TNF- $\alpha$, TNF- $\beta$, iNOS and COX-2. Abbreviations: LPS, Lipopolysaccharide; TLR4, Toll-like receptor 4; MyD88, myeloid differentiation primary response gene 88; IRAK1 and IRAK4, interleukin 1-associated kinases-1 and 4; TRAF6, tumor necrosis factor associated factor 6; TAK1, transforming growth factor- $\beta$-activated kinase- 1 ; TABs, TAK1-binding proteins; MAPK, mitogen-activated protein kinases; IKK $\alpha$ and IKK $\beta$, inhibitory IkB kinases; AP-1, activation of the transcription factor activator protein 1; TRIF, TIR-domain-containing adapter-inducing interferon- $\beta$; TBK1,

TANK-binding kinase; IRF3, IKK, and interferon regulatory factor 3; iNOS, inducible nitric oxide synthase; COX-2, cyclooxygenase 2.

TNF- $\beta$, inducible nitric oxide synthase (iNOS), and inducible cyclooxygenase2 (COX-2; mediates prostaglandin synthesis; Figure 2; Blackwell and Christman, 1997; Poligone and Baldwin, 2001; Tak and Firestein, 2001; Aktan, 2004). In the brain, microglia and astrocytes express TLRs and can also play a major role in cytokine production (Kielian, 2006). 
Inflammatory Cytokines and Effects on the Brainstem LPS exposure upregulates IL- $1 \beta$ and IL- 6 mRNA expression within the brainstem of rat pups (Balan et al., 2012), which may in turn alter neuronal function within the $\mathrm{pBÖTC.}$ Electrophysiological recordings of pre-Bötzinger neurons from neonatal mice following intrauterine LPS administration has revealed functional changes of pacemaker neurons that are characterized as large amplitude bursts, at slow and irregular firing frequency (Ramirez et al., 2016). If IL-1 $\beta$ and IL-6 depress inhibitory synaptic transmission, and simultaneously elevate excitatory signaling in the pBÖTC, then this could explain the prolonged inspiratory drive and the absent expiratory activity that leads to apnea. Neuronal activity can be rapidly and differentially modulated by cytokines, and these functional changes may persist long-term (Vezzani and Viviani, 2015). The pro-inflammatory cytokines IL-1 $\beta$, IL- 6 and TNF- $\alpha$ can modulate neuronal function within the central nervous system, particularly by potentiating excitatory signaling, and depressing inhibitory transmission (Galic et al., 2012). These cytokines alter neuronal excitability through post-translational modifications of glutaminergic, GABAergic, and glycinergic receptors, ultimately affecting neurotransmission and synaptic plasticity (Galic et al., 2012; Vezzani and Viviani, 2015). Studies in rodents and in in vitro hippocampal neurons demonstrate that IL- $1 \beta$ alters neuronal function in a concentration-dependent manner; at low concentrations IL- $1 \beta$ inhibits voltage-gated calcium currents, and lowers intracellular calcium concentrations (thereby decreasing neurotransmitter release), whereas high concentrations of IL-1 $\beta$ increase ionotropic glutamate receptor expression (NMDA), and decreases transmission at GABAergic and glycinergic receptors in rat hippocampal and cerebellar cultures (Campbell and Lynch, 1998; Wang et al., 2000; Viviani et al., 2003; Galic et al., 2012). TNF- $\alpha$ alters neuronal excitability through the upregulation of NMDA and AMPA receptor expression, and induces GABA receptor endocytosis, as observed in the rat hippocampus and cerebellum (Beattie et al., 2002; Fourgeaud and Boulanger, 2010; Galic et al., 2012). This leads to an increase in excitatory output (and possibly excitotoxicity), as well as a decrease in inhibitory signaling. IL-6 has been shown to play both protective and destructive roles within the central nervous system. Research has shown that IL-6 decreases metabotropic glutamate receptor expression, but can also excessively activate NMDA receptors and induce excitotoxicity (D'Arcangelo et al., 2000; Conroy et al., 2004; Vereyken et al., 2007; Wang et al., 2007). Additionally, IL-6 has also demonstrated the capacity to decrease GABAergic and glycinergic neurotransmission in dorsal horn neurons of rat spinal cord (Kawasaki et al., 2008).

It is now well established that LPS-induced systemic infection/inflammation can alter breathing frequency and chemosensory responses, however the mechanisms underlying the changes in respiratory functions remain unclear (Huxtable et al., 2011). An imbalance between excitatory and inhibitory neurotransmission has been demonstrated previously in the brainstem of rats and piglets during hypoxic conditions (Kazemi and Hoop, 1991; Huang et al., 1994; McCormick et al., 1998; Hoop et al., 1999), but less is known about

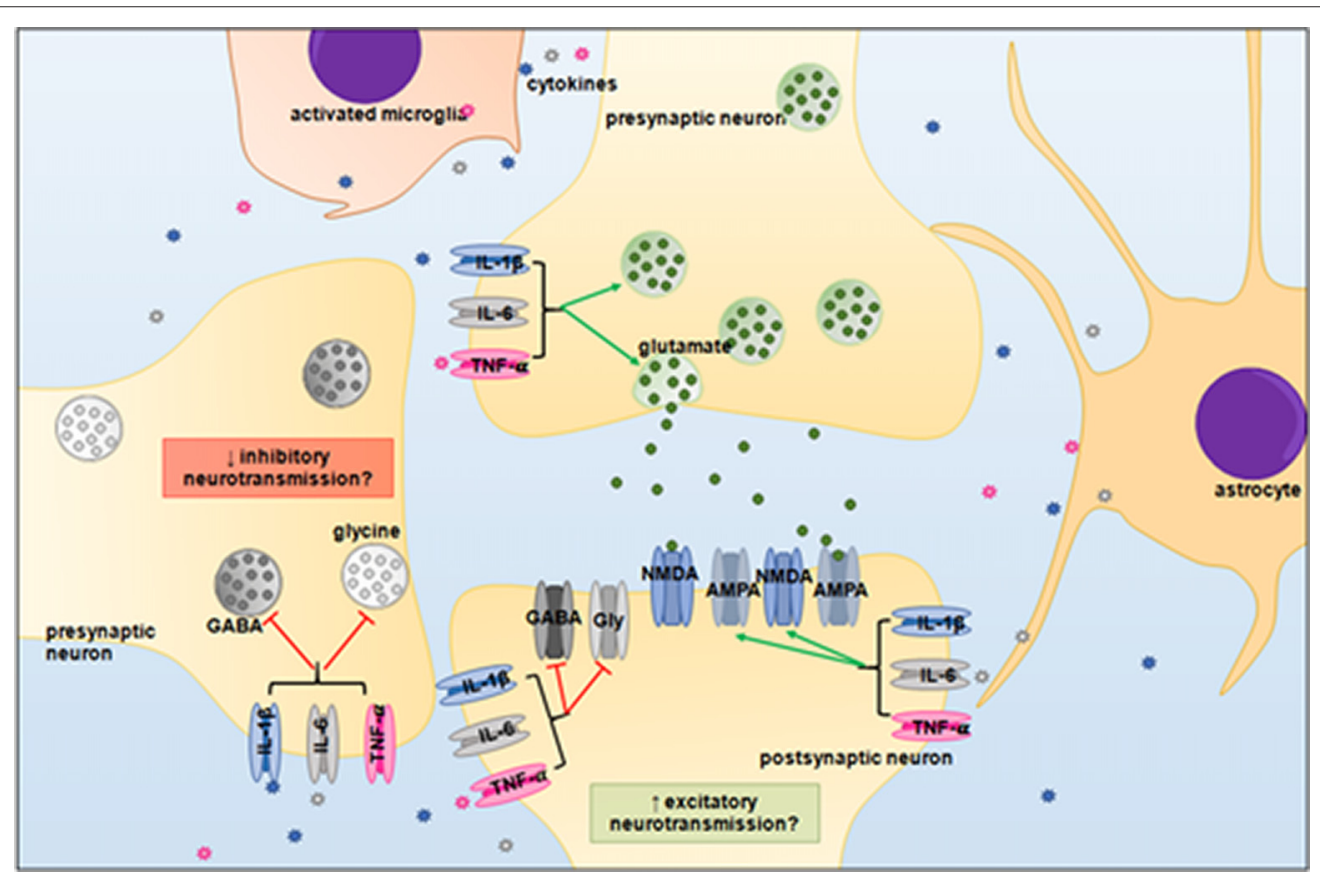

FIGURE 3 | Proposed neuromodulatory effects of pro-inflammatory cytokines in the brainstem. IL-1 $\beta$, IL-6 and TNF- $\alpha$ may upregulate glutaminergic receptor expression and potentiate excitatory signaling, whilst simultaneously depressing inhibitory GABAergic and glycinergic neurotransmission. An imbalance between excitatory and inhibitory signaling could desynchronize the neural circuitry of the brainstem respiratory centers. Abbreviations: GABA, gamma-Aminobutyric acid; Gly, glycine; NMDA, N-methyl-D-aspartate; AMPA, $\alpha$-amino-3-hydroxy-5-methyl-4-isoxazoleproprionc acid. 
the effects of inflammation on neurotransmitters within the brainstem. As neurons within the brainstem respiratory centers utilize glutamate, GABA and glycine, then pro-inflammatory cytokines could affect the balance of excitatory and inhibitory neurotransmitters, and thus, may alter neuronal function and respiratory responses (Figure 3).

Systemic administration of the pro-inflammatory cytokines IL-1 $\beta$, IL- 6 and TNF- $\alpha$ induces their own mRNA expression within the rat NTS (Churchill et al., 2006). IL-1 $\beta$ injection directly into the rat NTS increases inspiratory time as early as 20 min-post application, which is associated with delayed expiratory activity (observed after $80 \mathrm{~min}$; Gresham et al., 2011). Furthermore, the systemic administration of IL-1 $\beta$ leads to decreased respiratory frequency and induces apneustic episodes (Gresham et al., 2011). It is currently unclear whether IL-1 $\beta$ induces changes in excitatory neurotransmission of the NTS to modulate respiratory rhythm, or whether it does so by other downstream effects. Strong IL- $1 \beta$-immunoreactivity is observed in the rat NTS and area postrema following LPS exposure (Balan et al., 2011, 2012). Previous work has shown that the increase in IL-1 $\beta$ mRNA expression within the NTS can be abrogated by vagotomy (Balan et al., 2012).

Intraperitoneal and intrapulmonary injection of IL- $1 \beta$ to mouse and rat pups attenuates hypercapnic and hypoxic responses (Balan et al., 2012; Siljehav et al., 2014), indicative that chemosensory reflexes are impaired in response to inflammation. It is unclear whether altered chemosensory responses are due to compromised central chemoreceptor functions exclusively (RTN/pFRG neurons), or whether there are changes to vagal afferent signaling to the NTS, and/or impaired propagation of peripheral chemosensory information to brainstem respiratory centers by second-order NTS neurons. It is likely that infection/inflammation would induce considerable changes to both central and peripheral chemoreflexes.

In addition to IL- $1 \beta$ expression in the NTS, strong immunoreactivity is also observed in the area postrema following LPS exposure (Balan et al., 2011, 2012). Most research to date has attributed the loss of blood brain barrier integrity as the main pathway for brain inflammation and injury following systemic endotoxin or cytokine exposure. However, the NTS forms connections with the area postrema which is a circumventricular region of the brainstem that could be another entrance for peripheral inflammation.

LPS administration induces c-Fos immunoreactivity in neurons from the rostral ventrolateral medulla, NTS, and the $\mathrm{KF} / \mathrm{PB}$ nuclei in the rat (Zhang et al., 2000). This suggests that neurons from these respiratory nuclei are responsive to infection/inflammation, but the exact mechanisms for how (and which) inflammatory mediators affect the functions of respiratory-related neurons remain unclear.

\section{Prostaglandins and Effects on the Brainstem}

In addition to gene transcription of pro-inflammatory cytokines, it is well-established that NF- $\mathrm{B}$ and mitogenactivated protein kinases (MAPK) signaling can enhance COX isozyme expression leading to elevated prostaglandin synthesis
(Ricciotti and FitzGerald, 2011). The two main isoforms of COX are COX-1 and COX-2. COX-1 is constitutively expressed, whilst COX-2 is induced upon tissue injury and inflammation. COX isozymes convert arachidonic acid to the precursor substrate prostaglandin $\mathrm{H} 2\left(\mathrm{PGH}_{2}\right.$; Poligone and Baldwin, 2001; Simmons et al., 2004). $\mathrm{PGH}_{2}$ is utilized for the synthesis of prostaglandin E2 $\left(\mathrm{PGE}_{2}\right)$, prostaglandin $\mathrm{D} 2$, prostaglandin F2 $\alpha$, and prostacyclin (Ricciotti and FitzGerald, 2011). Specifically, microsomal prostaglandin E2 synthase-1 (mPEGS-1) catalyzes the synthesis of $\mathrm{PGE}_{2}$ from $\mathrm{PGH}_{2}$ (Murakami et al., 2000; Bahia et al., 2014). There is increasing evidence that COX-2-mediated $\mathrm{PGE}_{2}$ production plays a role in inflammation-induced preterm and neonatal brain injury, and altered function of respiratory-related neurons (Malaeb and Dammann, 2009; Fathali et al., 2010; Strunk et al., 2014; Jin et al., 2015). Similar to pro-inflammatory cytokines, $\mathrm{PGE}_{2}$ can alter neuronal excitability and neurotransmission. $\mathrm{PGE}_{2}$ has been shown to both enhance and inhibit glutaminergic transmission, depress glycingeric signaling, and modulate GABAergic receptor expression, and although the exact mechanisms remain unclear, neuromodulation is thought to be dependent on the type of eicosanoid prostaglandin PGE2 receptors (EPRs) stimulated (Ahmadi et al., 2002; Chen and Bazan, 2005; Laaris and Weinreich, 2007; Marty et al., 2008; Lin et al., 2014; Yang et al., 2015).

$\mathrm{PGE}_{2}$ has been associated with irregular breathing movements in vivo, as well as inhibitory effects on brainstem centers generating respiratory rhythm and chemosensory responses in vitro in slices generated from rat and mouse pups (Hofstetter et al., 2007; Siljehav et al., 2014; Forsberg et al., 2016). Moreover, $\mathrm{PGE}_{2}$ has been shown to depress FBMs, induce hypoventilation, reduce respiratory frequency and cause apnea in fetal sheep (Kitterman et al., 1983; Guerra et al., 1988). When directly injected into the mouse pBÖTC at a low concentration $(<200 \mathrm{nM}), \mathrm{PGE}_{2}$ increases sigh frequency with no apparent effects on eupneic breathing (Koch et al., 2015). High concentrations of $\mathrm{PGE}_{2}$, however, are shown to promote eupneic breathing (Koch et al., 2015). Conflicting results suggest multiple roles for $\mathrm{PGE}_{2}$ in the brainstem respiratory centers, and that there may be differential effects on breathing which may be context dependent. COX inhibition by indomethacin has been shown to stimulate breathing movements in fetal sheep which strengthens the notion that prostaglandins modulate respiratory activity (Jansen et al., 1984).

$\mathrm{PGE}_{2}$ is implicated in a number of neuropathological conditions which may be due to its capacity to bind to several G-protein coupled receptors. These include the EPRs 1-4 (EP1R, EP2R, EP3R, EP4R). Binding to these receptors can result in distinct signaling pathways (Figure 4). These signaling pathways may induce neuronal damage, dysfunction, or protection and may also potentiate or ameliorate inflammation, and alter cerebral blood flow.

$\mathrm{PGE}_{2}$ binding to EP1R leads to increases in intracellular calcium. Alterations in calcium homeostasis as a result of EP1R signaling has been associated with excitotoxicity and neuronal death in mice (Kawano et al., 2006; Shimamura et al., 2013). Furthermore, EP1R activation 


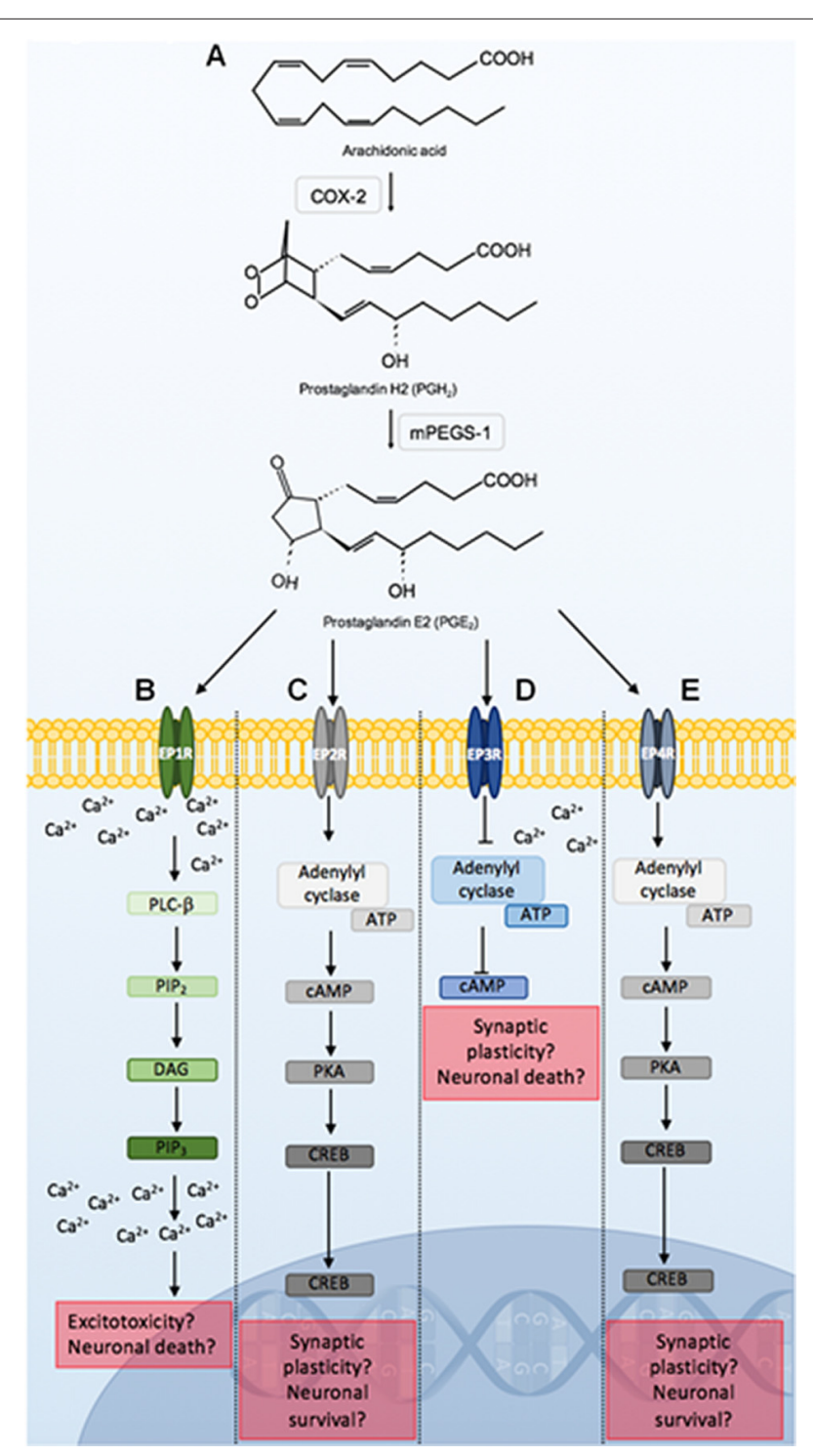

FIGURE 4 | Prostaglandin E2 ( $\left.\mathrm{PGE}_{2}\right)$ signaling through EPRs 1-4. COX-2 conversion of arachidonic acid to $P \mathrm{PH}_{2}$, is utilized by mPEGS-1 to synthesize $\mathrm{PGE}_{2}$ (A). $\mathrm{PGE}_{2}$ binding to EPRs initiates distinct signaling pathways that may lead to alterations in neuronal function, or result in neuroprotection or death. $\mathrm{PGE}_{2}$ ligation to EP1R leads to PLC- $\beta$ activation, which hydrolyzes $\mathrm{PIP}_{2}$, DAG and $\mathrm{PIP}_{3}$. $\mathrm{PIP}_{3}$ binds to respective receptors on the endoplasmic reticulum leading to further increases in intracellular calcium. Alterations in calcium homeostasis as a result of EP1R signaling can lead to excitotoxicity and neuronal death (B). PGE 2 binding to the EP2R initiates adenylyl cyclase activation of CAMP and PKA. PKA activates CREB which is a major transcription factor that can lead to synaptic plasticity, and neuroprotection (C). PGE 2 binding to EP3R inhibits ATP catalyzation by adenylyl cyclase, causing a reduction in CAMP, as well as an increase in intracellular calcium. This can modulate neuronal excitability and firing rate, and lead to cell death. $\mathrm{PGE}_{2}$ signaling through the EP4R is similar to the EP2R pathway (D). PGE2 binding to EP4R results in similar signaling cascades observed following EP2R stimulation (E). Abbreviations: EP1-4, eicosanoid prostanoid receptors 1-4; PLC- $\beta$, phospholipase C- $\beta$; PIP 2 , phosphatidylinositol-4,5-biphosphate; DAG, Diacylglycerol; $\mathrm{PIP}_{3}$, inositol-1,4,5-triphosphate; ATP, adenosine triphosphate; CAMP, cyclic adenosine monophosphate; PKA, protein kinase A; CREB, cAMP-response element binding; mPEGS-1, microsomal prostaglandin E2 synthase-1. stimulates vasoconstriction which can limit cerebral blood flow and potentiate hypoxic-ischemic events. In a neonatal rat model of hypoxic-ischemic encephalopathy, a selective EP1R antagonist significantly reduces cerebral injury (Taniguchi et al., 2011). The role of EP1R in chorioamnionitis-induced brainstem injury remains unknown.

$\mathrm{PGE}_{2}$ binding to EP2R leads to cAMP-response element binding (CREB) activation which is a major transcription factor that can induce synaptic plasticity, and neuroprotection (Carlezon et al., 2005; Kalinski, 2011; Liang et al., 2011; Sakamoto et al., 2011). PGE 2 binding to the EP2R has demonstrated neuroprotective effects in states of cerebral ischemia and excitotoxicity in the rat, and in mouse models (EP2R knockout, and middle cerebral artery occlusion; McCullough et al., 2004; Li et al., 2008). However, upon LPS exposure, COX-2 and iNOS-mediated neurotoxicity is abrogated by microglial EP2R deletion (Shie et al., 2005). It appears that the effects of $\mathrm{PGE}_{2}$ ligation to EP2R may be context dependent, but in circumstances of systemic inflammation, it is presumed that activation of the EP2R may play a deleterious role in the brainstem.

EP3R has the greatest affinity for $\mathrm{PGE}_{2}$ and has previously been implicated in neuroinflammation and neuronal dysfunction (Nakamura et al., 2000; Hofstetter et al., 2007; Hein and O'Banion, 2009; Leclerc et al., 2015). PGE 2 binding to EP3R leads to a reduction in cyclic adenosine monophosphate (cAMP), and an increase in intracellular calcium which can affect neuronal excitability and firing rate (Bos et al., 2004; Mohan et al., 2012). Downstream IL- $1 \beta$-mediated $\mathrm{PGE}_{2}$ production and subsequent binding to EP3R has been shown to reduce excitatory vagal neurotransmission to the NTS in rats (Marty et al., 2008). This would likely impact peripheral chemosensory input to the brainstem.

EP4R is highly expressed within the hypothalamus and brainstem, and functions similarly to EP2R (Andreasson, 2010; Taniguchi et al., 2014). $\mathrm{PGE}_{2}$ signaling through the EP4R is similar to the EP2R pathway as it offers neuroprotection in models of excitotoxic or hypoxic-ischemic injury, however, it may can also modulate pro-inflammatory and anti-inflammatory responses, as observed in the rat, and in mouse EP4R knockout studies (Zhang and Rivest, 1999; Andreasson, 2010; Shi et al., 2010). EP4R has been localized to the NTS and ventrolateral medulla of the brainstem, and its expression appears to increase following systemic IL-1 $\beta$ administration (Zhang and Rivest, 2000). However, further work is required to determine its role in chorioamnionitis-associated brainstem injury.

\section{CONCLUSION}

Preterm birth is associated with suboptimal development of the brainstem, and subsequently reduced respiratory control. Further, chorioamnionitis is strongly associated with preterm birth, and leads to an increased risk and severity of respiratory complications. There is extensive research on chorioamnionitisinduced white matter brain injury, however, the effects 
of inflammation on the brainstem, which contains central respiratory centers, remains unclear. Inflammation is associated with elevated prostaglandin synthesis, and $\mathrm{PGE}_{2}$ specifically has been shown to cause functional changes of respiratoryrelated neurons within the brainstem. Dysregulation and damage to these brainstem centers may be implicated in the multifaceted pathophysiology of respiratory depression in preterm neonates. Understanding how chorioamnionitis may affect these central respiratory centers could lead to effective therapeutic interventions within the delivery room, with the goal of reducing brain injury and preserving the neural circuitry controlling rhythmic and coordinated respiratory functions.

\section{REFERENCES}

Ahmadi, S., Lippross, S., Neuhuber, W. L., and Zeilhofer, H. U. (2002). PGE2 selectively blocks inhibitory glycinergic neurotransmission onto rat superficial dorsal horn neurons. Nat. Neurosci. 5, 34-40. doi: 10.1038/nn778

Aktan, F. (2004). iNOS-mediated nitric oxide production and its regulation. Life Sci. 75, 639-653. doi: 10.1016/j.lfs.2003.10.042

Alheid, G. F., Milsom, W. K., and McCrimmon, D. R. (2004). Pontine influences on breathing: an overview. Respir. Physiol. Neurobiol. 143, 105-114. doi: 10.1016/j.resp.2004.06.016

Amin, S. B., and Guillet, R. (2007). Auditory neural maturation after exposure to multiple courses of antenatal betamethasone in premature infants as evaluated by auditory brainstem response. Pediatrics 119, 502-508. doi: 10.1542/peds. 2006-2174

Amin, S. B., Orlando, M. S., Dalzell, L. E., Merle, K. S., and Guillet, R. (2003). Brainstem maturation after antenatal steroids exposure in premature infants as evaluated by auditory brainstem-evoked response. J. Perinatol. 23, 307-311. doi: 10.1038/sj.jp.7210898

Andreasson, K. (2010). Emerging roles of PGE2 receptors in models of neurological disease. Prostaglandins Other Lipid Mediat. 91, 104-112. doi: 10.1016/j.prostaglandins.2009.04.003

Baguma-Nibasheka, M., Gugic, D., Saraga-Babic, M., and Kablar, B. (2012). Role of skeletal muscle in lung development. Histol. Histopathol. 27, 817-826. doi: 10.14670/HH-27.817

Bahia, M. S., Katare, Y. K., Silakari, O., Vyas, B., and Silakari, P. (2014). Inhibitors of microsomal prostaglandin $\mathrm{E}_{2}$ synthase-1 enzyme as emerging anti-inflammatory candidates. Med. Res. Rev. 34, 825-855. doi: 10.1002/med. 21306

Bailey, E. F., and Fregosi, R. F. (2004). Coordination of intrinsic and extrinsic tongue muscles during spontaneous breathing in the rat. J. Appl. Physiol. 96, 440-449. doi: 10.1152/japplphysiol.00733.2003

Balan, K. V., Kc, P., Hoxha, Z., Mayer, C. A., Wilson, C. G., and Martin, R. J. (2011). Vagal afferents modulate cytokine-mediated respiratory control at the neonatal medulla oblongata. Respir. Physiol. Neurobiol. 178, 458-464. doi: 10.1016/j. resp. 2011.03.003

Balan, K. V., Kc, P., Mayer, C. A., Wilson, C. G., Belkadi, A., and Martin, R. J. (2012). Intrapulmonary lipopolysaccharide exposure upregulates cytokine expression in the neonatal brainstem. Acta Paediatr. 101, 466-471. doi: 10.1111/j.1651-2227.2011.02564.x

Barton, S. K., Moss, T. J. M., Hooper, S. B., Crossley, K. J., Gill, A. W., Kluckow, M., et al. (2014). Protective ventilation of preterm lambs exposed to acute chorioamnionitis does not reduce ventilation-induced lung or brain injury. PLoS One 9:e112402. doi: 10.1371/journal.pone.0112402

Beattie, E. C., Stellwagen, D., Morishita, W., Bresnahan, J. C., Ha, B. K., Von Zastrow, M., et al. (2002). Control of synaptic strength by glial TNF $\alpha$. Science 295, 2282-2285. doi: 10.1126/science.1067859

Beck, S., Wojdyla, D., Say, L., Betran, A. P., Merialdi, M., Requejo, J. H., et al. (2010). The worldwide incidence of preterm birth: a systematic review of maternal mortality and morbidity. Bull. World Health Organ. 88, 31-38. doi: 10.2471/BLT.08.062554

\section{AUTHOR CONTRIBUTIONS}

VS: wrote the manuscript and made figures. SLM, SBH and GRP: revised the manuscript.

\section{FUNDING}

This research was supported by a National Health and Medical Research Council (NHMRC) and National Heart Foundation Research Fellowship (GRP:1105526), a NHMRC fellowship (SBH:545921), an Australian Research Council Future Fellowship (SLM:FT130100650) and the Victorian Government's Operational Infrastructure Support Program.

Bell, M. J., and Hallenbeck, J. M. (2002). Effects of intrauterine inflammation on developing rat brain. J. Neurosci. Res. 70, 570-579. doi: 10.1002/jnr.10423

Benarroch, E. E. (2007). Brainstem respiratory control: substrates of respiratory failure of multiple system atrophy. Mov. Disord. 22, 155-161. doi: 10.1002/mds. 21236

Beutler, B. (2003). Innate immune responses to microbial poisons: discovery and function of the Toll-like receptors. Annu. Rev. Pharmacol. Toxicol. 43, 609-628. doi: 10.1146/annurev.pharmtox.43.100901.135729

Blackwell, T. S., and Christman, J. W. (1997). The role of nuclear factor-кB in cytokine gene regulation. Am. J. Respir. Cell Mol. Biol. 17, 3-9. doi: 10.1165/ajrcmb.17.1.f132

Bocking, A., Adamson, L., Cousin, A., Campbell, K., Carmichael, L., Natale, R., et al. (1982). Effects of intravenous glucose injections on human fetal breathing movements and gross fetal body movements at 38 to 40 weeks' gestational age. Am. J. Obstet. Gynecol. 142, 606-611. doi: 10.1016/s0002-9378(16)32427-9

Boddy, K., and Mantell, C. (1973). Human foetal breathing in utero. J. Physiol. 231, 105P-106P.

Bos, C. L., Richel, D. J., Ritsema, T., Peppelenbosch, M. P., and Versteeg, H. H. (2004). Prostanoids and prostanoid receptors in signal transduction. Int. J. Biochem. Cell Biol. 36, 1187-1205. doi: 10.1016/j.biocel.2003.08.006

Bowes, G., Adamson, T. M., Ritchie, B. C., Dowling, M., Wilkinson, M. H., and Maloney, J. E. (1981). Development of patterns of respiratory activity in unanesthetized fetal sheep in utero. J. Appl. Physiol. Respir. Environ. Exerc. Physiol. 50, 693-700. doi: 10.1152/jappl.1981.50.4.693

Burton, M. D., and Kazemi, H. (2000). Neurotransmitters in central respiratory control. Respir. Physiol. 122, 111-121. doi: 10.1016/s0034-5687(00) 00153-5

Butera, R. J. Jr., Rinzel, J., and Smith, J. C. (1999). Models of respiratory rhythm generation in the pre-Bötzinger complex. I. Bursting pacemaker neurons. J. Neurophysiol. 82, 382-397. doi: 10.1152/jn.1999.82.1.382

Campbell, V., and Lynch, M. A. (1998). Biphasic modulation of intracellular $\mathrm{Ca}^{2+}$ concentration by interleukin- $1 \beta$ in cortical synaptosomes: involvement of a pertussis toxin-sensitive G-protein and mitogen-activated protein kinase. Neuroreport 9, 1923-1927. doi: 10.1097/00001756-199806220-00002

Carlezon, W. A. Jr., Duman, R. S., and Nestler, E. J. (2005). The many faces of CREB. Trends Neurosci. 28, 436-445. doi: 10.1016/j.tins.2005.06.005

Carroll, J. L., and Agarwal, A. (2010). Development of ventilatory control in infants. Paediatr. Respir. Rev. 11, 199-207. doi: 10.1016/j.prrv.2010. 06.002

Chen, C., and Bazan, N. G. (2005). Endogenous PGE2 regulates membrane excitability and synaptic transmission in hippocampal CA1 pyramidal neurons. J. Neurophysiol. 93, 929-941. doi: 10.1152/jn.00696.2004

Church, M., Adams, B., Anumba, J., Jackson, D., Kruger, M., and Jen, K. (2012). Repeated antenatal corticosteroid treatments adversely affect neural transmission time and auditory thresholds in laboratory rats. Neurotoxicol. Teratol. 34, 196-205. doi: 10.1016/j.ntt.2011.09.004

Churchill, L., Taishi, P., Wang, M., Brandt, J., Cearley, C., Rehman, A., et al. (2006) Brain distribution of cytokine mRNA induced by systemic administration of interleukin-1 $\beta$ or tumor necrosis factor $\alpha$. Brain Res. 1120, 64-73. doi: 10.1016/j.brainres.2006.08.083 
Clewlow, F., Dawes, G. S., Johnston, B. M., and Walker, D. W. (1983). Changes in breathing, electrocortical and muscle activity in unanaesthetized fetal lambs with age. J. Physiol. 341, 463-476. doi: 10.1113/jphysiol.1983.sp014817

Cohen, M. I., and Shaw, C.-F. (2004). Role in the inspiratory off-switch of vagal inputs to rostral pontine inspiratory-modulated neurons. Respir. Physiol. Neurobiol. 143, 127-140. doi: 10.1016/j.resp.2004.07.017

Connors, G., Hunse, C., Carmichael, L., Natale, R., and Richardson, B. (1988). The role of carbon dioxide in the generation of human fetal breathing movements. Am. J. Obstet. Gynecol. 158, 322-327. doi: 10.1016/0002-9378(88)90147-0

Connors, G., Hunse, C., Carmichael, L., Natale, R., and Richardson, B. (1989). Control of fetal breathing in the human fetus between 24 and 34 weeks' gestation. Am. J. Obstet. Gynecol. 160, 932-938. doi: 10.1016/00029378(89)90313-x

Conroy, S. M., Nguyen, V., Quina, L. A., Blakely-Gonzales, P., Ur, C., Netzeband, J. G., et al. (2004). Interleukin-6 produces neuronal loss in developing cerebellar granule neuron cultures. J. Neuroimmunol. 155, 43-54. doi: 10.1016/j.jneuroim.2004.06.014

D'Arcangelo, G., Tancredi, V., Onofri, F., D'Antuono, M., Giovedì, S., and Benfenati, F. (2000). Interleukin-6 inhibits neurotransmitter release and the spread of excitation in the rat cerebral cortex. Eur. J. Neurosci. 12, 1241-1252. doi: 10.1046/j.1460-9568.2000.00011.x

Darnall, R. A. (2010). The role of $\mathrm{CO}_{2}$ and central chemoreception in the control of breathing in the fetus and the neonate. Respir. Physiol. Neurobiol. 173, 201-212. doi: 10.1016/j.resp.2010.04.009

Davis, S. E., Solhied, G., Castillo, M., Dwinell, M., Brozoski, D., and Forster, H. V. (2006). Postnatal developmental changes in $\mathrm{CO}_{2}$ sensitivity in rats. J. Appl. Physiol. 101, 1097-1103. doi: 10.1152/japplphysiol.003 78.2006

Dawes, G. S. (1984). The central control of fetal breathing and skeletal muscle movements. J. Physiol. 346, 1-18. doi: 10.1113/jphysiol.1984.sp015003

Dawes, G. S., Fox, H. E., Leduc, B. M., Liggins, G. C., and Richards, R. T. (1970). Respiratory movements and paradoxical sleep in the foetal lamb. J. Physiol. 210, $47 \mathrm{P}-48 \mathrm{P}$.

Dawes, G. S., Fox, H. E., Leduc, B. M., Liggins, G. C., and Richards, R. T. (1972). Respiratory movements and rapid eye movement sleep in the foetal lamb. J. Physiol. 220, 119-143. doi: 10.1113/jphysiol.1972.sp009698

Dessardo, N. S., Mustać, E., Dessardo, S., Banac, S., Peter, B., Finderle, A., et al. (2012). Chorioamnionitis and chronic lung disease of prematurity: a path analysis of causality. Am. J. Perinatol. 29, 133-140. doi: 10.1055/s-00311295654

Dong, X. W., and Feldman, J. L. (1995). Modulation of inspiratory drive to phrenic motoneurons by presynaptic adenosine A1 receptors. J. Neurosci. 15, 3458-3467.

Duncan, J. R., Cock, M. L., Scheerlinck, J. P., Westcott, K. T., McLean, C., Harding, R., et al. (2002). White matter injury after repeated endotoxin exposure in the preterm ovine fetus. Pediatr. Res. 52, 941-949. doi: 10.1203/00006450-200212000-00021

Dutschmann, M., and Herbert, H. (2006). The kölliker-fuse nucleus gates the postinspiratory phase of the respiratory cycle to control inspiratory off-switch and upper airway resistance in rat. Eur. J. Neurosci. 24, 1071-1084. doi: 10.1111/j.1460-9568.2006.04981.x

Ecevit, A., Anuk-İnce, D., Yapakci, E., Kupana-Ayva, S., Kurt, A., Yanik, F. F., et al. (2014). Association of respiratory distress syndrome and perinatal hypoxia with histologic chorioamnionitis in preterm infants. Turk. J. Pediatr. 56, 56-61.

Ezure, K., and Tanaka, I. (2006). Distribution and medullary projection of respiratory neurons in the dorsolateral pons of the rat. Neuroscience 141, 1011-1023. doi: 10.1016/j.neuroscience.2006.04.020

Fathali, N., Ostrowski, R. P., Lekic, T., Jadhav, V., Tong, W., Tang, J., et al. (2010). Cyclooxygenase-2 inhibition provides lasting protection against neonatal hypoxic-ischemic brain injury. Crit. Care Med. 38, 572-578. doi: 10.1097/ccm. 0b013e3181cb1158

Forsberg, D., Horn, Z., Tserga, E., Smedler, E., Silberberg, G., Shvarev, Y., et al. (2016). $\mathrm{CO}_{2}$-evoked release of PGE2 modulates sighs and inspiration as demonstrated in brainstem organotypic culture. Elife 5:e14170. doi: 10.7554/elife.14170

Forster, H., Bonis, J., Krause, K., Wenninger, J., Neumueller, S., Hodges, M., et al. (2014). Contributions of the pre-Bötzinger complex and the Köllikerfuse nuclei to respiratory rhythm and pattern generation in awake and sleeping goats. Prog. Brain Res. 209, 73-89. doi: 10.1016/B978-0-444-63274-6. 00005-9

Fortuna, M. G., West, G. H., Stornetta, R. L., and Guyenet, P. G. (2008). Botzinger expiratory-augmenting neurons and the parafacial respiratory group. J. Neurosci. 28, 2506-2515. doi: 10.1523/jneurosci.5595-07.2008

Fourgeaud, L., and Boulanger, L. M. (2010). Role of immune molecules in the establishment and plasticity of glutamatergic synapses. Eur. J. Neurosci. 32, 207-217. doi: 10.1111/j.1460-9568.2010.07342.x

Fraser, J., Walls, M., and McGuire, W. (2004). Respiratory complications of preterm birth. BMJ 329, 962-965. doi: 10.1136/bmj.329.7472.962

Freeman, C. I., Hezelgrave, N. L., and Shennan, A. H. (2015). Antenatal steroids for fetal lung maturity: time to target more frequent doses to fewer women? Obstet. Med. 8, 172-176. doi: 10.1177/1753495x15601772

Frøen, J. F., Akre, H., Stray-Pedersen, B., and Saugstad, O. D. (2000). Adverse effects of nicotine and interleukin- $1 \beta$ on autoresuscitation after apnea in piglets: implications for sudden infant death syndrome. Pediatrics 105:E52. doi: 10.1542/peds.105.4.e52

Galic, M. A., Riazi, K., and Pittman, Q. J. (2012). Cytokines and brain excitability. Front. Neuroendocrinol. 33, 116-125. doi: 10.1016/j.yfrne.2011.12.002

Galinsky, R., Polglase, G. R., Hooper, S. B., Black, M. J., and Moss, T. J. M. (2013). The consequences of chorioamnionitis: preterm birth and effects on development. J. Pregnancy 2013:412831. doi: 10.1155/2013/412831

Gallacher, D. J., Hart, K., and Kotecha, S. (2016). Common respiratory conditions of the newborn. Breathe 12, 30-42. doi: 10.1183/20734735.000716

Garcia, A. J., Zanella, S., Koch, H., Doi, A., and Ramirez, J. M. (2011). Networks within networks: the neuronal control of breathing. Prog. Brain Res. 188, 31-50. doi: 10.1016/B978-0-444-53825-3.00008-5

Gestreau, C., Dutschmann, M., Obled, S., and Bianchi, A. L. (2005). Activation of XII motoneurons and premotor neurons during various oropharyngeal behaviors. Respir. Physiol. Neurobiol. 147, 159-176. doi: 10.1016/j.resp.2005. 03.015

Gourine, A. V., Kasymov, V., Marina, N., Tang, F., Figueiredo, M. F., Lane, S., et al. (2010). Astrocytes control breathing through pH-dependent release of ATP. Science 329, 571-575. doi: 10.1126/science.1190721

Gray, P. A., Janczewski, W. A., Mellen, N., McCrimmon, D. R., and Feldman, J. L. (2001). Normal breathing requires preBötzinger complex neurokinin-1 receptor-expressing neurons. Nat. Neurosci. 4, 927-930. doi: 10.1038/ nn0901-927

Greer, J. J., Funk, G. D., and Ballanyi, K. (2006). Preparing for the first breath: prenatal maturation of respiratory neural control. J. Physiol. 570, 437-444. doi: 10.1113/jphysiol.2005.097238

Gresham, K., Boyer, B., Mayer, C., Foglyano, R., Martin, R., and Wilson, C. G. (2011). Airway inflammation and central respiratory control: results from in vivo and in vitro neonatal rat. Respir. Physiol. Neurobiol. 178, 414-421. doi: 10.1016/j.resp.2011.05.008

Grether, J. K., and Nelson, K. B. (1997). Maternal infection and cerebral palsy in infants of normal birth weight. JAMA 278, 207-211. doi: 10.1001/jama.1997. 03550030047032

Grether, J. K., Nelson, K. B., Walsh, E., Willoughby, R. E., and Redline, R. W. (2003). Intrauterine exposure to infection and risk of cerebral palsy in very preterm infants. Arch. Pediatr. Adolesc. Med. 157, 26-32. doi: 10.1001/archpedi. 157.1.26

Guerra, F. A., Savich, R. D., Wallen, L. D., Lee, C. H., Clyman, R. I., Mauray, F. E., et al. (1988). Prostaglandin E2 causes hypoventilation and apnea in newborn lambs. J. Appl. Physiol. 64, 2160-2166. doi: 10.1152/jappl.1988.64. 5.2160

Guyenet, P. G., Bayliss, D. A., Stornetta, R. L., Fortuna, M. G., Abbott, S. B., and Depuy, S. D. (2009). Retrotrapezoid nucleus, respiratory chemosensitivity and breathing automaticity. Respir. Physiol. Neurobiol. 168, 59-68. doi: 10.1016/j. resp.2009.02.001

Guyenet, P. G., and Mulkey, D. K. (2010). Retrotrapezoid nucleus and parafacial respiratory group. Respir. Physiol. Neurobiol. 173, 244-255. doi: 10.1016/j.resp. 2010.02.005

Haas, D. M. (2011). Preterm birth. BMJ Clin. Evid. 2011:1404.

Hansen-Pupp, I., Harling, S., Berg, A. C., Cilio, C., Hellström-Westas, L., and Ley, D. (2005). Circulating interferon-gamma and white matter brain damage in preterm infants. Pediatr. Res. 58, 946-952. doi: 10.1203/01.pdr.0000182592. 76702.e8 
Harding, R. (1994). "Development of the respiratory system," in Textbook of Fetal Physiology, eds R. Harding and G. D. Thorburn, (Oxford: Oxford University Press), 40-167.

Harding, R. (1997). Fetal pulmonary development: the role of respiratory movements. Equine Vet. J. 29, 32-39. doi: 10.1111/j.2042-3306.1997.tb0 5076.x

Heep, A., Behrendt, D., Nitsch, P., Fimmers, R., Bartmann, P., and Dembinski, J. (2003). Increased serum levels of interleukin 6 are associated with severe intraventricular haemorrhage in extremely premature infants. Arch. Dis. Child. Fetal Neonatal Ed. 88, F501-F504. doi: 10.1136/fn.88.6.f501

Hein, A. M., and O'Banion, M. K. (2009). Neuroinflammation and memory: the role of prostaglandins. Mol. Neurobiol. 40, 15-32. doi: 10.1007/s12035-0098066-z

Herlenius, E. (2011). An inflammatory pathway to apnea and autonomic dysregulation. Respir. Physiol. Neurobiol. 178, 449-457. doi: 10.1016/j.resp. 2011.06 .026

Herlenius, E., Lagercrantz, H., and Yamamoto, Y. (1997). Adenosine modulates inspiratory neurons and the respiratory pattern in the brainstem of neonatal rats. Pediatr. Res. 42, 46-53. doi: 10.1203/00006450-199707000-00008

Hilaire, G., Voituron, N., Menuet, C., Ichiyama, R. M., Subramanian, H. H., and Dutschmann, M. (2010). The role of serotonin in respiratory function and dysfunction. Respir. Physiol. Neurobiol. 174, 76-88. doi: 10.1016/j.resp. 2010.08.017

Hillman, N., Kallapur, S. G., and Jobe, A. (2012). Physiology of transition from intrauterine to extrauterine life. Clin. Perinatol. 39, 769-783. doi: 10.1016/j.clp. 2012.09.009

Hofstetter, A. O., Saha, S., Siljehav, V., Jakobsson, P. J., and Herlenius, E. (2007). The induced prostaglandin E2 pathway is a key regulator of the respiratory response to infection and hypoxia in neonates. Proc. Natl. Acad. Sci. U S A 104, 9894-9899. doi: 10.1073/pnas.0611468104

Hoop, B., Beagle, J. L., Maher, T. J., and Kazemi, H. (1999). Brainstem amino acid neurotransmitters and hypoxic ventilatory response. Respir. Physiol. 118, 117-129. doi: 10.1016/s0034-5687(99)00072-9

Hooper, S. B., Polglase, G. R., and Roehr, C. C. (2015). Cardiopulmonary changes with aeration of the newborn lung. Paediatr. Respir. Rev. 16, 147-150. doi: 10.1016/j.prrv.2015.03.003

Huang, W. L., Beazley, L. D., Quinlivan, J. A., Evans, S. F., Newnham, J. P., and Dunlop, S. A. (1999). Effect of corticosteroids on brain growth in fetal sheep. Obstet. Gynecol. 94, 213-218. doi: 10.1016/s0029-7844(99)00265-3

Huang, J., Suguihara, C., Hehre, D., Lin, J., and Bancalari, E. (1994). Effects of GABA receptor blockage on the respiratory response to hypoxia in sedated newborn piglets. J. Appl. Physiol. 77, 1006-1010. doi: 10.1152/jappl.1994.77.2. 1006

Huckstepp, R. T. R., Id Bihi, R., Eason, R., Spyer, K. M., Dicke, N., Willecke, K., et al. (2010). Connexin hemichannel-mediated $\mathrm{CO}_{2}$-dependent release of ATP in the medulla oblongata contributes to central respiratory chemosensitivity. J. Physiol. 588, 3901-3920. doi: 10.1113/jphysiol.2010. 192088

Huxtable, A., Vinit, S., Windelborn, J., Crader, S., Guenther, C., Watters, J., et al. (2011). Systemic inflammation impairs respiratory chemoreflexes and plasticity. Respir. Physiol. Neurobiol. 178, 482-489. doi: 10.1016/j.resp. 2011.06.017

Inder, T. E., Wells, S. J., Mogridge, N. B., Spencer, C., and Volpe, J. J. (2003). Defining the nature of the cerebral abnormalities in the premature infant: a qualitative magnetic resonance imaging study. J. Pediatr. 143, 171-179. doi: $10.1067 / \mathrm{s} 0022-3476(03) 00357-3$

Ireland, D. J., Kemp, M. W., Miura, Y., Saito, M., Newnham, J. P., and Keelan, J. A. (2015). Intra-amniotic pharmacological blockade of inflammatory signalling pathways in an ovine chorioamnionitis model. Mol. Hum. Reprod. 21, 479-489. doi: 10.1093/molehr/gav005

Jansen, A. H., De Boeck, C., Ioffe, S., and Chernick, V. (1984). Indomethacininduced fetal breathing: mechanism and site of action. J. Appl. Physiol. Respir. Environ. Exerc. Physiol. 57, 360-365. doi: 10.1152/jappl.1984.57.2.360

Jin, C., Londono, I., Mallard, C., and Lodygensky, G. A. (2015). New means to assess neonatal inflammatory brain injury. J. Neuroinflammation 12:180. doi: 10.1186/s12974-015-0397-2

Kalinski, P. (2011). Regulation of immune responses by prostaglandin $E_{2}$. J. Immunol. 188, 21-28. doi: 10.4049/jimmunol.1101029
Kallapur, S. G., Presicce, P., Rueda, C. M., Jobe, A. H., and Chougnet, C. A. (2014). Fetal immune response to chorioamnionitis. Semin. Reprod. Med. 32, 56-67. doi: $10.1055 / \mathrm{s}-0033-1361823$

Kamaluddeen, M., Lodha, A., and Akierman, A. (2009). Non-rotavirus infection causing apnea in a neonate. Indian J. Pediatr. 76, 1051-1052. doi: 10.1007/s12098-009-0199-6

Kaufman, D., and Fairchild, K. D. (2004). Clinical microbiology of bacterial and fungal sepsis in very-low-birth-weight infants. Clin. Microbiol. Rev. 17, 638-680. doi: 10.1128/cmr.17.3.638-680.2004

Kaukola, T., Herva, R., Perhomaa, M., Pääkkö, E., Kingsmore, S., Vainionpää, L., et al. (2006). Population cohort associating chorioamnionitis, cord inflammatory cytokines and neurologic outcome in very preterm, extremely low birth weight infants. Pediatr. Res. 59, 478-483. doi: 10.1203/01.pdr. 0000182596.66175.ee

Kawano, T., Anrather, J., Zhou, P., Park, L., Wang, G., Frys, K. A., et al. (2006). Prostaglandin $\mathrm{E}_{2}$ EP1 receptors: downstream effectors of COX-2 neurotoxicity. Nat. Med. 12, 225-229. doi: 10.1038/nm1362

Kawasaki, Y., Zhang, L., Cheng, J. K., and Ji, R. R. (2008). Cytokine mechanisms of central sensitization: distinct and overlapping role of interleukin- $1 \beta$, interleukin- 6 , and tumor necrosis factor- $\alpha$ in regulating synaptic and neuronal activity in the superficial spinal cord. J. Neurosci. 28, 5189-5194. doi: 10.1523/JNEUROSCI.3338-07.2008

Kazemi, H., and Hoop, B. (1991). Glutamic acid and $\gamma$-aminobutyric acid neurotransmitters in central control of breathing. J. Appl. Physiol. 70, 1-7. doi: 10.1152/jappl.1991.70.1.1

Kielian, T. (2006). Toll-like receptors in central nervous system glial inflammation and homeostasis. J. Neurosci. Res. 83, 711-730. doi: 10.1002/jnr.20767

Kitterman, J. A., Liggins, G. C., Fewell, J. E., and Tooley, W. H. (1983). Inhibition of breathing movements in fetal sheep by prostaglandins. J. Appl. Physiol. Respir. Environ. Exerc. Physiol. 54, 687-692. doi: 10.1152/jappl.1983.54.3.687

Koch, H., Caughie, C., Elsen, F. P., Doi, A., Garcia, A. J. III., Zanella, S., et al. (2015). Prostaglandin $\mathrm{E}_{2}$ differentially modulates the central control of eupnoea, sighs and gasping in mice. J. Physiol. 593, 305-319. doi: 10.1113/jphysiol.2014. 279794

Koos, B. J., and Rajaee, A. (2014). Fetal breathing movements and changes at birth. Adv. Exp. Med. Biol. 814, 89-101. doi: 10.1007/978-1-4939-1031-1_8

Koos, B. J., Sameshima, H., and Power, G. G. (1986). Fetal breathing movement, sleep state and cardiovascular responses to an inhibitor of mitochondrial atpase in sheep. J. Dev. Physiol. 8, 67-75.

Koshiya, N., and Smith, J. C. (1999). Neuronal pacemaker for breathing visualized in vitro. Nature 400, 360-363. doi: 10.1038/22540

Kubin, L., Alheid, G. F., Zuperku, E. J., and McCrimmon, D. R. (2006). Central pathways of pulmonary and lower airway vagal afferents. J. Appl. Physiol. 101, 618-627. doi: 10.1152/japplphysiol.00252.2006

Laaris, N., and Weinreich, D. (2007). Prostaglandin $E_{2}$ depresses solitary tractmediated synaptic transmission in the nucleus tractus solitarius. Neuroscience 146, 792-801. doi: 10.1016/j.neuroscience.2007.01.053

Lahra, M. M., Gordon, A., and Jeffery, H. E. (2007). Chorioamnionitis and fetal response in stillbirth. Am. J. Obstet. Gynecol. 196, 229.e1-229.e4. doi: 10.1016/j. ajog.2006.10.900

Lai, J., Shao, X. M., Pan, R. W., Dy, E., Huang, C. H., and Feldman, J. L. (2001). RT-PCR reveals muscarinic acetylcholine receptor mRNA in the preBötzinger complex. Am. J. Physiol. Lung Cell. Mol. Physiol. 281, L1420-L1424. doi: 10.1152/ajplung.2001.281.6.11420

Lawn, J. E., Cousens, S., and Zupan, J. (2005). 4 million neonatal deaths: when? Where? Why?. Lancet 365, 891-900. doi: 10.1016/s0140-6736(05)71048-5

Lazarenko, R. M., Milner, T. A., Depuy, S. D., Stornetta, R. L., West, G. H., Kievits, J. A., et al. (2009). Acid sensitivity and ultrastructure of the retrotrapezoid nucleus in Phox2b-EGFP transgenic mice. J. Comp. Neurol. 517, 69-86. doi: 10.1002/cne.22136

Leclerc, J. L., Lampert, A. S., Diller, M. A., and Doré, S. (2015). Genetic deletion of the prostaglandin E2 E prostanoid receptor subtype 3 improves anatomical and functional outcomes after intracerebral hemorrhage. Eur. J. Neurosci. 41, 1381-1391. doi: 10.1111/ejn.12909

Li, J., Liang, X., Wang, Q., Breyer, R. M., McCullough, L., and Andreasson, K. (2008). Misoprostol, an anti-ulcer agent and $\mathrm{PGE}_{2}$ receptor agonist, protects against cerebral ischemia. Neurosci. Lett. 438, 210-215. doi: 10.1016/j.neulet. 2008.04.054 
Liang, X., Lin, L., Woodling, N. S., Wang, Q., Anacker, C., Pan, T., et al. (2011). Signaling via the prostaglandin $\mathrm{E}_{2}$ receptor EP4 exerts neuronal and vascular protection in a mouse model of cerebral ischemia. J. Clin. Invest. 121, 4362-4371. doi: 10.1172/JCI46279

Lin, T. Y., Lu, C. W., Wang, C. C., Huang, S. K., and Wang, S. J. (2014). Cyclooxygenase 2 inhibitor celecoxib inhibits glutamate release by attenuating the PGE2/EP2 pathway in rat cerebral cortex endings. J. Pharmacol. Exp. Ther. 351, 134-145. doi: 10.1124/jpet.114.217372

Liu, L., Johnson, H. L., Cousens, S., Perin, J., Scott, S., Lawn, J. E., et al. (2012). Global, regional, and national causes of child mortality: an updated systematic analysis for 2010 with time trends since 2000. Lancet 379, 2151-2161. doi: 10.1016/s0140-6736(12)60560-1

Liu, Q., and Wong-Riley, M. T. T. (2005). Postnatal developmental expressions of neurotransmitters and receptors in various brain stem nuclei of rats. J. Appl. Physiol. 98, 1442-1457. doi: 10.1152/japplphysiol.01301.2004

LoMauro, A., and Aliverti, A. (2016). Physiology masterclass: extremes of age: newborn and infancy. Breathe 12, 65-68. doi: 10.1183/20734735.013315

Lorea-Hernández, J. J., Morales, T., Rivera-Angulo, A. J., Alcantara-Gonzalez, D., and Peña-Ortega, F. (2016). Microglia modulate respiratory rhythm generation and autoresuscitation. Glia 64, 603-619. doi: 10.1002/glia.22951

Lu, Y. C., Yeh, W. C., and Ohashi, P. S. (2008). LPS/TLR4 signal transduction pathway. Cytokine 42, 145-151. doi: 10.1016/j.cyto.2008.01.006

Ludlow, C. L. (2015). Central nervous system control of voice and swallowing. J. Clin. Neurophysiol. 32, 294-303. doi: 10.1097/WNP.00000000000 00186

Machado, B. H., Castania, J. A., Bonagamba, L. G. H., and Salgado, H. C. (2000). Neurotransmission of autonomic components of aortic baroreceptor afferents in the NTS of awake rats. Am. J. Physiol. Heart Circ. Physiol. 279, H67-H75. doi: 10.1152/ajpheart.2000.279.1.h67

Malaeb, S., and Dammann, O. (2009). Fetal inflammatory response and brain injury in the preterm newborn. J. Child Neurol. 24, 1119-1126. doi: $10.1177 / 0883073809338066$

Mallard, C., Welin, A. K., Peebles, D., Hagberg, H., and Kjellmer, I. (2003). White matter injury following systemic endotoxemia or asphyxia in the fetal sheep. Neurochem. Res. 28, 215-223. doi: 10.1023/a:1022368915400

Martelli, D., Stanić, D., and Dutschmann, M. (2013). The emerging role of the parabrachial complex in the generation of wakefulness drive and its implication for respiratory control. Respir. Physiol. Neurobiol. 188, 318-323. doi: 10.1016/j. resp.2013.06.019

Martin, R. J., Abu-Shaweesh, J. M., and Baird, T. M. (2004). Apnoea of prematurity. Paediatr. Respir. Rev. 5, S377-S382. doi: 10.1016/S15260542(04)90067-X

Marty, V., El Hachmane, M., and Amédée, T. (2008). Dual modulation of synaptic transmission in the nucleus tractus solitarius by prostaglandin E2 synthesized downstream of IL-1ß. Eur. J. Neurosci. 27, 3132-3150. doi: 10.1111/j.1460-9568. 2008.06296.x

McCormick, A., Suguihara, C., Huang, J., Devia, C., Hehre, D., Bruce, J. H., et al. (1998). Depressed ventilatory response to hypoxia in hypothermic newborn piglets: role of glutamate. J. Appl. Physiol. 84, 830-836. doi: 10.1152/jappl.1998. 84.3.830

McCullough, L., Wu, L., Haughey, N., Liang, X., Hand, T., Wang, Q., et al. (2004). Neuroprotective function of the PGE2 EP2 receptor in cerebral ischemia. J. Neurosci. 24, 257-268. doi: 10.1523/JNEUROSCI.4485-03.2004

McKay, L. C., Janczewski, W. A., and Feldman, J. L. (2005). Sleep-disordered breathing after targeted ablation of preBötzinger complex neurons. Nat. Neurosci. 8, 1142-1144. doi: 10.1038/nn1517

Miall, L., and Wallis, S. (2011). The management of respiratory distress in the moderately preterm newborn infant. Arch. Dis. Child. Educ. Pract. Ed. 96, 128-135. doi: 10.1136/adc.2010.189712

Mohan, S., Ahmad, A., Glushakov, A., Chambers, C., and Dore, S. (2012). Putative role of prostaglandin receptor in intracerebral hemorrhage. Front. Neurol. 3:145. doi: 10.3389/fneur.2012.00145

Moore, J. D., Kleinfeld, D., and Wang, F. (2014). How the brainstem controls orofacial behaviors comprised of rhythmic actions. Trends Neurosci. 37, 370-380. doi: 10.1016/j.tins.2014.05.001

Morgado-Valle, C., Baca, S. M., and Feldman, J. L. (2010). Glycinergic pacemaker neurons in prebotzinger complex of neonatal mouse. J. Neurosci. 30, 3634-3639. doi: 10.1523/JNEUROSCI.3040-09.2010
Moscuzza, F., Belcari, F., Nardini, V., Bartoli, A., Domenici, C., Cuttano, A., et al. (2011). Correlation between placental histopathology and fetal/neonatal outcome: chorioamnionitis and funisitis are associated to intraventricular haemorrage and retinopathy of prematurity in preterm newborns. Gynecol. Endocrinol. 27, 319-323. doi: 10.3109/09513590.2010.487619

Moss, T. J. (2006). Respiratory consequences of preterm birth. Clin. Exp. Pharmacol. Physiol. 33, 280-284. doi: 10.1111/j.1440-1681.2006.04359.x

Mulkey, D. K., Stornetta, R. L., Weston, M. C., Simmons, J. R., Parker, A., Bayliss, D. A., et al. (2004). Respiratory control by ventral surface chemoreceptor neurons in rats. Nat. Neurosci. 7, 1360-1369. doi: 10.1038/nn1357

Murakami, M., Naraba, H., Tanioka, T., Semmyo, N., Nakatani, Y., Kojima, F., et al. (2000). Regulation of prostaglandin $\mathrm{E}_{2}$ biosynthesis by inducible membrane-associated prostaglandin $\mathrm{E}_{2}$ synthase that acts in concert with cyclooxygenase-2. J. Biol. Chem. 275, 32783-32792. doi: 10.1074/jbc. M003505200

Murphy, D. J., Sellers, S., MacKenzie, I. Z., Yudkin, P. L., and Johnson, A. M. (1995). Case-control study of antenatal and intrapartum risk factors for cerebral palsy in very preterm singleton babies. Lancet 346, 1449-1454. doi: 10.1016/s0140-6736(95)92471-x

Nakamura, K., Kaneko, T., Yamashita, Y., Hasegawa, H., Katoh, H., and Negishi, M. (2000). Immunohistochemical localization of prostaglandin EP3 receptor in the rat nervous system. J. Comp. Neurol. 421, 543-569. doi: 10.1002/(sici)1096-9861(20000612)421:4<543::aid-cne6>3.0.co;2-3

Natale, R. (1980). Maternal plasma glucose concentration and fetal breathing movements: a review. Semin. Perinatol. 4, 287-293.

Natale, R., Nasello-Paterson, C., and Connors, G. (1988). Patterns of fetal breathing activity in the human fetus at 24 to 28 weeks of gestation. Am. J. Obstet. Gynecol. 158, 317-321. doi: 10.1016/0002-9378(88)90146-9

Nattie, E. E., and Li, A. (2002). Substance P-saporin lesion of neurons with NK1 receptors in one chemoreceptor site in rats decreases ventilation and chemosensitivity. J. Physiol. 544, 603-616. doi: 10.1113/jphysiol.2002. 020032

Nitsos, I., Rees, S. M., Duncan, J., Kramer, B. W., Harding, R., Newnham, J. P., et al. (2006). Chronic exposure to intra-amniotic lipopolysaccharide affects the ovine fetal brain. J. Soc. Gynecol. Investig. 13, 239-247. doi: 10.1016/j.jsgi.2006. 02.011

Nock, M. L., Difiore, J. M., Arko, M. K., and Martin, R. J. (2004). Relationship of the ventilatory response to hypoxia with neonatal apnea in preterm infants. J. Pediatr. 144, 291-295. doi: 10.1016/j.jpeds.2003.11.035

Olsson, A., Kayhan, G., Lagercrantz, H., and Herlenius, E. (2003). IL-1 $\beta$ depresses respiration and anoxic survival via a prostaglandin-dependent pathway in neonatal rats. Pediatr. Res. 54, 326-331. doi: 10.1203/01.pdr.0000076665. 62641.a2

Pålsson-McDermott, E. M., and O’Neill, L. A. J. (2004). Signal transduction by the lipopolysaccharide receptor, toll-like receptor-4. Immunology 113, 153-162. doi: 10.1111/j.1365-2567.2004.01976.x

Patrick, J., Campbell, K., Carmichael, L., Natale, R., and Richardson, B. (1980). Patterns of human fetal breathing during the last 10 weeks of pregnancy. Obstet. Gynecol. 56, 24-30.

Phillips, W. S., Herly, M., Del Negro, C. A., and Rekling, J. C. (2016). Organotypic slice cultures containing the preBötzinger complex generate respiratory-like rhythms. J. Neurophysiol. 115, 1063-1070. doi: 10.1152/jn.00904.2015

Poligone, B., and Baldwin, A. S. (2001). Positive and negative regulation of NF-кB by COX-2: roles of different prostaglandins. J. Biol. Chem. 276, 38658-38664. doi: 10.1074/jbc.M106599200

Polglase, G. R., Miller, S. L., Barton, S. K., Kluckow, M., Gill, A. W., Hooper, S. B., et al. (2014). Respiratory support for premature neonates in the delivery room: effects on cardiovascular function and the development of brain injury. Pediatr. Res. 75, 682-688. doi: 10.1038/pr.2014.40

Polglase, G. R., Nitsos, I., Baburamani, A. A., Crossley, K. J., Slater, M. K., Gill, A. W., et al. (2012). Inflammation in utero exacerbates ventilationinduced brain injury in preterm lambs. J. Appl. Physiol. 112, 481-489. doi: 10.1152/japplphysiol.00995.2011

Ramirez, S. C., Koschnitzky, J. E., Baertsch, N. A., Anderson, T. M., Smith, C. V., and Ramirez, J.-M. (2016). Disturbances in central respiratory rhythm generation may contribute to breathing disturbances in prematurely born mice. FASEB J. 30:987.4. 
Ramirez, J. M., Schwarzacher, S. W., Pierrefiche, O., Olivera, B. M., and Richter, D. W. (1998). Selective lesioning of the cat pre-Bötzinger complex in vivo eliminates breathing but not gasping. J. Physiol. 507, 895-907. doi: 10.1111/j.1469-7793.1998.895bs.x

Ricciotti, E., and FitzGerald, G. A. (2011). Prostaglandins and inflammation. Arterioscler. Thromb. Vasc. Biol. 31, 986-1000. doi: 10.1161/ATVBAHA. 110.207449

Richerson, G. B., Wang, W., Tiwari, J., and Bradley, S. R. (2001). Chemosensitivity of serotonergic neurons in the rostral ventral medulla. Respir. Physiol. 129, 175-189. doi: 10.1016/s0034-5687(01)00289-4

Rybak, I. A., Abdala, A. P. L., Markin, S. N., Paton, J. F. R., and Smith, J. C. (2007). Spatial organization and state-dependent mechanisms for respiratory rhythm and pattern generation. Prog. Brain Res. 165, 201-220. doi: 10.1016/s00796123(06)65013-9

Sakamoto, K., Karelina, K., and Obrietan, K. (2011). Creb: a multifaceted regulator of neuronal plasticity and protection. J. Neurochem. 116, 1-9. doi: 10.1111/j. 1471-4159.2010.07080.x

Shalak, L. F., Laptook, A. R., Jafri, H. S., Ramilo, O., and Perlman, J. M. (2002). Clinical chorioamnionitis, elevated cytokines, and brain injury in term infants. Pediatrics 110, 673-680. doi: 10.1542/peds.110.4.673

Shao, X. M., and Feldman, J. L. (2009). Central cholinergic regulation of respiration: nicotinic receptors. Acta Pharmacol. Sin. 30, 761-770. doi: 10.1038/aps.2009.88

Shatrov, J. G., Birch, S. C., Lam, L. T., Quinlivan, J. A., McIntyre, S., and Mendz, G. L. (2010). Chorioamnionitis and cerebral palsy: a meta-analysis. Obstet. Gynecol. 116, 387-392. doi: 10.1097/AOG.0b013e3181e90046

Shi, J., Johansson, J., Woodling, N. S., Wang, Q., Montine, T. J., and Andreasson, K. (2010). The prostaglandin $\mathrm{E}_{2}$ EP4 receptor exerts anti-inflammatory effects in brain innate immunity. J. Immunol. 184, 7207-7218. doi: 10.4049/jimmunol. 0903487

Shie, F. S., Montine, K. S., Breyer, R. M., and Montine, T. J. (2005). Microglial EP2 is critical to neurotoxicity from activated cerebral innate immunity. Glia 52, 70-77. doi: 10.1002/glia.20220

Shimamura, M., Zhou, P., Casolla, B., Qian, L., Capone, C., Kurinami, H., et al. (2013). Prostaglandin $E_{2}$ type 1 receptors contribute to neuronal apoptosis after transient forebrain ischemia. J. Cereb. Blood Flow Metab. 33, 1207-1214. doi: $10.1038 /$ jcbfm.2013.69

Shvarev, Y. N., Lagercrantz, H., and Yamamoto, Y. (2002). Biphasic effects of substance $\mathrm{p}$ on respiratory activity and respiration-related neurones in ventrolateral medulla in the neonatal rat brainstem in vitro. Acta Physiol. Scand. 174, 67-84. doi: 10.1046/j.1365-201x.2002.00926.x

Siljehav, V., Shvarev, Y., and Herlenius, E. (2014). Il-1 $\beta$ and prostaglandin E2 attenuate the hypercapnic as well as the hypoxic respiratory response via prostaglandin E receptor type 3 in neonatal mice. J. Appl. Physiol. 117, 1027-1036. doi: 10.1152/japplphysiol.00542.2014

Simmons, D. L., Botting, R. M., and Hla, T. (2004). Cyclooxygenase isozymes: the biology of prostaglandin synthesis and inhibition. Pharmacol. Rev. 56, 387-437. doi: $10.1124 /$ pr.56.3.3

Simonsen, K. A., Anderson-Berry, A. L., Delair, S. F., and Davies, H. D. (2014). Early-onset neonatal sepsis. Clin. Microbiol. Rev. 27, 21-47. doi: 10.1128/CMR. 00031-13

Smith, J. C., Abdala, A. P. L., Borgmann, A., Rybak, I. A., and Paton, J. F. R. (2013). Brainstem respiratory networks: building blocks and microcircuits. Trends Neurosci. 36, 152-162. doi: 10.1016/j.tins.2012.11.004

Smith, J. C., Abdala, A. P., Koizumi, H., Rybak, I. A., and Paton, J. F. (2007). Spatial and functional architecture of the mammalian brain stem respiratory network: a hierarchy of three oscillatory mechanisms. J. Neurophysiol. 98, 3370-3387. doi: 10.1152/jn.00985.2007

Smith, J. C., Abdala, A. P. L., Rybak, I. A., and Paton, J. F. R. (2009). Structural and functional architecture of respiratory networks in the mammalian brainstem. Philos. Trans. R. Soc. Lond. B Biol. Sci. 364, 2577-2587. doi: 10.1098/rstb.2009. 0081

Smith, J. C., Ellenberger, H. H., Ballanyi, K., Richter, D. W., and Feldman, J. L. (1991). Pre-Bötzinger complex: a brainstem region that may generate respiratory rhythm in mammals. Science 254, 726-729. doi: 10.1126/science. 1683005

Smith, C. A., Rodman, J. R., Chenuel, B. J., Henderson, K. S., and Dempsey, J. A. (2006). Response time and sensitivity of the ventilatory response to $\mathrm{CO}_{2}$ in unanesthetized intact dogs: central vs. peripheral chemoreceptors. J. Appl. Physiol. (1985) 100, 13-19. doi: 10.1152/japplphysiol.00926.2005

Speer, C. P. (2006). Inflammation and bronchopulmonary dysplasia: a continuing story. Semin. Fetal Neonatal Med. 11, 354-362. doi: 10.1016/j.siny.2006. 03.004

Stock, C., Teyssier, G., Pichot, V., Goffaux, P., Barthelemy, J. C., and Patural, H. (2010). Autonomic dysfunction with early respiratory syncytial virus-related infection. Auton. Neurosci. 156, 90-95. doi: 10.1016/j.autneu.2010.03.012

Stokowski, L. A. (2005). A primer on Apnea of prematurity. Adv. Neonatal Care 5 , 155-170; quiz 171-174. doi: 10.1016/j.adnc.2005.03.001

Stornetta, R. L. (2008). Identification of neurotransmitters and co-localization of transmitters in brainstem respiratory neurons. Respir. Physiol. Neurobiol. 164, 18-27. doi: 10.1016/j.resp.2008.07.024

Stornetta, R. L., Moreira, T. S., Takakura, A. C., Kang, B. J., Chang, D. A., West, G. H., et al. (2006). Expression of Phox2b by brainstem neurons involved in chemosensory integration in the adult rat. J. Neurosci. 26, 10305-10314. doi: 10.1523/JNEUROSCI.2917-06.2006

Strunk, T., Inder, T., Wang, X., Burgner, D., Mallard, C., and Levy, O. (2014). Infection-induced inflammation and cerebral injury in preterm infants. Lancet Infect. Dis. 14, 751-762. doi: 10.1016/S1473-3099(14)70710-8

Surbek, D., Drack, G., Irion, O., Nelle, M., Huang, D., and Hoesli, I. (2012). Antenatal corticosteroids for fetal lung maturation in threatened preterm delivery: indications and administration. Arch. Gynecol. Obstet. 286, 277-281. doi: 10.1007/s00404-012-2339-x

Sweeney, E. L., Kallapur, S. G., Gisslen, T., Lambers, D. S., Chougnet, C. A., Stephenson, S. A., et al. (2016). Placental infection with ureaplasma species is associated with histologic chorioamnionitis and adverse outcomes in moderately preterm and late-preterm infants. J. Infect. Dis. 213, 1340-1347. doi: 10.1093/infdis/jiv587

Tak, P. P., and Firestein, G. S. (2001). NF-кB: a key role in inflammatory diseases. J. Clin. Invest. 107, 7-11. doi: 10.1172/JCI11830

Takakura, A. C., Moreira, T. S., Colombari, E., West, G. H., Stornetta, R. L., and Guyenet, P. G. (2006). Peripheral chemoreceptor inputs to retrotrapezoid nucleus (RTN) $\mathrm{CO}_{2}$-sensitive neurons in rats. J. Physiol. 572, 503-523. doi: 10.1113/jphysiol.2005.103788

Taniguchi, H., Anacker, C., Suarez-Mier, G. B., Wang, Q., and Andreasson, K. (2011). Function of prostaglandin $\mathrm{E}_{2} \mathrm{EP}$ receptors in the acute outcome of rodent hypoxic ischemic encephalopathy. Neurosci. Lett. 504, 185-190. doi: 10.1016/j.neulet.2011.09.005

Taniguchi, H., Anacker, C., Wang, Q., and Andreasson, K. (2014). Protection by vascular prostaglandin $\mathrm{E}_{2}$ signaling in hypoxic ischemic encephalopathy. Exp. Neurol. 255, 30-37. doi: 10.1016/j.expneurol.2014.02.012

Tapia, I. E., Shults, J., Doyle, L. W., Nixon, G. M., Cielo, C. M., Traylor, J., et al. (2016). Perinatal risk factors associated with the obstructive sleep apnea syndrome in school-aged children born preterm. Sleep 39, 737-742. doi: $10.5665 /$ sleep. 5618

Trudinger, B. J., and Knight, P. C. (1980). Fetal age and patterns of human fetal breathing movements. Am. J. Obstet. Gynecol. 137, 724-728. doi: 10.1016/s0002-9378(15)33249-x

Vereyken, E. J., Bajova, H., Chow, S., de Graan, P. N., and Gruol, D. L. (2007). Chronic interleukin-6 alters the level of synaptic proteins in hippocampus in culture and in vivo. Eur. J. Neurosci. 25, 3605-3616. doi: 10.1111/j.1460-9568. 2007.05615.x

Vezzani, A., and Viviani, B. (2015). Neuromodulatory properties of inflammatory cytokines and their impact on neuronal excitability. Neuropharmacology 96, 70-82. doi: 10.1016/j.neuropharm.2014.10.027

Viscardi, R. M. (2010). Ureaplasma species: role in diseases of prematurity. Clin. Perinatol. 37, 393-409. doi: 10.1016/j.clp.2009.12.003

Viviani, B., Bartesaghi, S., Gardoni, F., Vezzani, A., Behrens, M. M., Bartfai, T., et al. (2003). Interleukin-1 $\beta$ enhances NMDA receptor-mediated intracellular calcium increase through activation of the src family of kinases. J. Neurosci. 23 , 8692-8700.

Wang, S., Cheng, Q., Malik, S., and Yang, J. (2000). Interleukin-1 $\beta$ inhibits $\gamma$-aminobutyric acid type $A\left(G_{A B A}\right)$ receptor current in cultured hippocampal neurons. J. Pharmacol. Exp. Ther. 292, 497-504.

Wang, X. C., Qiu, Y. H., and Peng, Y. P. (2007). Interleukin-6 protects cerebellar granule neurons from NMDA-induced neurotoxicity. Sheng Li Xue Bao 59, $150-156$. 
Weber, M. A., Klein, N. J., Hartley, J. C., Lock, P. E., Malone, M., and Sebire, N. J. (2008). Infection and sudden unexpected death in infancy: a systematic retrospective case review. Lancet 371, 1848-1853. doi: 10.1016/S01406736(08)60798-9

WHO. (2012). Born Too Soon: The Global Action Report on Preterm Birth. Geneva: World Health Organization.

Wong-Riley, M. T. T., and Liu, Q. (2005). Neurochemical development of brain stem nuclei involved in the control of respiration. Respir. Physiol. Neurobiol. 149, 83-98. doi: 10.1016/j.resp.2005.01.011

Wong-Riley, M. T. T., and Liu, Q. (2008). Neurochemical and physiological correlates of a critical period of respiratory development in the rat. Respir. Physiol. Neurobiol. 164, 28-37. doi: 10.1016/j.resp.2008.04.014

Wu, Y. W. (2002). Systematic review of chorioamnionitis and cerebral palsy. Ment. Retard. Dev. Disabil. Res. Rev. 8, 25-29. doi: 10.1002/mrdd.10003

Wu, Y. W., Escobar, G. J., Grether, J. K., Croen, L. A., Greene, J. D., and Newman, T. B. (2003). Chorioamnionitis and cerebral palsy in term and near-term infants. JAMA 290, 2677-2684. doi: 10.1001/jama.290.20.2677

Yang, G., Dong, W. H., Hu, C. L., and Mei, Y. A. (2015). PGE 2 modulates $\mathrm{GABA}_{A}$ receptors via an EP1 receptor-mediated signaling pathway. Cell. Physiol. Biochem. 36, 1699-1711. doi: 10.1159/000430143

Yang, L., Sameshima, H., Yamaguchi, M., and Ikenoue, T. (2005). Expression of inducible nitric oxide synthase and cyclooxygenase- 2 mRNA in brain damage induced by lipopolysaccharide and intermittent hypoxia-ischemia in neonatal rats. J. Obstet. Gynaecol. Res. 31, 185-191. doi: 10.1111/j.1341-8076.2005. 00266.x

Yanowitz, T. D., Jordan, J. A., Gilmour, C. H., Towbin, R., Bowen, A., Roberts, J. M., et al. (2002). Hemodynamic disturbances in premature infants born after chorioamnionitis: association with cord blood cytokine concentrations. Pediatr. Res. 51, 310-316. doi: 10.1203/00006450-20020300000008

Yoon, B. H., Romero, R., Park, J. S., Kim, C. J., Kim, S. H., Choi, J. H., et al. (2000). Fetal exposure to an intra-amniotic inflammation and the development of cerebral palsy at the age of three years. Am. J. Obstet. Gynecol. 182, 675-681. doi: $10.1067 / \mathrm{mob} .2000 .104207$

Zhang, Y. H., Lu, J., Elmquist, J. K., and Saper, C. B. (2000). Lipopolysaccharide activates specific populations of hypothalamic and brainstem neurons that project to the spinal cord. J. Neurosci. 20, 6578-6586.

Zhang, J., and Rivest, S. (1999). Distribution, regulation and colocalization of the genes encoding the $\mathrm{EP}_{2}-$ and $\mathrm{EP}_{4}-\mathrm{PGE}_{2}$ receptors in the rat brain and neuronal responses to systemic inflammation. Eur. J. Neurosci. 11, 2651-2668. doi: 10.1046/j.1460-9568.1999.00682.x

Zhang, J., and Rivest, S. (2000). A functional analysis of EP4 receptorexpressing neurons in mediating the action of prostaglandin E2 within specific nuclei of the brain in response to circulating interleukin- $1 \beta$. J. Neurochem. 74, 2134-2145. doi: 10.1046/j.1471-4159.2000.074 2134.X

Zhao, J., Gonzalez, F., and Mu, D. (2011). Apnea of prematurity: from cause to treatment. Eur. J. Pediatr. 170, 1097-1105. doi: 10.1007/s00431-0111409-6

Zoccal, D. B., Furuya, W. I., Bassi, M., Colombari, D. S., and Colombari, E. (2014). The nucleus of the solitary tract and the coordination of respiratory and sympathetic activities. Front. Physiol. 5:238. doi: 10.3389/fphys.2014.00238

Conflict of Interest Statement: The authors declare that the research was conducted in the absence of any commercial or financial relationships that could be construed as a potential conflict of interest.

Copyright (C) 2018 Stojanovska, Miller, Hooper and Polglase. This is an open-access article distributed under the terms of the Creative Commons Attribution License (CC BY). The use, distribution or reproduction in other forums is permitted, provided the original author(s) and the copyright owner are credited and that the original publication in this journal is cited, in accordance with accepted academic practice. No use, distribution or reproduction is permitted which does not comply with these terms. 


\title{
Changes in the Peripheral Chemosensory System Drive Adaptive Shifts in Food Preferences in Insects
}

\author{
Ayako Wada-Katsumata ${ }^{1 *}$, Hugh M. Robertson ${ }^{2}$, Jules Silverman ${ }^{1}$ and Coby Schal ${ }^{1 *}$ \\ ${ }^{1}$ Department of Entomology and Plant Pathology, North Carolina State University, Raleigh, NC, United States, ${ }^{2}$ Department \\ of Entomology, University of Illinois at Urbana-Champaign, Urbana, IL, United States
}

OPEN ACCESS

Edited by:

Dieter Wicher,

Max-Planck-Institut für Chemische

Ökologie, Germany

Reviewed by:

Anupama Dahanukar

University of California, Riverside,

United States

Sean T. Sweeney,

University of York, United Kingdom

${ }^{*}$ Correspondence: Ayako Wada-Katsumata

akatsum@ncsu.edu

Coby Schal

coby@ncsu.edu

Received: 04 June 2018 Accepted: 09 August 2018 Published: 29 August 2018

Citation:

Wada-Katsumata A, Robertson HM,

Silverman J and Schal C (2018)

Changes in the Peripheral

Chemosensory System Drive Adaptive Shifts in Food Preferences

in Insects.

Front. Cell. Neurosci. 12:281. doi: 10.3389/fncel.2018.00281
A key challenge in understanding the evolution of animal behaviors is to identify cellular and molecular mechanisms that underlie the evolution of adaptive traits and behaviors in polymorphic populations under local selection pressures. Despite recent advances in fish, mice, and insects, there are still only a few compelling examples of major genes and cellular mechanisms associated with complex behavioral changes. Shifts in food or host preferences in insects, accompanied by changes in the peripheral chemosensory system, offer some of the best examples of adaptive behavioral evolution. A remarkable example is the German cockroach, Blattella germanica, a major indoor pest with a highly diverse omnivorous diet. Strong and persistent selection pressure with toxic-baits has induced rapid evolution of behavioral resistance in multiple cockroach populations. While typical cockroaches detect and accept the sugar glucose as a feeding-stimulant, behaviorally resistant cockroaches avoid eating glucose-containing toxic baits by sensing glucose as a deterrent. We review the peripheral gustatory neural mechanisms of glucose-aversion and discuss how the rapid emergence of taste polymorphisms can impede pest control efforts and affect foraging and mate-choice in adapted cockroach populations.

Keywords: chemoreception, gustation, sensilla, nutrient sensing, glucose-aversion

\section{STRONG ANTHROPOGENIC SELECTION DRIVES RAPID EVOLUTION OF PEST INSECTS}

Local selection pressures and genetic drift can lead to slow evolutionary divergence of allopatric populations; selection may be imposed by a wide variety of factors such as climate, resource availability, predation, and competition. It is often difficult, however, to disentangle how genetic traits respond to selection and lead to the evolution of adaptive behaviors in wild animals, because most behaviors are driven by multiple genes, and the evolutionary process is slow, requiring long-term observations. Anthropogenic selection, on the other hand, can impose much stronger evolutionary pressures over a shorter timeframe, often on genetically closed populations, leading to the rapid evolution of adaptive responses. Quintessential examples are the rapid evolution of resistance to antibiotics in medically important pathogens and to pesticides in agricultural pests, in response to human-imposed strong selection pressures (e.g., Gould et al., 2018).

The German cockroach, Blattella germanica, is a cosmopolitan synanthropic pest, living obligatorily in human environments such as homes, food processing facilities, restaurants, 
hospitals, transportation systems, and even farm buildings, among others (Schal, 2011). Cockroach control indoors is particularly challenging because of their close proximity to humans and pets, but in the early 1980s insecticide baits became a popular and highly effective strategy to control cockroach infestations. Because cockroaches must feed in preparation for nymphal development and adult reproduction, this requirement, coupled with their chewing mouthparts, made baits highly effective and ecologically safer than spray insecticides. Baits combine an insecticide with various phagostimulants, typically corn syrup composed of glucose and fructose in early bait formulations. However, within just a few years, bait performance was severely compromised as they rapidly induced physiological and behavioral resistance in cockroach populations (Silverman and Bieman, 1993; Wang et al., 2004). Most interesting among these was a population collected in Florida in 1989 that behaviorally shunned toxic baits; these field-collected cockroaches rejected glucose, a phagostimulant and nutrient ingredient in baits, but they had no metabolic resistance to the insecticide in the bait (Silverman and Bieman, 1993). This trait rendered all glucose-containing baits ineffective against these glucose-averse (GA) cockroaches (Silverman and Liang, 1999), and in response, bait manufacturers promptly reformulated bait products at considerable costs.

The GA trait is heritable, controlled by a single major gene that follows Mendelian inheritance patterns (Silverman and Bieman, 1993): Crosses of homozygous wild-type (WT) and homozygous GA cockroaches result in 100\% glucose-rejecting cockroaches, and in the F1 cross the GA trait is expressed in $75 \%$ of the progeny (50\% heterozygous GA, 25\% homozygous GA), while $25 \%$ are homozygous WT that never reject glucose at any concentration (Wada-Katsumata et al., 2014). Phenotyping of GA cockroaches is uncomplicated because homozygous GA individuals have 10-fold greater deterrence for glucose than heterozygous GA cockroaches (Silverman, 1995). Importantly, injection of high concentrations of glucose into the hemocoel did not adversely affect the physiology or behavior, including feeding preference for glucose, foraging, and sexual maturation of GA and WT cockroaches. These findings indicate that glucose-aversion is mediated by information processing via the chemosensory system (Wada-Katsumata et al., 2011) and not through toxic effects associated with glucose. Glucose-aversion thus confers an enormous advantage in the presence of toxic baits due to behavioral rejection of these pesticide-containing products. Recent studies indicate that even short-term selection with a glucose-containing toxic bait can rapidly increase the frequency of GA cockroaches (Wada-Katsumata et al., 2014; Figure 1). This trait is now common in multiple field populations (Wang et al., 2004; Wada-Katsumata et al., 2013). Importantly, because GA cockroaches consume less glucose-containing diet, this trait is maladaptive in bait-free environments as GA cockroaches must seek glucose-free foods. Two decades after its discovery, the neural basis of this fascinating taste polymorphism was demonstrated with electrophysiological studies (Wada-Katsumata et al., 2011, 2013), but its molecular mechanism remains to be determined.

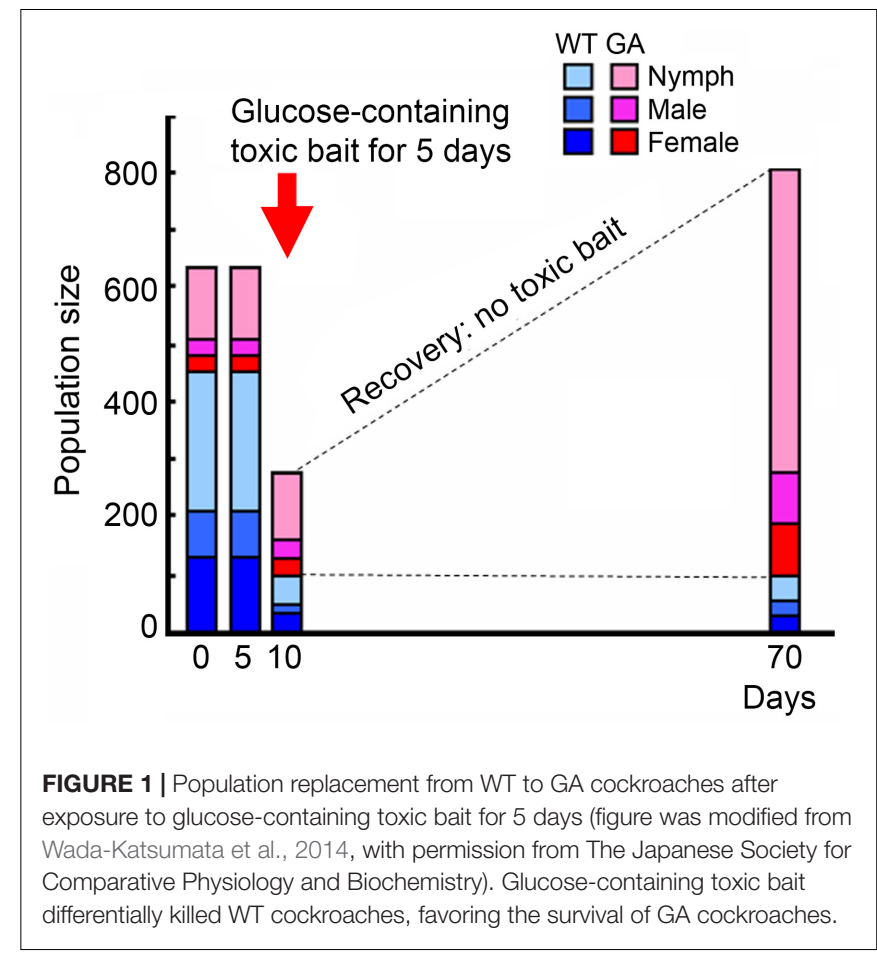

\section{THE INSECT GUSTATORY SYSTEM}

The ability to detect and discriminate tastants is essential because tastants convey important information about the quality and nutritional value of food, allowing animals to avoid potentially toxic or spoiled food, and in some instances guiding mate-choice decisions. As in humans and mice, the peripheral gustatory system of insects is mainly localized in the mouthparts, but other appendages such as the antennae, feet and wings may be involved as well. Detection and assessment of the quality and intensity of tastants occurs in bipolar Gustatory Receptor Neurons (GRNs) whose cell bodies and dendrites are housed within hair-like cuticle-lined sensilla with a pore at the tip (Newland et al., 2009). The organization of GRNs has been well described in the common fruit fly Drosophila melanogaster. The labellum contains approximately 31 sensilla which are grouped into morphological classes based on their length. Each sensillum contains two or four different types of GRNs, with each GRN expressing a specific taste modality. Therefore, GRNs are denoted sweet-, bitter-, water-, or salt-GRNs. While water- and salt-GRNs express Ionotropic Receptors (IRs) and pickpocket $(\mathrm{PPK})$ receptors, sweet- and bitter-GRNs are characterized by combinatorial sets of co-expressed Gustatory Receptors (GRs) which recognize particular tastant molecules (Montell, 2009; Freeman and Dahanukar, 2015; Scott, 2018). For example, Drosophila has 68 GRs, and sweet-GRNs express members of a conserved clade of sugar GRs. Bitter-GRNs, on the other hand, are tuned to aversive tastants such as noxious substances and are characterized by subsets of GRs that never overlap with GRs expressed in sweet-GRNs. Both sugar and bitter receptors are thought to be composed of multimeric GRs (Montell, 2009; 
Freeman and Dahanukar, 2015; Scott, 2018). The axons of GRNs with the same modal specificity (taste quality) project directly to the same region in the central nervous system (CNS). Quality, strength and duration of stimuli are represented as neuronal impulses by GRNs (Scott, 2018). The sweet-GRNs mediate appetitive behavior via CNS processing, whereas the responses of bitter-GRNs mediate rejection behavior. Therefore, modifications in tastant discrimination by GRs and GRNs, which represent the peripheral first stage in gustatory information processing, can critically impact the expression of gustatory behavior.

\section{GUSTATORY SYSTEM OF THE GERMAN COCKROACH AND GLUCOSE-AVERSION}

GRNs of the German cockroach are housed in sensilla on the mouthparts and antennae (Wada-Katsumata et al., 2009, 2011, 2013). While foraging, cockroaches discriminate food sources first with the antennae, the most distal appendages from the mouthparts, then with the maxillary and labial palps, and finally with the paraglossae, the gateway chemosensory structure to the mouth (Figure 2A); each of these can evaluate and discriminate nutrients from noxious substances. Adult females possess 2,380 gustatory sensilla (sensilla chaetica B) on the antenna, and each antenna of adult males house 2,360 gustatory sensilla (Ramaswamy and Gupta, 1981). The numbers and topologies of gustatory sensilla in the maxillary and labial palps are largely unknown. Behavioral assays with cockroaches whose taste organs were systematically ablated showed that differential inputs from these four sensory appendages (Figure 2A) mediate appetitive and aversive responses (Wada-Katsumata et al., 2011). While all four sensory appendages can stimulate acceptance and rejection of tastants, the paraglossae alone represent a minimal system for further investigations. They are the last checkpoint before ingestion and the paraglossae have the highest sensitivity to phagostimulants and deterrents (Wada-Katsumata et al., 2011).

Each paraglossa contains approximately 60 gustatory sensilla with no sexual dimorphism (Wada-Katsumata et al., 2009). At least four types of GRNs are housed in each sensillum (Figure 2A). Two GRNs have ligand specificity and are denoted sweet-GRN and bitter-GRN. The other two GRNs have no ligand specificity and are involved in sensing osmolality. Positive correlations among feeding responses, GRN chemosensation and the concentration of tastants suggest that chemosensation of the sweet-GRNs contributes to appetitive feeding responses to nutrient sugars such as glucose, fructose, sucrose, maltose, maltotriose, and trehalose. Bitter-GRNs contribute to aversive feeding responses to noxious substances such as caffeine (WadaKatsumata et al., 2013).

Glucose-aversion could result from heritable changes in the processing of chemosensory cues in the peripheral sensilla or in the CNS. Comparative electrophysiological analyses of GRN sensitivities to various tastants in homozygous WT, homozygous GA (Silverman and Bieman, 1993), backcrosses of
WT and GA, and two field-collected GA populations revealed that polymorphisms in GRN sensitivity drove glucose-aversion (Wada-Katsumata et al., 2013). Tip recordings from gustatory sensilla (Hodgson et al., 1995; Wada-Katsumata et al., 2009) revealed that in both WT and GA cockroaches phagostimulants (e.g., fructose) stimulated a sweet-GRN and guided appetitive behavior, whereas deterrents (e.g., caffeine) stimulated a bitterGRN and drove aversive behavior (Figure 2B; Wada-Katsumata et al., 2013). Glucose, like fructose, also simulated the sweetGRN in WT cockroaches, but in GA cockroaches, glucose stimulated both sweet- and bitter-GRNs. Three key features of the GRN responses are that: (a) the bitter-GRN responds to glucose in a concentration-dependent manner, (b) the electrophysiological responses of the sweet-GRN to glucose are greatly attenuated in GA cockroaches, and (c) the bitter-GRN response correlates with aversive behavior. This relationship between stimuli, electrophysiological responses, and behavioral responses suggests that the bitter-GRN acquired sensitivity to glucose, and this change is responsible for glucose-driven aversions.

These results suggest two major hypotheses, although others are possible as well. Mutations caused: (a) structural modification(s) of GRs in the bitter-GRN that enable detection of glucose, and/or (b) misexpression of native glucose GRs in the bitter-GRN (Figure 2C). Structure-activity studies using glucose-derivatives revealed that in WT cockroaches glucose and methyl $\alpha$-D-glucose stimulated the sweet-GRNs and appetitive feeding behavior. Methyl $\beta$-D-glucose did not stimulate either sweet- or bitter-GRNs and did not elicit a feeding response (Figure 2D; Wada-Katsumata et al., 2013). This indicates that sweet-GRNs of WT cockroaches have binding sites for glucose and methyl $\alpha$-D-glucose, and that both sweet- and bitter-GRNs have no binding sites for methyl $\beta$-D-glucose. On the other hand, in GA cockroaches, both glucose and its two methyl-derivatives stimulated the bitter-GRN and induced aversive feeding responses. In other words, bitter-GRNs of GA cockroaches have binding sites for methyl $\beta$-D-glucose and mediate aversive feeding behaviors to this compound. These results tentatively support the hypothesis that the glucose-sensitive GRs of the bitter-GRNs of GA cockroaches are differently tuned from the native glucose GRs on the sweet-GRNs. It is possible, however, that the ectopic expression of sugar GRs on the bitter GRNs was accompanied by modifications in their ligand affinities.

Our results show that a gain-of-function adaptation has emerged in the peripheral gustatory system. Namely, recognition of glucose by receptors on bitter-GRNs specifies glucose as a bitter tastant, changing its valence (taste quality) from sweet to bitter, and causing a novel adaptive behavior to emerge which protects the cockroach from the lethal effects of glucosecontaining toxic baits. Moreover, the aversion to glucose is further amplified by a pre-existing intrasensillar inhibition of sweet-GRN responses by deterrents. The gain-of-function of glucose receptors in bitter GRNs fits with the dominant nature of the genetics of this trait. Thus, glucose-aversion is a compelling example of a chemosensory-based adaptation that conferred 
A

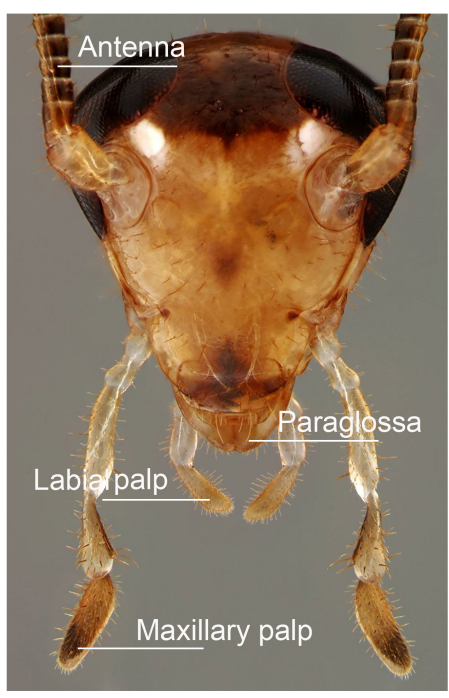

B

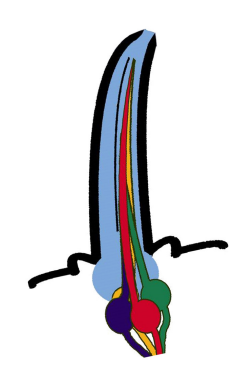

Tastant specificity

GRN1 Sugar

GRN2 Bitter

No tastant specificity

GRN3

GRN4

\begin{tabular}{|c|c|c|c|c|}
\hline \multirow{2}{*}{ Tastant } & \multicolumn{2}{|c|}{ Feeding behavior } & \multicolumn{2}{|c|}{ GRN response } \\
\hline & WT & GA & WT & GA \\
\hline $0.25 \mathrm{NaCl}$ & No effect & No effect & - & $\bullet$ \\
\hline D-fructose & Accept & Accept & - $\bullet$ & - ० \\
\hline Maltose & Accept & Accept & - $\bullet$ & - 00 \\
\hline Trehalose & Accept & Accept & - $\bullet$ & - 00 \\
\hline Sucrose & Accept & Accept & - $\bullet$ & - $\circ$ \\
\hline 3-o-methyl-D-glucose & Reject & Reject & $\bullet \bullet ০$ & $\bullet \bullet ০$ \\
\hline Caffeine & Reject & Reject & $\bullet \bullet ০$ & $\circ \bullet$ \\
\hline D-glucose & Accept & Reject & - 0 & 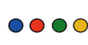 \\
\hline
\end{tabular}

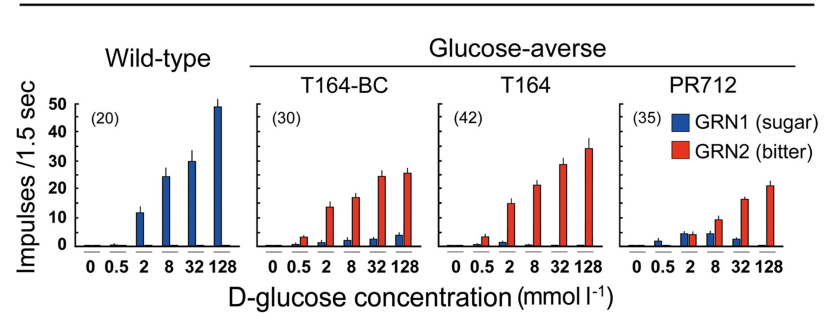

C

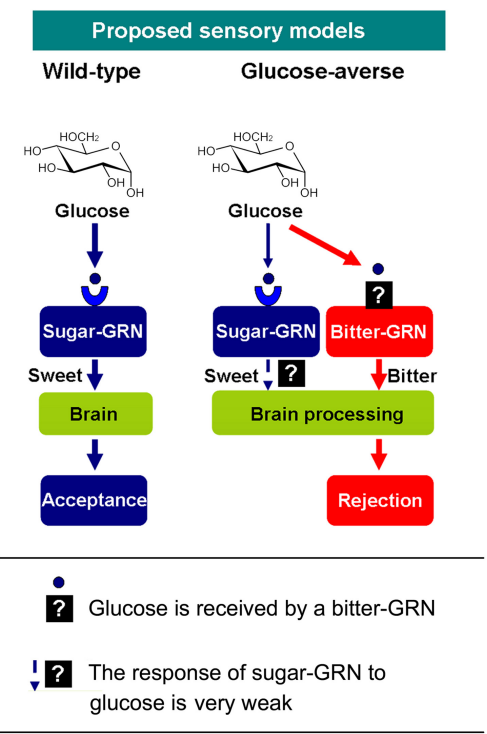

D

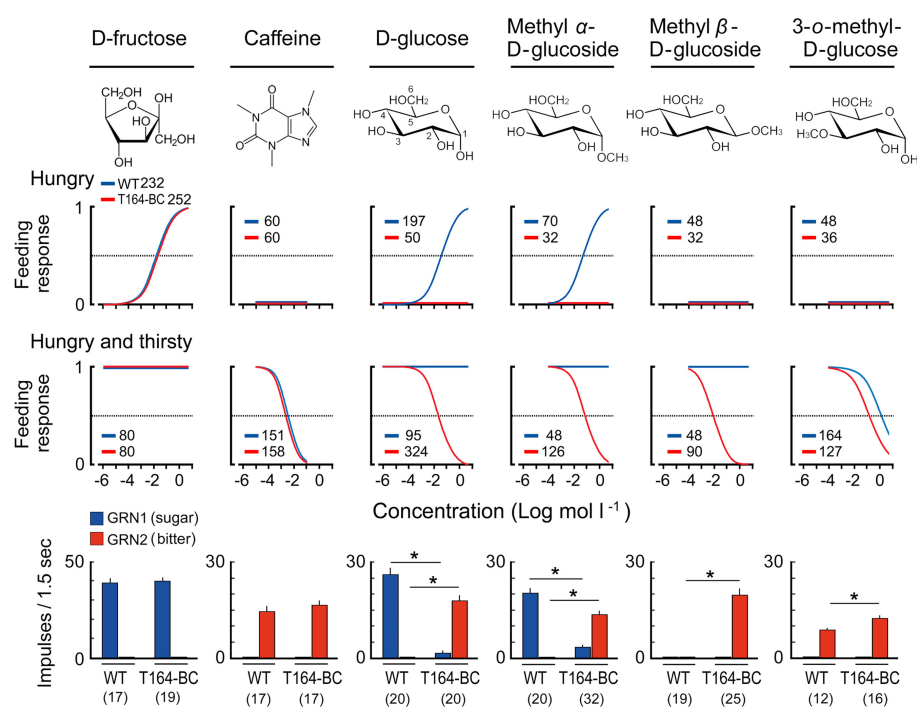

FIGURE 2 | Gustatory neural mechanism of glucose-aversion (figures are adapted from Wada-Katsumata et al., 2013, with permission from The American Association for the Advancement of Science). (A) Head of male German cockroach, Blattella germanica, showing the four sensory appendages (antennae, maxillary palps, labial palps, paraglossae) and schematic of a sensillum containing four gustatory receptor neurons (GRNs). (B) Summary of behavioral and GRN sensitivities of wild-type (WT) and GA (T164-Backcross) cockroaches to various tastants, and dose-GRN responses to glucose in a WT and three GA cockroach populations (T164-backcross with WT, T164, field-collected PR712 strain). Bitter-GRNs of GA cockroaches respond to glucose, whereas in WT cockroaches the bitter-GRN never responds to glucose. (C) Proposed model of glucose-aversion in the German cockroach. Glucose-aversion could be encoded by changes in glucose detection of bitter-GRNs of the paraglossa. Feeding responses in animals can be altered by genetic polymorphisms in gustatory receptors (GRs) over a finite range from highly sensitive to completely insensitive to a particular tastant. However, in bait-selected cockroach populations, the modal specificity of glucose, rather than sensitivity to glucose, has been strikingly transformed from "sweet" and highly phagostimulatory to "bitter" and highly deterrent. And these changes occurred at the sensory level. Generally, insect bitter-GRNs co-express a large number of gustatory receptors (GRs) and are broadly tuned to detect various deterrents. The co-expression of GRs accounts for the unique sensitivity of bitter-GRNs and their capacity to selectively respond to deterrents. Our studies suggest two major hypotheses: (a) Modifications of the structure of GRs on the bitter-GRN cause acceptance of glucose; (b) The mis-expression of native glucose GRs on the bitter-GRN result in glucose acceptance. Recruiting glucose as a bitter-GRN ligand expresses glucose-aversion as a novel adaptive behavior that offers protection against toxic baits. (D) Structure-activity experiments. Top, Chemical structures of phagostimulants and detterents. Middle, Dose-feeding responses of WT (blue), and GA (red, T164-Backcross) cockroaches. Hungry, cockroaches were motivated to accept phagostimulants but not water; Hungry and thirsty, cockroaches were motivated to take both phagostimulants and water. Feeding response is shown as the proportion of cockroaches ingesting the test solution. Legends indicate sample size. GA cockroaches rejected all tested compouds except fructose. Bottom, The sugar and bitter-GRNs of WT and GA cockroaches respond differentially to six compounds. Number in parentheses indicates tested sensilla. ( ${ }^{*} P<0.05$, Student's $t$-test). 
behavioral resistance to anthropogenic selection, protecting the German cockroach from insecticides.

\section{MOLECULAR MECHANISMS OF GLUCOSE-AVERSION: WORK IN PROGRESS}

Genomic and bioinformatic analyses of GR organization in holometabolous insects, including Diptera (Clyne et al., 2000; Scott et al., 2001; Hill et al., 2002; Robertson et al., 2003; Kent et al., 2008), Coleoptera (McKenna et al., 2016), Lepidoptera (Guo et al., 2017), and Hymenoptera (Robertson and Wanner, 2006) characterized 68 putatively functional GRs in D. melanogaster, 76 in the African malaria mosquito (Anopheles gambiae), 91 in the yellow fever mosquito (Aedes aegypti), 222 in the red flour beetle (Tribolium castaneum), 234 in the Asian longhorned beetle (Anoplophora glabripennis), 76 in the silkmoth (Bombyx mori), 5 in the fig wasp (Ceratosolen solmsi), and 12 in the European honey bee (Apis mellifera). The Drosophila GRs are functionally categorized for $\mathrm{CO}_{2}$ detection, sugar and amino acid detection (sweet), noxious substance detection (bitter) and pheromone detection. These patterns also suggest that diet specialists with narrow host ranges have few GRs, whereas generalist herbivores and omnivores evolved more diverse GRs. Indeed, the GR organization of omnivorous cockroaches support this pattern: the American cockroach Periplaneta americana and the German cockroach genomes encode 522 and 545 putatively functional GRs, respectively (Harrison et al., 2018; Li et al., 2018; Robertson et al., 2018). These GRs fall into the general clades of bitter receptors, sugar receptors and $\mathrm{CO}_{2}$ receptors, but most of the GRs are likely involved in the detection of bitter tastants. The German cockroach has 14 sugar GR candidates (BgerGr1-14). BgerGr431 is a divergent gene and a relative of the fructose receptor DmelGr43a lineage. It is present in all neopteran insects examined, but not in the dampwood termite (Zootermopsis nevadensis) (Terrapon et al., 2014; Robertson et al., 2018). Our ongoing functional analysis of the GRs of the German cockroach is a first step toward understanding the molecular mechanisms of glucose-aversion. Although, there is the awareness that GRN response to tastants could be supported by not only GRs, but also by other chemosensory proteins, such as IRs, OBPs, PPKs, and TRP channels, functional analysis using RNAi knockdown of candidate sugar receptor genes of WT and GA cockroaches will demonstrate if the misexpression of sugar receptor genes in bitter-GRNs of GA cockroaches mediates glucose-aversion.

\section{GLUCOSE-AVERSION MODIFIES FORAGING, MATING AND POPULATION DYNAMICS}

Foraging of GA cockroaches is impacted by associative olfactory learning. While WT cockroaches learn to associate bait odors with reinforcement from glucose, GA cockroaches associate the bait odors with punishment from glucose and promptly learn to avoid the bait (Wada-Katsumata et al., 2016). In a learning paradigm, a complex chocolate food odor was innately preferred over the complex odor of vanilla by both WT and GA cockroaches, but GA cockroaches quickly learned to associate chocolate odor with glucose as punishment and subsequently avoided chocolate while foraging. The avoidance response was retained for 3 days after only $1 \mathrm{~h}$ of self-training. On the other hand, odors paired with glucose positively reinforced the food preferences of WT cockroaches. Associative learning and memory thus amplify the adaptive response to glucose-containing toxic baits and exacerbate pest control efforts. As well, however, GA cockroaches learn to avoid glucose-containing non-toxic foods, lowering their overall fitness in a bait-free environment.

Glucose-aversion can drive other behavioral polymorphisms in German cockroach populations, even in bait-free natural environments. The population dynamics of WT and GA is significantly impacted by a difference in mate-choice of WT and GA females (Jensen et al., 2017). There are no differences in nymph development and female fecundity between GA and WT cockroaches provisioned with glucose-free food. However, although WT and GA males do not preferentially court GA or WT females, WT females tend to accept WT males more than GA males. The GA females, on the other hand, mate equally with WT and GA males. A potential explanation for this assortative mating by females is that gustatory preferences affect courtship behavior. During courtship, the female mounts the male and evaluates his quality by tasting a nuptial secretion that he offers on his tergum (Gemeno and Schal, 2004; WadaKatsumata et al., 2009); the secretion contains sugars, including maltose and maltotriose (Kugimiya et al., 2003), which stimulate the female's sweet-GRNs (Wada-Katsumata et al., 2009). This nuptial gift evolved under sexual selection to lure the female and position her for copulation. The differential mating success observed in this study suggests that WT and GA males have evolved nuptial secretion components that suit the gustatory preferences of the respective females in their population. Our model is that as WT males increase the amount of glucoserelated sugars in their nuptial secretion in response to WT female preferences, nuptial feeding by GA females on WT males is interrupted by the taste of glucose. This hypothesis, that the reversed modal specificity of glucose causes GA females to sense the nuptial gift components as deterrents, impeding the completion of the courtship sequence, will be tested by comparative chemical analysis of the nuptial secretions of WT and GA males.

The difference in mate preferences also impacts the demography of populations initiated with equal numbers of WT and GA females and males. When provisioned with glucose-containing food, the proportion of WT cockroaches increased over 12 months because of high mortality and delayed nymph development of GA cockroaches (Jensen et al., 2017). But even when provisioned with rodent chow or chow supplemented with fructose, the proportion of WT cockroaches increased gradually. These results suggest that population growth in GA cockroaches is slower than in WT populations, partly because of assortative mating preferences. 


\section{CONCLUSION}

Glucose-aversion is expressed as a reversal in the modal quality of glucose from sweet and appetitive to bitter and aversive. This gain-of-function change results from a modification of the peripheral gustatory system wherein glucose stimulates not only sweet-GRNs, but also bitter-GRNs. Glucose-aversion can profoundly affect food choice and other traits that are under sexual selection, resulting in changes in sexual communication, mate choice and population dynamics. Under natural conditions, this trait is maladaptive and probably maintained in heterozygotes as a low frequency gustatory polymorphism. However, under the strong anthropogenic selection of insecticide- and glucose-containing baits, this trait is highly adaptive and supports behavioral resistance to insecticidal products. The glucose-aversion trait of the German cockroach

\section{REFERENCES}

Clyne, P. J., Warr, C. G., and Carlson, J. R. (2000). Candidate taste receptors in Drosophila. Science 287, 1830-1834. doi: 10.1126/science.287.5459.1830

Freeman, E. G., and Dahanukar, A. (2015). Molecular neurobiology of Drosophila taste. Curr. Opin. Neurobiol. 34, 140-148. doi: 10.1016/j.conb.2015.06.001

Gemeno, C., and Schal, C. (2004). Sex pheromones of cockroaches. in Advances in Insect Chemical Ecology eds R. T. Cardé and J. Millar (New York, NY: Cambridge University Press), 179-247.

Gould, F., Brown, Z. S., and Kuzma, J. (2018). Wicked evolution: can we address the sociobiological dilemma of pesticide resistance? Science 360, 728-732. doi: 10.1126/science.aar3780

Guo, H., Cheng, T., Chen, Z., Jiang, L., Guo, Y., Liu, J., et al. (2017). Expression map of a complete set of gustatory receptor genes in chemosensory organs of Bombyx mori. Insect Biochem. Mol. Biol. 82, 74-82. doi: 10.1016/j.ibmb.2017. 02.001

Harrison, M. C., Jongepier, E., Robertson, H. M., Arning, N., Bitard-Feildel, T., Chao, H., et al. (2018). Hemimetabolous genomes reveal molecular basis of termite eusociality. Nat. Ecol. Evol. 2, 557-566. doi: 10.1038/s41559-0170459-1

Hill, C. A., Fox, A. N., Pitts, R. J., Kent, L. B., Tan, P. L., Chrystal, M. A., et al. (2002). $\mathrm{G}$ protein-coupled receptors in Anopheles gambiae. Science 298, 176-178. doi: 10.1126/science.1076196

Hodgson, E. E., Lettvin, J. Y., and Roeder, K. D. (1995). Physiology of a primary chemoreceptor unit. Science 122, 417-418. doi: 10.1126/science.122.3166.417-a

Jensen, K., Wada-Katsumata, A., Schal, C., and Silverman, J. (2017). Persistence of a sugar-rejecting cockroach genotype under various dietary regimes. Sci. Rep. 7:46361. doi: 10.1038/srep46361

Kent, L. B., Walden, K. K., and Robertson, H. M. (2008). The Gr family of candidate gustatory and olfactory receptors in the yellow-fever mosquito Aedes aegypti. Chem. Senses 33, 79-93. doi: 10.1093/chemse/bjm067

Kugimiya, S., Nishida, R., Sakuma, M., and Kuwahara, Y. (2003). Nutritional phagostimulants function as male courtship pheromone in the German cockroach. Blattella germanica. Chemoecology 13, 169-175. doi: 10.1007/ s00049-003-0245-1

Li, S., Zhu, S., Jia, Q., Yuan, D., Ren, C., Li, K., et al. (2018). The genomic and functional landscapes of developmental plasticity in the American cockroach. Nat. Commun. 9:1008. doi: 10.1038/s41467-018-03281-1

McKenna, D. D., Scully, E. D., Pauchet, Y., Hoover, K., Kirsch, R., Geib, S. M., et al. (2016). Genome of the Asian longhorned beetle (Anoplophora glabripennis), a globally significant invasive species, reveals key functional and evolutionary innovations at the beetle-plant interface. Genome Biol. 17:227. doi: 10.1186/ s13059-016-1088-8

Montell, C. (2009). A taste of the Drosophila gustatory receptors. Curr. Opin. Neurobiol. 19, 345-353. doi: 10.1016/j.conb.2009.07.001

Newland, P. L., Cobb, M., and Marion-Poll, F. (2009). Insect Taste. New York, NY: Taylor \& Francis Group. is a remarkable example of how the evolution of a single altered gustatory trait under anthropogenic selection can shape the foraging ecology, sexual communication and population dynamics of populations.

\section{AUTHOR CONTRIBUTIONS}

\author{
AW-K, HMR, JS, and CS wrote the manuscript.
}

\section{FUNDING}

This study was supported by the United States National Science Foundation (IOS-1557864) and the Blanton J. Whitmire Endowment at North Carolina State University.

Ramaswamy, S. B., and Gupta, P. (1981). Sensilla of the antennae and the labial and maxillary palps of Blattella germanica (L.) (Dictyoptera: Blattellidae): their classification and distribution. J. Morphol. 168, 269-279. doi: 10.1002/jmor. 1051680303

Robertson, H. M., Baits, R. L., Walden, K. K. O., Wada-Katsumata, A., and Schal, C. (2018). Enormous expansion of the chemosensory gene repertoire in the omnivorous German cockroach Blattella germanica. J. Exp. Zool. B Mol. Dev. Evol doi: 10.1002/jez.b.22797 [Epub ahead of print].

Robertson, H. M., and Wanner, K. W. (2006). The chemoreceptor superfamily in the honey bee, Apis mellifera: expansion of the odorant, but not gustatory, receptor family. Genome Res. 16, 1395-1403. doi: 10.1101/gr.5057506

Robertson, H. M., Warr, C. G., and Carlson, J. R. (2003). Molecular evolution of the insect chemoreceptor gene superfamily in Drosophila melanogaster. Proc. Natl. Acad. Sci. U.S.A. 100, 14537-14542. doi: 10.1073/pnas.2335847100

Schal, C. (2011). "Cockroaches," in Handbook of Pest Control, ed. D. Moreland (Cleveland, OH: Mallis Handbook), 150-291.

Scott, K. (2018). Gustatory processing in Drosophila melanogaster. Annu. Rev. Entomol. 63, 15-30. doi: 10.1146/annurev-ento-020117-043331

Scott, K., Brady, R. Jr., Cravchik, A., Morozov, P., Rzhetsky, A., Zuker, C., et al. (2001). A chemosensory gene family encoding candidate gustatory and olfactory receptors in Drosophila. Cell 104, 661-673. doi: 10.1016/S00928674(01)00263-X

Silverman, J. (1995). Effects of glucose-supplemented diets on food intake, nymphal development, and fecundity of glucose-averse, non-glucose-averse, and heterozygous strains of the German cockroach, Blattella germanica. Entomol. Exp. Appl. 76, 7-14. doi: 10.1111/j.1570-7458.1995.tb01941.x

Silverman, J., and Bieman, D. N. (1993). Glucose aversion in the German cockroach. Blattella germanica. J. Insect Physiol. 39, 925-933. doi: 10.1016/00221910(93)90002-9

Silverman, J., and Liang, D. (1999). Effect of fipronil on bait formulation-based aversion in the German cockroach (Dictyoptera: Blattellidae). J. Econ. Entomol. 92, 886-889. doi: 10.1093/jee/92.4.886

Terrapon, N., Li, C., Robertson, H. M., Ji, L., Meng, X., Booth, W., et al. (2014). Molecular traces of alternative social organization in a termite genome. Nat. Commun. 5:3636. doi: 10.1038/ncomms4636

Wada-Katsumata, A., Ozaki, M., Yokohari, F., Nishikawa, M., and Nishida, R. (2009). Behavioral and electrophysiological studies on the sexually biased synergism between oligosaccharides and phospholipids in gustatory perception of nuptial secretion by the German cockroach. J. Insect Physiol. 55, 742-750. doi: 10.1016/j.jinsphys.2009.04.014

Wada-Katsumata, A., Silverman, J., and Schal, C. (2011). Differential inputs from chemosensory appendages mediate feeding responses to glucose in wild-type and glucose-averse German cockroaches. Blattella germanica. Chem. Senses 36, 589-600. doi: 10.1093/chemse/bjr023

Wada-Katsumata, A., Silverman, J., and Schal, C. (2013). Changes in taste neurons support the emergence of an adaptive behavior in cockroaches. Science 340, 972-975. doi: 10.1126/science.1234854 
Wada-Katsumata, A., Silverman, J., and Schal, C. (2014). Sugar aversion: a newly-acquired adaptive change in gustatory receptor neurons in the German cockroach. Comp. Physiol. Biochem. 31, 220-230. doi: 10.3330/hikakuseiriseika. 31.220

Wada-Katsumata, A., Silverman, J., and Schal, C. (2016). Sugar-aversion: polymorphism of the peripheral gustatory system drives adaptive foraging behavior in the German cockroach. Chem. Senses 41:E117. doi: 10.1093/chemse/ bjw091

Wang, C., Scharf, M. E., and Bennett, G. W. (2004). Behavioral and physiological resistance of the German cockroach to gel baits (Blattodea: Blattellidae). J. Econ. Entomol. 97, 2067-2072. doi: 10.1093/jee/97.6.2067
Conflict of Interest Statement: The authors declare that the research was conducted in the absence of any commercial or financial relationships that could be construed as a potential conflict of interest.

Copyright (c) 2018 Wada-Katsumata, Robertson, Silverman and Schal. This is an open-access article distributed under the terms of the Creative Commons Attribution License (CC BY). The use, distribution or reproduction in other forums is permitted, provided the original author(s) and the copyright owner(s) are credited and that the original publication in this journal is cited, in accordance with accepted academic practice. No use, distribution or reproduction is permitted which does not comply with these terms. 


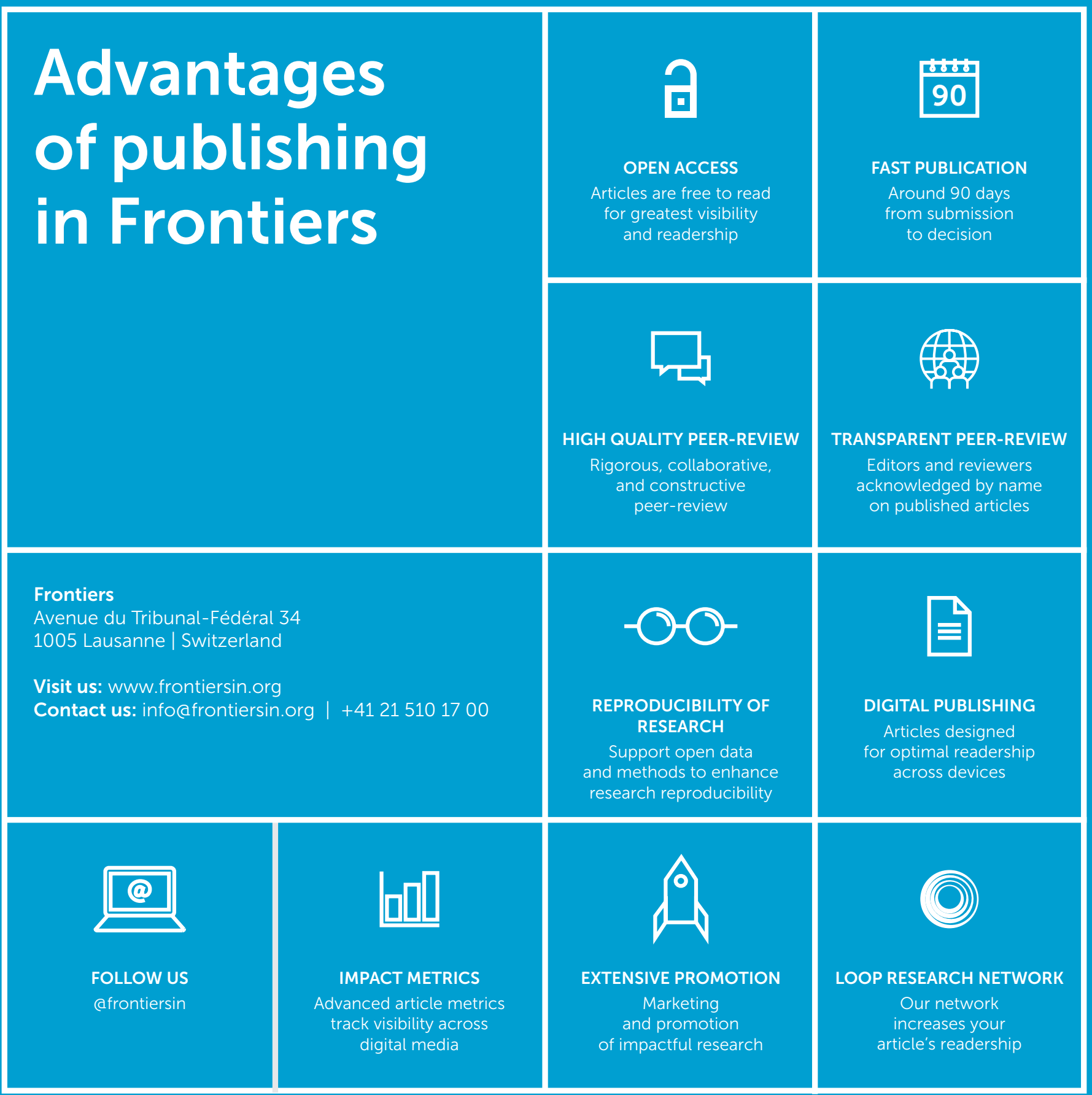

